Jimmy Jair Cabra García

Revisão e análise filogenética do gênero Glenognatha Simon, 1887 (Araneae, Tetragnathidae)

Revision and phylogenetic analysis of the spider genus Glenognatha Simon, 1887

(Araneae, Tetragnathidae) 
Jimmy Jair Cabra García

Revisão e análise filogenética do gênero

Glenognatha Simon, 1887 (Araneae,

Tetragnathidae)

\section{Revision and phylogenetic analysis of the spider genus Glenognatha Simon, 1887}

(Araneae, Tetragnathidae)

Dissertação apresentada ao Instituto de Biociências da Universidade de São Paulo, para a obtenção de Título de Mestre em Ciências Biológicas, na Área de Zoologia.

Orientador(a): Antonio D. Brescovit

São Paulo 


\section{Ficha Catalográfica}

Cabra García, Jimmy Jair

Revisão e análise filogenética do gênero Glenognatha Simon, 1887 (Araneae, Tetragnathidae). 400p.

Dissertação (Mestrado) - Instituto de Biociências da Universidade de São Paulo. Departamento de Zoologia.

1. Aranhas 2. Taxonomia 3.

Sistemática I. Universidade de São Paulo. Instituto de Biociências. Departamento de Zoologia.

\section{Comissão Julgadora:}

Prof. Dr.

Prof. Dr.

Prof. Dr. Antonio D. Brescovit

Orientador 


\section{DISCLAIMER}

New taxon names and nomenclatural changes referred to this dissertation are disclaimed and unavailable for nomenclatural purposes (ICZN Art. 8.3) 
To my parents, Jaime and Patriciat, my aunt Fanny, my grandmother Mery, my brothers Alex and Jhon, Alexandra and my beloved Viviana for their unconditional love and support. 


\section{Acknowledgments}

First, I would like to express my immense gratitude to Dr. Antonio D. Brescovit for his advice, patience, support and encouragement since the first time I contacted him.

I am grateful to my lab mates Yuji Lemos, André Marsola Giroti, João L. Chavari, Victor Passanha, Hector Gonzalez-Filho, Amanda Coronato, Rafael Prezzi Indicatti and Alexander Sánchez-Ruiz for the many rich discussions on systematics and morphology, and for their valuable friendship and support. Special thanks to Yuji and André for his generosity and intelligent advice and to João for his spontaneity and continuous encouragement.

I am specially thankful to Dr. Gustavo Hormiga for receiving me at the George Washington University and sharing with me ideas, advice, literature and encouragement. Thanks to Ligia Benavides, Jesus Ballesteros, Thiago Moreira, Daniela Andriamalala and Bob Kallal for their patient and help at Hormiga Lab. I deeply thank Ligia for hosting me in Washington and for her help and support.

I am sincerely grateful to Dr. Fernando Álvarez-Padilla and Dr. Dimitar Dimitrov for discussion on Glenognatha characters and encouragement. Dr. Dimitrov also kindly share unpublished SEM images of his own research. Special thanks go to Dr. Martin Ramírez, Cristian Grismado and Matías Izquierdo for sharing with me ideas and morphology knowledge in my visit to Argentina. I deeply thank Lina Almeida for her advice in the SEM mounting techniques and Dra. Cristina A. Rheims for her advice in drawing techniques. Thanks to Flávio Yamamoto and Rafael Prezzi Indicatti for sharing their excellent Glenognatha photographs.

I would like to give special thanks to Nancy Lo Man Hung and David Candiani for hosting me in my visit to Porto Alegre and for their help and support. I am sincerely grateful to Cristiano Sampaio and Tarciana Barbosa for their friendship and support.

I also would like to thank the following curators, staff and museums for the loan of specimens: N. I. Platnick (AMNH), C. Griswold (CAS), F. Zhang (HU), D. Battesti 
(IBSP), E. Flórez (ICN), C. L. Scioscia, M. Ramírez, C. Grismado and M. Izquierdo (MACN), E. H. Buckup, Maria Aparecida Marques and R. Ott (MCN), A. A. Lise (MCTP), L. Leibensperger and G. Giribet (MCZ), C. Rollard (MNHN), A. B. Kury and Carla Barros (MNRJ), A. B. Bonaldo (MPEG), R. Jocqué (MRAC), D. Silva (MUSM), R. Pinto da Rocha (MZSP), L. Baert (RBINS), L. Q. Matias (UFCE), A. Santos (UFMG), J. Coddington and Dana DeRoche (USNM) and J. Dunlop (ZMB).

Special thanks to J. Mendez and D. Rodríguez for their valuable help during fieldwork. I also would like to thank Jairo Moreno and Nicolas Hazzi for providing fresh material and natural history data.

I am grateful to my friends in Colombia Julian, Javier, Leonel, Carim, Maria, Luz Angela and Laura for their unconditional support and friendship.

Finally, I am immensely grateful to my father, aunt, grandmother, brothers and Alexandra for their unconditional love, confidence, support and encouragement. I could not have ended this dissertation without them. Also, I specially would like to thank Viviana for her infinite love, her patience and constant support.

Funding for this research have been provided by a FAPESP magister grant (processo No. 2011/14253-2), a FAPESP-BEPE grant (processo No. 2012/16412-3), a FAPESP thematic-project grant (processo No. 2011/50689-0) and an Ernst Mayr travel grant (Museum of Comparative Zoology, Harvard University). 


\section{Table of contents}

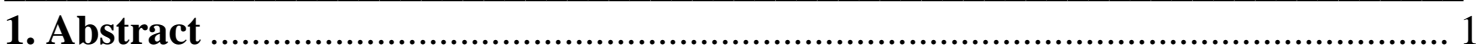

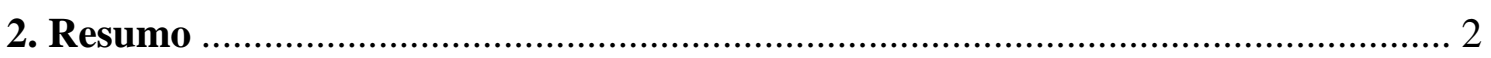

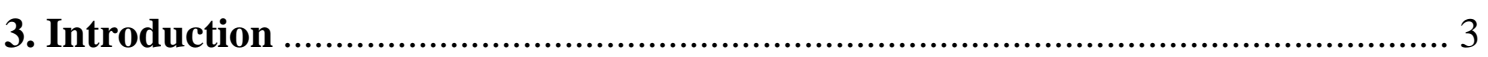

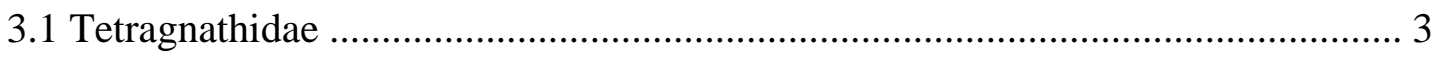

3.2 Taxonomic history of the genus Glenognatha …............................................... 4

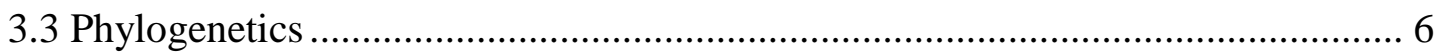

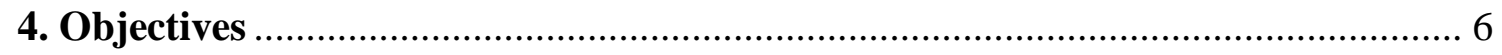

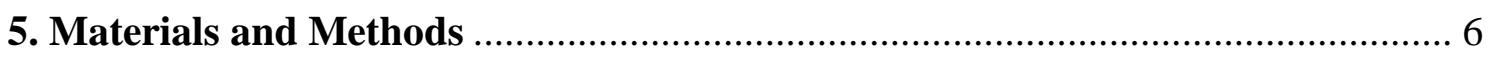

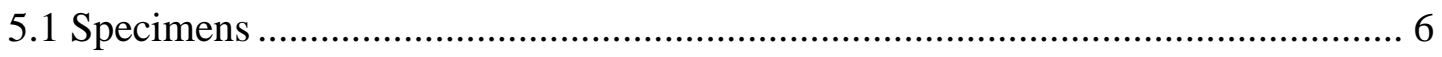

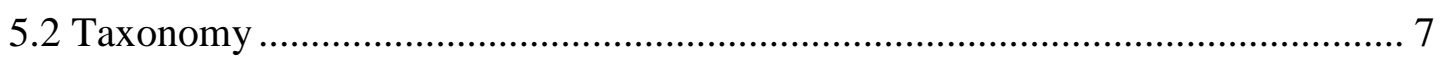

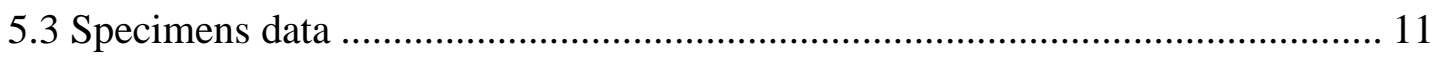

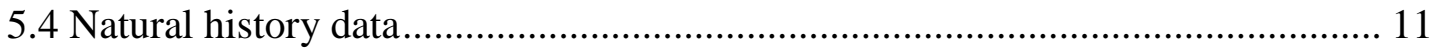

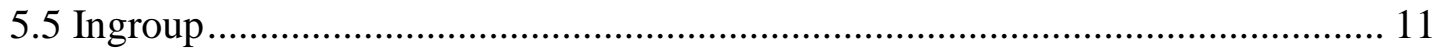

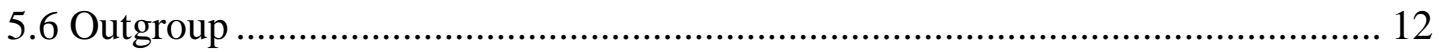

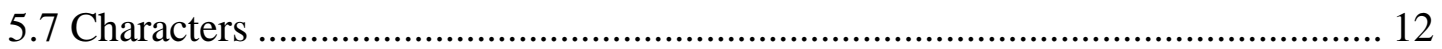

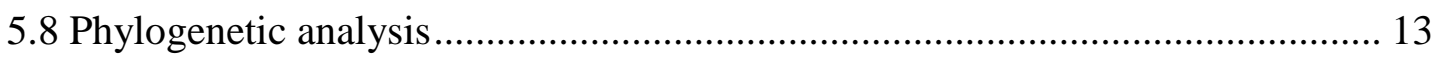

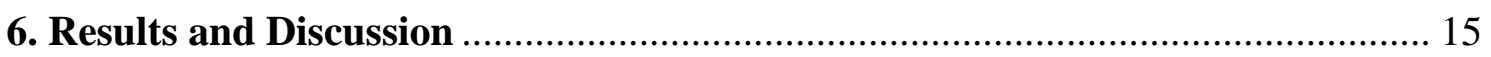

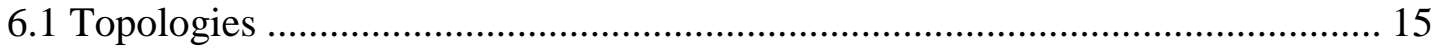

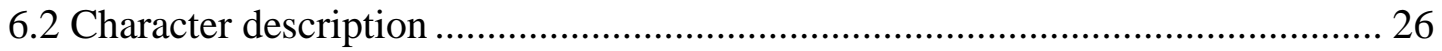

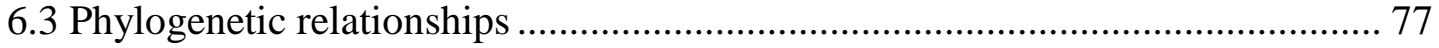

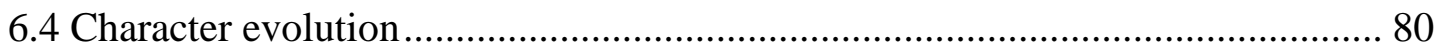

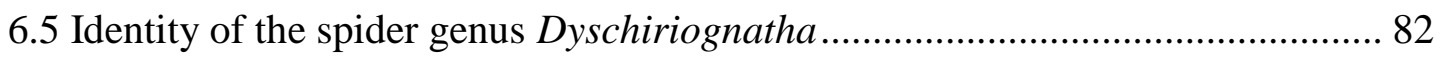

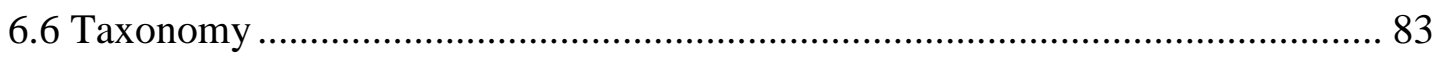

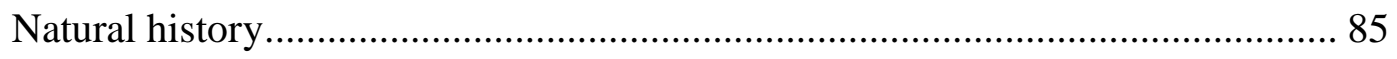

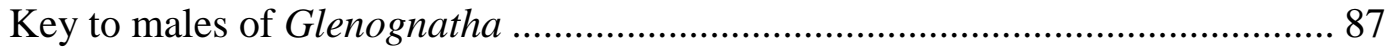

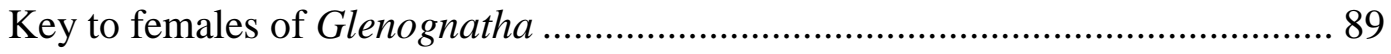

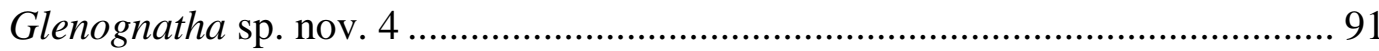

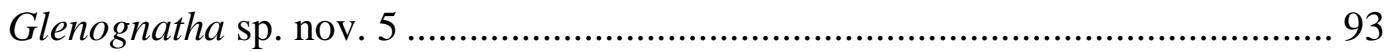

Glenognatha lacteovittata (Mello-Leitão, 1944) .............................................. 95

Glenognatha tangi (Zhu, Song \& Zhang, 2003) new combination ................... 100

Glenognatha argyrostilba (O. P.-Cambridge, 1876) new combination ............. 101

Glenognatha dentata (Zhu \& Wen, 1978) new combination ............................ 104 


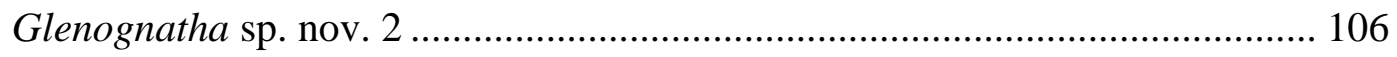

Glenognatha gaujoni Simon, 1895 .............................................................. 108

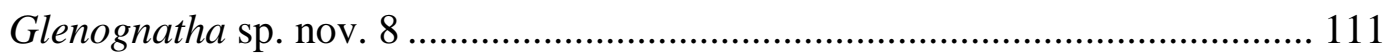

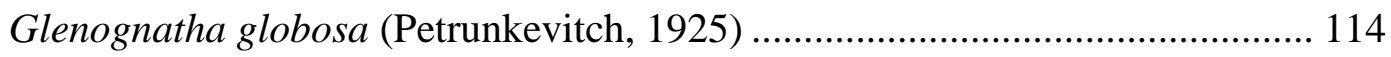

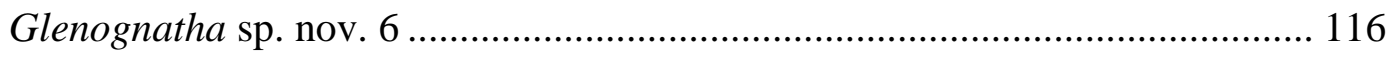

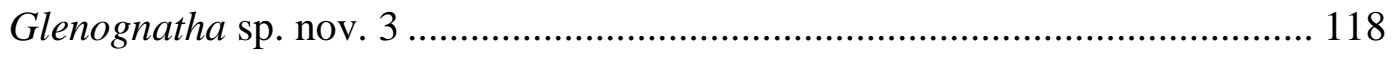

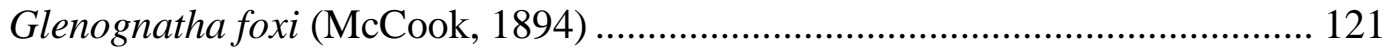

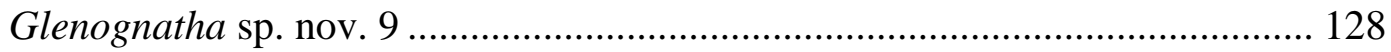

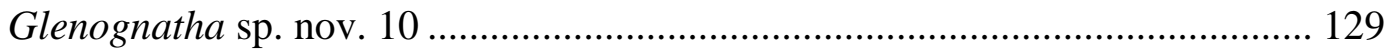

Glenognatha smilodon Bosmans \& Bosselaers, 1994 ..................................... 130

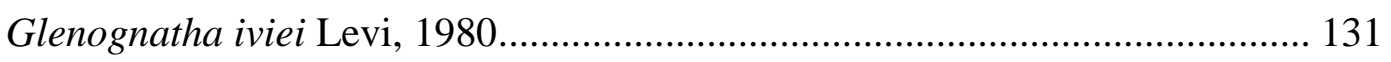

Glenognatha heleios Hormiga, 1990 ........................................................... 132

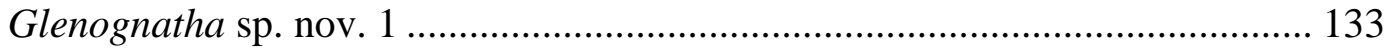

Glenognatha hirsutissima (Berland, 1935) ..................................................... 136

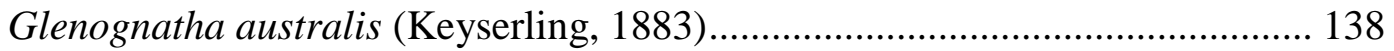

Glenognatha spherella Chamberlin \& Ivie, 1936............................................. 142

Glenognatha gloriae (Petrunkevitch, 1930) ................................................... 145

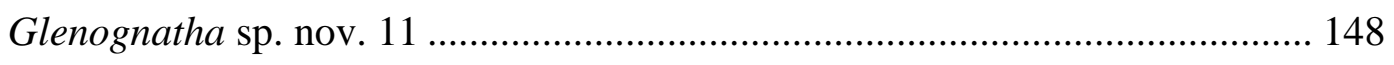

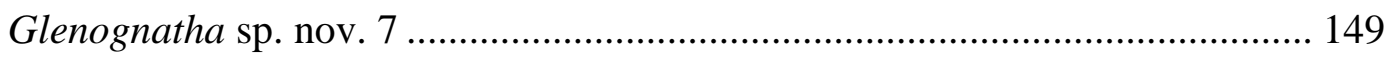

Glenognatha minuta Banks, 1898.............................................................. 151

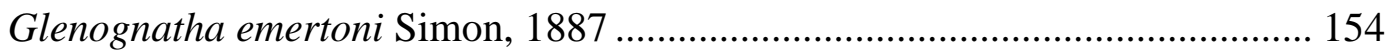

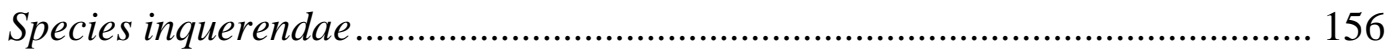

Glenognatha caporiaccoi Platnick, 1993........................................................... 156

Glenognatha argenteoguttata (Berland, 1935) .............................................. 156

Glenognatha chamberlini (Berland, 1942) ................................................... 156

Glenognatha nigromaculata (Berland, 1933) ................................................ 156

Glenognatha phalangiops (Berland, 1942) .................................................... 157

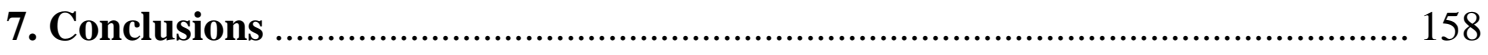

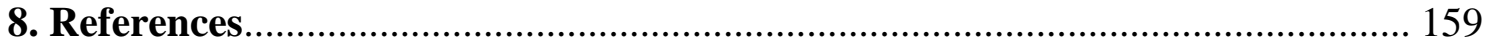

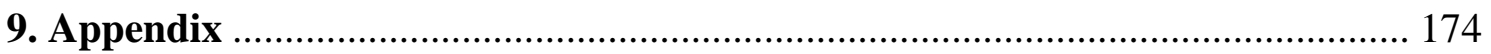

9.1 List of specimens examined for character scoring........................................ 174

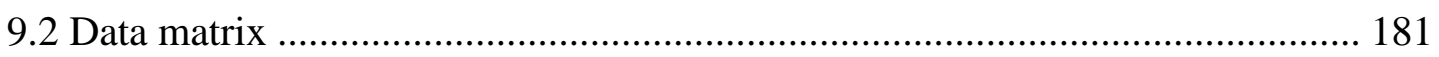




\section{List of Figures}

Figure 1. A, topological terms used in cheliceral descriptions. B, female chelicerae of Glenognatha gaujoni indicating terminology for teeth. At: anterior tooth, CFO: cheliceral fang outgrowth, EA: ectal apophysis, Prt: promarginal tooth, Ret:

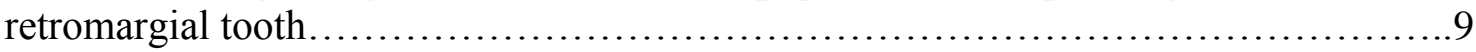

Figure 2. Strict consensus of 92 most parsimonious trees found under equal weights analyzing the complete matrix ( $\mathrm{L}=187, \mathrm{CI}=0.49, \mathrm{RI}=0.62)$. Numbers above branches are Jackknife percentages (left) and Symmetric resampling GC values (right). Numbers below branches are Bremer support values. .16

Figure 3. Strict consensus of eight most parsimonious trees found under equal weights analyzing the reduced matrix $(\mathrm{L}=173, \mathrm{CI}=0.52, \mathrm{RI}=0.64)$. Numbers above branches are Jackknife percentages (left) and Symmetric resampling GC values (right). Numbers below branches are Bremer support values. 17

Figure 4. Strict consensus of the most parsimonious trees obtained analyzing the complete matrix under implied weighting. A, under $k=1$ ( $\mathrm{L}=165, \mathrm{CI}=0.55, \mathrm{RI}=0.70)$, B, under $k=2(\mathrm{~L}=170, \mathrm{CI}=0.54, \mathrm{RI}=0.68)$.

Figure 5. Strict consensus of the most parsimonious trees obtained analyzing the complete matrix under implied weighting. $\mathbf{A}$, under $k=3-7 \quad(\mathrm{~L}=162, \mathrm{CI}=0.56$,

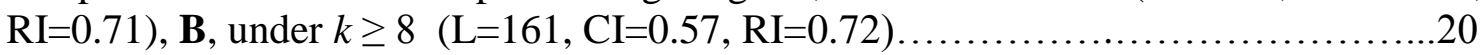

Figure 6. Strict consensus of the most parsimonious trees obtained analyzing the reduced matrix under implied weighting. A, under $k=1-2(\mathrm{~L}=162, \mathrm{CI}=0.55, \mathrm{RI}=0.69)$, B, Under $k=3(\mathrm{~L}=158, \mathrm{CI}=0.57, \mathrm{RI}=0.71), \mathrm{C}$, under $k \geq 4(\mathrm{~L}=154, \mathrm{CI}=0.58, \mathrm{RI}=0.72)$

Figure 7. Topology obtained analyzing the complete matrix under $k=8$. Numbers above branches are Jackknife percentages (left) and Symmetric resampling GC values (right). Numbers below branches are Bremer support values in units of fit (left) and

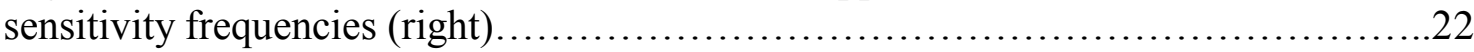

Figure 8. Topology obtained analyzing the complete matrix under $k=8$. Filled and open circles represent nonhomoplasious and homoplasious transformations, respectively. Character numbers are placed over the branches and the states are shown below the branches........................................................23-25

Figure 9. Glenognatha gaujoni. Female posterior median spinnerets (arrow,

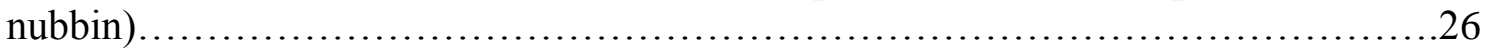

Figure 10. Glenognatha gaujoni. Female posterior lateral spinnerets (arrow, mesal

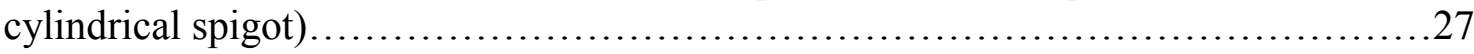

Figure 11. Female posterior median spinnerets. A, Glenognatha lacteovittata. B, Glenognatha gaujoni (arrow, aciniform spigots).... 
Figure 12. Glenognatha australis. Female posterior lateral spinnerets. AG: aggregate

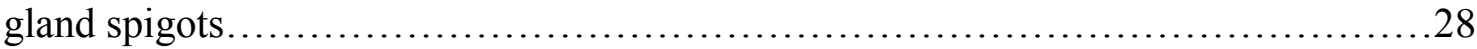

Figure 13. Glenognatha emertoni. Female posterior lateral spinnerets. FL: flagelliform

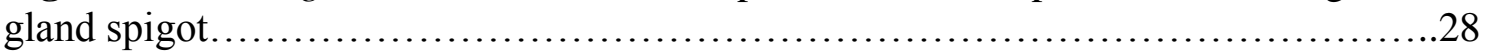

Figure 14. Female posterior lateral spinnerets. A, Glenognatha lacteovittata. B,

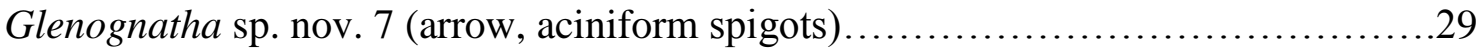

Figure 15. Epiandrous fusules. A, Glenognatha sp. nov. 5. B, Dyschiriognatha dentata................................................................... 30

Figure 16. Glenognatha sp. nov. 2. Epiandrous fusules. ........................... 30

Figure 17. Glenognatha globosa. Epiandrous fusules................................ 31

Figure 18. Glenognatha gaujoni. Epiandrous fusules.............................. 31

Figure 19. Glenognatha globosa. Epiandrous fusules (arrow, posterior edge)..........32

Figure 20. Glenognatha sp. nov. 5. Male left palp (arrow, cymbium constriction)......32

Figure 21. Glenognatha globosa. Male left palp. CB: cymbium...................33

Figure 22. Glenognatha sp. nov. 8. Male left palp (arrow, paracymbium)..............34

Figure 23. Character 16 states. The box plot shows median (squares), 50\% (box limits) and minimum and maximum (brackets) of all values. PDP: paracymbium distal portion.

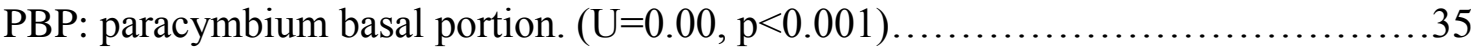

Figure 24. Paracymbium. A, Glenognatha lacteovittata. B, Glenognatha sp. nov. 6. PDP: Paracymbium distal portion. PBP: paracymbium basal portion. .35

Figure 25. Paracymbium. A, Glenognatha sp. nov. 7. B, Glenognatha heleios (arrow,

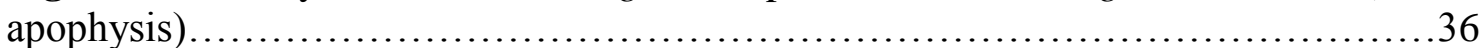

Figure 26. Glenognatha gaujoni. Male left palp (arrow, paracymbium setae)..........37

Figure 27. Glenognatha sp. nov. 4. Embolus and conductor. E: embolus. C:

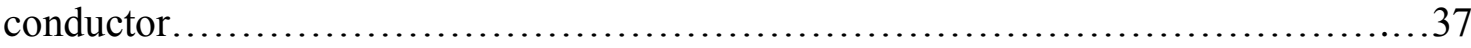

Figure 28. Glenognatha sp. nov. 3. Embolus and conductor. CRa: conductor retrolateral apophysis. 38

Figure 29. Glenognatha lacteovittata. Conductor. CRa: conductor retrolateral

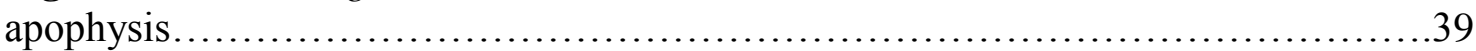

Figure 30. Embolus and conductor. A, Glenognatha sp. nov. 1. B, Glenognatha sp. nov. 6. C. Dyschiriognatha dentata. E: embolus. C: conductor. 
Figure 31. Embolus and conductor. A, Glenognatha sp. nov. 2. B, Dyschiriognatha dentata. E: embolus. C: conductor. .40

Figure 32. Conductor. A, Glenognatha sp. nov. 2. B, Glenognatha australis (arrow, retrolateral projection). E: embolus. C: conductor.

Figure 33. Conductor. A, Glenognatha sp. nov. 4. B, Glenognatha lacteovittata (arrow, prolateral fold)....

Figure 34. Embolus and conductor. A, Glenognatha sp. nov. 3. B, Glenognatha sp. nov. 8. E: embolus. C: conductor.

Figure 35. Embolus and conductor. A, Glenognatha sp. nov. 2. B, Glenognatha sp. nov. 1. E: embolus. C: conductor.

Figure 36. Embolus. A, Glenognatha sp. nov. 4. B, Glenognatha spherella. C. Glenognatha lacteovittata. E: embolus. C: conductor

Figure 37. A, Glenognatha minuta. B, Glenognatha sp. nov. 2 (arrow, projection beyond embolic opening). E: embolus. C: conductor.

Figure 38. Embolus. A, Glenognatha sp. nov. 2. B, Glenognatha gaujoni (arrow, scalelike projections). C, Glenognatha lacteovittata (tooth-like and filiform projections). 44

Figure 39. Glenognatha sp. nov. 1. Male left palp (arrow, embolic median

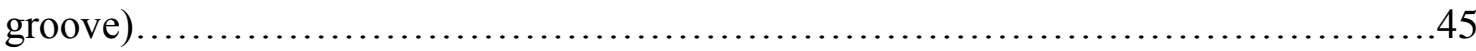

Figure 40. Male left palp. A, Glenognatha sp. nov. 5. B, Glenognatha sp. nov. 3 (arrow, embolic median groove) ........................................... 46

Figure 41. Glenognatha smilodon. Embolus (arrow, laminar projection).............46

Figure 42. Glenognatha sp. nov. 1. Male left palp.............................47

Figure 43. Character 36 states. The box plot shows median (squares), 50\% (box limits) and minimum and maximum (brackets) of all values, $(U=0.00, p<0.001) \ldots \ldots \ldots \ldots . . .48$

Figure 44. A, Glenognatha foxi. B, Glenognatha gaujoni...........................48

Figure 45. Glenognatha gaujoni. A, Gonopore. B, Body, ventral view (arrow,

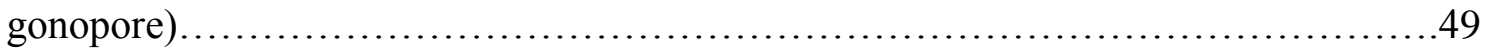

Figure 46. Internal genitalia. A, Glenognatha lacteovittata. B, Glenognatha gloriae. S: Spermathecae................................................................. 50

Figure 47. Glenognatha minuta. Internal genitalia. S: spermathecae. UE: uterus externus. 
Figure 48. Glenognatha foxi. Spermathecae. S: spermathecae. CD: copulatory duct. ...

Figure 49. Glenognatha minuta. Spermathecae (arrow, enlarged gland base). LSD: long-stem ducts.

Figure 50. Glenognatha heleios. Spermathecae and copulatory duct. CD: copulatory ducts. LSD: long-stem ducts. S: spermathecae.

Figure 51. Glenognatha argyrostilba. Internal genitalia. A, Dorsal view. B, Lateral view. S: spermathecae. UEs: uterus externus sac. .53

Figure 52. Uterus externus lateral view. A, Glenognatha argyrostilba. B, Glenognatha sp. nov. 2 (arrow, median projection). C, Glenognatha sp. nov. 1 (arrows, chambers). S: spermathecae.

Figure 53. Uterus externus distal chamber. A, Glenognatha sp. nov. 1 (arrow, distal chamber). B, Glenognatha sp. nov. 8 (arrow, distal chamber). S: spermathecae.........54

Figure 54. Dyschiriognatha dentata. Internal genitalia (arrows, uterus externus projections). .55

Figure 55. Glenognatha sp. nov. 1. Cephalothorax lateral view (arrow, lateral eyes) .56

Figure 56. Male cephalothorax, frontal view. A, Glenognatha globosa. B, Glenognatha hirsutissima. .56

Figure 57. Female cephalothorax, lateral view. A, Dyschiriognatha dentata (arrow,

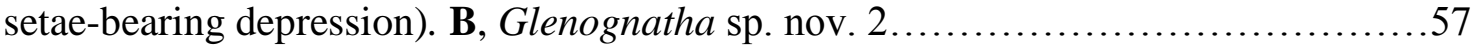

Figure 58. Female cephalothorax, lateral view. A, Glenognatha sp. nov. 2. B, Glenognatha argyrostilba (arrows, irregular depressions).

Figure 59. Female sternum A, Glenognatha sp. nov. 1. B, Glenognatha

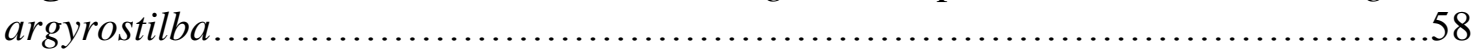

Figure 60. Female sternum A, Glenognatha lacteovittata. B, Glenognatha sp. nov. 8 (arrow, sternum projection)................................................58

Figure 61. Female chelicerae, lateral view. A, Glenognatha gaujoni. B, Dyschiriognatha dentata (arrow, setae bearing tubercles) .........................59

Figure 62. Male cephalothorax, lateral view. A, Glenognatha spherella. B,

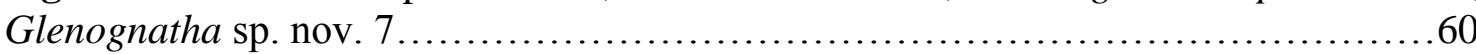

Figure 63. Male left chelicerae, anterior view. A, Glenognatha sp. nov. 7. B, Glenognatha foxi. AT: anterior tooth. 
Figure 64. Male left chelicerae, anterior view. A, Glenognatha heleios. B, Glenognatha sp. nov. 6. AT: anterior tooth.

Figure 65. Male left chelicerae, anterior view. A, Glenognatha sp. nov. 11. B, Glenognatha gaujoni. CFO: cheliceral fang outgrowth...........................62

Figure 66. Male left chelicerae, anterior view. A, Glenognatha sp. nov. 8. B, Glenognatha hirsutissima (arrow, macrosetae)...................................62

Figure 67. Male left chelicerae, anterior view. A, Glenognatha globosa. B, Glenognatha emertoni (arrow, anteromarginal projection)........................63

Figure 68. Male left chelicerae, anterior view. A, Glenognatha spherella. B, Glenognatha sp. nov. 3. prt1: promarginal tooth 1 .................................64

Figure 69. Male left chelicerae, anterior view. A, Glenognatha minuta. B, Glenognatha

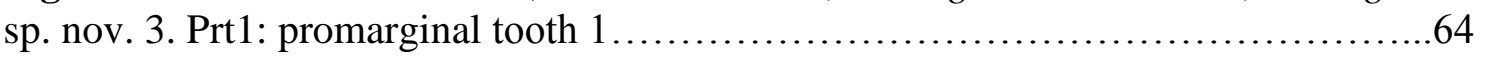

Figure 70. Character 63 states. The box plot shows median (squares), 50\% (box limits) and minimum and maximum (brackets) of all values, $(\mathrm{U}=0.00, \mathrm{p}<0.001)$. Ret1:

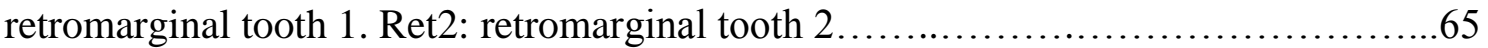

Figure 71. Male left chelicerae, posterior view. A, Glenognatha sp. nov. 6. B, Glenognatha emertoni. Ret1: retromarginal tooth 1. Ret2: retromarginal tooth 2......65

Figure 72. Male left chelicerae, posterior view. A, Glenognatha gaujoni. B,

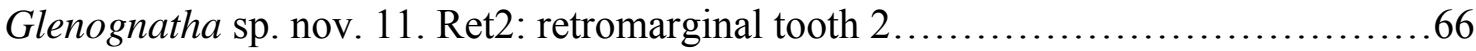

Figure 73. Character 65 states. The box plot shows median (squares), 50\% (box limits) and minimum and maximum (brackets) of all values, $(\mathrm{U}=0.00, \mathrm{p}<0.001)$. Ret1: retromarginal tooth 1. Ret3: retromarginal tooth 3

Figure 74. Male left chelicerae, posterior view. A, Glenognatha sp. nov. 3. B, Glenognatha sp. nov. 7. Ret1: retromarginal tooth 1. Ret3: retromarginal tooth 3 .

Figure 75. Male left chelicerae, mesal view. A, Glenognatha heleios. B, Glenognatha australis. Ret1: retromarginal tooth 1. Ret3: retromarginal tooth 3.

Figure 76. Male left chelicerae, anterior view. A, Glenognatha sp. nov. 3. B, Glenognatha argyrostilba. Prt1: promarginal tooth 1. Prt2: promarginal tooth 2. Prt3: promarginal tooth 3 .

Figure 77. Female left chelicerae, anterior view. A, Glenognatha sp. nov. 6. B, Glenognatha gaujoni. AT: anterior tooth.

Figure 78. Female left chelicerae, mesal view. A, Glenognatha sp. nov. 7. B, Glenognatha gaujoni. CFO: cheliceral fang outgrowth............................... 70 
Figure 79. Female left chelicerae, anterior view. A, Glenognatha globosa B, Glenognatha gaujoni. EA: ectal apophysis................................... 71

Figure 80. Female left chelicerae, mesal view. A, Glenognatha sp. nov. 6. B, G. emertoni (arrow, anterior bulge).............................................. 71

Figure 81. Female left chelicerae, anterior view. A, Glenognatha sp. nov. 11. B, Glenognatha emertoni (arrow, anteromarginal projection) ......................... 72

Figure 82. Female left chelicerae, mesal view. A, Glenognatha sp. nov. 6. B, Glenognatha sp. nov. 4 (arrows, posterior bulges)................................ 73

Figure 83. Glenognatha lacteovittata. Female left chelicerae, posterior view (arrows, cheliceral bulges) ............................................................. 73

Figure 84. Female left chelicerae, anterior view. A, Glenognatha sp. nov. 8. B, Glenognatha sp. nov. 7 (arrow, Prt1 base swollen). Prt1: promarginal tooth 1.........74

Figure 85. Female left chelicerae, mesal view. A, Glenognatha minuta. B, Glenognatha globosa. Ret: retromarginal teeth............................................ 74

Figure 86. Femur IV. A, Glenognatha tangi. B, Glenognatha sp. nov. 8. (arrows,

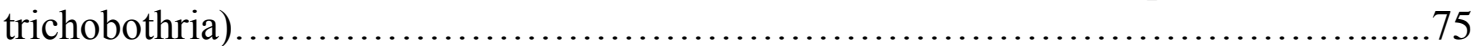

Figure 87. Glenognatha tangi. Femur IV (arrow, trichobothria)...................... 75

Figure 88. Glenognatha hirsutissima. Female ventral view (arrows, femur

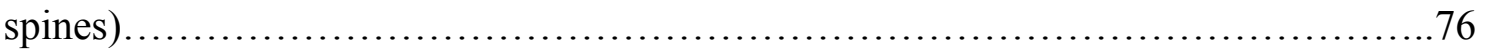

Figure 89. Glenognatha sp. nov. 1. Female abdomen cuticle.......................76

Figure 90. Glenognatha emertoni. Female tracheal system. MT: median trunks (arrow, lateral projection). LT: lateral trunks............................................. 77

Figure 91. Habitats of Glenognatha species. A, Glenognatha sp. nov. 2 and Glenognatha sp. nov. 8, Colombia, Valle del Cauca. B-C. Glenognatha gaujoni. B, Brazil, Rondônia. C. Peru, Loreto. Photos 2B-C by Rafael Prezzi Indicatti.............184

Figure 92. Webs of Glenognatha sp. nov. 2 from Colombia, Valle del Cauca.........184

Figure 93. Webs of Glenognatha sp. nov. 8. A-B, Colombia, Valle del Cauca. C, Colombia, Nariño. Photo $2 \mathrm{C}$ by Nicolas Hazzi................................ 185

Figure 94. Webs and habitus of Glenognatha gaujoni. A-B, Brazil, Rondônia. C-D, Peru, Loreto. Photos 4A-B by Flávio Yamamoto, 4C-D by Rafael Prezzi Indicatti.................................................................. 186

Figure 95. Glenognatha sp. nov. 4 from Brazil (MZSP 42538). Male habitus. A, dorsal. B, lateral. C, ventral. D, frontal. Scale bars, $200 \mu \mathrm{m}$. TS: tracheal spiracle... 187 
Figure 96. Glenognatha sp. nov. 4 from Brazil (MZSP 42538). Female habitus. A, dorsal. B, lateral (arrow, cheliceral bulge). C, ventral. D, frontal. Scale bars, $200 \mu \mathrm{m}$. TS: tracheal spiracle.

Figure 97. Glenognatha sp. nov. 4 from Brazil. A-C, Male left chelicerae (MZSP 42538). A, anterior. B, posterior. C, mesal. D-F, Female left chelicerae (MZSP 39047). D, anterior. E, posterior. F, mesal (arrow, cheliceral bulge). Scale bars, $100 \mu \mathrm{m}$. CFO: cheliceral fang outgrowth. Ret: retromarginal tooth

Figure 98. Glenognatha sp. nov. 4 from Brazil (MZSP 42538). Male left palp. A, ventral. B, dorsal. C, prolateral. D, retrolateral. Scale bars, $100 \mu \mathrm{m}$. C: conductor. CB: cymbium. CRa: conductor retrolateral apophysis. E: embolus. P: paracymbium. T: tegulum. TO: tarsal organ. 189

Figure 99. Glenognatha sp. nov. 4 from Brazil (MZSP 42538). Male left palp, schematic. A, ventral. B, conductor ventral. C, conductor dorsal. D, embolus ventral. Scale bars $100 \mu \mathrm{m}$. C: conductor. CB: cymbium. CRa: conductor retrolateral apophysis. E: embolus. ED: ejaculatory duct. P: paracymbium. T: tegulum

Figure 100. Glenognatha sp. nov. 4 from Brazil. Female genitalia. A, dorsal. B, lateral. Scale bars, $20 \mathrm{~mm}$. UEc: uterus externus chamber. UEs: uterus externus sac..........190

Figure 101. Glenognatha sp. nov. 4 from Brazil. Female tracheal system. A, dorsal. B, median trunk. C, lateral trunk. D, tracheal spiracle posterior view. Scale bars, $100 \mu \mathrm{m}$ (A), $10 \mu \mathrm{m}$ (B-D). LT: lateral tracheae. MT: median trunks. TAG: tracheal atrium gland.

Figure 102. Glenognatha sp. nov. 4 from Brazil. Male left palp. A, ventral. B, embolus and conductor ventral. C, embolus and conductor dorsal. Scale bars $100 \mu \mathrm{m}(\mathrm{A}), 50 \mu \mathrm{m}$ (B, C). C: conductor. CB: cymbium. CRa: conductor retrolateral apophysis. E: embolus. EMg: embolic medial groove. P: paracymbium. T: tegulum

Figure 103. Glenognatha sp. nov. 5 from Colombia (ICN 1973). Male habitus. A, dorsal. B, lateral. C, ventral. D, frontal. Scale bars, $200 \mu \mathrm{m}$. TS: tracheal spiracle.....192

Figure 104. Glenognatha sp. nov. 5 from Colombia (ICN 1973). Female habitus. A, dorsal. B, lateral. C, ventral. D, frontal. Scale bars, $200 \mu \mathrm{m}$. TS: tracheal spiracle.....192

Figure 105. Glenognatha sp. nov. 5 from Colombia (ICN 2141). A-C, Male left chelicerae. A, anterior. B, posterior. $\mathbf{C}$, mesal. D-F, Female left chelicerae. D, anterior. $\mathbf{E}$, posterior. F, mesal (arrow, cheliceral bulge). Scale bars, $100 \mu \mathrm{m}$. CFO: cheliceral fang outgrowth. Ret: retromarginal tooth.... .193

Figure 106. Glenognatha sp. nov. 5 from Colombia (ICN 1973). Male left palp. A, ventral. B, dorsal. C, prolateral. D, retrolateral. Scale bars, $100 \mu \mathrm{m}$. C: conductor. CB: cymbium. CRa: conductor retrolateral apophysis. E: embolus. P: paracymbium. T: tegulum. TO: tarsal organ. 
Figure 107. Glenognatha sp. nov. 5 from Colombia (Paratype ICN 2141). Male left palp, schematic. A, ventral. B, conductor ventral. C, conductor dorsal. D, embolus ventral. Scale bars $100 \mu \mathrm{m}$. C: conductor. CB: cymbium. CRa: conductor retrolateral apophysis. E: embolus. ED: ejaculatory duct. T: tegulum. 195

Figure 108. Glenognatha sp. nov. 5 from Colombia. Female genitalia. A, dorsal. B, lateral. $\mathbf{C}$, anterior D, uterus externus cuticle. E, posterior. Scale bars, $100 \mu \mathrm{m}$ (A-D), $20 \mu \mathrm{m}$ (E). UEc: uterus externus chamber. UEs: uterus externus sac. 196

Figure 109. Glenognatha sp. nov. 5 from Colombia. Female tracheal system. A, dorsal. $\mathbf{B}$, median and lateral trunks. $\mathbf{C}$, median trunk distal. D, tracheal spiracle posterior view. Scale bars, $100 \mu \mathrm{m}$ (A-C), $20 \mu \mathrm{m}$ (D). LT: lateral tracheae. MT: median trunks. TAG: tracheal atrium gland.

Figure 110. Glenognatha sp. nov. 5 from Colombia. Male left palp. A, ventral. B, embolus and conductor. C, embolus and conductor tip. D, paracymbium. Scale bars $200 \mu \mathrm{m}$ (A), $100 \mu \mathrm{m}$ (B, D) $20 \mu \mathrm{m}$ (C). C: conductor. CRa: conductor retrolateral apophysis. E: embolus. EMg: embolic medial groove. P: paracymbium. PBp: paracymbium basal portion. T: tegulum.

Figure 111. Glenognatha lacteovittata from Brazil (IBSP 141433). Male habitus. A, dorsal. B, lateral. C, ventral. D, frontal. Scale bars, $200 \mu \mathrm{m}$. TS: tracheal spiracle. 198

Figure 112. Glenognatha lacteovittata from Brazil (IBSP 163680). Female habitus. A, dorsal. B, lateral. C, ventral. D, frontal. Scale bars, $200 \mu \mathrm{m}$. TS: tracheal spiracle 198

Figure 113. Glenognatha lacteovittata from Uruguay (IBSP 44084). A-C, Male left chelicerae. A, anterior. B, posterior. C, mesal. D-F, Female left chelicerae. D, anterior. E, posterior (arrows, cheliceral bulges). F, mesal. Scale bars, $100 \mu \mathrm{m}$. CFO: cheliceral fang outgrowth. Ret: retromarginal tooth.

Figure 114. Glenognatha lacteovittata from Uruguay (IBSP 44084). Male left palp. A, ventral. B, dorsal. C, prolateral. D, retrolateral. Scale bars, $100 \mu \mathrm{m}$. C: conductor. CRa: conductor retrolateral apophysis. E: embolus. P: paracymbium. T: tegulum. TO: tarsal organ 200

Figure 115. Glenognatha lacteovittata from Brazil (IBSP 70038). Male left palp, schematic. A, ventral. B, conductor ventral. C, conductor dorsal. D, embolus ventral. Scale bars $100 \mu \mathrm{m}$. C: conductor. CB: cymbium. CRa: conductor retrolateral apophysis. E: embolus. ED: ejaculatory duct. P: paracymbium. T: tegulum. 201

Figure 116. Glenognatha lacteovittata from Brazil. Female genitalia. A, dorsal. B, lateral. C, ventral D-E, uterus externus cuticle. Scale bars, $100 \mu \mathrm{m}$ (A-D), $10 \mu \mathrm{m}$ (E). UEc: uterus externus chamber. UEs: uterus externus sac. 
Figure 117. Glenognatha lacteovittata from Brazil. Female tracheal system. A, dorsal. B, median trunk cuticle. C, lateral trunk cuticle. D, tracheal spiracle posterior view. Scale bars, $100 \mu \mathrm{m}$ (A-B), $10 \mu \mathrm{m}$ (C-D). LT: lateral tracheae. MT: median trunks. TAG: tracheal atrium gland. .203

Figure 118. Glenognatha lacteovittata from Uruguay. A-D, conductor. A, ventral. B, prolateral. C, dorsal. D, retrolateral. E-G, embolus (arrow, tooth-like projection). H, paracymbium. Scale bars $20 \mu \mathrm{m}$ (A-D), (E-F) $30 \mu \mathrm{m}, 10 \mu \mathrm{m}(\mathrm{G}), 100 \mu \mathrm{m}(\mathrm{H})$. CRa: conductor retrolateral apophysis. EMg: embolic medial groove. PDP: paracymbium distal portion. PBp: paracymbium basal portion. .204

Figure 119. Glenognatha tangi from Myanmar (USNM). Male habitus. A, dorsal (arrow, lateral depressions). B, lateral. C, ventral (arrow, pleural bar). D, frontal. Scale bars, $200 \mu \mathrm{m}$. TS: tracheal spiracle. 205

Figure 120. Glenognatha tangi from Myanmar (USNM). Female habitus. A, dorsal (arrow, lateral depressions). B, lateral. C, ventral (arrow, pleural bar). D, frontal. Scale bars, $200 \mu \mathrm{m}$. TS: tracheal spiracle .205

Figure 121. Glenognatha tangi from Myanmar (USNM). A-C, Male left chelicerae. A, anterior. B, posterior. C, mesal. D-F, Female left chelicerae. D, anterior. E, posterior. F, mesal. Scale bars, $100 \mu \mathrm{m}$. Prt: promarginal tooth 206

Figure 122. Glenognatha tangi from Myanmar (USNM). Male left palp. A, ventral. B, dorsal. C, prolateral. D, retrolateral. Scale bars, $100 \mu \mathrm{m}$. C: conductor. CB: cymbium. CRa: conductor retrolateral apophysis. E: embolus. EMg: embolic medial groove. P: paracymbium. T: tegulum. TO: tarsal organ. 207

Figure 123. Glenognatha tangi from Myanmar (USNM). Male left palp, schematic. A, ventral. B, conductor ventral. C, conductor dorsal. D, embolus ventral. Scale bars 100 $\mu \mathrm{m}$. C: conductor. $\mathrm{CB}$ : cymbium. CRa: conductor retrolateral apophysis. E: embolus. P: paracymbium. T: tegulum. .208

Figure 124. Glenognatha tangi from Myanmar. Female genitalia. A, dorsal. B, ventral. C, lateral. Scale bars, $100 \mu \mathrm{m}$. CD: copulatory duct. UEc: uterus externus chamber. UEs: uterus externus sac. S: spermathecae. .208

Figure 125. Glenognatha tangi from Myanmar. Female cephalothorax. A, lateral. B, frontal. C, ventral. E, dorsal. D, F carapace lateral cuticle. Scale bars $100 \mu \mathrm{m}$ (A-C, E),

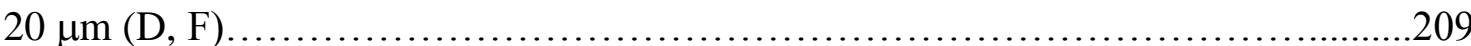

Figure 126. Glenognatha tangi from Myanmar. Male left palp. A, ventral. B, dorsal. C, embolus and conductor ventral. D, embolus and conductor dorsal. E, paracymbium. Scale bars $100 \mu \mathrm{m}$ (A-B), $10 \mu \mathrm{m}$ (C-E). C: conductor. CB: cymbium. CRa: conductor retrolateral apophysis. $\mathrm{E}$ : embolus. $\mathrm{EMg}$ : embolic medial groove. $\mathrm{P}$ : paracymbium. $\mathrm{T}$ : tegulum. 210 
Figure 127. Glenognatha argyrostilba from Nigeria (CAS). Male habitus. A, dorsal (arrow, lateral depressions). B, lateral. C, ventral (arrow, pleural bar). D, frontal. Scale bars, $200 \mu \mathrm{m}$. TS: tracheal spiracle.

Figure 128. Glenognatha argyrostilba from Nigeria (CAS). Female habitus. A, dorsal (arrow, lateral depressions). B, lateral. C, ventral (arrow, pleural bar). D, frontal. Scale bars, $200 \mu \mathrm{m}$. TS: tracheal spiracle

Figure 129. Glenognatha argyrostilba from Nigeria (CAS). A-C, Male left chelicerae. A, anterior. B, posterior. C, mesal. D-F, Female left chelicerae. D, anterior. E, posterior. F, mesal. Scale bars, $100 \mu \mathrm{m}$. Prt: promarginal tooth. CFO: cheliceral fang outgrowth.

Figure 130. Glenognatha argyrostilba from Nigeria (CAS). Male left palp. A, ventral. B, dorsal. C, prolateral. D, retrolateral. Scale bars, $100 \mu \mathrm{m}$. C: conductor. CB: cymbium. CRa: conductor retrolateral apophysis. E: embolus. EMg: embolic medial groove. P: paracymbium. T: tegulum. .213

Figure 131. Glenognatha argyrostilba from Nigeria (CAS). Male left palp, schematic. A, ventral. B, conductor ventral. C, conductor dorsal. D, embolus ventral. Scale bars $100 \mu \mathrm{m}$. C: conductor. CB: cymbium. CRa: conductor retrolateral apophysis. E: embolus. P: paracymbium. T: tegulum

Figure 132. Glenognatha argyrostilba from Nigeria. Female genitalia. A, dorsal. B, lateral. C, ventral. D-E, spermathecae and copulatory duct. Scale bars, $100 \mu \mathrm{m}$ (A, C), $20 \mu \mathrm{m}(\mathrm{B}), 10 \mu \mathrm{m}$ (D,E). CD: copulatory duct. LSD: long-stem gland ducts. UEc: uterus externus chamber. UEs: uterus externus sac. S: spermathecae.

Figure 133. Glenognatha argyrostilba from Nigeria. Female tracheal system. A, dorsal. $\mathbf{B}$, median and lateral trunks. $\mathbf{C}$, median trunk distal. D, tracheal spiracle posterior view. Scale bars, $100 \mu \mathrm{m}$ (A), $10 \mu \mathrm{m}$ (B-D). LT: lateral trachea. MT: median trunks. TAG: tracheal atrium gland...

216

Figure 134. Glenognatha argyrostilba from Ecuador (AMNH). Male habitus. A, dorsal (arrow, lateral depressions). B, lateral. C, ventral (arrow, pleural bar). D, frontal. Scale bars, $200 \mu \mathrm{m}$. TS: tracheal spiracle.

Figure 135. Glenognatha argyrostilba from Ecuador (MCZ 125476). Female habitus. A, dorsal (arrow, lateral depressions). B, lateral. C, ventral (arrow, pleural bar). D, frontal. Scale bars, $200 \mu \mathrm{m}$. TS: tracheal spiracle.

Figure 136. Glenognatha argyrostilba from Ecuador (AMNH). A-C, Male left chelicerae. A, anterior. B, posterior. C, mesal. D-F, Female left chelicerae. D, anterior. E, posterior. F, mesal. Scale bars, $100 \mu \mathrm{m}$. Prt: promarginal tooth. CFO: cheliceral fang outgrowth. Ret: retromarginal tooth. 
Figure 137. Glenognatha argyrostilba from Ecuador. Male left palp. A, ventral. B, dorsal. C, prolateral. D, retrolateral. Scale bars, $100 \mu \mathrm{m}$. C: conductor. CB: cymbium. CRa: conductor retrolateral apophysis. E: embolus. EMg: embolic medial groove. P: paracymbium. T: tegulum.

Figure 138. Glenognatha argyrostilba from Ecuador. Female genitalia. A, dorsal. B, lateral. C, ventral. D-E, spermathecae and copulatory duct. Scale bars, $100 \mu \mathrm{m}$ (A, C), $20 \mu \mathrm{m}(\mathrm{B}, \mathrm{D}), 10 \mu \mathrm{m}(\mathrm{E}) . \mathrm{CD}$ : copulatory duct. LSD: long-stem gland ducts. UEc: uterus externus chamber. UEs: uterus externus sac. S: spermathecae. .220

Figure 139. Glenognatha argyrostilba from Ecuador. Male left palp. A. retrolateral. B. ventral. Scale bars $100 \mu \mathrm{m}$. C: conductor. CB: cymbium. CRa: conductor retrolateral apophysis. E: embolus. EMg: embolic medial groove. P: paracymbium. T:

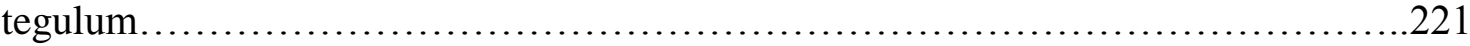

Figure 140. Glenognatha argyrostilba from Ecuador. Female cephalothorax. A, dorsal (arrow, lateral depressions). B, lateral (arrow, lateral depressions). C, ventral. Scale bars, $100 \mu \mathrm{m}$ .221

Figure 141. Glenognatha dentata from Myanmar (USNM). Male habitus. A, dorsal (arrow, lateral depressions). B, lateral. C, ventral (arrow, pleural bar). D, frontal. Scale bars, $200 \mu \mathrm{m}$. TS: tracheal spiracle 222

Figure 142. Glenognatha dentata from Myanmar (USNM). Female habitus. A, dorsal (arrow, lateral depressions). B, lateral. C, ventral (arrow, pleural bar). D, frontal. Scale bars, $200 \mu \mathrm{m}$. TS: tracheal spiracle.

222

Figure 143. Glenognatha dentata from Myanmar (USNM). A-C, Male left chelicerae. A, anterior. B, posterior. C, mesal (arrow, small tubercles). D-F, Female left chelicerae. D, anterior. E, posterior. F, mesal (arrow, small tubercles). Scale bars, $100 \mu \mathrm{m}$. CFO: cheliceral fang outgrowth. Prt: promarginal tooth.... .223

Figure 144. Glenognatha dentata from Myanmar (USNM). Male left palp. A, ventral. B, dorsal. C, prolateral. D, retrolateral. Scale bars, $100 \mu \mathrm{m}$. C: conductor. CB: cymbium. CRa: conductor retrolateral apophysis. E: embolus. EMg: embolic medial

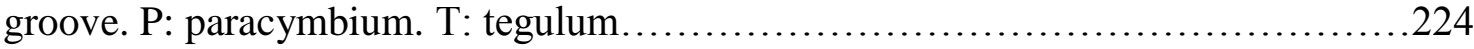

Figure 145. Glenognatha dentata from Vietnam (MCZ 84023). Male left palp, schematic. A, ventral. B, conductor ventral. C, conductor dorsal. D, embolus ventral. Scale bars $100 \mu \mathrm{m}$. C: conductor. CRa: conductor retrolateral apophysis. E: embolus. P: paracymbium. T: tegulum. .225

Figure 146. Glenognatha dentata from Myanmar. Female genitalia. A, dorsal (arrow, uterus externus chamber projection). B, lateral. C, ventral. D-E, spermathecae and copulatory duct. Scale bars, $20 \mu \mathrm{m}$ (A, C), $10 \mu \mathrm{m}$ (B), $10 \mu \mathrm{m}$ (D, E). CD: copulatory duct. LSD: long-stem gland ducts. UEc: uterus externus chamber. UEs: uterus externus

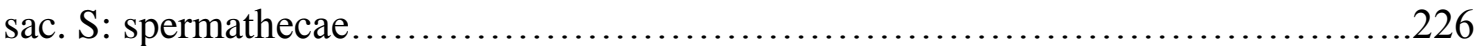


Figure 147. Glenognatha dentata from Myanmar. Female tracheal system. A, dorsal. B, median and lateral trunks. $\mathbf{C}$, median trunk cuticle. D, tracheal spiracle posterior view. Scale bars, $100 \mu \mathrm{m}$ (A), $20 \mu \mathrm{m}$ (B), $10 \mu \mathrm{m}$ (C, D). LT: lateral trachea. MT: median trunks. TAG: tracheal atrium gland.

Figure 148. Glenognatha dentata from Myanmar. Female cephalothorax. A, lateral. B, frontal. C, ventral. E, dorsal. D, F, carapace lateral cuticle. G, sternum cuticle. Scale

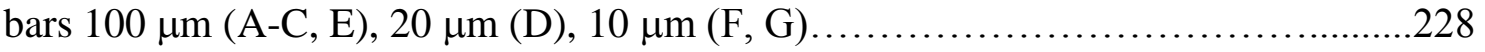

Figure 149. Glenognatha dentata from Myanmar. Male left palp. . A, ventral. B, ventroretorlateral. C, dorsoretrolateral. D, embolus and conductor ventral. E, embolus and conductor dorsal, $\mathbf{F}$, embolus and conductor apical. G, cymbium/paracymbium articulation. Scale bars, $100 \mu \mathrm{m}$ (A-C), $20 \mu \mathrm{m}$ (D-G). C: conductor. CB: cymbium. $\mathrm{CRa}$ : conductor retrolateral apophysis. E: embolus. EMg: embolic medial groove. P: paracymbium. T: tegulum.

Figure 150. Glenognatha sp. nov. 2 from Colombia (ICN 5982). Male habitus. A, dorsal. B, lateral. C, ventral. D, frontal. Scale bars, $200 \mu \mathrm{m}$. TS: tracheal spiracle. 230

Figure 151. Glenognatha sp. nov. 2 from Colombia (ICN 5982). Female habitus. A, dorsal. B, lateral. C, ventral. D, frontal. Scale bars, $200 \mu \mathrm{m}$. TS: tracheal spiracle..... .230

Figure 152. Glenognatha sp. nov. 2 from Colombia (ICN 5982). A-C, Male left chelicerae. A, anterior. B, posterior. C, mesal. D-F, Female left chelicerae. D, anterior. $\mathbf{E}$, posterior. F, mesal. Scale bars, $100 \mu \mathrm{m}$. CFO: cheliceral fang outgrowth. Ret: retromarginal tooth. .231

Figure 153. Glenognatha sp. nov. 2 from Colombia (ICN 5982). Male left palp. A, ventral. B, dorsal. C, prolateral. D, retrolateral. Scale bars, $100 \mu \mathrm{m}$. C: conductor. CB: cymbium. CRa: conductor retrolateral apophysis. E: embolus. EMg: embolic medial groove. P: paracymbium. T: tegulum. TO: tarsal organ .232

Figure 154. Glenognatha sp. nov. 2 from Colombia (ICN 5982). Male left palp, schematic. A, ventral. B, conductor ventral. C, conductor dorsal. D, embolus ventral. Scale bars $100 \mu \mathrm{m}$. C: conductor. CB: cymbium. CRa: conductor retrolateral apophysis. E: embolus. ED: ejaculatory duct. P: paracymbium. T: tegulum. TO: tarsal

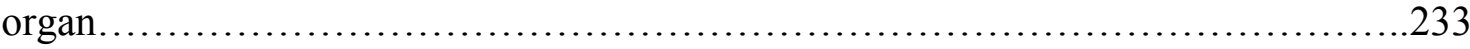

Figure 155. Glenognatha sp. nov. 2 from Colombia. Female genitalia. A, dorsal. B, lateral. C, ventral D-E, spermathecae and copulatory duct. Scale bars, $20 \mu \mathrm{m}$ (A-C), $10 \mu \mathrm{m}(\mathrm{D}, \mathrm{E})$. CD: copulatory duct. LSD: long-stem gland ducts. UEc: uterus externus chamber. UEs: uterus externus sac. S: spermathecae. .234

Figure 156. Glenognatha sp. nov. 2 from Colombia. Female tracheal system. A, dorsal. $\mathbf{B}$, median and lateral trunks. $\mathbf{C}$, median trunk distal. $\mathbf{D}$, tracheal spiracle posterior view. Scale bars, $100 \mu \mathrm{m}$ (A), $20 \mu \mathrm{m}$ (B-D). LT: lateral trachea. MT: median trunks. TAG: tracheal atrium gland. 
Figure 157. Glenognatha sp. nov. 2 from Colombia. Male left palp. A, ventral. B, retrolateral. C, conductor ventral. D, conductor dorsal. Scale bars $100 \mu \mathrm{m}(\mathrm{A}, \mathrm{B}) 10 \mu \mathrm{m}$ (C, D). C: conductor. CRa: conductor retrolateral apophysis. E: embolus. P: paracymbium. PBp: paracymbium basal portion. PDp: paracymbium distal portion. T:

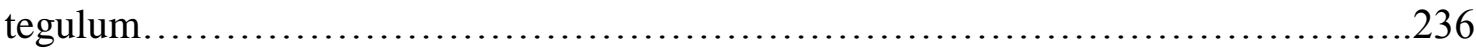

Figure 158. Glenognatha sp. nov. 2 from Colombia. Embolus. A, ventral (arrow, inner membrane). B, dorsoprolateral (arrow, inner membrane). C-D, distal. Scale bars $20 \mu \mathrm{m}$

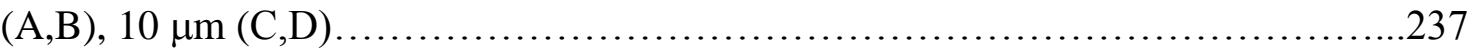

Figure 159. Glenognatha gaujoni from Brazil (IBSP 26965). Male habitus. A, dorsal. B, lateral. C, ventral (arrow, pleural bar). D, frontal. Scale bars, $500 \mu \mathrm{m}$. TS: tracheal spiracle.... .238

Figure 160. Glenognatha gaujoni from Brazil (IBSP 55545). Female habitus. A, dorsal. B, lateral. C, ventral (arrow, pleural bar). D, frontal. Scale bars, $500 \mu \mathrm{m}$. TS: tracheal spiracle. .238

Figure 161. Glenognatha gaujoni from Brazil. A-C, Male left chelicerae (IBSP 12182). A, anterior. B, posterior. C, mesal. D-F, Female left chelicerae (IBSP 12612). D, anterior. E, posterior. F, mesal. Scale bars, $500 \mu \mathrm{m}$. AT: anterior tooth. CFO: cheliceral fang outgrowth. EA: ectal apophysis. Prt: promarginal tooth. Ret: retromarginal tooth.

Figure 162. Glenognatha gaujoni from Brazil (IBSP 26965). Male left palp. A, ventral. B, dorsal. C, prolateral. D, retrolateral. Scale bars, $100 \mu \mathrm{m}$. C: conductor. CB: cymbium. CRa: conductor retrolateral apophysis. E: embolus. EMg: embolic medial groove. P: paracymbium. T: tegulum...................................... 240

Figure 163. Glenognatha gaujoni from Peru (MUSM 0504920). Male left palp, schematic. A, ventral. B, conductor ventral. C, conductor dorsal. D, embolus ventral. Scale bars $100 \mu \mathrm{m}$. C: conductor. CB: cymbium. CRa: conductor retrolateral apophysis. E: embolus. ED: ejaculatory duct. P: paracymbium. T: tegulum...................241

Figure 164. Glenognatha gaujoni from Peru. Female genitalia. A, dorsal. B, lateral. C, ventral. D-E, uterus externus cuticle. Scale bars, $100 \mu \mathrm{m}$ (A-C), $20 \mu \mathrm{m}$ (D), $10 \mu \mathrm{m}$ (E). UEc: uterus externus chamber. UEs: uterus externus sac .242

Figure 165. Glenognatha gaujoni from Brazil. Female tracheal system. A, dorsal. B-C, median and lateral trunks. D, tracheal spiracle posterior view. Scale bars, $100 \mu \mathrm{m}$ (AC), $30 \mu \mathrm{m}$ (D). LT: lateral tracheae. MT: median trunks. TAG: tracheal atrium gland....

Figure 166. Glenognatha gaujoni from Brazil. Male left palp. A, ventral. B, ventroretrolateral. $\mathbf{C}$, retrolateral. D, dorsal. $\mathbf{E}$, prolateral. $\mathbf{F}$, anterior. $\mathbf{G}$, paracymbium. Scale bars $100 \mu \mathrm{m}(\mathrm{A}-\mathrm{F}), 30 \mu \mathrm{m}(\mathrm{G})$. C: conductor. CB: cymbium. CRa: conductor retrolateral apophysis. ED: ejaculatory duct. P: paracymbium. T: tegulum. TO: tarsal organ 
Figure 167. Glenognatha gaujoni from Brazil. A-C, Embolus. A, ventroretrolateral (arrow, inner membrane). B, ventral (arrow, inner membrane). C, dorsal (arrow, scale-

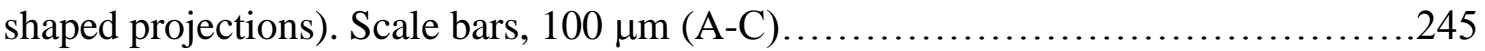

Figure 168. Glenognatha sp. nov. 8 from Colombia (ICN 5986). Male habitus. A, dorsal. B, lateral. C, ventral (arrow, pleural bar). D, frontal. Scale bars, $500 \mu \mathrm{m}$. TS: tracheal spiracle.........................................................246

Figure 169. Glenognatha sp. nov. 8 from Colombia (ICN 5986). Female habitus. A, dorsal. B, lateral. C, ventral (arrow, pleural bar). D, frontal. Scale bars, $500 \mu \mathrm{m}$. TS: tracheal spiracle ............................................................246

Figure 170. Glenognatha sp. nov. 8 from Colombia (ICN 5986). A-C, Male left chelicerae. A, anterior. B, posterior. C, mesal. D-F, Female left chelicerae. D, anterior. E, posterior. F, mesal. Scale bars, $100 \mu \mathrm{m}$. EA: ectal apophysis. Ret: retromarginal tooth.

Figure 171. Glenognatha sp. nov. 8 from Colombia (ICN 5986). Male left palp. A, ventral. B, dorsal. C, prolateral. D, retrolateral. Scale bars, $100 \mu \mathrm{m}$. C: conductor. CB: cymbium. CRa: conductor retrolateral apophysis. E: embolus. EMg: embolic medial groove. P: paracymbium. T: tegulum. TO: tarsal organ .248

Figure 172. Glenognatha sp. nov. 8 from Colombia (ICN 5986). Male left palp, schematic. A, ventral. B, conductor ventral. C, conductor dorsal. D, embolus ventral. Scale bars $100 \mu \mathrm{m}$. C: conductor. CB: cymbium. CRa: conductor retrolateral apophysis. E: embolus. ED: ejaculatory duct. P: paracymbium. T: tegulum. .249

Figure 173. Glenognatha sp. nov. 8 from Colombia. Female genitalia. A, dorsal. B, lateral. C, ventral D, uterus externus cuticle. Scale bars, $100 \mu \mathrm{m}$ (A-C), $20 \mu \mathrm{m}$ (D). UEc: uterus externus chamber. UEs: uterus externus sac. 250

Figure 174. Glenognatha sp. nov. 8 from Colombia. Female tracheal system. A, dorsal whole montage. B, dorsal. C, median and lateral trunks. D, tracheal spiracle posterior view. E, median trunk transversal cut. Scale bars, $200 \mu \mathrm{m}$ (A), $100 \mu \mathrm{m}$ (B, C), $20 \mu \mathrm{m}$ (D, E). LT: lateral tracheae. MT: median trunks. TAG: tracheal atrium gland .251

Figure 175. Glenognatha sp. nov. 8 from Colombia. Male left palp. A, ventral. B, embolus and conductor ventral. $\mathbf{C}$, embolus and conductor dorsal (arrow, inner membrane). Scale bars $200 \mu \mathrm{m}$ (A), $100 \mu \mathrm{m}$ (B, C). C: conductor. CRa: conductor retrolateral apophysis. E: embolus. EMg: embolic medial groove. P: paracymbium. T: tegulum. .252

Figure 176. Glenognatha globosa from Colombia (ICN 5991). Male habitus. A, dorsal. B, lateral. C, ventral (arrow, pleural bar). D, frontal. Scale bars, $500 \mu \mathrm{m}$. TS: tracheal

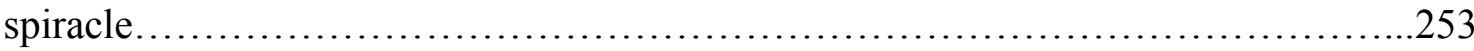

Figure 177. Glenognatha globosa from Colombia (ICN 5991). Female habitus. A, dorsal. B, lateral. C, ventral (arrow, pleural bar). D, frontal. Scale bars, $500 \mu \mathrm{m}$. TS: tracheal spiracle 
Figure 178. Glenognatha globosa from Colombia (ICN 5991). A-C, Male left chelicerae. A, anterior. B, posterior. C, mesal. D-F, Female left chelicerae. D, anterior. $\mathbf{E}$, posterior. F, mesal. Scale bars, $200 \mu \mathrm{m}$. AT: anterior tooth. Ret: retromarginal tooth

Figure 179. Glenognatha globosa from Colombia (ICN 5991). Male left palp. A, ventral. B, dorsal. C, prolateral. D, retrolateral. Scale bars, $100 \mu \mathrm{m}$. C: conductor. CB: cymbium. CRa: conductor retrolateral apophysis. E: embolus. EMg: embolic medial groove. $\mathrm{P}$ : paracymbium. T: tegulum. 255

Figure 180. Glenognatha globosa from Colombia (ICN 5991). Male left palp, schematic. A, ventral. B, conductor ventral. C, conductor dorsal. D, embolus ventral. Scale bars $100 \mu \mathrm{m}$. C: conductor. CB: cymbium. CRa: conductor retrolateral apophysis. E: embolus. ED: ejaculatory duct. P: paracymbium. T: tegulum. .256

Figure 181. Glenognatha globosa from Colombia. Female genitalia. A, dorsal. B, lateral. C, ventral. D-E, uterus externus cuticle. Scale bars, $100 \mu \mathrm{m}$ (A-C), $20 \mu \mathrm{m}$ (D, E). UEc: uterus externus chamber. UEs: uterus externus sac.......................257

Figure 182. Glenognatha globosa from Colombia. Female tracheal system. A, dorsal. $\mathbf{B}$, median and lateral trunks. C, median trunk distal. D, tracheal spiracle posterior view. Scale bars, $100 \mu \mathrm{m}$ (A-C), $10 \mu \mathrm{m}$ (D). LT: lateral tracheae. MT: median trunks. TAG: tracheal atrium gland .258

Figure 183. Glenognatha globosa from Colombia. Male left palp. A. retrolateral. B. embolus and conductor. C, paracymbium. Scale bars $100 \mu \mathrm{m}$. C: conductor. CRa: conductor retrolateral apophysis. E: embolus. EMg: embolic medial groove. PBp: paracymbium basal portion. PDp: paracymbium basal portion. T: tegulum...........258

Figure 184. Glenognatha sp. nov. 6 from Colombia (Holotype, ICN 5979). Male habitus. A, dorsal. B, lateral. C, ventral. D, frontal. Scale bars, $200 \mu \mathrm{m}$. TS: tracheal spiracle. .259

Figure 185. Glenognatha sp. nov. 6 from Venezuela (AMNH). Female habitus. A, dorsal. B, lateral. C, ventral. D, frontal. Scale bars, $200 \mu \mathrm{m}$. TS: tracheal spiracle. .259

Figure 186. Glenognatha sp. nov. 6 from Colombia (Paratype ICN 5980). A-C, Male left chelicerae. A, anterior. B, posterior. C, mesal. D-F, Female left chelicerae. D, anterior. E, posterior. F, mesal. Scale bars, $100 \mu \mathrm{m}$. AT: anterior tooth. CFO: cheliceral fang outgrowth. Ret: retromarginal tooth 260

Figure 187. Glenognatha sp. nov. 6 from Colombia (Holotype ICN 5979). Male left palp A-D. A, ventral. B, dorsal. C, prolateral. D, retrolateral. Scale bars, $100 \mu \mathrm{m}$. C: conductor. CB: cymbium. CRa: conductor retrolateral apophysis. E: embolus. EMg: embolic medial groove. P: paracymbium. T: tegulum. 261

Figure 188. Glenognatha sp. nov. 6 from Colombia (Paratype ICN 5980). Male left palp, schematic. A, ventral. B, conductor ventral. C, conductor dorsal. D, embolus 
ventral. Scale bars $100 \mu \mathrm{m}$. C: conductor. CB: cymbium. CRa: conductor retrolateral apophysis. E: embolus. ED: ejaculatory duct. P: paracymbium. T: tegulum.

Figure 189. Glenognatha sp. nov. 6 from Colombia. Female genitalia. A, dorsal. B, lateral. C, ventral (arrows, divisions of the uterus externus sac) D-E, spermathecae and copulatory duct. Scale bars, $20 \mu \mathrm{m}$ (A-C), $10 \mu \mathrm{m}$ (D, E). CD: copulatory duct. LSD: long-stem gland ducts. UEc: uterus externus chamber. UEs: uterus externus sac. S: spermathecae. 263

Figure 190. Glenognatha sp. nov. 6 from Colombia. Female tracheal system. A, dorsal. B, tracheal spiracle posterior view. C, median trunk cuticle. Scale bars, $100 \mu \mathrm{m}$ (A), $10 \mu \mathrm{m}$ (B, C). LT: lateral trachea. MT: median trunks. TAG: tracheal atrium gland.... 264

Figure 191. Glenognatha sp. nov. 6 from Peru. A-C, embolus and conductor. A, ventral. B, dorsal (arrow, pointed projections). C, anteroventral. D, paracymbium. Scale bars $20 \mu \mathrm{m}$ (A-C), $100 \mu \mathrm{m}$ (D). C: conductor. CRa: conductor retrolateral apophysis. E: embolus. EMg: embolus medial groove. PBp: paracymbium basal portion. PDp: paracymbium distal portion....................................................265

Figure 192. Glenognatha sp. nov. 3 from Brazil (MCTP 13771). Male habitus. A, dorsal. B, lateral. C, ventral. D, frontal. Scale bars, $200 \mu \mathrm{m}$. TS: tracheal spiracle. .266

Figure 193. Glenognatha sp. nov. 3 from Brazil (MCTP 17493). Female habitus. A, dorsal. B, lateral. C, ventral. D, frontal. Scale bars, $200 \mu \mathrm{m}$. TS: tracheal spiracle .266

Figure 194. Glenognatha sp. nov. 3 from Brazil. A-C, Male left chelicerae (IBSP 57091). A, anterior. B, posterior. C, mesal. D-F, Female left chelicerae (IBSP 57078). D, anterior. E, posterior. F, mesal. Scale bars, $100 \mu \mathrm{m}$. AT: Anterior tooth. CFO: cheliceral fang outgrowth. Prt: promarginal tooth 267

Figure 195. Glenognatha sp. nov. 3 from Brazil (MCTP 13771). Male left palp. A, ventral. B, dorsal. C, prolateral. D, retrolateral (arrow, paracymbium process). Scale bars, $100 \mu \mathrm{m}$. C: conductor. CB: cymbium. CRa: conductor retrolateral apophysis. E: embolus. EMg: embolic medial groove. P: paracymbium. T: tegulum..............268

Figure 196. Glenognatha sp. nov. 3 from Brazil (IBSP 57090). Male left palp, schematic. A, ventral. B, conductor ventral. C, conductor dorsal. D, embolus ventral. Scale bars $100 \mu \mathrm{m}$. C: conductor. CB: cymbium. CRa: conductor retrolateral apophysis. E: embolus. ED: ejaculatory duct. P: paracymbium. T: tegulum.....................269

Figure 197. Glenognatha sp. nov. 3 from Brazil. Female genitalia. A, dorsal. B, lateral (arrow, disc-shaped basal region). C, ventral (arrow, disc-shaped basal region). D-E, spermathecae and copulatory duct. Scale bars, $20 \mu \mathrm{m}$ (A-C), $10 \mu \mathrm{m}$ (D, E). CD: copulatory duct. LSD: long-stem gland ducts. UEc: uterus externus chamber. UEs: uterus externus sac. S: spermathecae. .270 
Figure 198. Glenognatha sp. nov. 3 from Brazil. Female tracheal system. A, dorsal. B, median trunk distal. Scale bars $100 \mu \mathrm{m}$ (A), $20 \mu \mathrm{m}$ (B). LT: lateral tracheae. MT: median trunks. TAG: tracheal atrium gland.

Figure 199. Glenognatha sp. nov. 3 from Brazil. Male left palp. A, ventral. B, embolus and conductor ventral. C, embolus and conductor dorsal. Scale bars $20 \mu \mathrm{m}$. C: conductor. CRa: conductor retrolateral apophysis. E: embolus. EMg: embolic medial groove. T: tegulum. 271

Figure 200. Glenognatha foxi from USA (AMNH). Male habitus. A, dorsal. B, lateral.

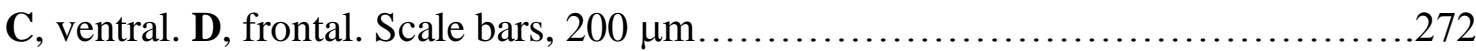

Figure 201. Glenognatha foxi from USA (AMNH). Female habitus. A, dorsal. B,

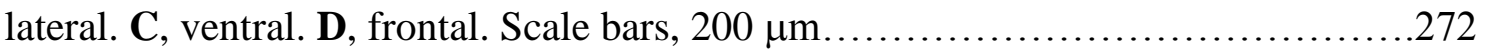

Figure 202. Glenognatha foxi from USA (AMNH). A-C, Male left chelicerae. A, anterior. B, posterior. C, mesal. D-F, Female left chelicerae. D, anterior. E, posterior. F, mesal. Scale bars, $100 \mu \mathrm{m}$. AT: anterior tooth. CFO: cheliceral fang outgrowth. .273

Figure 203. Glenognatha foxi from USA (AMNH). A-C, Male left chelicerae. A, anterior. B, posterior. C, mesal. D-F, Female left chelicerae. D, anterior. E, posterior. F, mesal. Scale bars, $100 \mu \mathrm{m}$. C: conductor. CB: cymbium. CRa: conductor retrolateral apophysis. E: embolus. EMg: embolic medial groove. P: paracymbium. T:

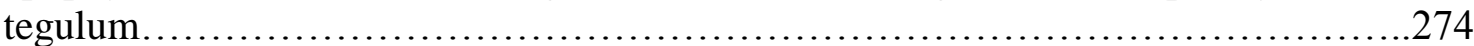

Figure 204. Glenognatha foxi from USA (AMNH). Male left palp, schematic. A, ventral. B, conductor ventral. C, conductor dorsal (arrow, excavation). D, embolus ventral. Scale bars $100 \mu \mathrm{m}$. C: conductor. CB: cymbium. CRa: conductor retrolateral apophysis. E: embolus. ED: ejaculatory duct. P: paracymbium. T:

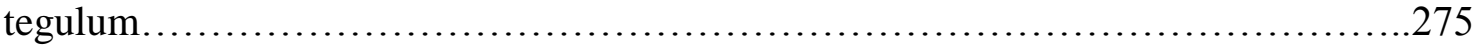

Figure 205. Glenognatha foxi from USA. Female genitalia. A, dorsal. B, lateral. C, anterior. D-E, spermathecae and copulatory duct. Scale bars, $20 \mu \mathrm{m}$ (A-C), $10 \mu \mathrm{m}$ (D, E). CD: copulatory duct. LSD: long-stem gland ducts. UEc: uterus externus chamber. UEs: uterus externus sac. S: spermathecae.................................276

Figure 206. Glenognatha foxi from USA. Female tracheal system. A, dorsal. B, median and lateral trunks. $\mathbf{C}$, median trunk distal. D, tracheal spiracle posterior view. Scale bars, $20 \mu \mathrm{m}$ (A), $10 \mu \mathrm{m}$ (B-D). LT: lateral tracheae. MT: median trunks. TAG: tracheal atrium gland....

277

Figure 207. Glenognatha foxi from USA. A, Male left palp ventral. B, Embolus and conductor ventroretrolateral. C, Paracymbium. D, Embolus and conductor dorsal (arrow, excavation). Scale bars $100 \mu \mathrm{m}$ (A, C), $10 \mu \mathrm{m}$ (B, D). C: conductor. CB: cymbium. CRa: conductor retrolateral apophysis. E: embolus. EMg: embolic medial groove. P: paracymbium. T: tegulum. 278 
Figure 208. Glenognatha sp. nov. 9 from Brazil (Holotype IBSP 120209). Male habitus. A, dorsal. B, lateral. C, ventral. D, frontal. Scale bars, $200 \mu \mathrm{m}$. TS: tracheal spiracle.

Figure 209. Glenognatha sp. nov. 9 from Brazil (IBSP120220). A-C, Male left chelicerae. A, anterior. B, posterior. C, mesal. Scale bars, $100 \mu \mathrm{m}$. AT: anterior tooth.

Figure 210. Glenognatha sp. nov. 9 from Brazil (Holotype IBSP 120209). Male left palp. A, ventral. B, dorsal. C, prolateral. D, retrolateral. Scale bars, $100 \mu \mathrm{m}$. C: conductor. CB: cymbium. CRa: conductor retrolateral apophysis. E: embolus. EMg: embolic medial groove. P: paracymbium. $\mathrm{T}$ : tegulum. TO: tarsal

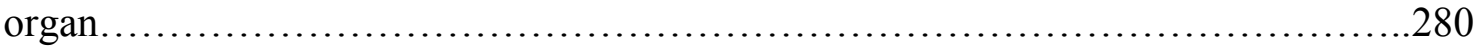

Figure 211. Glenognatha sp. nov. 9 from Brazil (IBSP 120220). Male left palp, schematic. A, ventral. B, conductor ventral. C, conductor dorsal. D, embolus ventral. Scale bars $100 \mu \mathrm{m}$. C: conductor. CB: cymbium. CRa: conductor retrolateral apophysis. $\mathrm{E}$ : embolus. P: paracymbium. T: tegulum. TO: tarsal

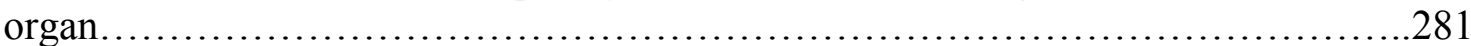

Figure 212. Glenognatha sp. nov. 10 from Peru (MUSM 054913). Male habitus. A, dorsal. B, lateral. C, ventral. D, frontal. Scale bars, $200 \mu \mathrm{m}$. TS: tracheal

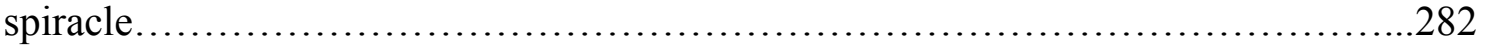

Figure 213. Glenognatha sp. nov. 10 from Peru (MUSM 0504905). A-C, Male left chelicerae. A, anterior. B, posterior. C, mesal. D-F, Female left chelicerae. D, anterior. $\mathbf{E}$, posterior. F, mesal. Scale bars, $100 \mu \mathrm{m}$. AT: anterior tooth......................282

Figure 214. Glenognatha sp. nov. 10 from Peru (Holotype MUSM 050913). Male left palp. A, ventral. B, dorsal. C, prolateral. D, retrolateral. Scale bars, $100 \mu \mathrm{m}$. C: conductor. CB: cymbium. CRa: conductor retrolateral apophysis. E: embolus. EMg: embolic medial groove. P: paracymbium. T: tegulum. TO: tarsal organ. .283

Figure 215. Glenognatha sp. nov. 10 from Peru (Holotype MUSM 050913). Male left palp, schematic. A, ventral. B, conductor ventral. C, conductor dorsal. D, embolus ventral. Scale bars $100 \mu \mathrm{m}$. C: conductor. CRa: conductor retrolateral apophysis. E: embolus. P: paracymbium. T: tegulum. TO: tarsal organ.......................284

Figure 216. Glenognatha sp. nov. 10 from Peru. Male left palp. A, ventroretrolateral. B, dorsal. C, anterior. . Scale bars $100 \mu \mathrm{m}$. C: conductor. CB: cymbium. CRa: conductor retrolateral apophysis. E: embolus. EMg: embolic medial groove. P: paracymbium. T:

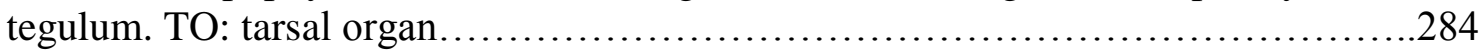

Figure 217. Glenognatha smilodon from Cameroon (Holotype MRAC 202710). Male habitus. A, dorsal. B, lateral. C, ventral. D, frontal. Scale bars, $200 \mu \mathrm{m}$. TS: tracheal spiracle .285

Figure 218. Glenognatha smilodon from Cameroon (Holotype MRAC 202710). Male left palp. A, ventral. B, dorsal. C, prolateral. D, retrolateral. Scale bars, $100 \mu \mathrm{m}$. C: 
conductor. CB: cymbium. CRa: conductor retrolateral apophysis. E: embolus. EMg: embolic medial groove. P: paracymbium. T: tegulum. TO: tarsal organ. ...

.286

Figure 219. Glenognatha smilodon from Cameroon (Holotype MRAC 202710). Male left palp, schematic. A, ventral. B, conductor ventral. C, conductor dorsal. D, embolus ventral. Scale bars $100 \mu \mathrm{m}$. C: conductor. CB: cymbium. CRa: conductor retrolateral apophysis. E: embolus. ED: ejaculatory duct. P: paracymbium. T: tegulum 287

Figure 220. Glenognatha iviei from USA (Holotype AMNH). Male habitus. A, dorsal. B, lateral. C, ventral. D, frontal. Scale bars, $200 \mu \mathrm{m}$. AT: Anterior tooth. Prt: promarginal tooth. TS: tracheal spiracle. .288

Figure 221. Glenognatha iviei from USA (Holotype AMNH). Male left palp, schematic. A, ventral. B, conductor ventral. C, conductor dorsal. D, embolus ventral. Scale bars $100 \mu \mathrm{m}$. C: conductor. CB: cymbium. CRa: conductor retrolateral apophysis. E: embolus. P: paracymbium. T: tegulum. .288

Figure 222. Glenognatha iviei from USA (Holotype AMNH). Male left palp. A, ventral. B, dorsal. C, prolateral. D, retrolateral (arrow, paracymbium process). Scale bars, $100 \mu \mathrm{m}$. C: conductor. CB: cymbium. CRa: conductor retrolateral apophysis. E: embolus. EMg: embolic medial groove. P: paracymbium. T: tegulum. 289

Figure 223. Glenognatha heleios from USA (AMNH). Male habitus. A, dorsal. B, lateral. C, ventral. D, frontal (Paratype). Scale bars, $200 \mu \mathrm{m}$. TS: tracheal spiracle. .290

Figure 224. Glenognatha heleios from USA (Paratype AMNH). Female habitus. A, dorsal. B, lateral. C, ventral. D, frontal. Scale bars, $200 \mu \mathrm{m}$. TS: tracheal spiracle. .290

Figure 225. Glenognatha heleios from USA (AMNH). A-C, Male left chelicerae. A, anterior. B, posterior. C, mesal. D-F, Female left chelicerae. D, anterior. E, posterior. F, mesal. Scale bars, $100 \mu \mathrm{m}$. AT: anterior tooth. CFO: cheliceral fang outgrowth. Prt: promarginal tooth.

Figure 226. Glenognatha heleios from USA (Paratype AMNH). Male left palp. A, ventral. B, dorsal (arrow, paracymbium process). C, prolateral. D, retrolateral. Scale bars, $100 \mu \mathrm{m}$. C: conductor. CB: cymbium. CRa: conductor retrolateral apophysis. E: embolus. EMg: embolic medial groove. P: paracymbium. T: tegulum. .292

Figure 227. Glenognatha heleios from USA (Paratype AMNH). Male left palp, schematic. A, ventral. B, conductor ventral. C, conductor dorsal. D, embolus ventral. Scale bars $100 \mu \mathrm{m}$. C: conductor. CB: cymbium. CRa: conductor retrolateral apophysis. E: embolus. ED: ejaculatory duct. P: paracymbium. T: tegulum .293

Figure 228. Glenognatha heleios from USA. Female genitalia. A, dorsal. B, lateral. C, ventral (arrow, constricted base). D-E, spermathecae and copulatory duct. Scale bars, $20 \mu \mathrm{m}$ (A-C), $10 \mu \mathrm{m}$ (D, E). CD: copulatory duct. LSD: long-stem gland ducts. UEc: uterus externus chamber. UEs: uterus externus sac. S: spermathecae. 
Figure 229. Glenognatha heleios from USA. Female tracheal system. A, dorsal. B, median and lateral trunks. C, median trunk distal. D, tracheal spiracle posterior view. Scale bars, $100 \mu \mathrm{m}$ (A), $10 \mu \mathrm{m}$ (B-D). LT: lateral tracheae. MT: median trunks. TAG: tracheal atrium gland.

Figure 230. Glenognatha heleios from USA. A-D, Male left palp. A, ventral. B, dorsal. $\mathbf{C}$, retrolateral. D, anterior. E-G, embolus and Conductor. E, ventral. F, dorsal. $\mathbf{G}$, retrolateral. H, paracymbium (arrow, paracymbium process). Scale bars $100 \mu \mathrm{m}$ (A-D), $20 \mu \mathrm{m}(\mathrm{E}-\mathrm{H})$. C: conductor. CB: cymbium. CRa: conductor retrolateral apophysis. E: embolus. EMg: embolic medial groove. PBp: paracymbium basal portion. T:

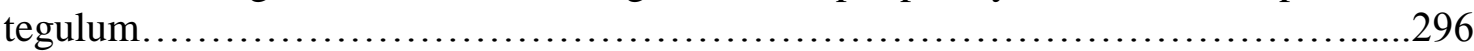

Figure 231. Glenognatha sp. nov. 1 from Brazil (IBSP 147809). Male habitus. A, dorsal. B, lateral. C, ventral. D, frontal. Scale bars, $200 \mu \mathrm{m}$. TS: tracheal spiracle.

Figure 232. Glenognatha sp. nov. 1 from Brazil (IBSP 147809). Female habitus. A, dorsal. B, ventral. C, lateral. D, frontal. Scale bars, $200 \mu \mathrm{m}$. TS: tracheal spiracle.

Figure 233. Glenognatha sp. nov. 1 from Brazil (IBSP 147861). A-C, Male left chelicerae. A, anterior. B, posterior. C, mesal. D-F, Female left chelicerae. D, anterior. E, posterior. F, mesal. Scale bars, $100 \mu \mathrm{m}$. Ret: retromarginal tooth .298

Figure 234. Glenognatha sp. nov. 1 from Brazil (IBSP 147809). Male left palp. A, ventral. B, dorsal. C, prolateral. D, retrolateral. Scale bars, $100 \mu \mathrm{m}$. C: conductor. CB: cymbium. CRa: conductor retrolateral apophysis. E: embolus. EMg: embolic medial groove. P: paracymbium. T: tegulum. TO: tarsal organ.

.299

Figure 235. Glenognatha sp. nov. 1 from Brazil (Paratype IBSP 147861). Male left palp, schematic. A, ventral. B, conductor ventral. C, conductor dorsal. D, embolus ventral. Scale bars $100 \mu \mathrm{m}$. C: conductor. CB: cymbium. CRa: conductor retrolateral apophysis. E: embolus. ED: ejaculatory duct. P: paracymbium. T: tegulum. TO: tarsal organ 300

Figure 236. Glenognatha sp. nov. 1 from Brazil. Female genitalia. A, dorsal (arrows, disc-shaped portions). B, lateral. C, ventral D-E, spermathecae and copulatory duct. Scale bars, $20 \mu \mathrm{m}$ (A-C), $10 \mu \mathrm{m}$ (D-E). CD: copulatory duct. LSD: long-stem gland ducts. UEc: uterus externus chamber. UEs: uterus externus sac. $S$ : spermathecae .......................................................... 301

Figure 237. Glenognatha sp. nov. 1 from Brazil. Female tracheal system. A, dorsal. B, median trunk distal. $\mathbf{C}$, median trunk basal. D, tracheal spiracle posterior view. Scale bars, $100 \mu \mathrm{m}$ (A), $10 \mu \mathrm{m}$ (B-D). LT: lateral trachea. MT: median trunks. TAG: tracheal atrium gland .302 
Figure 238. Glenognatha sp. nov. 1 from Brazil. Male left palp. A, ventral. B, embolus and conductor ventral. $\mathbf{C}$, embolus tip. D, embolus and conductor retrolateral. E, anteroretrolateral. F, cymbium. Scale bars, $100 \mu \mathrm{m}$ (A), $50 \mu \mathrm{m}$ (B, D-F), $10 \mu \mathrm{m}$ (C). C: conductor. CRa: conductor retrolateral apophysis. E: embolus. T: tegulum. TO: tarsal organ. .303

Figure 239. Glenognatha hirsutissima from Marquesas Islands (USNM). Male habitus. A, dorsal. B, lateral. C, ventral. D, frontal. Scale bars, $200 \mu \mathrm{m}$. TS: tracheal spiracle. .304

Figure 240. Glenognatha hirsutissima from Marquesas Islands (USNM). Female habitus. A, dorsal. B, lateral. C, ventral. D, frontal. Scale bars, $200 \mu \mathrm{m}$. TS: tracheal spiracle

Figure 241. Glenognatha hirsutissima from Marquesas Islands (USNM). A-C, Male left chelicerae. A, anterior (arrow, anterior macrosetae). B, posterior. C, mesal. D-F, Female left chelicerae. D, anterior (arrow, anterior spine socket). E, posterior. F, mesal. Scale bars, $100 \mu \mathrm{m}$. .305

Figure 242. Glenognatha hirsutissima from Marquesas Islands (USNM). Male left palp. A, ventral. B, dorsal. C, prolateral. D, retrolateral. Scale bars, $100 \mu \mathrm{m}$. C: conductor. CB: cymbium. CRa: conductor retrolateral apophysis. E: embolus. EMg: embolic medial groove. P: paracymbium. T: tegulum.......................... 306

Figure 243. Glenognatha hirsutissima from Marquesas Islands (USNM). Male left palp, schematic. A, ventral. B, conductor ventral. C, conductor dorsal. D, embolus ventral. Scale bars $100 \mu \mathrm{m}$. C: conductor. CB: cymbium. CRa: conductor retrolateral apophysis. E: embolus. ED: ejaculatory duct. P: paracymbium. T: tegulum. 307

Figure 244. Glenognatha hirsutissima from Marquesas Islands (USNM). Female genitalia. A, dorsal. B, ventral. C, lateral. Scale bars, $200 \mu \mathrm{m}$. CD: copulatory duct. UEc: uterus externus chamber. UEs: uterus externus sac. S: spermathecae. 307

Figure 245. Glenognatha australis from Peru (CAS 9039650). A-B, Male habitus. A, dorsal. B, ventral. C-D, Female habitus. C, dorsal. D, ventral. Scale bars $200 \mu \mathrm{m}$. TS: tracheal spiracle. .308

Figure 246. Glenognatha australis from Brazil (MCN 46671). Male habitus. A, dorsal. B, lateral. C, ventral. D, frontal. Scale bars, $200 \mu \mathrm{m}$. TS: tracheal

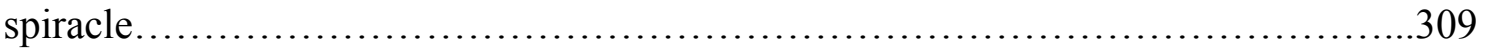

Figure 247. Glenognatha australis from Brazil (MCN 46671). Female habitus. A, dorsal. B, lateral. C, ventral. D, frontal. Scale bars, $200 \mu \mathrm{m}$. TS: tracheal spiracle.....

Figure 248. Glenognatha australis. A-C, Male left chelicerae from Argentina (MCT 838). A, anterior. B, posterior. C, mesal. D-F, Female left chelicerae from Brazil (MCN 42102). D, anterior. E, posterior. F, mesal. Scale bars, $100 \mu \mathrm{m}$. Ret: retromarginal tooth. 
Figure 249. Glenognatha australis from Brazil (MCN 46671). Male left palp. A, ventral. B, dorsal. C, prolateral. D, retrolateral. Scale bars, $100 \mu \mathrm{m}$. C: conductor. CB: cymbium. CRa: conductor retrolateral apophysis. E: embolus. EMg: embolic medial groove. P: paracymbium. T: tegulum. TO: tarsal organ......................... 311

Figure 250. Glenognatha australis from Brazil (MCTP 841). Male left palp, schematic. A, ventral. B, conductor ventral. C, conductor dorsal. D, embolus ventral. Scale bars $100 \mu \mathrm{m}$. C: conductor. CB: cymbium. CRa: conductor retrolateral apophysis. E: embolus. ED: ejaculatory duct. P: paracymbium. T: tegulum. TO: tarsal organ .312

Figure 251. Glenognatha australis from Brazil. Female genitalia. A, dorsal (arrows, disc-shaped portions). B, lateral. C, ventral. D-E, spermathecae and copulatory duct. Scale bars, $100 \mu \mathrm{m}$ (A-C), $10 \mu \mathrm{m}$ (D, E). CD: copulatory duct. LSD: long-stem gland ducts. UEc: uterus externus chamber. UEs: uterus externus sac. $S$ :

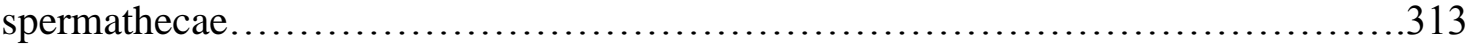

Figure 252. Glenognatha australis from Brazil. Female tracheal system. A, dorsal. B, median and lateral trunks. C, median trunk distal. D, tracheal spiracle posterior view. Scale bars, $100 \mu \mathrm{m}$ (A), $20 \mu \mathrm{m}$ (B-C), $10 \mu \mathrm{m}$ (D). LT: lateral tracheae. MT: median trunks. TAG: tracheal atrium gland 314

Figure 253. Glenognatha australis from Brazil. A, male left palp frontal. B, conductor ventral (tip broken). C, embolus and conductor ventral. D, embolus (arrow, inner membrane). E, embolus and conductor dorsal. Scale bars $100 \mu \mathrm{m}$ (A), $20 \mu \mathrm{m}$ (B,D), 50 $\mu \mathrm{m}(\mathrm{C}, \mathrm{E}) . \mathrm{CB}$ : cymbium. CRa: conductor retrolateral apophysis. E: embolus. EMg: embolic medial groove. P: paracymbium. T: tegulum. TO: tarsal organ .315

Figure 254. Glenognatha spherella from Mexico (CAS 9046858). Male habitus. A, dorsal. B, lateral. C, ventral. D, frontal. Scale bars, $200 \mu \mathrm{m}$. TS: tracheal spiracle. 316

Figure 255. Glenognatha spherella from Mexico (CAS 9046857). Female habitus. A, dorsal. B, lateral. C, ventral. D, frontal. Scale bars, $200 \mu \mathrm{m}$. TS: tracheal spiracle. .316

Figure 256. Glenognatha spherella from Mexico. A-C (CAS 9046858), Male left chelicerae. A, anterior. B, posterior. C, mesal. D-F (CAS 9046857), Female left chelicerae. D, anterior. E, posterior (arrow, posterolateral bulge). F, mesal (arrow, posterolateral bulge). Scale bars, $100 \mu \mathrm{m}$. Ret: retromarginal tooth 317

Figure 257. Glenognatha spherella from Mexico (CAS 9046858). Male left palp. A, ventral. B, dorsal. C, prolateral. D, retrolateral. Scale bars, $100 \mu \mathrm{m}$. C: conductor. CB: cymbium. CRa: conductor retrolateral apophysis. E: embolus. EMg: embolic medial groove. P: paracymbium. T: tegulum. TO: tarsal organ .........................318

Figure 258. Glenognatha spherella from Mexico (AMNH). Male left palp, schematic. A, ventral. B, conductor ventral. C, conductor dorsal. D, embolus ventral. Scale bars $100 \mu \mathrm{m}$. C: conductor. CB: cymbium. CRa: conductor retrolateral apophysis. E: embolus. ED: ejaculatory duct. P: paracymbium. T: tegulum. 
Figure 259. Glenognatha spherella from Costa Rica. Female genitalia. A, dorsal. B, lateral. C, ventral D-E, spermathecae and copulatory duct. Scale bars, $20 \mu \mathrm{m}$ (A, C), $10 \mu \mathrm{m}$ (B, D-E). CD: copulatory duct. LSD: long-stem gland ducts. UEc: uterus externus chamber. UEs: uterus externus sac. S: spermathecae....

Figure 260. Glenognatha spherella from Mexico. Female tracheal system. A, dorsal. B, median and lateral trunks. C, median trunk distal. D, tracheal spiracle posterior view. Scale bars, $100 \mu \mathrm{m}$ (A), $20 \mu \mathrm{m}$ (B, C), $10 \mu \mathrm{m}$ (D). LT: lateral tracheae. MT: median trunks. TAG: tracheal atrium gland. 321

Figure 261. Glenognatha spherella from Costa Rica. A, male left palp ventral. B, embolus and conductor ventral. $\mathbf{C}$, embolus and conductor dorsal. D, embolus and conductor retrolateral. E, paracymbium. Scale bars $20 \mu \mathrm{m}$ (A), $10 \mu \mathrm{m}$ (B-E). C: conductor. CRa: conductor retrolateral apophysis. E: embolus. EMg: embolic medial groove. P: paracymbium. PBp: paracymbium basal portion. PDp: paracymbium distal portion. T: tegulum 322

Figure 262. Glenognatha gloriae from Puerto Rico (MCZ 125485). Male habitus. A, dorsal. B, lateral. C, ventral. D, frontal (MCZ 125482). Scale bars, $200 \mu \mathrm{m}$ (A-C), 100 $\mu \mathrm{m}$ (D). TS: tracheal spiracle.

Figure 263. Glenognatha gloriae from Puerto Rico (MCZ 125485). Female habitus. A, dorsal. B, lateral. C, ventral. D, frontal. Scale bars, $200 \mu \mathrm{m}$ (A-C), $100 \mu \mathrm{m}$ (D). TS: tracheal spiracle .323

Figure 264. Glenognatha gloriae from Puerto Rico. A-C, Male left chelicerae (MCZ 125482). A, anterior. B, posterior. C, mesal. D-F, Female left chelicerae (MCZ 125482). D, anterior. E, posterior. F, mesal. Scale bars, $100 \mu \mathrm{m}$. Ret: retromarginal tooth.

Figure 265. Glenognatha gloriae from Puerto Rico (MCZ 125485). Male left palp. A, ventral. B, dorsal. C, prolateral. D, retrolateral. Scale bars, $100 \mu \mathrm{m}$. C: conductor. CB: cymbium. E: embolus. EMg: embolic medial groove. P: paracymbium. T: tegulum.

Figure 266. Glenognatha gloriae from Puerto Rico (MCZ 125485). Male left palp, schematic. A, ventral. B, conductor ventral. C, conductor dorsal. D, embolus ventral. Scale bars $100 \mu \mathrm{m}$. C: conductor. CRa: conductor retrolateral apophysis. E: embolus. ED: ejaculatory duct. P: paracymbium. T: tegulum. TO: tarsal organ. .326

Figure 267. Glenognatha gloriae from Puerto Rico. Female genitalia. A, dorsal. B, lateral. C, ventral. D-E, spermathecae and copulatory duct. Scale bars, $20 \mu \mathrm{m}$ (A-C), $10 \mu \mathrm{m}$ (D, E). CD: copulatory duct. LSD: long-stem gland ducts. UEc: uterus externus chamber. UEs: uterus externus sac. S: spermathecae. 
Figure 268. Glenognatha gloriae from Puerto Rico. A, Male left palp ventral. B-E, embolus and conductor. B, ventroretrolateral. C, ventroanterior. D, ventral. E, dorsal. F, paracymbium. Scale bars $20 \mu \mathrm{m}$. C: conductor. CRa: conductor retrolateral apophysis. E: embolus. PBp: paracymbium basal portion. PDp: paracymbium distal portion. T:

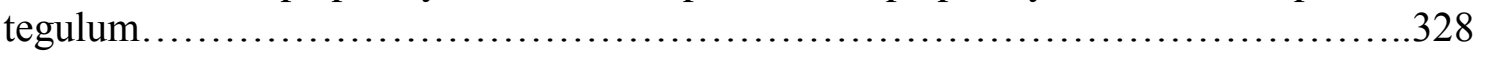

Figure 269. Glenognatha sp. nov. 11 from USA (Paratype, USNM). Male habitus. A, dorsal. B, lateral. C, ventral. D, frontal. Scale bars, $500 \mu \mathrm{m}$. TS: tracheal spiracle. 329

Figure 270. Glenognatha sp. nov. 11 from USA (Paratype, USNM). Female habitus. A, dorsal. B, lateral. C, ventral. D, frontal. Scale bars, $500 \mu \mathrm{m}$. TS: tracheal spiracle.

Figure 271. Glenognatha sp. nov. 11 from USA (Paratypes, USNM). A-C, Male left chelicerae. A, anterior. B, posterior. C, mesal. D-F, Female left chelicerae. D, anterior. E, posterior. F, mesal. Scale bars, $200 \mu \mathrm{m}$. Prt: promarginal tooth. Ret: retromarginal tooth. .330

Figure 272. Glenognatha sp. nov. 11 from USA (Paratype, USNM). Male left palp. A, ventral. B, dorsal. C, prolateral. D, retrolateral. Scale bars, $100 \mu \mathrm{m}$. C: conductor. CB: cymbium. CRa: conductor retrolateral apophysis. E: embolus. EMg: embolic medial groove. P: paracymbium. T: tegulum. TO: tarsal organ

Figure 273. Glenognatha sp. nov. 11 from USA (USNM). Male left palp, schematic. A, ventral. B, conductor ventral. C, conductor dorsal. D, embolus ventral. Scale bars 100 $\mu \mathrm{m}$. C: conductor. CB: cymbium. CRa: conductor retrolateral apophysis. E: embolus. ED: ejaculatory duct. P: paracymbium. T: tegulum. TO: tarsal organ. .332

Figure 274. Glenognatha sp. nov. 11 from USA (Paratype, USNM). Female genitalia. Female genitalia. A, dorsal. B, ventral. Scale bars, $100 \mu \mathrm{m}$. UEc: uterus externus chamber. UEs: uterus externus sac.... .332

Figure 275. Glenognatha sp. nov. 7 from Brazil (MNRJ 6598). Male habitus. A, dorsal. B, lateral. C, ventral (arrow, pleural bar). D, frontal. Scale bars, $500 \mu \mathrm{m}$. TS: tracheal spiracle

Figure 276. Glenognatha sp. nov. 7 from Brazil (MNRJ 6598). Female habitus. A, dorsal. B, lateral. C, ventral (arrow, pleural bar). D, frontal. Scale bars, $500 \mu \mathrm{m}$. TS: tracheal spiracle. .333

Figure 277. Glenognatha sp. nov. 7 from Brazil (MNRJ 6598). A-C, Male left chelicerae. A, anterior. B, posterior. C, mesal. D-F, female left chelicerae. D, anterior. E, posterior. F, mesal. Scale bars, $100 \mu \mathrm{m}$. Prt: promarginal tooth. Ret: retromarginal tooth.

Figure 278. Glenognatha sp. nov. 7 from Brazil (MNRJ 6598). Male left palp. A, ventral. B, dorsal. C, prolateral. D, retrolateral. Scale bars, $100 \mu \mathrm{m}$. C: conductor. CB: cymbium. CRa: conductor retrolateral apophysis. E: embolus. EMg: embolic medial groove. P: paracymbium. T: tegulum. TO: tarsal organ 
Figure 279. Glenognatha sp. nov. 7 from Brazil (MNRJ 6598). Male left palp, schematic. A, ventral. B, conductor ventral. C, conductor dorsal. D, embolus ventral. Scale bars $100 \mu \mathrm{m}$. C: conductor. CB: cymbium. CRa: conductor retrolateral apophysis. E: embolus. ED: ejaculatory duct. P: paracymbium. T: tegulum.....................336

Figure 280. Glenognatha sp. nov. 7 from Brazil. Female genitalia. A, dorsal. B, lateral. C, ventral. D-E, uterus externus cuticle. Scale bars, $100 \mu \mathrm{m}$ (A-C), $30 \mu \mathrm{m}$ (D), $20 \mu \mathrm{m}$ (E). UEc: uterus externus chamber. UEs: uterus externus sac. 337

Figure 281. Glenognatha sp. nov. 7 from Brazil. Female tracheal system. A, dorsal. B, median and lateral trunks. C, median trunk distal. D, tracheal spiracle posterior view. Scale bars, $100 \mu \mathrm{m}$ (A, B), $20 \mu \mathrm{m}$ (C), $10 \mu \mathrm{m}$ (D). LT: lateral tracheae. MT: median trunks. TAG: tracheal atrium gland.... .338

Figure 282. Glenognatha minuta from Mexico (AMNH). Male habitus. A, dorsal. B, lateral. C, ventral. D, frontal. Scale bars, $200 \mu \mathrm{m}$. TS: tracheal spiracle. 339

Figure 283. Glenognatha minuta from Mexico (AMNH). Female habitus. A, dorsal. B, lateral. C, ventral. D, frontal. Scale bars, $200 \mu \mathrm{m}$. TS: tracheal spiracle. 339

Figure 284. Glenognatha minuta. A-C, male left chelicerae from Panama (AMNH). A, anterior. B, posterior. C, mesal. D-F, female left chelicerae from Mexico (AMNH). D, anterior (arrow, cheliceral basal segment projection). E, posterior. F, mesal (arrow, cheliceral bulge). Scale bars, $100 \mu \mathrm{m}$. Ret: retromarginal tooth. 340

Figure 285. Glenognatha minuta from Mexico (AMNH). Male left palp. A, ventral. B, dorsal. C, prolateral. D, retrolateral. Scale bars, $100 \mu \mathrm{m}$. C: conductor. CB: cymbium. CRa: conductor retrolateral apophysis. E: embolus. EMg: embolic medial groove. P: paracymbium. T: tegulum. 341

Figure 286. Glenognatha minuta from Mexico (AMNH). Male left palp, schematic. A, ventral. B, conductor ventral. C, conductor dorsal. D, embolus ventral. Scale bars 100 $\mu \mathrm{m}$. C: conductor. CB: cymbium. CRa: conductor retrolateral apophysis. E: embolus. ED: ejaculatory duct. P: paracymbium. T: tegulum. .342

Figure 287. Glenognatha minuta from Mexico. Female genitalia. A, dorsal (arrow, tubiform projection). B, lateral. C, ventral D, anterior. E, spermathecae. Scale bars, $100 \mu \mathrm{m}$ (A-D), $10 \mu \mathrm{m}$ (E). CD: copulatory duct. LSD: long-stem gland ducts. UEc: uterus externus chamber. UEs: uterus externus sac. S: spermathecae. 343

Figure 288. Glenognatha minuta from Mexico. Female tracheal system. A, dorsal. B, median and lateral trunks. C, tracheal spiracle posterior view. Scale bars, $100 \mu \mathrm{m}$ (AB), $20 \mu \mathrm{m}$ (C). LT: lateral tracheae. MT: median trunks. TAG: tracheal atrium gland. 
Figure 289. Glenognatha minuta from Panama. Male left palp. A, ventral. B, embolus and conductor ventral. $\mathbf{C}$, embolus and conductor dorsal. D, paracymbium. Scale bars $100 \mu \mathrm{m}$ (A, D), $20 \mu \mathrm{m}$ (B-C). C: conductor. CRa: conductor retrolateral apophysis. E: embolus. EMg: embolic median groove. PBp: paracymbium basal portion. PDp: paracymbium distal portion. T: tegulum..................................... 344

Figure 290. Glenognatha emertoni from USA (AMNH). Male habitus. A, dorsal. B, lateral. C, ventral. D, frontal. Scale bars, $500 \mu \mathrm{m}$. TS: tracheal spiracle. .345

Figure 291. Glenognatha emertoni from USA (AMNH). Female habitus. A, dorsal. B, lateral. C, ventral. D, frontal. Scale bars, $500 \mu \mathrm{m}$. TS: tracheal spiracle. .345

Figure 292. Glenognatha emertoni from USA (AMNH). A-C, Male left chelicerae. A, anterior (arrow, cheliceral basal segment projection). B, posterior. C, mesal. D-F, Female left chelicerae. D, anterior (arrow, cheliceral basal segment projection). E, posterior. F, mesal (arrow, cheliceral bulge). Scale bars, $500 \mu \mathrm{m}$. Prt: Promarginal tooth. Ret: retromarginal tooth. .346

Figure 293. Glenognatha emertoni from USA (AMNH). Male left palp. A, ventral. B, dorsal. C, prolateral. D, retrolateral. Scale bars, $100 \mu \mathrm{m}$. C: conductor. CB: cymbium. CRa: conductor retrolateral apophysis. E: embolus. EMg: embolic medial groove. P: paracymbium. T: tegulum. TO: tarsal organ

Figure 294. Glenognatha emertoni from USA (AMNH). Male left palp, schematic. A, ventral. B, conductor ventral. C, conductor dorsal. D, embolus ventral. Scale bars 100 $\mu \mathrm{m}$. C: conductor. $\mathrm{CB}$ : cymbium. CRa: conductor retrolateral apophysis. E: embolus. ED: ejaculatory duct. P: paracymbium. T: tegulum............................. 348

Figure 295. Glenognatha emertoni from USA. Female genitalia. A, dorsal. B, lateral. C, ventral. D-E, spermathecae and copulatory duct. Scale bars, $100 \mu \mathrm{m}$ (A-C), $10 \mu \mathrm{m}$ (D,E). CD: copulatory duct. LSD: long-stem gland ducts. UEc: uterus externus chamber. UEs: uterus externus sac. S: spermathecae. .349

Figure 296. Glenognatha emertoni from USA. Female tracheal system. A, dorsal. B, median and lateral trunks. C, median trunk distal. D, tracheal spiracle posterior view. Scale bars, $100 \mu \mathrm{m}$ (A, B), $20 \mu \mathrm{m}$ (C, D). LT: lateral tracheae. MT: median trunks. TAG: tracheal atrium gland .350

Figure 297. Epiandrous fusules. A, Glenognatha dentata. B, Glenognatha tangi. C, Glenognatha australis. D, Glenognatha gaujoni. E, Glenognatha globosa. F, Glenognatha sp. nov. 2 G, Glenognatha sp. nov. 3 H, Glenognatha sp. nov. 10. I, Glenognatha lacteovittata. J, Glenognatha sp. nov. 5. Scale bars $10 \mu \mathrm{m} . . . \ldots \ldots \ldots . . . .351$

Figure 298. Female anterior lateral spinnerets. A, Glenognatha sp. nov. 1 B, Glenognatha sp. nov. 2 C, Glenognatha sp. nov. 7 D, Glenognatha australis. E, Glenognatha emertoni. F, Glenognatha gaujoni. G, Glenognatha globosa. H,

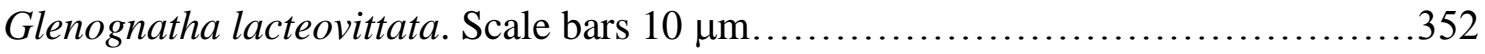


Figure 299. Male anterior lateral spinnerets. A, Glenognatha sp. nov. 1 B, Glenognatha sp. nov. 2 C, Glenognatha australis. D, Glenognatha gaujoni. E, Glenognatha globosa. F, Glenognatha lacteovittata. G, Glenognatha dentata. H, Glenognatha tangi. Scale

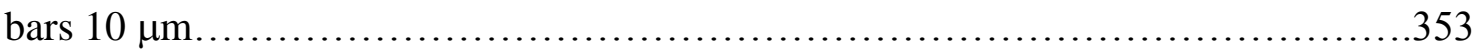

Figure 300. Female posterior median spinnerets. A, Glenognatha sp. nov. 2 B, Glenognatha sp. nov. 7 C, Glenognatha australis. D, Glenognatha emertoni. D, Glenognatha gaujoni. E, Glenognatha globosa. F, Glenognatha lacteovittata. Scale

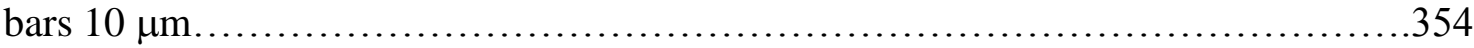

Figure 301. Male posterior median spinnerets. A, Glenognatha sp. nov. 2 B, Glenognatha australis. C, Glenognatha gaujoni. D, Glenognatha globosa. E, Glenognatha lacteovittata. F, Glenognatha tangi. Scale bars $10 \mu \mathrm{m} . \ldots \ldots \ldots \ldots \ldots \ldots . \ldots . \ldots 55$

Figure 302. Female posterior lateral spinnerets. A, Glenognatha sp. nov. 1 B, Glenognatha sp. nov. 2 C, Glenognatha sp. nov. 7 D, Glenognatha australis. E, Glenognatha emertoni. F, Glenognatha gaujoni. G, Glenognatha globosa. H,

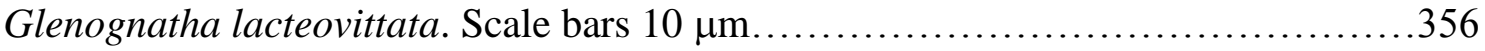

Figure 303. Male posterior lateral spinnerets. A, Glenognatha sp. nov. 2 B, Glenognatha australis. C, Glenognatha gaujoni. D, Glenognatha globosa. E, Glenognatha lacteovittata. F, Glenognatha tangi. Scale bars $10 \mu \mathrm{m} . \ldots \ldots \ldots \ldots \ldots \ldots . \ldots 357$

Figure 304. Distribution of G. emertoni, G. heleios and G. iviei...................358

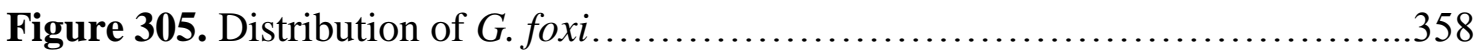

Figure 306. Distribution of G. minuta, G. spherella and G. sp. nov. 11...............359

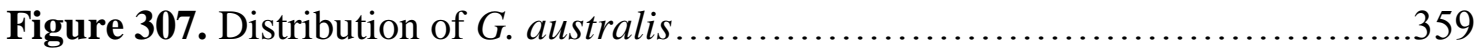

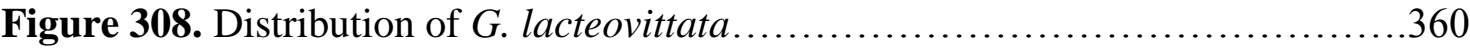

Figure 309. Distribution of G. gaujoni, G. sp. nov. 6, G. sp. nov. 9 and $G$. sp. nov. 10. 360

Figure 310. Distribution of G. globosa, G. sp. nov. 8, G. sp. nov. 5 and G. sp. nov. 2

Figure 311. Distribution of G. gloriae.... .361

Figure 312. Distribution of $G$. sp. nov. 4, G. sp. nov. 7, G. sp. nov. 1 and $G$. sp. nov. 3 .

Figure 313. Distribution of G. argyrostilba and G. smilodon ......................362

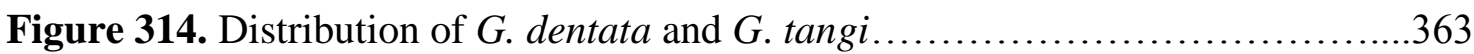

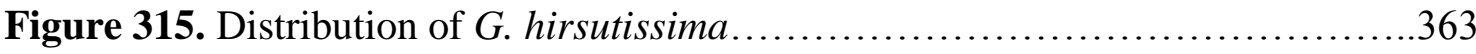




\section{List of Tables}

Table 1. Summary statistics from the implied weighting analysis. K: concavity constant, N: number of most parsimonious trees, L: tree length, F: total fit............18

Table 2. Unambiguous synapomorphies supporting major clades within Glenognatha

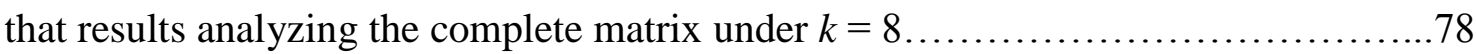




\section{ABSTRACT}

A taxonomic revision and phylogenetic analysis of the spider genus Glenognatha Simon, 1887 is presented. The analysis is based on a data set including 24 Glenognatha species plus eight outgroup representatives of three additional tetragnathine genera and one metaine, scored for 82 morphological characters. Eight unambiguous synapomorphies support the monophyly of Glenognatha, all free of homoplasy. Some internal clades within the genus are well-supported and its relationships are discussed. The genus Glenognatha has a broad distribution occupying the Neartic, Neotropic, Afrotropic, Indo-Malaya and Oceania ecozones. As revised here, Glenognatha comprises 27 species, four of them only know from males. New morphological data are provided for the description of thirteen previously described species. Eleven species are newly described: $G$. sp. nov. $1, G$. sp. nov. 3, G. sp. nov. 4 and $G$. sp. nov. 7 from southeast Brazil, G. sp. nov. 6, G. sp. nov. 9 and G. sp. nov. 10 from the Amazonian region, G. sp. nov. 2, G. sp. nov. 5 and G. sp. nov. 8 from northern Andes and G. sp. nov. 11 from central Mexico and Southern United States. Females of G. minuta Banks, 1898, G. gaujoni Simon, 1895 and G. gloriae (Petrunkevitch, 1930) and males of $G$. globosa (Petrunkevitch, 1925) and G. hirsutissima (Berland, 1935) are described for the first time. Three new combinations are proposed in congruence with the phylogenetic results: G. argyrostilba (O. P.-Cambridge, 1876), G. dentata (Zhu \& Wen, 1978) and G. tangi (Zhu, Song \& Zhang, 2003), all previously included in Dyschiriognatha. The following taxa are newly synonymized: Dyschiriognatha montana Simon, 1897, Glenognatha mira Bryant, 1945 and Glenognatha maelfaiti Baert, 1987 with Glenognatha argyrostilba (O. P.-Cambridge, 1876) and Glenognatha centralis Chamberlin, 1925 with Glenognatha minuta Banks, 1898. A key to species of Glenognatha and distribution maps are provided. 


\section{RESUMO}

A revisão taxonômica e análise cladística do gênero Glenognatha Simon, 1887 é apresentada. A análise é baseada em uma matriz que inclui 24 espécies de Glenognatha, oito representantes do grupo externo, incluindo três gêneros da subfamília Tetragnathinae e um de Metainae, e 82 caracteres morfológicos. Oito sinapomorfias não ambíguas sustentam a monofilia de Glenognatha, todas não homoplásticas. Alguns clados internos dentro do gênero são bem suportados e suas relações são discutidas. O gênero Glenognatha apresenta uma ampla distribuição ocupando as regiões Neártica, Neotropical, Afrotropical, Indo-Malaya, Australasia e Oceania. Vinte e sete espécies de Glenognatha são reconhecidas, quatro delas somente conhecidas por machos. Novos dados morfológicos são fornecidos para a descrição de treze espécies previamente conhecidas. Onze espécies novas são descritas: $G$. sp. nov. 1, G. sp. nov. 3, G. sp. nov. 4 e $G$. sp. nov. 7 do sudeste do Brasil, G. sp. nov. 6, G. sp. nov. 9 e $G$. sp. nov. 10 da região Amazônica, G. sp. nov. 2, G. sp. nov. 5 e $G$. sp. nov. 8 do norte da cordilheira dos Andes e G. sp. nov. 11 da região central do México e sul dos Estados Unidos. As fêmeas de G. minuta Banks, 1898, G. gaujoni Simon, 1895 e G. gloriae (Petrunkevitch, 1930) e os machos de G. globosa (Petrunkevitch, 1925) e G. hirsutissima (Berland, 1935) são descritos por primeira vez. São propostas três novas combinações em congruência com os resultados da análise cladística: Glenognatha argyrostilba (O. P.Cambridge, 1876), Glenognatha dentata (Zhu \& Wen, 1978) e Glenognatha tangi (Zhu, Song \& Zhang, 2003), todas previamente incluídas no gênero Dyschiriognatha. Quatro sinonímias são propostas: Dyschiriognatha montana Simon, 1897, Glenognatha mira Bryant, 1945 e Glenognatha maelfaiti Baert, 1987 com Glenognatha argyrostilba (O. P.-Cambridge, 1876) e Glenognatha centralis Chamberlin, 1925 com Glenognatha minuta Banks, 1898. É apresentada uma chave para a identificação e mapas de distribuição para as espécies. 


\section{INTRODUCTION}

\subsection{Tetragnathidae}

The orb-weaving spider family Tetragnathidae currently includes 47 genera and 947 species (Platnick, 2013). This family is particularly diverse in the Neotropical region which has $47 \%$ of the described genera. Most members of the family build horizontal orbicular webs on vegetation adjacent to rivers or streams (Levi, 2005), yet the group presents great morphological and ethological diversity (Levi, 1980; Eberhard, 1982; Hormiga et al., 1995; Álvarez-Padilla et al., 2009). Although tetragnathids have received increasing attention from systematic biologists in the last ten years, there is still great gaps of knowledge concerning the real richness of the family; probably only half or fewer of the species of Tetragnathidae have been described, as is the case in many other spider families (Alvarez-Padilla \& Hormiga, 2011).

Recent phylogenetic analyzes (Álvarez-Padilla, 2007; Dimitrov \& Hormiga, 2009, Álvarez-Padilla et al., 2009; Alvarez-Padilla \& Hormiga, 2011; Dimitrov \& Hormiga, 2011), support the monophyly of Tetragnathidae by the following synapomorphies: copulatory duct length less than half of the spermathecae length, femora IV mesal surface without basal macrosetae, male palpal patella with one macrosetae, male palpal tibia length approximately two times the widest point of the tibia, piriform gland spigot bases separated from the spigot shaft leaving a torus, tegulum oval to spherical and cymbial tarsal organ larger than the bases of surrounding macrosetae.

Based mainly on genitalia characters tetragnathids can be divided into four subfamilies: Tetragnathinae, Leucauginae, Metainae and Nanometinae (Álvarez-Padilla et al., 2009; Álvarez-Padilla \& Hormiga, 2011). The taxonomic composition of these subfamilies (except Metainae) has been rather stable in phylogenetic analysis regardless of data partitions and analytical approaches (Álvarez-Padilla \& Hormiga, 2011). The subfamily Tetragnathinae which includes the genera Antillognatha Bryant, 1945; Cyrtognatha Keyserling, 1881; Doryonychus Simon, 1900; Dyschiriognatha Simon, 1893; Glenognatha Simon, 1887; Hispanognatha Bryant, 1945; Tetragnatha Latreille, 1804 and Pachygnatha Sundevall, 1823, is particularly interesting among tetragnathids due to the evolution of a secondary haplogyne condition, in which fertilization ducts 
have been lost and there has been an overall simplification of female genitalia (Dimitrov et al., 2007).

Taxonomic revisions of tetragnathid genera with species distributed in the Neotropical region are scarce. In the case of Tetragnathinae, the only current revision of a group distributed in the Neotropics, was made by Dimitrov \& Hormiga (2009) for the genus Cyrtognatha. These authors established eight new combinations, described 11 new species and proposed Agriognatha O. P. Cambridge, 1896 as a junior synonym of Cyrtognatha. In addition, the monophyly of Cyrtognatha was tested and its position within Tetragnathinae discussed. The results obtained in this study highlight the importance of conducting systematic studies within Tetragnathinae.

\subsection{Taxonomic history of the genus Glenognatha}

The genus Glenognatha was erected by Simon (1887) with the type species $G$. emertoni Simon 1887, based on a male specimen collected in Arizona. Simon (1887) recognized the affinity of this species with species of the genus Pachygnatha, but stated that the well-developed tracheal spiracle, analogous with Anyphaena Sundevall, 1833, distinguished this new species from Pachygnatha. Before the erection of Glenognatha, Keyserling (1883) described the species Pachygnatha australis from males and females collected in Pacasmayo, Peru, which were subsequently transferred to Glenognatha by Simon (1894).

After these pioneers works, in the late nineteenth century and the first half of the twentieth century, 15 Glenognatha species were proposed. Seven of these species were described in Islands; five in French Polynesia: G. nigromaculata (Berland, 1933); G. hirsutissima (Berland, 1935); G. argenteoguttata (Berland, 1935); G. chamberlini (Berland, 1942) and G. phalangiops (Berland, 1942), and two in Caribbean islands: G. gloriae (Petrunkevitch, 1930) (Puerto Rico) and G. mira Bryant, 1945 (Hispaniola). The eight remaining species were described in Argentina ( $G$. lacteovittata (Mello-Leitão, 1944)), Ecuador (G. gaujoni Simon, 1895), United States of America (G. foxi (McCook, 1894)), Guyana (G. caporiaccoi Platnick, 1993), Mexico (G. minuta Banks, 1898 and G. spherella Chamberlin \& Ivie, 1936) and Panama (G. centralis Chamberlin, 1925 and

G. globosa (Petrunkevitch, 1925)). Twelve of the previously named species are known only from the type locality. Moreover, several species were initially described in other 
genera such as Dyschiriognatha (G. nigromaculata), Hivaoa Berland, 1935 ( $G$. nigromaculata, $G$. hirsutissima, $G$. argenteoguttata, $G$. chamberlini and $G$. phalangiops), Diplocephalus Bertha, 1883 (G. foxi and G. gloriae), Mimognatha Banks, 1929 (G. foxi and G. gloriae), Oedothorax Bertkau, 1883 (G. lacteovittata), Theridion Walckenaer, 1805 (G. foxi), Mysmena Simon, 1894 (G. foxi) and Cyrtognatha (G. globosa).

In the second half of the twentieth century the last four species of the genus were described, comprising 21 known species to date. Levi (1980) described G. iviei Levi, 1980 from Mississippi (USA) and conducted a taxonomic revision of Glenognatha species from north of Mexico. In this paper, an updated diagnosis of the genus and a key to the identification of species in the study area (G. foxi, G. emertoni and G. iviei) were provided. Levi's diagnosis highlights the unusual tracheal system of Glenognatha (divided median trunks) and the advance position of the tracheal spiracle, as important characters to differentiate the genus. Detailed drawings of the male genitalia and the first schematic illustrations of female genitalia were also presented. Baert (1987) described G. maelfaiti Baert, 1987 from Galapagos Islands, highlighting the importance of the conductor and cheliceral teeth to distinguish the species. Hormiga \& Döbel (1990) described G. heleios Hormiga, 1990 from New Jersey (USA) and provided illustrations of the type material of $G$. centralis and G. minuta. These authors stated that the diagnosis and description of Glenognatha might change with the study of new material, since the analysis of an undescribed species from Venezuela, revealed morphological variation underrepresented in the description suggested by Levi (1980): higher number of cheliceral teeth and presence of pleural bars. Finally, Bosmans \& Bosselaers (1994) described G. smilodon Bosmans \& Bosselaers, 1994 from Cameroon and suggested the possible synonymy between Dyschiriognatha and Glenognatha.

In the morphological atlas of Tetragnathidae (Álvarez-Padilla \& Hormiga, 2011), new diagnosis and description of Glenognatha were provided, including scanning electron micrographs of somatic and genital structures. Nevertheless, as stated by the authors, the description and diagnosis were based on G. foxi plus information included in Levi (1980) and Hormiga \& Döbel (1990) and do not cover all the variation observed across the described species. 


\subsection{Phylogenetics}

Although the monophyly of Glenognatha has never been tested, the species $G$. foxi has been widely used in studies dealing with intra-family phylogenetic relationships of Tetragnathidae (Álvarez-Padilla, 2007; Dimitrov \& Hormiga, 2009, Álvarez-Padilla et al., 2009; Alvarez-Padilla \& Hormiga, 2011; Dimitrov \& Hormiga 2011). In all cases Glenognatha fall inside the Tetragnathinae subfamily and Pachygnatha is recovered as its sister group, regardless of different data partitions (morphology, behavior, molecules) and analytical approaches (equal weights, successive and implied weighting).

\section{OBJECTIVES}

Due to the lack of a complete taxonomic revision, the differentiation between the described species of Glenognatha is ambiguous, the proportion of nominal species that could be synonymous is unknown and there is not a solid base to test the monophyly of the genus. In addition, the only antecedent of revisionary work within the genus (Levi, 1980; Hormiga \& Döbel, 1990) suggests a largely undescribed richness of the group, especially in the tropics. The aims of the present dissertation are revise the species of Glenognatha, propose a phylogenetic hypothesis among the species, develop a dichotomous key for identification of the species and elaborate maps of the geographical distribution.

\section{MATERIALS AND METHODS}

\subsection{Specimens}

A total of 5255 Glenognatha specimens (2150,$+ 2601 \precsim$ and 504 immatures), from the major arachnological collections around the world were examined. Research visits were conducted in eight museums in order to separate Glenognatha specimens form unsorted material.

Collections examined:
AMNH American Museum of Natural History, New York (N. I. Platnick)
BMNH The Natural History Museum, London (J. Beccaloni)
CAS California Academy of Sciences, San Francisco (C. Griswold)
HU Hebei University, Hebei (F. Zhang) 
IBSP Instituto Butantan, São Paulo (D. Battesti)

ICN Instituto de Ciencias Naturales, Bogotá (E. Flórez)

MACN Museo Argentino de Ciencias Naturales Bernardino Rivadavia, Buenos Aires (C. L. Scioscia)

MCN Museu de Ciências Naturais, Fundação Zoobotânica do Rio Grande do Sul, Porto Alegre (E. H. Buckup)

MCTP Museu de Ciências e Tecnologia, PUCRS, Porto Alegre (A. A. Lise)

MCZ Museum of Comparative Zoology, Harvard University, Cambridge (L. Leibensperger; G. Giribet)

MNHN Muséum National d'Historie Naturelle, Paris (C. Rollard)

MNRJ Museu Nacional Universidade Federal do Rio de Janeiro, Rio de Janeiro (A. B. Kury)

MPEG Museu Paraense Emilio Goeldi, Belém (A. B. Bonaldo)

MRAC Musée Royal de l'Afrique Centrale, Tervuren (R. Jocqué)

MUSM Museo de Historia Natural, Universidad Nacional Mayor de San Marcos, Lima (D. Silva)

MZSP Museu de Zoologia, Universidade de São Paulo, São Paulo (R. Pinto da Rocha)

RBINS Royal Belgian Institute of Natural Sciences, Brussels (L. Baert)

UFCE Universidade Federal do Ceará, (Fortaleza L. Q. Matias)

UFMG Universidade Federal de Minas Gerais, Belo Horizonte (A. Santos)

USNM National Museum of Natural History, Smithsonian Institution, Washington, D.C (J. Coddington)

ZMB Museum für Naturkunde der Humboldt- Universität, Berlim (J. Dunlop)

\subsection{Taxonomy}

General taxonomic methods and description formats follow Hormiga (1994; 2000; 2002) and Dimitrov \& Hormiga (2009). Specimens were examined and illustrated using a Leica MZ12 stereoscopic microscope with a camera lucida; further details were studied using a Zeiss Axio Scope compound microscope with a drawing tube. Nonchitinous abdominal tissue was digested with the SIGMA Pancreatin LP1750 enzyme complex (Álvarez-Padilla \& Hormiga, 2008) or Ultrazyme® enzymatic eye lens cleaner to study internal structures such as tracheae and female genitalia. Expansion of the male palp was accomplished by immersing it in a concentrated 
solution of $\mathrm{KOH}(10 \%)$ and then transferring the palp to tap water for five minutes. The male and female genitalia were examined using lactic acid as a clearing agent, then positioned for illustration on a temporary slide (Coddington, 1983). Depending on the available material, at least five female specimens were dissected for each species.

For the male palp illustrations, the left palp was used. Hairs and macrosetae are not depicted in the final drawings. All drawings were scanned and edited with the help of the program Inkscape 0.48 . Digital images of the specimens were taken using a Leica MZ 16A stereoscopic microscope with a LEICA DFC 500 digital camera. Extended focal range images were composed with Leica Application Suite version 2.5.0. In addition, Scanning Electron micrographs (SEM) were taken using a Zeiss DSM 940 at Instituto de Biociências - Universidade de São Paulo and a LEO 1430 VP at the Biological Department - George Washington University. For SEM, abdomen, cephalothorax, male palps and internal structures like trachea and female genitalia, were cleaned ultrasonically, transferred to $100 \%$ ethanol, and left to dehydrate for ca. 24 hours. Then were critically point dried, mounted, and coated with gold-palladium for observation.

Measurements (in millimeters) were taken using a scale reticle in the dissecting microscope. As stated by Hormiga (2002), the measurements of the abdomen and the total length are only approximations, because the abdomen size changes more easily in preserved specimens and its relative position in relation with the cephalothorax could vary across individuals. The distance between tracheal spiracle and spinnerets was measured from the posterior margin of the spiracle up to the posterior margin of the abdominal cuticle (Ramírez, 2003). Coloration patterns are described based on specimens preserved in $80 \%$ ethanol.

Topological terms for cheliceral views and terms to express the position of the cheliceral teeth have not been standardized in Glenognatha taxonomy (see Levi, 1980; Hormiga \& Döbel, 1990; Ramírez, 1996; Bosmans \& Bosselaers, 1994). In this work I used a standardized terminology for the teeth on the cheliceral margins and employed standardized topological terms for cheliceral views (Fig. 1), considering Okuma (1987) and Gillespie (2002). This standardization was made to facilitate hypothesis of primary homology among structures. 


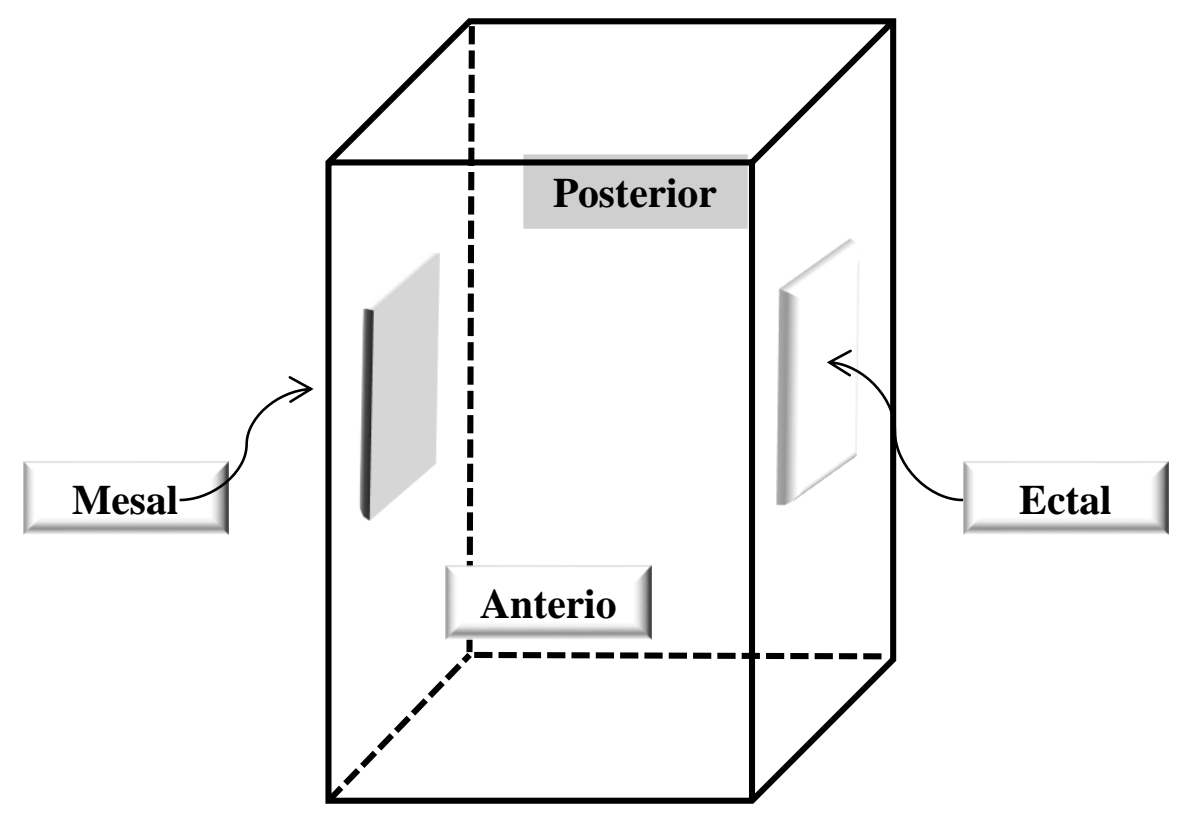

A.
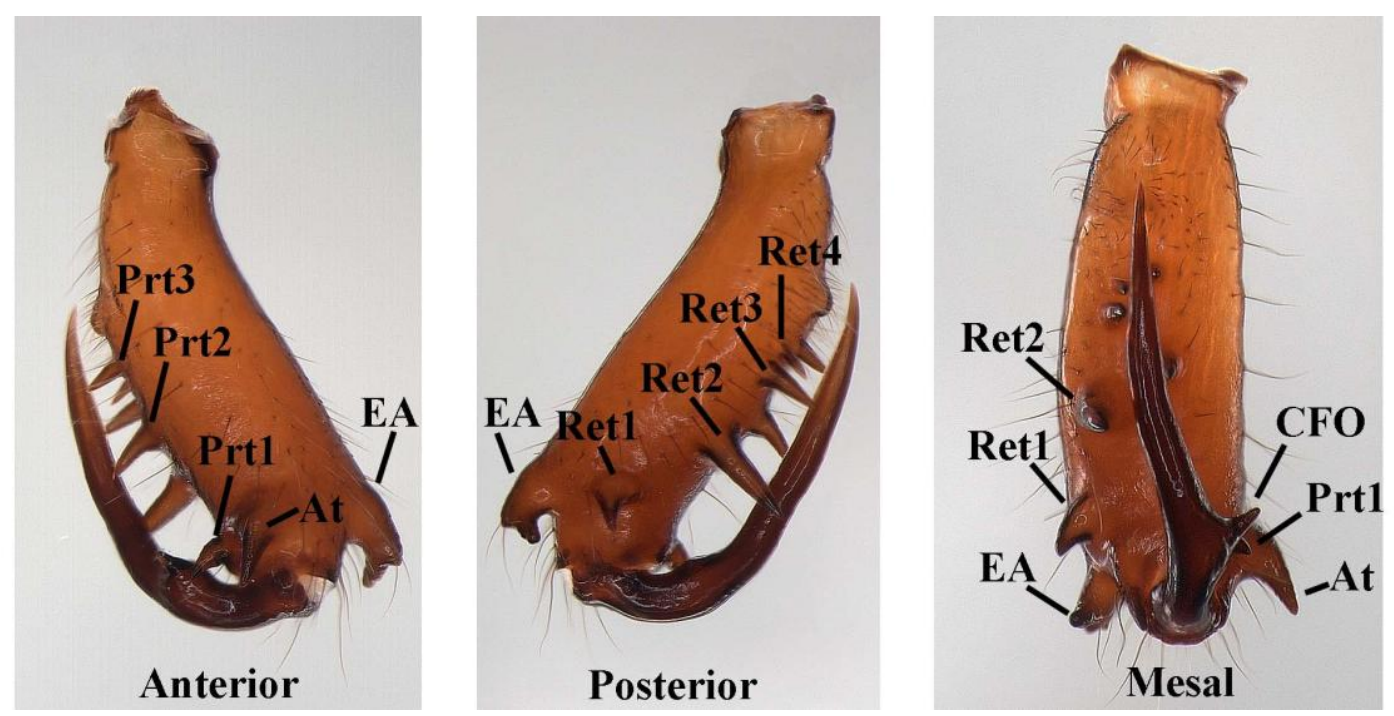

B.

Figure 1. A, topological terms used in cheliceral descriptions. B, female chelicerae of Glenognatha gaujoni indicating terminology for teeth. At: anterior tooth, CFO: cheliceral fang outgrowth, EA: ectal apophysis, Prt: promarginal tooth, Ret: retromargial tooth.

The anatomical abbreviations used in text and figures are:

Male palp

C conductor

CB cymbium

CRa conductor retrolateral apophysis 
E embolus

EMg embolic medial groove

ED ejaculatory duct

$\mathrm{F}$ fundus

$\mathrm{P}$ paracymbium

PBp paracymbium basal portion

PDp paracymbium distal portion

$\mathrm{T}$ tegulum

TO Tarsal organ

$\underline{\text { Female genitalia }}$

CD copulatory duct

Go Gonopore

LSD Long-stem ducts

$\mathrm{S}$ spermathecae

UEc uterus externus chamber

UEs uterus externus sac

$\underline{\text { Spinnerets }}$

AC aciniform gland spigots

AG aggregate gland spigots

ALS anterior lateral spinnerets

CY cylindrical gland spigots

FL flagelliform spigot

MAP major ampullate gland spigot

mAP minor ampullate gland spigot

PI piriform gland spigots

PLS posterior lateral spinnerets

PMS posterior median spinnerets

Somatic morphology

ALE anterior lateral eyes

AME anterior median eyes

AT Anterior tooth 
BL book lung

CFO Cheliceral fang outgrowth

CB Cheliceral boss

DTS Distance between tracheal spiracle and spinnerets

DTE Distance between tracheal spiracle and epigastric furrow

EA Ectal apophysis

LT Lateral tracheae

MT Median trunks

PLE posterior lateral eyes

PME posterior median eyes

Prt1 - Prt $n$ Numbers of promarginal teeth from apical end

PT Posterior tooth

Ret1 - Ret $n$ Numbers of retromarginal teeth from apical end

TAG Tracheal atrium gland

TS Posterior tracheal spiracle

\subsection{Specimens data}

Names for districts, provinces, altitude and coordinates, not included in the original labels, were obtained from Google Earth (Lat/Lon-WGS84). Data inferred from a secondary source are in [square brackets] in the material examined section.

\subsection{Natural history data}

In order to observe and document natural history aspects of Glenognatha species, field trips were conducted into three municipalities in southwestern Colombia. Webs were dusted with cornstarch (Eberhard, 1976) and photographed with a Sony Cybershot DSC-W610 camera. In addition, a comprehensive literature search was made in order to compile previous known data.

\subsection{Ingroup}

After the taxonomic revision conducted in this work, the known richness of Glenognatha was reduced from 21 to 18 species, due to three synonymies. Because of lost types and unavailable material in the examined collections, five described species of Glenognatha (G. caporiaccoi, G. phalangiops, G. nigromaculata, G. chamberlini and $G$. argenteoguttata) were not included in the ingroup. The final sample consists of 24 
Glenognatha species (13 described and 11 undescribed). Characters were scored following direct observation on specimens. See appendix 1 for a list of examined material for character scoring.

\subsection{Outgroup}

The outgroup selection was based on the phylogenetic hypothesis of ÁlvarezPadilla \& Hormiga (2011). In the preferred topology of this study Glenognatha falls into the subfamily Tetragnathinae and Pachygnatha is obtained as its sister group. In addition, the genus Tetragnatha is recovered as sister of the clade Glenognatha + Pachygnatha and the genus Cyrtognatha as sister to all these genera. The outgroup consists of a total of seven species, four representing the aforementioned genera (Pachygnatha autumnalis Marx, 1884, Pachygnatha clercki Sundevall, 1823, Tetragnatha versicolor Marx, 1884 and Cyrtognatha espanola (Bryant, 1945)), and three species of the genus Dyschiriognatha (D. argyrostilba (O. P.-Cambridge, 1876), D. dentata Zhu \& Wen, 1978 and D. tangi Zhu, Song \& Zhang, 2003), that has been suggested as closely related to Glenognatha (Bosmans \& Bosselaers, 1994). Chrysometa alajuela Levi, 1986 was used to root the cladogram according with its position in the Metainae subfamily (Álvarez-Padilla \& Hormiga, 2011). Characters were scored following direct observation on specimens, with the exception of $C$. espanola and Ch. alajuela that were scored according with the detailed studies of Álvarez-Padilla \& Hormiga (2011) and Dimitrov \& Hormiga (2009). See appendix 1 for a list of examined material for character scoring.

\subsection{Characters}

The data set for phylogenetic analysis comprises 42 new characters here proposed and 40 previously used (Miller \& Hormiga, 2004; Dimitrov \& Hormiga, 2009; Álvarez-Padilla \& Hormiga, 2011) (Appendix 2). Characters were grouped into five sets: spinnerets spigots and epiandrous fusules, male papal morphology, female genitalia, cephalothorax morphology (including legs) and abdomen morphology (including tracheal system). Four continuous characters were included in the analysis. Non-parametric tests and visualization of graphed data were conducted in an attempt to discretize these characters in valid states (Ramírez, 2003; Scharanschkin \& Doyle, 2006; de Bivort et al., 2010) For this purpose, measurements of three specimens per species, covering the known range of distribution and depending on the available 
material, were took. All the measurements were size-corrected by dividing by the carapace length. Kruskal-Wallis and Mann-Whitney tests were conducted in Statistica 7.0 (Statsfot, 2004) in order to test significant differences $(\mathrm{p}<0.05)$ between character states.

Mesquite v. 2.75 (Maddison \& Maddison, 2011) was used to edit the data matrix. Winclada v. 1.00 .08 (Nixon 2002) was used for characters state optimizations and tree editing. The nine multistate characters were treated as non-additive (Fitch, 1971) and ambiguous character optimizations were resolved to favor reversal or secondary loss over convergence (ACCTRAN). Character definitions follow Sereno (2007). Due to its descriptive value and its possible utility in future phylogenetic studies autapomorphies were considered in the analysis.

\subsection{Phylogenetic analysis}

Analysis were run in TNT v. 1.1 (Goloboff et al., 2008) using the traditional search and new technology search options. In order to facilitate the reproduction of each analysis, script files with commands and instructions for each case were created (Ojanguren-Affilastro \& Ramírez, 2009).

Heuristic tree searches. Heuristic searches, under equal weights (EW), consisted of 5000 replicates of random addition sequences (RAS), followed by tree bisectionreconnection branch swapping (TBR), holding 100 trees per iteration (commands: mult=tbr repl 5000 hold 100;). Minimum length $=0$ was used as a collapsing rule in all searches. If the most parsimonious resolutions were found in few replications, more aggressive searches iterating with cycles of the Parsimony Ratchet were used (Ojanguren-Affilastro \& Ramírez, 2009, Frick \& Sharff, 2013) (commands: ratchet:iter 1000; mult=ratchet repl 1000 tbr hold 500;).

Sensitivity analysis. In order to assess the clade sensitivity to the variation in analytical parameters (Giribet, 2003; Goloboff et al., 2008), parsimony analysis under implied weights (IW) were performed. This weighting method assigns higher weight to those characters with less homoplasy (higher fit), and the sum of weights over all characters (total fit) is maximized during tree search (Ramírez, 2003). The fit of a character is calculated as: $\mathrm{f}=k /(\mathrm{s}+k)$, where $\mathrm{s}=$ number of homoplastic steps and $\mathrm{k}$ is a user-defined concavity constant. Although Ramírez (2003) used a sensitivity analysis as 
a metacriterion to determine an optimal value for $k$, recent publications agree to explore different concavities in order to produce more conservative conclusions (Goloboff et al., 2008; Mirande, 2009; Ojanguren-Affilastro \& Ramírez, 2009; Vega et al., 2009; Mendes, 2011; Daza et al., 2012; Magalhães \& Santos 2012).

The sensitivity of the results to differences in the strength of the weighting function was analyzed performing heuristic searches with $k=1-15,20,50$ and 100. The search parameters were the same as the equal weight analysis. The frequency of each group under these weighting regimes was reported in the preferred tree.

Measures of tree support. Three measures of group support were calculated: Absolute Bremer support (ABS) (Bremer, 1988, 1994), group frequencies under jackknifing (JK) and symmetric resampling (SR) (Goloboff et al., 2003). For ABS, heuristic searches by TBR swapping retaining suboptimal trees were conducted. Under equal weight analysis, a rough precedent search setting suboptimal to 20 was made to find the upper limit of supports (commands: hold 10000; sub 20; bb = fillonly tbr; bsupport;). Then 20 cycles were run increasing the suboptimal stepwise in 1 and the tree buffer in 1000 each cycle (Gravish-Regev et al., 2013) (commands: sub 1; hold 1000; bb = fillonly tbr; sub 2; hold 2000; bb = fillonly tbr; ... sub 20; hold 20000; bb= fillonly tbr; bsupport;). For ABS under implied weights analysis, values are reported in units of fit. A rough precedent search setting suboptimal to 2 was made to find the upper limit of supports (commands: hold 10000; sub 2; bb = fillonly tbr; bsupport;). Then 20 cycles were run increasing the suboptimal stepwise in 0.1 units of fit and the tree buffer in 1000 each cycle (commands: sub 0.1; hold 1000; bb = fillonly tbr; sub 0.2; hold 2000; bb = fillonly tbr; ... sub 2; hold 20000; bb = fillonly tbr; bsupport;).

For the Jackknife support, I performed 1000 pseudoreplicates of 10 random sequence additions, keeping 10 trees each, using TBR swapping (commands: mult:noratchet repl 100 tbr hold 10; resample jak repl 1000 freq from 0 [mult];). A probability of elimination $\mathrm{P}=0.36$ was used. Symmetrical resampling was calculated as described for Jackknife support and the results are reported in differences of group frequency values (GC) (Goloboff et al., 2003). 
Taxon removal. In order to explore the effect of high percentages of missing data per taxon in the analysis, two matrices were analyzed: complete matrix, which include all terminals and reduced matrix, which exclude species only known from males $(G$. iviei, G. smilodon, G. sp. nov. 9 and G. sp. nov. 10).

\section{RESULTS AND DISCUSSION}

\subsection{Topologies}

Equal weight analysis. Heuristic searches for the complete matrix resulted in 92 most parsimonious trees (MPTs), with 161 steps, $\mathrm{CI}=0.57$ and $\mathrm{RI}=0.72$ (143 steps, $\mathrm{CI}=0.51, \mathrm{RI}=0.72$ removing uninformative characters). As evidenced by the strict consensus tree (Fig. 2), all topological differences between MPTs are the consequence of dissimilar hypothesis of relationships within Glenognatha. Searches for the reduced matrix, generated eight MPTs with 154 steps, $\mathrm{CI}=0.58$ and $\mathrm{RI}=0.72(137$ steps, $\mathrm{CI}=$ $0.53, \mathrm{RI}=0.73$ removing uninformative characters). The strict consensus of these eight trees (Fig. 3) is quite similar to that obtained with the complete matrix (Fig. 2).

Despite conflicting relationships within Glenognatha, equally weighted analysis recovered its monophyly with high support values (Figs. 2-3). In addition, several monophyletic groups are recovered within the genus; one including the three representatives of the genus Dyschiriognatha.

Sensitivity analysis. Results of the heuristic searches with the defined $k$ values are presented in Table 1. The analysis of the complete matrix under implied weighting resulted in four similar topologies (Figs. 4-5). The main differences across these trees are related with the position of $G$. foxi, G. hirsutissima, G. sp. nov. 1, G. sp. nov. 9 and G. sp. nov. 10. Only with $k \geq 8$ a fully resolved tree was found (Fig. 5B), this topology was also obtained under equal weights. The analysis of the reduced matrix resulted in three alternative topologies (Fig. 6). Only with $k \geq 4$ a fully resolved tree was obtained (Fig. 6C). Overall, these hypotheses were very similar to that found with the complete matrix.

Considering the stability of the results and the full compatibility with the most stable topology obtained with the reduced matrix analysis (Fig. 6C), the tree generated under $k=8$ for the complete matrix was chose as the preferred hypothesis. Support 
values and sensitivity of this tree to concavity changes are presented in figure 7. Character optimizations are presented in figure 8.

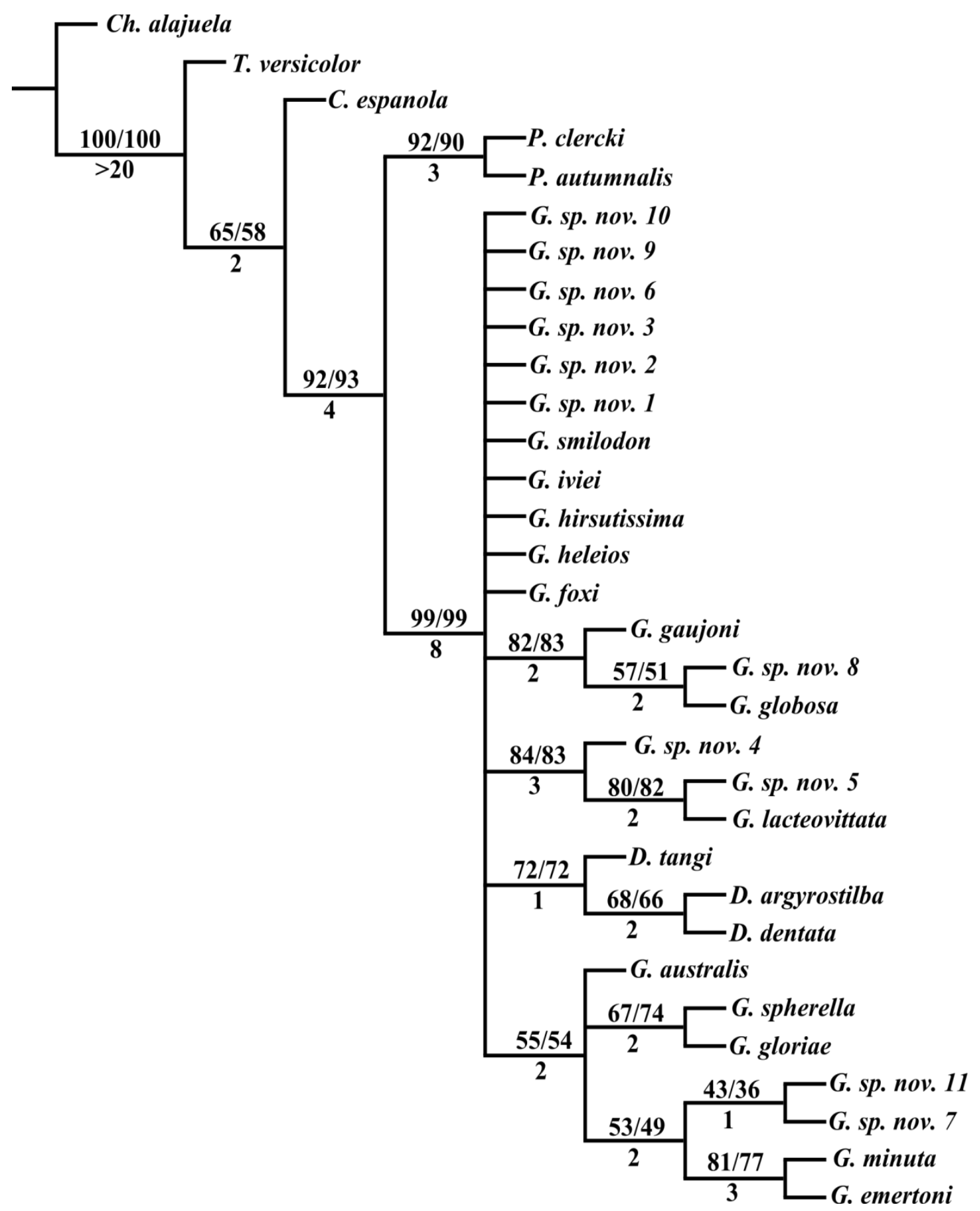

Figure 2. Strict consensus of 92 most parsimonious trees found under equal weights analyzing the complete matrix $(\mathrm{L}=187, \mathrm{CI}=0.49, \mathrm{RI}=0.62)$. Numbers above branches are Jackknife percentages (left) and Symmetric resampling GC values (right). Numbers below branches are Bremer support values. 


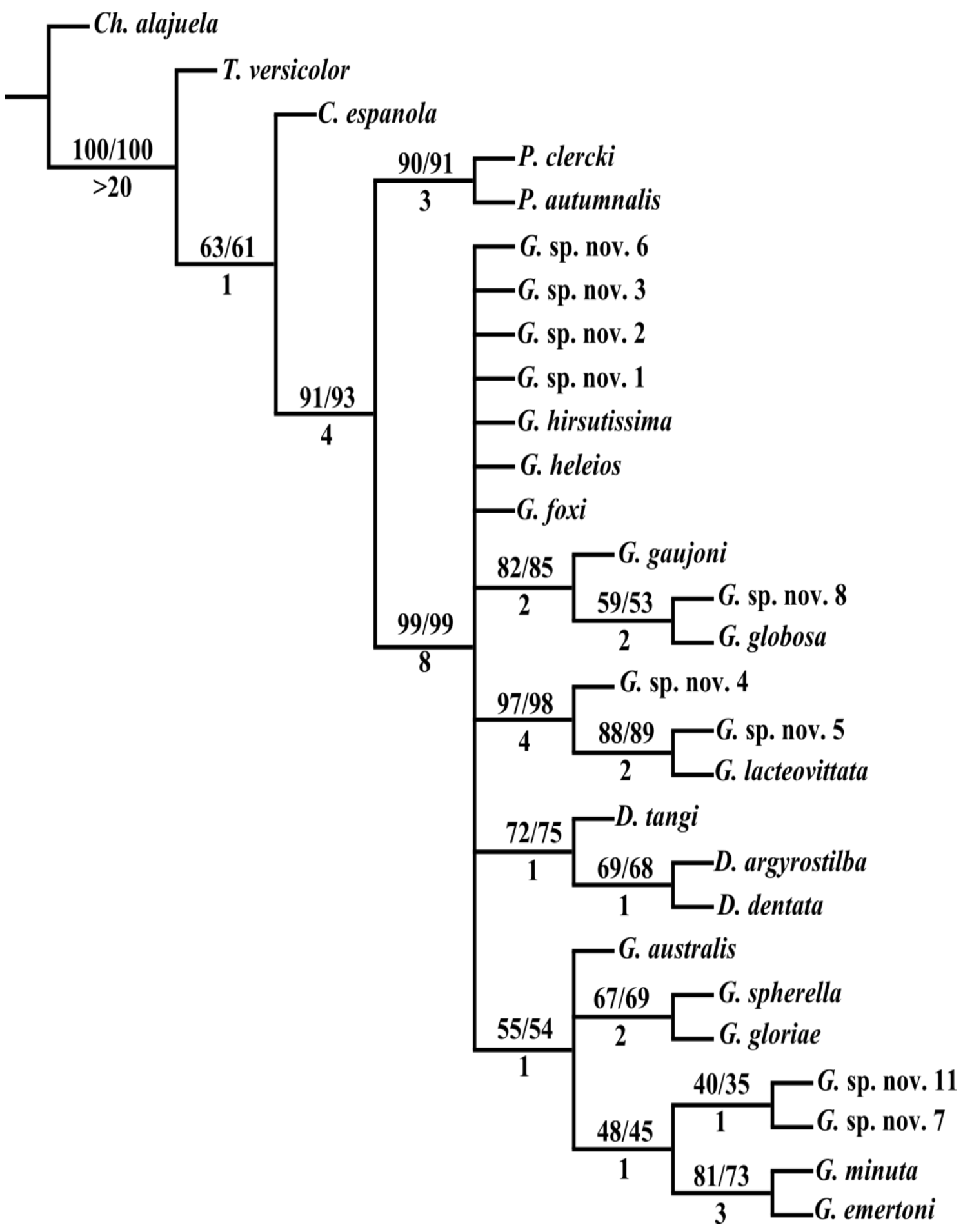

Figure 3. Strict consensus of eight most parsimonious trees found under equal weights analyzing the reduced matrix $(\mathrm{L}=173, \mathrm{CI}=0.52, \mathrm{RI}=0.64)$. Numbers above branches are Jackknife percentages (left) and Symmetric resampling GC values (right). Numbers below branches are Bremer support values. 
Table 1. Summary statistics from the implied weighting analysis. K: concavity constant, N: number of most parsimonious trees, L: tree length, F: total fit.

\begin{tabular}{|c|c|c|c|c|c|c|}
\hline & \multicolumn{3}{|c|}{ Complete matrix } & \multicolumn{3}{|c|}{ Reduced matrix } \\
\hline $\mathrm{K}$ & $\mathrm{N}$ & $\mathrm{L}$ & $\mathrm{F}$ & $\mathrm{N}$ & $\mathrm{L}$ & $\mathrm{F}$ \\
\hline 1 & 2 & 164 & 45.75 & 6 & 157 & 44.86 \\
\hline 2 & 8 & 164 & 50.34 & 6 & 157 & 49.15 \\
\hline 3 & 1 & 162 & 53.07 & 2 & 155 & 51.74 \\
\hline 4 & 1 & 162 & 54.91 & 1 & 154 & 53.49 \\
\hline 5 & 1 & 162 & 56.24 & 1 & 154 & 54.77 \\
\hline 6 & 1 & 162 & 57.25 & 1 & 154 & 55.73 \\
\hline 7 & 1 & 162 & 58.05 & 1 & 154 & 56.49 \\
\hline 8 & 1 & 161 & 58.69 & 1 & 154 & 57.10 \\
\hline 9 & 1 & 161 & 59.23 & 1 & 154 & 57.61 \\
\hline 10 & 1 & 161 & 59.68 & 1 & 154 & 58.03 \\
\hline 11 & 1 & 161 & 60.06 & 1 & 154 & 58.40 \\
\hline 12 & 1 & 161 & 60.40 & 1 & 154 & 58.71 \\
\hline 13 & 1 & 161 & 60.69 & 1 & 154 & 58.98 \\
\hline 14 & 1 & 161 & 60.94 & 1 & 154 & 59.22 \\
\hline 15 & 1 & 161 & 61.17 & 1 & 154 & 59.43 \\
\hline 20 & 1 & 161 & 62.01 & 1 & 154 & 60.21 \\
\hline 50 & 1 & 161 & 63.70 & 1 & 154 & 61.79 \\
\hline 100 & 1 & 161 & 64.33 & 1 & 154 & 62.38 \\
\hline
\end{tabular}



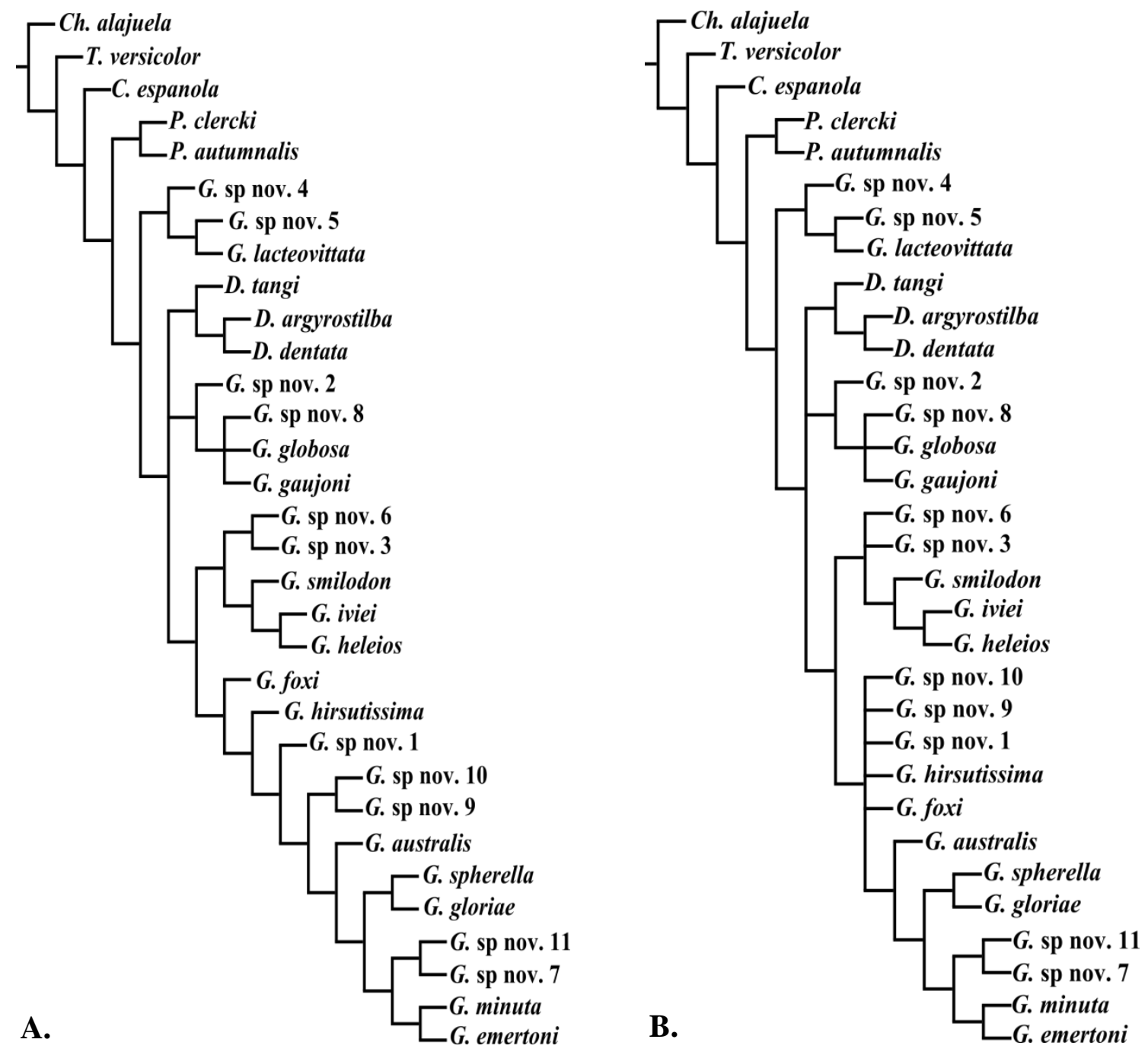

Figure 4. Strict consensus of the most parsimonious trees obtained analyzing the complete matrix under implied weighting. A, under $k=1(\mathrm{~L}=165, \mathrm{CI}=0.55, \mathrm{RI}=0.70)$, B, under $k=2(\mathrm{~L}=170, \mathrm{CI}=0.54, \mathrm{RI}=0.68)$. 

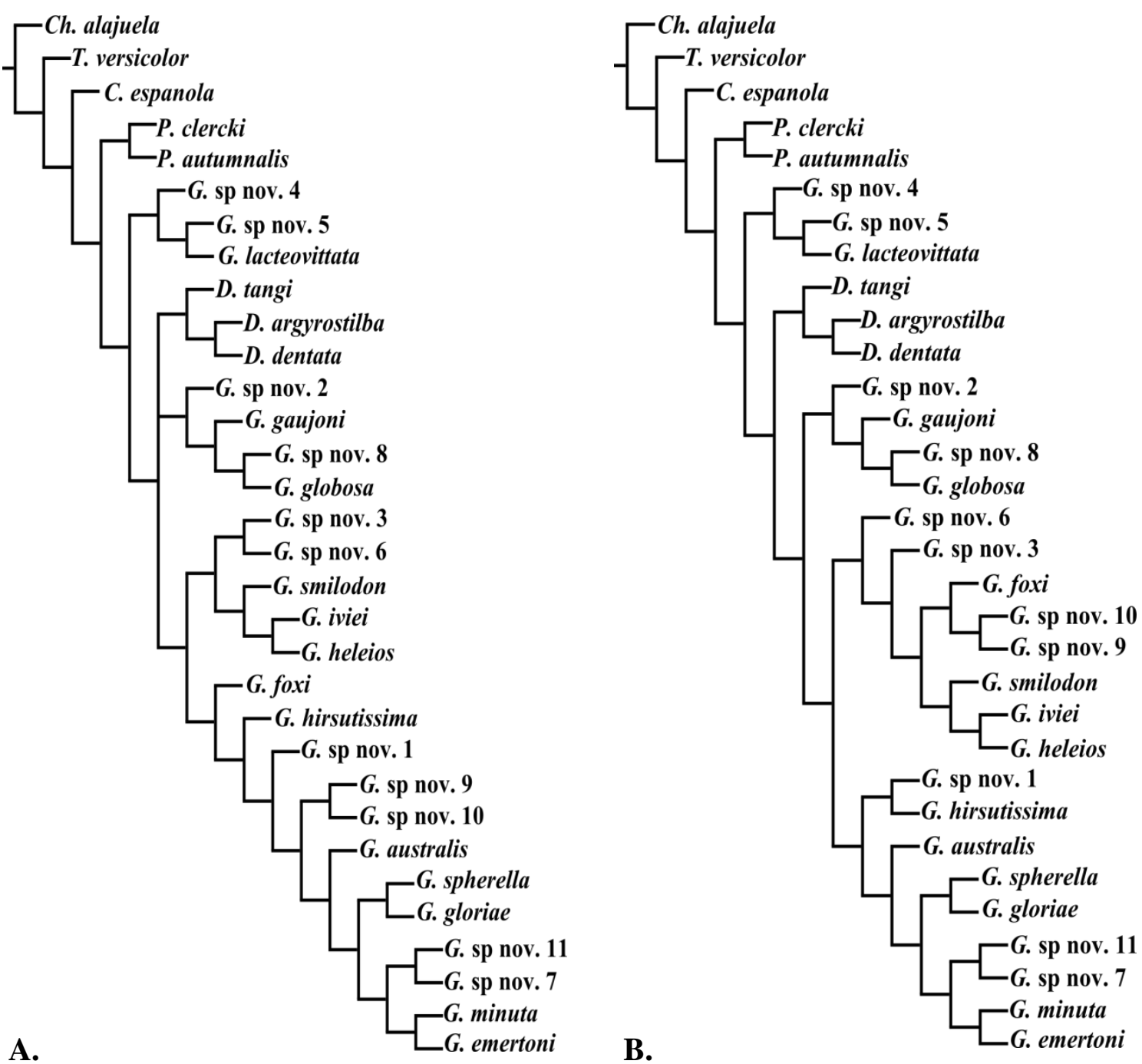

Figure 5. Strict consensus of the most parsimonious trees obtained analyzing the complete matrix under implied weighting. A, under $k=3-7(\mathrm{~L}=162, \mathrm{CI}=0.56$, $\mathrm{RI}=0.71), \mathrm{B}$, under $k \geq 8 \quad(\mathrm{~L}=161, \mathrm{CI}=0.57, \mathrm{RI}=0.72)$. 

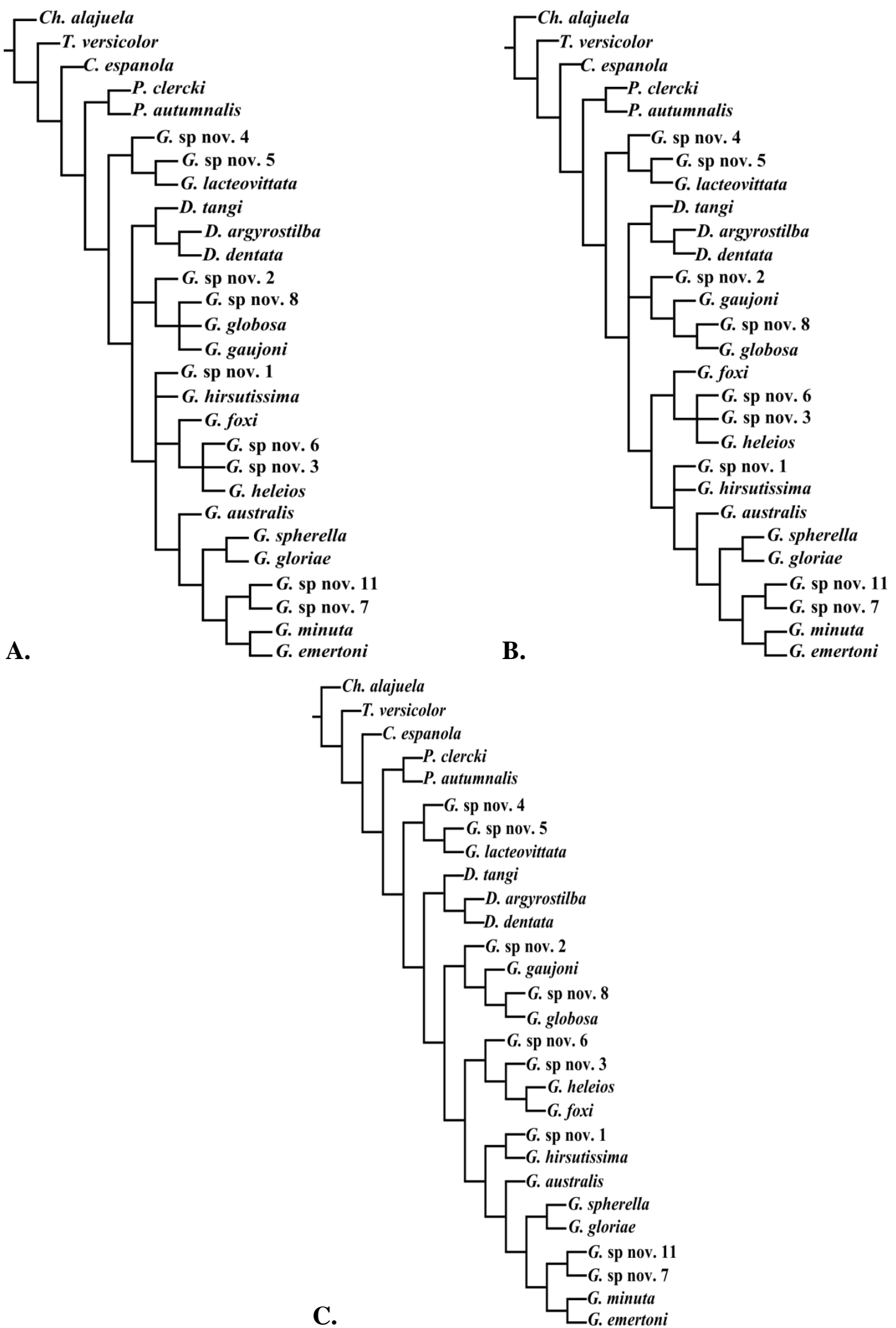

Figure 6. Strict consensus of the most parsimonious trees obtained analyzing the reduced matrix under implied weighting. $\mathbf{A}$, under $k=1-2(\mathrm{~L}=162, \mathrm{CI}=0.55, \mathrm{RI}=0.69)$, B, under $k=3(\mathrm{~L}=158, \mathrm{CI}=0.57, \mathrm{RI}=0.71), \mathrm{C}$. Under $k \geq 4(\mathrm{~L}=154, \mathrm{CI}=0.58, \mathrm{RI}=0.72)$. 


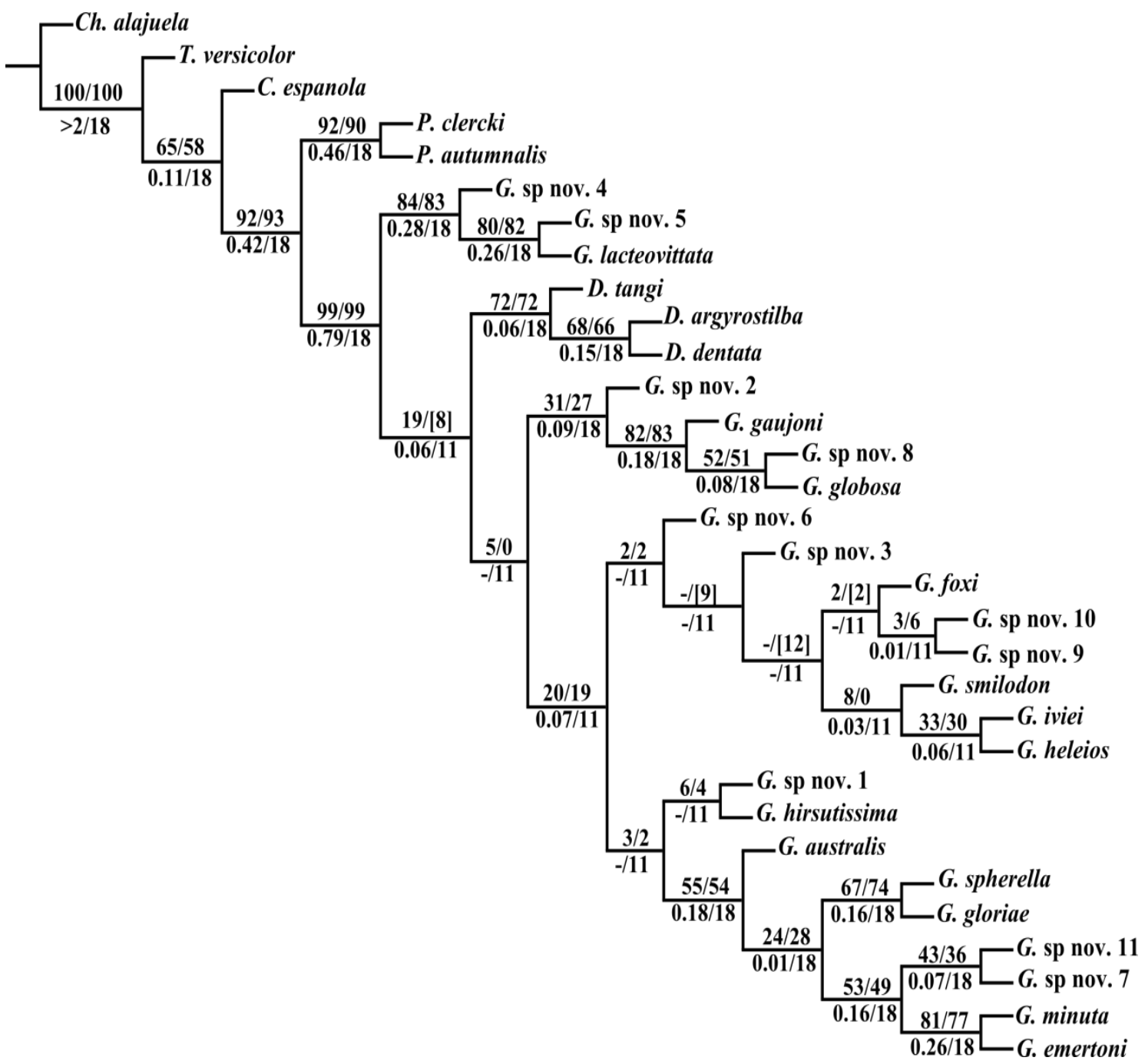

Figure 7. Topology obtained analyzing the complete matrix under $k=8$. Numbers above branches are Jackknife percentages (left) and Symmetric resampling GC values (right). Numbers below branches are Bremer support values in units of fit (left) and sensitivity frequencies (right). 


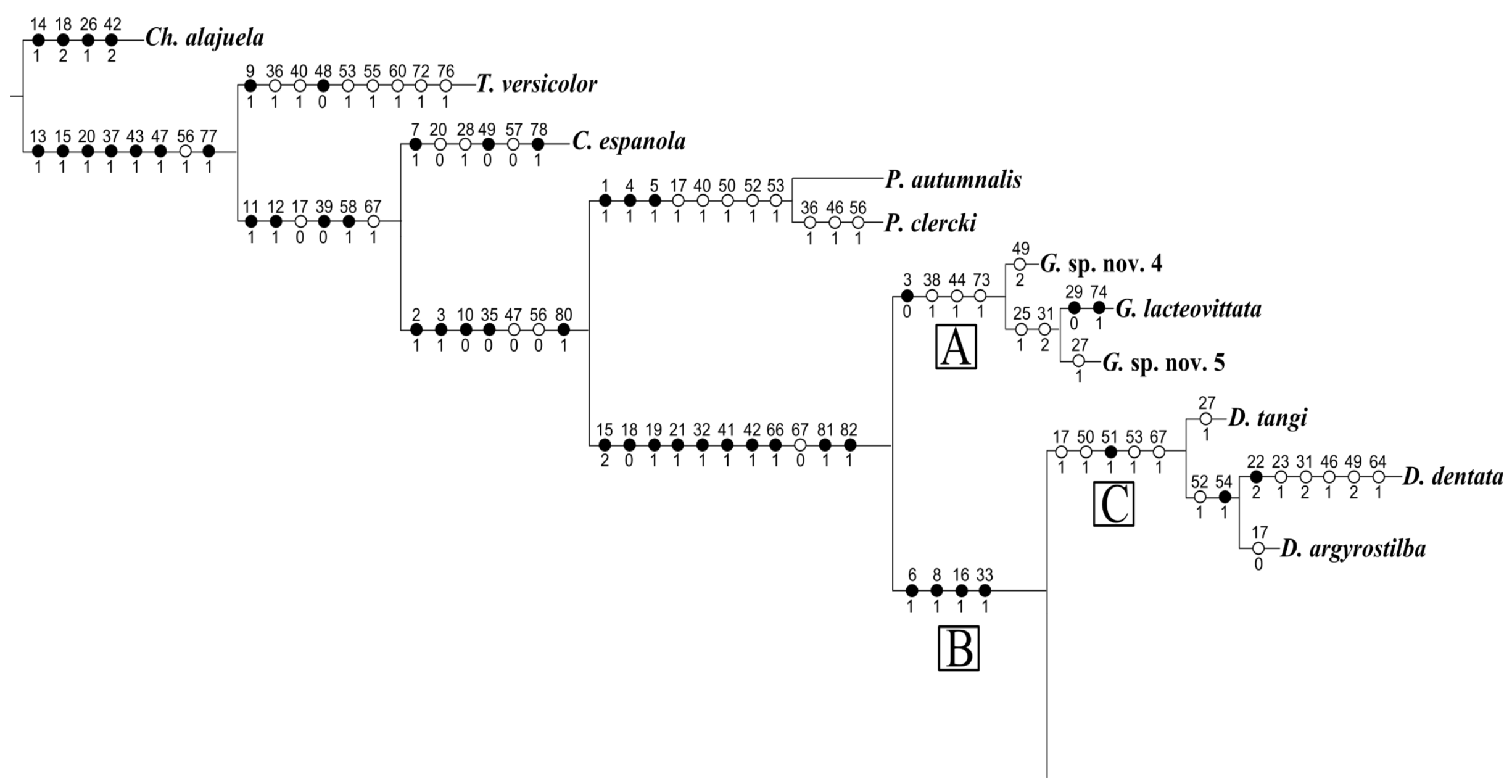

Figure 8. Topology obtained analyzing the complete matrix under $k=8$. Filled and open circles represent nonhomoplasious and homoplasious transformations, respectively. Character numbers are placed over the branches and the states are shown below the branches. Letters denote clade names. 


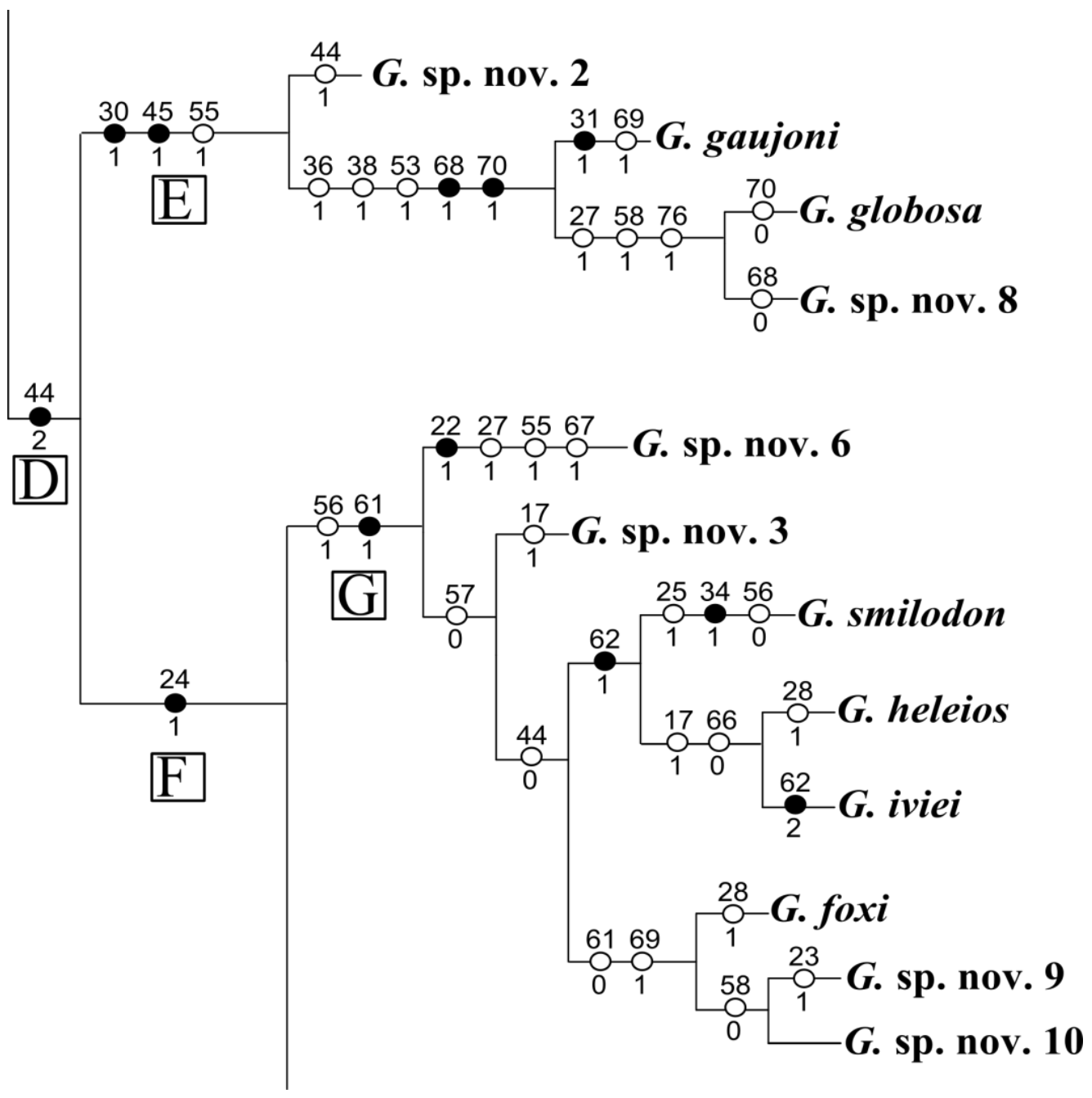

Figure 8. Continued. 


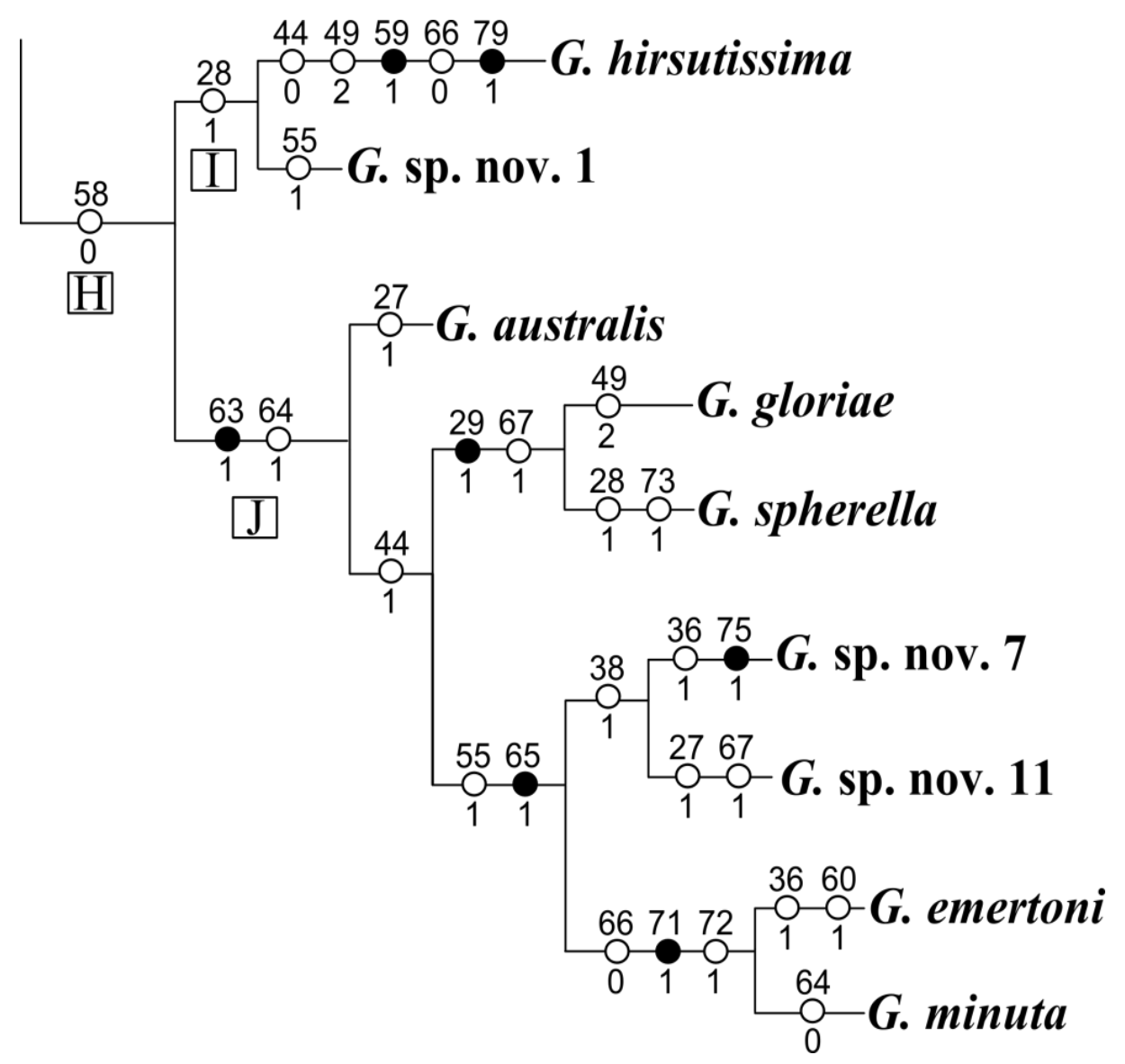

Figure 8. Continued. 


\subsection{Character description}

Character descriptions include length $(\mathbf{L})$, consistency index $(\mathbf{C I})$, retention index (RI) and Fit (F) values for the preferred tree (complete matrix analysis under $\mathrm{k}=8$ ). For characters used in previous works I added the reference to its most recent usage by means of the following code: Miller \& Hormiga, 2004=MH04, Arnedo et al., 2009=AHS09, Dimitrov \& Hormiga, 2009=DH09, Lopardo et al., 2011= LGH11 and Álvarez-Padilla \& Hormiga, 2011 =APH11. New characters are marked with an asterisk symbol (*). Underlined characters correspond to those where a state have been added, erased or changed.

\section{Spinnerets spigots and epiandrous fusules}

1. PMS, nubbin: ( $\mathrm{L}=1, \mathrm{CI}=1, \mathrm{RI}=1)(\mathrm{APH} 11)$

(0) present (Fig. 9).

(1) absent.

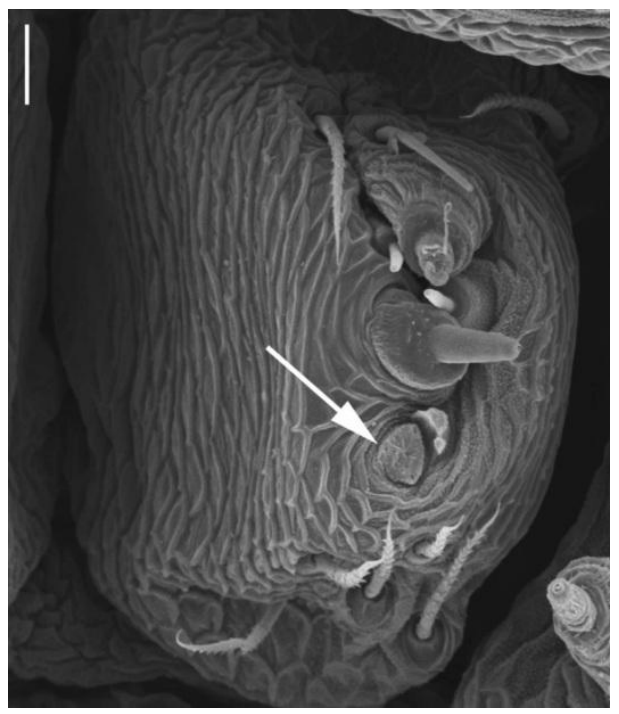

Figure 9. Glenognatha gaujoni. Female posterior median spinnerets (arrow, nubbin).

Two types of cuticular protuberances can be distinguished in the spinning field of adult araneoid spiders: nubbins and tartipores. The former refer to a partially formed nonfunctional vestigial spigot and the latter to a cuticular scar with a collared opening after molting (Moon 2012). Considering my taxonomic sampling, the loss of the PMS nubbin optimizes as synapomorphic for the genus Pachygnatha. Nevertheless, several independent losses have been occurred outside Tetragnathidae in members of the "reduced piriform clade" (symphytognathoid, linyphioids, theridioids, and cyatholipoids families) (Álvarez-Padilla \& Hormiga 2011). 


\section{Female, PLS mesal cylindrical spigot base, size relative to the distal spigot:}

$(\mathrm{L}=1, \mathrm{CI}=1, \mathrm{RI}=1, \mathrm{~F}=1)(\mathrm{APH} 11)$

(0) same size (Álvarez-Padilla \& Hormiga, 2011: fig. 26D);

(1) larger (Fig. 10).

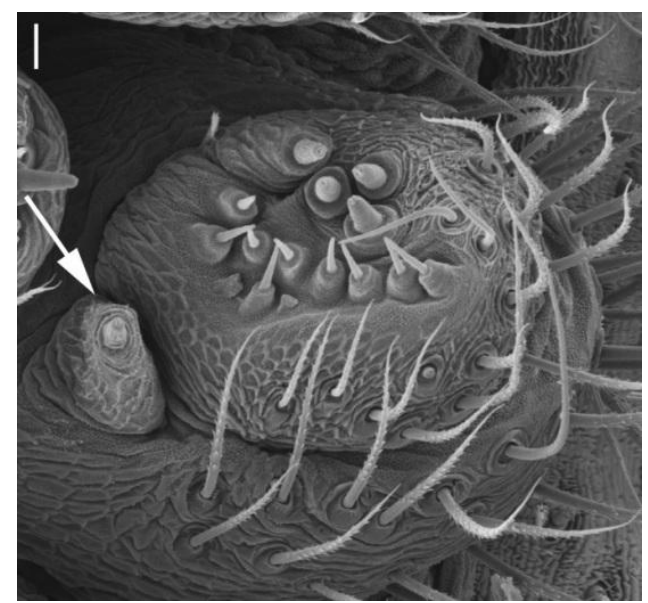

Figure 10. Glenognatha gaujoni. Female posterior lateral spinnerets (arrow, mesal cylindrical spigot).

An enlarged mesal cylindrical spigot is a synapomorphy for Pachygnatha + Glenognatha. However, this condition is also found outside Tetragnathidae in Symphytognathidae + Anapidae and Linyphiidae + Pimoidae (Griswold et al., 1998).

3. PMS, aciniform gland spigots, quantity: $(\mathrm{L}=2, \mathrm{CI}=1, \mathrm{RI}=1, \mathrm{~F}=1)$ (AHS09)

(0) one (Fig.11A);

(1) two (Fig. 11B);

(2) three.

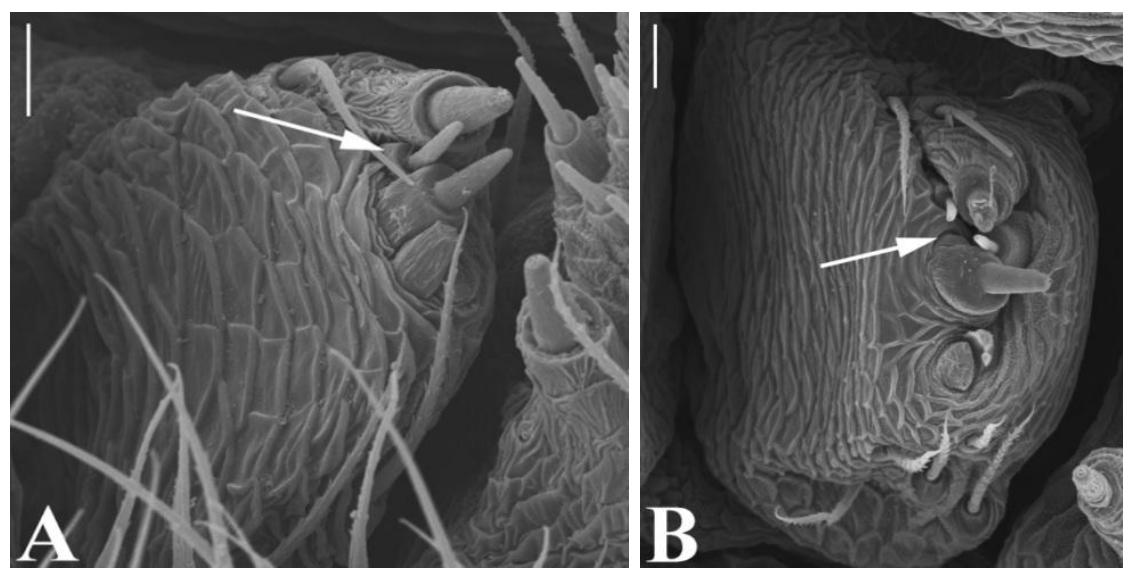

Figure 11. Female posterior median spinnerets. A, Glenognatha lacteovittata. B, Glenognatha gaujoni. (arrow, aciniform spigots). 
The number of aciniform gland spigots is variable in Tetragnathidae (ÁlvarezPadilla \& Hormiga 2011) and other araneoid families like Linyphiidae (Miller \& Hormiga 2004). In the present analysis, two AC spigots is a synapomorphy for Pachygnatha + Glenognatha. The reduction to one spigot supports the clade A.

4. Female, aggregate spigots: $(\mathrm{L}=1, \mathrm{CI}=1, \mathrm{RI}=1, \mathrm{~F}=1)(\mathrm{APH} 11)$

(0) present (Fig. 12).

(1) absent.

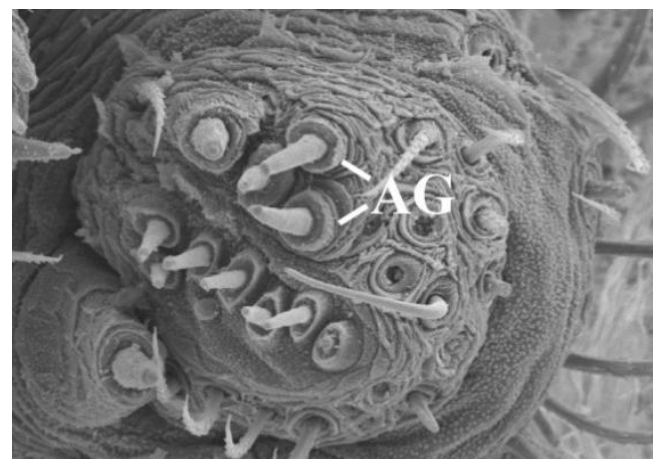

Figure 12. Glenognatha australis. Female posterior lateral spinnerets. AG: aggregate gland spigots.

The aggregate gland spigots coat the flagelliform fiber with sticky glue as it is spun (Griswold et al., 2005). Aggregate glands are synapomorphic for Araneoidea with several secondary losses (Griswold et al., 2005). The absence of aggregate spigot supports Pachygnatha.

5. Female, flagelliform spigots: $(\mathrm{L}=1, \mathrm{CI}=1, \mathrm{RI}=1, \mathrm{~F}=1)(\mathrm{APH} 11)$.

(0) present (Fig. 13).

(1) absent.

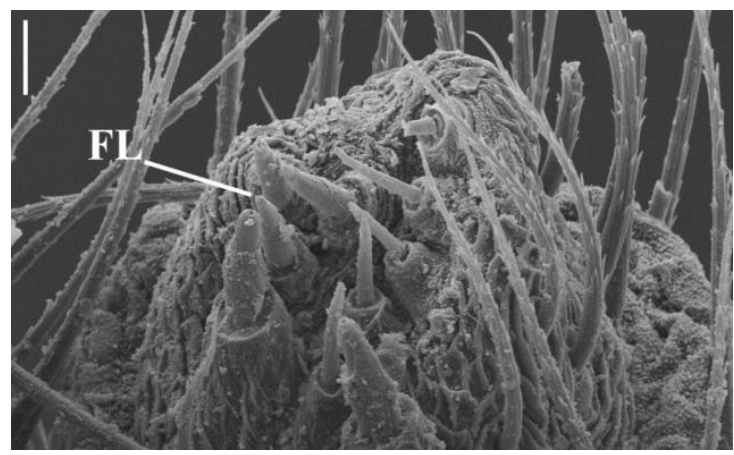

Figure 13. Glenognatha emertoni. Female posterior lateral spinnerets. FL: flagelliform gland spigot. 
Flagelliform glands produce the axial fibers onto which the viscid silk is dropped (Álvarez-Padilla \& Hormiga, 2011). In the present analysis, the absence of flagelliform spigot supports Pachygnatha.

\section{PLS aciniform gland spigots, arrangement*: $(\mathrm{L}=1, \mathrm{CI}=1, \mathrm{RI}=1, \mathrm{~F}=1)$}

(0) two or more irregular lines (Fig. 14A);

(1) one almost straight line (Figs. 14B).
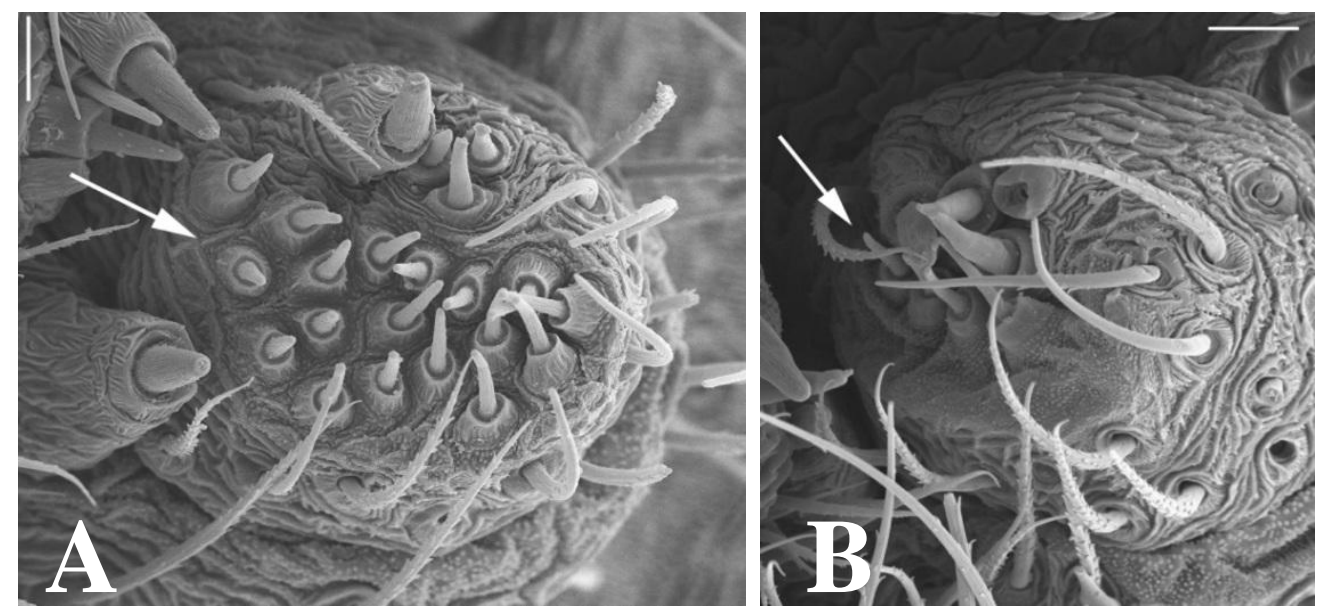

Figure 14. Female posterior lateral spinnerets. A, Glenognatha lacteovittata. B, Glenognatha sp. nov. 7 (arrow, aciniform spigots).

Most tetragnathids have two or more irregular lines of aciniform spigots in the PLS spinning field (Álvarez-Padilla \& Hormiga, 2011 figs. 56D, 77E, 100D, 109F, 114G). PLS aciniform gland spigots arranged in an almost straight line is synapomorphic for the clade B. Specimens with one or two mesally displaced spigots were coded as (1) (Fig. 12). The disposition of aciniform spigots in the PLS could be related with web architecture as noted by Hormiga et al. (2007) and Eberhard (2010). Detailed web-building behavioral data could reveal the possible functional significance of these contrasting arrangements within Glenognatha.

7. PLS, macrosetae on the retrolateral surface: $(\mathrm{L}=1, \mathrm{CI}=1, \mathrm{RI}=1)(\mathrm{DH} 09)$

(0) absent;

(1) present (Dimitrov \& Hormiga, 2009: figs. 28G, 28J, 35E).

The presence of conspicuous macrosetae on the retrolateral surface of the PLS is autapomophic for the genus Cyrtognatha (Dimitrov \& Hormiga, 2009) 
8. Epiandrous fusules, quantity: $(\mathrm{L}=1, \mathrm{CI}=1, \mathrm{RI}=1, \mathrm{~F}=1)(\mathrm{LGH} 11)$

(0) six or more (Fig. 15A).

(1) four (Fig. 15B).
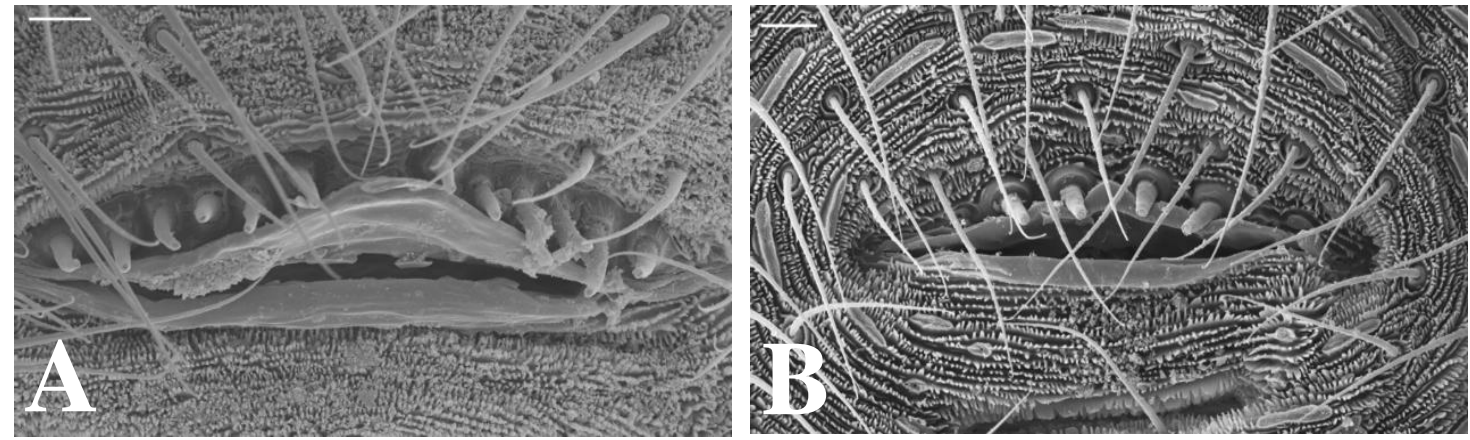

Figure 15. Epiandrous fusules. A, Glenognatha sp. nov. 5. B, Dyschiriognatha dentata.

A high number (six or more, state 0 ) of epiandrous fusules is the widespread pattern in tetragnathids (Álvarez-Padilla \& Hormiga, 2011 figs. 56F, 82G, 100F, 109F, 114F). A reduced number of these fusules (state 1) optimizes as a synapomorphy of the clade B.

9. Epiandrous fusules, arrangement: ( $L=1, \mathrm{CI}=1, \mathrm{RI}=1)(\mathrm{APH} 11)$

(0) one transverse line (Fig. 16);

(1) two main clusters (Álvarez-Padilla \& Hormiga, 2011: fig. 109C).

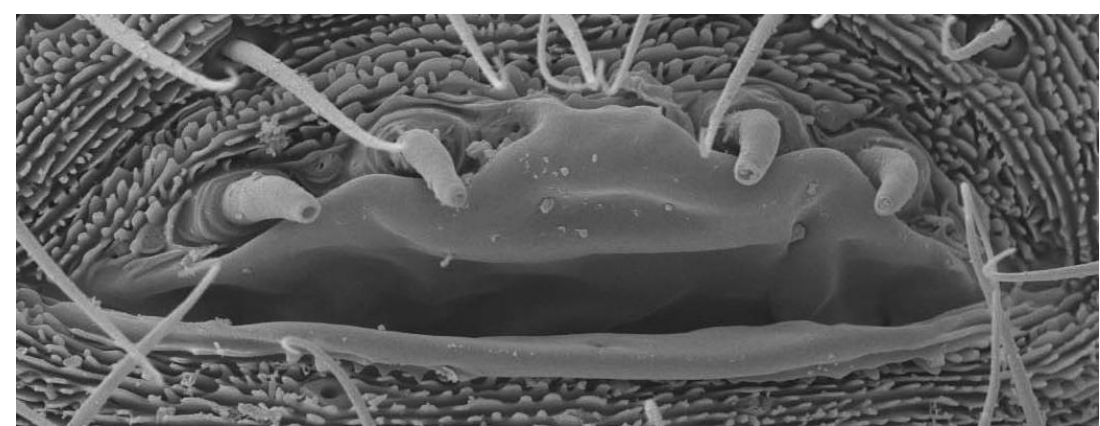

Figure 16. Glenognatha sp. nov. 2. Epiandrous fusules.

Epiandrous fusules arranged in two main cluster optimizes as an autapomorphy for $T$. versicolor. 
10. Epiandrous fusules, pits: $(\mathrm{L}=1, \mathrm{CI}=1, \mathrm{RI}=1, \mathrm{~F}=1)(\mathrm{APH} 11)$

(0) absent (Fig. 17);

(1) present.

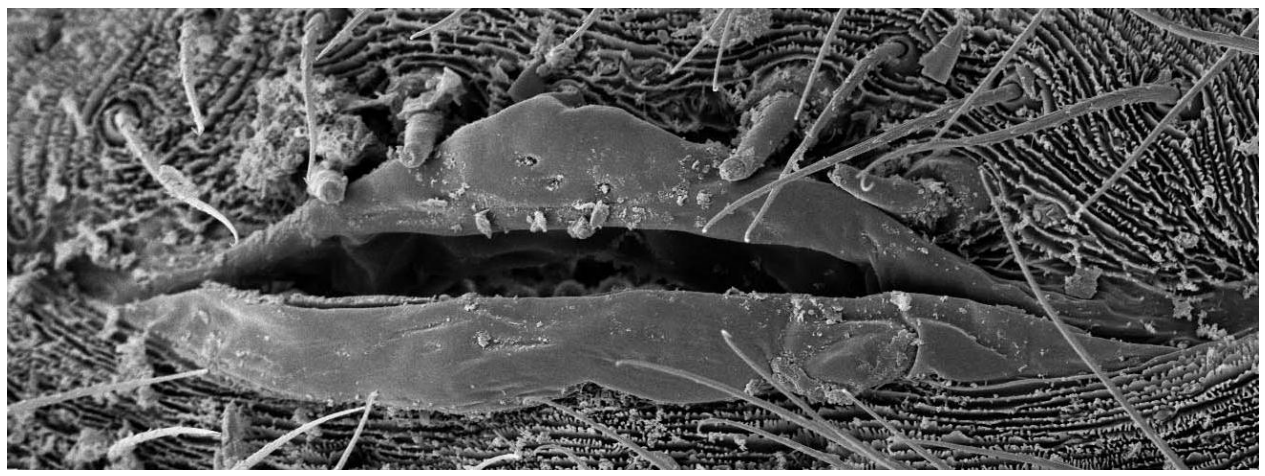

Figure 17. Glenognatha globosa. Epiandrous fusules.

Epiandrous fusules bases not immersed in pits (state 0) optimizes as synapomorphic for Pachygnatha + Glenognatha.

11. Epiandrous fusules, base distal margin, shape: $(L=1, C I=1, R I=1, F=1)$ (APH11)

(0) sharp edge;

(1) round edge (Fig. 18).

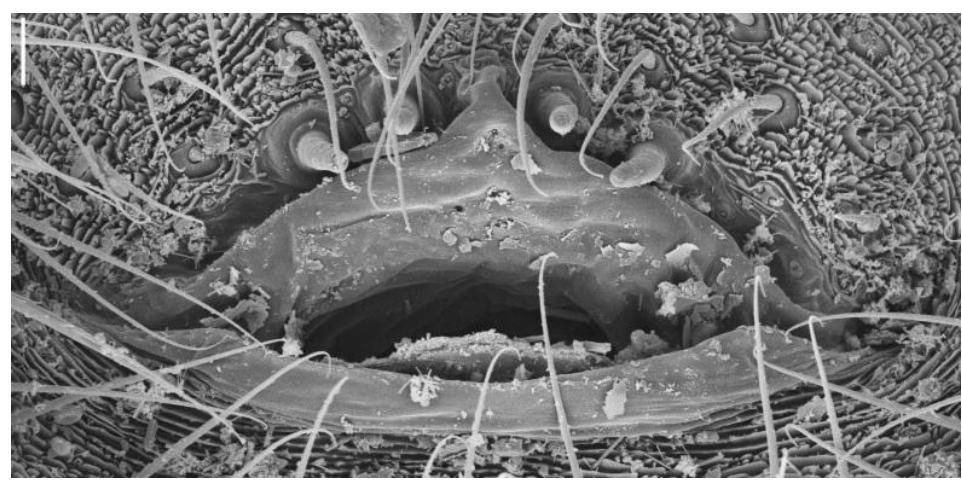

Figure 18. Glenognatha gaujoni. Epiandrous fusules.

Rounded edge of the base of the fusules (state 1) optimizes as synapomorphic for the clade including Cyrtognatha, Tetragnatha, Pachygnatha and Glenognatha. 
12. Epiandrous plate, posterior edge, elevation relative to the surrounding cuticle: $(\mathrm{L}=1, \mathrm{CI}=1, \mathrm{RI}=1, \mathrm{~F}=1)(\mathrm{APH} 11)$

(0) not swollen;

(1) swollen (Fig. 19).

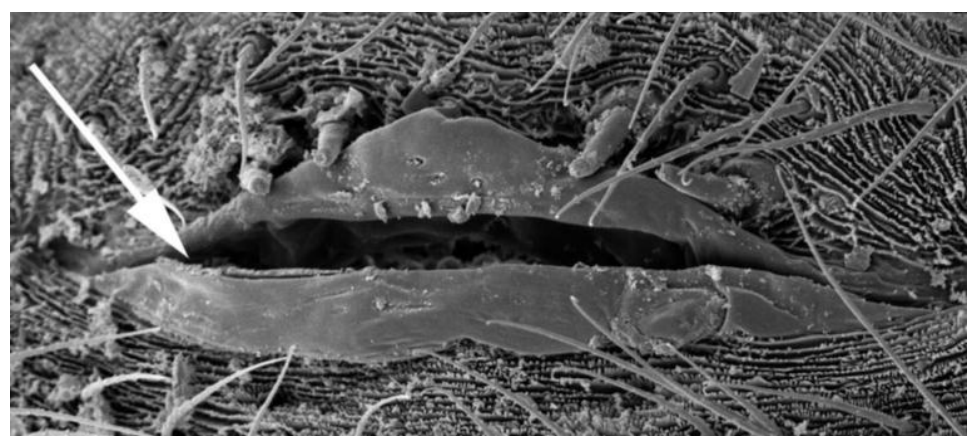

Figure 19. Glenognatha globosa. Epiandrous fusules (arrow, posterior edge).

Posterior edge differentiated from the surrounding cuticle (state 1), optimizes as synapomorphic for the clade including Cyrtognatha, Tetragnatha, Pachygnatha and Glenognatha.

\section{Male papal morphology}

13. Cymbium, median region, form: $(\mathrm{L}=1, \mathrm{CI}=1, \mathrm{RI}=1)$ ( $\mathrm{APH} 11)$

(0) entire, not constricted;

(1) constricted (Fig. 20).

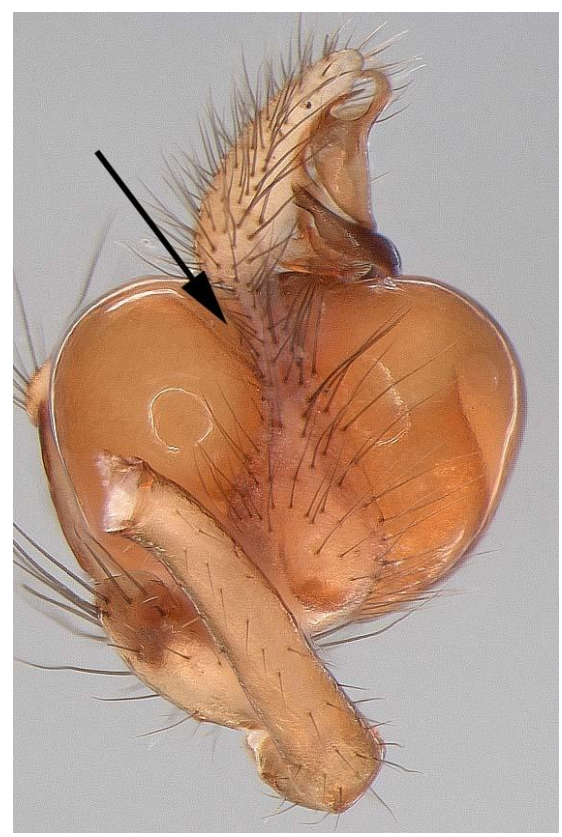

Figure 20. Glenognatha sp. nov. 5. Male left palp (arrow, cymbium constriction). 
Constricted cymbium is synapomorphic for the subfamily Tetragnathinae (Dimitrov \& Hormiga, 2009; Álvarez-Padilla \& Hormiga, 2011).

14. Cymbium, process: $(\mathrm{L}=1, \mathrm{CI}=1, \mathrm{RI}=1)(\mathrm{APH} 11)$

(0) absent (Fig. 21);

(1) present (Álvarez-Padilla \& Hormiga, 2011: fig. 21A).

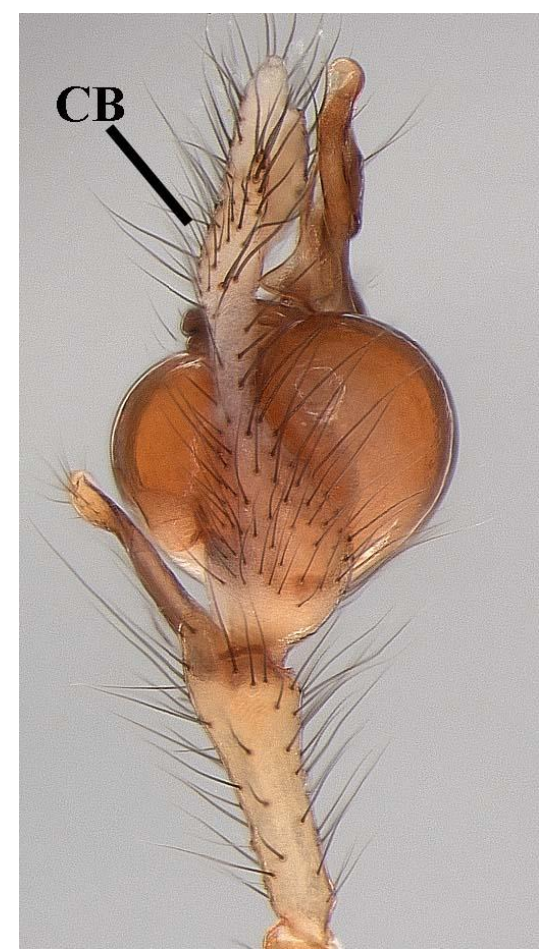

Figure 21. Glenognatha globosa. Male left palp. CB: cymbium.

In Tetragnathidae the cymbium could carry a wide variety of processes located in specific regions and variable in form (Álvarez-Padilla \& Hormiga, 2011). Besides the paracymbium, no other cymbial processes is present in the subfamily Tetragnathinae. The presence of cymbium process optimizes as an autapomorphy for Chrysometa. 
15. Paracymbium, shape: $(\mathrm{L}=2, \mathrm{CI}=1, \mathrm{RI}=1, \mathrm{~F}=1)(\mathrm{APH} 11)$

(0) semicircular, with protuberances;

(1) cylindrical, longer than wide;

(2) 1-shaped, with two nearly perpendicular portions, the distal one in opposite direction to the cymbium (Fig. 22).

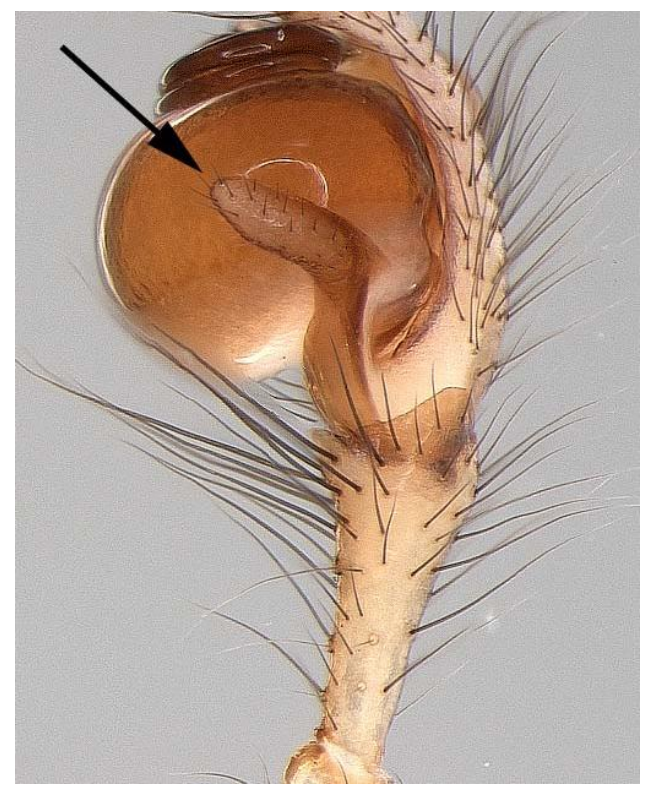

Figure 22. Glenognatha sp. nov. 8. Male left palp (arrow, paracymbium).

The variable morphology of the paracymbium has been widely used in phylogenetic studies within Araneoidea (Hormiga et al. 1995; Griswold et al. 1998; Hormiga, 2000; Agnarsson 2004; Miller \& Hormiga 2004; Kuntner et al., 2008; Álvarez-Padilla \& Hormiga, 2011; Lopardo et al., 2011). Nevertheless, character states definitions have been challenging and in most cases different states have been created to encompass the morphological diversity (Kuntner et al., 2008). In my taxonomic sample, a three state character statement covers all the observed diversity. A paracymbium compose of two nearly perpendicular portions provides unambiguous support for Glenognatha.

16. Paracymbium, distal portion, length*: $(\mathrm{L}=1, \mathrm{CI}=1, \mathrm{RI}=1, \mathrm{~F}=1)$

(0) short, shorter than 1.4 times the basal portion maximum width (Fig. 23, 24A);

(1) long, longer than 2 times the basal portion maximum width (Fig. 23, 24B). 


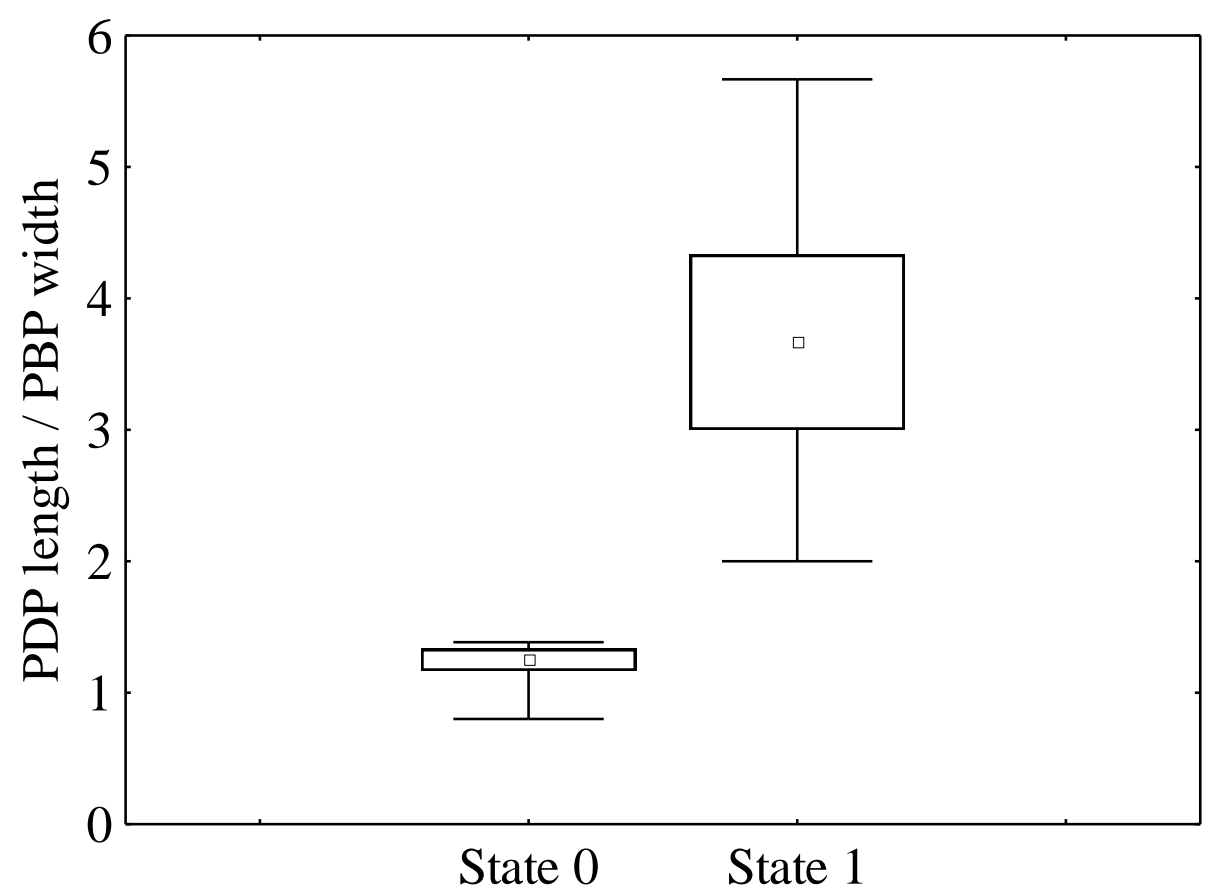

Figure 23. Character 16 states. The box plot shows median (squares), 50\% (box limits) and minimum and maximum (brackets) of all values. PDP: paracymbium distal portion. PBP: paracymbium basal portion. $(\mathrm{U}=0.00, \mathrm{p}<0.001, \mathrm{n}=75)$.

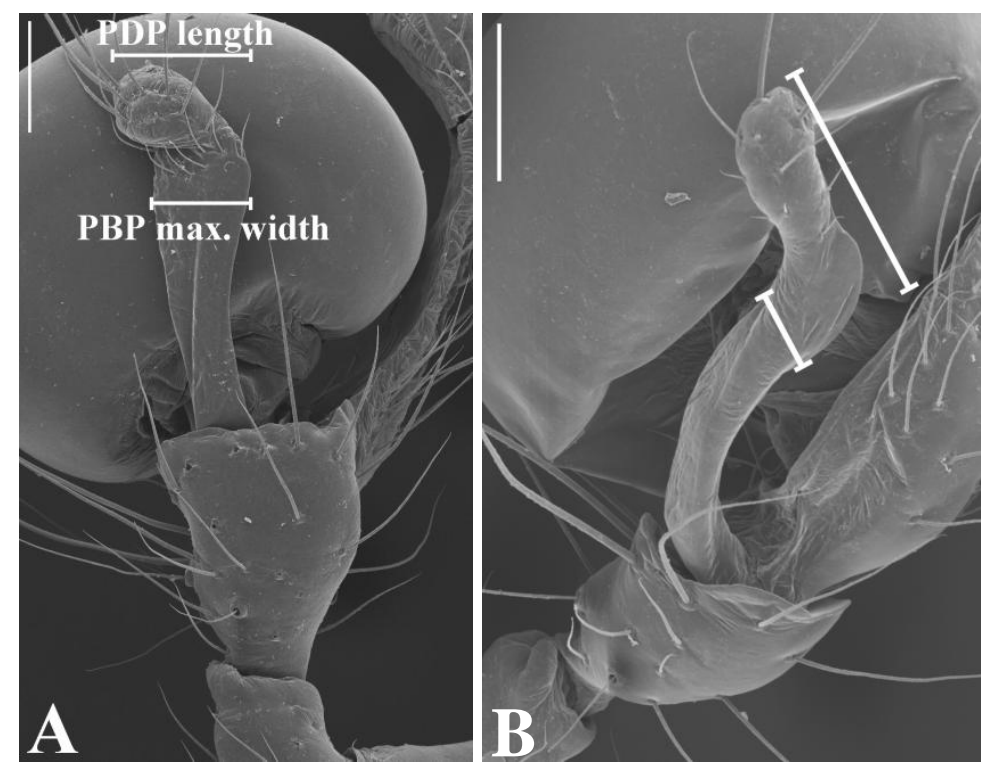

Figure 24. Paracymbium. A, Glenognatha lacteovittata. B, Glenognatha sp. nov. 6. PDP: paracymbium distal portion. PBP: paracymbium basal portion.

In Glenognatha the paracymbium is composed of two nearly perpendicular portions (Fig. 24). The basal one is articulated with the cymbium base and is nearly parallel to the cymbium axis in retrolateral view. The distal portion is in opposite 
direction in relation with the cymbium. The length of the distal portion is taken relative to the maximum width of the basal portion as depicted in figure $24 \mathrm{~A}$. Long distal portion of the paracymbium optimizes as a synapomorphy of clade B.

17. Paracymbium, apophysis: $(\mathrm{L}=6, \mathrm{CI}=0.16, \mathrm{RI}=0.37, \mathrm{~F}=0.615)(\mathrm{APH} 11)$

(0) absent (Fig. 25A);

(1) present (Fig. 25B).
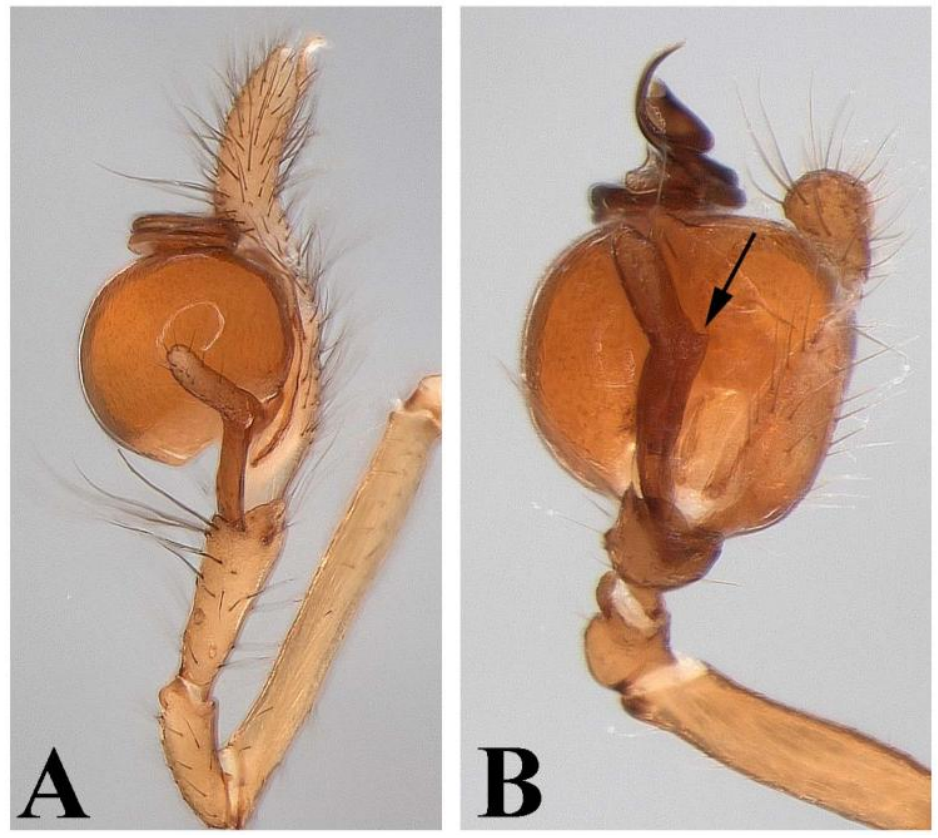

Figure 25. Paracymbium. A, Glenognatha sp. nov. 7. B, Glenognatha heleios (arrow, apophysis).

Highly homoplastic. The presence of apophysis in the paracymbium appears as the plesiomorphic condition. There have been several reversals to this condition within Glenognatha in the clade $\mathrm{C}$ and $\mathrm{G}$.

18. Paracymbium, setae, distribution: $(\mathrm{L}=2, \mathrm{CI}=1, \mathrm{RI}=1, \mathrm{~F}=1)(\mathrm{APH} 11)$

(0) only on the distal portion (Fig. 26);

(1) evenly distributed;

(2) only on the margins. 


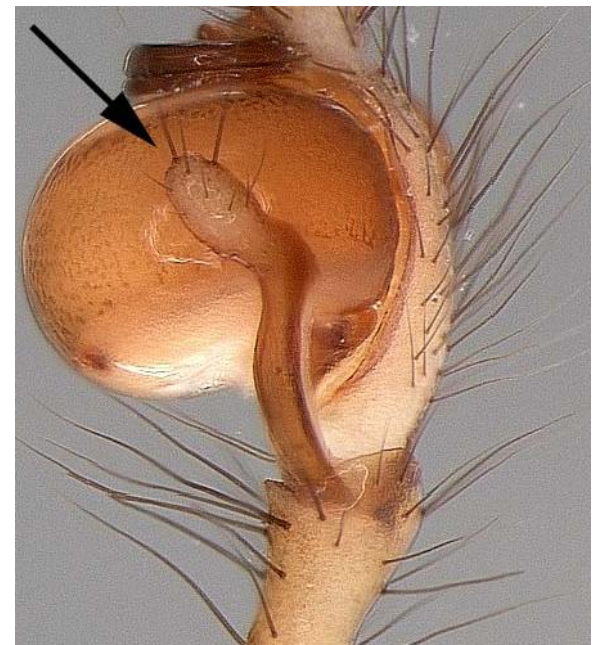

Figure 26. Glenognatha gaujoni. Male left palp (arrow, paracymbium setae).

Evenly distributed setae is the plesiomorphic condition. Setae distributed only in the distal region optimizes as synapomorphic for Glenognatha.

19. Conductor, basal apophysis: $(\mathrm{L}=1, \mathrm{CI}=1, \mathrm{RI}=1, \mathrm{~F}=1)(\mathrm{DH} 09)$

(0) present (Dimitrov \& Hormiga, 2009 fig. 6)

(1) absent (Fig. 27);

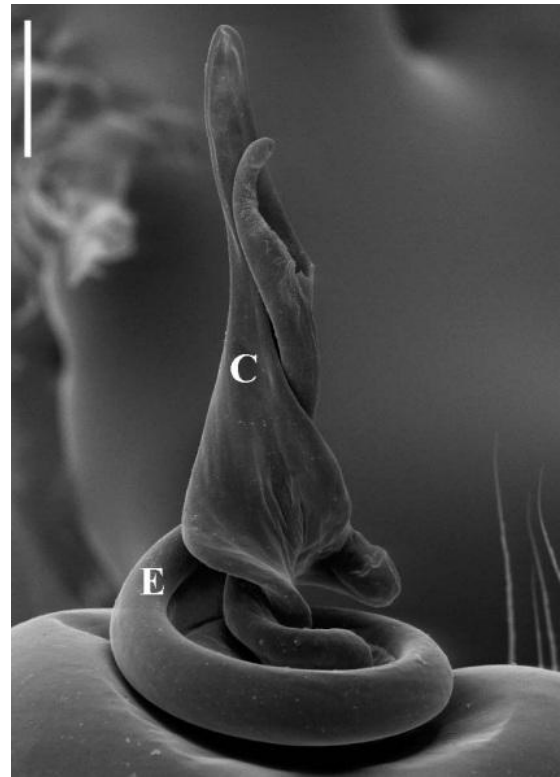

Figure 27. Glenognatha sp. nov. 4. Embolus and conductor. E: embolus. C: conductor.

As defined by Dimitrov \& Hormiga (2009) the conductor basal apophysis starts near the conductor base and runs under the embolus. It is interesting to note that these authors suggest the presence of this apophysis in Glenognatha (Dimitrov \& Hormiga, 
2009, p. 13), however the character was coded as absent in the data matrix of the paper (state 0 ). I agree with this codification and I considered the conductor apophysis present in Glenognatha as a different character due to its relative position (see character 20). Representatives of Tetragnatha and Pachygnatha examined, present a prolateral extension of the conductor base that extends under the embolus. I considered this projection homologous to the conductor basal apophysis defined by Dimitrov \& Hormiga (2009).

20. Conductor, retrolateral apophysis: $(\mathrm{L}=1, \mathrm{CI}=0.5, \mathrm{RI}=0, \mathrm{~F}=0.889)(\mathrm{DH} 09)$

(0) absent;

(1) present (Fig. 28).

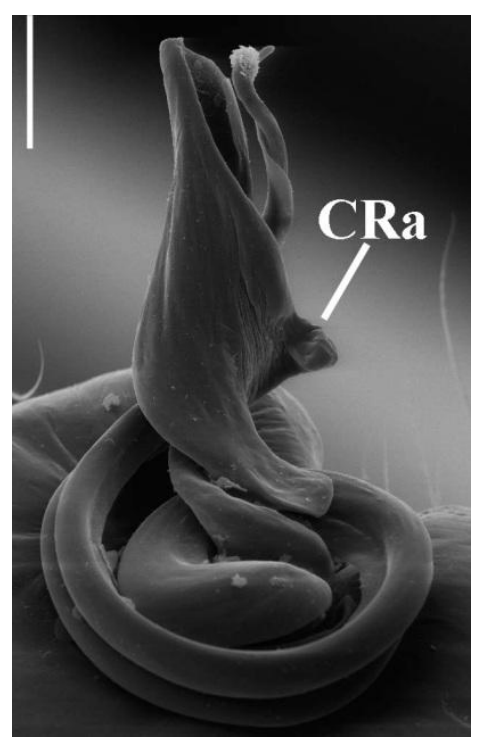

Figure 28. Glenognatha sp. nov. 3. Embolus and conductor. CRa: conductor retrolateral apophysis.

The presence of a retrolateral apophysis optimizes as synapomorphic for Tetragnathinae with a reversal in Cyrtognatha. This apophysis is located near the conductor base and emerges as a retrolateral projection above the embolus.

21. Conductor, retrolateral apophysis, morphology*: $(\mathrm{L}=1, \mathrm{CI}=1, \mathrm{RI}=1, \mathrm{~F}=1)$

(0) rounded, not folded (Álvarez-Padilla \& Hormiga, 2011: figs. 108C, F);

(1) prolaterally folded (Fig. 29). 


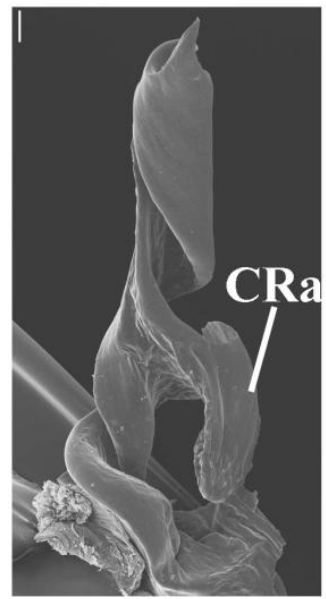

Figure 29. Glenognatha lacteovittata. Conductor. CRa: conductor retrolateral apophysis.

Unfolded apophysis is the plesiomorphic condition. A folded retrolateral apophysis optimizes as synapomorphic for Glenognatha.

22. Conductor, texture*: $(\mathrm{L}=2, \mathrm{CI}=1, \mathrm{RI}=1)$

(0) smooth (Fig. 30A);

(1) with small pointed projections (Fig. 30B)

(2) with folds (Fig. 30C)
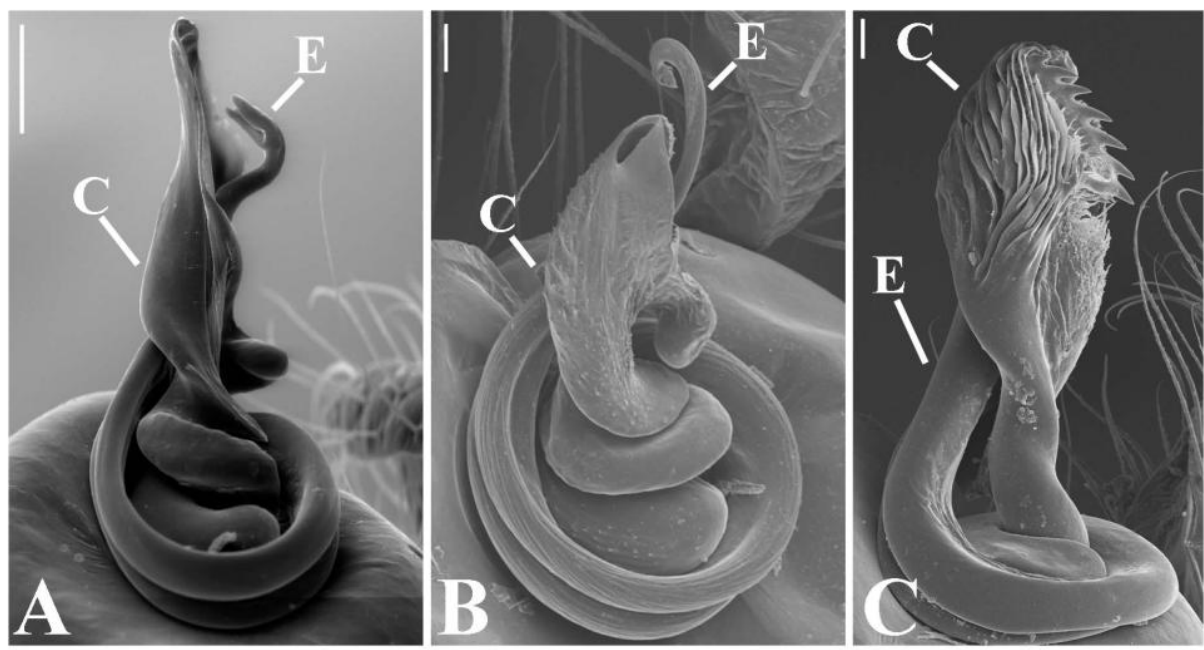

Figure 30. Embolus and conductor. A, Glenognatha sp. nov. 1. B, Glenognatha sp. nov. 6. C, Dyschiriognatha dentata. E: embolus. C: conductor.

Smooth conductor is the widespread condition in Glenognatha species and members of the outgroup. Conductor with folds is autapomorphic for D. dentata and with small pointed projections is autapomorphic for G. sp. nov. 6 . 
23. Conductor, retrolateral margin, morphology* $(\mathrm{L}=2, \mathrm{CI}=0.5, \mathrm{RI}=0, \mathrm{~F}=0.889)$

(0) entire (Fig. 31A);

(1) serrated (Fig. 31B).

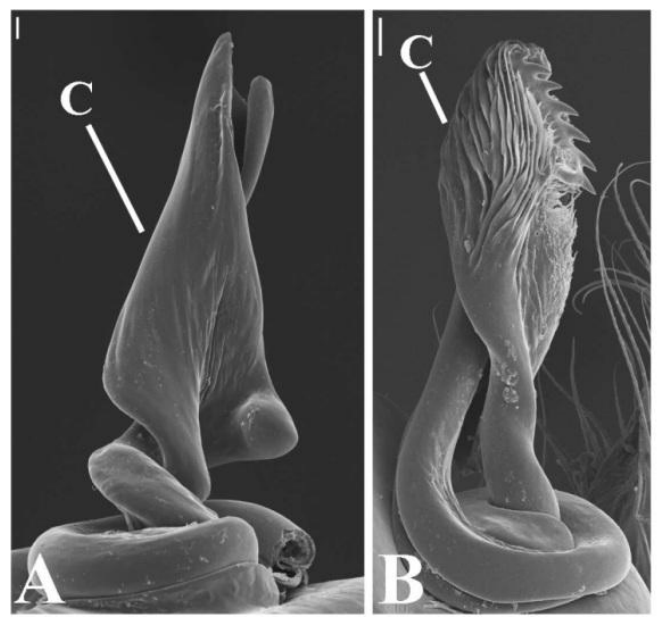

Figure 31. Embolus and conductor. A, Glenognatha sp. nov. 2. B, Dyschiriognatha dentata. E: embolus. C: conductor.

Serrated retrolateral margin of the conductor appears independently in $D$. dentata and G. sp. nov. 9.

24. Conductor, retrolateral projection*: $(\mathrm{L}=1, \mathrm{CI}=1, \mathrm{RI}=1, \mathrm{~F}=1)$

(0) absent (Fig. 32A);

(1) present (Fig. 32B).

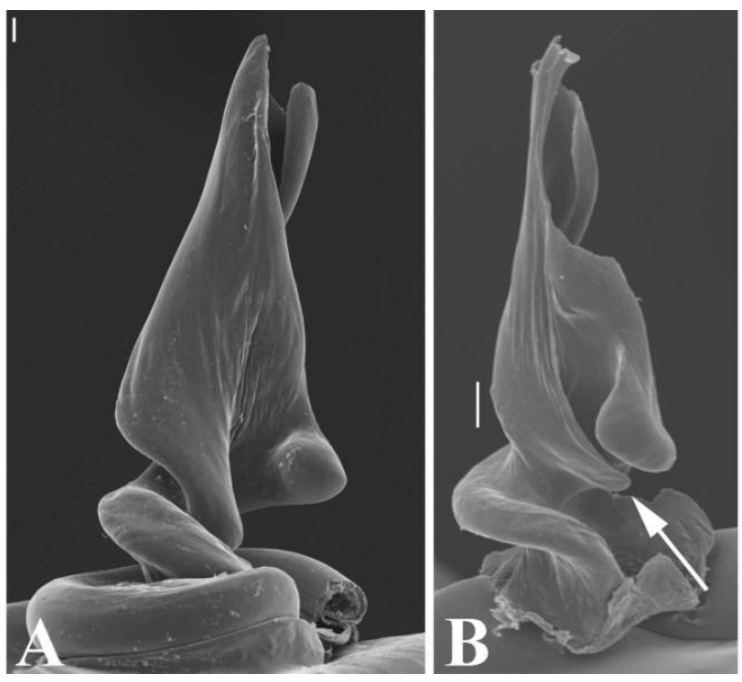

Figure 32. Conductor. A, Glenognatha sp. nov. 2. B, Glenognatha australis (arrow, retrolateral projection). E: embolus. C: conductor. 
The presence of a retrolateral projection in the conductor optimizes as a synapomorphy of clade $\mathrm{F}$. This projection emerges in the region where the cylindrical conductor base flattens into a folded lamina and is located below the retrolateral apophysis (Fig. 32B).

25. Conductor, apical portion, morphology*: $(\mathrm{L}=2, \mathrm{CI}=0.5, \mathrm{RI}=0.5, \mathrm{~F}=0.889)$

(0) without prolateral fold or if present wider than long (Fig. 33A);

(1) with a prolateral fold longer than wide (Fig. 33B).

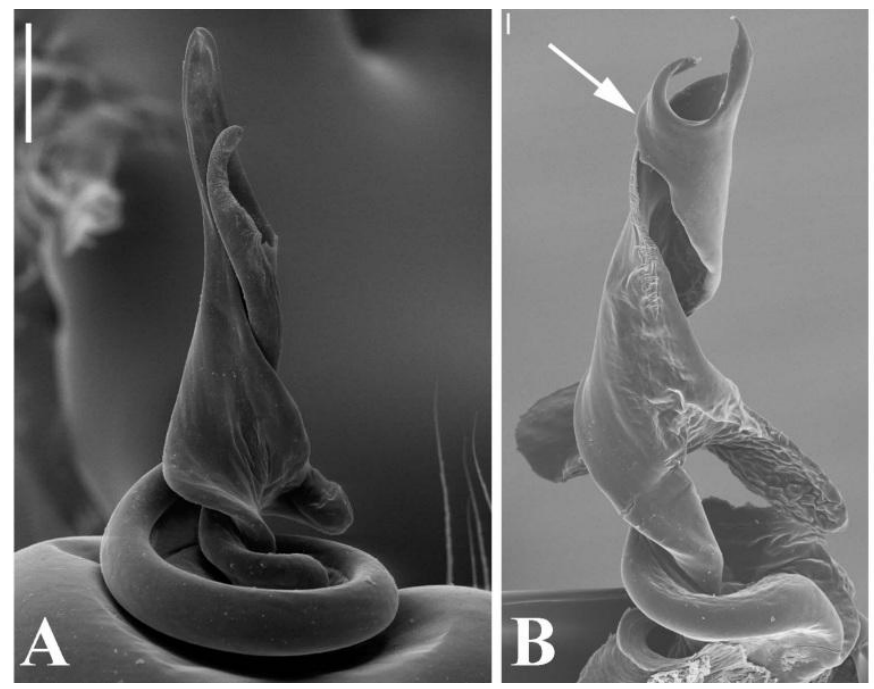

Figure 33. Conductor. A, Glenognatha sp. nov. 4. B, Glenognatha lacteovittata (arrow, prolateral fold).

Long prolateral fold in the apical portion of the conductor appears independently in the clade G. lacteovittata + G. sp. nov. 5 and in the species G. smilodon.

26. Embolus, metine embolic apophysis $(\mathrm{L}=2, \mathrm{CI}=1, \mathrm{RI}=1)(\mathrm{APH} 11)$

(0) absent;

(1) present (Álvarez-Padilla \& Hormiga, 2011: figs. 60A-B).

The presence of metine embolic apophysis optimizes as an autapomorphy for Ch. alajuela. 
27. Embolus, tip, curvature relative to the palp longitudinal axis*: $(\mathrm{L}=6, \mathrm{CI}=0.16$, $\mathrm{RI}=0.16, \mathrm{~F}=0.615)$

(0) straight to $90^{\circ}$ curved (Fig. 34A);

(1) $135^{\circ}$ to $180^{\circ}$ curved (Fig. $34 \mathrm{~B}$ ).

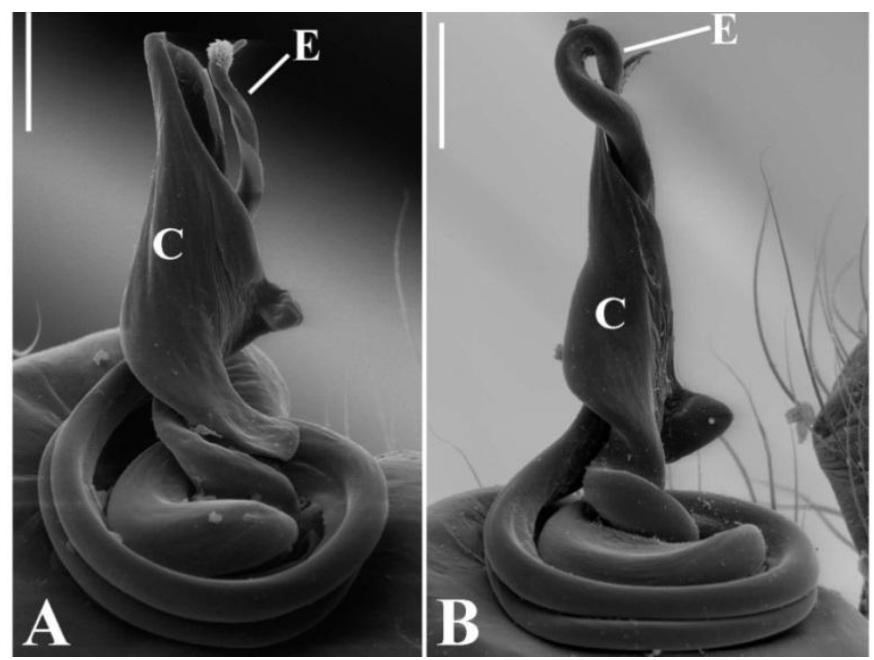

Figure 34. Embolus and conductor. A, Glenognatha sp. nov. 3. B, Glenognatha sp. nov. 8. E: embolus. C: conductor.

Highly homoplastic. A strong curved embolic tip appears independently in members of clades A, C, E, G and J.

28. Embolus, tip, orientation*: $(\mathrm{L}=5, \mathrm{CI}=0.2, \mathrm{RI}=0.2, \mathrm{~F}=0.667)$

(0) anterior to retrolateral (Fig. 35A);

(1) prolateral (Fig. 35B).
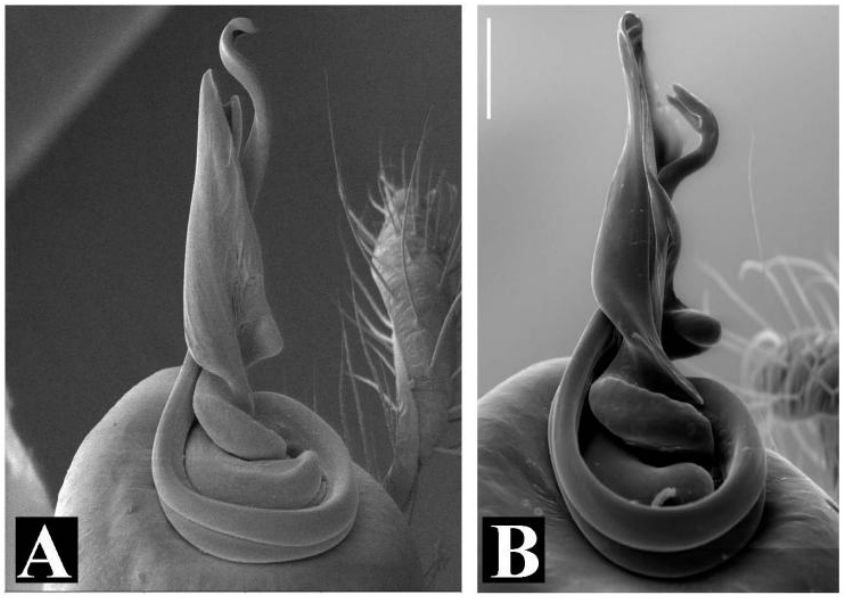

Figure 35. Embolus and conductor. A, Glenognatha sp. nov. 2. B, Glenognatha sp. nov. 1. E: embolus. C: conductor. 
Highly homoplastic. Embolic tip prolaterally oriented appears independently in members of clades $\mathrm{G}$ and $\mathrm{H}$.

29. Embolus, subapical, region*: $(\mathrm{L}=2, \mathrm{CI}=1, \mathrm{RI}=1, \mathrm{~F}=1)$

(0) uncoiled (Fig. 36A);

(1) coiled (Fig. 36B);

(2) twice coiled (Fig. 36C).
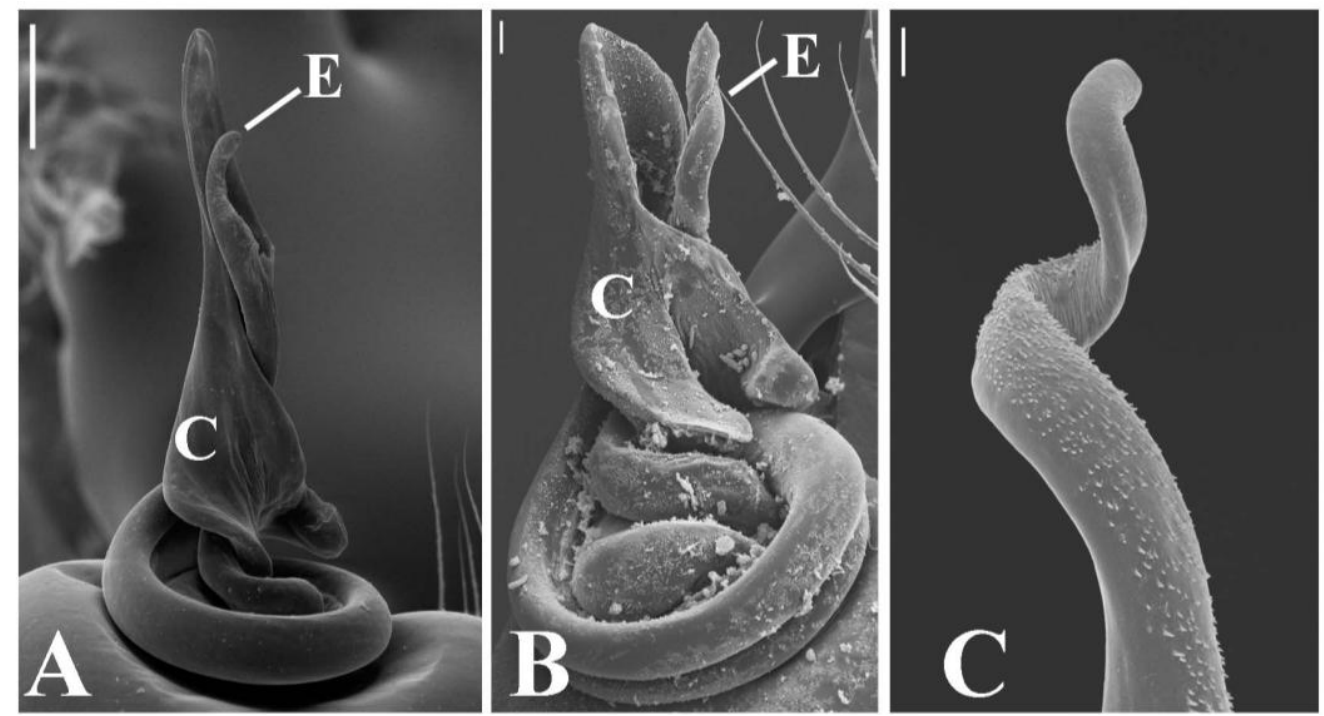

Figure 36. Embolus. A, Glenognatha sp. nov. 4. B, Glenognatha spherella. C, Glenognatha lacteovittata. E: embolus. C: conductor.

Uncoiled embolus tip is the plesiomorphic condition. Embolus twice coiled optimizes as autapomorphic for G. lacteovittata and coiled embolus as synapomorphic for G. gloriae + G. spherella.

30. Embolus, tip, morphology: $(\mathrm{L}=1, \mathrm{CI}=1, \mathrm{RI}=1, \mathrm{~F}=1)(\mathrm{MH} 04)$

(0) terminating in embolic opening (Fig. 37A);

(1) with projection beyond embolic opening (Fig. 37B).

Embolus tip with a projection beyond embolic opening optimizes as synapomorphic for the clade E. 


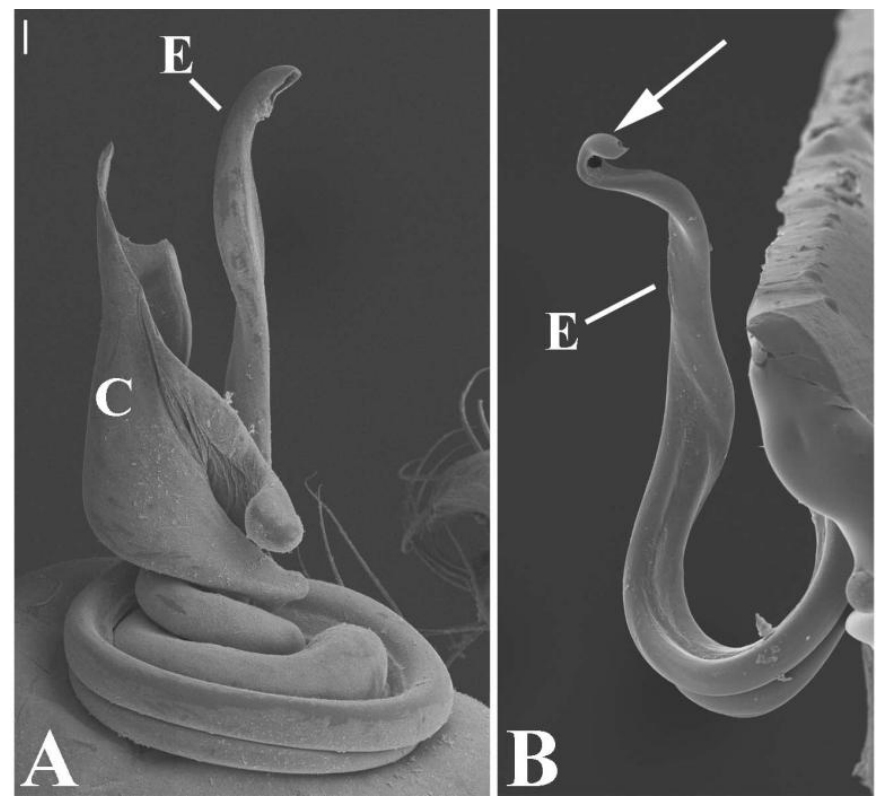

Figure 37. A, Glenognatha minuta. B, Glenognatha sp. nov. 2 (arrow, projection beyond embolic opening). E: embolus. C: conductor.

31. Embolus, texture*: $(\mathrm{L}=3, \mathrm{CI}=0.66, \mathrm{RI}=0.5, \mathrm{~F}=0.88)$

(0) smooth (Fig. 38A);

(1) with scale-like projections (Fig. 38B);

(2) with pointed projections (Fig. 38C).

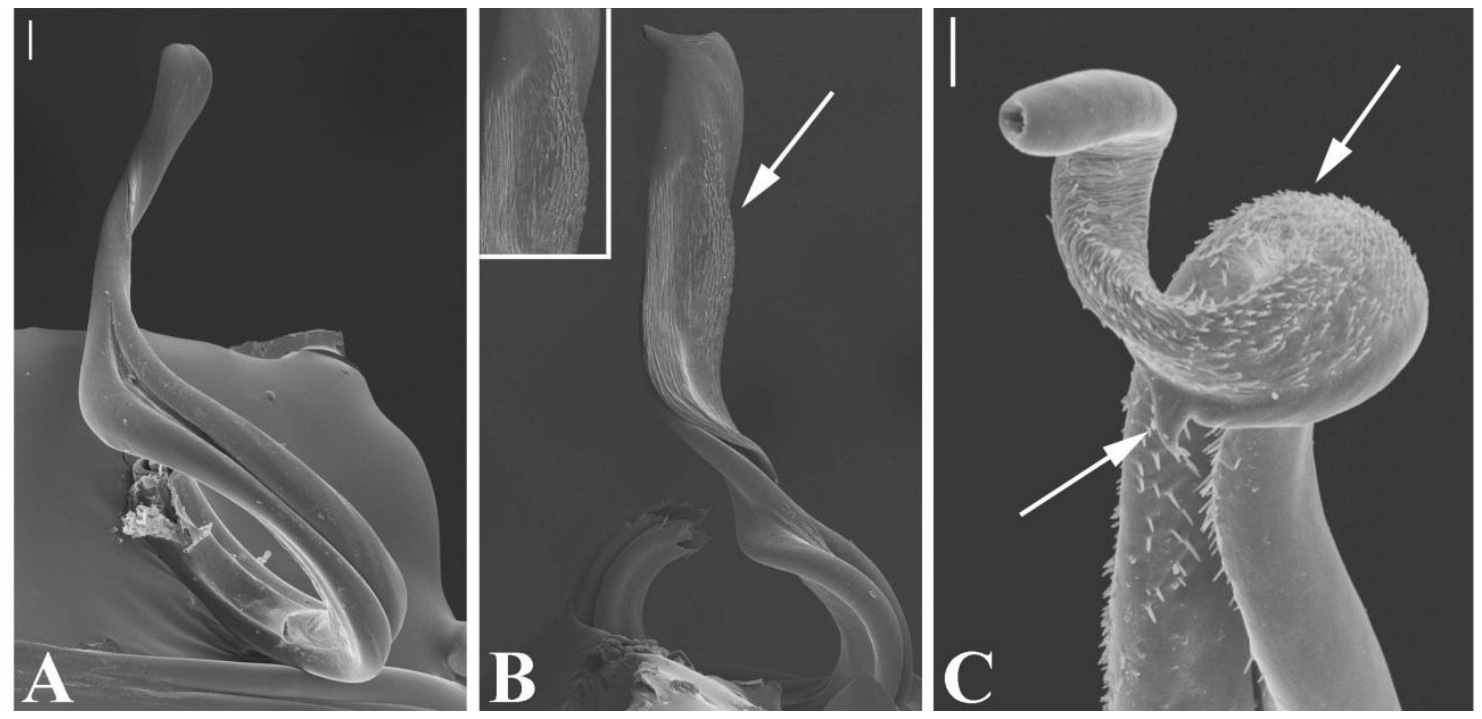

Figure 38. Embolus. A, Glenognatha sp. nov. 2. B, Glenognatha gaujoni (arrow, scalelike projections). C, Glenognatha lacteovittata (tooth-like and filiform projections). 
Smooth embolus is the plesiomorphic condition. Scale-like projections optimizes as autapomorphic for G. gaujoni and pointed projections appears independently in $G$. lacteovittata $+G$. sp. nov. 5 and D. dentata.

32. Embolic medial groove*: $(\mathrm{L}=1, \mathrm{CI}=1, \mathrm{RI}=1, \mathrm{~F}=1)$

(0) absent;

(1) present (Fig. 39).

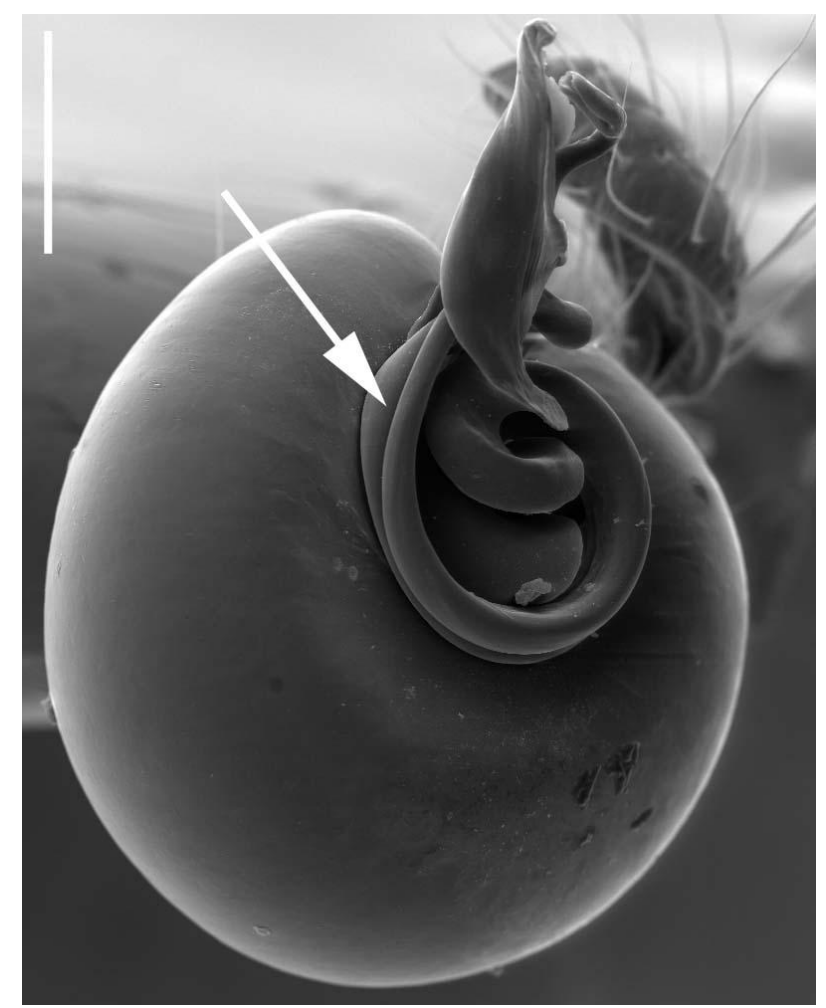

Figure 39. Glenognatha sp. nov. 1. Male left palp (arrow, embolic median groove)

The presence of an embolic medial groove optimizes as synapomorphic for Glenognatha. This groove originates near the embolus base and runs medially until the subapical portion (Fig. 38A).

33. Embolic medial groove, configuration*: $(\mathrm{L}=1, \mathrm{CI}=1, \mathrm{RI}=1, \mathrm{~F}=1)$

(0) limited to the inner surface of the embolus (Fig. 40A).

(1) expanded to the outer surface of the embolus (Fig. 40B); 


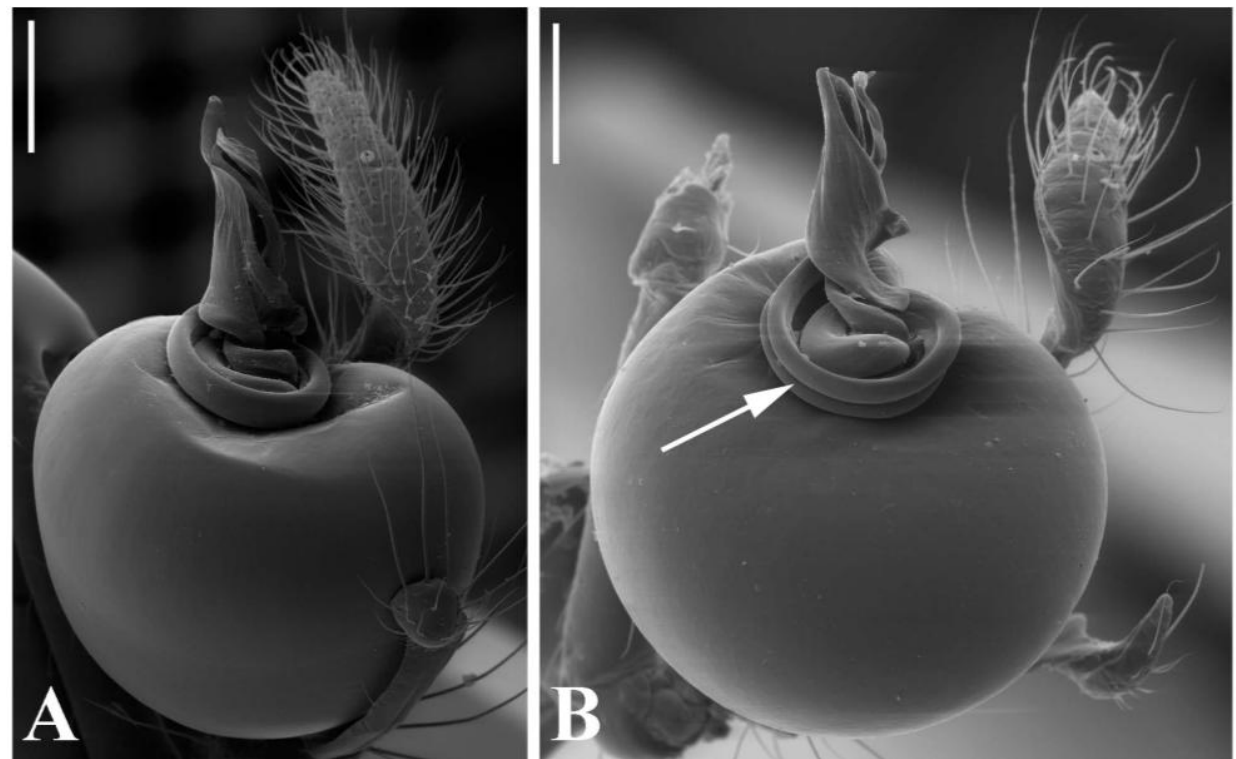

Figure 40. Male left palp. A, Glenognatha sp. nov. 5. B, Glenognatha sp. nov. 3 (arrow, embolic median groove).

An embolic groove limited to the inner surface is the plesiomorphic condition. Expanded groove optimizes as synapomorphic for the clade B. It is interesting to note that species coded with the state 1 also exhibit a well-developed inner membrane that covers the groove (Fig. 38A). The functional and evolutionary significance of these contrasting configurations among Glenognatha species are unknown. Copulatory behavior and histological data are needed in order to suggest possible selection pressures shaping this morphological variation.

34. Embolus, laminar projection*: $(\mathrm{L}=1, \mathrm{CI}=1, \mathrm{RI}=1)$

(0) absent;

(1) present (Fig. 41).

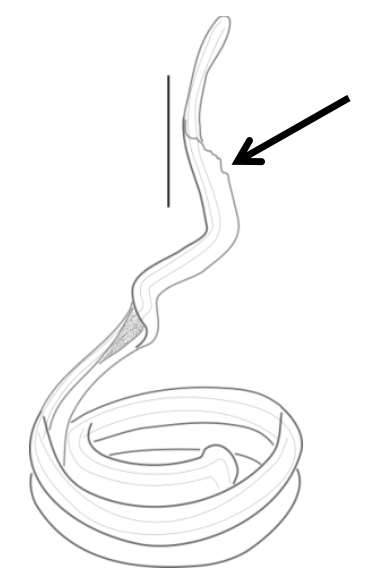

Figure 41. Glenognatha smilodon. Embolus (arrow, laminar projection). 
A laminar projection in the embolus is an autapomorphic character of $G$. smilodon.

35. Male, palpal patella, macrosetae: $(\mathrm{L}=1, \mathrm{CI}=1, \mathrm{RI}=1, \mathrm{~F}=1)(\mathrm{APH} 11)$

(0) none (Fig. 42);

(1) one.

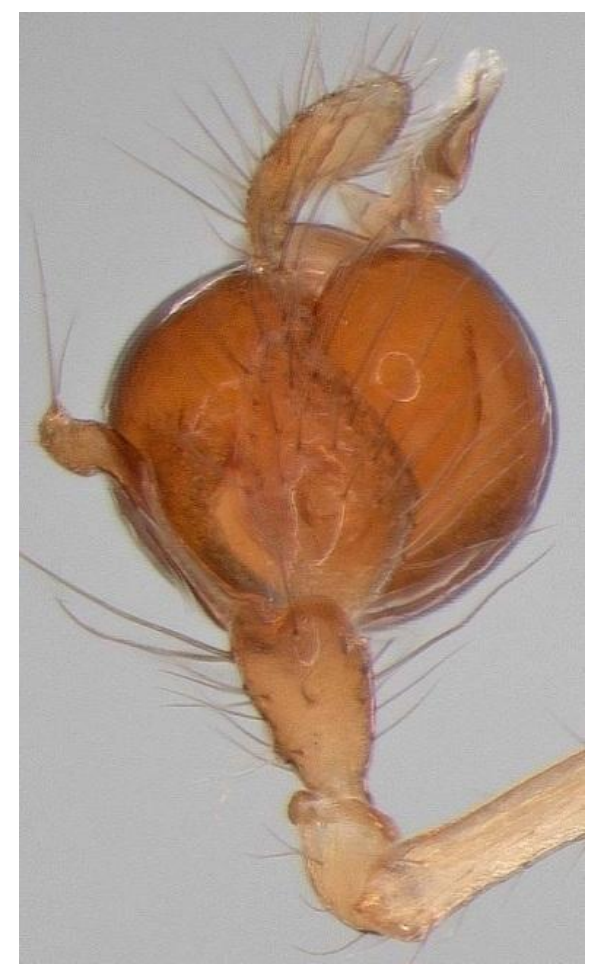

Figure 42. Glenognatha sp. nov. 1. Male left palp.

Absence of male palpal patella macrosetae optimizes as synapomorphic for Pachygnatha + Glenognatha. Nevertheless, as noted by Hormiga et al. (1995), coding this character for Pachygnatha is problematic due to interspecific variation within the genus. These authors mentions that $P$. clercki present macrosetae, but this was not observed in the examined specimens of the present study. A phylogenetic revision of the genus Pachygnatha is needed in order to establish the plesiomorphic condition and clarify the evolution of the character in the clade Pachygnatha + Glenognatha.

36. Male, palpal tibia, length: $(\mathrm{L}=5, \mathrm{CI}=0.2, \mathrm{RI}=0.33, \mathrm{~F}=0.66)(\mathrm{APH} 11)$

(0) short, shorter than 1.5 times the maximum width of the tibia (Fig. 43, 44A);

(1) long, longer than 1.9 times the maximum width of the tibia (Fig. 43, 44B). 


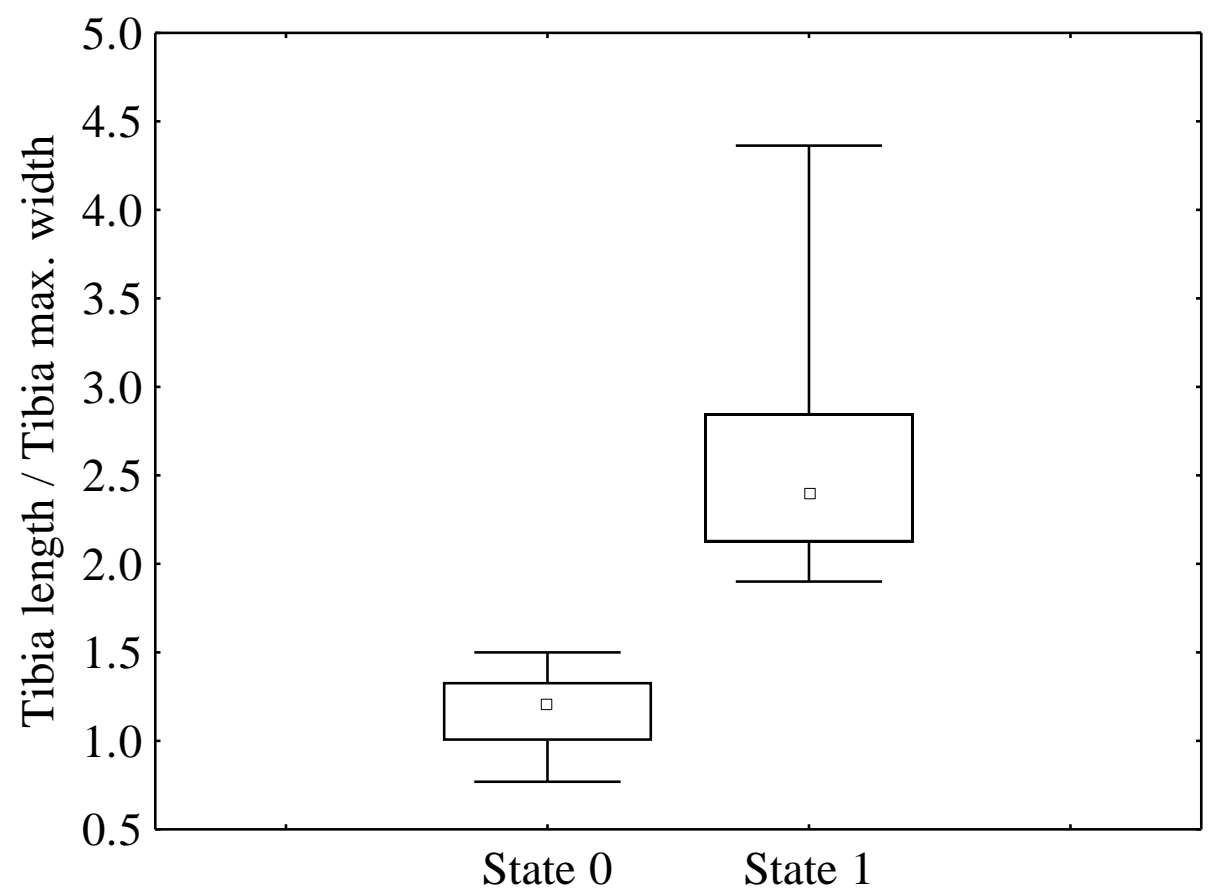

Figure 43. Character 36 states. The box plot shows median (squares), 50\% (box limits) and minimum and maximum (brackets) of all values, $(\mathrm{U}=0.00, \mathrm{p}<0.001, \mathrm{n}=85)$.

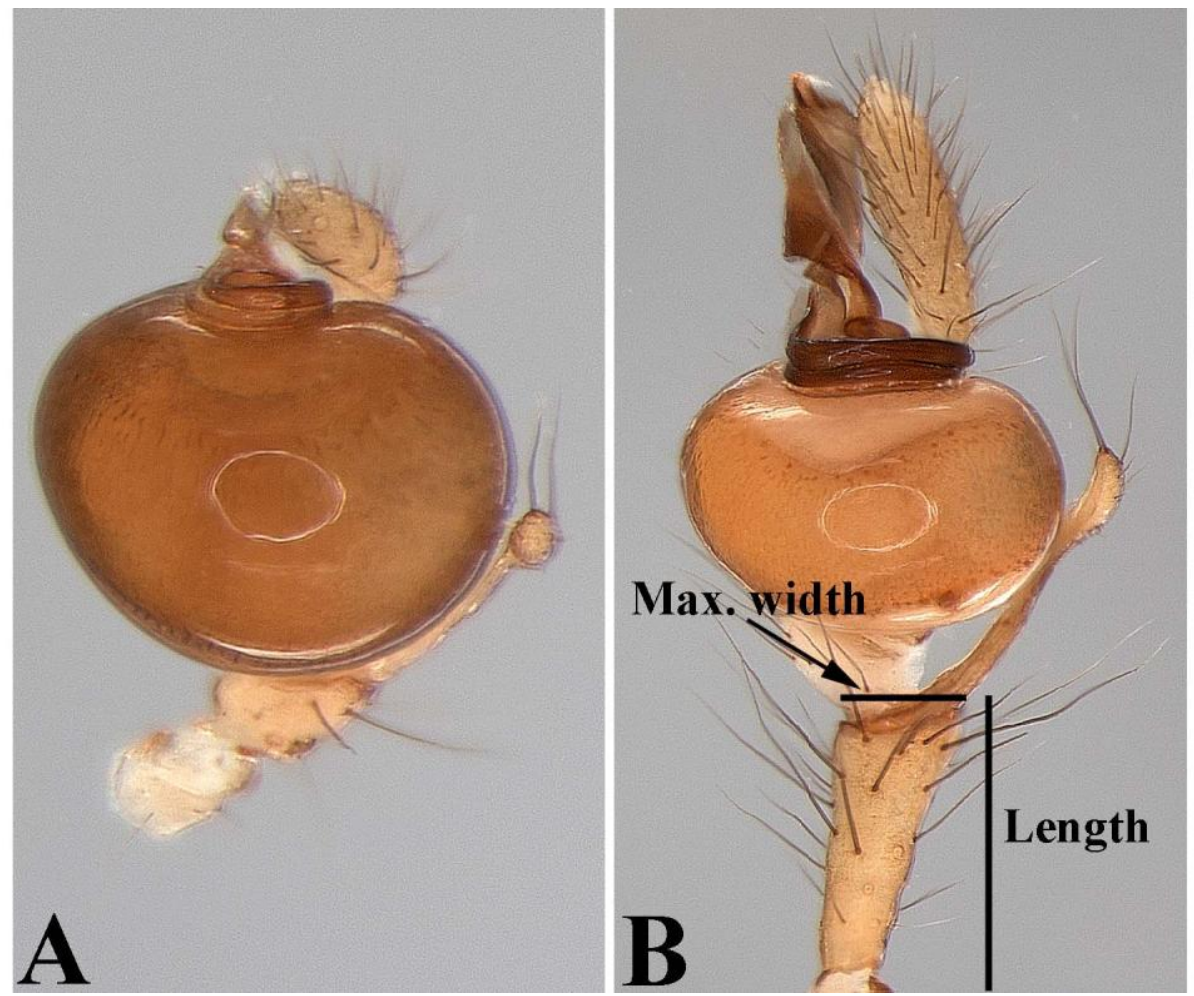

Figure 44. A, Glenognatha foxi. B, Glenognatha gaujoni. 
The length of the tibia is taken relative to its maximum width as depicted in figure 44B. Long palpal tibia appear independently in T. versicolor, $P$. clercki, clade E and $\mathrm{J}$.

\section{Female genitalia}

37. Epigynum: $(\mathrm{L}=1, \mathrm{CI}=1, \mathrm{RI}=1)(\mathrm{APH} 11)$

(0) present

(1) absent (Fig. 45A, B);
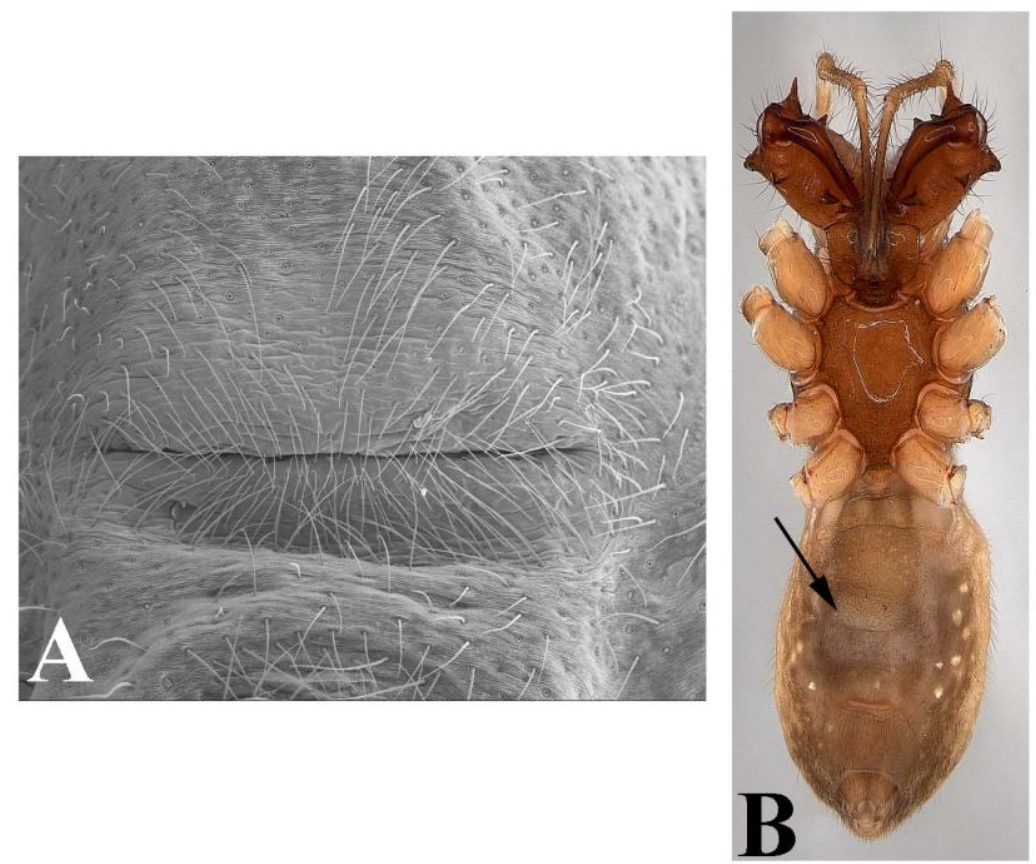

Figure 45. Glenognatha gaujoni. A, Gonopore. B, Body, ventral view (arrow, gonopore).

The secondary haplogyne condition is a synapomorphy for Tetragnathinae (Dimitrov et al., 2007; Álvarez \& Hormiga, 2011). In this condition, a general simplification of female genital structures have been occurred, including the loss of copulatory ducts and epigynum. In the present analysis only one character (epigynum) have been coded to characterize this condition.

38. Spermathecae: $(\mathrm{L}=3, \mathrm{CI}=0.33, \mathrm{RI}=0.72, \mathrm{~F}=0.8)(\mathrm{APH} 11)$

(0) present (Fig. 46A).

(1) absent (Fig. 46B); 


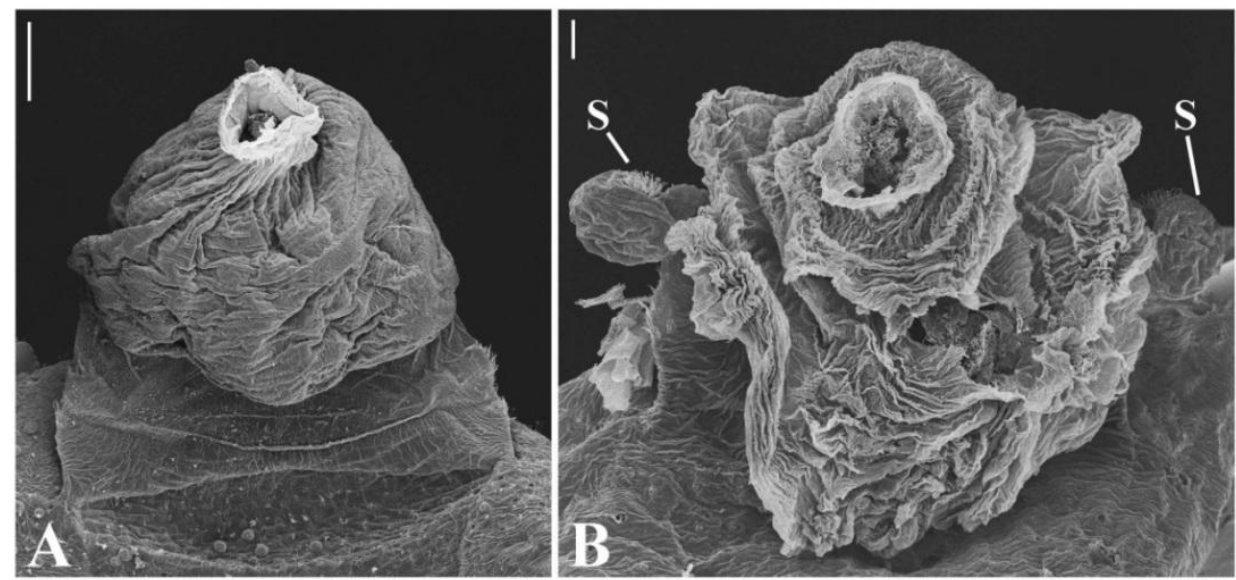

Figure 46. Internal genitalia. A, Glenognatha lacteovittata. B, Glenognatha gloriae. S: spermathecae.

Spermathecae have been lost independently in three clades within Glenognatha. According with dissection and observation of species without spermathecae after copulation ( $G$. globosa and $G$. sp. nov. 8), the sperm storage function appears to be assumed by the enlarged uterus externus sac.

39. Spermathecae, separation: $(\mathrm{L}=1, \mathrm{CI}=1, \mathrm{RI}=1, \mathrm{~F}=1)(\mathrm{APH} 11)$

(0) separated more than two spermathecae diameters (Fig. 47);

(1) under two diameters apart.

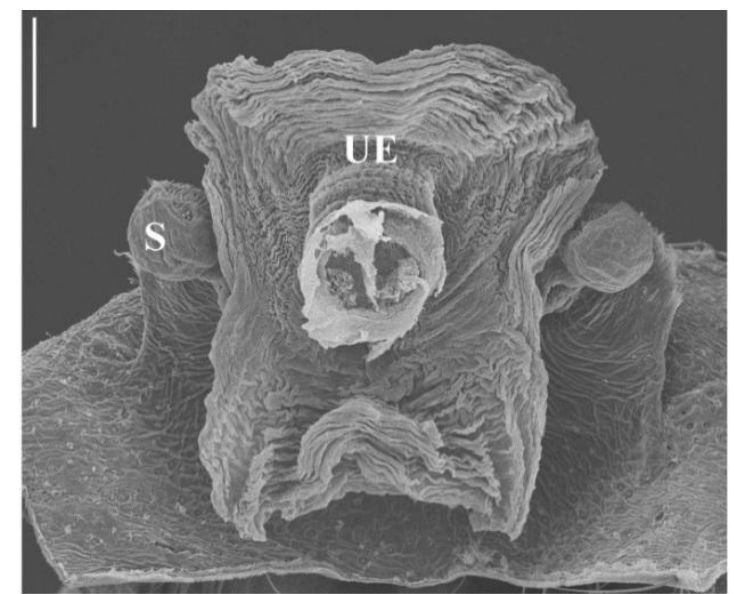

Figure 47. Glenognatha minuta. Internal genitalia. S: spermathecae. UE: uterus externus.

Separated spermathecae optimizes as synapomorphic for the clade Cyrtognatha (Pachygnatha, Glenognatha). Under DELTRAN optimizes as synapomorphic for Pachygnatha + Glenognatha . 
40. Spermathecae, chambers, quantity $(\mathrm{L}=2, \mathrm{CI}=0.5, \mathrm{RI}=0.5, \mathrm{~F}=0.88)(\mathrm{APH} 11)$

(0) each spermathecae consisting of a single chamber (Fig. 48);

(1) two chambers

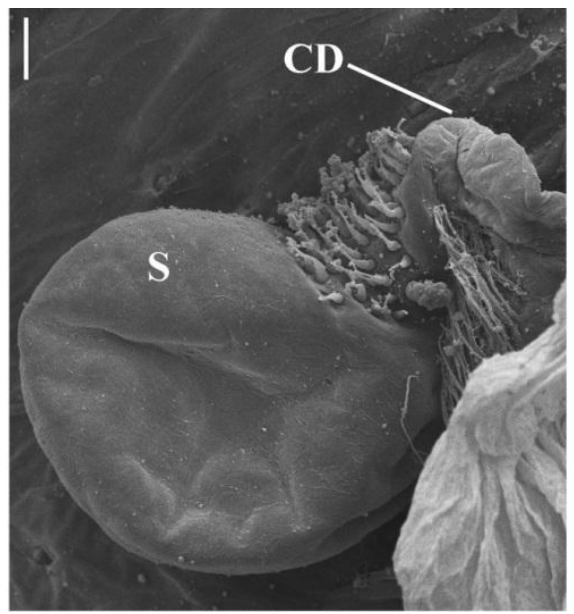

Figure 48. Glenognatha foxi. Spermathecae. S: Spermathecae. CD: copulatory duct.

Spermathecae divided into two chambers appears independently in T. versicolor and Pachygnatha.

41. Accessory gland base, form $(\mathrm{L}=1, \mathrm{CI}=1, \mathrm{RI}=1, \mathrm{~F}=1)(\mathrm{APH} 11)$

(0) subequal to the duct;

(1) widely enlarged (Fig. 49).

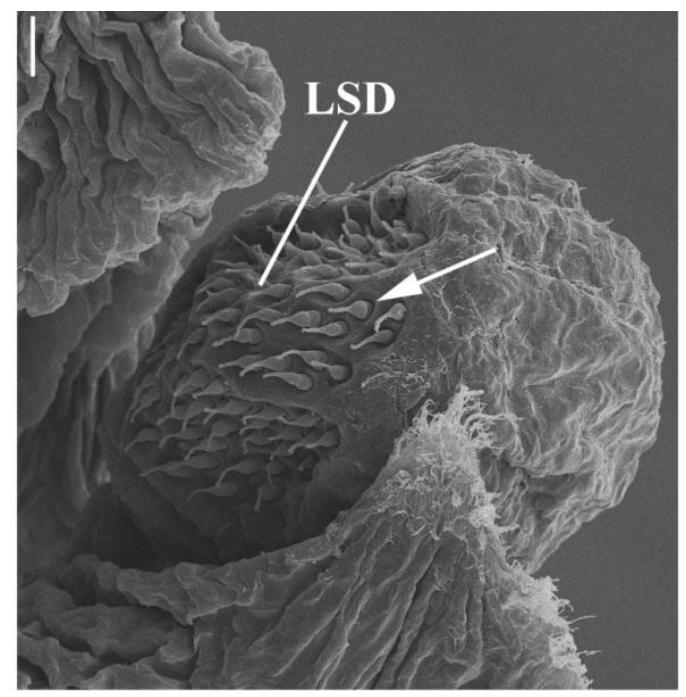

Figure 49. Glenognatha minuta. Spermathecae (arrow, enlarged gland base). LSD: long-stem ducts. 
Accessory glands base subequal to duct is the plesiomorphic condition. Enlarged accessory gland bases optimizes as synapomorphic for Glenognatha. If DELTRAN optimization is used the character is synapomorphic for the clade B.

42. Accessory gland, distribution $(\mathrm{L}=2, \mathrm{CI}=1, \mathrm{RI}=1, \mathrm{~F}=1)(\mathrm{APH} 11)$

(0) evenly over the spermathecae;

(1) near the copulatory ducts (Fig. 50);

(2) near the fertilization ducts.

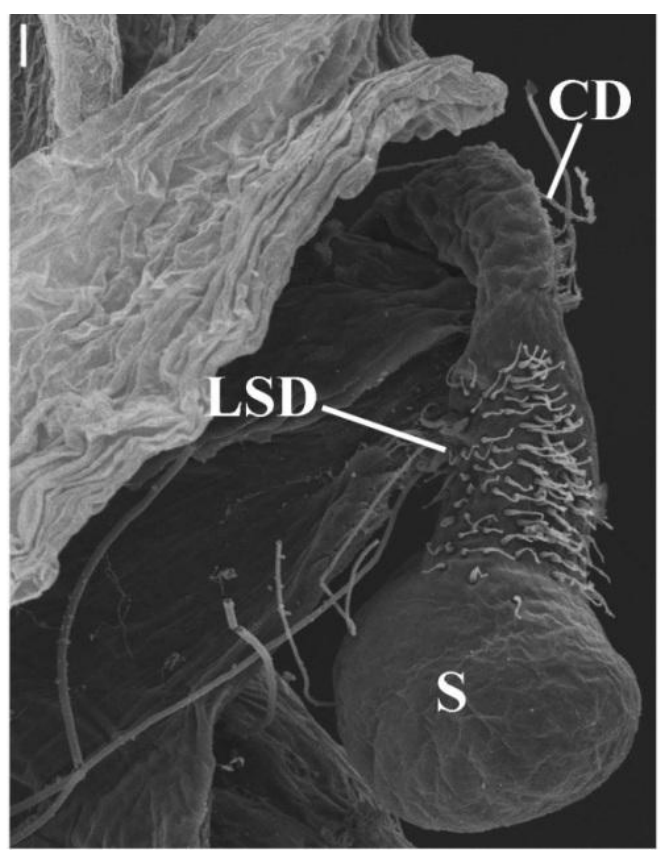

Figure 50. Glenognatha heleios. Spermathecae and copulatory duct. CD: copulatory ducts. LSD: long-stem ducts. S: spermathecae.

Accessory gland ducts located near the copulatory ducts optimizes as a synapomorphy for Glenognatha. Coding this character is difficult since the limit between copulatory duct and spermathecae can only be objectively assigned using histological techniques. In the present analysis, this differentiation was based in the cuticle texture observed through SEM images or light microscopy montages. If DELTRAN optimization is used the character is synapomorphic for the clade B.

43. Uterus externus, enlarged sac $(\mathrm{L}=1, \mathrm{CI}=1, \mathrm{RI}=1)(\mathrm{APH} 11)$

(0) absent;

(1) present (Fig. 51A, B). 


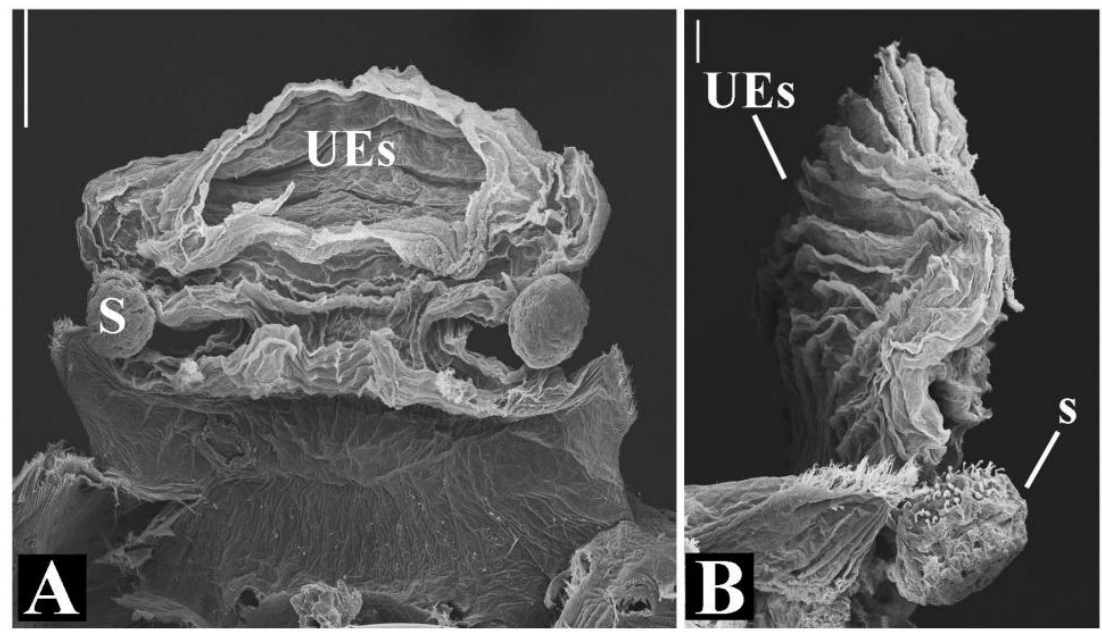

Figure 51. Glenognatha argyrostilba. Internal genitalia. A, Dorsal view. B, Lateral view. S: Spermathecae. UEs: uterus externus sac.

The presence of an enlarged uterus externus sac is synapomorphic for Tetragnathinae.

44. Uterus externus sac, morphology*: $(\mathrm{L}=6, \mathrm{CI}=0.33, \mathrm{RI}=0.73, \mathrm{~F}=0.66)$

(0) entire (Fig. 52A);

(1) with a median projection (Fig. 52B)

(2) divided into two or more distinct chambers (Fig. 52C).
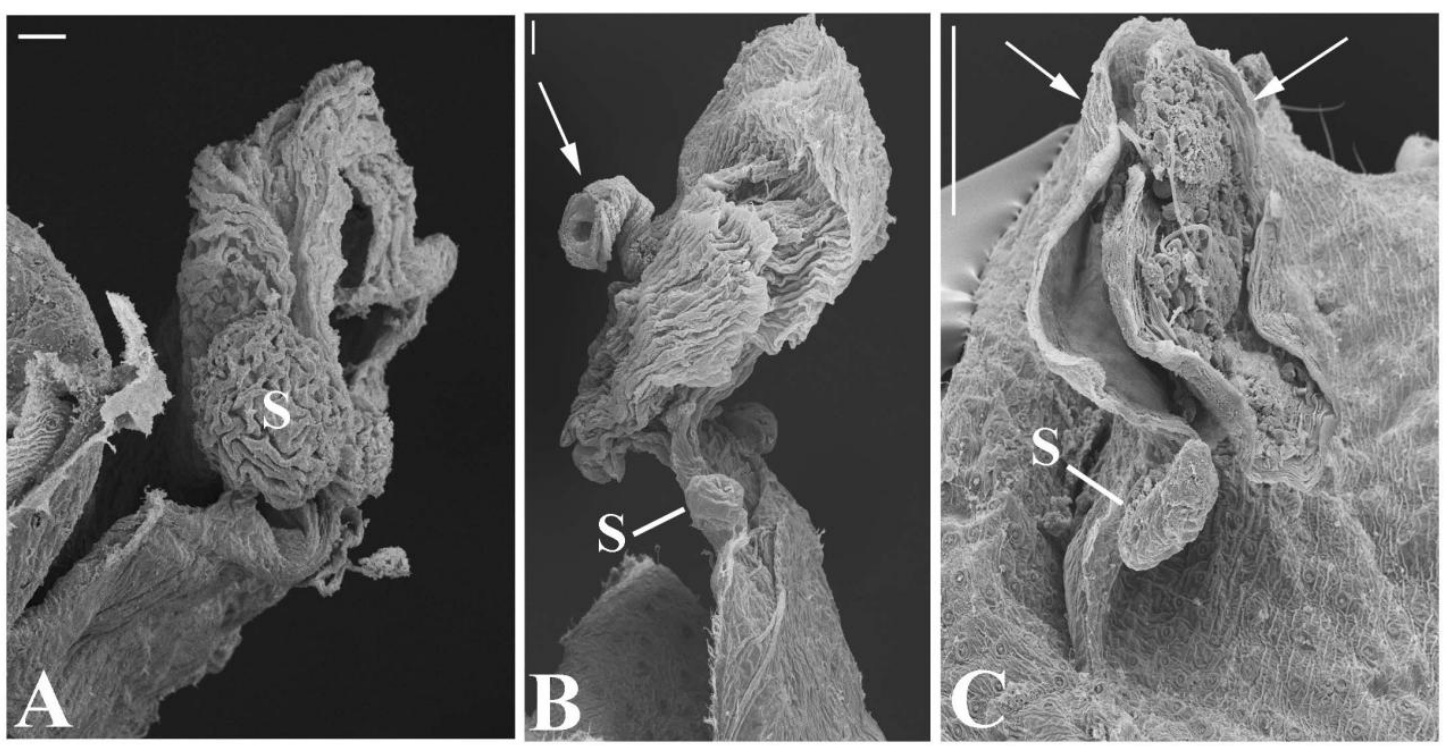

Figure 52. Uterus externus lateral view. A, Glenognatha argyrostilba. B, Glenognatha sp. nov. 2 (arrow, median projection). C, Glenognatha sp. nov. 1 (arrows, chambers). S: spermathecae. 
Haplogyne female spiders typically possess an ectodermal genital cavity (also called uterus externus, bursa copulatrix, vulva or vagina) and spatially separated spermathecae (receptacula seminis) in which a single duct connects the spermathecal lumen with the genital cavity ("cul-de-sac" type) (Uhl, 1994; Uhl, 2000). In Glenognatha the uterus externus can be divided in two regions: a) the uterus externus chamber (ectodermal genital cavity) which carries tendons for muscular attachment and the copulatory ducts and b) the uterus externus sac (UEs) which exhibit a variable morphology. In the present analysis, three states were created to cover this variation. Uterus externus sac divided in chambers (state 2) optimizes as synapomorphic for the clade D, although within this clade entire UEs (state 0 ) and with median projection (state 1) have appeared independently.

45. Uterus externus sac, apical chamber, form*: $(\mathrm{L}=1, \mathrm{CI}=1, \mathrm{RI}=1, \mathrm{~F}=1)$

(0) laminar (Fig. 53A).

(1) tubiform (Fig. 53B).
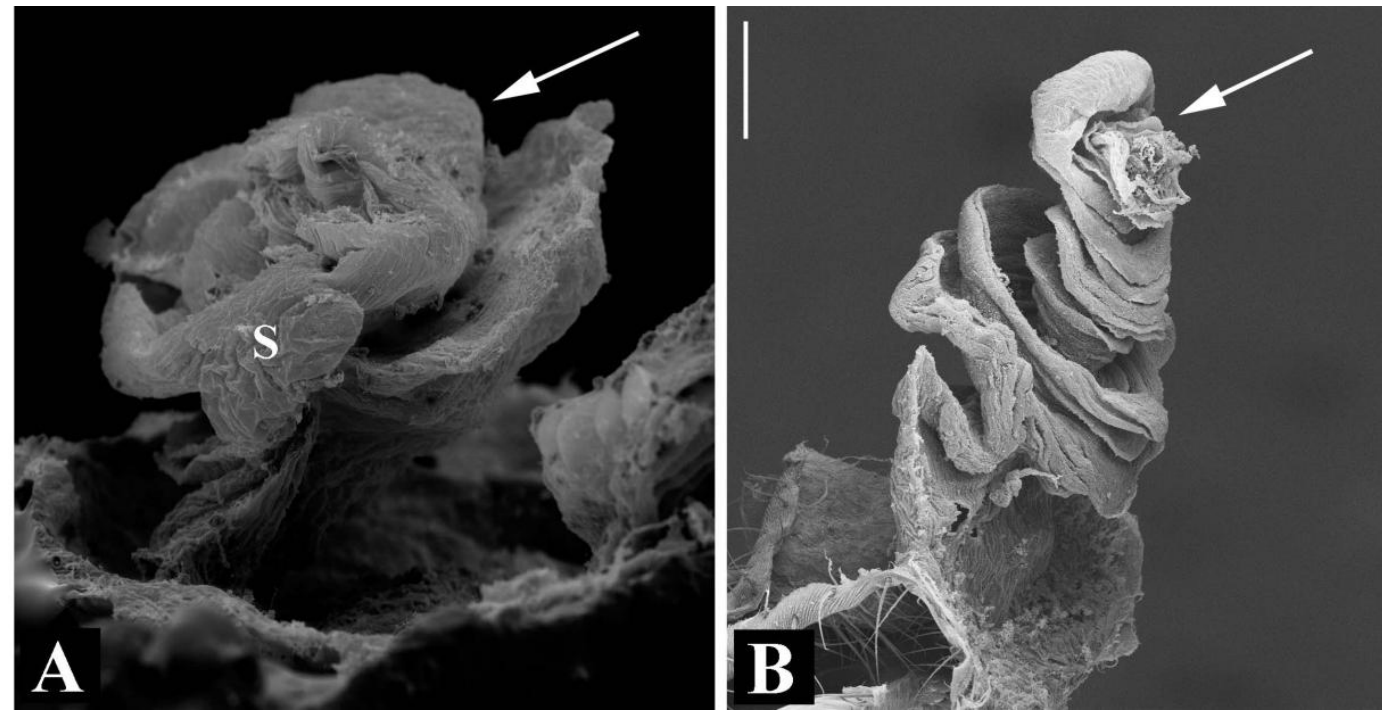

Figure 53. Uterus externus distal chamber. A, Glenognatha sp. nov. 1 (arrow, distal chamber). B, Glenognatha sp. nov. 8 (arrow, distal chamber). S: spermathecae.

Uterus externus sac with a tubiform apical chamber optimizes as synapomorphic for the clade E. If DELTRAN optimization is used the character appears as synapomorphic for the group G. gaujoni (G. globosa, G. sp. nov. 8). 
46. Uterus externus, thin projections*: $(\mathrm{L}=2, \mathrm{CI}=0.5, \mathrm{RI}=0, \mathrm{~F}=0.88)$

(0) absent;

(1) present (Fig. 54).

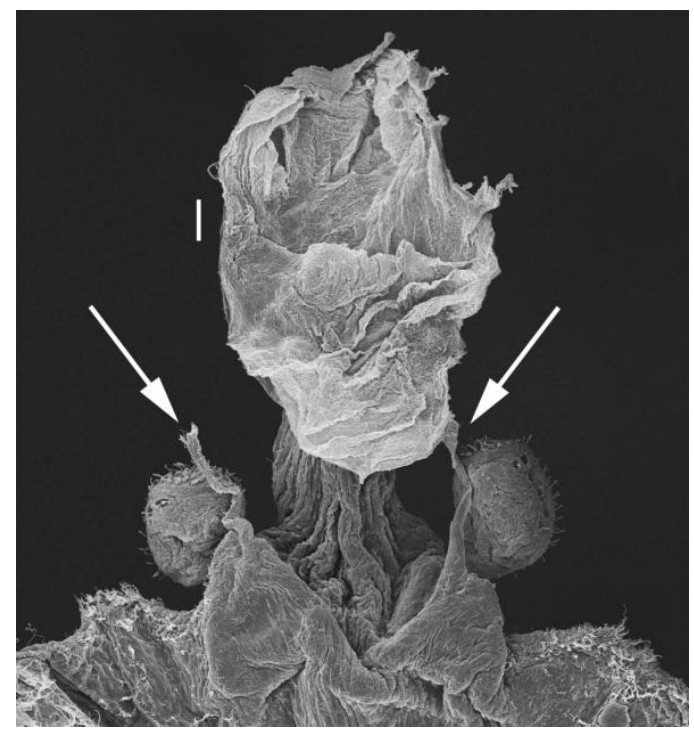

Figure 54. Dyschiriognatha dentata. Internal genitalia (arrows, uterus externus projections).

The presence of filiform projections in the uterus externus appears independently in D. dentata and P. clercki.

47. Female genitalia, posterior sac: $(\mathrm{L}=2, \mathrm{CI}=0.5, \mathrm{RI}=0, \mathrm{~F}=0.88)(\mathrm{APH} 11)$

(0) absent;

(1) present (Álvarez-Padilla \& Hormiga, 2011: fig. 24D).

Posterior sac in the female genitalia optimizes at the base of Tetragnathinae with a reversal in the clade Pachygnatha + Glenognatha. Under DELTRAN optimization posterior sac appears independently in Tetragnatha and Cyrtognatha.

\section{Cephalothorax morphology, chelicerae and legs}

48. Female, lateral eyes, position relative to each other: $(L=1, C I=1, R I=1)(A P H 11)$

(0) separated;

(1) together on a single tubercle (Fig. 55). 


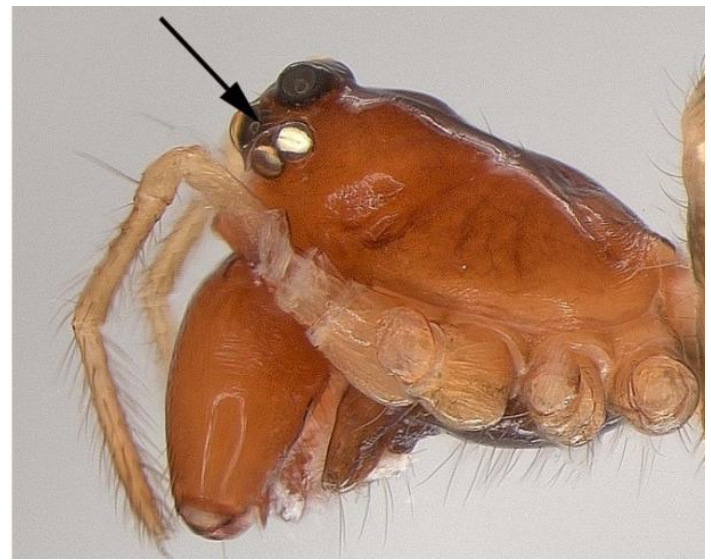

Figure 55. Glenognatha sp. nov. 1. Cephalothorax lateral view (arrow, lateral eyes).

Lateral eyes together is the plesiomorphic condition. Separated female lateral eyes is an autapomorphy of $T$. versicolor.

49. Male clypeus, height relative to AME diameter: $(\mathrm{L}=5, \mathrm{CI}=0.4, \mathrm{RI}=0, \mathrm{~F}=0.72)$ (APH11).

(0) less than one diameter;

(1) between one and two diameters (Fig. 56A);

(2) more than 2.5 diameters (Fig. 56B).
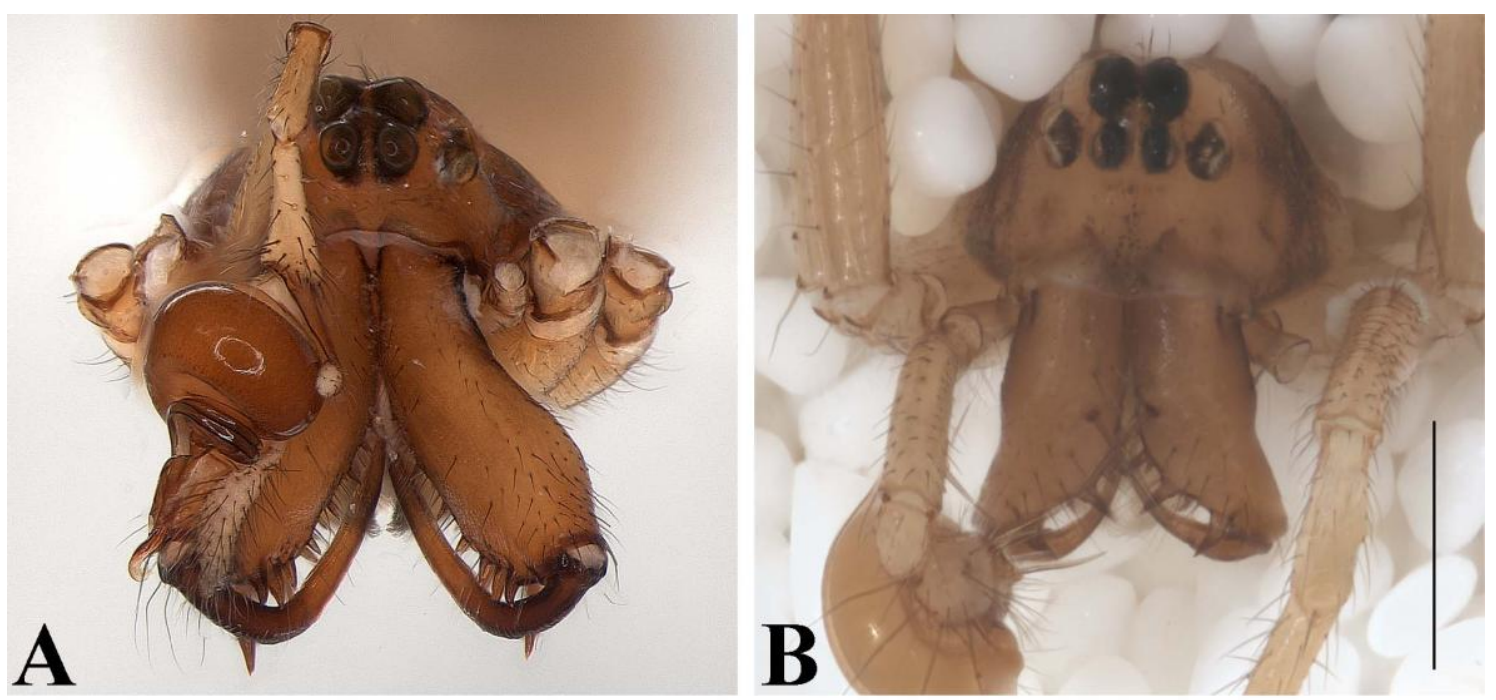

Figure 56. Male cephalothorax, frontal view. A, Glenognatha globosa. B, Glenognatha hirsutissima.

Highly homoplastic. Low clypeus (state 0) is autapomorphic for C. espanola, highest clypeus (state 2) appear independently in the clade A, C and $\mathrm{H}$. 
50. Carapace, lateral surface, setae-bearing depressions*: $(L=2, C I=0.5, R I=0.75$, $\mathrm{F}=0.88)$

(0) absent (Fig. 57A);

(1) present (Fig. 57B).

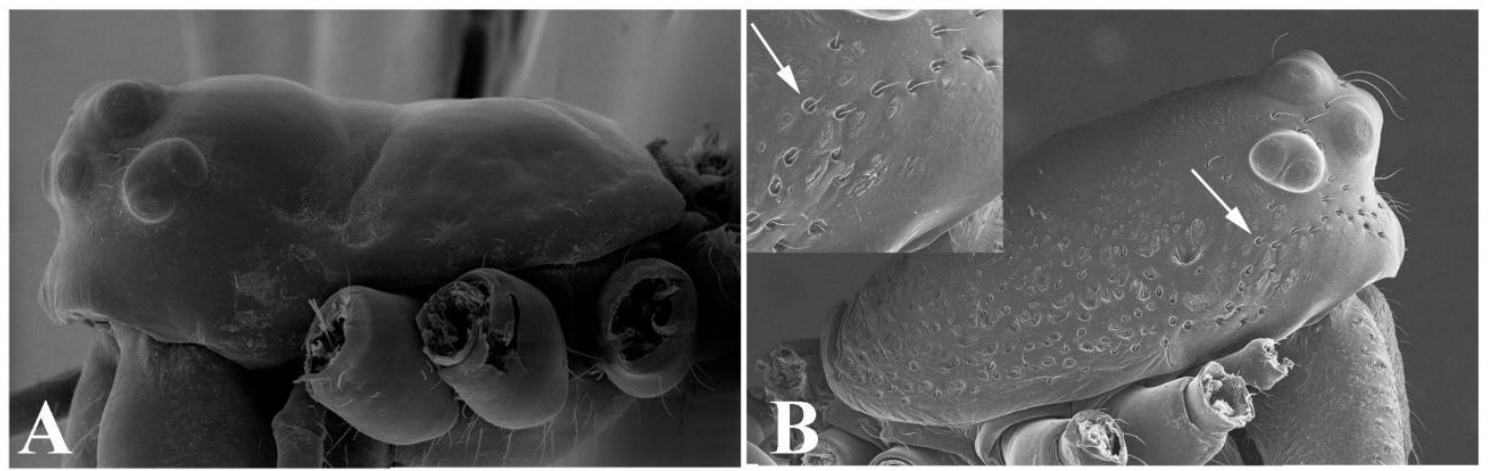

Figure 57. Female cephalothorax, lateral view. A, Dyschiriognatha dentata (arrow, setae-bearing depression). B, Glenognatha sp. nov. 2.

Setae-bearing depressions occur independently in Pachygnatha and the clade C. In the latter group these depressions are scattered in the lateral surfaces of the carapace and on the clypeus.

51. Carapace, lateral surface, irregular depressions*: $(L=1, C I=1, R I=1, F=1)$

(0) absent (Fig. 58A);

(1) present (Fig. 58B).
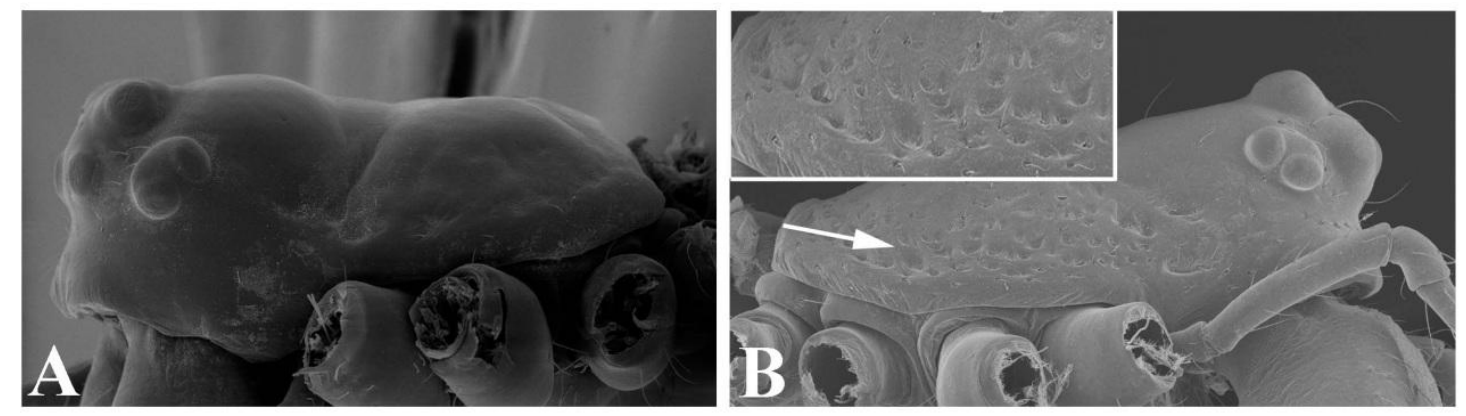

Figure 58. Female cephalothorax, lateral view. A, Glenognatha sp. nov. 2. B, Glenognatha argyrostilba (arrows, irregular depressions).

In members of the clade $\mathrm{C}$, besides rounded setae-bearing depressions, there are irregular depressions scattered on the lateral surfaces of the carapace. The presence of such depressions optimizes as a synapomorphy for this clade. 
52. Sternum, texture*: $(\mathrm{L}=2, \mathrm{CI}=0.5, \mathrm{RI}=0.66, \mathrm{~F}=0.88)$

(0) smooth (Fig. 59A);

(1) with rounded setae-bearing depressions (Fig. 59B).
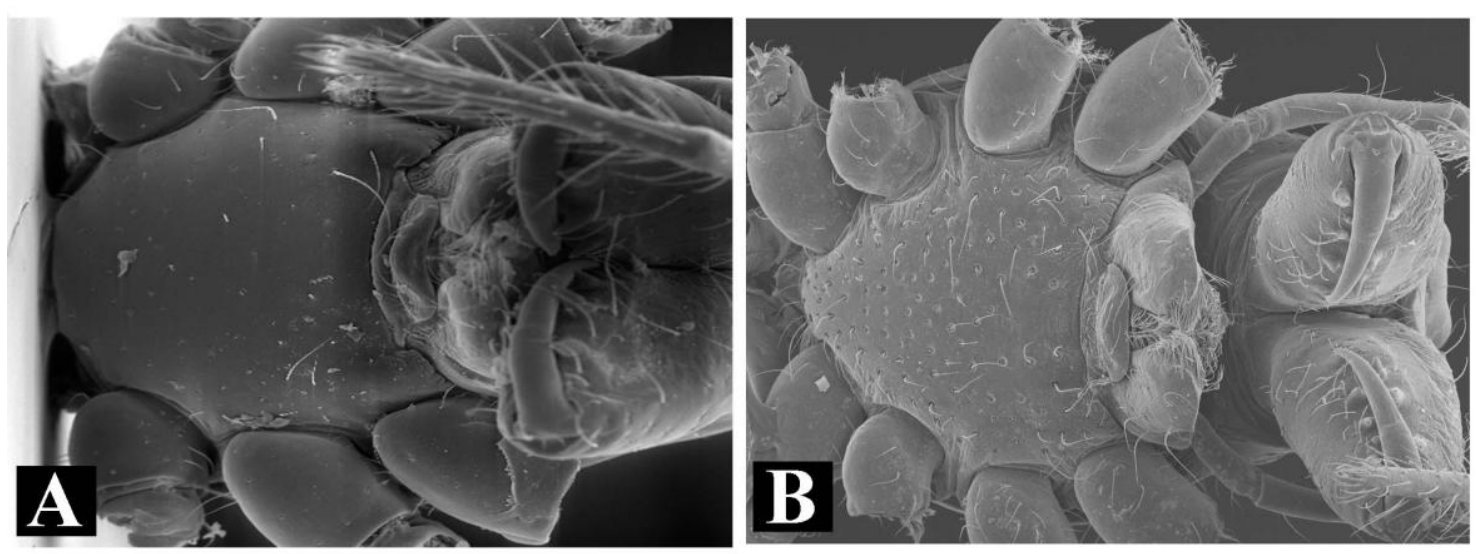

Figure 59. Female sternum A, Glenognatha sp. nov. 1. B, Glenognatha argyrostilba.

Rounded setae-bearing depressions in the sternum occur independently in Pachygnatha and the clade D. dentata + D. argyrostilba.

53. Sternum, configuration*: $(\mathrm{L}=4, \mathrm{CI}=0.25, \mathrm{RI}=0.62, \mathrm{~F}=0.72)$

(0) not projected between coxae (Fig. 60A);

(1) projected between coxae II-III (Fig. 60B).
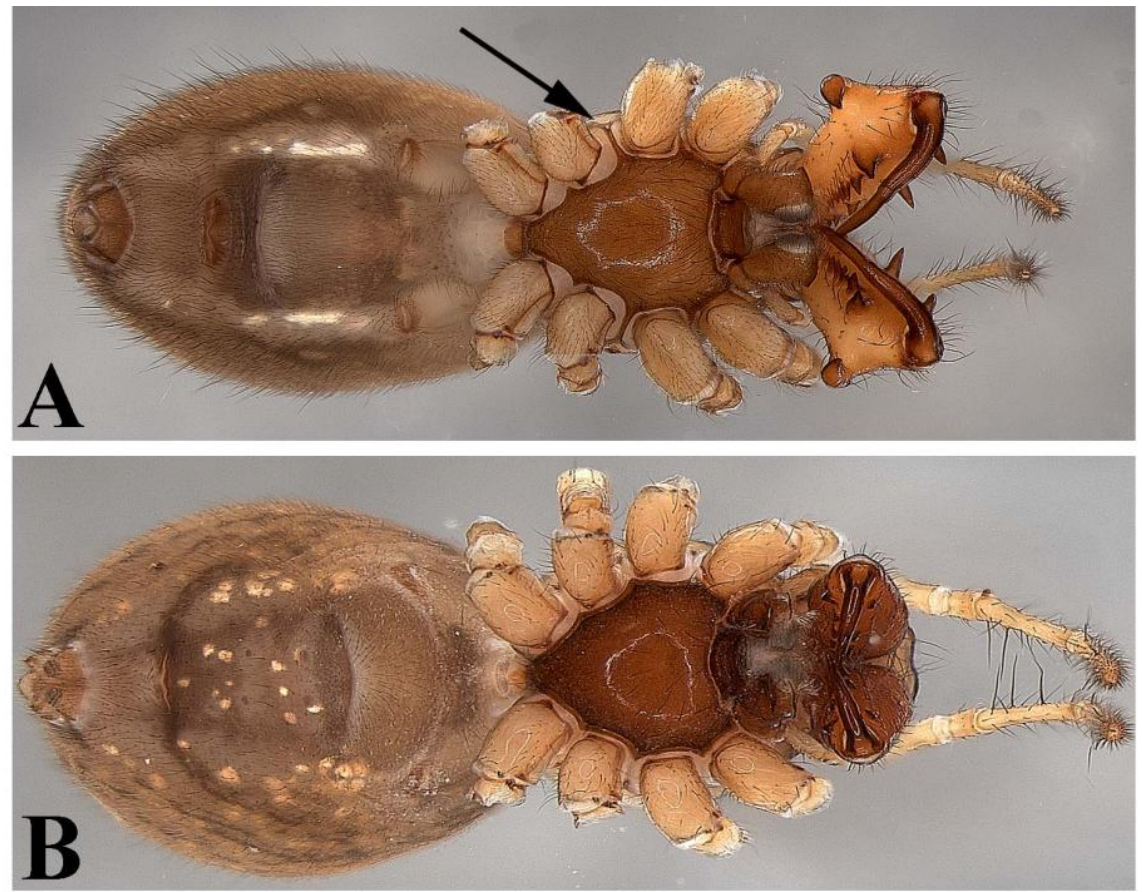

Figure 60. Female sternum A, Glenognatha lacteovittata. B, Glenognatha sp. nov. 8 (arrow, sternum projection). 
Highly homoplastic. Sternum projections occur independently in T. versicolor, Pachygnatha, clade C and the group G. gaujoni (G. globosa, G. sp. nov. 8).

54. Chelicerae, anteroectal surface, setae-bearing tubercles: $(L=1, C I=1, R I=1, F=1)$ (AHS 2009)

(0) absent (Fig. 61A);

(1) present (Fig. 61B).

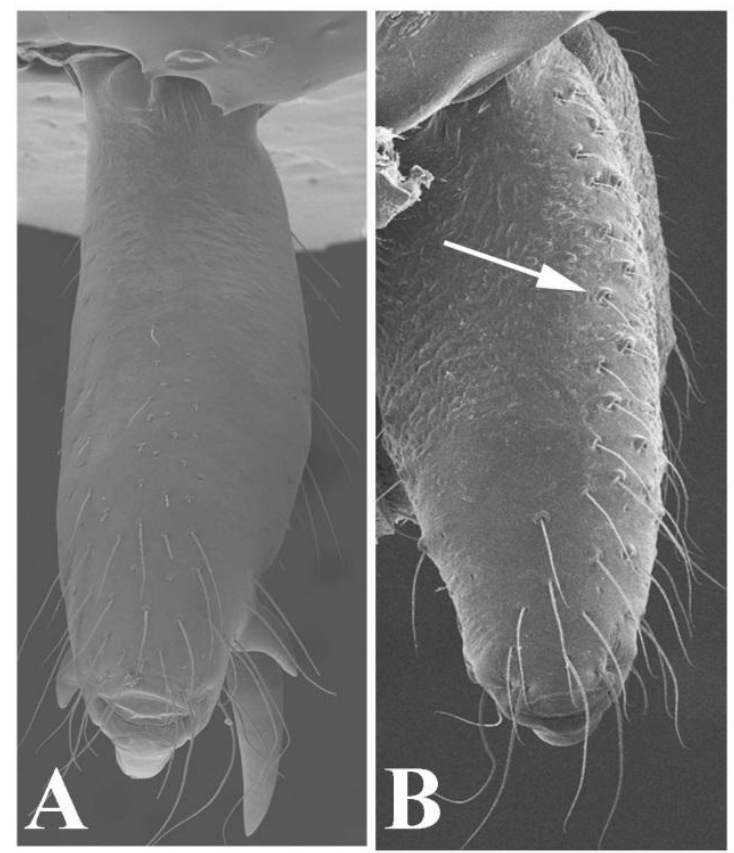

Figure 61. Female chelicerae, lateral view. A, Glenognatha gaujoni. B, Dyschiriognatha dentata (arrow, setae bearing tubercles).

Cheliceral anteroectal setae-bearing tubercles optimizes as synapomorphic for the group D. dentata + D. argyrostilba.

55. Male, chelicerae, orientation: $(\mathrm{L}=5, \mathrm{CI}=0.2, \mathrm{RI}=0.6, \mathrm{~F}=0.66)(\mathrm{DH} 09)$

(0) not projected (Fig. 62A);

(1) projected (Fig. 62B). 

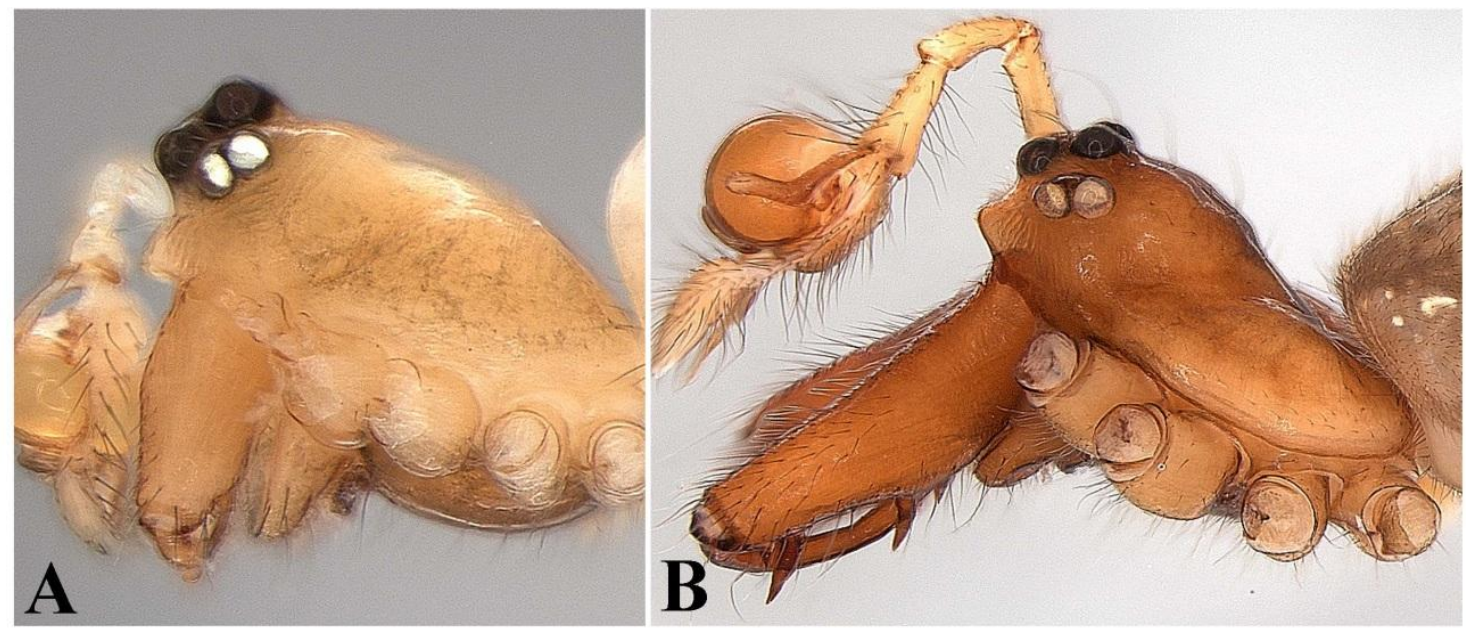

Figure 62. Male cephalothorax, lateral view. A, Glenognatha spherella. B, Glenognatha sp. nov. 7.

Highly homoplastic. Projected male cephalothorax appear independently in $T$. versicolor, clade E, G. sp. nov. 6, G. sp. nov. 1 and the group ((G. sp. nov. 7, G. sp. nov. 11) (G. emertoni, G. minuta)).

56. Male, chelicerae, anterior tooth: $(\mathrm{L}=5, \mathrm{CI}=0.2, \mathrm{RI}=0.55, \mathrm{~F}=0.66)(\mathrm{DH} 09)$

(0) absent (Fig. 63A);

(1) present (Fig. 63B).

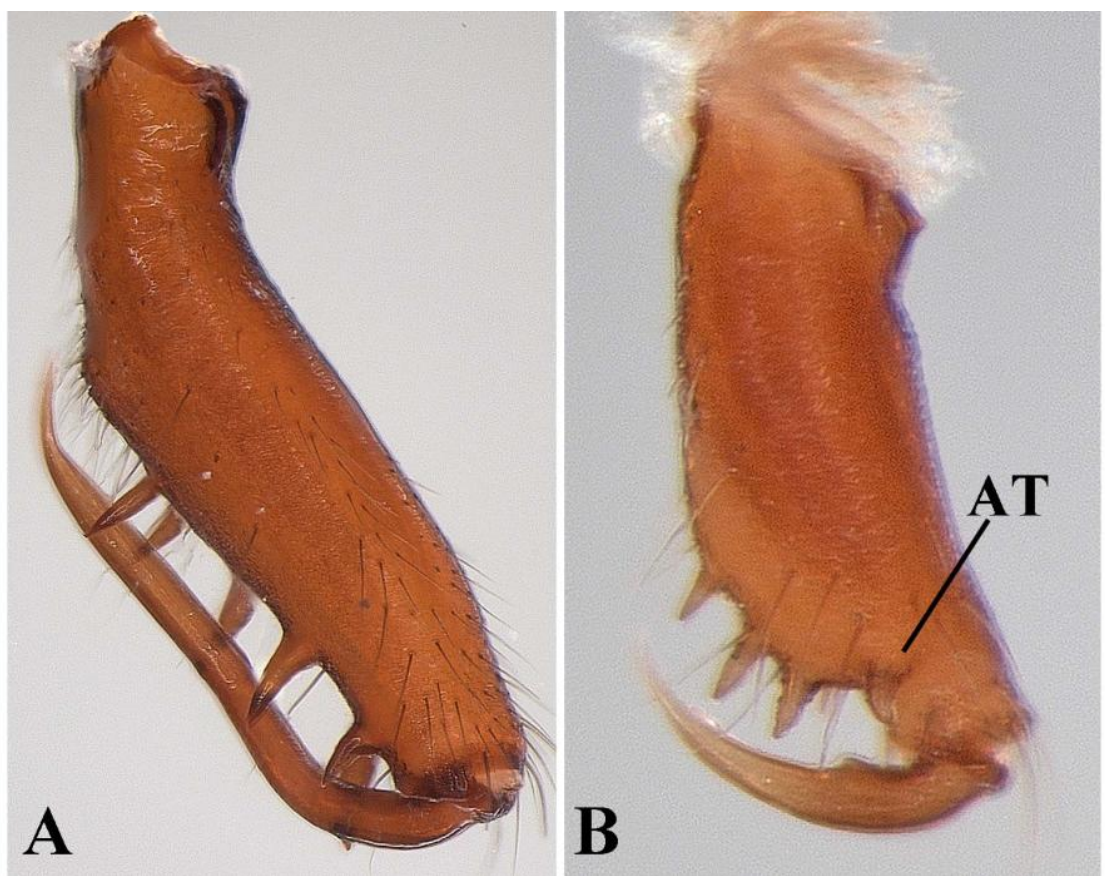

Figure 63. Male left chelicerae, anterior view. A, Glenognatha sp. nov. 7. B, Glenognatha foxi. AT: anterior tooth. 
Male anterior tooth optimizes at the base of Tetragnathinae with a reversal in the group Pachygnatha + Glenognatha. Within the latter clade the anterior tooth appears as a secondary condition in $P$. clercki and in the clade $G$ with a subsequent reversal in $G$. smilodon. Under DELTRAN optimization the anterior tooth appears independently in $T$. versicolor, C. espanola, P. clercki and the clade G. According with the topological convention defined (Fig. 1), I changed the name dorsal tooth proposed by Dimitrov \& Hormiga (2009) to anterior tooth in the character definition.

57. Male, chelicerae, anterior tooth size relative to the $\operatorname{Prt} 1^{*}:(L=2, C I=0.5, R I=0.5$, $\mathrm{F}=0.88)$

(0) subequal or shorter (Fig. 64A);

(1) longer (Fig. 64B).
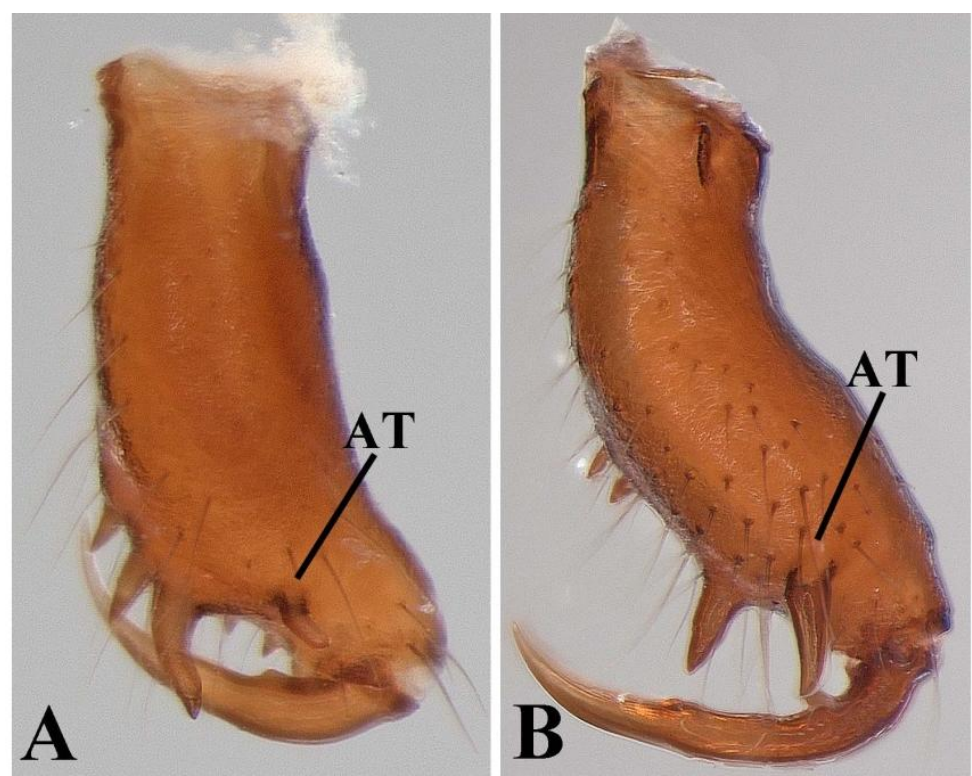

Figure 64. Male left chelicerae, anterior view. A, Glenognatha heleios. B, Glenognatha sp. nov. 6. AT: anterior tooth.

Short anterior tooth (state 1) appears independently in C. espanola and within the clade $\mathrm{G}$.

58. Male, chelicerae, fang outgrowth: $(\mathrm{L}=4, \mathrm{CI}=0.25, \mathrm{RI}=0.78, \mathrm{~F}=0.72)(\mathrm{DH} 09)$

(0) absent (Fig. 65A);

(1) present (Fig. 65B). 


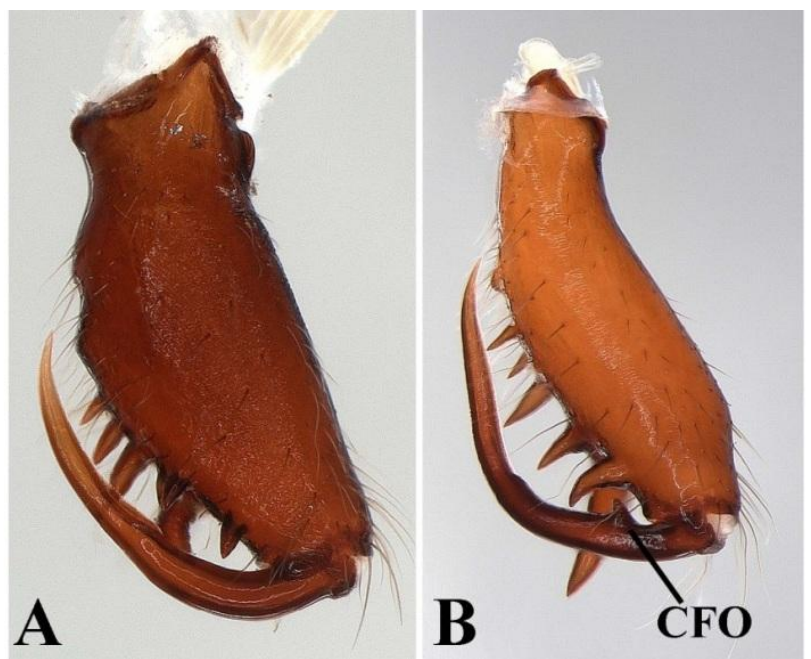

Figure 65. Male left chelicerae, anterior view. A, Glenognatha sp. nov. 11. B, Glenognatha gaujoni. CFO: cheliceral fang outgrowth.

Male cheliceral fang outgrowth optimizes as synapomorphic for C. espanola + Pachygnatha + Glenognatha. Independent reversals occurred in G. globosa + G. sp. nov. $8, G$. sp. nov. $9+G$. sp. nov. 10 and the clade H.

59. Male, chelicerae, anteromesal macrosetae*: $(\mathrm{L}=1, \mathrm{CI}=1, \mathrm{RI}=1)$

(0) absent (Fig. 66A);

(1) present (Fig. 66B).
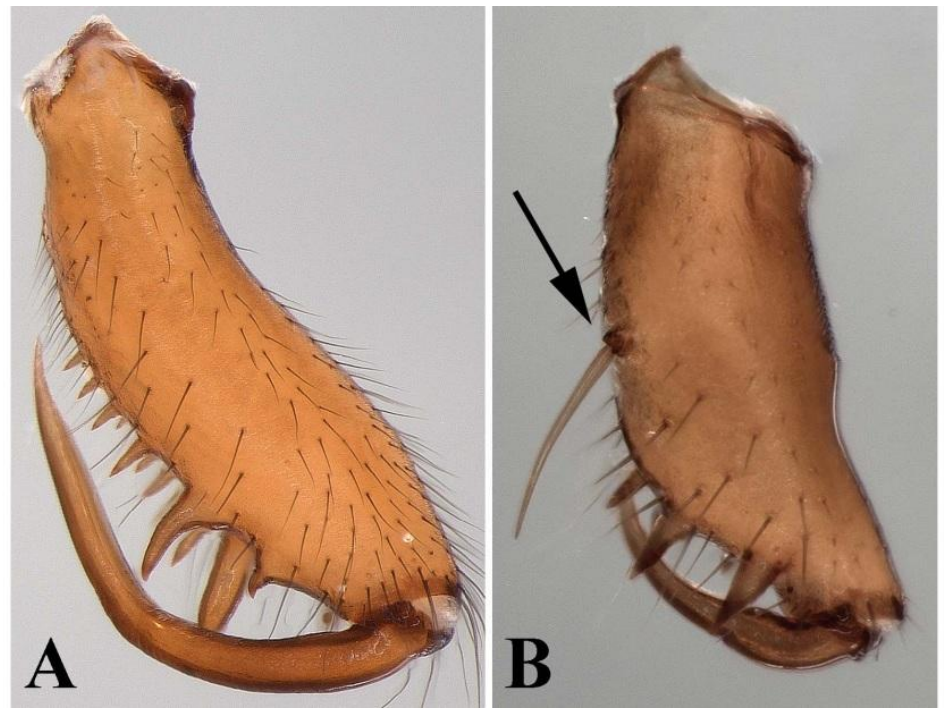

Figure 66. Male left chelicerae, anterior view. A, Glenognatha sp. nov. 8. B, Glenognatha hirsutissima (arrow, macrosetae). 
Anteromesal macrosetae on the male chelicerae is autapomorphic for $G$. hirsutissima.

60. Male, chelicerae, anteromarginal projection*: $(\mathrm{L}=2, \mathrm{CI}=0.5, \mathrm{RI}=0 ; \mathrm{F}=0.88)$

(0) absent (Fig. 67A);

(1) present (Fig. 67B).
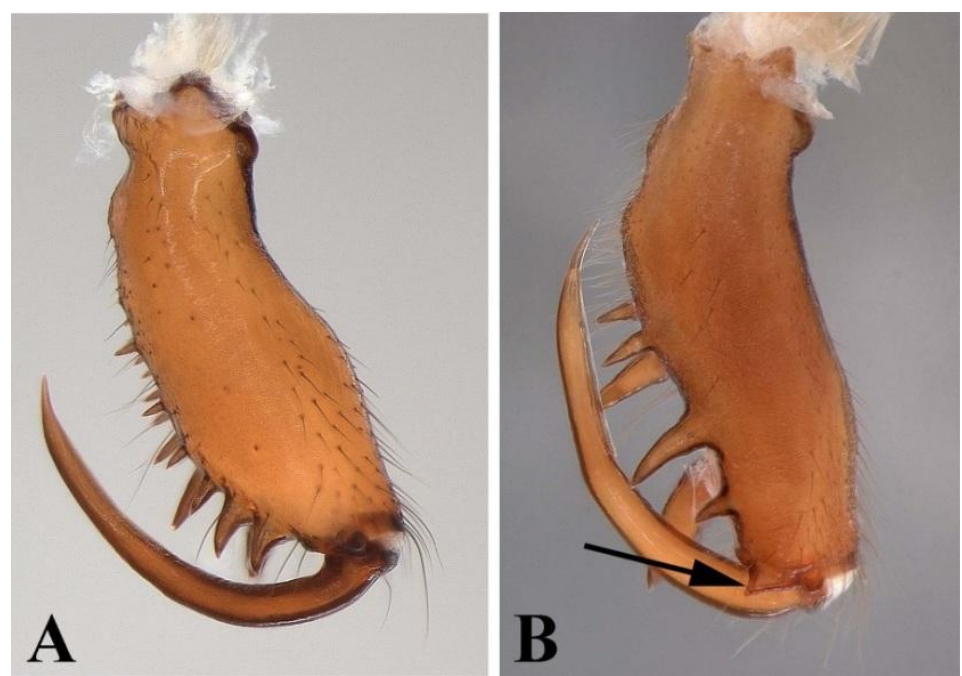

Figure 67. Male left chelicerae, anterior view. A, Glenognatha globosa. B, Glenognatha emertoni (arrow, anteromarginal projection).

Anteromarginal projection in the male chelicerae appears independently in $T$. versicolor and G. emertoni.

61. Male, Prt1, size relative to other promarginal teeth*: $(L=2, C I=0.5, R I=0.75$; $\mathrm{F}=0.88$ )

(0) subequal or shorter (Fig. 68A);

(1) conspicuously enlarged (Fig. 68B). 

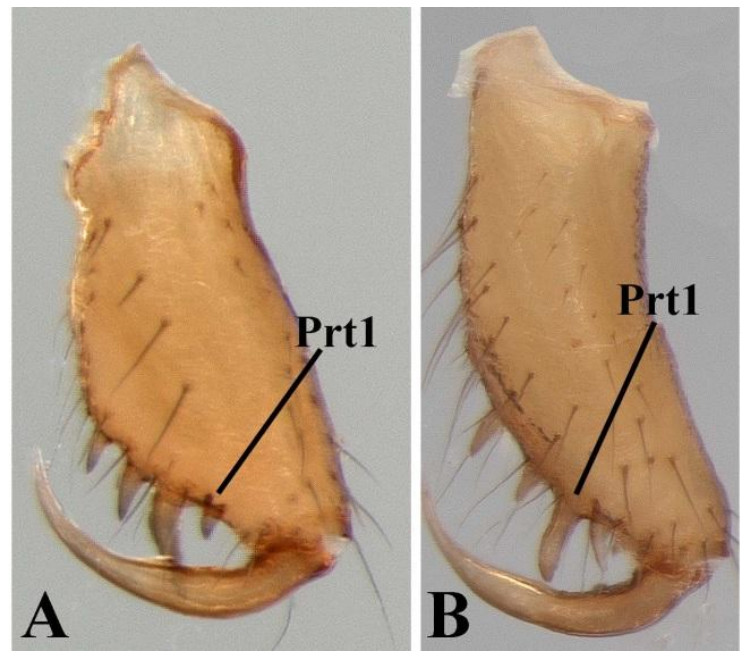

Figure 68. Male left chelicerae, anterior view. A, Glenognatha spherella. B, Glenognatha sp. nov. 3. Prt1: promarginal tooth 1.

Conspicuously enlarged promarginal tooth 1 support the clade G. Reversal to the plesiomorphic state occur in the group G. foxi (G. sp. nov. 9, G. sp. nov. 10).

62. Male, Prt1, shape*: $(\mathrm{L}=2, \mathrm{CI}=1, \mathrm{RI}=1 ; \mathrm{F}=1)$

(0) straight to moderately curved (Fig. 69A);

(1) curved, with two direction changes from the base to the apical portion (Fig. 69B);

(2) apical portion strongly curved (Fig. 69C).
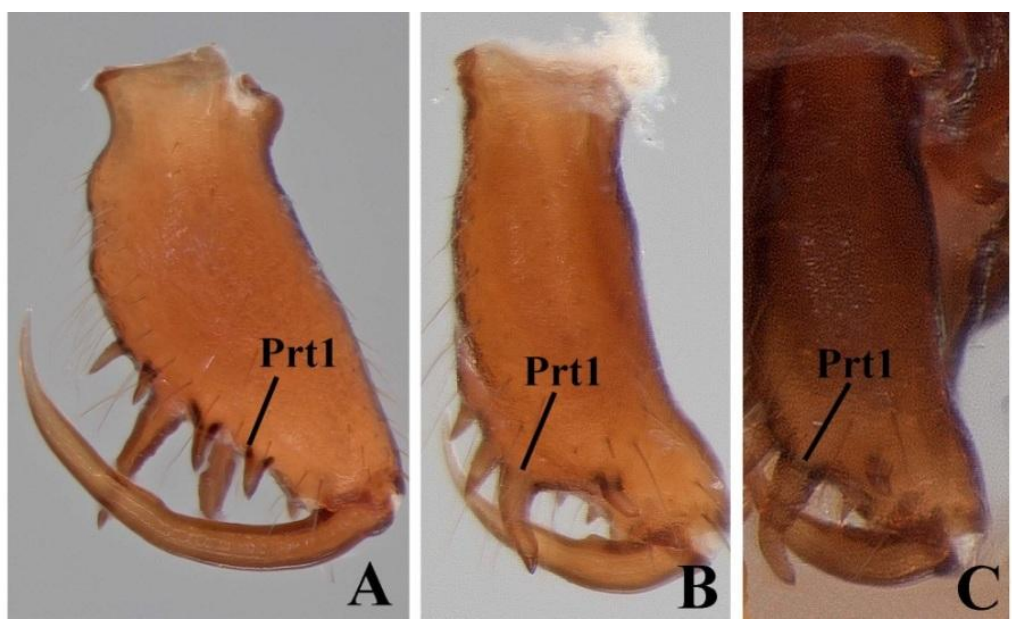

Figure 69. Male left chelicerae, anterior view. A, Glenognatha minuta. B, Glenognatha heleios. C, Glenognatha iviei. Prt1: promarginal tooth 1.

Straight Prt1 is the widespread condition in Glenognatha. Curved tooth optimizes as synapomorphic for the group G. smilodon (G. heleios, G. iviei), within this clade a strongly curved tooth is autapomorphic for G. iviei. 
63. Male, Ret2, size relative to $\operatorname{Ret} 1 *$ : $(L=2, C I=1, R I=1 ; F=1)$

(0) small, shorter to 2.8 times the Ret1 length (Fig. 70, 71A);

(1) big, at least 3.8 times longer than the Ret1 length (Fig. 70, 71B).

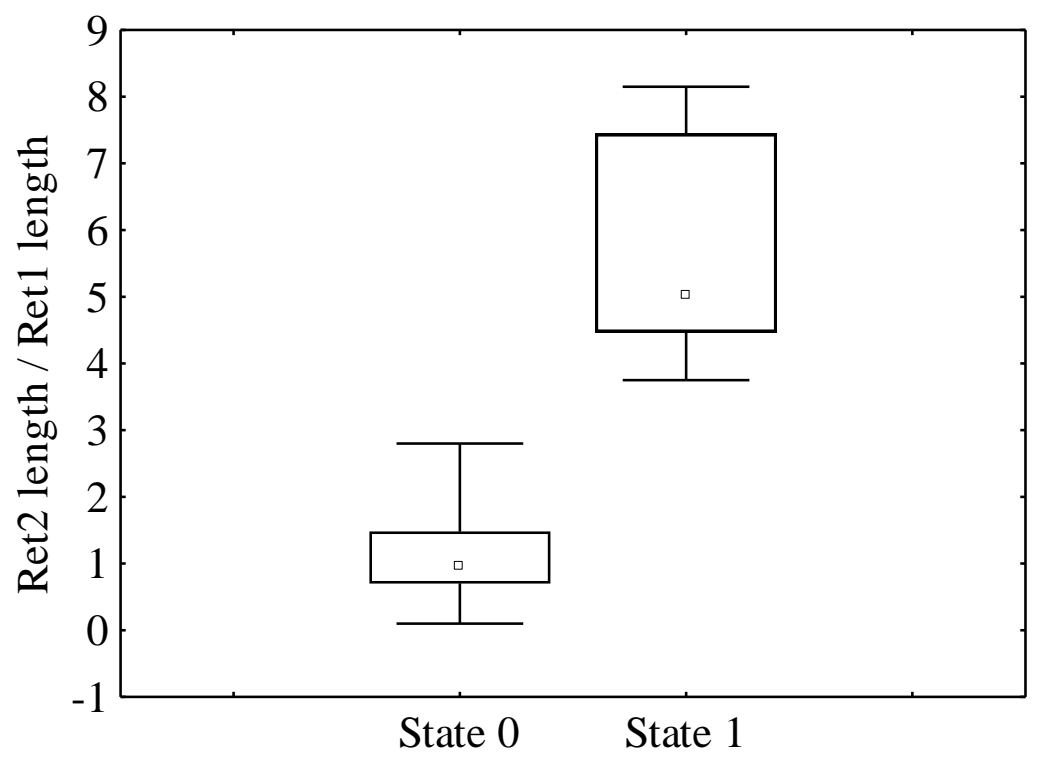

Figure 70. Character 63 states. The box plot shows median (squares), 50\% (box limits) and minimum and maximum (brackets) of all values, $(\mathrm{U}=0.00, \mathrm{p}<0.001, \mathrm{n}=85)$. Ret 1 : Retromarginal tooth 1. Ret2: Retromarginal tooth 2.
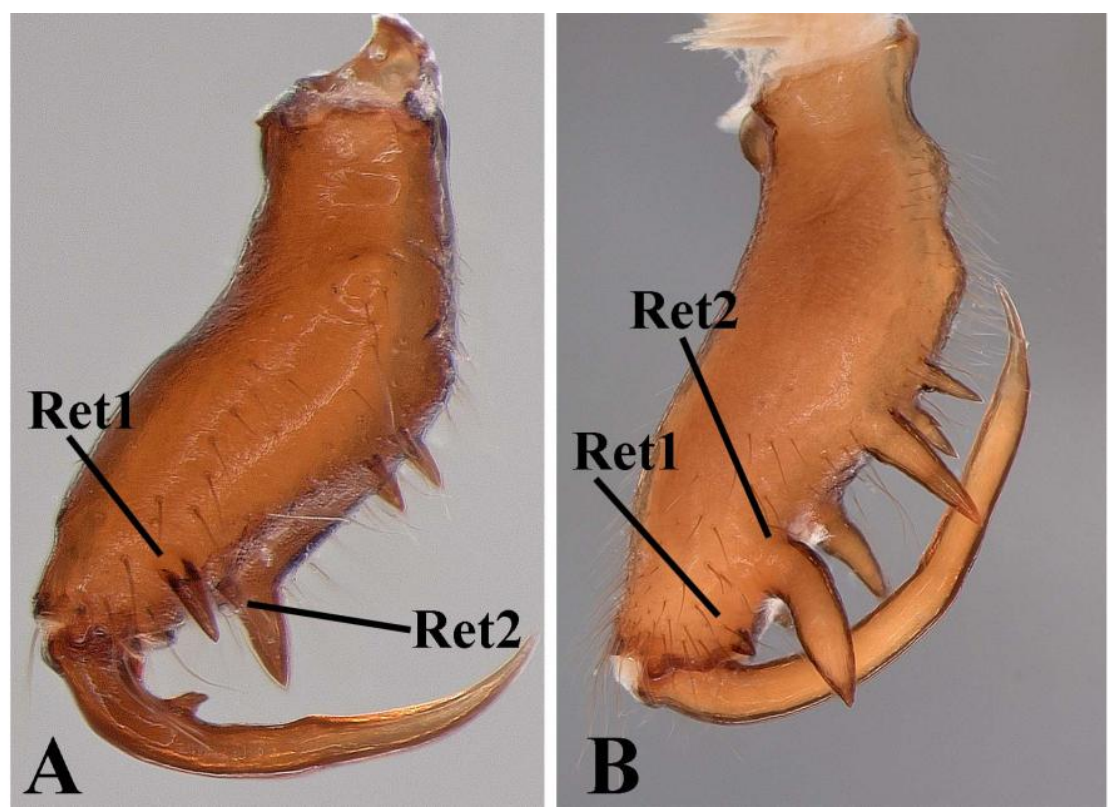

Figure 71. Male left chelicerae, posterior view. A, Glenognatha sp. nov. 6. B, Glenognatha emertoni. Ret1: retromarginal tooth 1. Ret2: retromarginal tooth 2. 
The length of the Ret 2 is taken relative to the length of the Ret1. Teeth length was measured along the midline from the base to the tip. Long Ret2 optimizes as synapomorphy for the clade $\mathrm{J}$.

64. Male, Ret2, shape in posterior view*: $(\mathrm{L}=3, \mathrm{CI}=0.33, \mathrm{RI}=0.66 ; \mathrm{F}=0.8)$

(0) straight or mesally curved (Fig. 72A);

(1) ectally curved (Fig. 72B).
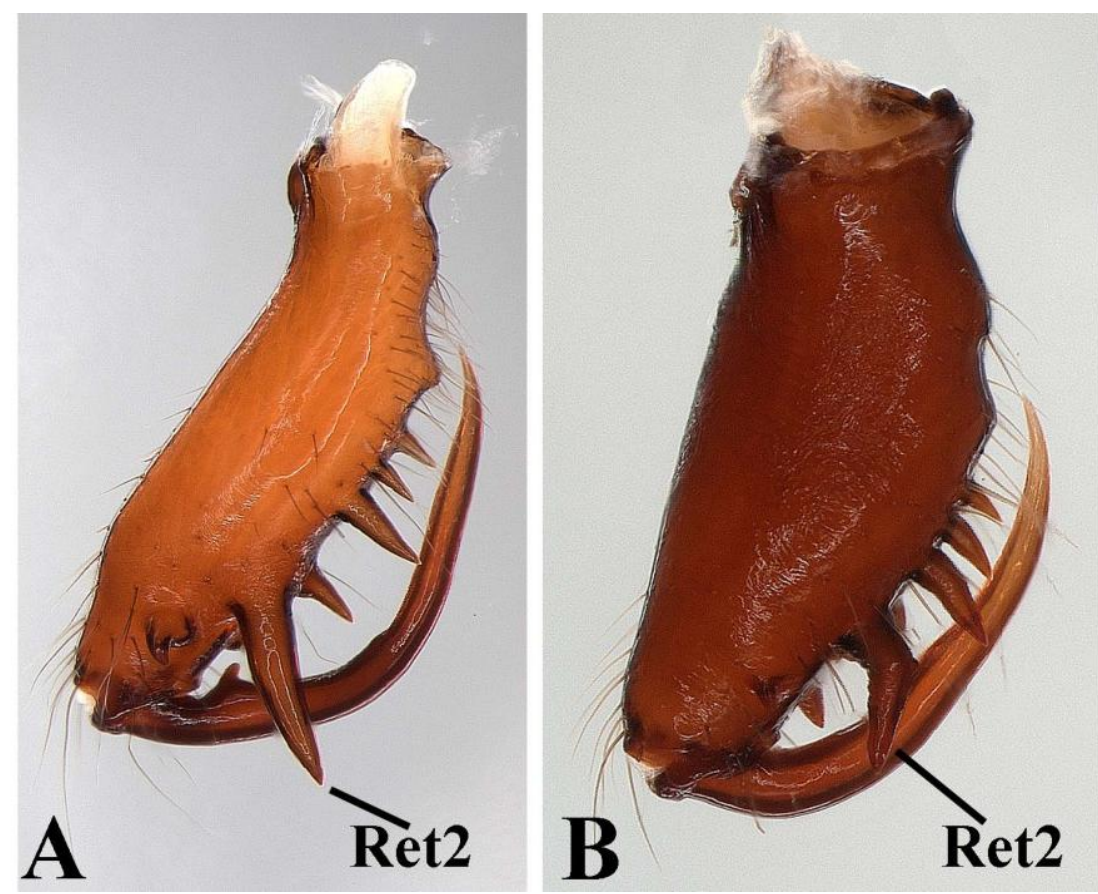

Figure 72. Male left chelicerae, posterior view. A, Glenognatha gaujoni. B, Glenognatha sp. nov. 11. Ret2: retromarginal tooth 2.

Retromarginal tooth 2 ectally curved appears independently in D. dentata and the clade $\mathrm{J}$.

65. Male, Ret 3, size relative to $\operatorname{Ret} 1 *:(L=1, C I=1, R I=1 ; F=1)$

(0) small, shorter to two times Ret1 length (Fig. 73, 74A);

(1) big, at least three times longer than the Ret1 length (Fig. 73, 74B). 


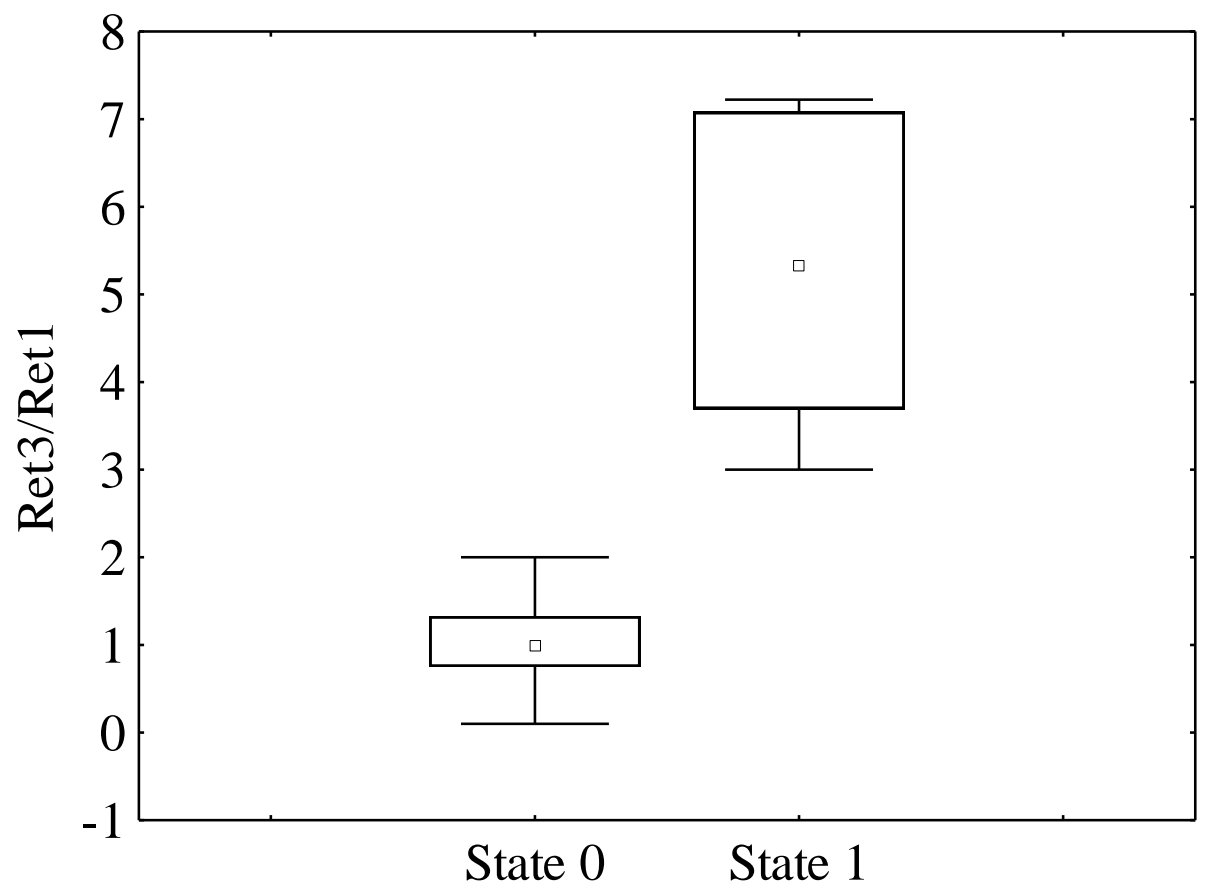

Figure 73. Character 65 states. The box plot shows median (squares), 50\% (box limits) and minimum and maximum (brackets) of all values, $(\mathrm{U}=0.00, \mathrm{p}<0.001, \mathrm{n}=85)$. Ret1: Retromarginal tooth 1. Ret3: Retromarginal tooth 3.

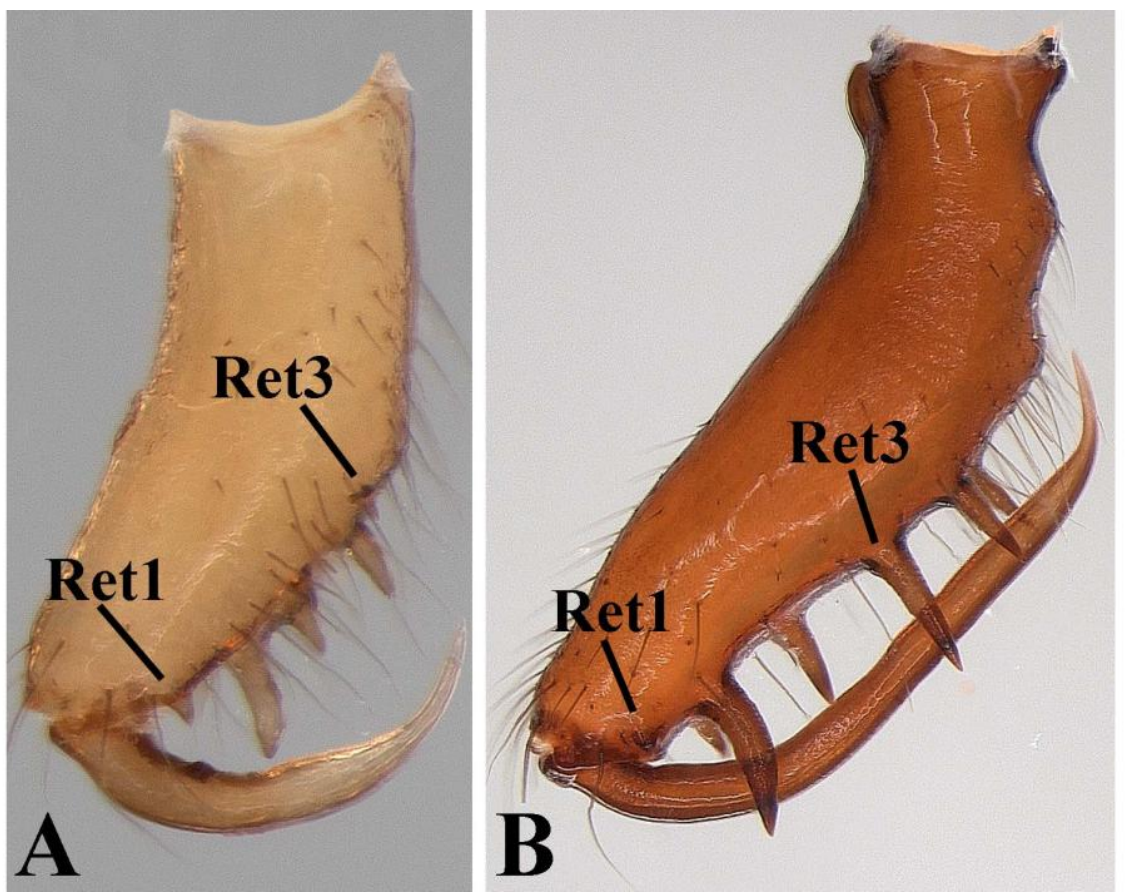

Figure 74. Male left chelicerae, posterior view. A, Glenognatha sp. nov. 3. B, Glenognatha sp. nov. 7. Ret1: retromarginal tooth 1. Ret3: retromarginal tooth 3. 
The length of the Ret3 is taken relative to the length of the Ret1. Teeth length was measured along the midline from the base to the tip. Long Ret3 optimizes as synapomorphy for the group (G. sp. nov. 7, G. sp. nov. 11) (G. emertoni, G. minuta).

66. Male, Rte1, position relative to the $R t 2 *(\mathrm{~L}=4, \mathrm{CI}=0.25, \mathrm{RI}=0.66 ; \mathrm{F}=0.72)$

(0) aligned (Fig. 75A);

(1) posteriorly displaced (Fig. 75B).

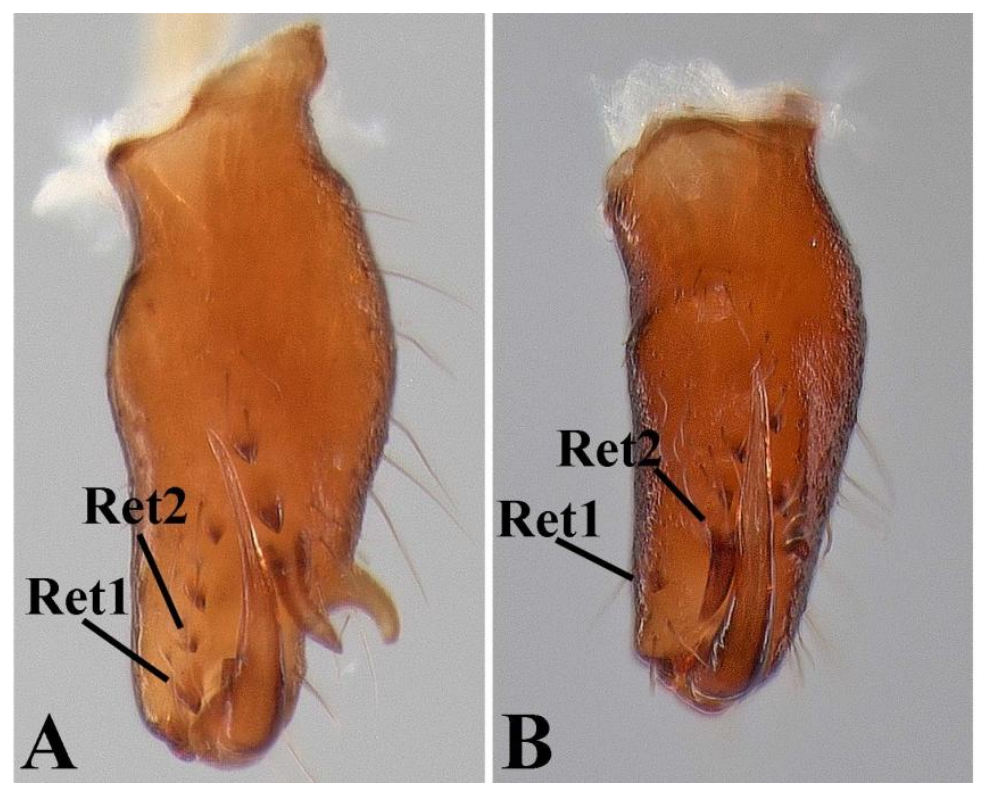

Figure 75. Male left chelicerae, mesal view. A, Glenognatha heleios. B, Glenognatha australis. Ret1: retromarginal tooth 1. Ret3: retromarginal tooth 3.

Aligned retromarginal teeth is the plesiomorphic condition. Ret1 posteriorly displaced appears as synapomorphic for Glenognatha. Within the genus, several reversals occurred in G. heleios + G. iviei, G. hirsutissima and G. emertoni + G. minuta.

67. Male, Prt2, position relative to Prt1 and Prt3*: $(\mathrm{L}=6, \mathrm{CI}=0.16, \mathrm{RI}=0.44 ; \mathrm{F}=0.61)$

(0) closer to Prt1 than to Prt3 or subequally distant from both (Fig. 76A);

(1) closer to Prt3 than to Prt1 (Fig. 76B); 


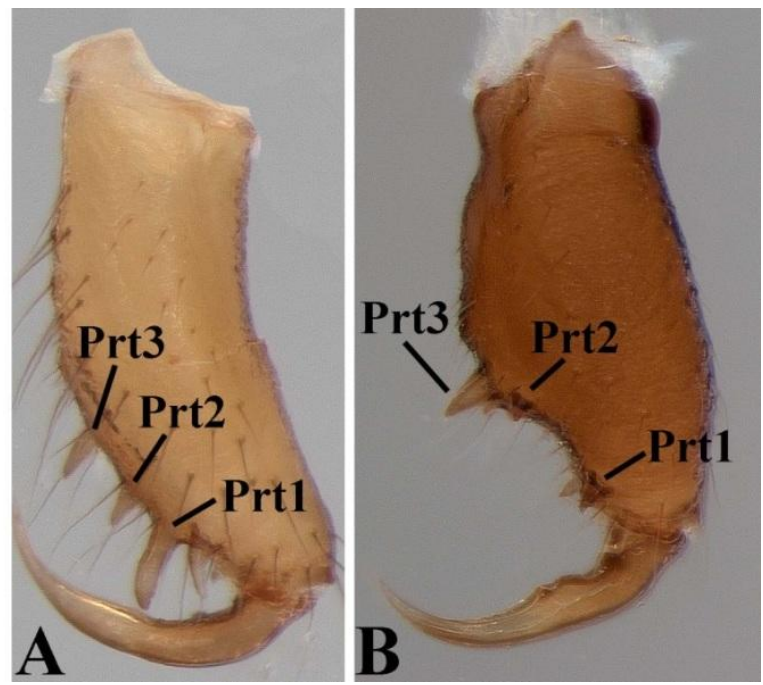

Figure 76. Male left chelicerae, anterior view. A, Glenognatha sp. nov. 3. B, Glenognatha argyrostilba. Prt: promarginal tooth.

Highly homoplastic. Male Prt2 closer to Prt3 than to Prt1 optimizes ambiguously as synapomorphic for Cyrtognatha (Pachygnatha, Glenognatha). Within the latter genus a basal reversal occurred. Under DELTRAN optimization this condition appears independently several times.

68. Female, chelicerae, anterior tooth*: $(\mathrm{L}=2, \mathrm{CI}=0.5, \mathrm{RI}=0 ; \mathrm{F}=0.88)$

(0) absent (Fig. 77A);

(1) present (Fig. 77B).

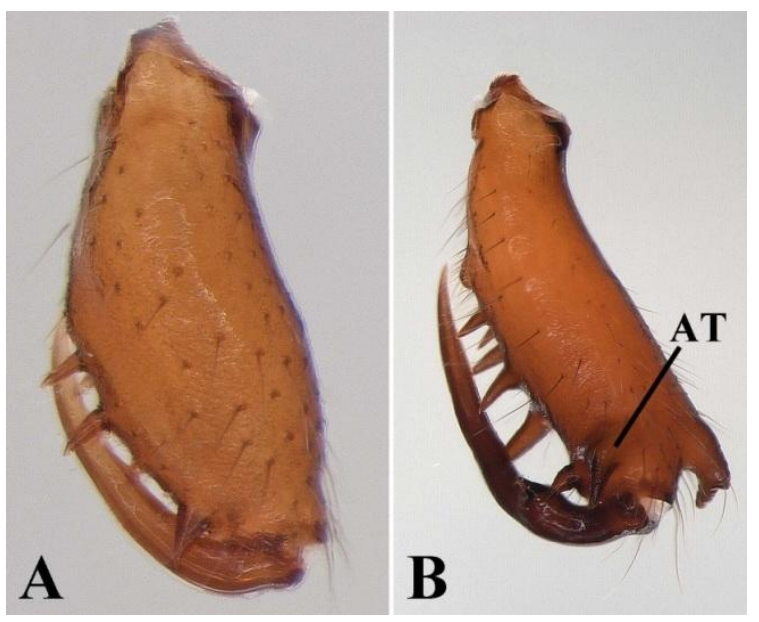

Figure 77. Female left chelicerae, anterior view. A, Glenognatha sp. nov. 6. B, Glenognatha gaujoni. AT: anterior tooth. 
Female anterior tooth optimizes as synapomorphic for the clade G. gaujoni ( $G$. globosa, $G$. sp. nov. 8,) with a reversal in the latter species.

69. Female, chelicerae, fang outgrowth*: $(\mathrm{L}=2, \mathrm{CI}=0.5, \mathrm{RI}=0 ; \mathrm{F}=0.88)$

(0) absent (Fig. 78A);

(1) present (Fig. 78B).

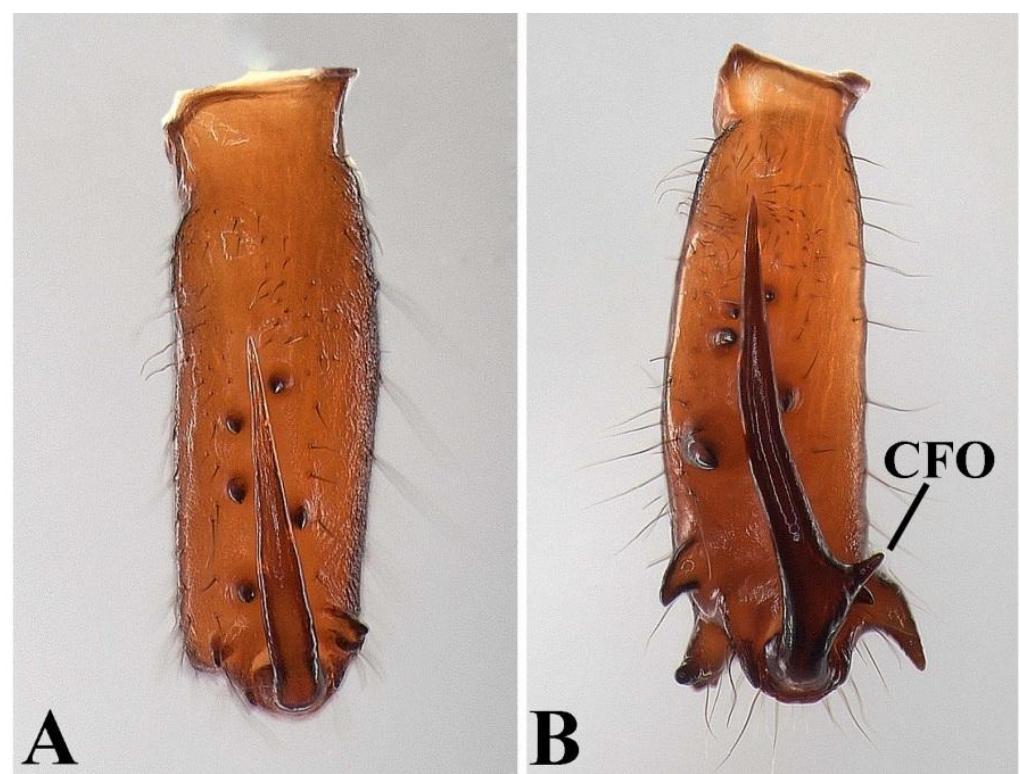

Figure 78. Female left chelicerae, mesal view. A, Glenognatha sp. nov. 7. B, Glenognatha gaujoni. CFO: cheliceral fang outgrowth.

Female cheliceral fang outgrowth appears independently in G. gaujoni and the clade G. foxi (G. sp. nov. 9, G. sp. nov. 10).

70. Female, chelicerae, ectal apophysis*: $(\mathrm{L}=2, \mathrm{CI}=0.5, \mathrm{RI}=0 ; \mathrm{F}=0.88)$

(0) absent (Fig. 79A);

(1) present (Fig. 79B). 


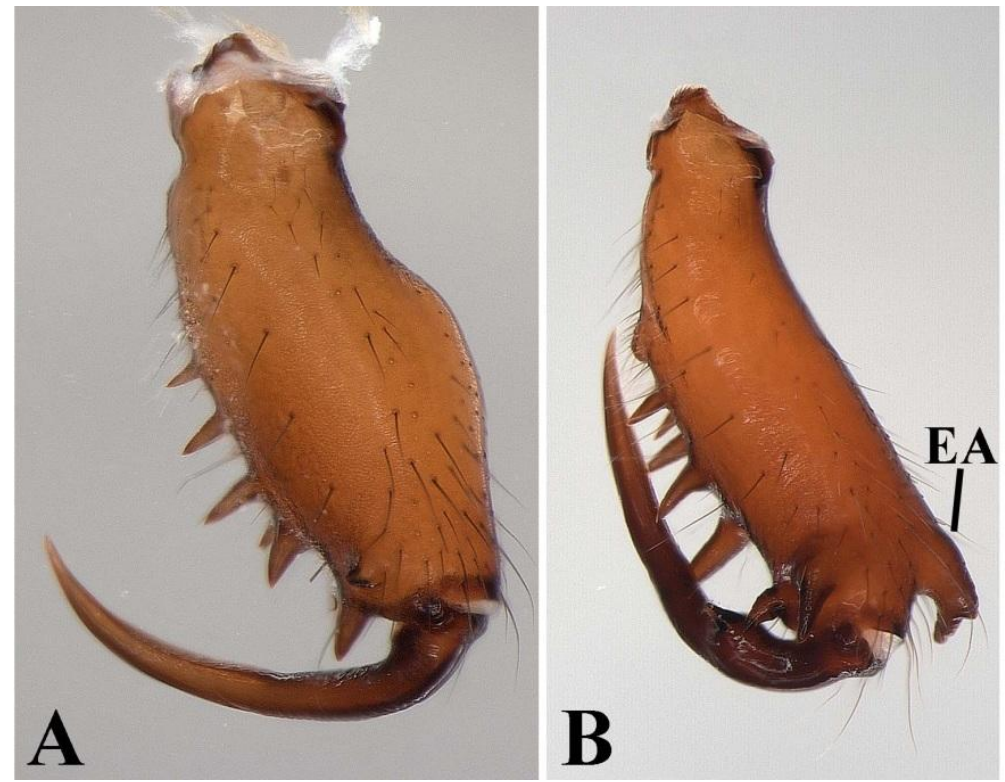

Figure 79. Female left chelicerae, anterior view. A, Glenognatha globosa B, Glenognatha gaujoni. EA: ectal apophysis.

Female ectal apophysis optimizes as synapomorphic for the clade $G$. gaujoni ( $G$. globosa, G. sp. nov. 8,) with a reversal in G. globosa.

71. Female, chelicerae, anterior bulge*: $(\mathrm{L}=1, \mathrm{CI}=1, \mathrm{RI}=1 ; \mathrm{F}=1)$

(0) absent (Fig. 80A);

(1) present (Fig. 80B).

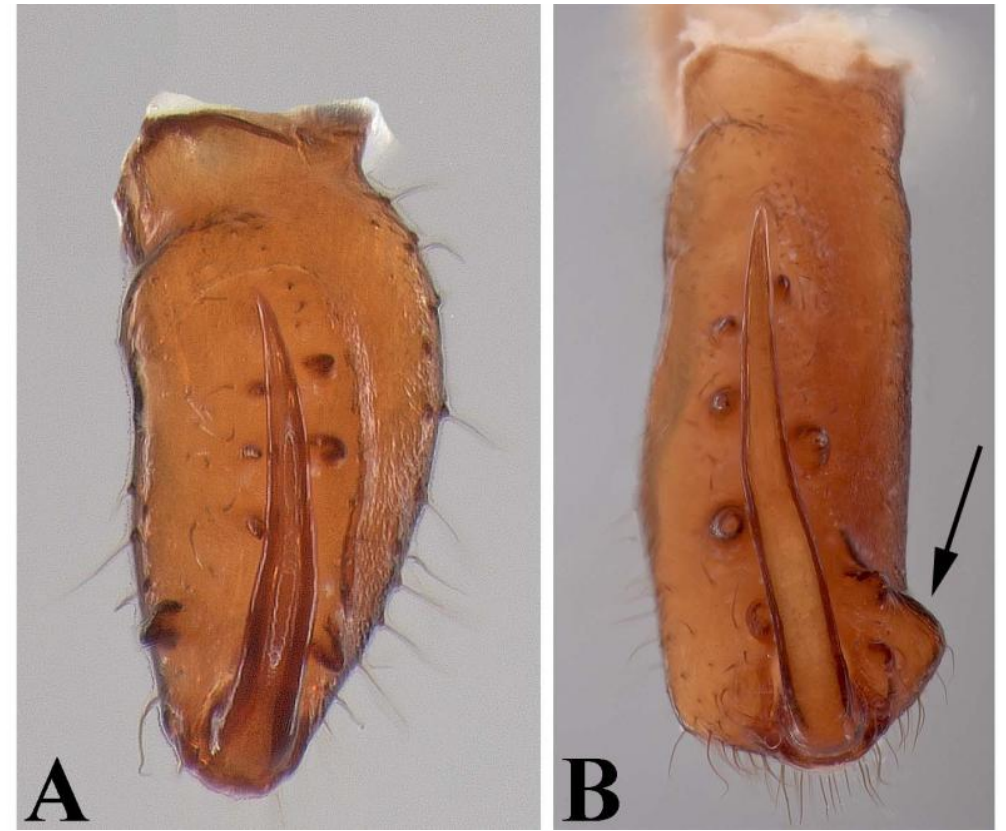

Figure 80. Female left chelicerae, mesal view. A, Glenognatha sp. nov. 6. B, G. emertoni (arrow, anterior bulge). 
Female cheliceral anterior bulge optimizes as synapomorphic for G. emertoni + G. minuta.

72. Female, chelicerae, anteromarginal projection*: $(\mathrm{L}=2, \mathrm{CI}=0.5, \mathrm{RI}=0.5 ; \mathrm{F}=0.88)$

(0) absent (Fig. 81A);

(1) present (Fig. 81B).

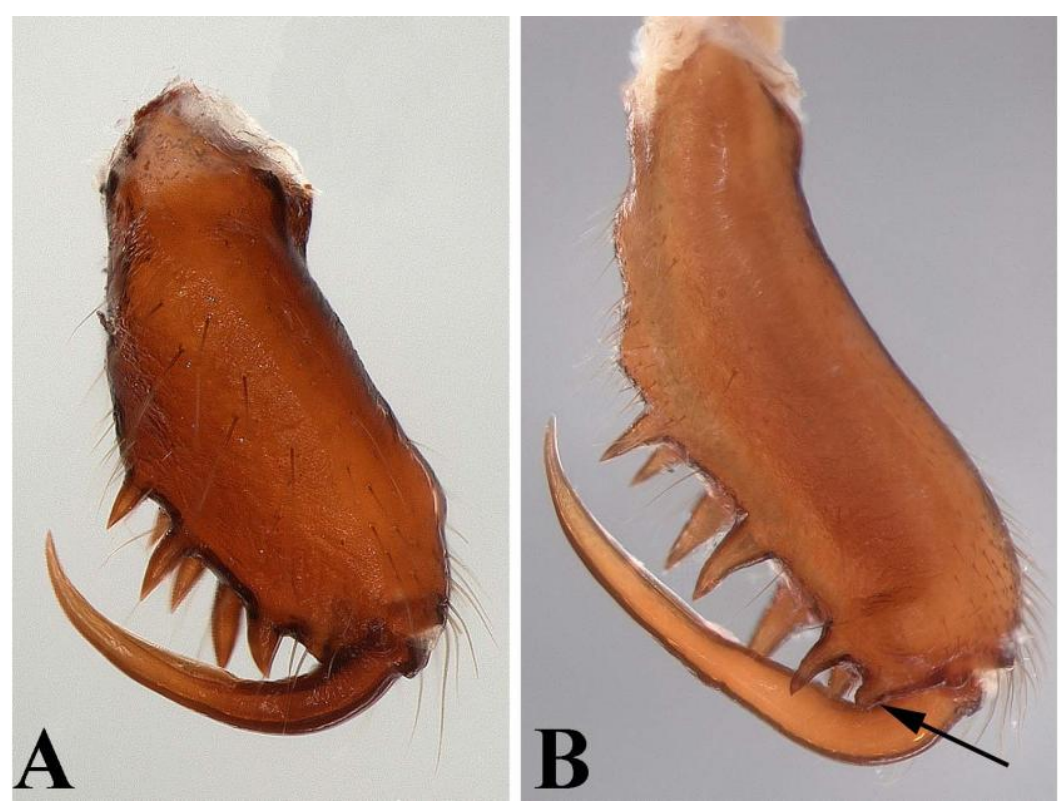

Figure 81. Female left chelicerae, anterior view. A, Glenognatha sp. nov. 11. B, Glenognatha emertoni (arrow, anteromarginal projection).

Female cheliceral anteromarginal projection appears independently in $T$. versicolor and the group G. emertoni + G. minuta.

73. Female, chelicerae, posterior bulge*: $(\mathrm{L}=2, \mathrm{CI}=0.5, \mathrm{RI}=0.66 ; \mathrm{F}=0.88)$

(0) absent (Fig. 82A);

(1) present (Fig. 82B). 


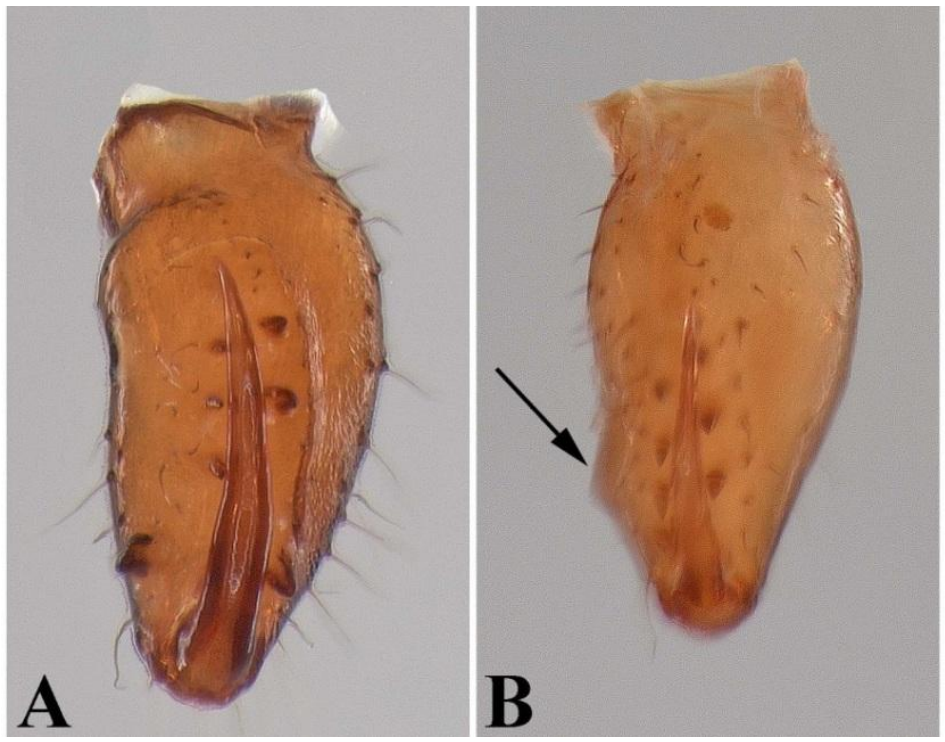

Figure 82. Female left chelicerae, mesal view. A, Glenognatha sp. nov. 6. B, Glenognatha sp. nov. 4 (arrows, posterior bulges).

Female cheliceral posterior bulge appears independently in clade A and $G$. spherella.

74. Female, chelicerae, posterior bulge, quantity*: $(\mathrm{L}=1, \mathrm{CI}=1, \mathrm{RI}=1)$

(0) one;

(1) two (Fig. 83).

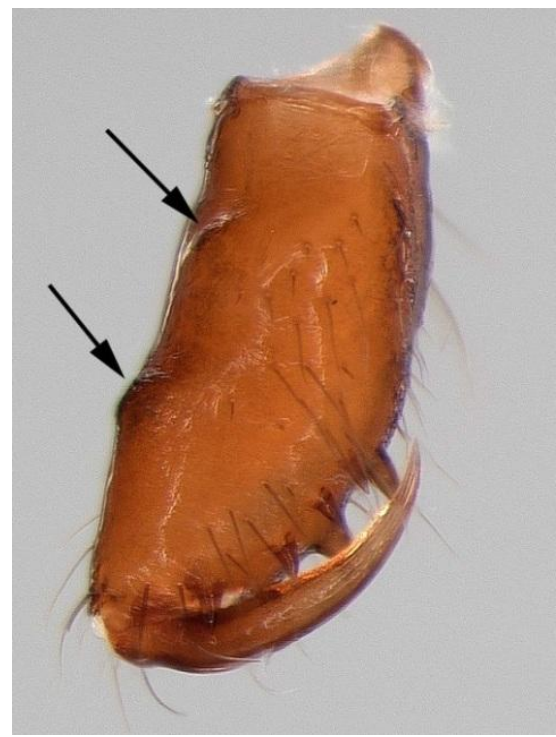

Figure 83. Glenognatha lacteovittata. Female left chelicerae, posterior view (arrows, cheliceral bulges).

Two cheliceral posterior bulges is autapomorphic for G. lacteovittata. 
75. Female, Prt1, base*: $(\mathrm{L}=1, \mathrm{CI}=1, \mathrm{RI}=1)$

(0) not swollen (Fig. 84A);

(1) swollen (Fig. 84B).

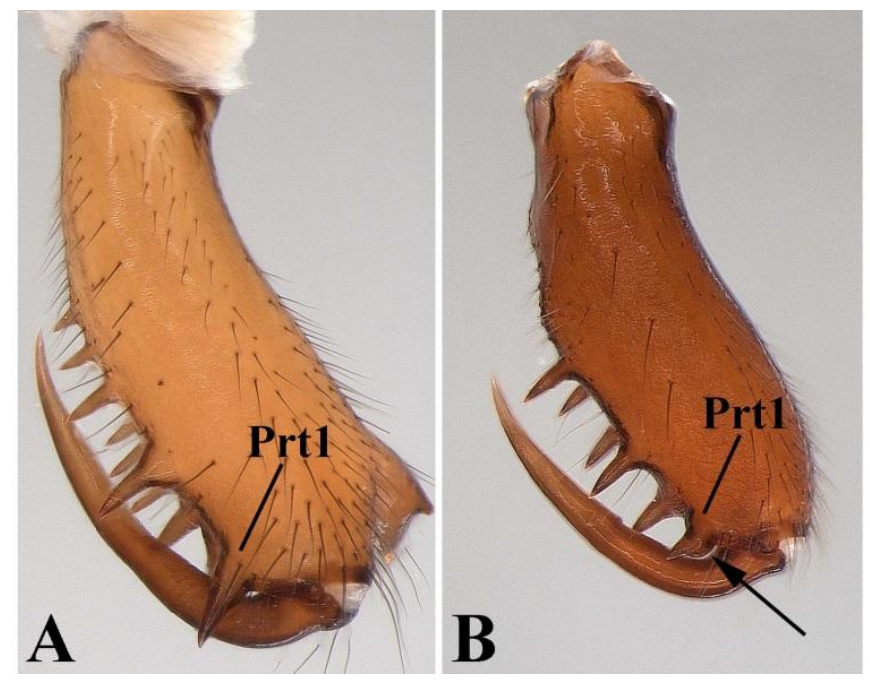

Figure 84. Female left chelicerae, anterior view. A, Glenognatha sp. nov. 8. B, Glenognatha sp. nov. 7 (arrow, Prt1 base swollen). Prt1: promarginal tooth 1.

Enlarged Prt1 base is autapomorphic for G. sp. nov. 7 .

76. Chelicerae, retromarginal teeth, quantity: ( $\mathrm{L}=2, \mathrm{CI}=0.5, \mathrm{RI}=0.5 ; \mathrm{F}=0.88)$ (AHS09)

(0) three to five (Fig. 85A);

(1) seven or more (Fig. 85B).

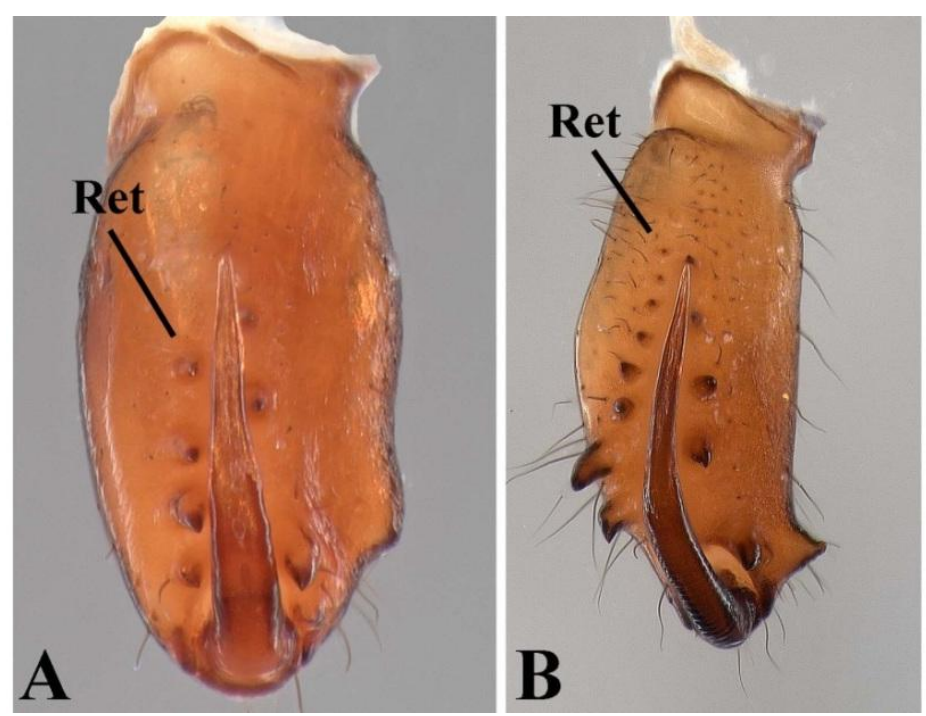

Figure 85. Female left chelicerae, mesal view. A, Glenognatha minuta. B, Glenognatha globosa. Ret: retromarginal teeth. 
High number of retromarginal teeth appears independently in $T$. versicolor and the clade G. globosa + G. sp. nov. 8 .

77. Femur IV, trichobothria: $(\mathrm{L}=1, \mathrm{CI}=1, \mathrm{RI}=1)(\mathrm{APH} 11)$

(0) absent;

(1) present (Fig. 86A-B).
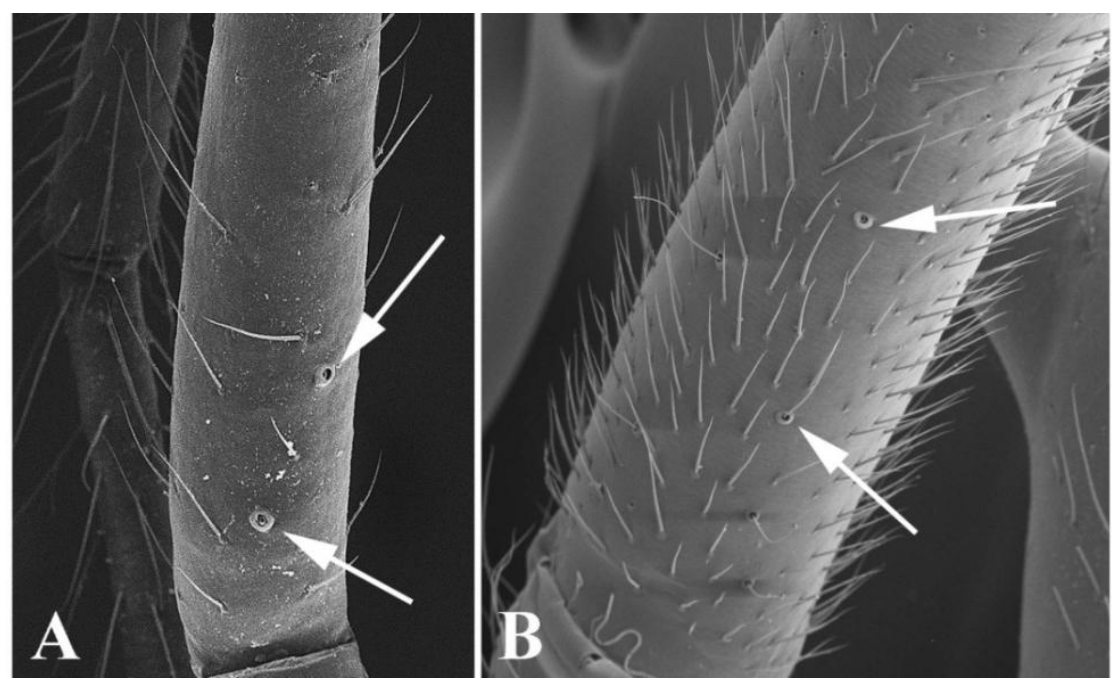

Figure 86. Femur IV. A, Glenognatha tangi. B, Glenognatha sp. nov. 8. (arrows, trichobothria).

In the present analysis, trichobothria on the femur IV is synapomorphic for Tetragnathinae. Nevertheless, this condition also appear in Leucauginae members (Alvarez-Padilla \& Hormiga, 2011).

78. Femur IV, trichobothria, distribution: (L=1, CI=1, RI=1) (APH11)

(0) less than one third of femur (Fig. 87);

(1) more than one third of femur.

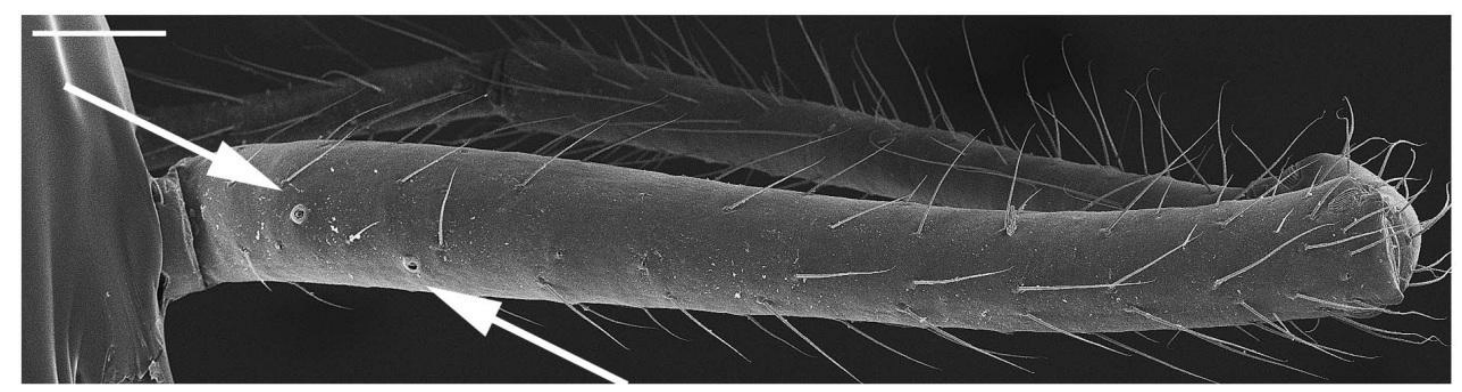

Figure 87. Glenognatha tangi. Femur IV (arrow, trichobothria). 
Trichobothria occupying more than one third of the femur IV is autapomorphic for C. espanola. However, this condition is also present in Leucauginae members (Alvarez-Padilla \& Hormiga, 2011).

79. Femur, ventral macrosetae*: $(\mathrm{L}=1, \mathrm{CI}=1, \mathrm{RI}=1)$

(0) absent;

(1) present (Fig. 88).

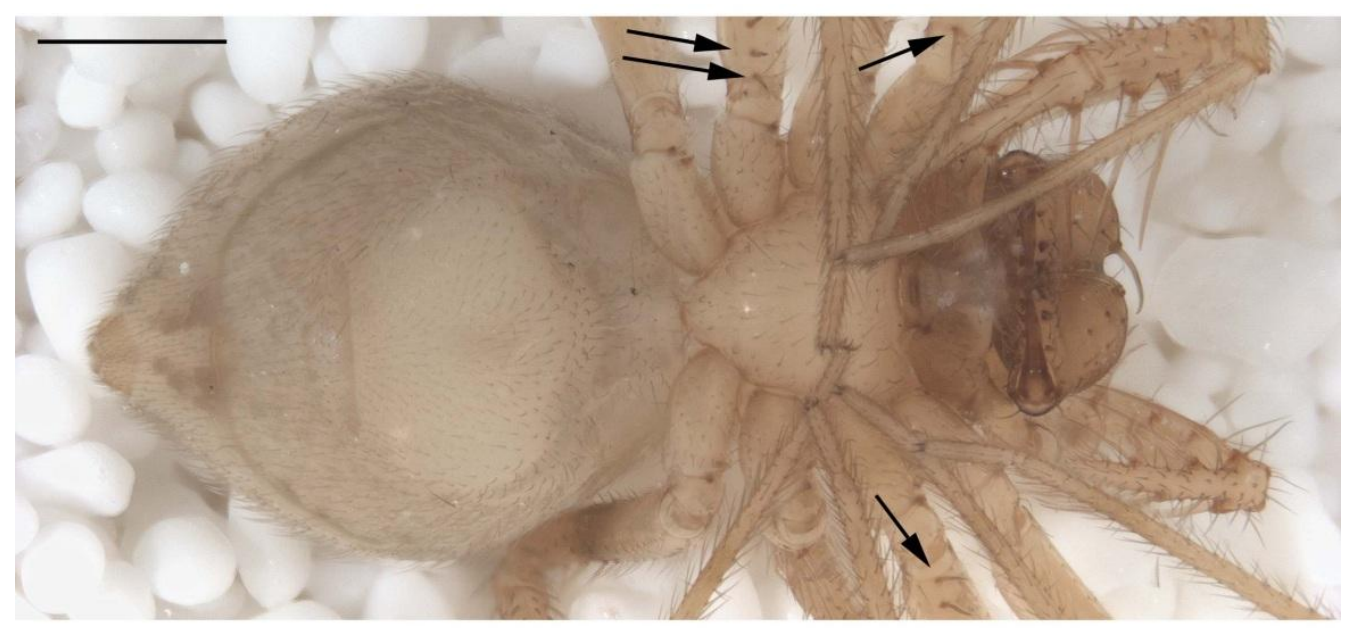

Figure 88. Glenognatha hirsutissima. Female ventral view (arrows, femur spines).

Strong ventral macrosetae in the femur is autapomorphic for G. hirsutissima.

\section{Abdomen morphology and tracheal system}

80. Abdomen, cuticle, microsculpture: $(\mathrm{L}=1, \mathrm{CI}=1, \mathrm{RI}=1, \mathrm{~F}=1)(\mathrm{APH} 11)$

(0) made of flaps;

(1) made of cylinders (Fig. 89).

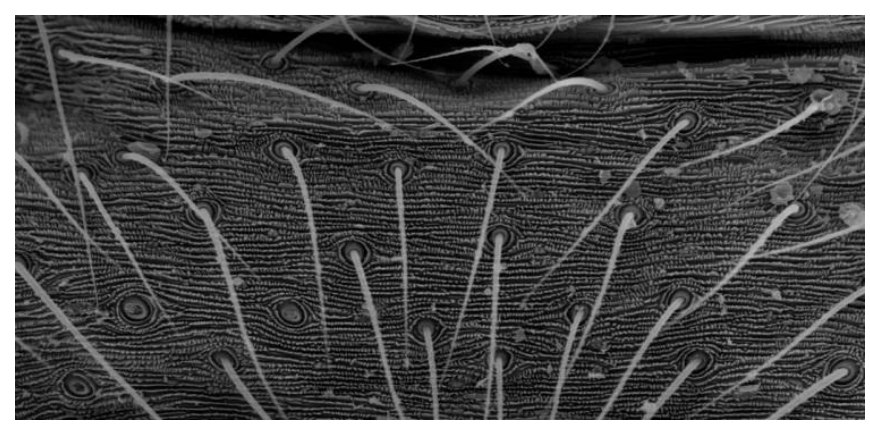

Figure 89. Glenognatha sp. nov. 1. Female abdomen cuticle.

Cuticle microsculpture made of cylinders optimizes as synapomorphic for Pachygnatha + Glenognatha. 
81. Median tracheal trunks, configuration $(\mathrm{L}=1, \mathrm{CI}=1, \mathrm{RI}=1, \mathrm{~F}=1)(\mathrm{APH} 11)$

(0) entire (not branched) or bifurcated;

(1) ramified into numerous tracheoles (Fig. 90).

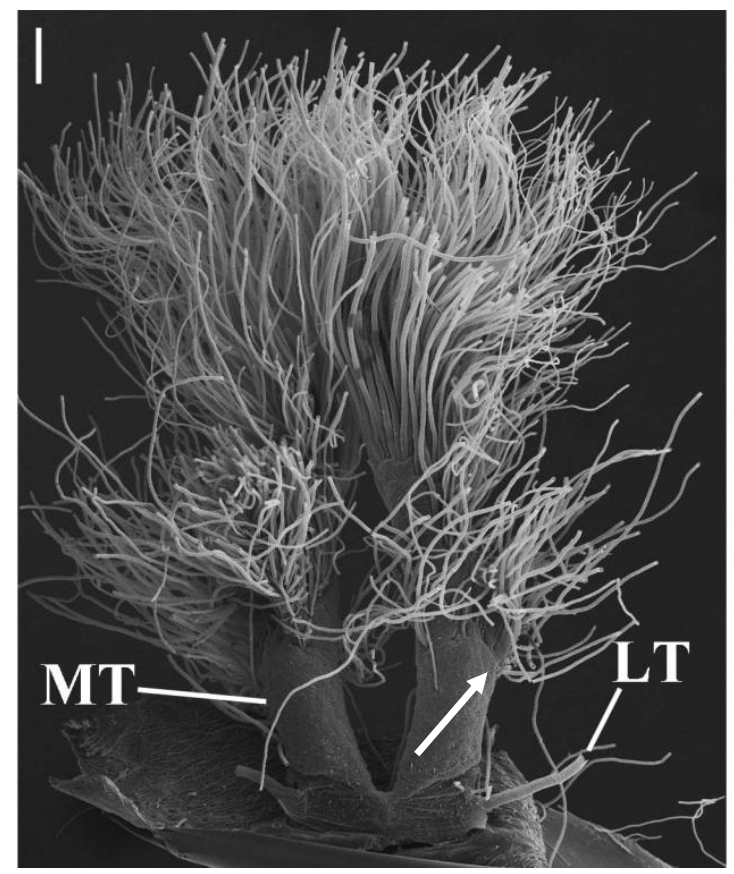

Figure 90. Glenognatha emertoni. Female tracheal system. MT: median trunks (arrow, lateral projection). LT: lateral trunks.

Ramified median tracheal trunks optimizes as synapomorphic for Glenognatha. All the examined Glenognatha species have a similar branching pattern with tracheoles emerging from distinct lateral projections of the median trunks and at the apical end (Fig. 90), entering into the cephalothorax.

82. Median tracheal trunks, width $(\mathrm{L}=1, \mathrm{CI}=1, \mathrm{RI}=1, \mathrm{~F}=1)(\mathrm{MH} 04)$

(0) about as wide as laterals;

(1) at least one a half times wider than laterals (Fig. 90).

Large median trunks relative to laterals optimizes as synapomorphic for Glenognatha.

\subsection{Phylogenetic relationships}

Although this study did not planned to reconstruct the intergeneric relationships within the subfamily Tetragnathinae, as this issue requires a denser taxonomic sample, 
some comments about the relationships obtained here are pertinent. According with previous investigations (Álvarez-Padilla, 2007; Kuntner et al. 2008; Dimitrov \& Hormiga, 2009; Álvarez-Padilla et al., 2009; Álvarez-Padilla \& Hormiga, 2011; Dimitrov \& Hormiga, 2011), Tetragnathinae is recovered as a highly supported monophyletic clade (Fig. 7). Nevertheless, the genus Cyrtognatha is obtained as sister to Pachygnatha + Glenognatha, contrary to the hypothesis found in the aforementioned works where Cyrtognatha appears either as sister to Tetragnatha or sister to a clade composed by Tetragnatha, Pachygnatha and Glenognatha. This difference could be related with the lower taxon sampling in the present study and the exclusion of some characters, used by previous authors (Álvarez-Padilla \& Hormiga, 2011 and references therein), uninformative to solve Glenognatha relationships. As previously suggested (Álvarez-Padilla \& Hormiga, 2011 and references therein), Pachygnatha is recovered as the sister group of Glenognatha (Figs. 7-8). In the present study, five unambiguous synapomorphies, free of homoplasy support Pachygnatha + Glenognatha (Fig. 8).

The monophyly of Glenognatha is strongly supported by high values of resampling measures and Bremer support (Fig. 7). Eight unambiguous synapomorphies support the monophyly of Glenognatha, all free of homoplasy: 1-shaped paracymbium (char. 15[state 2]), setae distributed only in the distal portion of the paracymbium (char. 18[0]), conductor without basal apophysis (char. 19[1]), conductor retrolateral apophysis prolaterally folded (char. 21[1]), embolus with medial groove (char. 32[1]), male Ret1 posteriorly displaced (char. 66), medial tracheal trunks branched (char. 81[1]) and large relative to lateral tracheae (char. 82 [1]). Additional synapomorphies (although of ambiguous optimization) are: large accessory gland base (char. 41 [1]), accessory glands located near the copulatory ducts (char. 42 [1]) and male Prt2 closer to Prt1 than to Prt3 or subequally distant (char. 67 [0], homoplasious).

Within Glenognatha several monophyletic groups are recovered, though most of them with low support values ( 15 of 25 internal nodes with resampling values $<50$ ) (Fig. 8). These results are congruent with that obtained in generic systematic revisions among other taxa, where low support values are also reported (Lopardo, 2005; Izquierdo \& Ramírez, 2008; Dimitrov \& Hormiga, 2009; Ojanguren \& Ramírez, 2009; Werenkraut \& Ramírez, 2009; Daza et al., 2012; Soto \& Ramírez 2012). The amount of support for a group is the result of the interaction between the characters that favor the 
group and those that contradict it (Goloboff et al., 2003). Low support values are obtained when the same characters that support a group also contradict it (Daza et al., 2012). Considering the geographic distribution of Glenognatha, the unknown females in some species and the high probability of finding undescribed species, future addition of taxa and characters might improve some support values.

Unambiguous synapomorphies supporting the major internal clades within Glenognatha are given in Table 2. Clade A is recovered as the most basal lineage. The three representatives of the genus Dyschiriognatha included in the outgroup sample, form a monophyletic group nested within Glenognatha (clade C). These species present all the diagnostic character of Glenognatha and therefore they are transfer to this genus (see taxonomy section). The morphology of the uterus externus sac provides support for the clade D which includes most Glenognatha species. Within this group male genitalia and chelicerae characters provide support for internal clades (Fig. 8, Table 2).

The Cameroon endemic species G. smilodon, is recovered as sister to G. heleios + G. iviei, the shape of the Prt1 provides unambiguous support to this clade. Glenognatha hirsutissima, the only Polynesian species available for this study, is recovered as sister group of $G$. sp. nov. 1, but with very low support. According with Berland (1933, 1935, 1942), other Glenognatha species could be found in the French Polynesia. However, coding these taxa from the original descriptions and drawings, which lack details of crucial character systems as genitalia and spinnerets morphology, might produce numerous missing data and greater instability in the relationships. Therefore, examination of new material is necessary in order to assess the position of these Polynesian species within Glenognatha. Perhaps these species could form a monophyletic group as suggested in several taxa radiations registered in the Marquesas islands, including the tetragnathine genus Tetragnatha (Gillespie et al., 2008). 
Table 2. Unambiguous synapomorphies supporting major clades within Glenognatha that results analyzing the complete matrix under $k=8$.

\begin{tabular}{llc}
\hline Clade & Unambiguous synapomorphies & Character No. \\
\hline A & PMS with one aciniform gland spigots & 3 \\
B & PLS aciniform gland spigots arranged in one line & 6 \\
& Four epiandrous fusules & 8 \\
& Paracymbium distal portion long & 16 \\
& Embolic medial groove expanded & 33 \\
C & Carapace with irregular depressions on lateral surface & 51 \\
D & Uterus externus sac divided & 44 \\
E & Embolus tip with projection beyond embolic opening & 30 \\
F & Conductor with retrolateral projection & 24 \\
G & Male Prt1 conspicuously enlarged & 61 \\
J & Male Ret2 long & 63 \\
\hline
\end{tabular}

PLS: posterior lateral spinnerets. PMS: posterior median spinnerets. Prt: promarginal tooth. Ret: retromarginal tooth.

\subsection{Character evolution}

\section{Genitalia}

The male palpal morphology of Glenognatha is very characteristic; five of the eight unambiguous synapomorphies that support the monophyly of the genus are related with this character system. According with my preferred hypothesis (Fig. 8), the plesiomorphic condition in Glenognatha correspond to a short distal portion of the paracymbium (Figs. 98D, 106D, 110D) and an embolic groove limited to the inner surface of the embolus (Fig. 102A, 110A) (Clade A). Long distal portion of the paracymbium and expanded embolic groove have an unique origin at clade B, although with low support. These contrasting male genitalia configurations are not correlated with clear morphological patterns in female genitalia, a fact that could be related with the absence of a "lock-and-key" fit between male and female genitalia in haplogyne taxa (Huber \& Senglet, 1997; Danielson-François, 2006). Mating behavioral data and histological preparations could provide functional significance for the contrasting configuration of male genitalia within Glenognatha, clarifying crucial aspects as which sclerites besides the embolus are inserted in the female genital cavity and which kind of insertion (ipsilateral or contralateral) is implemented. 
Within Glenognatha several clades have lost independently the copulatory ducts and the spermathecae. As stated by Eberhard \& Huber (2010), this condition could be explained by an untested hypothesis: males under sexual selection may have "shortcircuited" female storage organs and introduced sperm directly into the oviduct. In accordance with this hypothesis, sperm masses were detected in the uterus externus sac of females without spermathecae after copula (G. globosa and G. sp. nov. 8). However, histological preparations are necessary in order to confirm where the sperm is deposited in females with and without spermathecae and if this locations is phylogenetically informative.

\section{Chelicerae}

Cheliceral morphology within Glenognatha is highly variable amongst species and this variation is phylogenetically informative. Conspicuous asymmetry in the size of cheliceral teeth appears to be an apomorphic condition; enlarged Prt1 support clade G and enlarged Ret2 clade J. Available published data for Glenognatha species (Barrows, 1919; Edwards \& Senske, 2001; Danielson-François; 2006) and observations of mating behavior in G. globosa, G. sp. nov. 8 and G. lacteovittata, confirm that cheliceral clasp occur prior to insemination in Glenognatha. Therefore, the apparently rapid divergent evolution of chelicerae could be related with sexual selection. However, modifications of the cheliceral teeth might also be related with prey specialization as suggested for the genus Tetragnatha (Gillespie et al., 1997).

\section{Tracheal system}

One of the most striking characteristic of the genus Glenognatha is the morphology of the tracheal system (Figs. 101, 109, 174). In the present analysis, this character provides unambiguous support for the monophyly of the genus. However, it should be noted that ramified median trunks are also present in members of the Nanometinae subfamily (Álvarez-Padilla \& Hormiga, 2011) and some Pachygnatha species (Bosmans \& Bosselaers, 1994), but with a totally different configuration. Similar branching patterns to those of Glenognatha, have been reported in distant related groups as some linyphiids (“desmitracheate" condition) and uloborids (Opell, 1979, 1990; Arnedo et al., 2009). In both cases, a relation of the branched tracheal pattern and the active monitoring of webs have been proposed. In the case of Glenognatha, natural history data are necessary in order to suggest a possible functional 
significance of the tracheal morphology. No discrete variation in the general morphology of the tracheal system was detected across Glenognatha species

\subsection{Identity of the spider genus Dyschiriognatha}

The three representatives of the genus Dyschiriognatha included in the outgroup sample are nested within Glenognatha (Fig. 7). These species form a monophyletic group strongly supported by high resampling values and Bremer support (Fig. 7). In addition, D. montana Simon, 1897 is suggested as junior synonym of D. argyrostilba after examination of the type material (see taxonomic section). The remaining four described Dyschiriognatha species, including the type species, were not available for this study and the lack of morphological details in the original descriptions and drawings prevent me to include this species in the phylogenetic analysis, in order to avoid the effects of high proportion of missing data. However, some information included in the original descriptions suggest the possibility of including these species in the genus Glenognatha.

Simon (1893) described D. bedoti, the type species of the genus Dyschiriognatha, from a single female specimen collected in Borneo. Despite its evident similarity with the previously described genus Glenognatha, as stated by Simon in the original description, the author decided to proposed a new genus based on highly variable somatic characters as the eye and leg size to classify this species. Although the original description do not include information about the internal female genitalia or tracheal system, Simon (1893) states that this species present an anteriorly displaced spiracle as in Glenognatha and mentions two characters that might place this species in the clade C within Glenognatha: "cephalothorax.....versus marginem subtiler granosus" and "sternum....laeve sed parce granosum", these conditions could be the apomorphic states of characters number 51 and 52 used in the present analysis.

Vellard (1926) described D. lobata, from a single male specimen collected in Lagoa de Bom Successo, Rio de Janeiro, Brazil. Unfortunately the type material is lost and there is great uncertainty about the exact location of the type locality. In addition, the original description would apply similar to different species and therefore the species could be considered as a nomen dubium. However, it should be noted that the 
morphology of the paracymbium depicted by Vellard (1926) correspond to the diagnostic morphology of Glenognatha.

Dyschiriognatha oceanica Berland, 1929 and D. upoluensis Marples, 1955, were described from male specimens from Upolu, Samoa. According with the original drawings, these species exhibit the typical Glenognatha male genitalia morphology and in D. upoluensis the characteristic female tracheal system (Marples, 1955 pl. 59 fig. 17). The presence of anterior tooth in D. oceanica (Berland, 1929 fig. 43) suggest the inclusion of this species in the clade $\mathrm{G}$.

Examination of new material collected in the type locality of $D$. bedoti is necessary in order to propose objectively Dyschiriognatha as junior synonym of Glenognatha. In addition, the inclusion of this species and the other described Dyschiriognatha species, unavailable for this study, within the data matrix could improve the stability and support of some internal clades.

\subsection{Taxonomy}

\section{Glenognatha Simon, 1887}

Mimognatha, Banks, 1929: 90, type species by monotypy Mimognatha foxi Banks, 1929. Synonymized by Levi, 1980: 64.

Hivaoa, Berland, 1935: 50, type species by original designation Hivaoa argenteoguttata Berland, 1935. Synonymized by Levi, 1980: 64.

Type species: Glenognatha emertoni Simon, 1887, type by monotypy.

Diagnosis: Males of Glenognatha can be distinguished from all other tetragnathid genera by having a prolaterally folded apophysis on the conductor (Figs. 118A-D, $126 \mathrm{~A}-\mathrm{D}, 157 \mathrm{C}-\mathrm{D}, 166 \mathrm{~A}-\mathrm{F})$. The presence of a medial groove in the basal portion of the embolus (Figs. 118E, 158A, 171A, 175B, 199A-B) and the paracymbium with a distal portion oriented in opposite direction to the cymbium are also diagnostic characters (Figs. 98D, 106D, 114D, 122D, 153D, 187D, 214D). Females differ from other members of the Tetragnathinae subfamily, with which they share the secondary haplogyne condition (Figs. 116, 124, 155, 189, 197, 205, 228, 236), by having tracheal system composed of two median trunks divided into numerous tracheoles (on the sides 
and on the distal end) and two simple (unbranched) lateral tracheae (Figs. 174, 182, $237,281)$.

Description: total length 1.25-4.60 in males and 1.40-4.80 in females. Carapace longer than wide, 0.62-2.4 long in males, 0.61-2.05 in females; pale yellow to dark brown (Figs. 95, 96, 103, 127, 150, 151, 208, 212). Sternum as wide as long and prolonged between coxae in some species (Figs. 119C, 127C, 159C, 168C). Labium trapezoidal, wider than long, and rebordered. PME and AME larger than the other eyes. Lateral eyes with nearly the same diameter. Clypeus higher than one AME diameter. Chelicerae large, sexually dimorphic, with 3-5 promarginal teeth and 3-10 retromarginal teeth (Figs. 97, 161, 170, 178, 194). The number of cheliceral teeth usually varies between and within species. Some individual presents asymmetry in the number of retromarginal teeth. Male chelicerae are usually divergent and in some species with an anterior tooth (spur sensu Levi, 1980) (Figs. 186A, 194A, 202A, 209C, 123C). Female chelicerae are less divergent and could carry ectal apophysis (Figs. 161D-F, 170D-F). Cheliceral boss well developed. Cheliceral fangs with retrolateral outgrowth (Figs. 105A, 113B, 143A, 161F, 186B), in some species can be small or absent (Figs. 152A, 170, 178, 233). Legs without spines (except in G. hirsutissima), slightly longer in males than in females. Femur I length varies from 0.75 to 3.45 in males and from 0.67 to 3.4 in females. Leg formula I $>$ II $>$ IV $>$ III. Dorsally the base of the femur has one to six trichobothria and the tibia five to ten . On the first half of the metatarsi there is one trichobothrium (two in $G$. hirsutissima) longer than the diameter of the article. The fourth metatarsus lacks trichobothria except in $G$. sp. nov. 5, G. sp. nov. 10, G. sp. nov. 11, G. tangi, $G$. emertoni and G. hirsutissima. Abdomen oval to spherical with silver guanine patches, in some species with irregular patterns of dark longitudinal bands that join medially.

Tracheal system with two median trunks branching into several tracheoles at the tip and on the sides and two unbranched lateral tracheae (Figs. 101, 109, 117, 133, 147, 174, 237). Spiracle atrium glands occupying both sides of the atrium and the basal region of the median trunks (Figs. 117, 237, 252, 281). Posterior tracheal spiracle wider than long and anteriorly displaced in most species. ALS with more than 12 piriform spigots (Figs. 298-299). PMS with one to two aciniform spigot between the cylindrical and the minor ampullate spigots (Figs. 300-301). PLS with 5-18 AC spigots (Figs. 302-303). Epiandrous fusules arranged linearly (Fig. 297). 
Male palp tibia with 3-6 trichobothria. Cymbium medially constricted. Paracymbium with two perpendicular portions, the distal with its apex in opposite direction in relation with the cymbium (Figs. 98D, 106D, 114D, 122D, 153D, 187D, 214D, 257D). Tegulum spherical, with a cuplike apical depression where the embolus and the conductor rest. Embolus surrounding the conductor base and with a well-differentiated medial groove that originates near the base and ends near the apical region (Figs. 118E, 158A, 171A, 175B, 199A-B, 253E). Conductor emerging from the center of the tegulum as a cylindrical tube that bends prolaterally and flattens into a lamina, which is retrolaterally folded and carries a distinct apophysis (Figs. 102A, 110A, 118A, 126A-D, 157A, C-D, 166, 175, 191A-C, 230A-G). Females lack epigynum. Copulatory opening spiracleshaped (Figs. 112C, 120C, 117C) leading to the uterus externus chamber (Figs. 100B, 108B, 116B, 155A-C, 280B). This chamber has tendons in its lateral borders that serve as muscle attachment points and carries the copulatory ducts that lead to spermathecae. In some species the spermathecae and copulatory ducts are absent (Figs. 100, 108, 116, $164,173,181,274,280)$. Uterus externus chamber connected with a membranous sac of variable morphology across the species (Figs. 100, 132, 146, 189, 228, 251, 259, 295).

Composition: Glenognatha currently contains 27 species: G. argyrostilba (O. P.Cambridge, 1876) comb. nov., G. australis (Keyserling, 1883), G. dentata (Zhu \& Wen, 1978) comb. nov. , G. emertoni Simon, 1887, G. foxi (McCook, 1894), G. gaujoni Simon, 1895, G. globosa (Petrunkevitch 1925), G. gloriae (Petrunkevitch, 1930), G. heleios Hormiga, 1990, G. hirsutissima (Berland, 1935), G. iviei Levi, 1980, G. lacteovittata (Mello-Leitão, 1944), G. minuta Banks, 1898, G. smilodon Bosmans \& Bosselaers, 1994, G. spherella Chamberlin \& Ivie, 1936, G. tangi (Zhu, Song \& Zhang, 2003) comb. nov. and 11 new species described herein.

Distribution: Glenognatha species have a broadly distribution with records in Nearctic, Neotropic, Afrotropic, Indomalaya and Oceania ecozones. It occupies a broad altitudinal range from 6 masl (G. iviei) to 2700 masl (G. sp. nov. 5) (Figs. 304-315).

Natural history: The natural history of Glenognatha species is still little known and published data are mainly focused on Nearctic species. 
Glenognatha foxi is recorded as an abundant spider in a wide variety of crops in the United States: soybean (Young \& Edwards, 1990; Balfour \& Rypstra, 1998), alfalfa (Young \& Edwards, 1990; Birkhofer et al., 2007), rice, sugarcane, peanuts and cotton (Young \& Edwards, 1990). Chapman et al. (2012) recorded Collembola, Aphididae and Brachycera as prey taxa for G. foxi in winter wheat. Phenology of G. foxi in Georgia was assessed by Draney (1997), who suggested a possible annual reproductive cycle with mating in the early summer. $G$. foxi is recorded as the most abundant aerial species in one of the two sampling stations assessed by Dean \& Sterling (1985). Some aspects of the reproductive biology and habits of G. foxi were described by Barrows (1919). According with this author, G. foxi is common in meadows and waste-lands in hot dry situations. The mating occurs in the middle of the web with cheliceral clasp and each male palp is inserted alternately every 5 minutes. The overall copula has a duration of 15 minutes.

Hormiga \& Döbel (1990) and Döbel et al. (1990) provided phenology and web architecture data for $G$. heleios. According with these authors, G. heleios is an univoltine species in intertidal salt marshes of New Jersey, with greater abundance in short and intermediate forms of Spartina alterniflora. Webs are placed close to the soil surface with the sticky spiral very closely spaced. Some aspects of the reproductive biology of G. heleios were described by Edwards \& Senske (2001). These authors recorded the insertion of only one palp and a copula duration of two hours.

Danielson-François (2006) carried on a detailed study of habits, sperm release patterns and reproductive behavior of $G$. emertoni. In this work, specimens were found in vegetation overhanging streams and under stream-side rocks in Arizona. Avoidance behavior after disturbance and mating behavior were described. In the former, the spider dropped into the water beneath and float certain distance until the extended legs allow climbed up some streamside vegetation. The mating behavior involved cheliceral clasp, vibratory courtship through legs movements and palps insertions.

Glenognatha lacteovittata is recorded as a common species in alfalfa and wheat crops in Argentina (Armendano \& González, 2010; 2011) and G. maelfaiti is collected preferentially in humid habitats of Santa Cruz island in Galápagos, Ecuador (Baert et al., 1991). 
Adults and juveniles of Glenognatha sp. nov. 2 were collected in low mountain humid forests in western Colombian Andes (Fig. 91). This species builds its webs in vegetation adjacent to streams about $25 \mathrm{~cm}$ to $2 \mathrm{~m}$ above ground level (Figs. 92), although some individuals were found far away from any current in low vegetation. In both cases, the web is almost horizontal. It has closed hub and few sticky spiral turns and radii. The web frame can be rectangular or triangular. Some webs were observed under leaves (Figs. 92B).

Glenognatha sp. nov. 8 lives in the same habitats as Glenognatha sp. nov. 2 (Fig. 91). Nevertheless, this species builds its webs a few centimeters $(1-5 \mathrm{~cm})$ above streams (Fig. 93). Rocks are used as the principles attachment points. Web aggregations with shared radii and numerous hubs were commonly observed (Figs. 93A). In this structures different individuals cohabit, each occupying one hub. No aggression between conspecifics was recorded. A similar avoidance behavior, as that described for $G$. emertoni by Danielson-François (2006), was observed after disturbance. Diptera and Ephemeroptera were preyed during observations.

Glenognatha gaujoni builds its webs a few centimeters $(5-15 \mathrm{~cm})$ above streams in Amazonian rainforests (Fig. 91). The web is horizontal with closed hub, few radii and sticky spiral turns (Fig. 94).

\section{Key to males of Glenognatha}

1. Femur III with a row of strong ventral macrosetae (Fig. 240C)......... G. hirsutissima

- Femur III without macrosetae.................................................. 2

2. Chelicerae with anterior tooth (Figs. 186A, 194A, 202A, 209C, 225A)............... 3

- Chelicerae without anterior tooth (Figs. 97A, 152A, 170A, 256A).................. 9

3. Ret1 displaced to the posterior surface (Figs. 186C)................... G. sp. nov. 6

- Ret1 not displaced (Figs. 194C, 202C, 209C, 213C)............................ 4

4. Prt1 enlarged relative to Prt2 and Prt3 (Figs. 194A, 220D, 225A) ...................... 5

- Prt1 not enlarged (Figs. 202A, 209A, 213A)........................................ 7

5. Paracymbium with a rounded projection on the anterior margin (Fig. 195D)...

G. sp. nov. 3 
- Paracymbium with a pointed projection on the anterior margin (Figs. 222D, 226B) 6

6. Embolus coiled, Prt1 strongly curved (Figs. 220A, 221, 222). G. iviei

- Embolus not coiled, Prt1 slightly curved (Figs. 225A, 226, 227). G. heleios

7. Paracymbium distal portion as long or longer than the tegulum in retrolateral view (Fig. 214D). G. sp. nov. 10

- Paracymbium distal portion shorter than the tegulum in retrolateral view........... 8

8. Conductor lamina excavated in dorsal view, margin entire, Canada to Panama (Figs. 204C, 207D). G. foxi

- Conductor lamina not excavated, margin serrated, Brazil (Figs. 211BC). G. sp. nov. 9

9. Embolic medial groove not visible in ventral view, paracymbium distal portion short (Figs. 98D, 102A, 106D, 110A, 110D). 10

- Embolic medial groove visible in ventral view, paracymbium distal portion long (Figs. 122A, 137A, 171A, D, 187A, D, 203C-D). 12

10. Conductor lamina with distal prolateral fold (Figs. 110A, 118A) 11

- Conductor lamina without distal prolateral fold (Figs. 99B-C, 102B)..... G. sp. nov. 4 11. Embolus tip twice coiled, with filiform projections (Figs. 115E, 118EG). G. lacteovittata

- Embolus tip uncoiled, without filiform projections (Figs. 107D, 110AC) G. sp. nov. 5

12. Carapace with irregular lateral depressions (Figs. 119A, 127A, 141A)............ 13

- Carapace without such depressions (Figs. 150A, 159A, 168A) .................... 15

13. Conductor with tooth-like projections (Figs. 144B, 145B-C, 149D)....... G. dentata - Conductor without such projections (Figs. 126A-D, 139A-B)..................... 14 14. Conductor lamina sinuous, sternum with rounded setae-bearing depressions (Figs. 131B-C). G. argyrostilba

- Conductor lamina almost rectangular, sternum smooth (Fig. 123B-C)......... G. tangi 15. Chelicerae with enlarged Ret2 and Ret3, the latter being longer (Fig. 284B) G. minuta

- Chelicerae otherwise (Figs. 248B, 256B), if Ret2 and Ret3 are enlarged, the latter is shorter (Fig. 271B, 277B, 292B). 16

16. Chelicerae with enlarged Prt1, curved (Fig. 217D). G. smilodon

- Chelicerae with Prt1 not enlarged (Figs. 248A, 256A, 271A). 17 
17. Ret1 adjacent to the fang articulation, longer than the fang width (Figs. 152AC). G. sp. nov. 2

- Ret1 not adjacent to the fang articulation (Figs. 161B, 170B, 178B, 233B, 271B), if near to the fang articulation smaller than the fang width (Figs. 256B-C, 264BC). 18

18. Embolus tip curved towards the conductor (Figs. 238A-C)............ G. sp. nov. 1

- Embolus tip otherwise....................................................... 19

19. Ret2 straight (Figs. 170B, 178B), if curved, apical portion mesally oriented (Fig. 161B). 20

- Ret2 curved, apical portion ectally oriented (Figs. 248B, 256B, 264B, 271B, 277B). 22

20. Cheliceral anterior margin with three teeth (Figs. 161A, C) G. gaujoni

Cheliceral anterior margin with at least five teeth (Figs. 170A, 178A). 21

21. Conductor with a long apical projection (Fig. 172B-C, 175B). G. sp. nov. 8

Conductor without such projection (Figs. 180B-C). G. globosa

22. Cheliceral basal segment with antero-marginal projection (Fig. 292A).

G. emertoni

- Cheliceral basal segment without such projection .... 23

23. Ret2 abruptly curved (Fig. 271B) G. sp. nov. 11

- Ret2 gradually curved (Figs. 277B, 248B, 256B, 264B). 24

24. Embolus longer than the conductor in ventral view (Fig. 278A). G. sp. nov. 7

- Embolus subequal to the conductor in ventral view (Figs. 249A, 250A, 258A, 261A, 268A-B). 25

25. Conductor with a thin apical projection (Figs. 250A-B, 253C). G. australis

- Conductor apical portion otherwise (Figs. 258A-B, 266A-B) 26

26. Clypeus strongly projected (Fig. 262B). G. gloriae

- Clypeus slightly projected (Fig. 254B) G. spherella

\section{Key to females of Glenognatha}

1. Spermathecae absent (Figs. 100, 108, 116, 164, 173)........................... 2

- Spermathecae present (Figs. 124, 132, 146, 155, )............................ 9

2. Chelicerae with ectal apophysis (Figs. 161D-F, 170D-F)........................ 3

- Ectal apophysis absent (Figs. 97D-F, 271D-F, 277D-F)........................ 4

3. Chelicerae with anterior tooth (Fig. 161D)............................. G. gaujoni 
- Anterior tooth absent (Fig. 170D).................................... sp. nov. 8

4. Chelicerae with anterior tooth (Figs. 178D, F)......................... G. globosa

- Anterior tooth absent (Fig. 97D, 271D, 277D).................................. 5

5. Prt 1 basal region enlarged (Fig. 277D) .......................... G. sp. nov. 7

- Prt 1 basal region not enlarged (Figs. 105D, 271D)............................ 6

6. Ret2 enlarged, strongly curved (Fig. 271E-F)........................ . sp. nov. 11

- Ret2 not enlarged, moderately curved (Figs. 97E, 105E, 113E) $\ldots \ldots \ldots \ldots \ldots \ldots \ldots \ldots . \ldots$

7. Chelicerae with two posterior bulges (Fig. 113E)................... G. lacteovittata

- Chelicerae with one posterior bulge (Figs. 97F, 105F)......................... 8

8. Cheliceral posterior bulge located at the level of the Ret2 and Ret3 (Fig. 97F).

G. sp. nov. 4

- Cheliceral posterior bulge located at the level of the Ret3 and Ret4 (Fig.

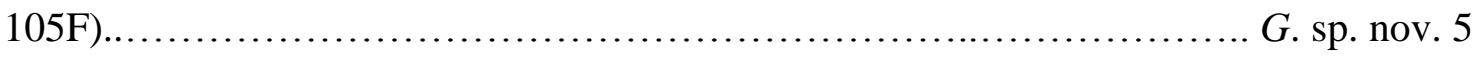

9. Cheliceral with anterior bulge (Figs. 284F, 292F) .......................... 10

- Chelicerae without such bulge (Figs. 186D, 194D, 202D, 225D, 233D).............. 11

10. Spermathecae and copulatory ducts well-developed (Fig. 287)............ G. minuta

- Spermathecae and copulatory ducts reduced (Fig. 295)..................... G. emertoni

11. Carapace with conspicuous lateral depressions (Figs. 125A, D-F, 140A-B)........ 12

- Carapace without such depressions ...................................... 14

12. Sternum smooth (Fig. 125C) ........................................ G. tangi

- Sternum with setae-bearing depressions (Figs. 148C, 140C)..................... 13

13. Uterus externus chamber with filiform projections (Fig. 146)............. G. dentata

- Uterus externus chamber without such projections (Figs. 132, 138).... G. argyrostilba

14. Femur III with a row of strong ventral macrosetae (Fig. 240C)....... G. hirsutissima

- Femur III without such macrosetae.......................................... 15

15. Ret1 enlarged (Figs. 152E, 186E) ..................................... 16

- Ret1 not enlarged (Figs. 233E, 248E, 264E).............................. 17

16. Prt2 closer to Prt1 than to Prt3 (Fig. 152D).......................... G. sp. nov. 2

- Prt 2 closer to Prt3 than to Prt1 (Fig. 186D)............................... G. sp. nov. 6

17. Spermathecae bean-shaped (Figs. 236, 251)................................ 18

- Spermathecae otherwise (Figs. 205, 267, 259)............................. 19

18. Prt1 and Prt2 subequal in size (Fig. 233D)........................... sp. nov. 1

- Prt2 enlarged relative to Prt1 (Fig. 248D)............................. G. australis

19. Cheliceral fang outgrowth well-developed (Fig. 202E).................... G. foxi 
- Cheliceral fang outgrowth absent (Figs. 264E, 256E) .......................... 20

20. Clypeus strongly projected (Fig. 263B)................................ G. gloriae

- Clypeus slightly projected (Figs. 193B, 224B, 255B) ............................ 21

21. Chelicera with posterior bulge, Ret2 enlarged (Figs. 256E-F)............ G. spherella

- Chelicerae without posterior bulge (Figs. 194D-F, 225D-F)................... 22

22. Chelicerae with 3 promarginal and 3 retromarginal teeth, southeastern Brazil (Fig. 194D-F).......................................................... . nov. 3

- Chelicerae with 3 promarginal and 4 retromarginal teeth, New Jersey, USA (Fig.

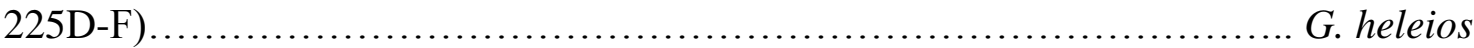

\section{Glenognatha sp. nov. 4}

(Figs. 95-102, 312)

Type material: male holotype from Estação Biológica Boracéia, Salesópolis, São Paulo, Brazil, 2339`20.6”S,4553`28.6”W, elev. [900m], 1.iv.2001, S.T.P. Amarante e Equipe leg., deposited in MZSP 41270; paratypes: 4ત 1 ㅇ same data as the holotype, deposited in MZSP 42538; $1 \delta^{\Uparrow}$ from the type locality, 9.v.2003, G. R. S. Ruiz leg., deposited in IBSP 38325.

Diagnosis: males of G. sp. nov. 4 resemble those of G. lacteovittata and G. sp. nov. 5 by the short distal portion of the paracymbium (Fig. 98D) and the embolic medial groove confined to the inner surface of the embolus (Fig. 102A). It can be distinguished from the two latter species by the absence of a prolateral fold enclosing partially the embolus tip (Figs. 99, 102B). Females resemble those of G. lacteovittata and G. sp. nov. 5 by the median projection of the uterus externus sac (Fig. 100A); distinguished from the former by the presence of one posterior bulge on the cheliceral basal segment (Figs. $96 \mathrm{~B}, 97 \mathrm{~F}$ ) and from the latter by the position of the posterior bulge, which is located at the level of Ret2 and Ret3 in mesal view (Fig. 97F).

Description: Male (Holotype): habitus as in figure 95. Carapace yellow-brownish, darker along furrows. Sternum dusky yellow-brownish with darker edges. Abdomen oval, strongly depigmentated, dorsally with two diffused black patches at posterior region. Silver guanine spots absent. Ventrally with diffused black areas posterior to the tracheal spiracle. Legs light yellow-brownish. Total length 2.00. Cephalothorax 1.02 long, 0.80 wide, 0.62 high. Abdomen 1.00 long, 0.80 wide, 0.80 high. Sternum 0.50 
long, 0.55 wide. Eye diameters and interdistances: AME 0.07. ALE 0.06. PME 0.09. PLE 0.04. AME-AME 0.04, AME-ALE 0.06, PME-PME 0.10, PME-PLE 0.09, ALEPLE 0.01. Clypeus height $2.67 \times$ AME diameter. Chelicerae divergent, with the same coloration of the cephalothorax. Three promarginal and four retromarginal teeth. (Figs. 97A-C). CFO present (Fig. 97A). Leg measurements: I: femur 1.57, patella 0.30, tibia 1.55, metatarsus 1.32 , tarsus 0.57 , total 5.32 ; II: $1.45,0.27,1.50,1.25,0.55$, 5.02; III: 0.95, 0.25, 0.75, 0.75, 0.42, 3.12; IV: $1.22,0.27,1.05,0.97,0.50,4.02$. DTS 0.11. DTE 0.55. Palp as in figures 98-99. Paracymbium with short distal portion (Fig. 98D). Conductor sinuous without distal prolateral fold (Figs. 99B, 102B-C). Embolus smooth without projections (Fig. 102). Medial groove not evident in the outer surface of the embolus (Figs. 102A-B).

Female (Paratype MZSP 41270): coloration and general habitus as in male (Fig. 96). Abdomen with scattered silver guanine spots dorsally along the median region and in the lateral surfaces. Venter with diffused greyish patches between the spinnerets and the tracheal spiracle. Total length 2.62. Cephalothorax 1.05 long, 0.80 wide, 0.70 high. Abdomen 1.47 long, 1.28 wide, 1.25 high. Sternum 0.5 long, 0.55 wide. Eye diameters and interdistances: AME 0.07. ALE 0.05. PME 0.09. PLE 0.06. AME-AME 0.06, AME-ALE 0.05, PME-PME 0.11, PME-PLE 0.07, ALE-PLE 0.01. Clypeus height 2.10 $\times$ AME diameter. Chelicerae with the same coloration of the cephalothorax, with three promarginal and four retromarginal teeth on left chelicera (Figs. 97D-F) and three teeth on each margin on right chelicera. Basal segment of the chelicerae with a distinct posterior bulge (Figs. 96B, 97F). Leg measurements: I: femur 1.50, patella 0.35, tibia 1.40, metatarsus 1.20, tarsus 0.55 , total 5.00; II: 1.42, 0.32, 1.35, 1.15, 0.67, 4.92; III: 1.00, 0.25, 0.65, 0.77, 0.42, 3.10; IV: 1.27, 0.25, 1.05, 1.02, 0.47, 4.07. DTS 0.20. DTE 0.77. Tracheal system as figure 101. Internal genitalia without spermathecae (Fig. 100).

Variation: male total length 1.7-2.0 $(N=10$; average 1.9$)$, cephalothorax length $0.8-1.2$ $(N=10$; average 0.9$)$, femur I length 1.3-1.6 $(N=10$; average 1.5). Female total length 2.1-2.6 ( $N=3$; average 2.2), cephalothorax length 0.7-1.0 $(N=3$; average 0.9$)$, femur I length 1.3-1.5 $(N=3$; average 1.4).

Distribution: known only from the type locality in southeastern Brazil (Fig. 312). 
Additional material examined (N = 13): BRAZIL: São Paulo: Salesópolis, Estação

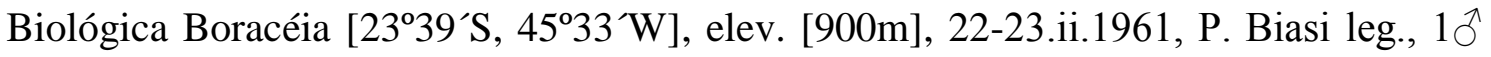

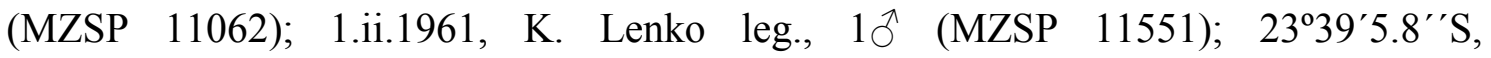

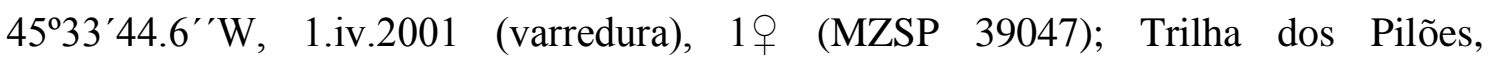

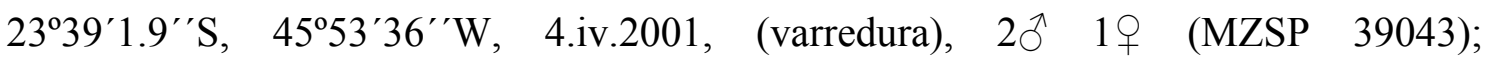

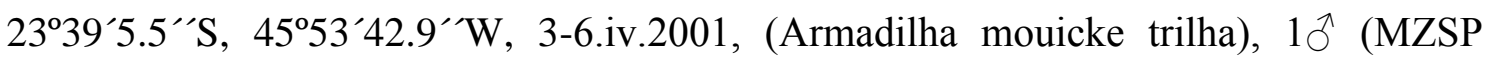

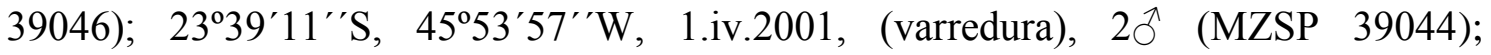

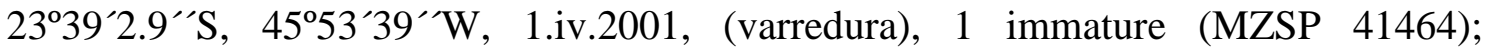

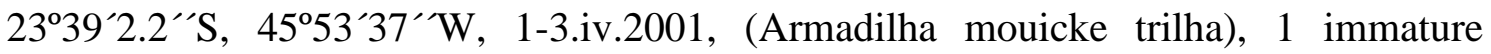

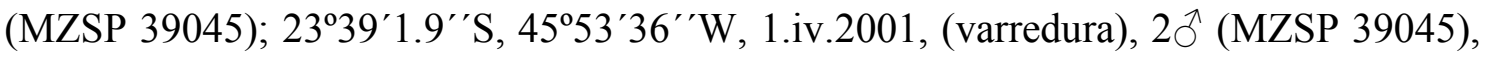
all collected by , S.T.P. Amarante e Equipe.

\section{Glenognatha sp. nov. 5}

(Figs. 103-110, 297J, 310)

Type material: Male holotype from Laguna La Herrera, Mosquera, Cundinamarca,

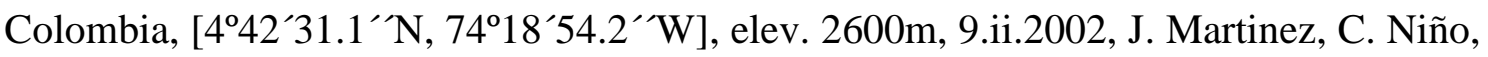
A. Castañeda, G. Mora leg., (Vegetación macrófita emergentes, suelo, trampa Pitfall) deposited in ICN 5976; paratypes: $24 \hat{\jmath} 14 q$ same data as the holotype, deposited in ICN 5977; 4ð 4 ㅇ same data as the holotype, deposited in IBSP 163855.

Diagnosis: males of $G$. sp. nov. 5 resemble those of $G$. lacteovittata by the distal prolateral fold of the conductor lamina (Figs. 107B, 110A). They are distinguished from the latter by the absence of filiform projections in the distal portion of the embolus (Figs. 107D, 110B-C). Females resemble those of G. lacteovittata and G. sp. nov. 4 by the median projection of the uterus externus sac (Figs. 108A-B); distinguished from the former by the presence of one posterior cheliceral bulge (Fig. 105F) and from the latter by the position of the posterior bulge, which is located at the level of Ret 3 and Ret 4 in mesal view (Fig. 105F).

Description: Male (Holotype): habitus as in figure 103. Carapace pale brown, darker along sides and median region. Sternum dusky brown. Abdomen oval, dorsally with irregular pattern of two longitudinal black bands that join medially forming thin transverse bands in the posterior region. Silver guanine spots scattered in dorsal and lateral surfaces. Venter with a dusky median band that ramifies towards the lateral 
surface into thin irregular bands. Legs light yellow-brownish with distal parts of segments darker. Tracheal spiracle well-sclerotized. Total length 3.90. Cephalothorax 1.62 long, 1.27 wide, 1.07 high. Abdomen 2.35 long, 1.87 wide, 1.75 high. Sternum 0.72 long, 0.82 wide. Eye diameters and interdistances: AME 0.11. ALE 0.09. PME 0.11. PLE 0.08. AME-AME 0.08, AME-ALE 0.07, PME-PME 0.10, PME-PLE 0.14, ALE-PLE 0.01. Clypeus height $2.40 \times$ AME diameter. Chelicerae with the same coloration of the cephalothorax. Three promarginal and four retromarginal teeth. (Figs. 105A-C). CFO well-developed (Fig. 105A). Leg measurements: I: femur 1.72, patella 0.52 , tibia 1.67 , metatarsus 1.22 , tarsus, total 0.72 ; II: $1.67,0.52,1.60,1.22,0.72,5.75$; III: $1.15,0.40,0.97,0.65,0.52,3.70$; IV: $1.50,0.45,1.57,1.20,0.70,5.42$. DTS 0.27. DTE 0.95. Epiandrous fusules as in figure 297J. Palp as in figures 106-107. Paracymbium with short distal portion (Figs. 106D, 110D). Embolus with tooth-like projections (Fig. 107D). Medial groove not evident in the outer surface of the embolus (Fig. 107A, 110A).

Female (Paratype ICN 2141): coloration and general habitus as in male (Fig. 104). Abdomen with three longitudinal black bands, one median, bifurcated at the anterior region and two laterals. Total length 3.85. Cephalothorax 1.60 long, 1.30 wide, 0.80 high. Abdomen 2.65 long, 2.00 wide, 1.75 high. Sternum 0.80 long, 0.82 wide. Eye diameters and interdistances: AME 0.10. ALE 0.08. PME 0.11. PLE 0.08. AME-AME 0.1, AME-ALE 0.07, PME-PME 0.12, PME-PLE 0.11, ALE-PLE 0.01. Clypeus height $2.20 \times$ AME diameter. Chelicerae with the same coloration of the cephalothorax. Three promarginal and four retromarginal teeth (Figs. 105D-F). Leg measurements: femur 1.45, patella 0.55 , tibia 1.25 , metatarsus 1.07 , tarsus 0.57 , total 4.90; II: $1.47,0.50,1.15$, 1.07, 0.65, 4.85; III: 1.07, 0.42, 0.87, 0.60, 0.50, 3.47; IV: 1.50, 0.30, 1.37, 1.07, 0.45, 4.70. DTS 0.52. DTE 0.87. Tracheal system as in figure 109. Internal genitalia without spermathecae (Fig. 108).

Variation: Male total length 3.2-4.1 $(N=10$; average 3.6), cephalothorax length 1.5-1.7 $(N=10$; average 1.6), femur I length 1.5-1.8 $(N=10$; average 1.7). Female total length 3.5-4.2 ( $N=10$; average 3.8), cephalothorax length 1.5-1.7 $(N=10$; average 1.6), femur I length 1.2-1.7 ( $N=10$; average 1.4). 
Distribution: Known only from Cundinamarca and Boyacá states in Colombian central Andes (Fig. 310).

Additional material examined (N = 178): COLOMBIA: Boyacá: Paipa, Laguna de

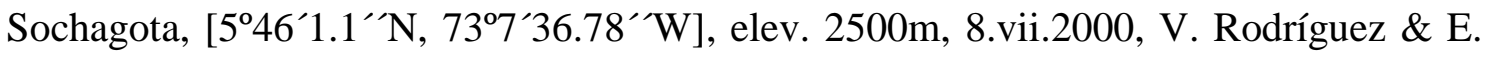
Flórez leg., (Debajo de tronco caído), $10 \widehat{~(I C N ~ 5102) . ~ C u n d i n a m a r c a: ~ M o s q u e r a, ~ L a ~}$ Herrera, [44ㄴ $31.1^{\prime \prime} \mathrm{N}, 74^{\circ} 18^{\prime} 54.2^{\prime \prime} \mathrm{W}$ ], elev. 2600m, 9.ii.2002, J. Martinez, C. Niño, A. Castañeda, G. Mora leg., $43 \hat{\jmath} 18 \bigcirc 53$ immature (ICN 2136); $43 \hat{\jmath} 4 \uparrow 8$ immature

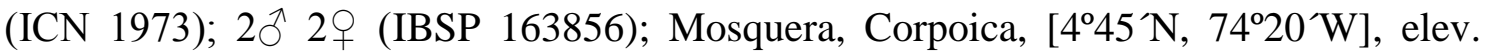
2600m, v-vi.1997, L. Benavides leg., (Cultivo y pastos Kituyo y Falsa Poa), 2 ๙ (ICN

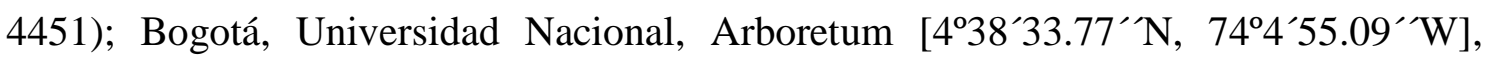
elev. 2600m, 21.ii.2009, F. Bagon leg., (colecta manual en suelo y hojarasca), 1 ( (ICN 5100); Bogotá, Universidad Nacional, Instituto de Ciencias Naturales [ $4^{\circ} 38^{`} \mathrm{~N}, 74^{\circ} 4^{`} \mathrm{~W}$ ], elev. 2600m, 9.ix.1992, C. Martinez \& A. Ordoñez leg., $1 \bigcirc$ (ICN 6433).

Glenognatha lacteovittata (Mello-Leitão, 1944)

(Figs. 111-118, 297I, 298H, 299F, 300F, 301E, 302H, 303E, 308)

Oedothorax lacteovittatus Mello-Leitão, 1944: 327, fig. 9 (Male and female lectotypes from La Plata, Buenos Aires, Argentina, R. Costa leg., deposited in MNRJ 1963, examined).

Glenognatha lacteovittata: Ramírez, 1996: 35, figs. 1-10; Platnick, 2013.

Diagnosis: males of G. lacteovittata resemble those of $G$. sp. nov. 4 and G. sp. nov. 5 by the short distal portion of the paracymbium (Figs. 114D, 118H) and the embolic medial groove confined to the embolus inner surface (Fig. 114A). It can be distinguished from the two latter species by the presence of filiform projections in the embolus distal region (Figs. 115E, 118F-G). Females are diagnosed by the presence of two posterior cheliceral bulges (Fig. 113E).

Description: male and female described by Ramírez (1996). Additional data are presented here.

Male (IBSP 44111): habitus as in figure 111. Carapace brown, with three longitudinal dusky bands (one median and two lateral). Sternum brown, darker on sides. Abdomen spherical, dorsally with irregular pattern of two longitudinal black bands on sides. Black 
patches scattered around and between these bands. Silver guanine spots concentrated on the median regions of the dorsal and lateral surface. Venter with a pale black median band that ramifies into irregular thin bands towards the lateral surface. Legs yellowbrownish. Total length 2.72. Cephalothorax 1.30 long, 1.03 wide, 0.80 high. Abdomen 1.71 long, 1.3 wide, 1.22 high. Sternum 0.80 long, 0.70 wide. Eye diameters and interdistances: AME 0.09. ALE 0.08. PME 0.08. PLE 0.08. AME-AME 0.08, AMEALE 0.06, PME-PME 0.13, PME-PLE 0.08, ALE-PLE 0.01. Clypeus height $1.90 \times$ AME diameter. Chelicerae brown. Three promarginal and four retromarginal teeth (Figs. 113A-C). Ret1 on the posterior surface (Fig. 113B). CFO well-developed (Fig. 113B). Leg measurements: femur 1.92, patella 0.42, tibia 1.92, metatarsus 1.50 , tarsus 0.82, total 6.60; II: $1.85,0.40,1.82,1.37,0.75,6.20$; III: 1.25, 0.30, 1.05, 0.85, 0.50, 3.95; IV: 1.92, 0.32, 1.77, 1.33, 0.52, 5.88. DTS 0.22. DTE 0.72. Epiandrous fusules as in figure 297I. Spinnerets as in figures 299F, 301E and 303E. Palp as in figures 114115. Paracymbium with short distal portion (Figs. 114D, 118H). Conductor lamina distal end prolaterally folded (Figs. 115B, 118A). Embolus with subapical tooth-like projections (Fig. 118G) and filiform projections (Figs. 118E-G). Medial groove restricted to the inner surface of the embolus (Fig. 114A).

Female (IBSP 44084): coloration and general habitus as in male (Fig. 112). Abdomen with two large dorso-lateral patches of silver guanine spots. Venter with grey-brownish area anterior to the gonopore and scattered silver guanine spots. Total length 2.77. Cephalothorax 1.32 long, 1.05 wide, 0.75 high. Abdomen 2.00 long, 1.60 wide, 1.40 high. Sternum 0.65 long, 0.70 wide. Eye diameters and interdistances: AME 0.08. ALE 0.08. PME 0.09. PLE 0.08. AME-AME 0.08, AME-ALE 0.07, PME-PME 0.14, PMEPLE 0.09, ALE-PLE 0.01. Clypeus height $1.9 \times$ AME diameter. Three promarginal and four retromarginal teeth. (Figs. 113D-F). Leg measurements: I: femur 1.85, patella 0.45, tibia 1.72 , metatarsus 1.45 , tarsus 0.75 , total 6.22 ; II: $1.67,0.42,1.72,1.32,0.67,5.82$; III: $1.07,0.32,0.92,0.65,0.37,3.35$; IV: 1.92, 0.32, 1.67, 1.25, 0.56, 5.73. DTS 0.17. DTE 0.60. Tracheal system as in figure 117. Spinnerets as in figures $298 \mathrm{H}, 300 \mathrm{~F}$ and 302H. Internal genitalia without spermathecae (Fig. 116).

Variation: Male total length 2.3-3.7 ( $N=10$; average 2.7), cephalothorax length 1.1-1.6 $(N=10$; average 1.3), femur I length 1.6-2.3 $(N=10$; average 2$)$. Female total length 
2.6-3.5 $(N=10$; average 3.1), cephalothorax length 1.1-1.6 $(N=10$; average 1.3$)$, femur I length 1.6-2.2 $(N=10$; average 1.8).

Distribution: Known in Peru, Brazil, Uruguay and Argentina (Fig. 308).

Additional material examined ( $=834)$ : ARGENTINA: Buenos Aires: Bella Vista,

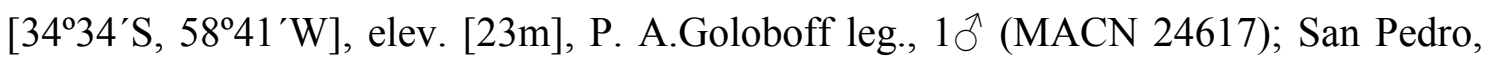

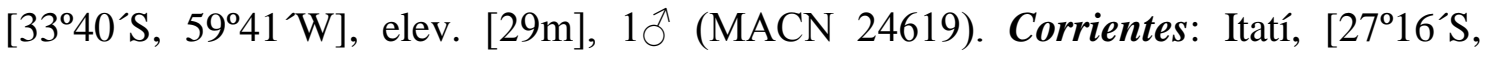
58 $14^{\circ}$ W], elev. [60m], M. E. Galiano leg., 19 1 $\delta^{\Uparrow}$ (MACN 24618). Entre Ríos: Río Gualeguaychú, [334’S, 58²6W], elev. [6m], 10.xii.1982, Ramírez \& Goloboff leg., $1 \delta^{\Uparrow}$ (MACN 24620). Santa Fe: Las Gamas, 20Km W Vera, [29²3’S, 60³2`W], elev. [54m], 27-30.x.1994, M. Ramírez \& J. Faibovich leg., 1 (MACN 24616).

New records: ARGENTINA: Buenos Aires: Campana, Reserva Natural Otamendi, Estación Río Luján, [349`S, 5856`W], elev. [4m], 30.vi.2007, C. J. Grismado, L. Damer, N. López, S. Trivero \& N, Olejnik leg., $2 q 1$ immature (MACN 12722); $1 \overbrace{}^{\Uparrow}$

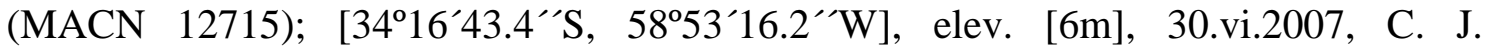
Grismado, L. Damer, N. López, S. Trivero \& N, Olejnik leg., 2 ๆ 2 (MACN 12750); 34.2774S, 58.888W, elev. 5m, 9.ix.2010, M. Ramírez, C. Grismado, L. Piacentini, E. Soto, M. E. González-Márquez, M. Guala, J. M. Andia Navarro, N. López, I. Ortega Insaurralde y P. Zermoglio leg., 4 ㅇ $3 \overbrace{}^{\Uparrow} 2$ immature (MACN); Delta, Arroyo Caraguatá,

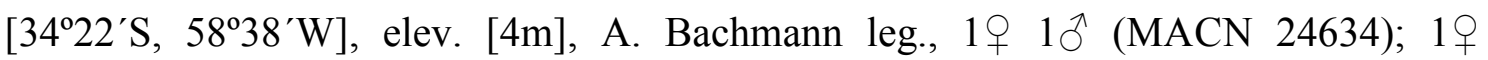

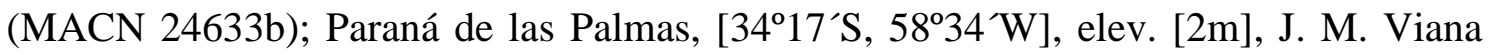
leg., $1{ }^{\Uparrow}$ (MACN 26004). Chaco: 100NW Resistencia, Chaco NP, [2649`N, 5940`W], elev. [76m], 12-17.xii.1990, S. \& J. Peck leg., 1ð̄ (AMNH). Ciudad Autónoma de

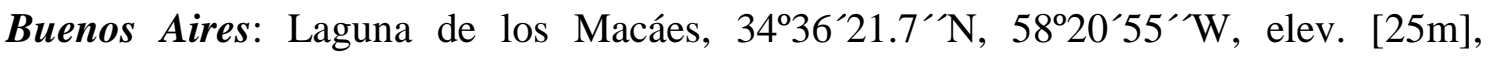
12.v.2008, M. Turienzo leg., trampa de caída, $2 \widehat{\jmath} 1$ immature (MACN); 10.xi.2008, M.

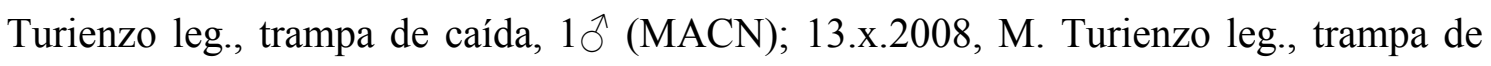
caída, $194 \hat{\jmath}(\mathrm{MACN})$; 27.x.2008, M. Turienzo leg., trampa de caída, $2 \widehat{\circlearrowleft}(\mathrm{MACN})$; 1.ix.2008, M. Turienzo leg., trampa de caída, $195 \widehat{\text { }}$ (MACN); 15.ix.2008, M. Turienzo leg., trampa de caída, 1 + $3 ð 1$ immature (MACN); 29.ix.2008, M. Turienzo leg., trampa de caída, $1 \uparrow 1{ }^{\Uparrow}(\mathrm{MACN}) ; 19.1 .2009$, M. Turienzo leg., trampa de caída, $1 ð$ (MACN); 5.i.2009, M. Turienzo leg., trampa de caída, $1 ð$ (MACN); 2.ii.2009, M. Turienzo leg., trampa de caída, $1 \delta^{\Uparrow}(\mathrm{MACN})$. Entre Ríos: San Justo, [32²6`S, $58^{\circ} 25^{\prime} \mathrm{W}$ ], elev. [44m], A. M. Julieta leg., $1910^{\Uparrow} 1$ immature (MACN 24636). Río

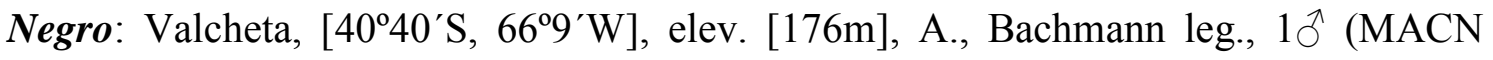


24637). BRAZIL: Acre: Rio Branco, campus UFAC, [957’S, 6752 W], elev. [162m], 4.iv.1996, A. D. Brescovit \& A. B. Bonaldo leg., 1 ㅇ 1 đ 1 immature (IBSP 6978).

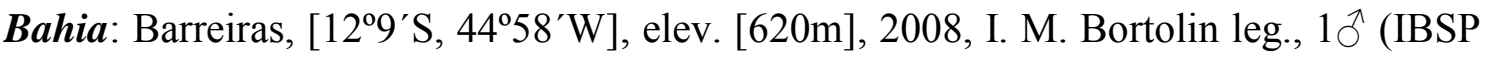

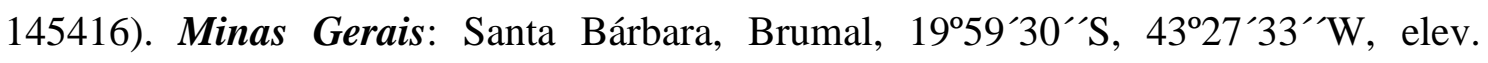
[730m], 28-29.viii.2008, I.L.F. Magalhães leg., 1 + 1ठ (UFMG 4773). Paraná: Boa

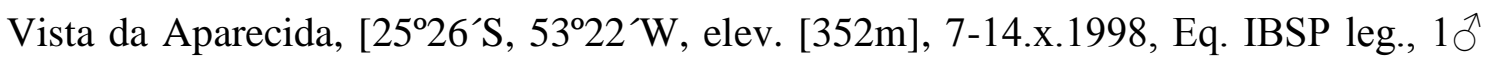
(IBSP 21102); Candói/Mangueirnha, Usina Hidrelétrica de Segredo, Reservatório do

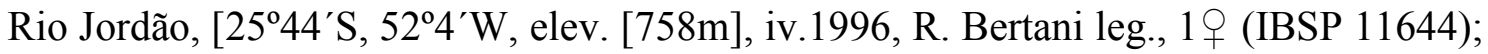

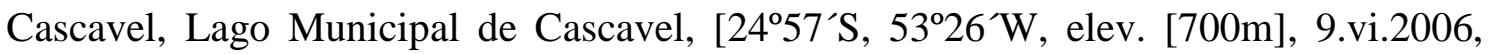

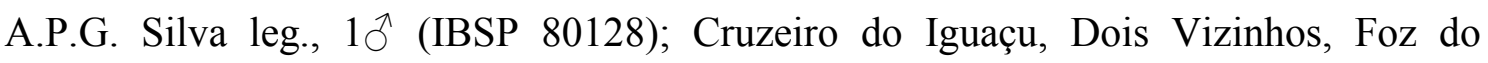

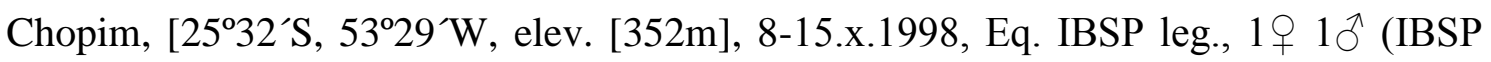
21057); $1 \delta^{\Uparrow}$ (IBSP 21113); 19 (IBSP 21313); $2 q 1 \delta^{\Uparrow} 1$ immature (IBSP 21209); 1 + (IBSP 21129); 19 (IBSP 21249); 19 (IBSP 21264); 19 (IBSP 21228); $1 \hat{\jmath}$ (IBSP

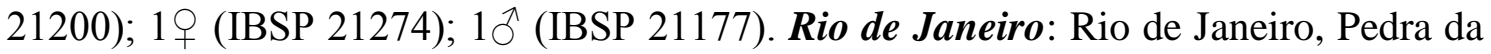

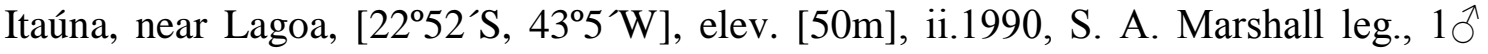
$(\mathrm{AMNH})$; Bom Jesus do Itabapuana, São José do Calçado, Rio Itabapuana, Usina

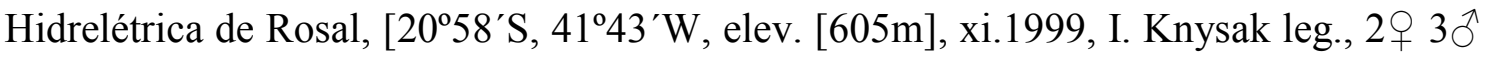

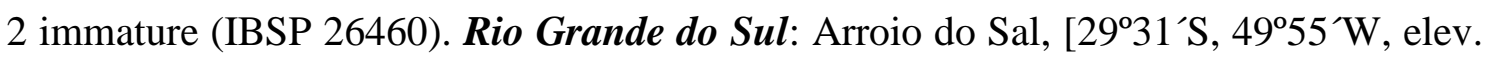
[23m], 24.viii.1998, J. Romanzin leg., 2 q $1 \hat{\jmath} 2$ immature (MCTP 10320); Bagé,

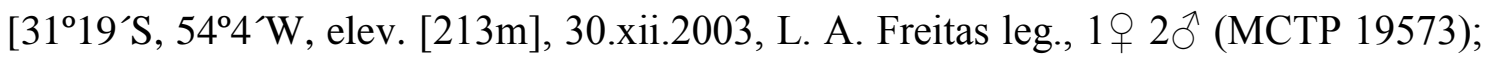

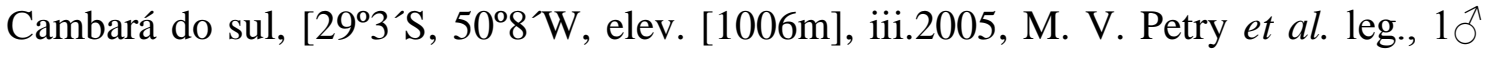
(MCTP 30986); vii.2005, M. V. Petry et al. leg., 1 đ (MCTP 30987); viii.2005, M. V. Petry et al. leg., 1ठ઼ (MCTP 30989); iii.2006, M. V. Petry et al. leg., $1{ }^{\Uparrow}$ (MCTP

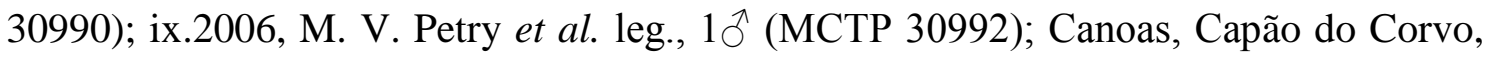
[295' S, 51 ${ }^{\circ} 13^{\prime} \mathrm{W}$, elev. [5m], 23.iv.1966, A. Lise leg., $15 q$ (MCN 699); Caxias do

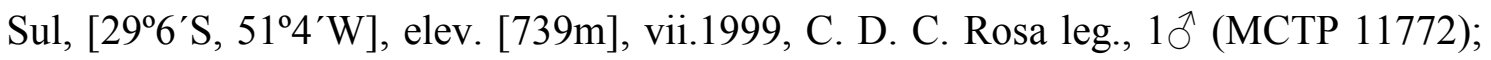
Derrubadas, Parque Estadual do Turvo, [2710`S, 5355`W], elev. [313m], 4-8.v.2004, R. Ott et al. leg., 2 (MCN 38786); 27-31.x.2003, R. Ott et al. leg., 5 ふ (MCN 38778); Planalto, [27 $19^{\prime} \mathrm{S}, 53^{\circ} 4^{\prime} \mathrm{W}$ ], elev. [563m], 23.i.1994, R. Oliveira leg., $3{ }^{\wedge} 1$ immature (MCTP 4859); Porto Alegre, [304`S, 51ㅇ' W], elev. [174m], 10.iv.2000, A. Braul leg.,

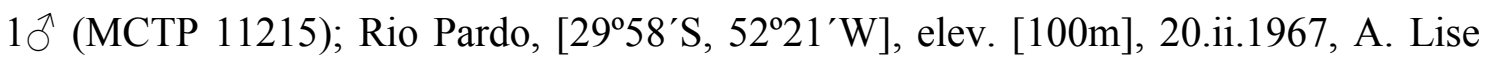
leg., 1 ( (MCN 48507); 6.vii.1966, A. Lise leg., 10ð 3 immature (MCN 266); 12 ๙ 2

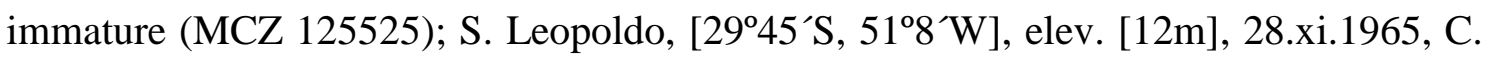

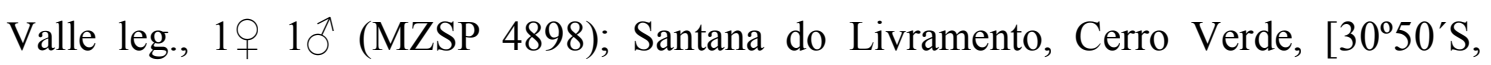




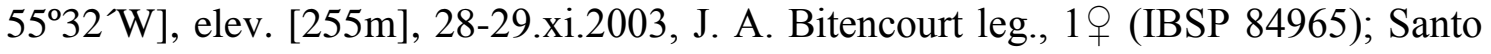
Ângelo, [28 $18^{\prime} \mathrm{S}, 5^{\circ} 17^{\prime} \mathrm{W}$ ], elev. [204m], 12-25.ii.2004, I. Renardi leg., $1 \delta^{\wedge}$ (MCTP

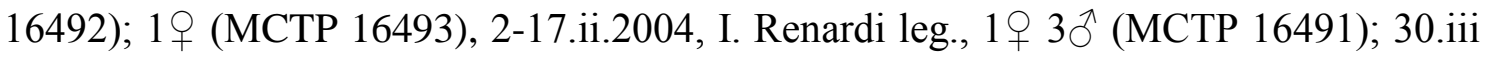
- 9.iv.2004, F. D' Agostini 3 q 1ô (MCTP 22620); São Francisco de Paula, Potreiro

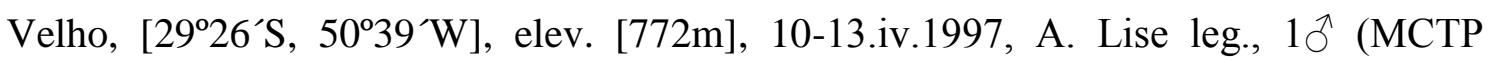
14278); Tapes, Fazenda São Miguel, [3040’S, 51²5`W], elev. [9m], 14.v.2003, Equipe Probio leg., $1 \delta^{\Uparrow}(\mathrm{MCN}$ 35926); Torres, [2920'S, 4944'W], elev. [13m], 26.iii.2006, E. L. C. da Silva leg., 1 đ 2 immature (MCN 1972); Triunfo, Estaçao Braskem de Proteçao

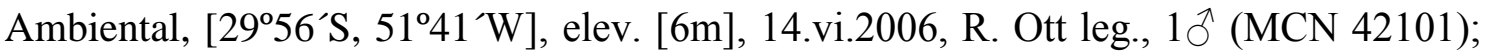
Triunfo, Faz. Pontal, Parque Estadual Delta do Jacuí, [2959`S, 51²9`W], elev. [15m],

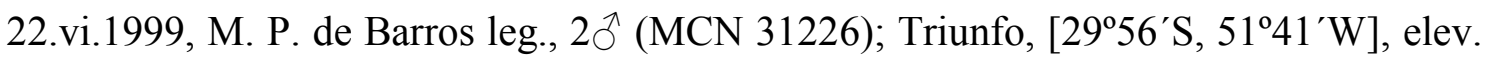
[6m], 4.vi.1987, C. Marros leg., 1 (MCN 16934); 18.x.1993, L. Moura leg., $1 \overbrace{}^{\Uparrow}(\mathrm{MCN}$

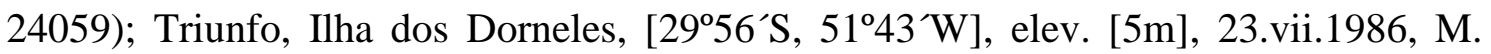

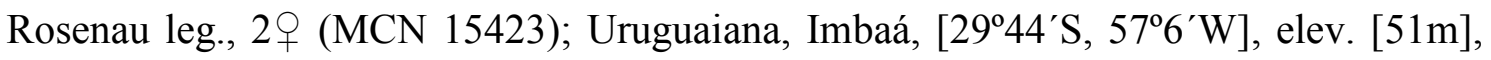
24.vi.2009, R. Alves leg., 1 $\overbrace{}^{\Uparrow}$ (MCN 26490). Rio Grande do Sul / Santa Catarina: Rio

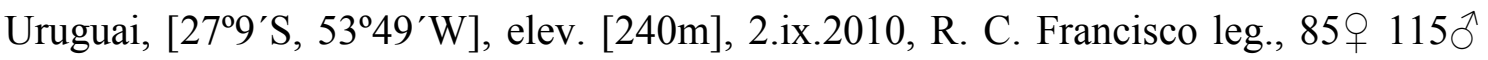
(MCTP). Santa Catarina: Bombinhas, Arie Costeira de Zimbros, [27오 $\mathrm{S}, 48^{\circ} 31^{\prime} \mathrm{W}$ ], elev. [74m], 4.vii.2011, A. L. Trivia \& L. Malta leg., 1 ( (IBSP 163680); Chapecó,

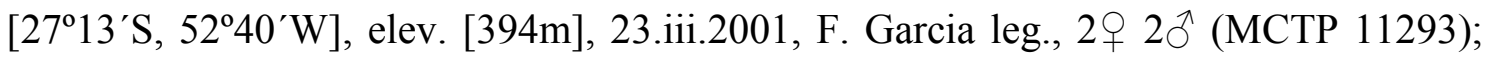
Florianápolis, Ilha do Árvoredo, Reserva Biológica Marinha de Arvoredo, [235ํㅇ, $46^{\circ} 10^{\prime} \mathrm{W}$ ], elev. [3m], 2003, Noili leg., $1911^{\Uparrow}$ (IBSP 75324); 1 (IBSP 75354); 1 ㅇ (IBSP 75323), Itá, Rio Uruguai, [27 $\left.16^{\prime} \mathrm{S}, 52^{\circ} 19^{\prime} \mathrm{W}\right]$, elev. [457m], 1.x.2001, $1 \delta^{\wedge}$ (MCTP 849); Rio Negrinho, [26¹3`S, 49³1`W], elev. [797m], iv.2004 - vii.2005, M. A. Nickel leg., $1 q 3 \hat{\partial} 1$ immature (IBSP 75995); M. A. Nickel leg., $153+163 ð 56$

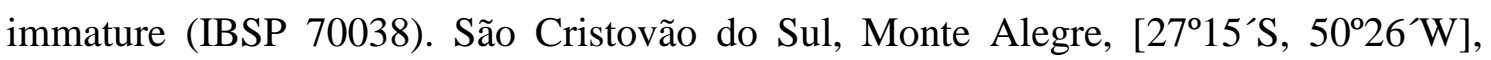
elev. [1019m], 2001-2002, J. M. Moreira leg., $1 q$ 1 $\delta^{\Uparrow}$ (IBSP 141433); $1 q$ (IBSP 141429); 2 (IBSP 141427); 19 (IBSP 141431); $1 \hat{\sigma}$ (IBSP 141432); $2 \hat{0}$ (IBSP 141428); 19 (IBSP 141426); $1 \hat{\jmath}$ (IBSP 141430); 1ð (IBSP 141425); São Miguel do Oeste, [26 $43^{\prime} \mathrm{S}, 53^{\circ} 30^{\prime} \mathrm{W}$ ], elev. [603m], iii-viii.2004, J. Barp leg., 1 \% $1 \overbrace{}^{\Uparrow}$ (IBSP

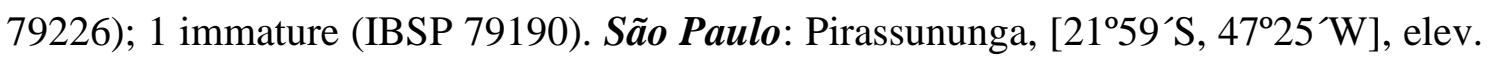
[630m], 8.vii.1940, Schubart leg., 1ð̋ (MZSP 6432); Rosana, Usina Hidrelétrica Sérgio

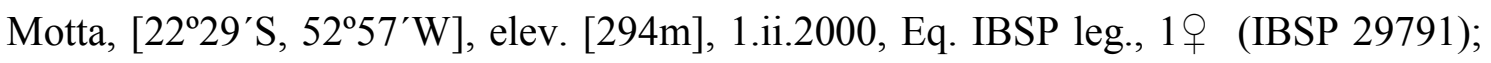

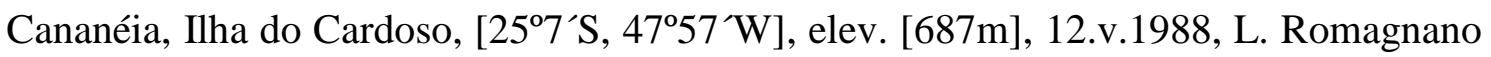
\& E. Venticinque leg., 19 (MCN 23205). ECUADOR: Napo: Lago Agrio, [05’S, 


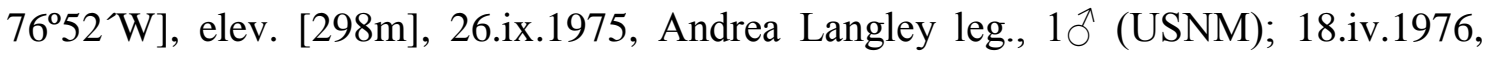
Langley leg., $1 q$ (USNM). PARAGUAY: Central: $15 \mathrm{Km}$ NE Asunción, [2515’S,

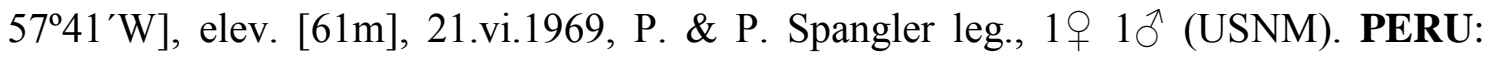
Loreto: San Miguel, arenas movedizas, ca. boca del Rio Samiria, entre el Río Marañón

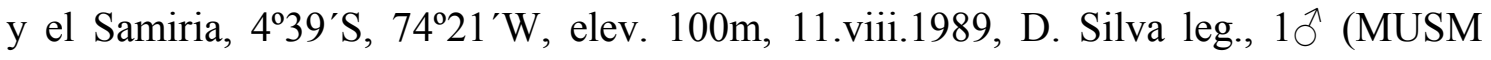

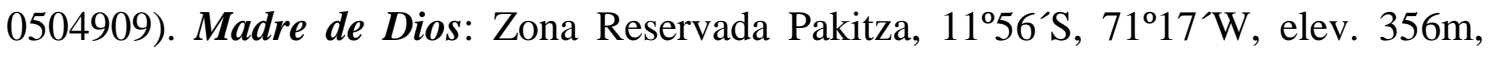

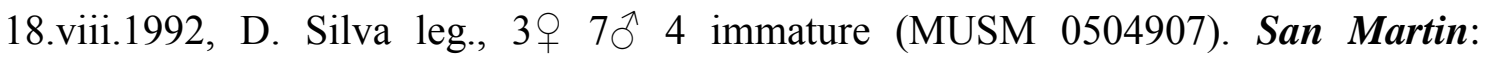

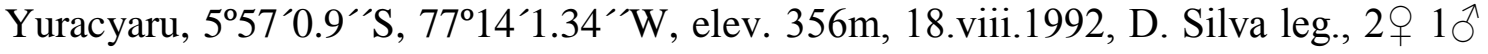

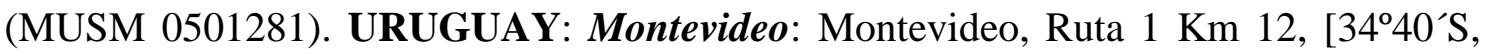
56 $29^{`} \mathrm{~W}$ ], elev. [3m], 2001, M. A. Benamu leg., 3 (IBSP 44074); 1 + $10^{\Uparrow}$ (IBSP 44084); $1 q$ (IBSP 44081); $3 q$ (IBSP 44085); $1 q 1 \hat{\jmath} 1$ immature (IBSP 44111); $3 \delta^{\Uparrow}$ (IBSP 44082); 2 ㅇ (IBSP 44083); 4 ㅇ (IBSP 44072).

Glenognatha tangi (Zhu, Song \& Zhang, 2003) new combination

(Figs. 119-126, 297B, 299H, 301F, 303F, 314)

Dyschiriognatha tangi Zhu, et al., 2003: 207, figs. 113A-G, 114A-F (female holotype from Yongning county, Guangxi province, China, 6.xii.1982, Zhang Yongquiang leg., deposited in HU, not examined; paratypes: $1 \overbrace{}^{\lambda}$ from Jinghong county, Yunnan province, China, 10.vii.1983, Zhu Chuandian leg., deposited in HU, not examined, $1 ð$ paratype from Nanning city, Guangxi province, China, 11.xii.1986 Zhang Yongquiang leg., deposited in HU, examined); Platnick, 2013.

Transfer justification: Detailed study of $D$. tangi male paratype and female specimens from Myanmar showed that this species has all the diagnostic characters of the genus Glenognatha. The conductor (Figs. 123B-C, 126C), the embolus (Fig. 123D, 126C), the paracymbium (Fig 126E) and the female genitalia (Fig. 124) exhibit the typical Glenognatha morphology.

Diagnosis: males of $G$. tangi resemble those of $G$. dentata and G. argyrostilba by the presence of irregular and rounded setae-bearing depressions on the lateral surface of the carapace (Fig. 125D-F). They can be distinguished from the latter species by the shape of the conductor lamina in ventral view, which is rectangular and without tooth-shaped projections (Figs. 123B-C, 126C). Females are diagnosed by the rounded UEs distal portion (Fig. 124) and the smooth sternum (Fig. 125C). 
Description: male and female described by Zhu et al. (2003), additional data are presented here.

Male (Myanmar, USNM): habitus as in figure 119. Cephalothorax with lateral depressions (Fig. 119A). Cephalothorax 0.70 high. Abdomen 1.05 high. Sternum 0.45 long, 0.50 wide. Chelicerae as in figures 121A-C. Cheliceral fang outgrowth welldeveloped (Fig. 121A). Epiandrous fusules as in figure 297B. Spinnerets as in figures 299H, 301F and 303F. Palp as in figures 122-123.

Female (Myanmar, USNM): habitus as in figure 120. Cephalothorax with lateral depressions (Figs. 125A, D-F ). Cephalothorax 0.75 high. Abdomen 1.47 high. Sternum 0.50 long, 0.50 wide. Chelicerae as in figures 121D-F. Internal genitalia with welldeveloped spermathecae (Fig. 124).

Variation: male total length 1.9-2.2 $(N=4$; average 2.1$)$, cephalothorax length $0.8-1.1$ $(N=4$; average 0.9$)$, femur I length 0.9-1.2 $(N=4$; average 1.1).

Distribution: known from Guangxi and Yunnan provinces in China and Sagaing region in Myanmar (Fig. 314).

Additional material examined $(\mathbf{N}=5)$ : New records: MYANMAR: Sagaing: Chattin Wildlife Sanct. Takontaing, 2337’20`N, 95³1'52”E, elev. [179m], 7-12.x1998, Coddington \& Baptista leg., 1 ㅇ $3 \hat{\jmath} 1$ immature (USNM).

Glenognatha argyrostilba (O. P.-Cambridge, 1876) new combination

(Figs. 127-140, 313)

Pachygnatha argyrostilba O. P.-Cambridge, 1876: 573, pl. 59, fig. 8 (male lectotype and female paralectotype from Alexandria, Egypt, deposited in Hope Entomological Collection, Oxford, not examined).

Dyschiriognatha argyrostilba: Simon, 1893: 324; Bosmans \& Bosselaers, 1994: 346, fig. 132-147; Saaristo, 2003: 22, fig. 19-20; Saaristo, 2010: 233, fig. 36.24-26; Platnick, 2013.

Pachygnatha (Dyschiriognatha) argyrostilba: Pavesi, 1895: 504. 
Dyschiriognatha montana Simon, 1897: 868 (male and female syntypes from St. Vincent Islands, Lesser Antilles, deposited in BMNH, examined through photographs of the habitus and male genitalia). New Synonymy.

Dyschiriognatha atlantica Holm, 1969: 63, fig. 1-5 (male holotype from Sandy bay beach, St. Helena island, 11.i.1966, P. Basilewsky, P. Benoit \& N. Leleup leg., deposited in MRAC, not examined); Benoit, 1977: 160, figs. 69a-e. Synonymized by Bosmans \& Bosselaers, 1994.

Glenognatha mira Bryant, 1945: 405 (female holotype from Miragoane, Haiti, 2.xi.1934, Darlington leg., deposited in MZC, examined). New synonymy.

Glenognatha maelfaiti Baert, 1987: 141, figs. 1-7 (male holotype from Cerro Maternidad, Santa Cruz, Galápagos, Ecuador, 15.ii-1.iii-1986, deposited in

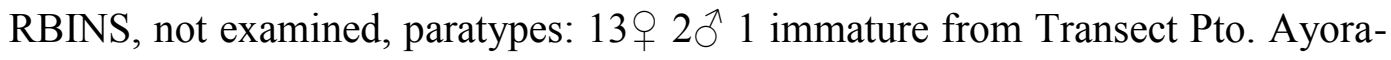
Itabaca, z-flank, Santa Cruz, Galápagos, Ecuador, 15.ii-1.iii-1986 Baert, Maelfait et Desender leg., deposited in RBINS 27047, examined; 36 † 19 5 immature from Santa Cruz, Galápagos, Ecuador, deposited in RBINS, not examined. New synonymy.

Transfer and Synonymy justification: Detailed study of D. argyrostilba specimens collected in Africa and drawings of the type material (see Bosmans \& Bosselaers, 1994), showed that this species has all the diagnostic characters of the genus Glenognatha. Conductor (Figs. 131 B-C, 139A-B), embolus (Figs. 131D, 139B), paracymbium (Figs. 130D, 137D), female genitalia (Figs. 132, 138) and tracheal system (Fig. 133) exhibit the typical Glenognatha morphology.

Meticulous examination of the available type material of D. montana, G. mira and $G$. maelfaiti, showed that these species have the diagnostic characters of $D$. argyrostilba and should be considered as synonyms. Male palps of D. montana and G. maelfaiti have the conductor lamina sinuous in ventral view and the conductor retrolateral apophysis tip sharpened as observed in D. argyrostilba. In addition, the uterus externus sac of $G$. mira and G. maelfaiti is wider than long as characteristic for D. argyrostilba. It was not possible to examine the internal female genitalia of D. montana, nevertheless based on the male palp and the female chelicerae morphology I consider it as a synonym of $G$. argyrostilba. 
Diagnosis: males of $G$. argyrostilba resemble those of $G$. dentata and $G$. tangi by the presence of irregular and rounded setae-bearing depressions on the lateral surface of the carapace (Figs. 127A, 134A, 140A-B). They can be distinguished from the latter species by the pointed conductor retrolateral apophysis (Figs. 131B-C). Females are diagnosed by the morphology of the UEs, which is wider than long (Figs. 132, 138).

Description: male and female described by Bosmans \& Bosselaers (1994), additional data are presented here.

Male (CAS 9056025): habitus as in figure 127. Cephalothorax with lateral depressions (Fig. 127A). Chelicerae with reduced CFO (Fig. 129A). Prt2 and Prt3 closer to each other than to Prt1 (Figs. 129A, C). Ret2 and Ret3 closer to each other than to Ret1 (Fig. 129B). Palp as in figures 130-131. Conductor enclosing the embolus (Figs. 130A, 131A).

Female (CAS 9056025): habitus as in figure 128. Cephalothorax with lateral depressions (Fig. 128A). Sternum with setae-bearing depressions (Fig. 140C). Chelicerae as in figures 129D-F. Tracheal system as in figure 133. Internal genitalia as in figure 132. Spermathecae well-developed (Fig. 132).

Variation: male total length 1.6-2.2 $(N=10$; average 2.0), cephalothorax length $0.8-1.2$ $(N=10$; average 1.0), femur I length 1.0-1.3 $(N=10$; average 1.2). Female total length 2.1-2.6 $(N=10$; average 2.4), cephalothorax length 0.9-1.1 $(N=10$; average 1.0$)$, femur I length 1.1-1.4 ( $N=10$; average 1.2). Habitus and chelicerae of Ecuadorian specimens as in figures $134-136$.

Distribution: known in the Neotropics and Afrotropics (Fig. 313).

Additional material examined $(\mathbf{N}=31)$ : ECUADOR: Galápagos: Isabela, $\left[0^{\circ} 55^{\prime} \mathrm{S}\right.$,

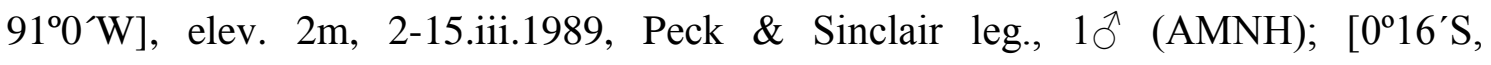
$90^{\circ} 41^{\prime} \mathrm{W}$ ], elev. [300m], 10.iv.1982, Y. Lubin leg., 2 (MCZ 125476).

New records: BRAZIL: Bahia: Mucugê, Reserva Particular do Patrimônio Natural

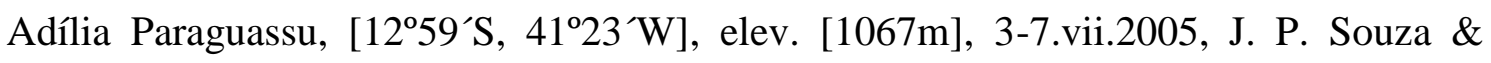
Alves leg., 19 (IBSP 56557). Ceará: Fortaleza, UFCE, Campus de Itaperi [3º7`S, $38^{\circ} 33^{`} \mathrm{~W}$ ], elev. [26m], 8.vii.2011, R. Azevedo leg., 19 (UFCE 852); 1 (UFCE 841). 


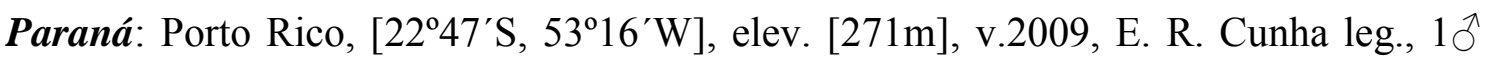

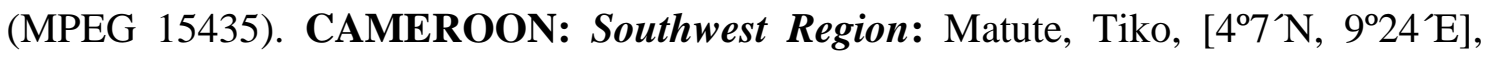
elev. [30m], 14.iv.-6.v.1949, B. Malkin 1ð̋ (CAS 9039595). CUBA: Pinar del Río:

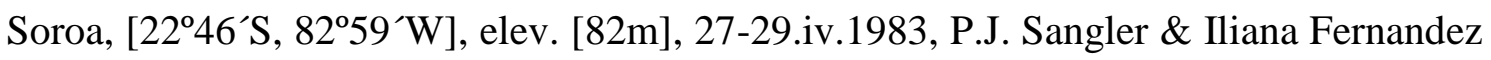

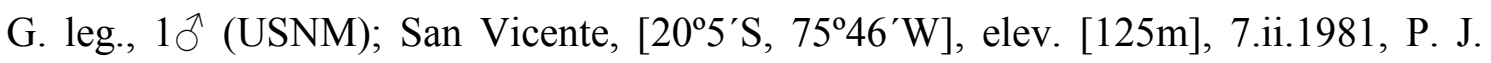
Spangler \& A. Vega leg., $2{ }^{\Uparrow} 6 \propto$ (USNM). Sancti Spiritus: Gaviña, Sierra Escambray, [21 $\left.1^{\circ} 56^{\prime} \mathrm{S}, 7^{\circ} 26^{\prime} \mathrm{W}\right]$, elev. [67m], 13.ii.1981, P.J. Spangler \& A. Vega leg., 1 q

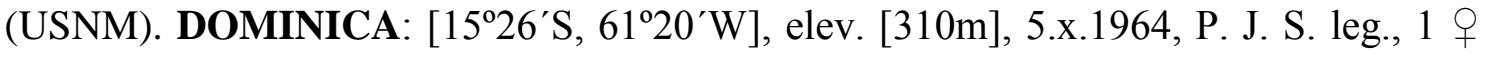
(USNM). ECUADOR: El Oro: Arenillas (18Km SE), La Cuca. Exp. Sta., [3³3’S, 80² W], elev. [77m], 22.v.1979, Jos J. Anderson leg., $1910^{\Uparrow}$ (USNM); Guayas: Naranjal (30Km N), [240’S, 79³6`W], elev. [24m], 24.xii.1977, Jos J. Anderson leg., $1 q 10^{\Uparrow}$ (USNM); Manabí: Chone, [041’S, 805`W], elev. [65m], 7.i.1978, P. J. Spangler \& J. Anderson leg., $1 \delta^{\Uparrow}$ (USNM). HAITI: Sud: Lévy (1 Km NE), [18¹8`N, 735 51 W], elev. [185m], 10-11.ix.1981, P. Spangler \& R. Faitoute leg., $1 \overbrace{}^{\Uparrow}$ (USNM).

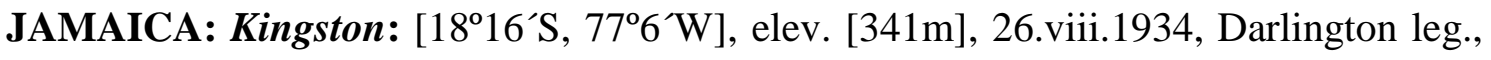
$1 \delta^{\Uparrow}$ (MCZ 125504). NIGERIA : Kabba: Kabba, : [750`N, 6º $\left.{ }^{\circ} \mathrm{E}\right]$, elev. [458m], 1923.ii.1949, B. Malkin leg., $2 \uparrow 2 \Uparrow$ (CAS). REPUBLIC OF CÔTE D'IVOIRE: Vallée du Bandama region: Bouaké, [701N N, 5² ${ }^{`} \mathrm{~W}$ ], elev. [350m], 7.x.1995, Russel-Smith A. leg., $1 \overbrace{}^{\circledR}$ (MRAC 227412).

\section{Glenognatha dentata (Zhu \& Wen, 1978) new combination}

(Figs. 141-149, 297A, 299G, 314)

Dyschiriognatha dentata Zhu \& Wen, 1978: 16, figs. 1-9 (female holotype from China, 24.x.1975, presumably deposited in the Jilin University, not examined; paratypes:

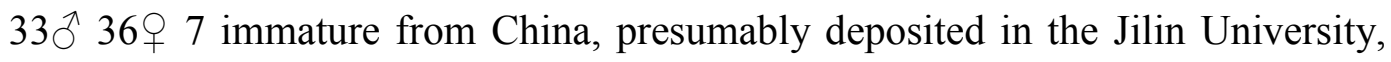
not examined); Hu, 1984: 133, figs. 135.1-8; Chen \& Gao, 1990: 74, figs. 93A-E; Okuma, 1991: 15, figs. 1, 2A-F, 3A; Okuma et al., 1993: 31, figs. 28A-C; Song et al., 1999: 213, figs. 1190-R; Zhu et al., 2003: 204, figs. 111A-K, 112A-G, pl. VIIA-D; Tanikawa, 2007: 103, figs. 364-365, 812-814; Tanikawa, 2009: 412, figs. 49-51; Platnick, 2013.

D. hawigtenera Barrion \& Litsinger, 1995: 490, figs. 300A-E, 301A-G (female holotype from Luzon Island, Philippines, 10.viii.1979, A. T. Barrion leg., deposited in the International Rice Research Institute, not examined). Synonymized by Zhu et al., 2003: 204. 
Transfer justification: Detailed study of D. dentata specimens collected in Asia (Myanmar, China, Vietnam and Japan) and schematic drawings of the male genitalia and somatic characters in the original description (Zhu \& Wen, 1978), showed that this species has all the diagnostic characters of the genus Glenognatha. The conductor and the embolus although highly modified (Figs. 145B-D, 149), the female genitalia (Fig. 146) and the tracheal system (Fig. 147) exhibit the typical Glenognatha morphology.

Diagnosis: males of $G$. dentata can be easily distinguished from all other Glenognatha species by the prominent tooth-like projections on the distal retrolateral margin of the conductor (Figs. 144B, 145B-C, 149D). Females are diagnosed by the presence of filiform projections in the uterus externus chamber (Fig. 146).

Description: male and female described by Zhu \& Wen (1978), additional data are presented here.

Male (Myanmar, USNM): habitus as in figure 141. Cephalothorax with lateral depressions (Fig. 141A). Cephalothorax 0.77 high. Abdomen 0.88 high. Sternum 0.55 long, 0.55 wide. Chelicerae as in figures $143 \mathrm{~A}-\mathrm{C}$, with small setae-bearing tubercles on the antero-ectal surface (Fig. 143C). Cheliceral fang outgrowth well-developed (Fig. 143A). Epiandrous fusules as in figure 297A. ALS as in figure 299 G. Palp as in figures 144-145. Embolus and conductor highly modified (Figs. 149A-F).

Female (Myanmar, USNM): habitus as in figure 142. Cephalothorax with lateral depressions (Fig. 142A, 148A, D-F). Sternum with setae-bearing depressions (Figs. 148C, G). Cephalothorax 0.82 high. Abdomen 1.57 high. Sternum 0.52 long, 0.58 wide. Chelicerae as in figures 143D-F, with small setae-bearing tubercles on the antero-ectal surface (Figs. 143F, 148B). Tracheal system as in figure 147. Internal genitalia with well-developed spermathecae (Fig. 146).

Variation: male total length 1.6-2.2 $(N=4$; average 2.0), cephalothorax length $0.8-1.2$ $(N=4$; average 1.0), femur I length 1.0-1.3 $(N=4$; average 1.2). Female total length 2.5-3.0 ( $N=7$; average 2.7), cephalothorax length 1.1-1.3 ( $N=7$; average 1.2$)$, femur I length 1.0-1.2 $(N=7$; average 1.1). 
Distribution: known from India, Bangladesh, China, Myanmar, Japan, Philippines and Vietnam (Fig. 314).

Additional material examined (N = 18): CHINA: Hong kong: Tai Po Tsai, [22 $22^{\prime} \mathrm{N}$, 114 ${ }^{\circ} 15^{\prime} \mathrm{E}$ ], elev. [7m], 1.iii.1961, E. Shingler leg., 19 (AMNH). JAPAN: Okinawa: $\left[26^{\circ} 9^{\prime} \mathrm{N}, 127^{\circ} 53^{\prime} \mathrm{E}\right]$, elev. [15m], 22.vii.1945, C. T. Parsons \& F. G. Werner leg., $1 \delta^{\wedge}$ (MCZ 125497).

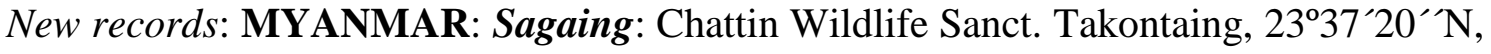
95³1'52”E, elev. [179m], 7-12.x1998, Coddington \& Baptista leg., 6 ㅇ $3 \bigcirc^{\wedge} 6$ immature (USNM). VIETMAN: 2-20.xi1966, $1 \widehat{\jmath}$ (MCZ 84023).

\section{Glenognatha sp. nov. 2}

(Figs. 150-158, 297F, 298B, 299B, 300A, 301A, 302B, 303A, 310)

Type material: male holotype from Hacienda San Pedro, Alto Queremal, Dagua, Valle del Cauca, Colombia, 3²9’5.42”N, 7642`W, elev. 1800m, 21.xi.2009, J. Mendez leg., hand collection, deposited in ICN 5981; paratypes: $12 \AA 9 q$ from the type locality, 21 26.xi.2009, J. Cabra leg., hand collection, deposited in ICN 5982; 1 q from Río Dagua,

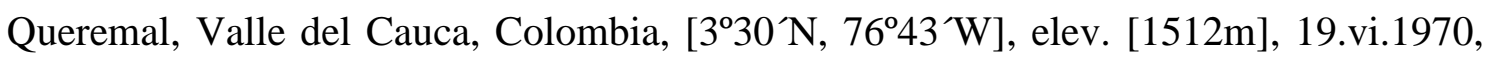
deposited in MZC 92671; $1 \delta^{\Uparrow} 19$ from Vereda Chicoral, La Cumbre, Valle del Cauca, Colombia, 3³4N, 76³5.6W, elev. 1800-2100m, 31.x.2009, J. Mendez leg., deposited in IBSP 163857; $3 \curvearrowright 1 q$ from Bosque Integral Otonga, $4.5 \mathrm{Km}$ from San Francisco de

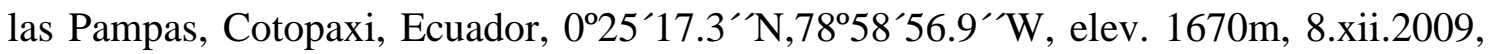
A. J. Santos leg., deposited in UFMG 8975.

Diagnosis: males of G. sp. nov. 2 can be distinguished from all other Glenognatha species by the morphology of the chelicerae, which has the Ret1 adjacent to the fang articulation (Fig. 152B). Females are diagnosed by having few gland ducts concentrated in the spermathecae (Figs. 155D-E).

Description: Male (Holotype): habitus as in figure 150. Carapace yellow-brownish. Sternum dusky brown with darker edges and dusky bands oriented to the median region. Abdomen oval, with irregular pattern of two lateral black bands darker at posterior region. Silver guanine spots scattered in two longitudinal bands dorsally and in lateral surfaces. Venter without guanine spots. Legs brownish tan. Total length 2.39 . 
Cephalothorax 0.97 long, 0.78 wide, 0.67 high. Abdomen 1.40 long, 0.88 wide, 0.92 high. Sternum 0.50 long, 0.52 wide. Eye diameters and interdistances: AME 0.06. ALE 0.05. PME 0.07. PLE 0.04. AME-AME 0.05, AME-ALE 0.1, PME-PME 0.09, PMEPLE 0.09, ALE-PLE 0.01. Clypeus height $2.50 \times$ AME diameter. Chelicerae with the same coloration of the cephalothorax, divergent, with three promarginal and four retromarginal teeth (Figs. 152A-C). Ret 1 adjacent to the fang articulation (Fig. 152B). Leg measurements: I: femur 1.80 , patella 0.35 , tibia 1.92 , metatarsus 1.72 , tarsus 0.90 , total 6.70; II: 1.72, 0.35, 1.77, 1.50, 0.80, 6.15; III: 1.15, 0.25, 0.77, 0.85, 0.47, 3.50; IV: $1.47,0.25,1.17,1.07,0.50,4.47$. DTS 0.18. DTE 0.67. Epiandrous fusules as in figure 297F. Spinnerets as in figures 299B, 301B and 303A. Palp as in figures 153, 154 and 157. Embolus tip S-shaped (Figs. 153A, 154D, 158), with projection beyond embolic opening (Fig. 158)

Female (Paratype ICN 5982): coloration and general habitus as in male (Fig. 151). Sternum dusky brown with darker edges. Venter with a lighter patch between the gonopore and the openings of the book lungs. Total length 2.27. Cephalothorax 0.95 long, 0.78 wide, 0.55 high. Abdomen 1.32 long, 1.15 wide, 1.20 high. Sternum 0.50 long, 0.52 wide. Eye diameters and interdistances: AME 0.06. ALE 0.05. PME 0.07. PLE 0.04. AME-AME 0.05, AME-ALE 0.09, PME-PME 0.1, PME-PLE 0.09, ALEPLE 0.01. Clypeus height $2.08 \times$ AME diameter. Chelicerae with the same coloration of the cephalothorax, with three promarginal and four retromarginal teeth (Figs. 152D-F). Leg measurements: I: femur 1.65 , patella 0.35 , tibia 1.77 , metatarsus 1.50 , tarsus 0.80 , total 6.07 ; II: $1.50,0.35,1.77,1.50,0.80,5.92$; III: 1.00, 0.25, 0.77, 0.97, 0.47, 3.47]; IV: $1.45,0.22,1.20,1.02,0.47,4.37$. DTS 0.16. DTE 0.39 . Tracheal system as in figure 156. Spinnerets as in figures $298 \mathrm{~B}, 300 \mathrm{~A}$ and $302 \mathrm{~B}$. Uterus externus sac with a tubiform median projection (Figs. 155A-B). Spermathecae with two gland ducts (Figs. 155D-E).

Variation: male total length 1.8-2.2 $(N=10$; average 2.0$)$, cephalothorax length $0.7-1.0$ ( $N=10$; average 0.9$)$, femur I length 1.3-2.0 $(N=10$; average 1.6). Female total length 1.7-2.4 $(N=10$; average 2.2), cephalothorax length 0.7-1.0 $(N=10$; average 0.9$)$, femur I length 1.2-1.6 $(N=10$; average 1.4). Male specimens from Costa Rica and Nariño state in Colombia have short and less divergent chelicerae. 
Distribution: known from southwestern Colombia, northwestern Ecuador and Costa Rica (Fig. 310).

Additional material examined $(\mathbf{N}=81)$ : COLOMBIA: Nariño: Barbacoas, Altaquer, Reserva Nambi, [1 $\left.{ }^{\circ} 15^{\prime} \mathrm{N}, 7^{\circ} 7^{\prime} \mathrm{W}\right]$, elev. 1450m, 10.iv.2010, E. Flórez y Est. Tax.

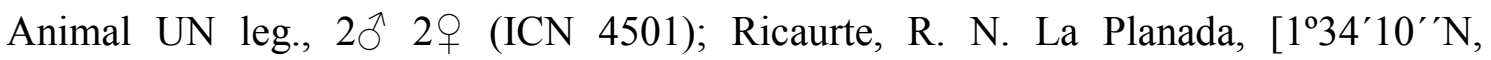

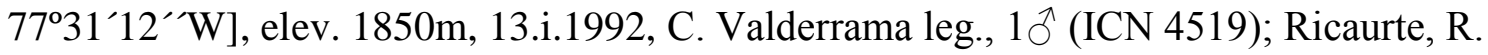

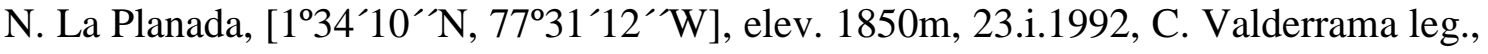
Bosque nublado, tela casi horizontal sobre hojas grandes a $130 \mathrm{~cm}$ del suelo, diámetro tela 7cm, 2 q (ICN 4495); [Ricaurte], La Planada, 7km S. Chocones, [1 $30^{\prime} 7.59^{\prime \prime} \mathrm{N}$,

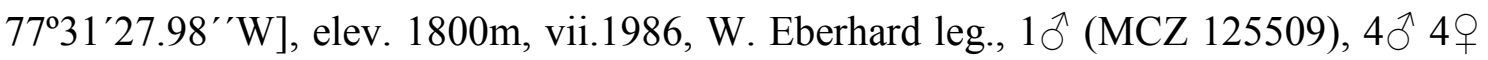
1 immature (MCZ 125508); 19 (MCZ 125507). Valle del Cauca: Cali, Bosque San Antonio, $3^{\circ} 37^{`} \mathrm{~N}, 7^{\circ} 34^{`} \mathrm{~W}$, elev. 1800 - 2100m, ix.2009, J. Cabra leg., $1{ }^{\Uparrow}$ (ICN 5983); La Cumbre, Vereda Chicoral, 33`N, 76º35.6`W, elev. 1800-2100m, ix.2009, J. Mendez leg., 4ત 4ㅇ 3 immature (ICN 5984); 31.x.2009, J. Mendez leg., $13 \hat{\jmath} 21 q 4$

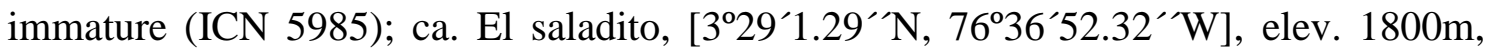
1ð̄ (MCZ 125506). COSTA RICA: Punta Arenas / Alajuelas: Monte Verde

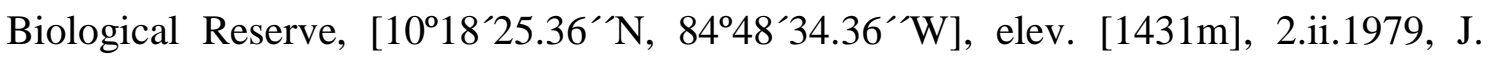

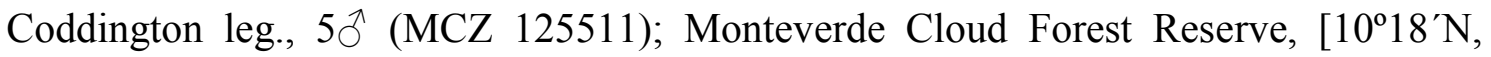

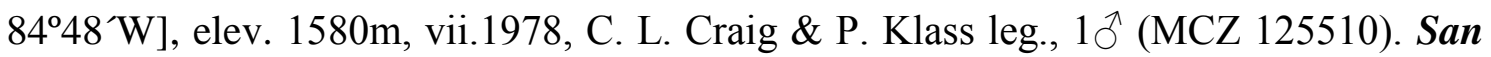
Jose: Rio Hondura, $10^{\circ} \mathrm{N}, 84^{\circ} \mathrm{W}$, elev. 1200m, 30.iii.1991, W. Eberhard leg., $3{ }^{\Uparrow}$ (MCZ 125522); iii.1986, W. Eberhard leg., 1 ㅇ (MCZ 125475).

Glenognatha gaujoni Simon, 1895

(Figs. 159-167, 297D, 298F, 299D, 300D, 301C, 302F, 303C, 309)

Glenognatha gaujoni Simon, 1895: 151 (male holotype from Loja (Gaujon), Ecuador, deposited in MNHN, examined); Platnick, 2013.

Diagnosis: males of G. gaujoni resemble those of G. globosa and G. sp. nov. 8 by the presence of thin pleural bars between coxae II-III (Fig. 159C). It can be distinguished from the two latter species by the presence of scale-shaped projections in the retrolateral surface of the embolus (Fig. 167C). Females are diagnosed by the presence of anterior tooth, ectal apophysis and CFO on the chelicerae (Figs. 161D-F). 
Description: Male (IBSP 26965): habitus as in figure 159. Carapace brown, with three dusky longitudinal bands (two lateral and one medial). Sternum pale brown. Abdomen greyish, dorsally with black patches arranged in two longitudinal bands. Silver guanine spots scattered in the dorsal and lateral surfaces. Venter with black median patch between the epigastric furrow and the petiole. Silver guanine spots scattered between the tracheal spiracle and the epigastric furrow . Tracheal spiracle well-sclerotized. Legs yellow-brownish. Pleural bars between coxae I-II, II-III and III-IV. Total length 3.60. Cephalothorax 1.55 long, 1.17 wide, 0.95 high. Abdomen 2.17 long, 1.37 wide, 1.22 high. Sternum 0.90 long, 0.70 wide. Eye diameters and interdistances: AME 0.14. ALE 0.10. PME 0.15. PLE 0.12. AME-AME 0.11, AME-ALE 0.08, PME-PME 0.13, PMEPLE 0.08, ALE-PLE 0.02. Clypeus height $1.10 \times$ AME diameter. Chelicerae brown. Three promarginal and four retromarginal teeth (Figs. 161A-C). Cheliceral fang outgrowth well-developed (Fig. 161A). Ret1 on the posterior surface (Fig. 161B). Leg measurements: I: femur 3.00, patella 0.65 , tibia 3.05 , metatarsus 2.97 , tarsus 1.47 , total 11.15; II: 2.72, 0.60, 2.57, 2.50, 1.22, 9.62; III: 1.75, 0.45, 1.42, 1.42, 0.55, 5.60; IV: $2.35,0.42,1.92,1.90,0.85,7.45$. DTS 0.52. DTE 0.62. Epiandrous fusules as in figure 297D. Spinnerets as in 299D, 301C and 303C. Palp as in figures 162, 163 and 166. Palp tibia longer than wide (Fig. 162, 163). Conductor lamina in dorsal view with a conspicuous excavation (Fig. 163C). Embolus widened subapically, with projection beyond embolic opening (Fig. 167).

Female (MUSM 0504906): coloration and general habitus as in male (Fig. 160). Abdomen greyish, dorsally with three longitudinal bands of black patches that forms thin transversal bands in the posterior region. Venter with a black median patch between the gonopore and the petiole. Silver guanine spots arranged in two longitudinal bands between the tracheal spiracle and the epigastric furrow. Tracheal spiracle wellsclerotized. Legs yellow-brownish. Pleural bars between coxae I-II, II-III and III-IV. Total length 4.35. Cephalothorax 1.85 long, 1.30 wide, 1.12 high. Abdomen 2.67 long, 1.70 wide, 1.82 high. Sternum 0.80 long, 0.80 wide. Eye diameters and interdistances AME 0.60. ALE 0.45. PME 0.70. PLE 0.55. AME-AME 0.50, AME-ALE 0.80, PMEPME 0.10, PME-PLE 0.77, ALE-PLE 0.02. Clypeus height $1.10 \times$ AME diameter. Chelicerae brown. Three promarginal and four retromarginal teeth (Figs. 161D-F). Anterior tooth present (Figs. 161D). Ectal apophysis well-developed (Figs. 161D-F). Cheliceral fang outgrowth well-developed (Fig. 161F). Ret1 on the posterior surface 
(Fig. 161E). Leg measurements: I: femur 3.25, patella 0.55, tibia 2.75, metatarsus 3.25, tarsus 1.37, total; II: 3.00, 0.65, 2.90, 2.85, 1.25, 10.65; III: 1.75, 0.37, 1.30, 1.55, 0.67, 5.65; IV: $2.60,0.50,2.10,2.10,0.85,8.15$. DTS 0.60. DTE 1.20. Tracheal system as in figure 165. Spinnerets as in figures $298 \mathrm{~F}, 300 \mathrm{D}$ and $302 \mathrm{~F}$. Internal genitalia without spermathecae (Fig. 164).

Variation: male total length 3.6-4.9 $(N=10$; average 4.2), cephalothorax length 1.5-2.0 ( $N=10$; average 1.7), femur I length 2.9-3.7 $(N=10$; average 3.1). Female total length 3.7-4.5 ( $N=10$; average 4.0), cephalothorax length 1.6-1.9 $(N=10$; average 1.7), femur I length 2.5-3.3 $(N=10$; average 3.0).

Distribution: known from Amazonian regions of Colombia, Ecuador, Peru and Brazil (Fig. 309).

Additional material examined $(\mathbf{N}=123)$ : New records: BRAZIL: Acre: Marechal Thaumaturgo, Parque Nacional da Serra do Divisor, [8 $17^{\prime}$ S, $73^{\circ} 19^{\prime} \mathrm{W}$ ], elev. [262m],

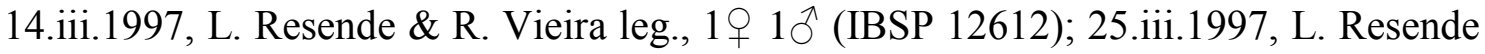
\& S. Vieira leg., $1 \uparrow 1 \hat{\jmath}$ (IBSP 12182). Amazonas: Manaus, Reserva do PDBFF,

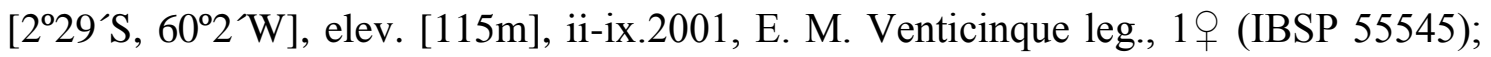
Manaus, Reserva Florestal Adolpho Ducke, [259`S, 5956`W], elev. [90m], vii.1999, A. J. Santos leg., $1 q 2 \hat{\jmath}$ (IBSP 26965); 10.iv.1992, S. Darwichleg, $1 q 1{ }^{\Uparrow}$ (MCTP 2715). Mato Grosso: Vale São Domingos/Pontes e Lacerda, Usina hidrelétrica de

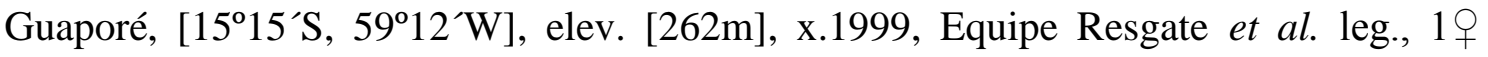
(IBSP 41537). Rondônia: Porto Velho, Mutum, [8³3’S, 6342 W], elev. [83m], 7.vi.2011, M. C. Silveira leg., $2{ }^{\AA}$ (MZSP 41003). Roraima: Pacaraima, [4²5’S, 61ㅇ' W], elev. [480m], 25.vi - 5.vii.1988, J. A. Rafael leg., $1 \delta^{\circ}$ (MCN 19742). São

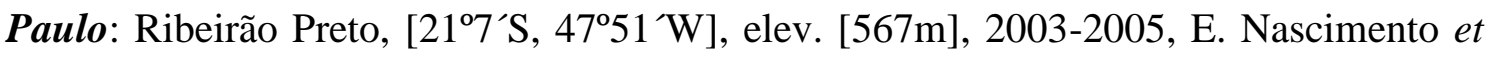
al. leg., $1 \delta^{\Uparrow}$ (IBSP 58162). COLOMBIA: Meta: Villavicencio, Bosque Bavaria, [4 $4^{\circ} \mathrm{N}$, 73³8`W], elev. [467m], 14.v.2005, Sistemática Animal leg., 1 ㅇ (ICN 4487).

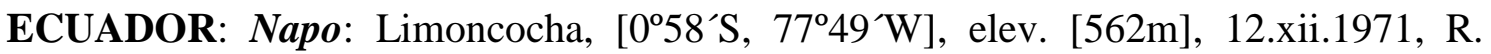
Buskirk leg., 39 (MCZ 125477). PERU: Cuzco: Convención, Cerro Dorado,

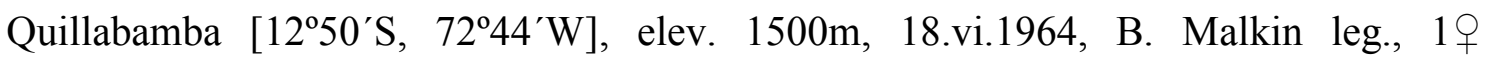

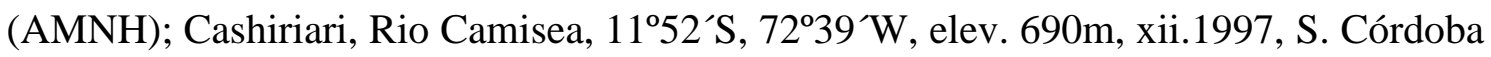

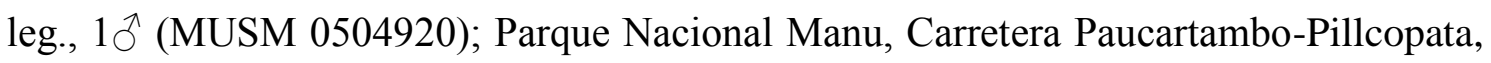


quebrada quita calzón, [132' S, $\left.71^{\circ} 31^{\prime} \mathrm{W}\right]$, elev. 1180m, ix.1991, B. Leon leg., $1 \delta^{\Uparrow}$ (MUSM 0504919). Huánuco: Sta. Teresa. Huallaga, [940`S, 75³0`W], elev. 600m, viii.1954, F. Woytkowski leg., 19 (CAS 9039649). Loreto: Jenaro Herrera, Qebrada el

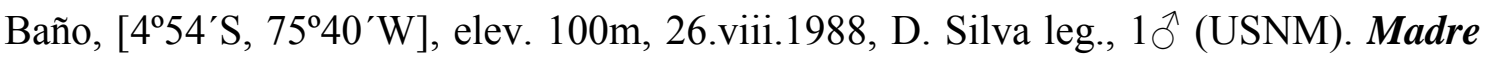
de Dios: 15 km E Puerto Maldonado, 12³3’S, 69³ W, elev. 200m, 17.vii.1989, D. Silva leg., $9 q 3 \jmath^{\Uparrow} 2$ immature (MUSM 0504926); Boca del rio los Amigos, $12^{\circ} 34^{\prime} \mathrm{S}$, 706’W, elev. 270m, 6.xii.2005, J. A. Ochoa et al. leg., 2 q (MUSM 0501093); Zona

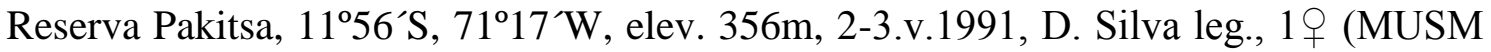
0504912); 9.v.1991, D. Silva leg., 1 (MUSM 0504915); 13.v.1991, D. Silva leg., 1 1ठ (MUSM 0504908); 25.v.1991, D. Silva leg., 3 + (MUSM 0504922); 6.x.1987, J. Coddinton \& D. Silva leg., $697 \widehat{0} 1$ immature (MUSM 0509928); Zona Reserva

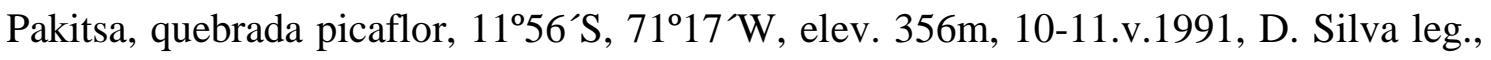
$4 \uparrow 2 \widehat{\jmath}$ (MUSM 0504914); 8.v.1991, D. Silva leg., $1 q 3 \widehat{\jmath}$ (MUSM 0504923); Zona

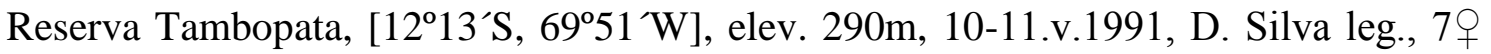
7ð (MUSM 0504927); Zona Reserva Tambopata, [1250'S, 69¹7'W], elev. 290m,

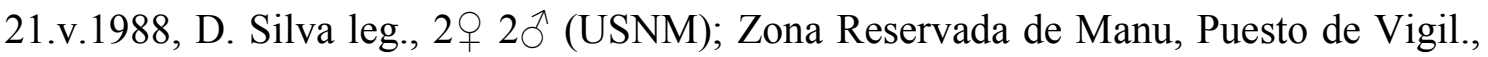

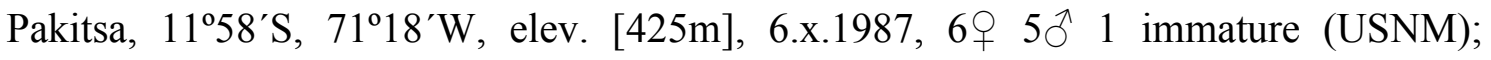
1.x.1987, $191{ }^{\Uparrow}$ (USNM); 28.ix.1987, 2 ㅇ (USNM); 1.x.1987, 3910 (USNM); 5.x.1987, 19 (USNM), all collected by D. Silva \& J. Coddington. Pasco:

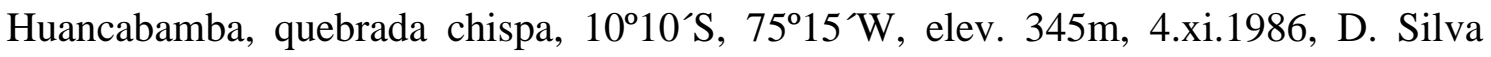

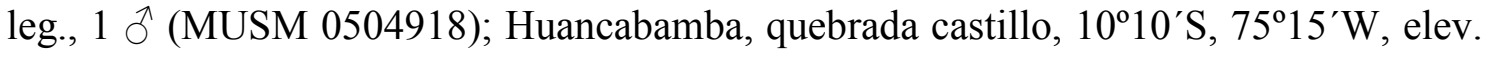
345m, 10.ix.1987, D. Silva leg., 1910 (MUSM 0504906); 2 ㅇ 1ð (USNM); Santa Rosa, río Palcazú, [10²7’S, 75²9’W], elev. 1776m, 24.ix.1991, P. Hocking leg., 1

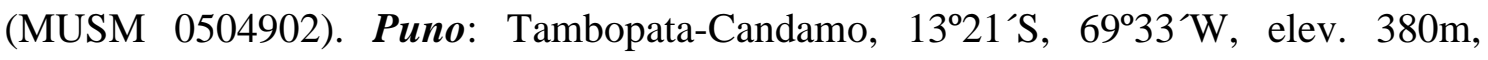
27.viii.1992, I. Bohorquez leg., 2 q 102 immature (MUSM 0504916). VENEZUELA: Amazonas: Cerro de la Neblina, 050`S, 669`W, elev. 140m, 4-12.ii.1984, D. Davis \& T. McCabe leg., 1 (USNM).

\section{Glenognatha sp. nov. 8}

(Figs. 168-175, 310)

Type material: male holotype from Hacienda San Pedro, Alto Queremal, Dagua, Valle del Cauca, Colombia, 32`N, 76² $42^{`}$ W, elev. 1800m, 21.ix.2009, J. Cabra leg., nocturnal hand collection, deposited in ICN 5987; paratypes: same data as the holotype, $4 \lesssim 19 q$ deposited in ICN 5986; $1 \lesssim 1 \uparrow$ deposited in IBSP 163858. 
Diagnosis: males of $G$. sp. nov. 8 resemble those of $G$. globosa by the nearly $180^{\circ}$ curved embolus tip (Figs. 171B, 172D, 175A). It can be distinguished from the latter species by the thin distal projection of the conductor lamina (Figs. 172B-C, 175). Females can be easily distinguished from all other Glenognatha species (except $G$. gaujoni) by the presence of cheliceral ectal apophysis (Figs. 170D-F); distinguished from G. gaujoni by the absence of anterior tooth (Fig. 170D).

Description: Male (Holotype): habitus as in figure 168. Carapace pale brown, darker along furrows. Sternum pale brown, darker on sides. Abdomen oval, dorsally with irregular pattern of two longitudinal dusky bands on sides. Silver guanine spots scattered in two median longitudinal bands and on lateral surfaces. Venter with a pale black median band and two lateral lighter bands with scattered silver guanine spots. Legs yellow-brownish. Tracheal spiracle well-sclerotized. Pleural bars between coxae III, II-III and III-IV. Total length 3.6. Cephalothorax 1.75 long, 1.30 wide, 0.90 high. Abdomen 2.15 long, 1.55 wide, 1.45 high. Sternum 0.90 long, 0.90 wide. Eye diameters and interdistances: AME 0.12. ALE 0.10. PME 0.15. PLE 0.10. AME-AME 0.10, AME-ALE 0.15, PME-PME 0.21, PME-PLE 0.12, ALE-PLE 0.01. Clypeus height 1.2 $\times$ AME diameter. Chelicerae slightly lighter than the cephalothorax and anteriorly projected (Fig. 168B). Six promarginal and eight retromarginal teeth. Ret1 on the posterior surface (Fig. 170B). Leg measurements: femur 3.20, patella 0.75, tibia 3.25, metatarsus 3.35, tarsus 1.65, total 12.20; II: 3.00, 0.65, 3.00, 2.90, 1.40, 10.95; III: 2.00, 0.40, 1.30, 1.40, 0.55, 5.65; IV: 2.55, 0.50, 2.05, 2.10, 0.87, 8.07. DTS 0.50. DTE 0.65. Palp as in figures 171, 172 and 175. Palp tibia longer than wide (Fig. 171). Conductor lamina with a thin distal end (Fig. 172B-C). Embolus tip nearly $180^{\circ}$ curved (Figs. 171B, 172D, 175A).

Female (Paratype ICN 5986): coloration and general habitus as in male (Fig. 169). Pleural bars between coxae I-II, II-III and III-IV. Total length 4.8. Cephalothorax 2.05 long, 1.50 wide, 1.05 high. Abdomen 3.25 long, 2.55 wide, 2.45 high. Sternum 0.95 long, 0.90 wide. Eye diameters and interdistances: AME 0.10. ALE 0.10. PME 0.17. PLE 0.12. AME-AME 0.10, AME-ALE 0.12, PME-PME, 0.17 PME-PLE 0.15, ALEPLE 0.02. Clypeus height $1.30 \times$ AME diameter. Chelicerae slightly lighter than the cephalothorax. Five promarginal and eight retromarginal teeth. Ectal apophysis well developed (Figs. 170D-F). Leg measurements: I: femur 3.40, patella 0.70, tibia 3.45, 
metatarsus 3.35 , tarsus 1.70 , total 12.60; II: 3.25, 0.70, 3.05, 3.00, 1.40, 11.40; III: 1.85, 0.50, 1.55, 1.35, 0.55, 5.80; IV: 2.50, 0.50, 2.00, 2.10, 0.90, 8.00. DTS 0.70. DTE 0.55. Tracheal system as in figure 174. Internal genitalia without spermathecae. Uterus externus sac with a tubiform projection (Fig. 173).

Variation: male total length 3.9-4.3 ( $N=10$; average 4.1), cephalothorax length 1.5-2.0 ( $N=10$; average 1.8), femur I length 3.2-3.7 $(N=10$; average 3.4). Female total length 3.5-5.0 $(N=10$; average 4.4), cephalothorax length 1.5-2.2 $(N=10$; average 1.8$)$, femur I length 3.0-4.0 $(N=10$; average 3.4).

Distribution: known from Valle del Cauca, Risaralda and Nariño states in Colombia (Fig. 310).

Material examined ( $\mathbf{N}=85$ ): COLOMBIA: Quindio: Filandia, [ $4^{\circ} 40^{\prime} \mathrm{N}, 75^{\circ} 40^{\prime} \mathrm{W}$ ], elev. [1758m], 4.viii.2012, N. Hazzi leg., 6 ㅇ $1 ð 4$ immature (ICN 5998); 2 ㅇ $2 \widehat{\jmath}$ (IBSP 163859). Nariño: Barbacoas, Altaquer, Reserva Río Nambi, [1¹8N, $\left.78^{\circ} 5^{`} \mathrm{~W}\right]$, elev. 1440m, 12.iv.2010, E. Flórez y Est. Tax. Animal UN leg., 2 ㅇ $1 \delta^{\Uparrow} 1$ immature (ICN 4505); 10.x.2009, E. Flórez y Est. Tax. Animal UN leg., 192 immature (ICN 4408);

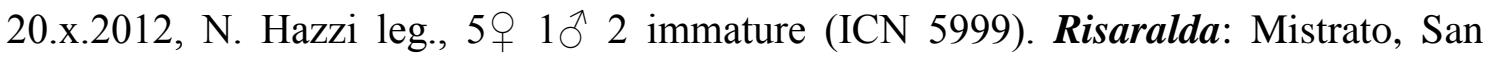

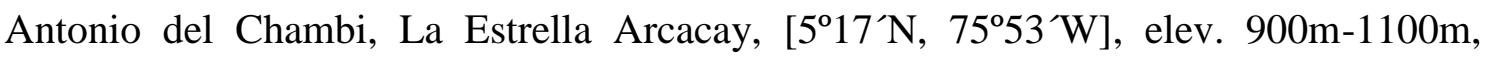
31.iii-1.iv.1992?, Estud. Biol. UNAL leg., 19 (ICN 1078b). Valle del Cauca: Cali, El

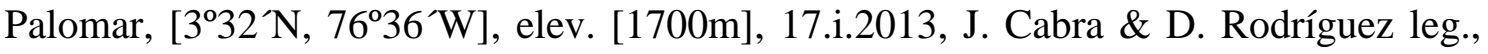

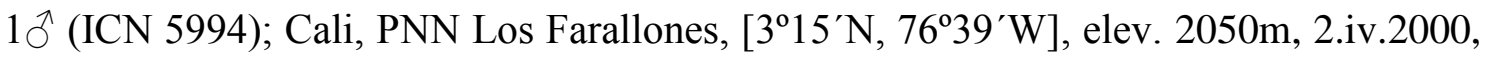

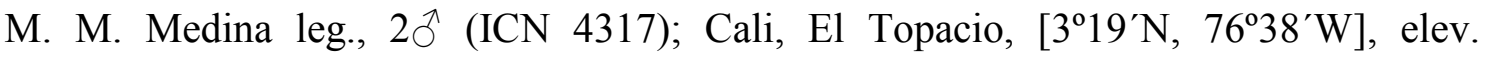
[1624m], 6.i.2011, N. Hazzi leg., $193 \overbrace{}^{\Uparrow}$ (ICN 5996); Dagua, same data as holotype, 37 immature (ICN 5988); La Cumbre, Vereda Chicoral, 3³4N, 76º35.6`W, elev. 1800m2100m, ix.2009, J. Mendez leg., 1 ( (ICN 5989); Pance, ca. Topacio, [3²0`10.73“N, 76 36'5.04'W], elev. [1430m], viii.2011, N. Hazzi leg., 1 ( $3^{\circ} 19^{\prime} 41^{\prime \prime} \mathrm{N}, 76^{\circ} 38^{\prime} 12^{\prime \prime} \mathrm{W}$, elev. 1624m, 2.i.2012, N. Hazzi leg., 2 ㅇ $3{ }^{\jmath} 4$ immatures (ICN 5997). 
Glenognatha globosa (Petrunkevitch, 1925)

(Figs. 176-183, 297E, 298G, 299E, 300E, 301D, 302G, 303D, 310)

Cyrtognatha globosa Petrunkevitch, 1925a: 123, fig. 42 (female holotype from San

Lorenzo, Panama, 4.xii.1924, A. \& W. Petrunkevitch leg., deposited in YPM, examined through photographs of the habitus and female genitalia).

Glenognatha globosa: Dimitrov \& Hormiga, 2009: 102, figs. 66A-F; Platnick, 2013.

Diagnosis: males of G. globosa resemble those of $G$. sp. nov. 8 by the nearly $180^{\circ}$ curved embolus tip (Figs. 179C, 183B). It can be distinguished from the latter species by the absence of a long apical projection in the conductor lamina (Figs. 180B-C). Females can be easily distinguished from all other Glenognatha species (except $G$. gaujoni) by the presence of anterior tooth (Fig. 178D); distinguished from G. gaujoni by the absence of CFO (Fig. 178F) and by having at least six retromarginal teeth (Fig. $178 \mathrm{~F})$.

Description: Male (ICN 5991): habitus as in figure 176. Carapace brown, darker along furrows and with a well-marked fovea. Sternum brown, darker on sides. Abdomen oval, dorsally with irregular pattern of two longitudinal dusky bands on sides and thin transverse bands on the posterior region. Silver guanine spots scattered in dorsal and lateral surfaces. Venter with a pale black median band that ramifies towards lateral surface from the tracheal spiracle and the spinnerets. Silver guanine spots scattered in pale bands between the tracheal spiracle and the epigastric furrow. Legs yellowbrownish. Tracheal spiracle well-sclerotized. Pleural bars between coxae I-II, II-III and III-IV. Total length 3.70. Cephalothorax 1.70 long, 1.40 wide, 1.02 high. Abdomen 2.12 long, 1.45 wide, 1.35 high. Sternum 0.90 long, 0.85 wide. Eye diameters and interdistances: AME 0.10. ALE 0.10. PME 0.15. PLE 0.10. AME-AME 0.06, AMEALE 0.1, PME-PME 0.15, PME-PLE 0.13, ALE-PLE 0.01. Clypeus height $1.60 \times$ AME diameter. Chelicerae slightly lighter than the cephalothorax. Six promarginal and ten retromarginal teeth. Ret1 on the posterior surface (Fig. 178B). Leg measurements: I: femur 2.90, patella 0.60 , tibia 2.80 , metatarsus 2.80 , tarsus 1.40 , total 10.50 ; II: 2.30 , 0.50, 2.85, 2.45, 1.25, 9.35; III: 1.70, 0.40, 2.05, 2.00, 0.80, 6.95; IV: 2.50, 0.50, 2.00, 2.05, 0.85, 7.90. DTS 0.40. DTE 0.50. Epiandrous fusules as in figure 297E. Spinnerets as in figures 299E, 301D and 303D. Palp as in figures 179, 180 and 183. Palp tibia longer than wide (Fig. 179). Embolus tip nearly $180^{\circ}$ curved (Figs. Figs. 179C, 183B). 
Female: described by Dimitrov \& Hormiga (2009). Additional data are presented here: (ICN 5991): habitus as in figure 177. Carapace with three dusky bands (one median and two lateral). Sternum dusky yellow-brownish. Abdomen greyish, dorsally with irregular pattern of four longitudinal dusky bands (two median and two on sides) and thin transverse bands on the posterior region. Silver guanine spots scattered in dorsal and lateral surfaces. Venter with a pale black median band that ramifies towards lateral surface from the tracheal spiracle and the spinnerets. Silver guanine spots scattered in pale bands around the black median band. Legs yellow-brownish. Tracheal spiracle well-sclerotized. Pleural bars between coxae I-II, II-III and III-IV. Total length 4.07. Cephalothorax 1.90 long, 1.40 wide, 1.10 high. Abdomen 2.45 long, 1.95 wide, 1.95 high. Sternum 1.05 long, 0.85 wide. Eye diameters and interdistances: AME 0.12. ALE 0.10. PME 0.15. PLE 0.10. AME-AME 0.08, AME-ALE 0.15, PME-PME 0.15, PMEPLE 0.15, ALE-PLE 0.01. Clypeus height $1.4 \times$ AME diameter. Five promarginal and eight retromarginal teeth. Anterior tooth present (Figs. 178D, F). Leg measurements: I: femur 3.15, patella 0.70 , tibia 3.20, metatarsus 3.10, tarsus 1.45, total 11.60; II: 2.80, 0.70, 2.85, 2.75, 1.25, 10.35; III: 1.90, 0.45, 1.40, 1.55, 0.65, 5.95; IV: 2.50, 0.45, 1.95, $2.00,0.85,7.75$. DTS 0.47. DTE 0.30. Tracheal system as in figure 182. Spinnerets as in figures 298G, 300E and 302G. Internal genitalia without spermathecae (Fig. 181). Uterus externus sac with a tubiform projection (Fig. 181).

Variation: male total length 3.0-4.6 $(N=10$; average 3.7), cephalothorax length 1.5-2.1 $(N=10$; average 1.7), femur I length 2.3-3.8 $(N=10$; average 3.2). Female total length 3.2-4.2 $(N=10$; average 3.9), cephalothorax length 1.6-2 $(N=10$; average 1.8), femur I length 2.7-3.2 ( $N=10$; average 3.0).

Distribution: known from Panama and Colombia (Fig. 310).

Additional material examined $(\mathbf{N}=\mathbf{5 8})$ : New records: COLOMBIA: Boyacá: Corinto,

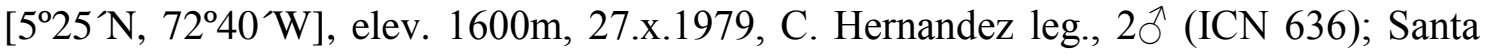
María, Vda. La Carbonera, cascada la Colorata, [7¹7N, $73^{\circ} 10^{\prime} \mathrm{W}$ ], elev. 600m, 19.v.2009, E. Flórez y Est. leg., 2 (ICN 4188). Cauca: Caloto, Vereda Morales,

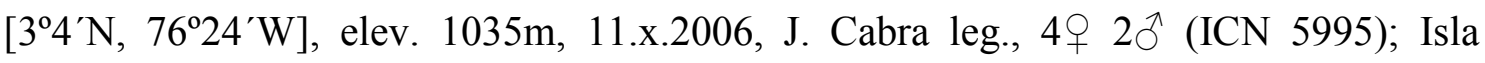

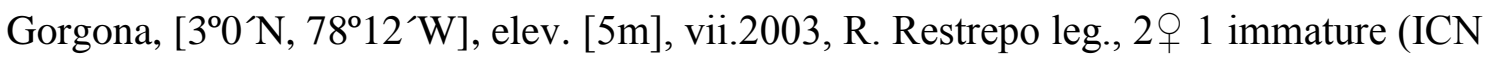
4270). Cundinarmaca: ca. La Vega. Río San Miguel, [50`N, 74²0`W], elev. 1480m, 
27.iv.1992, 1ठ (ICN 1080); Cachipay, [442’N, 74²9’W], elev. [1019m], 27.ii.2011, J. Cabra leg., 6 ㅇ $8 \AA 5$ immature (ICN 5991). Magdalena: SNSM Finza La Victoria, $11^{\circ} 7^{\prime} 45^{\prime \prime} \mathrm{N}, 74^{\circ} 5^{\prime} 42^{\prime \prime} \mathrm{W}$, elev. [900m], 19.vii.2006, L. Ferreira leg., 1ð (ICN 4123).

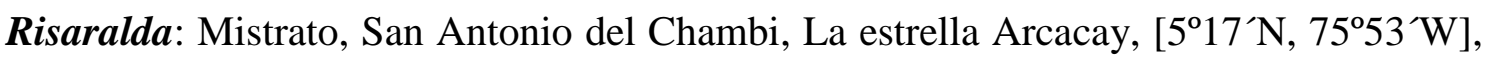
elev. 900m - 1100m, 31.iii - 1.iv.1992, Estud. Biol. UNAL leg., $3 q 1$ immature (ICN 1078a). Santander: Carmen de Chucurí, Vda. La Bodega, PNN Los Yariguies, [6 $6^{\circ} 41^{`} \mathrm{~N}$, 73³0`W], elev. [585m], 14-20.iii.2011, Est. Taxonomia animal leg., 1 ㅇ (ICN 6129).

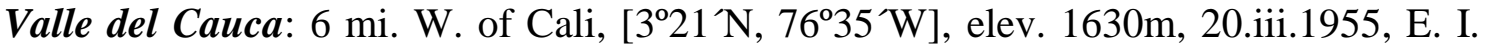
Schingler \& E. S. Ross leg., $1+1{ }^{\lambda}$ (CAS 9039648); Cali, El Palomar, [3³2’N, 76³6’W], elev. [1700m], 17.i.2013, J. Cabra \& D. Rodríguez leg., 1 ㅇ $1 \overbrace{}^{\Uparrow}$ (ICN 5992);

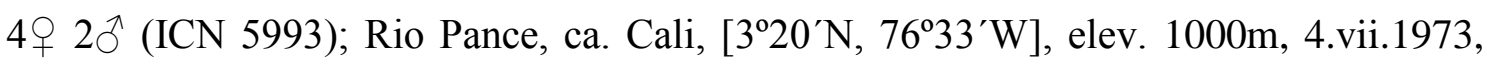
$1 q$ (MCZ 67453); vii.1973, $1 q 1$ immature (MCZ 67454). PANAMA: Chiriquí:

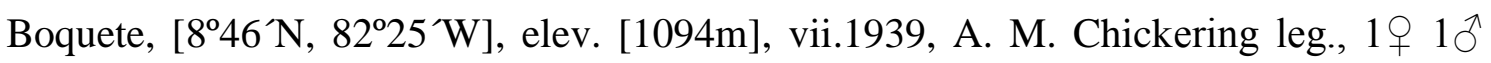
(MCZ 67470); Panamá: Barro Colorado. C. Zone, [99`N, 7950`W], elev. [150m], 10.ii.1936, W. J. Gertsch leg., 2 immature (AMNH); Canal Zone, Forest reserve, [99`N, 7950`W], elev. [150m], 29.i.1958, A. M. Chickering leg., 1 immature (MCZ 125490). VENEZUELA: Miranda: Santa Cruzita, Guatopo National Park., [105 N, 66 $25^{`} \mathrm{~W}$ ], elev. 450m, 13.ii.1984, J. Coddington leg., $1 \sigma^{\Uparrow}$ (USNM).

\section{Glenognatha sp. nov. 6}

(Figs. 184-191, 309)

Type material: male holotype from Estación Biológica Caparu, Lago Taraira. Taraira, Vaupés, Colombia, [1`S, 69²9`W], elev. [128m], 11.iv.2004, J. Pinzón leg., deposited in ICN 5979; paratypes: $2 \hat{\bigcirc} 19$ from the type locality, same data as the holotype, deposited in ICN 5980; $1 \widehat{O} 1$ from Igarapé forest of upper Rio Yaciba, [Amazonas], Venezuela, [1 ${ }^{\circ} 14^{`} \mathrm{~N}, 6^{\circ} 44^{`} \mathrm{~W}$ ], elev. [109m], 7.xii.1953, W. M. Beebe leg., deposited in AMNH; 1 đ from Estaçao Científica Ferreira Penna, Melgaço, Pará, Brazil, 143`21.6”S, 51²5’51.2’ W, elev. [40m], 1993-2009?, A. B. Bonaldo leg., deposited in MPEG 19083; $1 \hat{\jmath}$ from 15 km E Puerto Maldonado, Madre de Dios, Peru, 12³3`S, 69³ W, elev. 200m, 10.vii.1989, D. Silva leg., deposited in MUSM 0504904.

Diagnosis: males of G. sp. nov. 6 can be distinguished from all other Glenognatha species by the presence of small sharpened projections on the conductor (Figs. 191AC). Females resemble those of G. sp. nov. 2 and $G$. lacteovittata in having a 
conspicuously enlarged Ret1 (Fig. 186E). They are distinguished from the latter by the presence of disc-shaped portions in the uterus externus sac (Fig. 189C).

Description: Male (Holotype): habitus as in figure 184. Carapace yellow-brownish, darker along furrows. Sternum dark brown, with dusky bands oriented towards the median region. Abdomen spherical, dorsally with irregular pattern of two longitudinal black bands on sides and some dark patches on the postero-median region. Silver guanine spots scattered in the entire dorsal surface and the median lateral surfaces. Venter with a diffused dusky median band, darker around epigastric furrow and two lighter lateral bands with some silver guanine spots. Legs light yellow-brownish with distal parts of segments darker. Tracheal spiracle well-sclerotized. Total length 2.62. Cephalothorax 1.17 long, 0.85 wide, 0.62 high. Abdomen 1.60 long, 1.15 wide, 1.15 high. Sternum 0.52 long, 0.53 wide. Eye diameters and interdistances: AME 0.09. ALE 0.08. PME 0.08. PLE 0.07. AME-AME 0.08, AME-ALE 0.12, PME-PME 0.09, PMEPLE 0.11, ALE-PLE 0.01. Clypeus height $1.66 \times$ AME diameter. Chelicerae with the same coloration of the cephalothorax. Three promarginal and four retromarginal teeth (Figs. 186A-C) Anterior tooth present (Figs. 186A). Cheliceral fang outgrowth welldeveloped (Fig. 186B). Leg measurements: I: femur 1.45, patella 0.37, tibia 1.55, metatarsus 1.25 , tarsus 0.65 , total 5.27; II: 1.25 , 0.35, 1.27, 1.20, 0.55, 4.62; III: 0.92, 0.25, 0.63, 0.70, 0.45, 2.96; IV: 1.27, 0.27, 1.02, 1.00, 0.47, 4.05. DTS 0.17. DTE 0.52. Palp as in figures 187-188. Conductor with rugose texture (Figs. 191A-C). Embolus straight with $180^{\circ}$ curved tip (Fig. 191A-B).

Female (Paratype AMNH): habitus as in figure 185. Carapace brown, darker along furrows, with a median longitudinal dusky band. Sternum dark brown, darker on the edges. Abdomen spherical, strongly depigmentated, dorsally with dusky patches. Silver guanine spots absent. Venter with a diffused dusky median band, and two lighter bands on sides. Gonopore region and book lung covers lighter. Legs light yellow-brownish with distal parts of segments darker. Tracheal spiracle well-sclerotized. Total length 3.1. Cephalothorax 1.45 long, 0.97 wide, 0.70 high. Abdomen 1.67 long, 1.55 wide, 1.60 high. Sternum 0.65 long, 0.62 wide. Eye diameters and interdistances: AME 0.09. ALE 0.08. PME 0.08. PLE 0.06. AME-AME 0.08, AME-ALE 0.13, PME-PME 0.10, PMEPLE 0.12, ALE-PLE 0.01. Clypeus height $2.22 \times$ AME diameter. Chelicerae slightly darker than the cephalothorax. Three promarginal and four retromarginal teeth (Figs. 
186D-F). Ret1 enlarged (Fig. 186E). Leg measurements: I: femur 1.90, patella 0.40, tibia 1.90, metatarsus 1.72 , tarsus 0.82 , total 6.75 ; II: $1.93,0.42,1.65,1.25,0.75,6.01$; III: $1.17,0.42,0.77,0.90,0.47,3.75$; IV: 1.57, 0.32, 1.35, 1.15, 0.52, 4.92. DTS 0.30. DTE 0.50. Tracheal system as in figure 190. Uterus externus sac divided in three portions (Fig. 189).

Variation: male total length 2.0-2.7 $(N=10$; average 2.4), cephalothorax length 1.0-1.2 ( $N=10$; average 1.1), femur I length 1.3-1.6 $(N=10$; average 1.5). Female total length 2.3-3.1 ( $N=5$; average 2.6), cephalothorax length 1.0-1.4 ( $N=5$; average 1.2$)$, femur I length 1.2-1.9 $(N=5$; average 1.6).

Distribution: known from Amazonian regions of Colombia, Bolivia, Peru, Brazil, Venezuela and Suriname (Fig. 309).

Additional material examined ( $\mathbf{N}=15)$ : BOLIVIA: Beni: Rio Benicito, Chacobo Indian Village, $12^{\circ} 30^{\prime} \mathrm{S}, 6^{\circ} \mathrm{W}$ ], elev. [156m], 10.31.vii.1960, B. Malkin leg., 1 q (AMNH). BRAZIL: Rondônia: Guajará-Mirim, Parque Estadual Guajará, [1048 $\left.41.98^{\prime \prime} \mathrm{S}, 66^{\circ} 18^{\prime} 41.36^{\prime} \mathrm{W}\right]$, elev. [132m], 18.i - 13.iv.1998, Lawne J. Vitte \& T. C. Ávila-Pires leg., $1 \sigma^{\Uparrow} 19$ (MCTP 11373). PERU: Loreto: P.N. Pacaya-Samiria

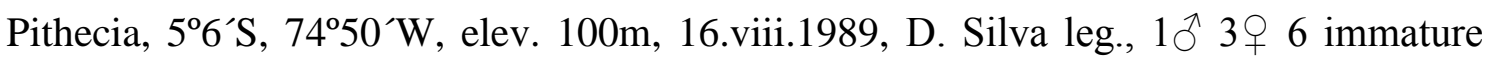
(MUSM 0504921). Madre de Dios: Zona Reservada de Manu, Puesto de Vigilancia., Pakitsa, 11 ${ }^{\circ} 58^{\prime} \mathrm{S}, 71^{\circ} 18^{\prime} \mathrm{W}$, elev. [425m], 6.x.1987, D. Silva \& J. Coddington leg., 1 q (USNM). SURINAME: Para: Carolina Creek, 10km from Zanderij, [5 $27^{\circ} \mathrm{N}$, $55^{\circ} 12^{\prime} \mathrm{W}$ ], elev. [27 m], B. Malkin leg., $1 \overbrace{}^{\wedge}(\mathrm{AMNH})$.

\section{Glenognatha sp. nov. 3}

(Figs. 192-199, 297G, 312)

Type material: male holotype from Potreiro Velho, São Francisco de Paula, Rio Grande do Sul, Brazil, [2928’S, 509`W], elev. [772m], 25.iii.1997, J. Ketterl leg., deposited in MCTP 17537, paratypes: $1 \delta^{\Uparrow} 1$, $, 29^{\circ} 28^{\prime} 26.619^{\prime \prime} \mathrm{S}, 50^{\circ} 9^{\prime} 22.4451^{\prime \prime} \mathrm{W}, 7 . \mathrm{iv} .2001$, deposited in $\mathrm{MCN}$; 2ᄋ, 29 28'26.619' $\mathrm{S}, 50^{\circ} 9^{\prime} 22.4451^{\prime \prime} \mathrm{W}, 14.1 \mathrm{v} .2002$, deposited in

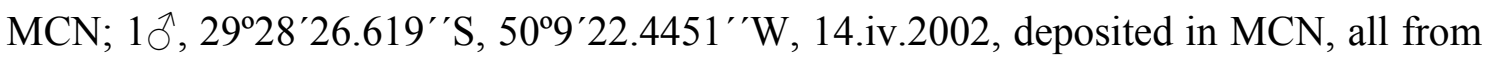
the type locality, R. Ott leg.; $1 \hat{\jmath}$ paratype (IBSP 57090) and $1 q$ paratype (IBSP 57080) 
from Parque Nacional da Serra da Bocaina, São José do Barreiro, São Paulo, Brazil,

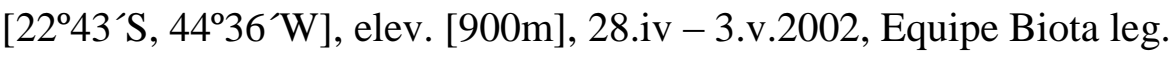

Diagnosis: males of G. sp. nov. 3 can be distinguished from all other Glenognatha species by the presence of a distinct rounded projection on the paracymbium (Fig. 195D). Females can be easily differentiated from all other Glenognatha (except $G$. gloriae) by having three promarginal and three retromarginal teeth (Figs. 194D-F); distinguished from G. gloriae by the slightly projected clypeus in lateral view (Fig. 193B).

Description: Male (Holotype): habitus as in figure 192. Carapace yellow-brownish, darker along furrows. Sternum brown with darker edges. Abdomen oval, strongly depigmentated, with irregular pattern of two diffused dusky bands that join medially at posterior region. Silver guanine spots scattered in two longitudinal bands dorsally and in lateral surfaces. Ventrally without silver spots. Legs light yellow-brownish. Total length 1.85. Cephalothorax 0.87 long, 0.80 wide, 0.57 high. Abdomen 0.97 long, 0.82 wide, 0.80 high. Sternum 0.50 long, 0.55 wide. Eye diameters and interdistances: AME 0.08. ALE 0.07. PME 0.13. PLE 0.08. AME-AME 0.08, AME-ALE 0.07, PME-PME 0.13, PME-PLE 0.07, ALE-PLE 0.01. Clypeus height $1.80 \times$ AME diameter. Chelicerae darker than cephalothorax, with a well-developed anterior tooth (Fig. 194A, C). Three promarginal and three retromarginal teeth. (Fig. 194A-C). Prt1 enlarged (Fig. 194A). CFO well-developed (Fig. 194C). Leg measurements: I: femur 1.30, patella 0.32, tibia 1.30, metatarsus 1.02 , tarsus 0.65 , total 4.60 : II: $1.15,0.30,1.15,0.87,0.55$, 4.02; III: 0.75, 0.27, 0.55, 0.57, 0.37, 2.52; IV: 1.05, 0.22, 0.90, 0.75, 0.45, 3.37. DTS 0.07. DTE 0.48. Epiandrous fusules as in figure 297G. Palp as in figures 195, 196 and 199. Paracymbium with distinct rounded projection (Fig. 195D). Median region of the embolus with helical configuration (Figs. 199B).

Female (Paratype MCN): coloration and general habitus as in male (Fig. 193). Venter with a lighter area between the gonopore and the openings of the book lungs. Total length 2.10. Cephalothorax 0.97 long, 0.85 wide, 0.65 high. Abdomen 1.35 long, 1.12 wide, 1.12 high. Sternum 0.50 long, 0.52 wide. Eye diameters and interdistances: AME 0.09. ALE 0.08. PME 0.13. PLE 0.07. AME-AME 0.08, AME-ALE 0.08, PME-PME 0.14, PME-PLE 0.09, ALE-PLE 0.01. Clypeus height $1.80 \times$ AME diameter. Chelicerae 
darker than cephalothorax, with three promarginal and three retromarginal teeth (Figs. 194D-F). Leg measurements: I: femur 1.27, patella 0.32, tibia 1.35, metatarsus 1.05, tarsus 0.67 , total 4.67; II: $1.25,0.32,1.22,0.80,0.62,4.22$; III: 0.82, 0.20, 0.52, 0.58 , $0.37,2.51$; IV: $1.07,0.25,0.80,0.75,0.43,3.31$. DTS 0.07. DTE 0.52. Tracheal system as in figure 198. Uterus externus sac with a disc-shaped basal projection (Fig. 197).

Variation: male total length 1.7-2.0 $(N=10$; average 1.8$)$, cephalothorax length $0.8-1.0$ ( $N=10$; average 0.9), femur I length 1.3-1.4 $(N=10$; average 1.3). Female total length 1.7-2.3 ( $N=10$; average 2.0), cephalothorax length 0.7-1.1 $(N=10$; average 0.9$)$, femur I length $0.8-1.3(N=10$; average 1.1).

Distribution: known from São Paulo, Santa Catarina and Rio Grande do Sul states in Brazil (312).

Additional material examined (N = 49): BRAZIL: Rio Grande do Sul: São Francisco de Paula, Potreiro Velho, [29²8’S, 509`W], elev. [772m], 31.iii.1997, J. Ketterl leg.,

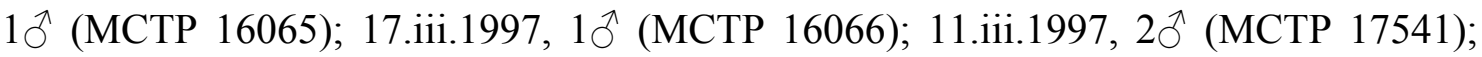
18.iii.1997, 1 (MCTP 17493), all collected by J. Ketterl; 12-15.vi.1997, A. Lise leg., $1 \delta^{\lambda}(\mathrm{MCTP} 13771) ; 29^{\circ} 28^{\prime} 27.121^{\prime \prime} \mathrm{S}, 50^{\circ} 9^{\prime} 25.0714^{\prime \prime} \mathrm{W}, 1 . v .2001,1{ }^{\lambda}$ (MCN); $29^{\circ} 29^{\prime} 15.898^{\prime \prime} \mathrm{S}, 50^{\circ} 10^{\prime} 59.5791^{\prime \prime} \mathrm{W}, 1 . v .2001,1$ immature (MCN); 29²8’27.121”'S,

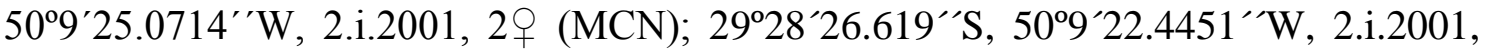
1 우 $\quad(\mathrm{MCN}) ; \quad 29^{\circ} 29^{\prime} 14.82^{\prime \prime} \mathrm{S}, \quad 50^{\circ} 10^{\prime} 57.8025^{\prime \prime} \mathrm{W}, \quad 2.1 \mathrm{i} .2001, \quad 1 \sigma^{\wedge} \quad(\mathrm{MCN})$;

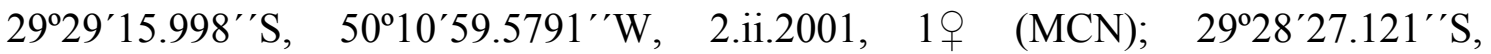

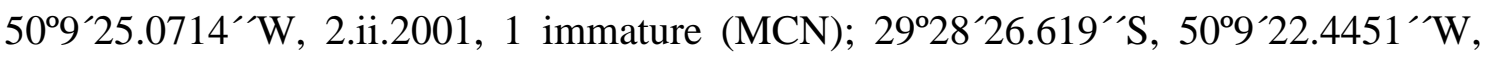
2.ii.2002, 2 ㅇ $(\mathrm{MCN}) ; 29^{\circ} 28^{\prime} 27.121^{\prime \prime} \mathrm{S}, 50^{\circ} 9^{\prime} 25.0714^{\prime \prime} \mathrm{W}, 2 . \mathrm{ii} .2002,2$ ( 2 (MCN); $29^{\circ} 29^{\prime} 14.82^{\prime \prime} \mathrm{S}, \quad 50^{\circ} 10^{\prime} 57.8025^{\prime \prime} \mathrm{W}, \quad 2 . \mathrm{ii} .2002,19 \quad(\mathrm{MCN}) ; 29^{\circ} 28^{\prime} 38.84296^{\prime \prime} \mathrm{S}$, $50^{\circ} 10^{\prime} 8.63689^{\prime \prime} \mathrm{W}, 2.1 \mathrm{i} .2002,1$, $(\mathrm{MCN}) ; 2^{\circ} 29^{\prime} 14.82048^{\prime \prime} \mathrm{S}, 50^{\circ} 10^{\prime} 57.80248^{\prime \prime} \mathrm{W}$,

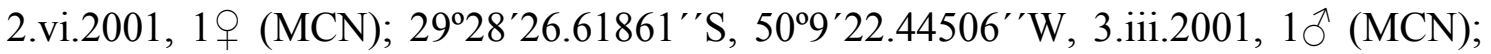
$29^{\circ} 28^{\prime} 38.84296^{\prime \prime} \mathrm{S}, 50^{\circ} 10^{\prime} 8.63689^{\prime \prime} \mathrm{W}, 7 . i v .2001,10^{\Uparrow}(\mathrm{MCN}) ; 2^{\circ} 29^{\prime} 14.82048^{\prime \prime} \mathrm{S}$, $50^{\circ} 10^{\prime} 57.80248^{\prime \prime} \mathrm{W}, 7 . i v .2001,1$ ㅇ $(\mathrm{MCN}) ; 29^{\circ} 28^{\prime} 26.61861^{\prime \prime} \mathrm{S}, 50^{\circ} 9^{\prime} 22.44506^{\prime \prime} \mathrm{W}$,

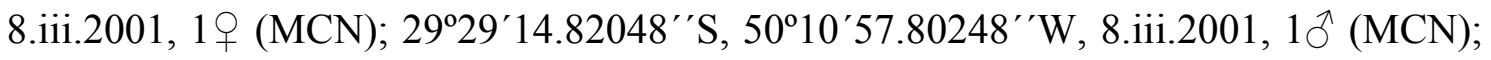
$29^{\circ} 28^{\prime} 38.84296^{\prime \prime} \mathrm{S}, 50^{\circ} 10^{\prime} 8.63689^{\prime \prime} \mathrm{W}, 9 . \mathrm{v} .2001$, 1 q (MCN); 2928'27.12072” S,

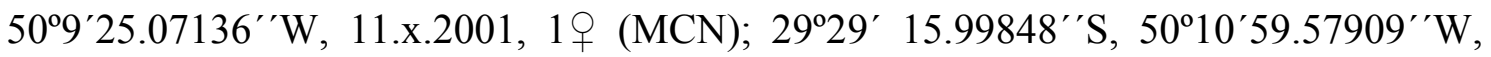

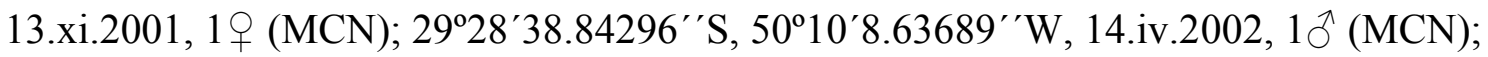




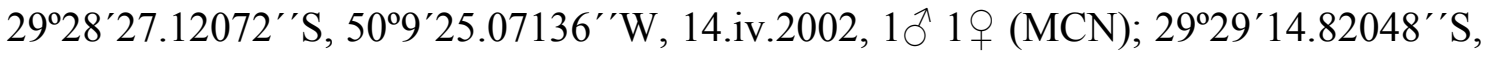
$50^{\circ} 10^{\prime} 57.80248^{\prime \prime} \mathrm{W}, 14.1 \mathrm{v} .2002,1 q(\mathrm{MCN}) ; 29^{\circ} 28^{\prime} 27.12072^{\prime \prime} \mathrm{S}, 50^{\circ} 9^{\prime} 25.07136^{\prime \prime} \mathrm{W}$,

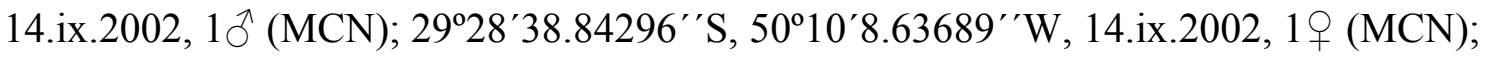
$29^{\circ} 28^{\prime} 27.12072^{\prime \prime} \mathrm{S}, 50^{\circ} 9^{\prime} 25.07136^{\prime \prime} \mathrm{W}, 18 . v .2002,19(\mathrm{MCN}) ; 29^{\circ} 28^{\prime} 26.61861^{\prime \prime} \mathrm{S}$,

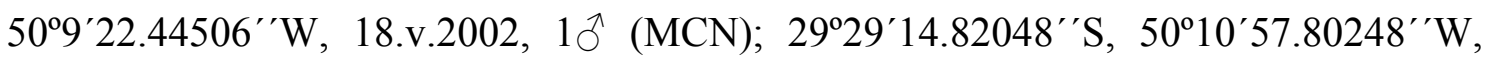

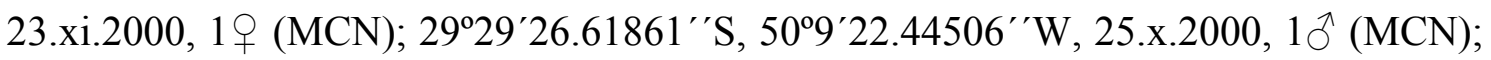

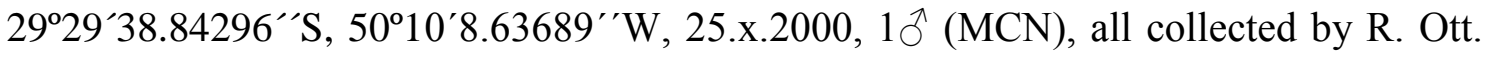

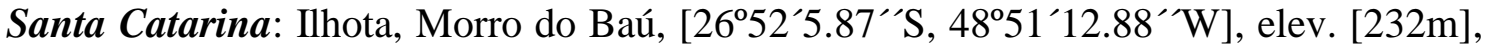
13.v.1996, C. N. Duchett leg., $2 \widehat{\jmath}$ (MCN 27631); São Paulo: São José do Barreiro,

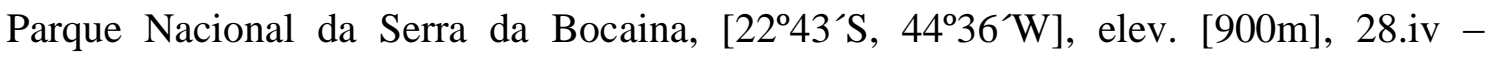
3.v.2002, Equipe Biota leg., Pitfall, 4 ( (IBSP 68552); $1 \delta^{\Uparrow} 1 q$ (IBSP 57091); $1 \overbrace{}^{\Uparrow}$ (IBSP 57078).

Glenognatha foxi (McCook, 1894)

(Figs. 200-207, 305)

Theridium foxi McCook, 1894: 402, pl. 29, fig. 1. Nomen nudum.

Mysmena bulbifera Banks, 1896: 66 (syntypes from United States of America, deposited in MCZ, examined); Banks, 1904: 126, pl. 7, figs. 12-13; Barrows, 1919: 210, figs. 1-2. Synonymized by Levi 1980: 68.

Diplocephalus crumbi Petrunkevitch, 1925b: 171, pl. 8, fig. 1 (syntypes from Tennessee, United States of America, deposited in YPM, not examined). Synonymized by Levi 1980: 68.

Glenognatha foxi: Crosby \& Bishop, 1928: 1055.

Mimognatha foxi: Banks, 1929: 90; Kaston, 1945: 7, fig. 27; Kaston, 1948: 264, figs. 834-835. Synonymized by Levi 1980: 68.

Glenognatha foxi: Levi, 1980: 68, figs. 272-284; Coddington, 1990: 16, fig. 48; Breene et al., 1993: 107, figs. 167A-C; Dimitrov et al., 2007: 765, figs. 8A-D, 11A-B; Álvarez-Padilla et al., 2009: 138, fig. 9b, Álvarez-Padilla \& Hormiga, 2011: 762, figs. 36A-I, 37A-G, 38A-D, 39A-F, 40A-B; Platnick, 2013.

Diagnosis: males of G. foxi resemble those of G. heleios, G. iviei, G. sp. nov. 3, G. sp. nov. 9 and G. sp. nov. 10 by the presence of anterior tooth (Figs. 202A,C). They are distinguished from the latter species by the widely excavated conductor lamina in dorsal 
view (Figs. 204C, 207D). Females are differentiated from the very similar species $G$. heleios by the presence of a well-developed CFO (Fig. 202E).

Description: male and female described by Levi (1980), additional data are presented here.

Male (USA, Pennsylvania, AMNH): habitus as in figure 200. Total length 1.47. Cephalothorax 060 long, 0.42 wide, 0.57 high. Abdomen 0.82 long, 0.75 wide, 0.72 high. Sternum 0.32 long, 0.35 wide. Eye diameters and interdistances: AME 0.05, ALE 0.05, PME 0.06, PLE 0.04. AME-AME 0.05, AME-ALE 0.07, PME-PME 0.06, PMEPLE 0.08, ALE-PLE 0.01. Clypeus height $2.00 \times$ AME diameter. Three promarginal and four retromarginal teeth (Fig. 202A-C). Anterior tooth present (Figs. 202A,C). Leg measurements: I: femur $0.82+$ patella 0.25 + tibia 0.75 + metatarsus $0.62+$ tarsus 0.40 , total 2.85; II: $0.70,0.22,0.65,0.45,0.30,2.32$; III: 0.45, 0.17, 0.32, 0.35, 0.27, 1.57; IV: 0.72, 1.50, 0.50, 0.45, 0.27, 3.45. DTS 0.06. DTE 0.37. Palp as in figures 203-204. Embolus tip widened (Figs. 204D, 207B). Conductor retrolateral apophysis rounded (Figs. 204B-C).

Female (USA, Arizona, AMNH): habitus as in figure 201. Total length 1.75. Cephalothorax 0.70 long, 0.55 wide, 0.55 high. Abdomen 1.00 long, 0.97 wide, 1.02 high. Sternum 0.35 long, 0.42 wide. Eye diameters and interdistances: AME 0.05, ALE 0.03, PME 0.05, PLE 0.03. AME-AME 0.06, AME-ALE 0.05, PME-PME 0.07, PMEPLE 0.07, ALE-PLE 0.01. Clypeus height $1.70 \times$ AME diameter. Three promarginal and four retromarginal teeth (Figs. 202D-F). Cheliceral fang outgrowth well-developed (Fig. 202E). Leg measurements: I: femur 0.67, patella 0.22, tibia 0.60, metatarsus 0.52 , tarsus 0.35 , total 2.37; II: $0.62,0.21,0.50,0.45,0.35,2.13$; III: $0.45,0.12,0.27,0.30$, 0.22, 1.37; IV: 0.67, 0.17, 0.45, 0.42, 0.36, 2.08. DTS 0.08. DTE 0.37. Tracheal system as in figure 206. Internal genitalia with well-developed spermathecae (Fig. 205).

Variation: male total length 1.2-1.8 $(N=10$; average 1.6), cephalothorax length 0.6-0.8 $(N=10$; average 0.7), femur I length 0.7-1.0 $(N=10$; average 0.8). Female total length 1.4-1.8 $(N=10$; average 1.6), cephalothorax length 0.6-0.7 $(N=10$; average 0.69), femur I length 0.6-0.8 $(N=10$; average 0.6$)$. 
Distribution: known from southern Canada to Panama (Fig. 305).

Additional material examined $(\mathbf{N}=684)$ : CANADA: Ontario: Pelee Island. Lake Erie, [41 $\left.45^{\circ} \mathrm{N}, 82^{\circ} 40^{\top} \mathrm{W}\right]$, elev. [179m], 4-16.vi.1950, W. Ivie \& T. B. Kurata leg., $1 \delta^{\Uparrow} 3$

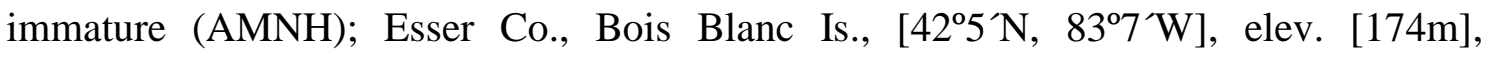
9.viii.1933, W. D. Shepard leg., (in roadside ditch), 10َ (AMNH). CUBA: Cienfuegos: Soledad, [Jardín Botánico de Cienfuegos], [22 ${ }^{\circ} \mathrm{N}, 8^{\circ} 20^{`} \mathrm{~W}$ ], elev. [50m], 111.viii.1934, P. J. Darlington leg., $4+\frac{+}{0}$ (AMNH). JAMAICA: Saint Andrews:

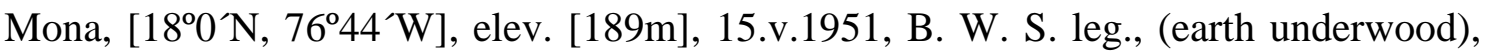
$1 \overbrace{}^{\Uparrow}$ (MCZ 125488). Saint Catherine: School of Agni, [1759`N, 7658`W], elev. [34m], 23.xi.1923, Chickering leg., 191 immature (MCZ 125486). MEXICO: Tamaulipas:

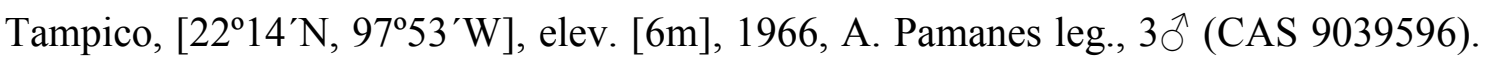
PANAMA: Colón: Santa Rosa, [852`N, 805W], elev. [272m], vi.1945, C. D. Michener leg., $1 \delta^{\Uparrow}$ (AMNH) Panamá: Barro Colorado, Canal zone, [99`N, 7950`W], elev. [150m], 10.iii.1936, W. J. Gertsch leg., 1 (AMNH); 2-14.vii.1979, E. Broadhead et al. leg., 1 ภ (USNM); viii.1950, 1 ㅇ 2へ (MCZ 125540); viii.1954, $1 \widehat{\jmath}$ (MCZ

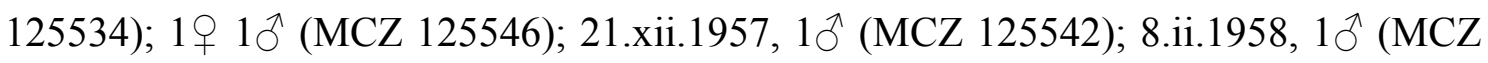
129047), all collected by A. M. Chickering; Canal Zone, Corozal, [8º ${ }^{`} \mathrm{~N}, 8^{\circ} 26^{\prime} \mathrm{W}$ ], elev. [200m], xii.1957 - i.1958, $5 q$ (MCZ 125492); 23.xii.1957, 2 + 3ð (MCZ

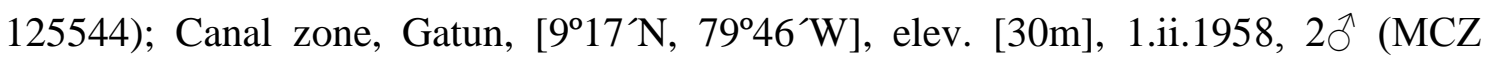

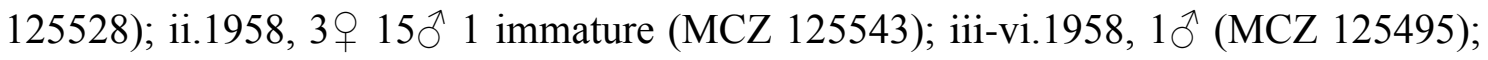
6.iii.1958, $3 q 1 \lesssim$ (MCZ 125493); 30.i.1958, 4 우 5ð 2 immatures (MCZ 125539), Canal Zone, Pedro Miguel, [9 $\left.{ }^{\circ} 1^{\prime} \mathrm{N}, 7^{\circ} 36^{\prime} \mathrm{W}\right]$, elev. [50m], 26.xii.1957, 1 ㅇ 2 immature (MCZ

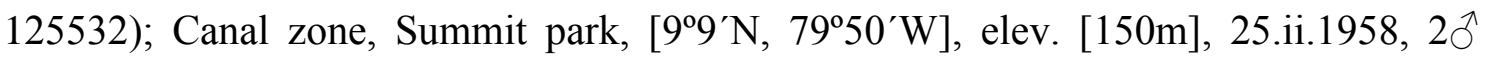

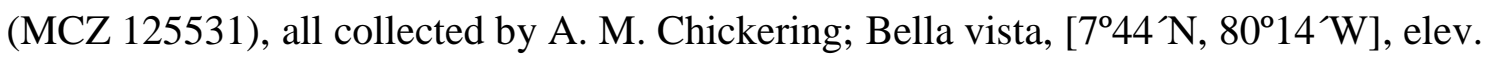
[30m], 6.vii, Banks leg., 1へ̂ (MCZ 125547). UNITED STATES OF AMERICA:

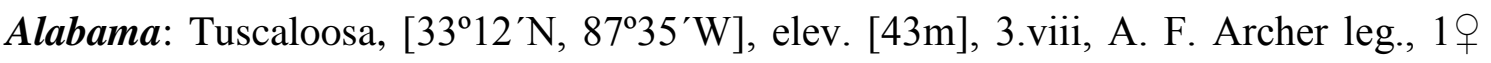

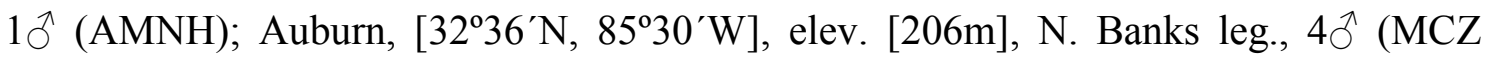

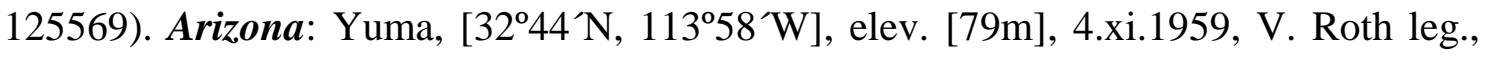
(Swp. Alfalfa), 3 immatures (AMNH). Arkansas: Sevier Cossatot River, [335ํN, 94 ${ }^{\circ} 13^{\prime} \mathrm{W}$ ], elev. [95m], 3.v.1966, W. Peck leg., 1ð̋ (CAS 9039594); Mississippi Co.

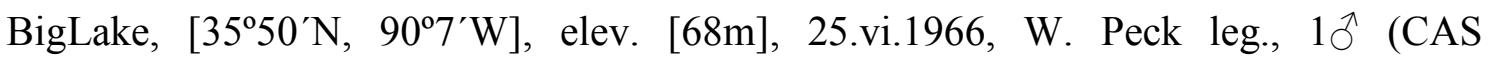

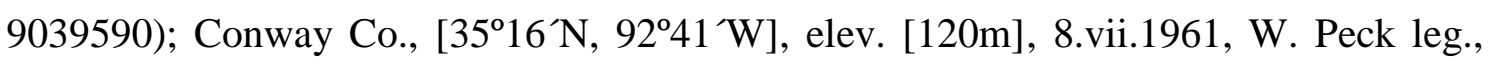
$1 \circlearrowleft 1$ (CAS 9039644); Washington Co., Cove creek valley, 15 mi. W of Prairie Grove 


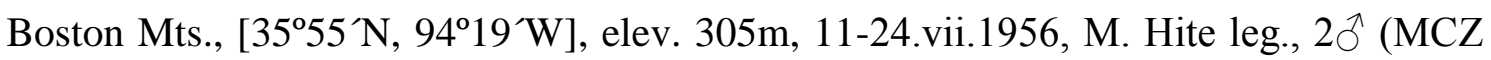
125576); Washington Co., Cove cr. valley, 15 mi. S. of Prairie Grove, Boston Mts.,

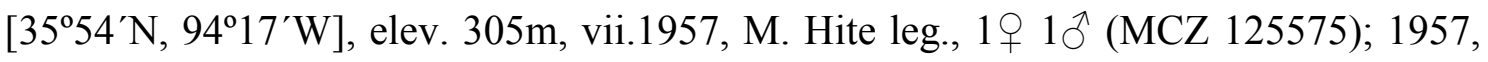

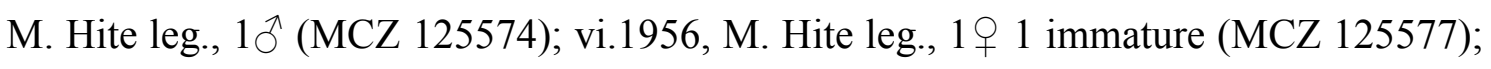
19.vi.1957, M. Hite leg., 1 ㅇ (MCZ 125572); Washington Co., Fayetteville, [363ㅈN, 94’W], elev. [388m], vii.1957, M. Hite leg., 1 ( Co. Imperial Dam., [32 $\left.50^{\prime} \mathrm{N}, 114^{\circ} 29^{\prime} \mathrm{W}\right]$, elev [48m], 28.ix.1957, B. Schick leg., $1 q$

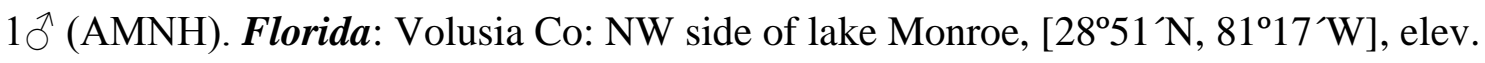
[5m], 30.iii.1942, W. H. \& L. F. Stieckel leg., (plant sweep), 1 q (AMNH); Archbold, biological station, $27^{\circ} 11^{\prime} \mathrm{N}, 81^{\circ} 21^{\prime} \mathrm{W}$, elev. [42m], 19.xii.1962, W. Ivie leg., 4 ㅇ $6 \bigcirc^{\lambda} 16$ immature (AMNH); 8 miles N.W. of Stuart, $27^{\circ} 17^{\prime} \mathrm{N}, 80^{\circ} 17^{`} \mathrm{~W}$, elev. [4m], 12.xii.1962, W. Ivie leg., 2 (AMNH); Collier Co., Naples, [26 $\left.6^{\prime} \mathrm{N}, 81^{\circ} 47^{\prime} \mathrm{W}\right]$, elev. [5m], 17.i12.ii.1946, Stowell Rounds leg., 1 ㅇ (AMNH); N. of Winter Park, [28 $43^{\prime} \mathrm{N}, 8^{\circ} 24^{\prime} \mathrm{W}$ ], elev. [5m], 11.iv.1938, W. J. Gertsch leg., 19 (AMNH); Miami, [2550’ N, 80²2’W],

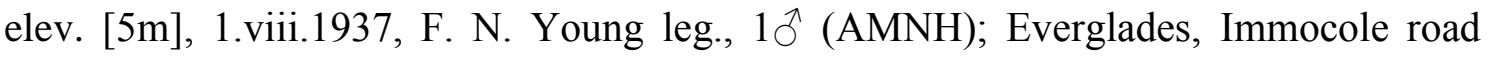
[26 $\left.29^{\prime} \mathrm{N}, 81^{\circ} 25^{\prime} \mathrm{W}\right]$, elev. [11m], 16.i.1935, W. M. B. leg., 1ठ (AMNH); Gainesville,

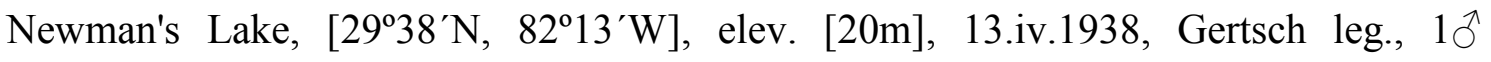
$(\mathrm{AMNH})$; Gainesville, $3 \mathrm{mi}$. S. Lake Istokpoga, [27 $11^{`} \mathrm{~N}, 81^{\circ} 17^{\top} \mathrm{W}$ ], elev. [18m], 28.ii.1951, A. M. Nadler leg., 1 (AMNH); Miakka River State Park, nr. Sarasota, [27 $\left.12^{\prime} \mathrm{N}, 82^{\circ} 15^{\prime} \mathrm{W}\right]$, elev. [9m], 6.iv.1938, Gertsch leg., 1 \% (AMNH); Okeechobee,

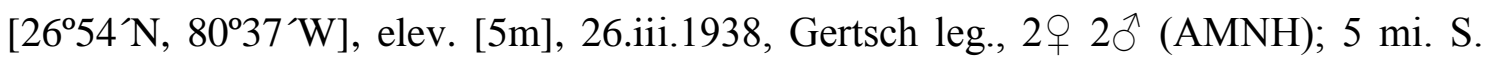

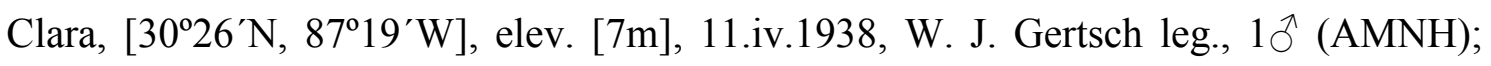

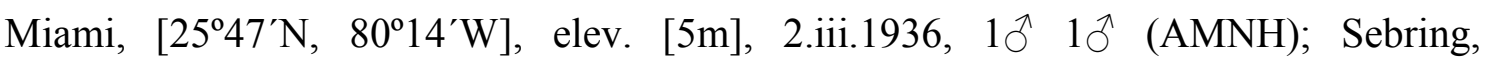

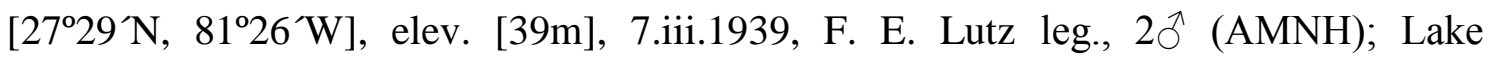

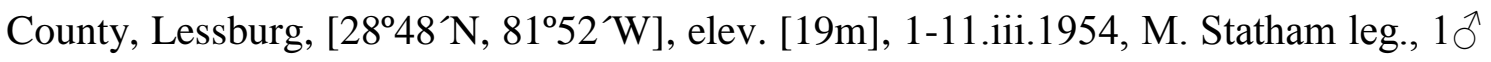

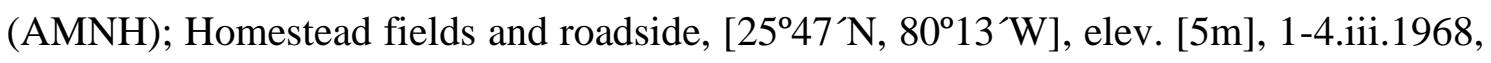

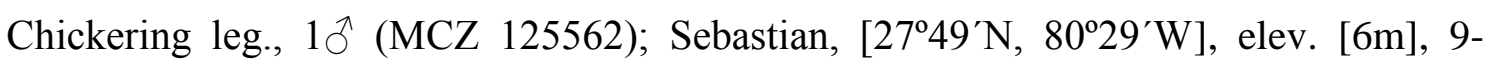
14.iv.1944, G. Nelson leg., $1 \delta^{\Uparrow}$ (MCZ 125567); Runnymede, [27ำ $\left.10^{\prime} \mathrm{N}, 80^{\circ} 18^{\prime} \mathrm{W}\right]$, elev.

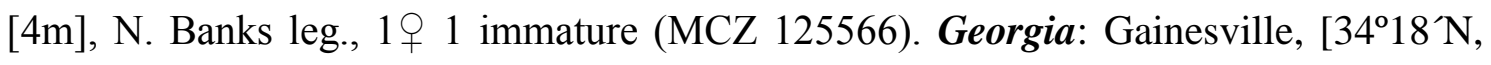
834’ W], elev. [369m], 31.iii.1949, E. Kaston leg., 1ठ (USNM); Rabun Co., S.W. end

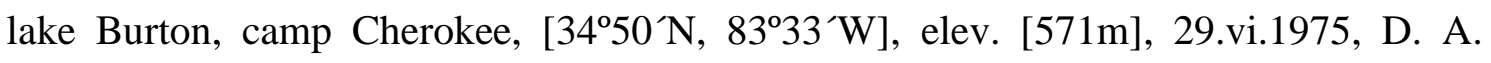
Rossman leg., $1 \delta^{\Uparrow}$ (AMNH); Sylvania to Sardis, $32^{\circ} 50^{\prime} \mathrm{N}, 81^{\circ} 40^{\prime} \mathrm{W}$, elev. [36m],

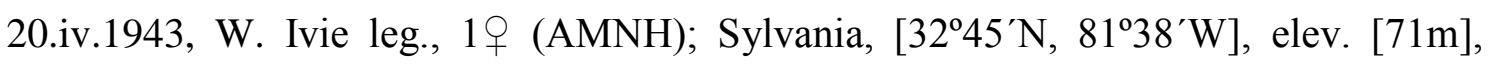
27.iii.1957, W. J. Gertsch \& R. Forster leg., 1 ( $(\mathrm{AMNH})$. Illinois: Old Ripley, along 


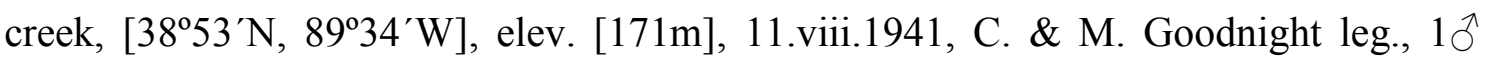
(AMNH); Grafton, along river, [385`N, 90²6`W], elev. [176m], 3.ix.1944, C. \& M.

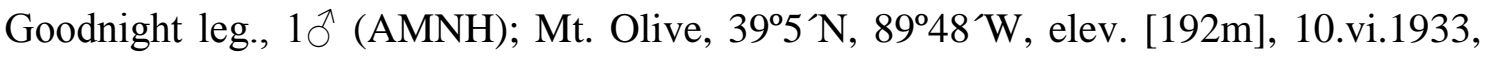
W. Ivie leg., $2 q 1 \delta^{\hat{}}(\mathrm{AMNH})$. Indiana: Fort Wayne (St. Marys River), $41^{\circ} 3^{\wedge} \mathrm{N}$, 85ㅇ' W, elev. [241m], 3.viii.1964, J. \& W. Ivie leg., $3 q 4{ }^{\circ} 1$ immature (AMNH). Kansas: 4 miles S.W. of Redfield, $37^{\circ} 45^{\prime} \mathrm{N}, 9^{\circ} 55^{`} \mathrm{~W}$, elev. [258m], 8.viii.1964, J. \&

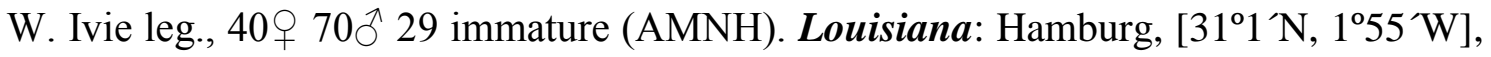
elev. [15m], 13.v.1963, 16우 25 9 immature (AMNH); Baton Rouge, [3027^N, 91 ${ }^{\circ} 8^{\prime} \mathrm{W}$, elev. [15m], ix.1958, 2 \% (AMNH); Farmerville, [31 $\left.{ }^{\circ} 45^{\prime} \mathrm{N}, 92^{\circ} 9^{\prime} \mathrm{W}\right]$, elev.

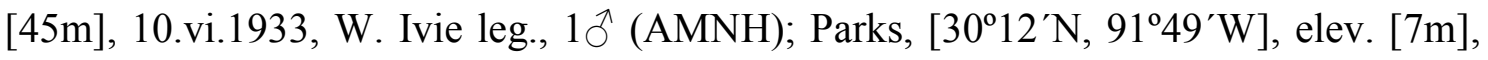
16.vii.1963, 27ㅇ 35ð 15 immature (CAS 9038090). Massachusetts: Nantucket,

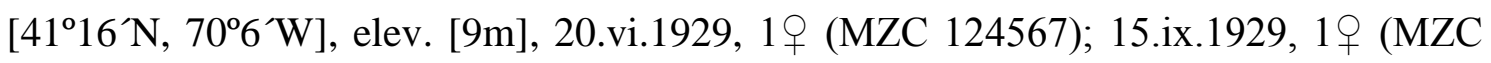
125466), 23.vi.1928, (saltmarsh), $1 \overbrace{}^{\Uparrow}$ (MZC 125465). Mississippi: Hattiesburg,

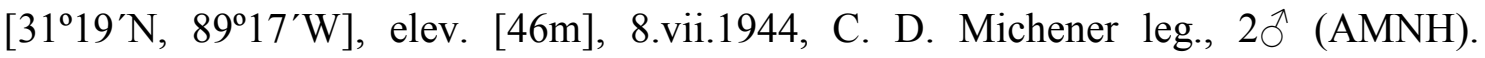

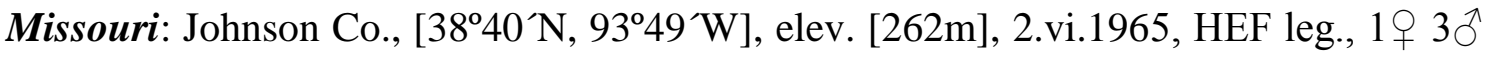

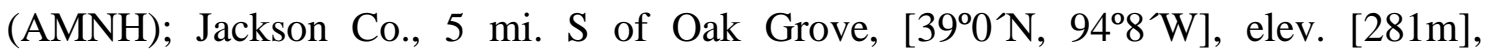

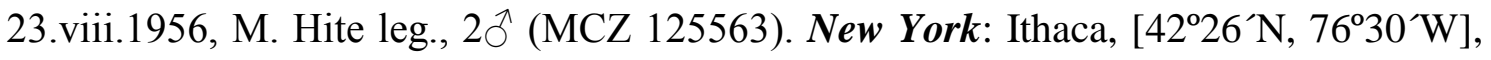

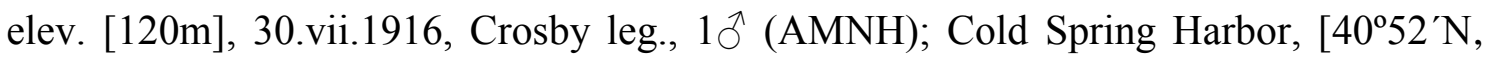
76 27'W], elev. [120m], 23.vii.1907, E. B. Bryant leg., 1ð (MCZ 125470). North

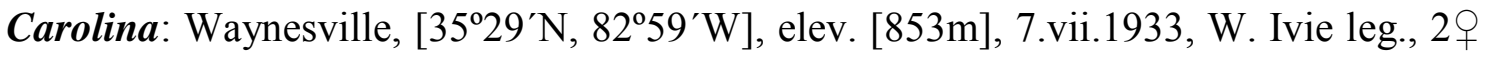

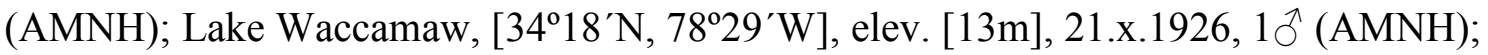

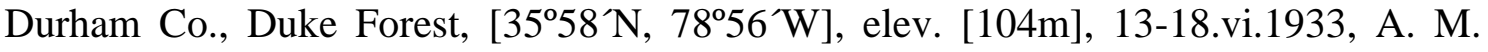

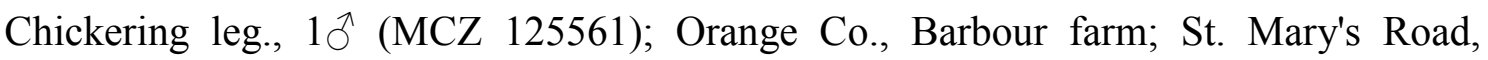
[35 $\left.59^{\prime} \mathrm{N}, 7^{\circ} 8^{\prime} \mathrm{W}\right]$, elev. [187m], 13.ix.1963, J. W. Berry leg., 1 ㅇ (MCZ 125570);

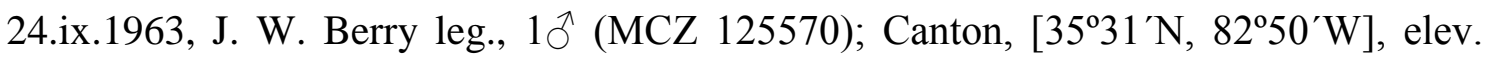
[809m], N. Banks leg., $1 \widehat{\text { }}$ (MCZ 125472). Pennsylvania: N.E. of Jamison, Horseshoe

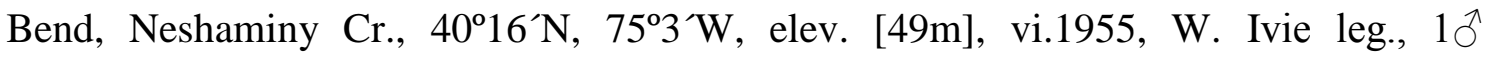
(AMNH); x.1954, W. Ivie leg., 9 + 12ð 4 immature (AMNH); ix. 1954, W. Ivie leg.,

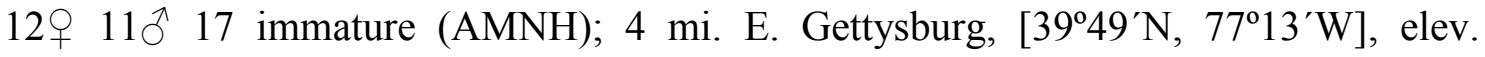
[168m], 2.vii.1960, J. R. D. Barnes leg., $3 \widehat{\jmath}$ (MCZ 125464). South Carolina: Long

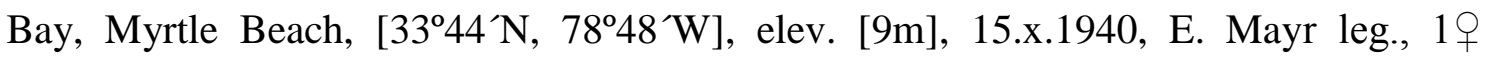

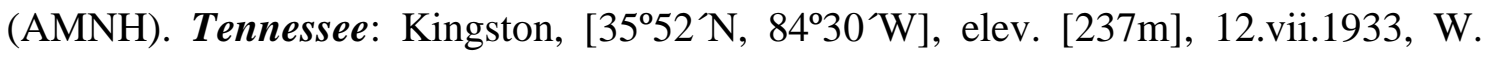

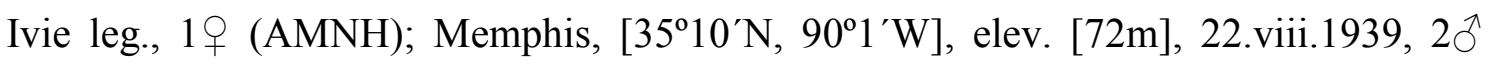
(AMNH); Little Pigeon Cr., Great Smoky Mts., 35³8`N, 8330`W, elev. [998m], 


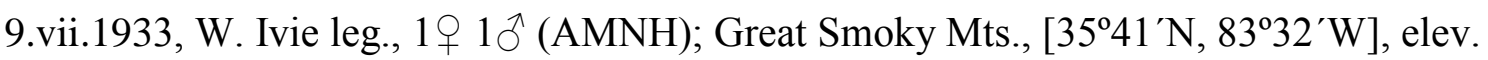
[450m], 8.vii.1933, Gertsch leg., 1 ㅇ (AMNH); 1871, J. Curtis leg., 1ठ̊ (MCZ 125568).

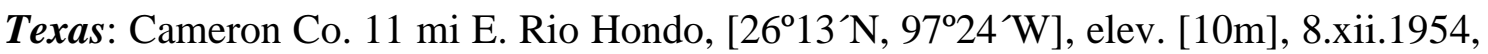

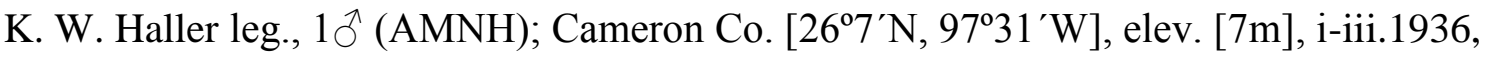

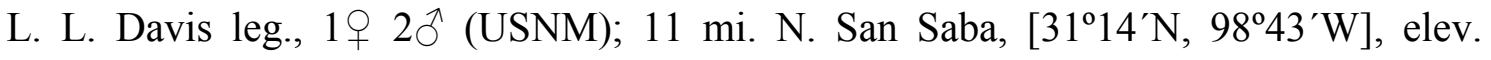

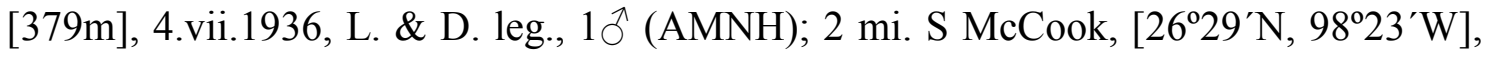
elev. [93m], 28.vi.1935, S.M. leg., 3 + (AMNH); 20 mi. S. Alpine, [30²16 $\mathrm{N}$,

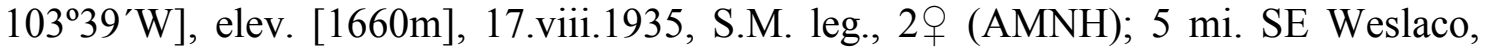
[26 $3^{\circ} \mathrm{N}, 97^{\circ} 51^{\prime} \mathrm{W}$ ], elev. [21m], 21.vii.1935, S.M. leg., $2 \delta^{\Uparrow} 1$ immature (AMNH); 6 mi.

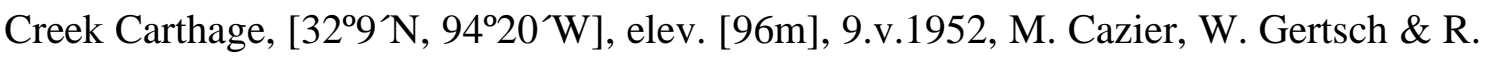
Schramme leg., $1 \delta^{\Uparrow}(\mathrm{AMNH}) ; 7 \mathrm{mi}$. E of Palestine, [31 $45^{\prime} \mathrm{N}, 95^{\circ} 33^{\prime} \mathrm{W}$ ], elev. [134m], 17.vii.1938, I. Davis leg., 2 + $1 \delta^{\Uparrow}(\mathrm{AMNH}) ; 7 \mathrm{mi}$. W of Higgins, [36 ${ }^{\circ} 7^{\prime} \mathrm{N}, 100^{\circ} 2^{\prime} \mathrm{W}$ ], elev. [779m], 16.vii.1939, L. I. Davis leg., 1 \% (AMNH); Bon Wier, [3044N, 933' W], elev. [23m], 18.viii.1940, A. M. \& L. I. Davis leg., 19 (AMNH); 1 mi NW

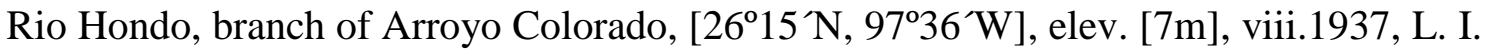

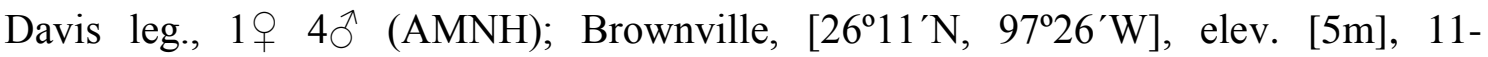
16.vi.1933, Darlington leg., $1{ }^{\Uparrow}$ (MCZ 124569); Cameron Co., [26º' N, 97²9’W], elev.

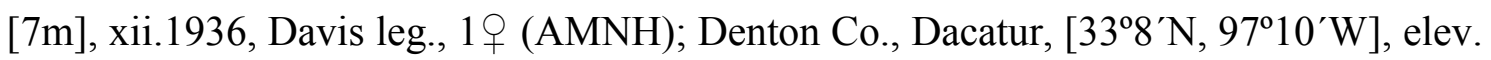
[196m], 8.viii.1945, 1ð (MCZ 124573); E. of Harlingen; [26¹0`N, 97³5’W], elev.

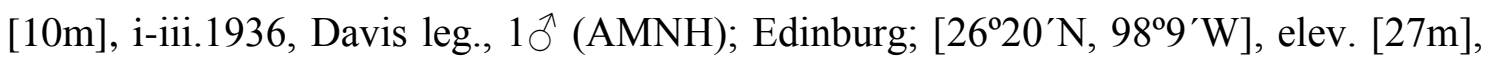

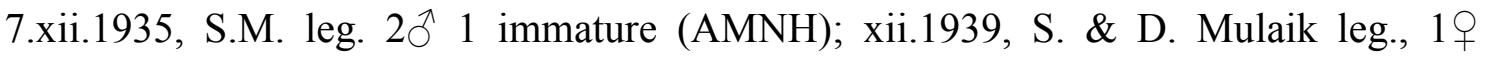
(AMNH); 12.x.1935, S. M. leg., 5ठ 1 immature (AMNH); xii.1939, S. \& D. Mulaik

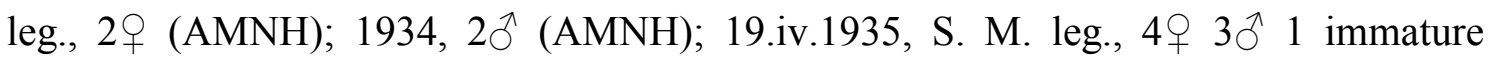
(AMNH); xi.1933, Mulaik leg., 1 ㅇ (AMNH); Harlingen, [26 $\left.11^{\circ} \mathrm{N}, 97^{\circ} 41^{\prime} \mathrm{W}\right]$, elev.

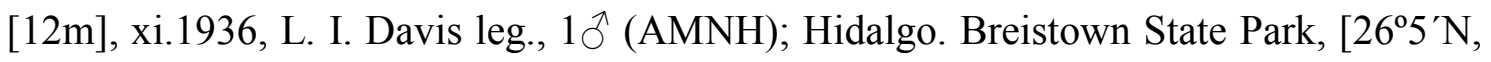

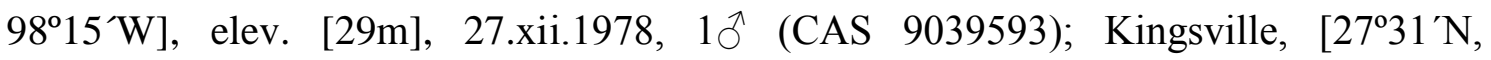

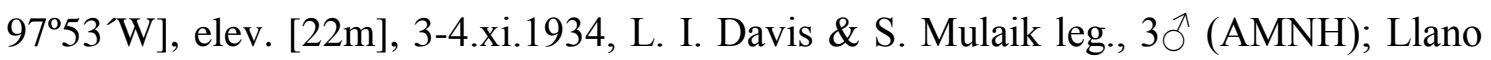

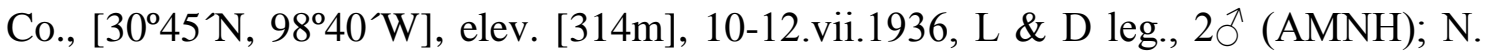

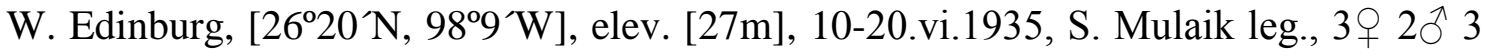
immature (AMNH); Nacogdoches, 2 miles south, $31^{\circ} 35^{\prime} \mathrm{N}, 94^{\circ} 40^{\prime} \mathrm{W}$, elev. [121m], 11.viii.1946, J. \& W. Ivie leg., $3 q 6 \hat{0} 6$ immature (AMNH); New Brownfield,

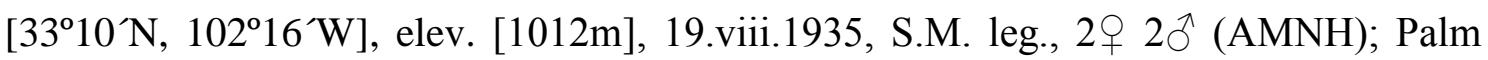

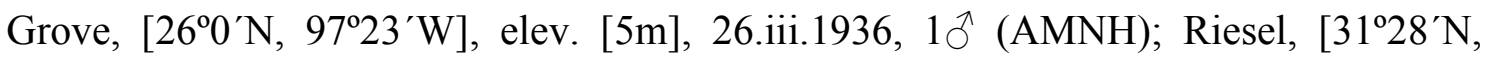
96 $55^{\prime}$ W], elev. [153m], 15.vii.1941, M. Kagen leg., 3 ㅇ (AMNH); Sanderson, [30² N, 
$102^{\circ} 23^{\prime} \mathrm{W}$, elev. [851m], 20.viii.1935, S. Mulaik leg., $4+6 \overbrace{}^{\lambda}$ (AMNH). Virginia:

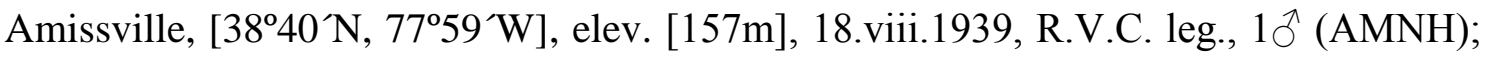
Douthat St. Pk., Bath Co., [37 53 N , 794 $48^{\prime}$ W], elev. [456m], R. L. Hoffman leg., (sweeping short grass) $1 \delta^{`}$ (AMNH); Great Falls, [38 $\left.59^{\prime} \mathrm{N}, 77^{\circ} 17^{\prime} \mathrm{W}\right]$, elev. [103m], J. H. Emerton leg., 1 + $1 \delta^{\wedge}$ (MCZ 125564); Falls Church, [38 $\left.52^{\prime} \mathrm{N}, 77^{\circ} 10^{\prime} \mathrm{W}\right]$, elev.

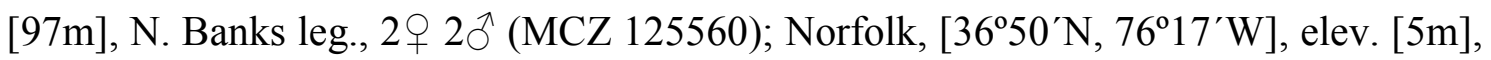
v.1900, J. H. Emerton leg., $1 \delta^{\widehat{C}}$ (MCZ 125565). West Virginia: Pocahontas Co., 2350'

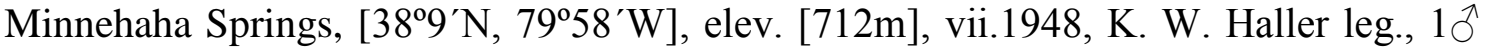

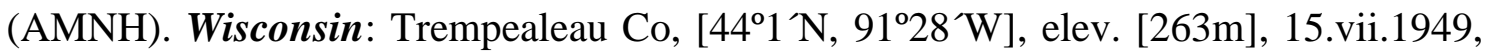

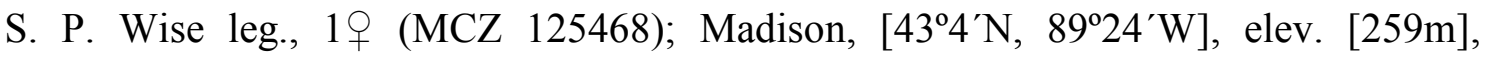
vi.1967, $1 \sigma^{\Uparrow}(\mathrm{MCZ} 125501)$.

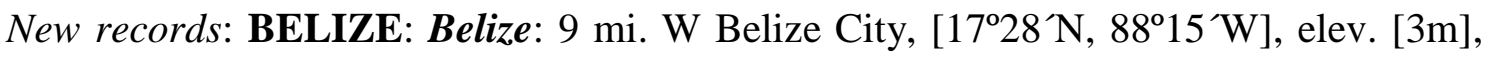
9.viii.1933, W. D. Shepard leg., (in roadside ditch), 1ð̋ 1 immature (CAS 9039646). COSTA RICA: San José: San José, [956№, 845W], elev. [1152m], E. Schmidt leg.,

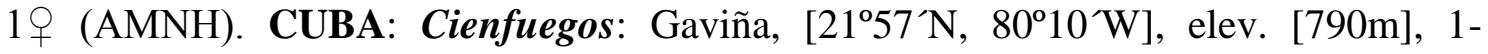
13.ii.1981, P. J. Spangler \& A. Vega leg., 1 (USNM). HAITI: Sud: Lévy (1 Km NE), [18 $\left.18^{\prime} \mathrm{N}, 7^{\circ} 51^{\prime} \mathrm{W}\right]$, elev. [185m], 10-11.ix.1981, P. Spangler \& R. Faitoute leg., 1 (USNM). MEXICO: Chiapas: 13 miles NW of Comitán, [16 $\left.6^{\circ} 16^{`} \mathrm{~N}, 92^{\circ} 10^{`} \mathrm{~W}\right]$, elev. [1945m], 17.viii.1972, G. P. Hevel leg., 19 (USNM). Guerrero: 1.5 miles W Acapulco, [16 $\left.52^{`} \mathrm{~N}, 9^{\circ} 53^{\top} \mathrm{W}\right]$, elev. [320m], 4.i.1948, (fresh lake shore), $1 \delta^{\Uparrow}(\mathrm{AMNH})$;

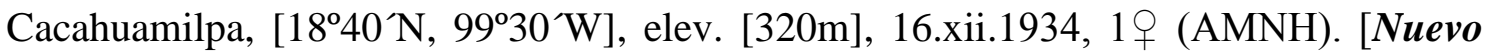

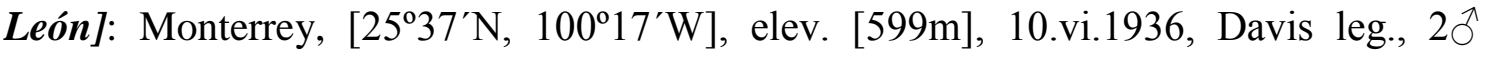
(AMNH). San Luis Potosí: Tamazunchale, arroyo la Chorrera, [21ำ $15^{\prime} \mathrm{N}, 98^{\circ} 47^{\prime} \mathrm{W}$ ], elev. [135m], 28.iii.1937, A. M. \& L. I. Davis leg., 2 (AMNH). Sinaloa: Burrion, $25^{\circ} 33^{\prime} \mathrm{N}, 108^{\circ} 25^{\prime} \mathrm{W}$, elev. [15m], 17.v.1963, W. J. Gertsch \& W. Ivie leg., 19 (AMNH). Sonora: Hermosillo, [295N, 11056`W], elev. [135m], 20.ix.1952, B. Malkin \& V.E. Thatcher leg., 19 (AMNH). Tamaulipas: San Fernando, [21 $1^{\circ} 15^{\prime} \mathrm{N}$,

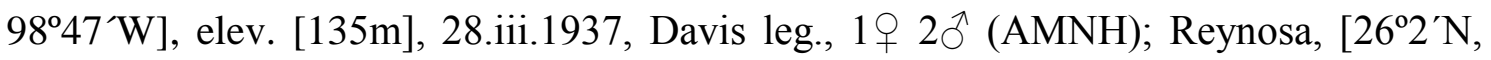
$98^{\circ} 17^{\prime} \mathrm{W}$ ], elev. [39m], 2.iv.1936, S. Mulaik leg., 2 (AMNH); Mante, $22^{\circ} 45^{\prime} \mathrm{N}$, 9858'W, elev. [89m], 17.iv.1963, W. J. Gertsch \& W. Ivie leg., 19 (AMNH).

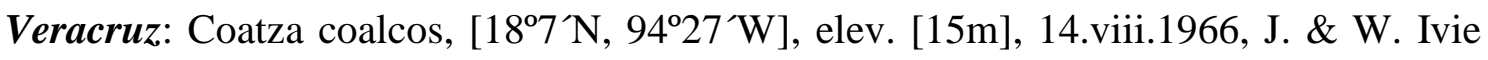

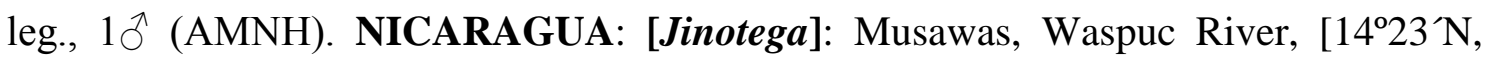

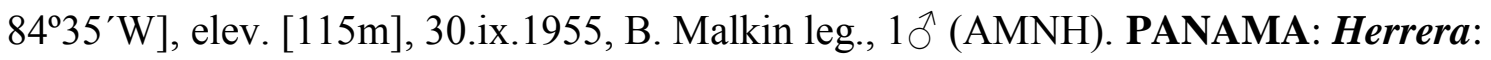

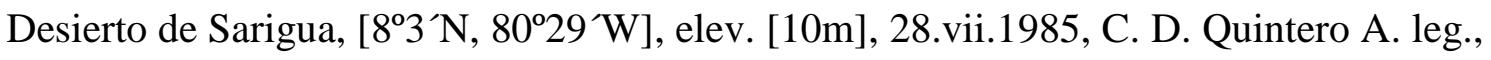




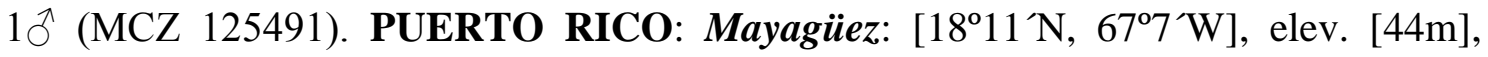
10.vi.1964, $1 ð$ (MCZ 125484). UNITED STATES OF AMERICA: Louisiana: St.

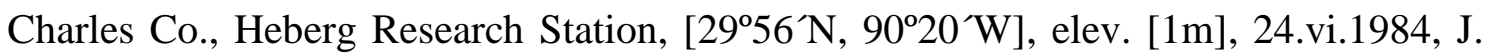

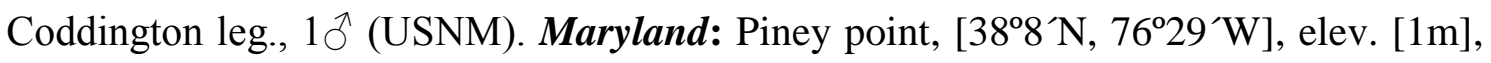
26.viii.1946, E. A. Chapain leg., $1 \hat{\jmath}$ (USNM). Missouri: Callaway Co., Tucker prairie, [38 53'N , 91 $54^{\circ}$ 'W], elev. [245m], 12.vi.1984, J. C. Weaver leg., 1ठ (USNM). North Carolina: Craven Co., Croatan, Croatan Nat. forest, [34 $54^{`} \mathrm{~N}, 7^{\circ} 3^{\prime} \mathrm{W}$ ], elev. [12m],

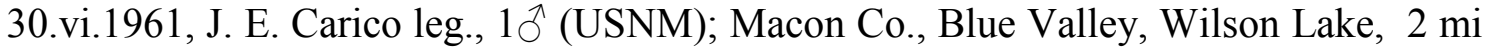
SW Highlands, [34 $57^{\prime} \mathrm{N}, 83^{\circ} 21^{\prime} \mathrm{W}$ ], elev. [680m], 15.vii.1998, M. van der Meiwe leg., $1 \lesssim$ (USNM).

\section{Glenognatha sp. nov. 9}

(Figs. 208-211, 309)

Type material: male holotype from Lago Janauari, Manaus, Amazonas, Brazil, $3^{\circ} 20^{\prime} \mathrm{S}$, 60¹7 W, elev. [12m], vi.1987-vii.1988, J. Adis et al. leg., deposited in IBSP 120209; paratypes: $1 \delta$ same data as the holotype deposited in IBSP 120266.

Diagnosis: males of $G$. sp. nov. 9 resemble those of G. heleios, G. iviei, G. sp. nov. 3 and G. sp. nov. 10 by the presence of anterior tooth (Fig. 209C). They are distinguished from the latter species by the serrated distal margin of the conductor (Fig. 211B-C).

Description: Male (Holotype): habitus as in figure 208. Carapace pale brown, darker on sides and with a diffused dusky patch from the median eyes to the cephalic fovea. Sternum dusky brown, with darker bands towards the median region. Abdomen spherical, strongly depigmentated, dorsally with irregular greyish patches and scattered silver guanine spots. Venter with a pale black median band. Legs yellow-brownish with darker bands at the base of the segments. Total length 1.77. Cephalothorax 0.77 long, 0.60 wide, 0.50 high. Abdomen 0.92 long, 0.75 wide, 0.75 high. Sternum 0.35 long, 0.42 wide. Eye diameters and interdistances: AME 0.08. ALE 0.05. PME 0.07. PLE 0.06. AME-AME 0.05, AME-ALE 0.05, PME-PME 0.07, PME-PLE 0.08, ALE-PLE 0.01. Clypeus height $1.20 \times$ AME diameter. Chelicerae slightly lighter than the cephalothorax. Three promarginal and four retromarginal teeth (Figs. 209A-C). Anterior tooth near the fang base (Fig. 209C). Leg measurements: I: femur 0.80, patella 0.22, tibia 0.75 , metatarsus 0.70 , tarsus 0.45 , total 2.92 ; II: $0.67,0.22,0.70,0.61,0.45,2.66$; 
III: $0.50,0.18,0.40,0.42,0.28,1.80$; IV: $0.75,0.17,0.60,0.55,0.30,2.37$. DTS 0.10. DTE 0.34. Palp as in figures 210-211. Embolus short, almost completely enclosed by the conductor (Figs. 211A).

Female: unknown.

Variation: male total length 1.65-1.77 $(N=4$; average 1.72), cephalothorax length 0.62$0.75(N=4$; average 0.72$)$, femur I length 0.8-0.85 $(N=4$; average 0.81$)$.

Distribution: known only from the type locality in the state of Amazonas, Brazil (Fig. 309).

Material examined ( $\mathbf{N}=2$ ): BRAZIL: Amazonas: Manaus, Lago Janauari, 320’ S,

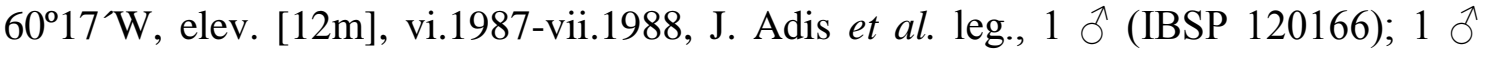
(IBSP 120220).

Glenognatha sp. nov. 10

(Figs. 212-216, 297H, 309)

Type material: male holotype from Río Camisea, La Convención, Cusco, Peru $11^{\circ} 47^{\prime} 9.8^{\prime \prime} \mathrm{S}, 72^{\circ} 42^{\prime} 5.3^{\prime \prime} \mathrm{W}$, elev. 474m, iii-iv.1997, S. Córdoba leg., deposited in MUSM 0505885; paratypes: $1 \hat{\jmath}$, same data as the holotype, deposited in MUSM 0504913.

Diagnosis: males of $G$. sp. nov. 10 can be distinguished from all other Glenognatha species by slender distal portion of the paracymbium (Fig. 214D).

Description: Male (Holotype): habitus as in figure 212. Carapace pale brown, darker on sides and along furrows, with a median lighter patch. Sternum brown, with darker bands towards the median region. Abdomen oval, dorsally with two dusky longitudinal bands on sides and one median posterior band that divides into two lateral bands that join medially in the posterior region. Silver guanine spots scattered in lighter dorsal regions and on lateral surfaces. Venter with a pale black median band and two lighter lateral bands. Legs yellow-brownish with distal parts of segments darker. Total length 1.62. Cephalothorax 0.80 long, 0.62 wide, 0.45 high. Abdomen 0.97 long, 0.80 wide, 0.82 
high. Sternum 0.40 long, 0.45 wide. Eye diameters and interdistances: AME 0.08. ALE 0.07. PME 0.07. PLE 0.06. AME-AME 0.06, AME-ALE 0.04, PME-PME 0.04, PMEPLE 0.05, ALE-PLE 0.01. Clypeus height $1.17 \times$ AME diameter. Chelicerae with the same coloration of the cephalothorax. Three promarginal and four retromarginal teeth (Figs. 213A-C). Anterior tooth well-developed (Fig. 213A,C). Leg measurements: I: femur 1.02, patella 0.25 , tibia 1.75 , metatarsus 0.80 , tarsus 0.57 , total 3.72 ; II: 0.90 , 0.25, 0.90, 0.81, 0.52, 3.38; III: 0.62, 0.20, 0.45, 0.52, 0.35, 2.15; IV: 0.85, 0.20, 0.71, 0.67, 0.42, 2.86. DTS 0.10. DTE 0.36. Epiandrous fusules as in figure 297H. Palp as in figures 214-216. Paracymbium distal portion slender (Figs. 214D).

Female: unknown.

Variation: male total length 1.62-1.75 $(N=6$; average 1.66), cephalothorax length 0.75 $0.85(N=6$; average 0.79$)$, femur I length 0.97-1.25 $(N=6$; average 1.08).

Distribution: Known from Cusco state in Peru (Fig. 309).

Material examined $(\mathbf{N}=4)$ : PERU: Cusco: La Convención, Río Camisea, $11^{\circ} 47^{\prime} 9.8^{\prime \prime} \mathrm{S}, 72^{\circ} 42^{\prime} 5.3^{\prime} \mathrm{W}$, elev. 474m, S. Córdoba leg., 1ð (MUSM 0504917); La

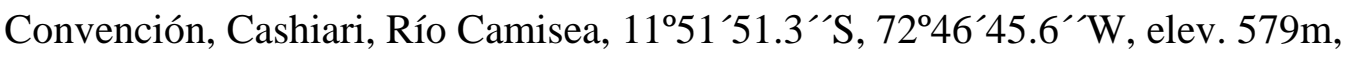

v-vi.1997, S. Córdoba leg., $1{ }^{\Uparrow}$ (MUSM 0504905); La Convención, Echarate, C. N.

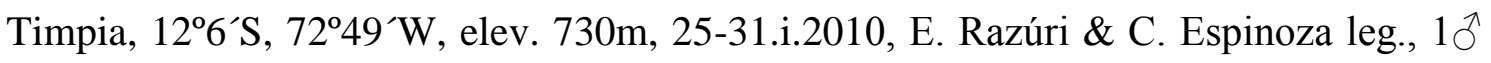

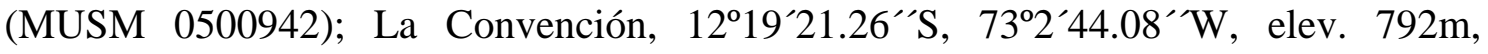
23.iv.2007, W. Paredes leg., $1 ð$ (MUSM 0504903).

Glenognatha smilodon Bosmans \& Bosselaers, 1994

(Figs. 217-219, 313)

Glenognatha smilodon Bosmans \& Bosselaers, 1994: 348, f. 148-157 (male holotype from Pic Hosseré Vokré, Poli Mountains, Cameroon, 17.iv.1983, elev. 900m, Bosmans R. \& Van Stelle leg., deposited in MRAC 202710, examined); Platnick, 2013.

Diagnosis: males of G. smilodon can be easily distinguished from all other Glenognatha species by the prominent curved apical portion of the embolus in lateral view (Figs. 218C-D). 
Description: holotype described by Bosmans \& Bosselaers (1994). Additional data are presented here: Habitus as in figure 217. Cephalothorax 0.75 high. Abdomen 1.17 high. Palp as in figures 218-219. Conductor lamina distal end prolaterally folded (Figs. 219AB). Embolus with a distinct laminar projection (Fig. 219D).

Female: unknown

Distribution: known only from the type locality (Fig. 313).

Glenognatha iviei Levi, 1980

(Figs. 220-222, 304)

Glenognatha iviei Levi, 1980: 69, figs. 285-289 (male holotype from Gulfport, Mississippi, United States of America, 18.viii.1922, W. Ivie leg., deposited in AMNH, examined); Platnick, 2013.

Diagnosis: males of G. iviei can be differentiated from all other Glenognatha species (except $G$. heleios) by the presence of a small pointed apophysis on the paracymbium (Fig. 222D); distinguished from G. heleios by the strongly curved Prt1 (Fig. 220D) and by the coiled distal portion of the embolus (Figs. 221A, D, 222A).

Description: male described by Levi (1980), additional data are presented here.

Male (Holotype): Habitus as in figure 220. Cephalothorax 0.42 high. Abdomen 0.78 long, 0.48 wide, 0.58 high. Sternum 0.38 long, 0.32 wide. Eye diameters and interdistances: AME 0.07, ALE 0.04, PME 0.08, PLE 0.04. AME-AME 0.07, AMEALE 0.03, PME-PME 0.05, PME-PLE 0.06, ALE-PLE 0.005. Clypeus height $1.80 \times$ AME diameter. Three promarginal and four retromarginal teeth. Anterior tooth present (Fig. 220D). Leg measurements: I: femur 0.75, patella 0.25, tibia 0.77, metatarsus 0.62 , tarsus 0.37 , total 2.77 ; II: $0.67,0.22,0.65,0.52,0.32,2.40$; III: $0.42,0.17,0.33,0.33$, 0.22, 1.50; IV: 0.58, 0.17, 0.47, 0.45, 0.25, 1.93. DTS 0.03. DTE 0.22. Palp as in figures 221-222. Conductor retrolateral apophysis rounded (Figs. 221B-C). Embolus distal portion coiled (Figs. 221A, D, 222A).

Female: unknown 
Distribution: known only from the type locality (Fig. 304).

Glenognatha heleios Hormiga, 1990

(Figs. 223-230, 304)

Glenognatha heleios Hormiga, in Hormiga \& Döbel, 1990: 197, figs. 1-14 (male holotype from Tuckerton, Ocean Co., New Jersey, United States of America, 1.xi.1984, H. Döbel leg., deposited in USNM, examined; paratypes from the same

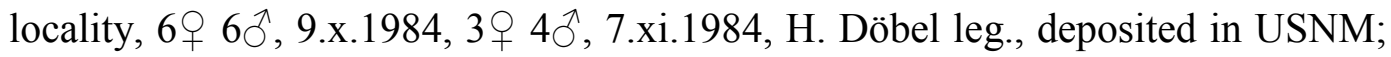
1 ㅇ 1ð̂, 9.x.1984, H. Döbel leg., deposited in AMNH; 1 + 1今̂, 9.x.1984 H. Döbel leg., deposited in MCZ, all examined); Hormiga et al., 1995: 326, figs. 7H-I; Platnick, 2013.

Diagnosis: males of G. heleios can be easily distinguished from all other Glenognatha species (except G. iviei) by the presence of a small pointed apophysis on the paracymbium (Figs. 226B, 230H); distinguished from G. iviei by the slightly curved Prt 1 (Figs. 225A) and by the uncoiled embolus distal portion (Figs. 227A, 230E-F). Females are distinguished from the very similar species $G$. foxi by the absence of CFO (Fig. 225D-F) and from G. sp. nov. 3 by having four retromarginal teeth (Fig. 225F).

Description: male and female described by Hormiga \& Döbel (1990), additional data are presented here: habitus as in figures 223-224. Male and female with three promarginal and four retromarginal teeth (Fig. 225). Male chelicerae with anterior tooth and CFO (Fig. 225A). Palp as in figures 226-227, 230. Conductor retrolateral apophysis rounded (227B-C). Embolus curved prolaterally (Figs. 227A, D, 230E). Female tracheal system as in figure 229. Internal genitalia with well-developed spermathecae (Figs. 228). Uterus externus sac large, with a conspicuous constriction at its base (Fig. 228C, arrow).

Variation: see Hormiga \& Döbel, (1990).

Distribution: known in New Jersey state in United States of America (Fig. 304).

Additional material examined ( $\mathbf{N}=18)$ : UNITED STATES OF AMERICA: New Jersey: Ocean Co., Tuckerton, [39³6’ N, 74²0W], elev. [1m], 7.xi.1984, H. Döbel 
leg., 4ð 7 (USNM); 28.vii.1984, H. Döbel leg., $3 \widehat{\jmath}$ (USNM); 25.ix.1984, H. Döbel leg., $2 \hat{\jmath}$ (USNM).

New records: UNITED STATES OF AMERICA: New Jersey: Oceanville, [39² $2{ }^{`} \mathrm{~N}$, $74^{\circ} 27^{\prime} \mathrm{W}$ ], elev. [12m], 26.ix.1949, P. F. Springer leg., $191 \delta^{\Uparrow}$ (AMNH).

\section{Glenognatha sp. nov. 1}

(Figs. 231-238, 298A, 299A, 302A, 312)

Type material: Male holotype and female paratype from Barragem do Rio Paraitinga, Salesópolis, São Paulo, Brazil, [2332'5.42”S, 4546’56.62”W], elev. [870m], 1118.vi.2002, Eq. IBSP leg., deposited in IBSP 163681; paratypes: $13 ð 10$ from the type locality, 11-18.vi.2002, Eq. IBSP leg., deposited in IBSP 147861; $1 \overbrace{}^{\Uparrow}$ from Reserva Natural da Cachoeira, Antonina, Paraná, Brazil, [25²4`2,4”S, 48 $41^{\prime} 18.75^{\prime} \mathrm{W}$ ], elev. [100m], 28.vi-2.vii.2003, H. Hofer et al. leg., deposited in IBSP 131182; $1 \overbrace{}^{\Uparrow} 2$ from Estaçao Braskem de Proteçao Ambiental, Triunfo, Rio Grande do

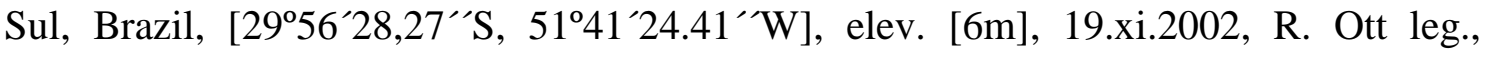
deposited in MCN 34952; $1 \widehat{\delta} 19$ from Parque do Estado de São Paulo, São Paulo, São

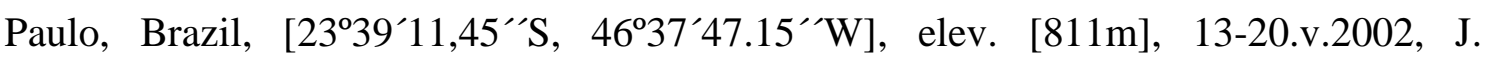
Valvassori leg., deposited in IBSP 59239.

Diagnosis: Males of $G$. sp. nov. 1 can be easily distinguished from all other Glenognatha species by the bifurcated embolus tip (Figs. 235D, 238C). Females resemble those of $G$. australis by the bean-shaped spermathecae (Fig. 236); distinguished from the latter species by having the Prt1 and Prt 2 subeaqual in size (Fig. 233D).

Description: Male (Holotype): Habitus as in figure 231. Carapace brown, darker along furrows. Sternum brown with darker edges. Abdomen oval, with two irregular dusky bands that join medially in the posterior region. Silver guanine spots scattered in two longitudinal bands dorsally and in lateral surfaces. Venter with a median dusky band that ramifies laterally from posterior tracheal spiracle, guanine silver spots absent. Legs pale yellow-brownish. Tracheal spiracle well-sclerotized. Total length 2.20. Cephalothorax 1.00 long, 0.81 wide, 0.72 high. Abdomen 1.25 long, 1.00 wide, 1.00 high. Sternum 0.52 long, 0.55 wide. Eye diameters and interdistances: AME 0.09. ALE 0.08. PME 0.10. PLE 0.08. AME-AME 0.10, AME-ALE 0.09, PME-PME 0.11, PME- 
PLE 0.09, ALE-PLE 0.01. Clypeus height $2.11 \times$ AME diameter. Chelicerae brownish. Three promarginal and four retromarginal teeth (Figs. 233A-C). Ret 1 on the posterior surface (Fig. 233B). Leg measurements: I: femur 1.50, patella 0.37, tibia 1.55, metatarsus 1.25, tarsus 0.72, total 5.40; II: 1.30, 0.37, 1.28, 1.00, 0.65, 4.61; III: 0.97, 0.25, 0.72, 0.65, 0.30, 2.90; IV: 1.25, 0.25, 1.05, 0.90, 0.47, 3.92. DTS 0.16. DTE 0.52. ALS as in figure 299A. Palp as in figures 234-235, 238. Conductor lamina apically rounded (Figs. 235B-C). Embolus tip bifurcated (Figs. 235D, 238C).

Female (Paratype IBSP 147809): Coloration and general habitus as in male (Fig. 232). Tracheal spiracle well-sclerotized. Total length 2.55. Cephalothorax 1.08 long, 0.93 wide, 0.8 high. Abdomen 1.45 long, 1.30 wide, 1.32 high. Sternum 0.6 long, 0.65 wide. Eye diameters and interdistances: AME 0.09. ALE 0.08. PME 0.09. PLE 0.08. AMEAME 0.08, AME-ALE 0.08, PME-PME 0.11, PME-PLE 0.11, ALE-PLE 0.01. Clypeus height $2.10 \times$ AME diameter. Chelicerae brownish, with three promarginal and four retromarginal teeth (Figs. 233D-F). Leg measurements: I: femur 1.52, patella 0.37, tibia 1.45, metatarsus 1.30 , tarsus 0.75 , total 5.40; II: $1.30,0.37,1.25,1.17,0.62$, 4.72; III: 1.07, 0.27, 0.62, 0.68, 0.38, 3.05; IV: 1.25, 0.27, 1.05, 0.92, 0.50, 4.00. DTS 0.18. DTE 0.60. ALS and PLS as in figures 298A, 302A. Tracheal system as in figure 237. Internal genitalia with bean-shaped spermathecae (Fig.236). Uterus externus sac divided into two disc-shaped portions (Fig. 236A).

Variation: Male total length 1.7-2.5 ( $N=10$; average 2.1), cephalothorax length 0.9-1.2 $(N=10$; average 1.0), femur I length 1.2-1.5 $(N=10$; average 1.4). Female total length 2.1-3.2 ( $N=10$; average 2.5), cephalothorax length 1.0-1.3 $(N=10$; average 1.1$)$, femur I length 1.1-1.5 ( $N=10$; average 1.3).

Distribution: Known from the states of São Paulo, Paraná and Rio Grande do Sul in Brazil (Fig. 312).

Additional material examined (N =1096): BRAZIL: São Paulo: São Paulo, Parque do

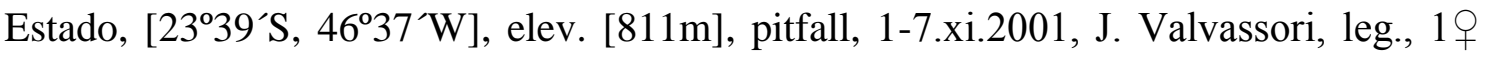
(IBSP 59154); $1 \hat{\jmath}$ (IBSP 59155); 1 (IBSP 59233); $1 \hat{\jmath}$ (IBSP 59234); $2 \hat{\jmath}$ (IBSP

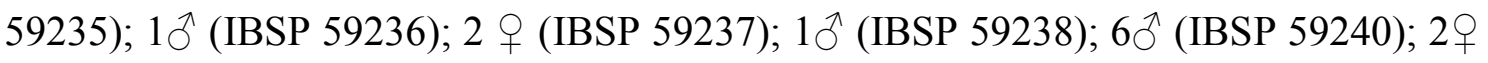
(IBSP 59242); 1ð (IBSP 59243); 13-20.v.2002, 1 (IBSP 59244); $2 \Uparrow 2$ (IBSP 
59245); $1 q$ (IBSP 59246); $1 ð$ (IBSP 59247); 19 (IBSP 59248); 1 (IBSP 59250);

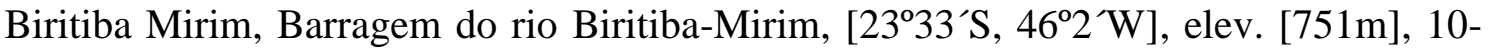
15.v.2003, Equipe Biota leg., 19 (IBSP 117427); 19 (IBSP 117428); 1 immature (IBSP

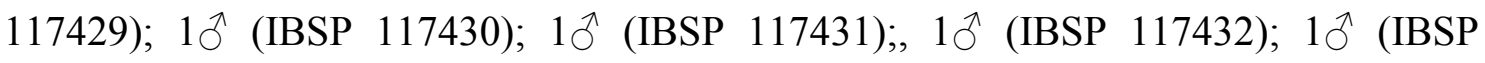
117433); $1 \lesssim$ (IBSP 117434); 1 immature (IBSP 117435); 1 immature (IBSP 117436); $1{ }^{\Uparrow}$ (IBSP 117437); $1 \delta^{\Uparrow}$ (IBSP 117438); 19 (IBSP 117439); 1 immature (IBSP 117440); 1 우 (IBSP 117441); 1 immature (IBSP 147790); 10-15.v.2003, 3 ㅇ (IBSP 147791); 1015.v.2003, $2 \widehat{\jmath}$ (IBSP 147792); 10-15.v.2003, 1ð 1ণ (IBSP 147793); Salesópolis,

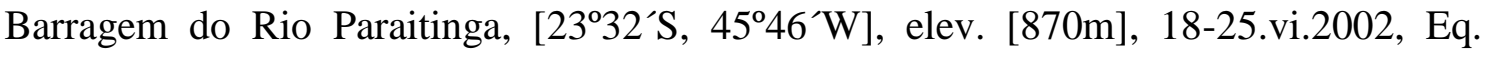
IBSP leg., 1 (IBSP 146596); 1ð (IBSP 147309); $1 \uparrow$ (IBSP 147311); 11-18.vi.2002,

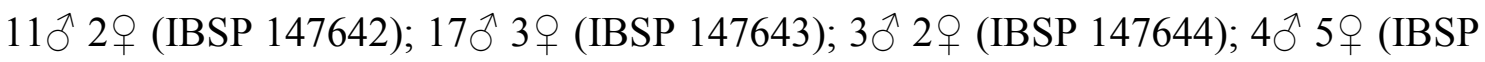
147645); $2 \hat{\jmath} 10$ (IBSP 147646); $10 \hat{\jmath} 4$ ㅇ (IBSP 147647); $5 \hat{\jmath} 7 q$ (IBSP 147648); $4 \hat{\jmath}$ $13 q$ (IBSP 147649); $90 \hat{0} 6$ (IBSP 147650); 13ð 19 (IBSP 147651); $7 ð 2 q$ (IBSP

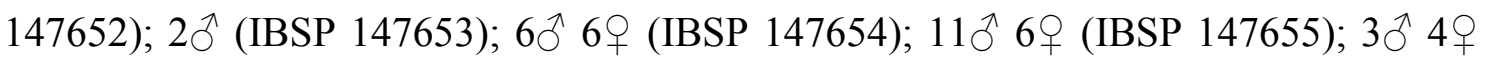

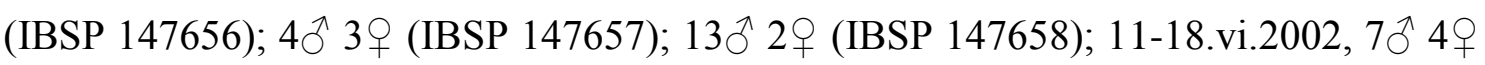

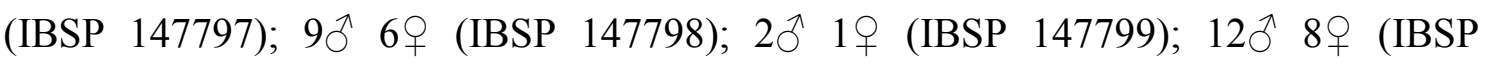

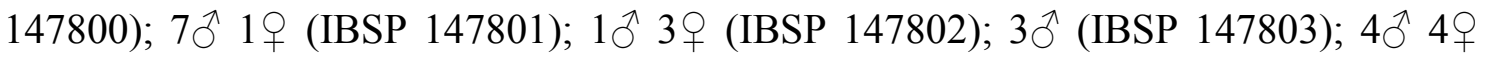
(IBSP 147804); $5 \hat{\jmath} 6$ ㅇ (IBSP 147805); $3 \hat{\jmath} 4$ 우 (IBSP 147806); $2 \hat{\jmath} 1 ㅇ$ (IBSP 147807);

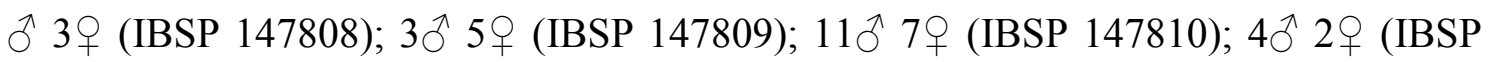
147811); $11 \hat{\jmath} 5$ ㅇ (IBSP 147812); $3 \hat{\jmath} 2$ ㅇ (IBSP 147813); $7 \hat{\jmath} 4$ ㅇ (IBSP 147814); $6 \hat{\jmath}$

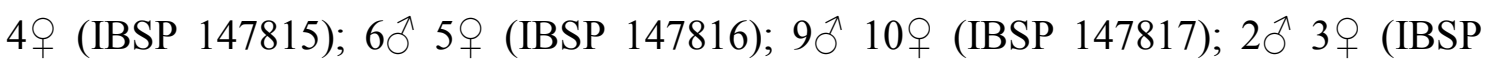
147818); $8 \hat{\delta} 4$ ㅇ 1 immature (IBSP 147819); $9 \hat{\jmath} 7$ ㅇ 1 immature (IBSP 147820); $7 \hat{o} 1 q$ (IBSP 147821); $2 \hat{\jmath}$ 5ㅇ (IBSP 147822); $5 \hat{\jmath} 2$ 우 (IBSP 147823); 1 (IBSP 147824); $3 \hat{\jmath}$ 32 immature (IBSP 147825); $9 \hat{0} 691$ immature (IBSP 147826); 12へ 17우 (IBSP

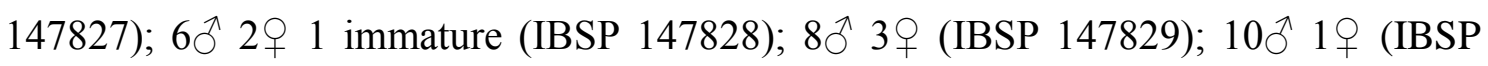
147830); $28 \hat{\jmath} 29 q 1$ immature (IBSP 147831); $6 \hat{\jmath} 5$ (IBSP 147832); $6 \AA 80$ (IBSP

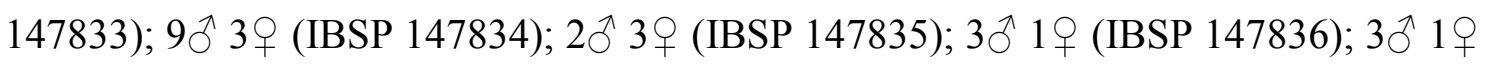
(IBSP 147837); $4 \hat{\sigma} 6 q$ (IBSP 147838); $10 \hat{\diamond} 4 q$ (IBSP 147839); $4 \hat{\jmath} 3 q$ (IBSP

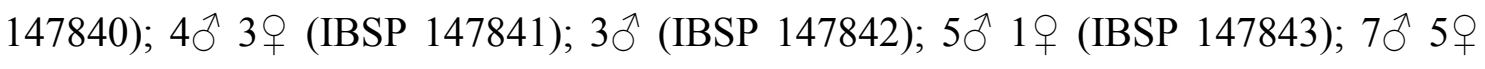
(IBSP 147844); $5 \hat{\jmath} 8$ ㅇ (IBSP 147845); $2 \hat{\jmath}$ (IBSP 147846); $9 \hat{\jmath} 1$ 우 (IBSP 147847); $9 \hat{\jmath}$

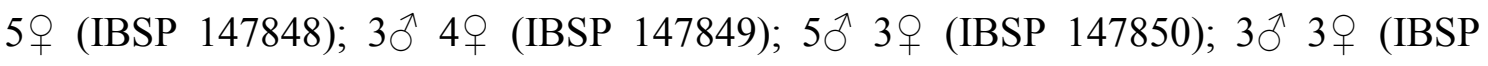

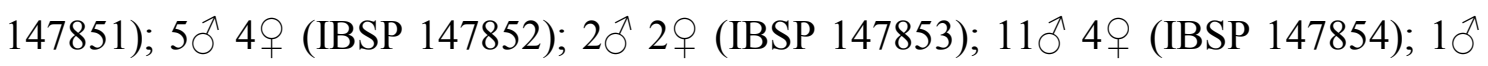

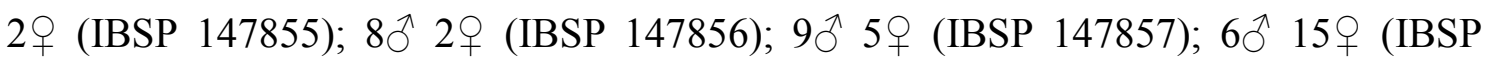
147858); $6 \overbrace{}^{\Uparrow} 2$ (IBSP 147859); $13 \overbrace{}^{\Uparrow} 8$ (IBSP 147860); $12 \hat{\jmath} 4 q$ (IBSP 147862); $12 \hat{\jmath}$ 
4 (IBSP 149115); $4 \hat{0} 10$ (IBSP 149202); $9 \hat{0} 6$ 6우 (IBSP 149203); $2 \hat{0}$ (IBSP

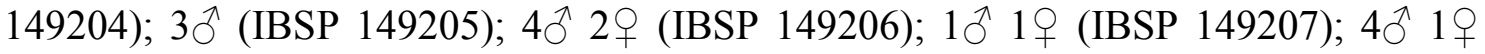
(IBSP 149208); $4 \hat{\delta} 4$ 우 (IBSP 149209); $3 \hat{\jmath} 4$ 우 (IBSP 149210); $7 \hat{\jmath} 6$ 우 (IBSP 149211); $3 \hat{\jmath} 3 q$ (IBSP 149212); $7 \hat{\jmath} 13 q$ (IBSP 149213); $13 \hat{\jmath} 3 q$ (IBSP 149214); $7 \hat{\jmath} 3 q$ (IBSP

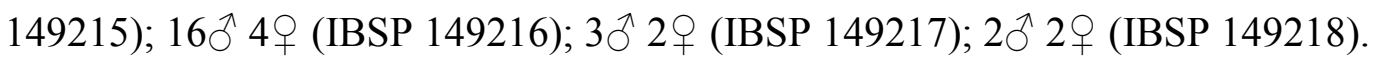

Glenognatha hirsutissima (Berland, 1935)

(Figs. 239-244, 315)

Hivaoa hirsutissima Berland, 1935: 52, fig. 20-23 (female holotype from Teavaituhai, Uapou, Marquesas Islands, 30.xi.1931, deposited in Bernice Bishop Museum, Honolulu, lost).

Glenognatha hirsutissima: Levi 1980: 64; Platnick, 2013.

Diagnosis: males and females of G. hirsutissima can be easily distinguished from all other Glenognatha species by the presence of spines on the ventral surface of femur III (Figs. 240C).

\section{Description:}

Male (Marquesas Islands, Nuku Hiva, USNM): habitus as in figure 239. Carapace yellow-brownish, with two dusky lateral bands. Sternum light yellow, darker on edges. Abdomen oval, dorsally with irregular pattern of longitudinal dusky bands that extend to the lateral surface. Thin transvers bands on the posterior region. Silver guanine spots scattered in the dorsal and lateral surfaces. Venter greyish with scattered silver guanine spots. Legs light yellow. Total length 2.45. Cephalothorax 1.17 long, 0.87 wide, 0.75 high. Abdomen 1.37 long, 0.87 wide, 0.85 high. Sternum 0.62 long, 0.57 wide. Eye diameters and interdistances: AME 0.07. ALE 0.05. PME 0.09. PLE 0.05. AME-AME 0.09, AME-ALE 0.09, PME-PME 0.11, PME-PLE 0.11, ALE-PLE 0.01. Clypeus height $3.21 \times$ AME diameter. Chelicerae with a strong macrosetae (Fig. 241A). Three promarginal and four retromarginal teeth (Figs. 241A-C). Leg measurements: I: femur 1.50, patella 0.37 , tibia 1.50 , metatarsus 1.45 , tarsus 0.37 , total 5.20 ; II: $1.42,0.35,1.50$, 0.92, 0.30, 4.50; III: 1.02, 0.32, 0.80, 0.72, 0.25, 3.12; IV: 1.37, 0.27, 1.17, 1.22, 0.30, 4.35. Femur III with a ventral row of strong macrosetae. DTS 0.20. DTE 0.45. Palp as in figures 242-243. Embolus and conductor slender (Figs. 242A, 243A). 
Female (Marquesas Islands, Nuku Hiva, USNM): Habitus and general coloration as in male (Fig. 240). Venter of abdomen with thin transverse dusky band originating from the tracheal spiracle towards the anterior region. Light patch anterior to the gonopore. Total length 2.87. Cephalothorax 1.20 long, 1.05 wide, 0.75 high. Abdomen 1.75 long, 1.45 wide, 1.50 high. Sternum 0.65 long 0.62 wide. Eye diameters and interdistances: AME 0.08. ALE 0.05. PME 0.09. PLE 0.06. AME-AME 0.06, AME-ALE 0.1, PMEPME 0.11, PME-PLE 0.11, ALE-PLE 0.01. Clypeus height $3.12 \times$ AME diameter. Chelicerae with a strong macrosetae (Fig. 240D) Three promarginal and four retromarginal teeth. (Figs. 241D-F). Leg measurements: I: femur 1.47, patella 0.45, tibia 1.50, metatarsus 1.17 , tarsus 0.27 , total 4.87 ; II: 1.42 , 0.45, 1.42, 1.10, 0.25, 4.65; III: 1.00, 0.25, 0.77, 0.82, 0.22, 3.07; IV: 1.47, 0.30, 1.20, 1.30, 0.27, 4.55. DTS 0.25. DTE 0.35. Femur I, II and III with a ventral row of strong macrosetae. Uterus externus chamber projected around uterus externus sac (Fig. 244).

Distribution: known only from Marquesas Islands (Fig. 315).

Remarks: Although the female holotype of G. hirsutissima (presumably lost) was not examined, a vial from USNM ( $2 \hat{\jmath} 1 \uparrow 2$ immature) collected near the type locality (30 $\mathrm{km} \mathrm{N}$ ) was available for study. After a careful comparison with the original description (Berland, 1935), the female specimen was identified as G. hirsutissima considering the presence of conspicuous spines on legs, palp and chelicerae as depicted by Berland (1935, figs. 21-23). Males examined also present such spines (Figs. 239, 241A). According with the original descriptions of the remaining Polynesian Glenognatha (Berland, 1933, 1935, 1942) and all the material examined in this work, the presence of these spines is autapomorphic for G. hirsutissima. Since the identity of the species is not in doubt the designation of a neotype is not necessary (ICZN, Art. 75).

Additional material examined ( $\mathrm{N}=5$ ): FRENCH POLYNESIA: Marquesas Islands: Nuku Hiva, Tekao Ridge, 851`39”S, 140¹0`24”'W, elev. 400m, 21.x.1999, D. A. \& J. T. Polhemus leg., $2 \lesssim 1 q 2$ immature (USNM). 
Glenognatha australis (Keyserling, 1883)

(Figs. 245-253, 297C, 298D, 299C, 300C, 301B, 302D, 303B, 307)

Pachygnatha australis Keyserling, 1883: 211 (syntypes from Pacasmayo, Peru, deposited in BMNH, examined through photographs of the habitus and male genitalia).

Glenognatha australis: Simon, 1894: 716; Platnick, 2013.

Diagnosis: males of G. australis resemble those of G. gloriae and G. spherella by the prominent Ret2 (Fig. 248B) and the absence of anterior tooth (Fig. 248A). It can be distinguished from the former by the shape of the clypeus in lateral view, which is not projected (Fig. 246B) and from the latter by the bigger size and the uncoiled embolus tip (Figs. 250D, 253C). Females resemble those of $G$. sp. nov. 1 by the bean-shaped spermathecae, but are distinguished from the latter by the enlarged Prt2 (Fig. 248D).

Description: Male (CAS 9039650): habitus as in figures 245A-B. Carapace brown, darker along furrows. Sternum pale brown, darker on edges. Abdomen tan, dorsally with two diffused longitudinal black bands that join medially forming thin transverse bands in the posterior region. Venter with a median diffused black band that ramifies to the lateral surfaces from the tracheal spiracle. Silver guanine spots absent. Legs pale tan with distal parts of segments darker. Total length 2.47. Cephalothorax 1.17 long, 0.97 wide, 0.57 high. Abdomen 1.47 long, 1.20 wide, 1.12 high. Sternum 0.57 long, 0.65 wide. Eye diameters and interdistances: AME 0.10. ALE 0.09. PME 0.09. PLE 0.10. AME-AME 0.06, AME-ALE 0.06, PME-PME 0.08, PME-PLE 0.10, ALE-PLE 0.01. Clypeus height $1.80 \times$ AME diameter. Three promarginal and four retromarginal teeth. Ret2 enlarged, with a subapical pointed projection. Leg measurements: I: femur 1.65, patella 0.42 , tibia 1.72 , metatarsus 1.35 , tarsus 0.80 , total 5.95 ; II: $1.52,0.42,1.5,1.15$, 0.52, 5.12; III: $1.02,0.30,0.75,0.80,0.45,3.32$; IV: 1.35, 0.32, 1.27, 1.10, 0.57, 4.62. DTS 0.15. DTE 0.50. Epiandrous fusules as in figure 297C. Spinnerets as in figures 299C, 301B, 303B. Palp as in figures 249-250, 253. Conductor lamina with thin distal end in ventral view (Fig. 250B). Embolus tip curved (Figs. 250D, 253C-E).

Female (CAS 9039650): coloration and general habitus as in male (Figs. 245C-D). Cephalothorax, abdomen and legs clearer than the male. Abdomen without clearly defined black bands. Silver guanine spots absent. Total length 2.65. Cephalothorax 1.30 
long, 0.95 wide, 0.75 high. Abdomen 1.55 long, 1.25 wide, 1.25 high. Sternum 0.57 long, 0.67 wide. Eye diameters and interdistances: AME 0.09. ALE 0.08. PME 0.08. PLE 0.08. AME-AME 0.07, AME-ALE 0.08, PME-PME 0.09, PME-PLE 0.11, ALEPLE 0.01. Clypeus height $1.80 \times \mathrm{AME}$ diameter. Three promarginal and four retromarginal teeth. Leg measurements: I: femur 1.62, patella 0.42, tibia 1.50, metatarsus 1.25 , tarsus 0.75 , total 5.55. II: 1.62 , $0.37,1.50,1.22,0.57,5.30$; III: 1.07 , 0.35, 0.75, 0.77, 0.50, 3.45; IV: 1.55, 0.35, 1.27, 1.22, 0.57, 4.97. DTS 0.17. DTE 0.40. Female tracheal system as in figure 252. Spinnerets as in figures 298D, 300C, 302D. Internal genitalia with well-developed spermathecae, bean-shaped (Fig. 251). Uterus externus sac divided into two disc-shaped regions (Fig. 251A).

Variation: male total length 1.8-2.6 $(N=10$; average 2.2), cephalothorax length 1.0-1.1 $(N=10$; average 1.0), femur I length 1.0-1.6 $(N=10$; average 1.3). Female total length 2.2-3.0 $(N=10$; average 2.6), cephalothorax length 1.0-1.3 $(N=10$; average 1.1$)$, femur I length 1.1-1.6 $(N=10$; average 1.2). Specimens from southern Brazil and Argentina exhibit a different coloration pattern (Figs. 246-247). In addition, the Ret 2 of the males lacks a pointed projection (Figs. 245A-C).

Distribution: known in northwestern Peru, northern Argentina and Brazil (Fig. 307).

Additional material examined (N = 295): New records: ARGENTINA: Buenos Aires: Campana, Estación Río Luján, [349’S, 5856`W], elev. [4m], C.J. Grismado, L. Damer, N. López, S. Trivero, I. Crudele \& N. Olejnik leg., 10 1 ㅇ (MACN 12382); Campana, C. J. Grismado, I. Crudele, N. Olejnik \& S. Trivero leg., $1 q$ (MACN 12557); Campana, C. J. Grismado, L. Damer, N. López, S. Trivero, I. Crudele \& N. Olejnik leg., 1 \% (MACN 12480); Delta del Paraná, Arroyo Sarmiento, [34²``S, 58³1`W], elev. [10m],

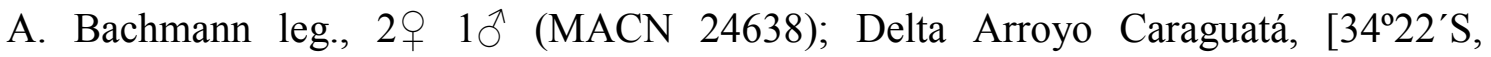
58 38 'W], elev. [4m], A. Bachmann leg., 2 (MACN 24633a); Paraná de las Palmas,

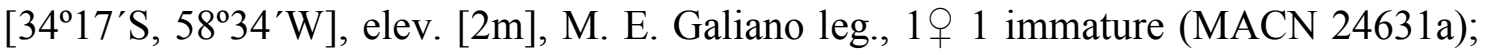

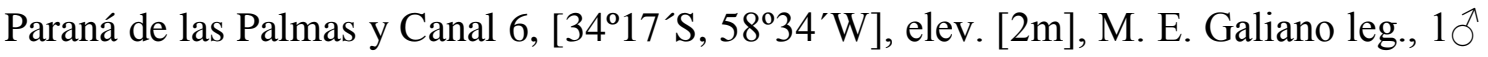
(MACN 24628a); Delta, Estación Experimental INTA, [342``S, 58³8`W], elev. [4m], A. Bachmann leg., 1 ㅇ $1 \hat{O}$ (MACN 24621); Campana, Reserva Natural Otamendi, Estación río Luján, 34.277352S, 58.888054W, elev. 5m, M. Ramírez, C. Grismado, L. Piacentini, E. Soto, M. E. González-Márquez, M. Guala, J. M. Andia Navarro, N. 
López, I. Ortega Insaurralde y P. Zermoglio leg. (pastizal y bañado) $7 \uparrow 13 \widehat{\overbrace{}}$ (MACN); Ciudad Autónoma de Buenos Aires: Laguna de los Macáes, 34³6”21.7”S, $58^{\circ} 20^{\prime} 55^{\prime \prime} \mathrm{W}$, elev. [5m], 1.ix.2008, M. Turienzo leg., (Trampa de caída) 1ठ (MACN);

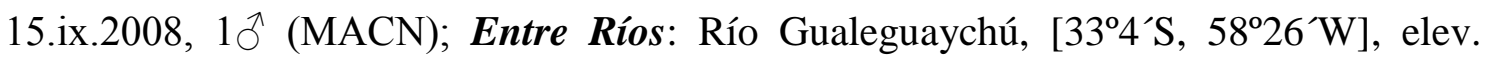
[6m], M. J. Ramírez \& P. A. Goloboff leg., 1 (MACN 24624); Jujuy: P. N Calilegua,

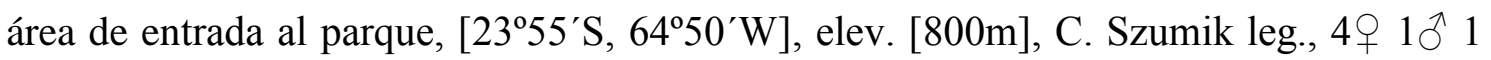
immature (MACN 24629); M. J. Ramírez, P. A. Goloboff \& C. Szumik leg., $393 \hat{\jmath} 1$

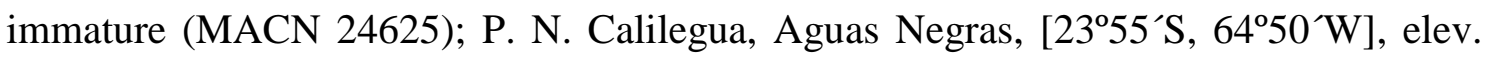
[800m], M.J. Ramírez \& L. Compagnuccileg., $1 \overbrace{}^{\Uparrow}$ (MACN 24627); Misiones: P. N.

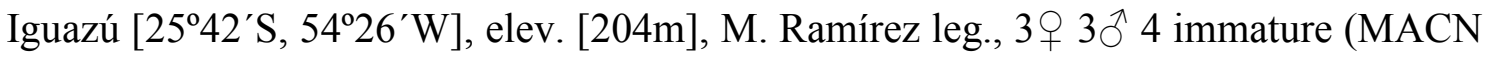
24627); Misiones y Corrientes: [27º $\left.30^{\prime} \mathrm{S}, 5^{\circ}{ }^{\circ}{ }^{`} \mathrm{~W}\right]$, elev. [100m], 11-21.iv.1989, 1 1

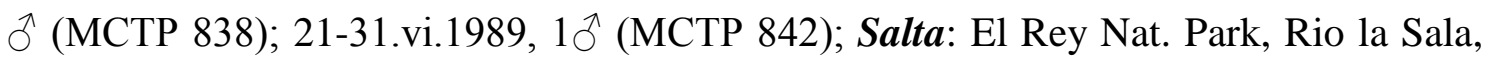

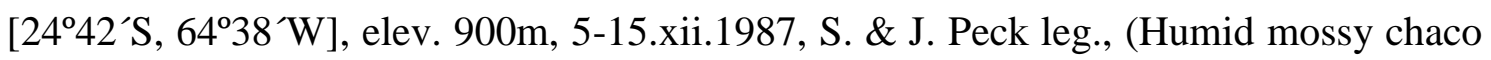
forest, Malaise FIT 87-137), 1ठ (AMNH); Santa Fe: Las Gamas, $20 \mathrm{~km} \mathrm{~W} \mathrm{de} \mathrm{Vera,}$

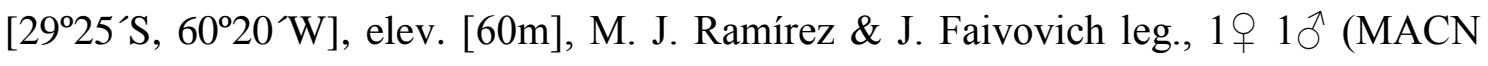
24623); Tucumán: Horco Molle, [2646’S, 65¹9`W], elev. [775m], M. E. Galiano leg., $3 \uparrow 4 \AA$ (MACN 24622). BOLIVIA: Beni: Estación Biológica Beni, [14³8`S, 66 ${ }^{\circ} 17^{`} \mathrm{~W}$ ], elev. [187m], 9.ix.1987, S. Larcher leg., 1q (USNM). BRAZIL: Ceará:

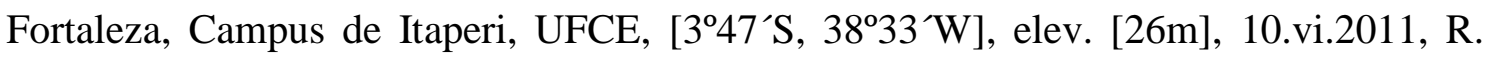

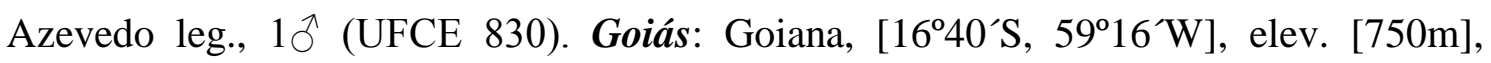
12.vii.1957, B. Malkin leg., $1 \overbrace{}^{\Uparrow}$ (AMNH). Paraná: Guaraqueçaba, Serra do Itaqui,

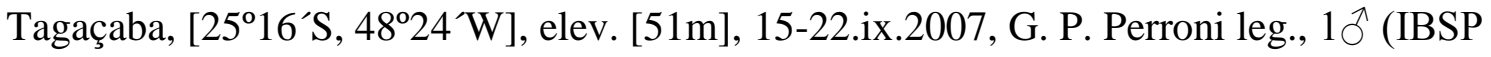

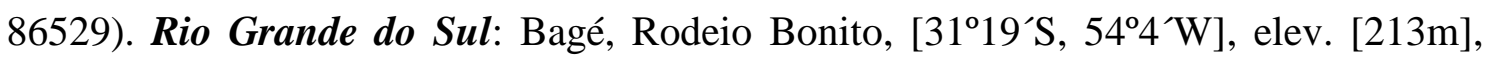
10.ii.1967, C. Oliveira leg., $1 \bigcirc^{\Uparrow}$ (MCN 489); Cachoeira do Sul, [300’S, 5258`W], elev. [29m], 2.iv.1977, M. E. L. Souza leg., $1 ð$ (MCN 5240); 13.ix.1996, 1ð (MCTP 9309); Canoas, Banhado Paquetá, Parque Estadual Delta do Jacuí, [2959`S, 51²9`W], elev. [15m], 8.vii.1999, A. B. Bonaldo leg., 3 + (MCN 31331); El dorado do Sul, [305’S, 51³6’W], elev. [36m], 14.i.2009, L. Fritz leg., 1 § (MCN 45914); 9.i.2008, L. Fritz

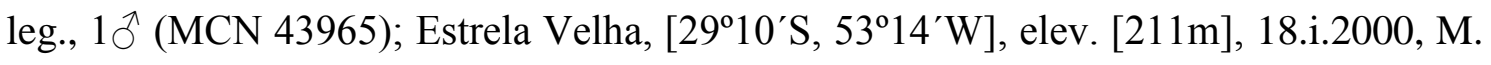

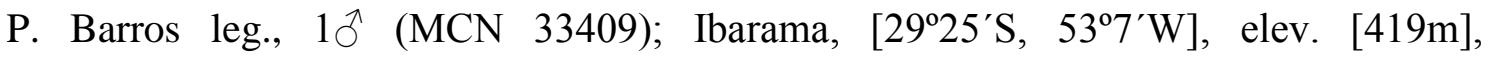
27.xi.1999, C. B. Kotzian leg., 1 $10^{\Uparrow}$ (MCTP 11248); Monte Negro, [29²41'S, 51²8`W], elev. [206m], 7.vii.1977, E. H. Buckup leg., $1 \delta^{\circledR}$ (MCN 6145); Nova Santa Rita, Banhado Paquetá, Parque Estadual Delta do Jacuí, [2959`S, 51²9`W], elev. [15m], 8.vii.1999, I. Heidrisch leg., $1 \overbrace{}^{\Uparrow}$ (MCN 31372); Novo Cabrais, Parque Witeck, 
[294`S, 5257`W], elev. [77m], 1.xi.2008, R. G. Buss leg., $1 \overbrace{}^{\Uparrow}$ (MCTP 27990); Pirapó,

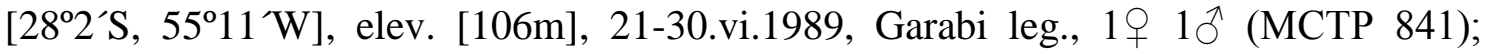

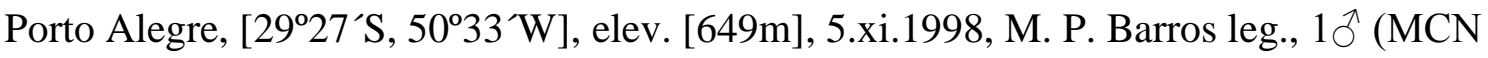

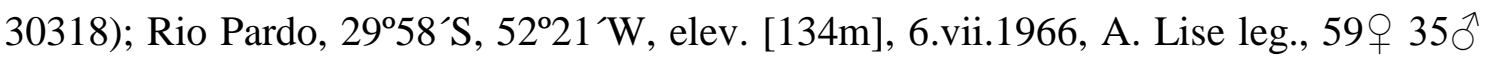
(MCN 267); 20.ii.1967, A. Lise leg., 2ð (MCN 562); 6.vii.1966, A. Lise leg., 24 q 18 ○ 2 immature (MCZ 125524); Salvador Sul, [29²6`S, 51³0`W], elev. [485m], 1964, Padre Celio leg., 1 † 2 ô (MZSP 4051); São Francisco de Paula, [29²6’S, 50³9`W],

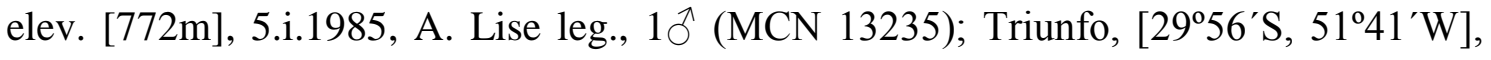
elev. [6m], 4.vi.1987, C. Marros leg., 1 ( (MCN 16935); 21.ix.1989, E. H. Buckup leg.,

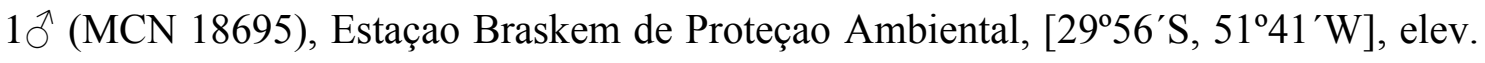
[6m], 15.ix.2009, P. E. S. Rodrigues leg., $1 q 1{ }^{\Uparrow}$ (MCN 46671); 31.viii.2007, M. C. Pairet Jr. leg., $1 \bigcirc^{\Uparrow}(\mathrm{MCN}$ 46804); 15.ix.2009, E. N. L. Rodrigues leg., $2 \overbrace{}^{\Uparrow}(\mathrm{MCN}$ 46622); 19.xi.2002, R. Ott leg., $1 q$ (MCN 48508); 14.vi.2006, R. Ott leg., $1 q 1 \sigma^{\Uparrow}$ (MCN 42102), Fazenda Pontal, Parque Estadual Delta do Jacuí, [2959’S, 51²9`W], elev. [6m], 22.vi.1999, A. B. Bonaldo leg., $1 ð$ (MCN 31244); Uruguaiana, [2944’S,

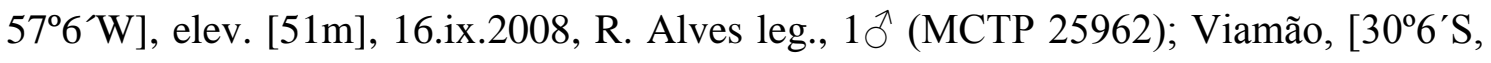

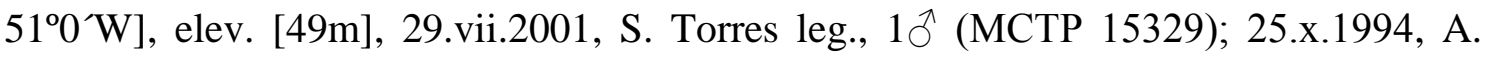

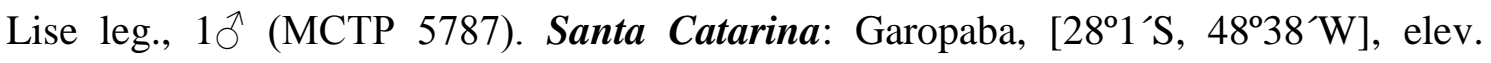
[18m], 22.x.1993, M. P. de Barros leg., 1ठ઼ (MCTP 4304). São Paulo: Paranapiacaba,

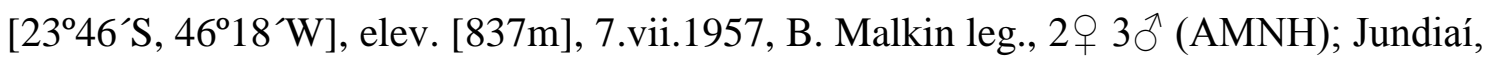

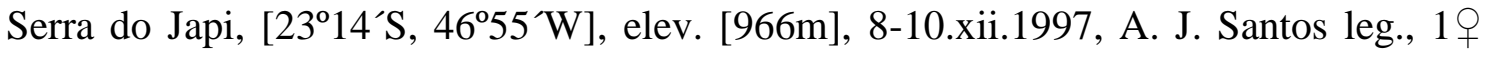
(IBSP 36559); São Paulo, Jaraguá, [23²7’S, 46²5`W], elev. [891m], 8.vii.1994, R. Baptista leg., $11 q 7 ð 2$ immature (MNRJ 3495). ECUADOR: Guayas: Nobol (5.5km S), [1 $\left.{ }^{\circ} 54^{\prime} \mathrm{S}, 80^{\circ} 0^{`} \mathrm{~W}\right]$, elev. [6m], 18.i.1978, P. J. Spangler \& J. Anderson leg., 19 (USNM); Boliche (14.5Km S), [2 $\left.12^{\prime} \mathrm{S}, 79^{\circ} 35^{\prime} \mathrm{W}\right]$, elev. [20m], 14.i.1978, P. J. Spangler \& J. Anderson leg., $1 \delta^{\Uparrow}$ (USNM). Los Ríos: Babahoyo, [148`S, 79³2`W], elev. [11m], 21.vi.1975, Lohen, Langley \& Monnig leg., $1 ð$ (USNM). PARAGUAY: Central: Itá, [2530`S, 57²1 W], elev. [141m], 12.iv.1980, P. J. Spangler leg., 1 \%

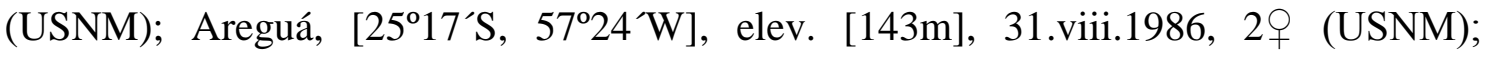

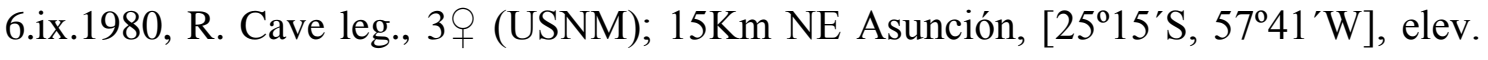
[61m], 21.vi.1969, P. \& P. Spangler leg., 1 ○ (USNM). Cordillera: San Bernardino,

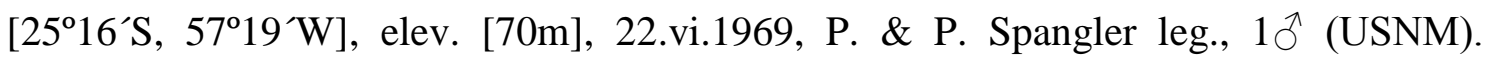
PERU: Piura: Quebrada Mogollón, [4³2`S, 81`W], elev. [700m], 24.ix.1939, HEF \& DLF leg., 1 1 1 (CAS 9039650). 
Glenognatha spherella Chamberlin \& Ivie, 1936

(Figs. 254-261, 306)

Glenognatha spherella Chamberlin \& Ivie, 1936: 66, pl. 17, fig. 154-156. (male holotype from Chilapa, Guerrero, Mexico, x.1929, female paratype from the type locality, both deposited in ZMB 26040, examined); Platnick, 2013.

Diagnosis: males of G. spherella resemble those of G. gloriae and G. australis by the prominent Ret2 (Fig. 256B) and the absence of anterior tooth (Fig. 256A). It can be distinguished from the former by the shape of the clypeus in lateral view, which is not projected (Fig. 254B) and from the latter by the smaller size and the coiled embolus tip (Figs. 258D, 261A-B). Females can be easily distinguished from all other Glenognatha species (except $G$. sp. nov. 4) by the presence of a postero-lateral cheliceral bulge (Figs. 256E-F); distinguished from G. sp. nov. 4 by the presence of spermathecae (Fig. 259).

Description: Male (CAS 9046859): habitus as in figure 254. Carapace yellowbrownish, darker along furrows, with a clear median square. Sternum with darker edges. Abdomen oval, dorsally with two irregular black bands that join medially, forming transverse thin bands. Silver guanine spots scattered in lateral surfaces. Venter with a median black band that ramifies laterally before the posterior tracheal spiracle, two guanine silver spots anterior to the spinnerets. Legs pale yellow-brownish. Total length 1.45. Cephalothorax 0.67 long, 0.57 wide, 0.50 high. Abdomen 0.80 long, 0.62 wide, 0.57 high. Sternum 0.35 long, 0.40 wide. Eye diameters and interdistances: AME 0.07, ALE 0.07, PME 0.08, PLE 0.05. AME-AME 0.05, AME-ALE 0.06, PME-PME 0.08, PME-PLE 0.07, ALE-PLE 0.01. Clypeus height $2.00 \times$ AME diameter. Chelicerae yellow-brownish, with three promarginal and four retromarginal teeth (Figs. 256A-C). Ret2 enlarged (Figs. 256B). Leg measurements: I: femur 1.00, patella 0.25, tibia 1.05, metatarsus 0.80 , tarsus 0.45 , total 3.91; II: 0.90, 0.22, 0.87, 0.67, 0.40, 3.07; III: 0.52, 0.20, 0.40, 0.45, 0.25, 1.82; IV: 0.85, 0.15, 0.71, 0.50, 0.28, 2.50. DTS 0.08. DTE 0.35. Palp as in figures 257-258, 261. Embolus distal portion coiled and pointed (Figs. 258D, 261A-B).

Female (CAS 9046857): coloration and general habitus as in male (Fig. 255). Cephalothorax, abdomen and legs darker than the male. Thin transverse bands formed in the posterior region of the abdomen. Total length 1.50. Cephalothorax 0.67 long, 0.62 
wide, 0.65 high. Abdomen 0.87 long, 0.77 wide, 0.80 high. Sternum 0.35 long, 0.41 wide. Eye diameters and interdistances: AME 0.07, ALE 0.06, PME 0.08, PLE 0.06. AME-AME 0.06, AME-ALE 0.07, PME-PME 0.09, PME-PLE 0.06, ALE-PLE 0.01. Clypeus height $1.7 \times$ AME diameter. Chelicerae brownish, with three promarginal and four retromarginal teeth (Figs. 256D-F). Leg measurements I: femur 0.82, patella 0.23, tibia 0.75 , metatarsus 0.67 , tarsus 0.42 , total 2.91 ; II: $0.72,0.18,0.60,0.60,0.37,2.48$; III: $0.6,0.15,0.42,0.33,0.25,1.76$; IV: $0.77,0.16,0.57,0.51,0.31,2.33$. DTS 0.07. DTE 0.36. Tracheal system as in figure 260. Internal genitalia as in figure 259. Spermathecae well-developed (Fig. 259).

Variation: male total length 1.3-1.6 $(N=10$; average 1.4), cephalothorax length 0.5-0.8 $(N=10$; average 0.7), femur I length 0.9-1.1 $(N=10$; average 1.0). Female total length 1.5-2.0 $(N=10$; average 1.6), cephalothorax length 0.6-0.8 $(N=10$; average 0.7$)$, femur I length 0.7-0.9 $(N=10$; average 0.8$)$.

Distribution: known from Mexico to Peru (Fig. 306).

Additional material examined $(\mathbf{N}=1252)$ : MEXICO: Guerrero: Chilapa, $\left[17^{\circ} 35^{\prime} \mathrm{N}\right.$, 99¹0`W], elev. [1400m], 29.x.1934, L. Schultze leg., 10ึ (AMNH).

New records: BELIZE: Cayo: Near Belmopan, hummingbird hwy., $\left[17^{\circ} 13^{`} \mathrm{~N}\right.$,

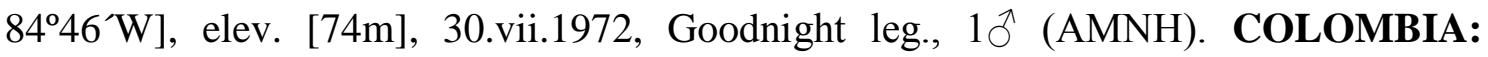

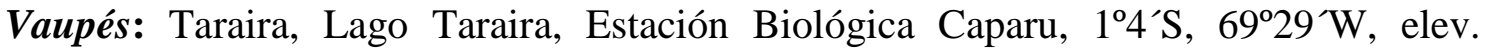
[128m], 11.iv.2004, J. Pinzón leg., $192 \widehat{~} \overbrace{}^{\Uparrow}$ (ICN 5978). COSTA RICA: Cartago:

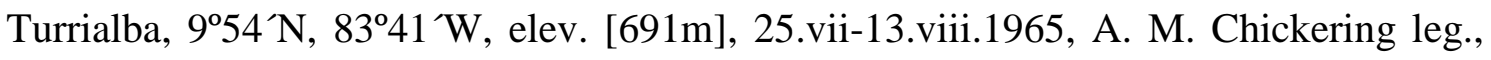

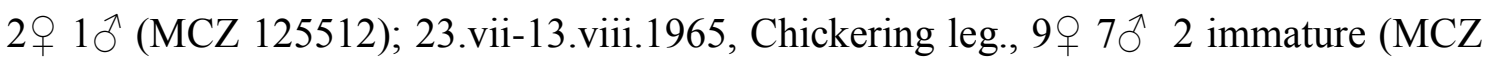

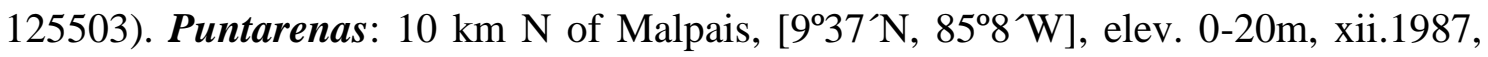

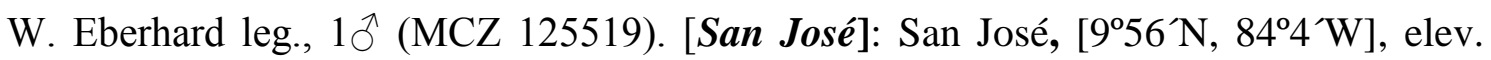

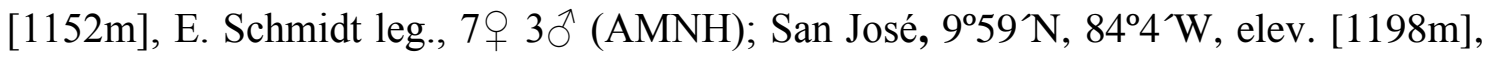

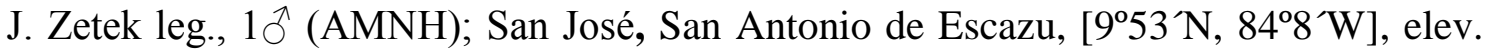
1300m, viii.1983, W. Eberhard leg., $1 ð$ (MCZ 125513); xii.1981, 1ð (MCZ 125514); vi.1983, 1 (MCZ 125474). GUATEMALA: Izabal: Los Amates, [15¹5`N, 895`W], elev. [89m], 1908, Kellerman leg., $1{ }^{\Uparrow}$ (MCZ 125479). MEXICO: Chiapas: Ocosingo, [16 $\left.6^{\circ} 54^{\prime} \mathrm{N}, 9^{\circ} 6^{\prime} \mathrm{W}\right]$, elev. [958m], 25.vi.1950, C. \& M. Goodnight, L. Stannard leg., 1 q

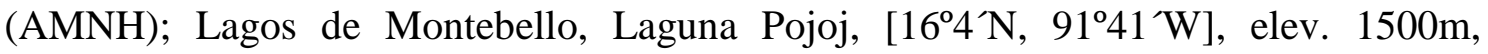




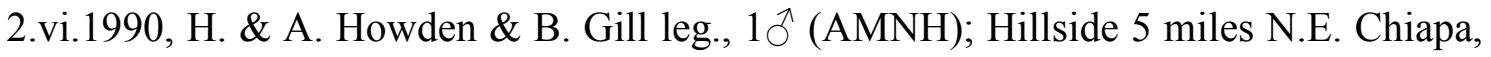
$16^{\circ} 45^{\prime} \mathrm{N}, 92^{\circ} 53^{\prime} \mathrm{W}$, elev. [1444m], 22.viii.1966, J.\& W. Ivie leg., 1 q (AMNH); Sumidero, Barranca, NE Tuxtla Gutierrez, [16 $\left.46^{\circ} \mathrm{N}, 93^{\circ} 5^{\prime} \mathrm{W}\right]$, elev. [648m], 19.viii.1966, J. \& W. Ivie leg., $1 \delta^{\Uparrow}$ (AMNH); Hillside 5 miles N.E. Chiapa, $16^{\circ} 45^{\prime} \mathrm{N}$, 92 $53^{\prime}$ W, elev. [1444m], 26.viii.1966, J. \& W. Ivie leg., 1 ( $(\mathrm{AMNH})$; 5800' Finca

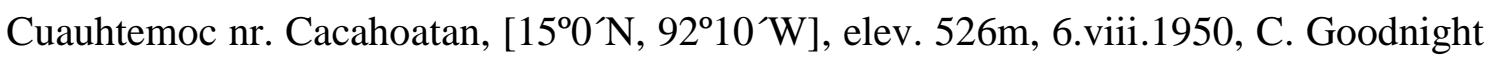

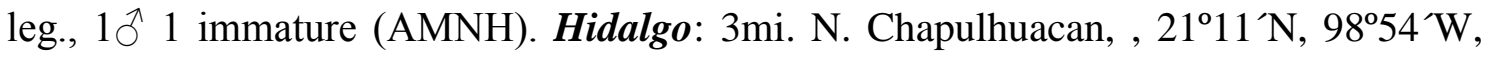
elev. [814m], 20.iv.1963, W. J. Gertsch \& W. Ivie leg., 1 ㅇ (AMNH). Michoacán: Tepetates, 19²${ }^{`}$ N, $100^{\circ} 45^{`}$ W, elev. [2428]m, 8.v.1963, W. J. Gertsch \& W. Ivie leg., $1 \delta^{\Uparrow}$ (AMNH) ; Zitacuaro, [19²5`N, 100²0`W], elev. [2011m], 27.vi.1936, Davis leg., 1 q (AMNH); Basencheve National Park, $19^{\circ} 25^{\prime} \mathrm{N}, 100^{\circ} 10^{\prime} \mathrm{W}$, elev. [2757m], 7.v.1963, W. J. Gertsch \& W. Ivie leg., $1 q 2{ }^{\Uparrow} 2$ immature (AMNH) ; Garnica Pass (summit,

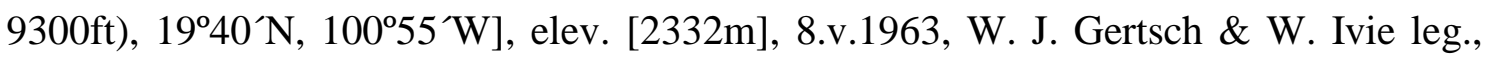

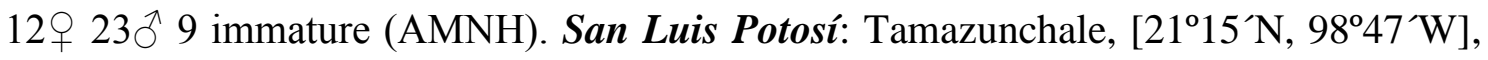
elev. [135m], 6.vii.1941, L. I. Davies leg., 1 (AMNH); 26.vi.1947, B. Malkin leg., 1 웅 $(\mathrm{AMNH})$; 19.iv.1963, W. J. Gertsch \& W. Ivie leg., $3 \sigma^{\Uparrow} 1$ immature (AMNH); 20.v.1944, F. Bonet leg., $1 \overbrace{}^{\Uparrow}(\mathrm{AMNH})$ 26.vi.1947, B. Malkin leg., $1 \overbrace{}^{\Uparrow}(\mathrm{AMNH}) ; 8 \mathrm{mi}$. NNW. Tamazunchale, $21^{\circ} 20^{\prime} \mathrm{N}, 98^{\circ} 50^{\prime} \mathrm{W}$, elev. [257m], 19.iv.1963, W. J. Gertsch \&

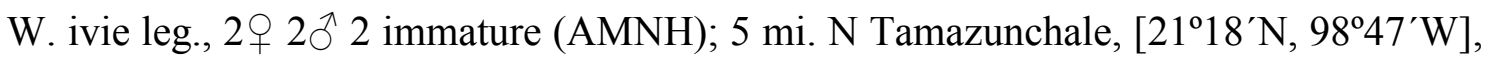
elev. [282m], 2.vii.1936, A. M. \& L. I. Davis leg., 1ठิ (AMNH); Huichihuayan, $21^{\circ} 19^{\prime} \mathrm{N}, 98^{\circ} 50^{\prime} \mathrm{W}$, elev. [423m], 24.vii.1966, J. \& W. Ivie leg., $1 \bigcirc^{\jmath}$ (AMNH); Xilitla, $21^{\circ} 23^{\prime} 50^{\prime} \mathrm{N}, 98^{\circ} 59^{\prime} 38^{\prime} \mathrm{W}$, elev. 689m, 27-31.viii.2011, FAPLAB leg., 1 ㅇ $3 \overbrace{}^{\Uparrow}$ (CAS 9046859); 14-18.xi.2011, FAPLAB leg., $1 \hat{\jmath}$ (CAS 9046858) ; $3 \hat{\jmath}$ (CAS 9046857).

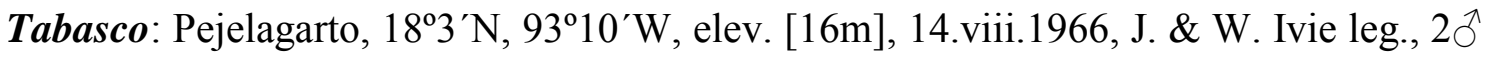
(AMNH). Tamaulipas: Miramar Beach, near Tampico, [22 $17^{\circ} \mathrm{N}, 97^{\circ} 48^{`} \mathrm{~W}$ ], elev. [3m],

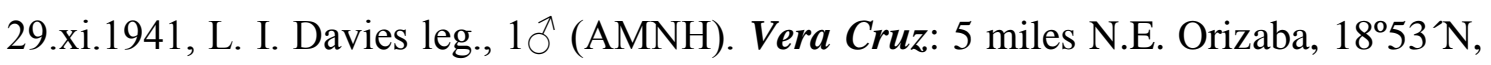
$97^{\circ} 2^{\prime} \mathrm{W}$, elev. [1280m], 25.iv.1963, W. J. Gertsch \& W. Ivie leg., $1 \delta^{\Uparrow}$ (AMNH); Istmo

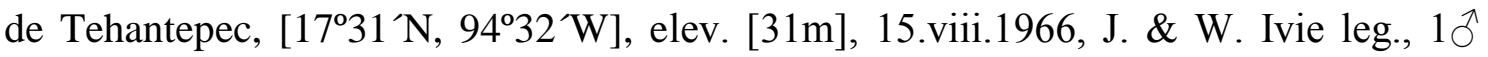

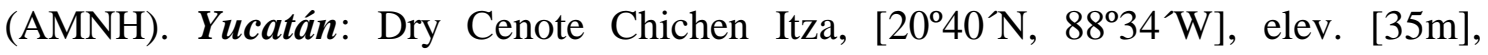
10.vii.1948, C. \& M. Goodnight leg., 19 (AMNH); Chichen-Itza, [2040`N, 88³4`W], elev. [35m], vi.1948, C. \& M. Goodnight leg., 1 ( (AMNH). PANAMA: 9-14.viii.1950, Chickering leg., $2 \bigcirc 2 \widehat{\jmath}$ (MCZ 125538). Chiriquí: Chiri. $2 \mathrm{Km}$ E Cerro Punta, [8 $8^{\circ} 51^{`} \mathrm{~N}$,

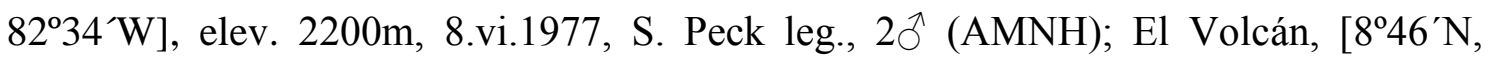

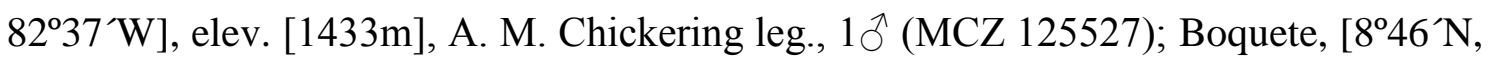


82 $25^{`} \mathrm{~W}$ ], elev. [1094m], 4-11.viii.1954, Chickering leg., 1ठ (MCZ 125537); 411.viii.1954, A. M. Chickering leg., 1 ( MCZ 125494). Panamá: Barro Colorado,

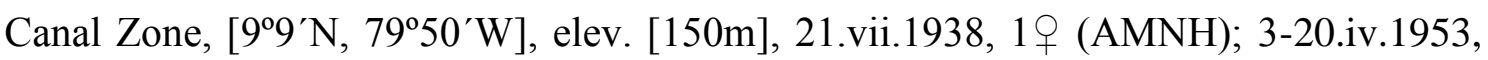

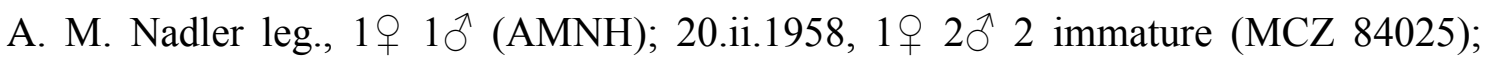
viii.1950, 21 ㅇ 18ðิ (MCZ 64707); vii.1950, 1 ㅇ (MCZ 125515); viii.1954, 2 ㅇ (MCZ 129044); i.1958, 43 37ð 4 immature (MCZ 125529); 20.ii.1958, 58q 89 13

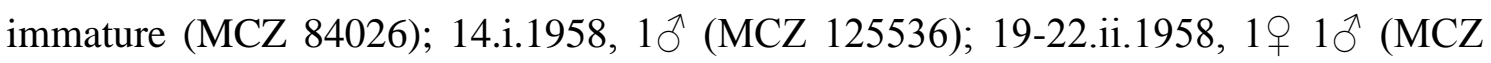

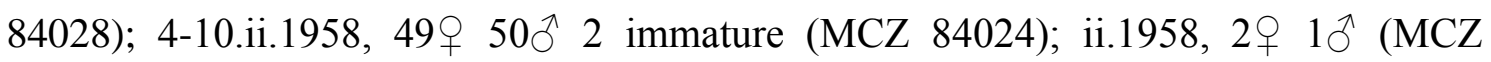
125552); viii.1954, 9 ㅇ 10^ 4 immature (MCZ 129045); 14.ii.1958, 2 ㅇ (MCZ 125516); 21.xii.1957, 9ㅇ 2ð (MCZ 125558); 21.vii.1954, 1ð (MCZ 125541); 6.ii.1958, 6 ㅇ 8ภ (MCZ 125551); ii.1958, $1 \hat{\jmath}$ (MCZ 125518); same data, 2

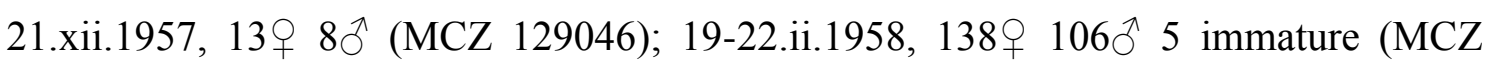
125554); 2.i.1958, 1 ㅇ (MCZ 125533); 8.ii.1958, 15 ㅇ 10^ 1 immature (MCZ 125553); 21.xii.1957, 1ð (MCZ 125555); 5.iii.1957, 20 ㅇ 17ð 7 immature (MCZ 125557); 18.ii.1958, 26ㅇ 22へ 4 immature (MCZ 125556); ix.1975, 1ð (MCZ 125517); 30.vii.1954, 2 + (MCZ 125535); ii.1958, 8 ○ $6{ }^{\Uparrow} 1$ immature (MCZ 125530); 28.ii.1958,

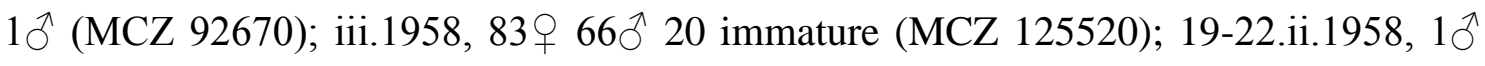

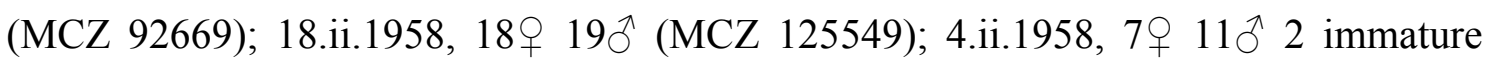
(MCZ 125559); 14.i-3.iii.1958, 3 ㅇ 3今 (MCZ 125550), all collected by A. M. Chickering; Barro Colorado, Canal Zone, Forest Reserve, [99`N, 7950`W], elev. [150m], 28.vii.1954, A. M. Chickering leg. 1ð̂ (MCZ 84027); 28.ii.1958, 2 q (MCZ 125545); 29.i.1958, 1ठ (MCZ 129049), all collected by A. M. Chickering; Gatun,

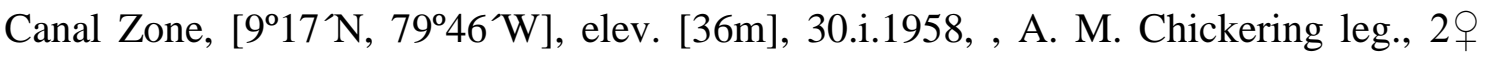
(MCZ 129048); Panama city, [859`N, 79³1`W], elev. [19m], 15-30.vii.1979, E.

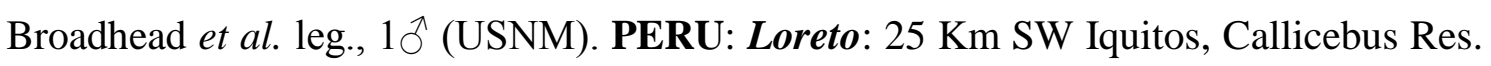

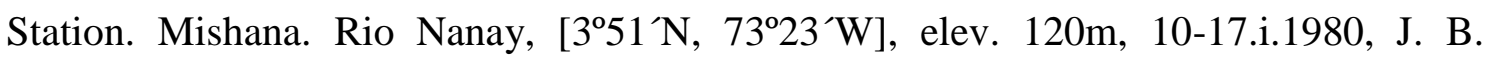
Heppener leg., $1 \delta^{\Uparrow}$ (USNM).

Glenognatha gloriae (Petrunkevitch, 1930)

(Figs. 262-268, 311)

Diplocephalus gloriae Petrunkevitch, 1930: 224, fig. 72-74 (male holotype from Luquillo Mountains, La Gloria, Puerto Rico, 30.i.1926, female paratype from El Yunque, Puerto Rico, 11.x.1925, both collected by A. Petrunkevitch, deposited in 
YPM, only the female paratype examined; male paratype from Adjuntas, Puerto Rico, 8.vi.1915, deposited in AMNH, not examined).

Mimognatha gloriae: Crosby \& Bishop, 1933: 108.

Glenognatha gloriae: Platnick, 2013.

Diagnosis: males of G. gloriae resemble those of G. spherella and G. australis by the prominent Ret2 (Figs. 264B) and the absence of anterior tooth (Fig. 264A). It can be distinguished from the latter two species by the strongly projected clypeus in lateral view (Figs. 262B, 263B). Females can be distinguished from $G$. spherella by the absence of cheliceral bulge (Figs. 264D-F) and from G. australis by having three retromarginal teeth (Fig. 264E-F).

Description: male described by Petrunkevitch (1930), additional data are presented here.

Male (MCZ 125482): habitus as in figure 262. Abdomen with scattered silver guanine spots in the dorsal, lateral and ventral surfaces. Total length 1.70. Cephalothorax 0.75 long, 0.62 wide, 0.52 high. Abdomen 0.92 long, 0.72 wide, 0.7 high. Sternum 0.36 long, 0.42 wide. Eye diameters and interdistances: AME 0.04. ALE 0.03. PME 0.06. PLE 0.03. AME-AME 0.04, AME-ALE 0.07, PME-PME 0.06, PME-PLE 0.07, ALE-PLE 0.01. Clypeus height $2.50 \times$ AME diameter. Chelicerae yellow-brownish. Three promarginal and three retromarginal teeth (Figs. 264A-C). Ret2 enlarged (Fig. 264B). Leg measurements: I: femur 1.15, patella 0.25 , tibia 1.00 , metatarsus 0.87 , tarsus 0.47 , 3.75 total; II: $1.10,0.25,1.02,0.87,0.40,3.65$; III: 0.75, 0.20, 0.55, 0.52, 0.30, 2.32; IV: 0.97, 0.20, 0.77, 0.72, 0.37, 3.05. DTS 0.10. DTE 0.50. Palp as in figures 265-266, 268. Conductor large enclosing almost totally the embolus (Figs. 266A, 268A-E). Embolus short, with coiled tip (Figs. 266D, 268D-E).

Female (MCZ 125485): habitus as in figure 263. Carapace pale yellow with two greyish longitudinal bands that join medially in the posterior region. Sternum dusky yellow, darker on edges. Abdomen greyish, dorsally with two diffused longitudinal black bands that join medially forming thin transverse bands. Silver guanine spots scattered in the dorsal and lateral surfaces Venter with two pale longitudinal bands covered with silver guanine spots. Legs pale yellow. Total length 1.55. Cephalothorax 0.67 long, 0.60 wide, 0.45 high. Abdomen 0.92 long, 0.72 wide, 0.65 high. Sternum 
0.42 long, 0.42 wide. Eye diameters and interdistances: AME 0.03. ALE 0.04. PME 0.07. PLE 0.04. AME-AME 0.05, AME-ALE 0.06, PME-PME 0.09, PME-PLE 0.04, ALE-PLE 0.01. Clypeus height $3.10 \times$ AME diameter. Chelicerae pale yellow. Three promarginal and three retromarginal teeth (Figs. 264D-F). Leg measurements: I: femur1.02, patella 0.30, tibia 0.87, metatarsus 0.82 , tarsus 0.42 , total 3.45; II: 0.90 , 0.25, 0.87, 0.80, 0.37, 3.20; III: 0.65, 0.20, 0.52, 0.52, 0.30, 2.20; IV: 0.87, 0.22, 0.72, $0.67,0.36,2.86$. DTS 0.07. DTE 0.32. Internal genitalia with well-developed spermathecae (Fig. 267).

Variation: male total length 1.5-2.0 $(N=7$; average 1.7), cephalothorax length 0.5-0.9 $(N=7$; average 0.7), femur I length 1.0-1.2 $(N=7$; average 1.1). Female total length 1.5-2.3 ( $N=4$; average 1.9), cephalothorax length 0.6-0.9 $(N=4$; average 0.7$)$, femur I length 0.7-1.3 $(N=4$; average 1.0).

Distribution: known only from Puerto Rico (Fig. 311).

Remarks: Some vials with couples captured near the collection sites of the type material were available for study. After a careful comparison of the male specimens with the original description (Petrunkevitch, 1930) and the female paratype, they were identified as G. gloriae considering the following characters: 1) all the individuals have a conspicuously projected clypeus as the female paratype and the illustration of the male holotype (Petrunkevitch, 1930 fig. 73), 2) the conductor is as depicted by Petrunkevitch (1930, fig. 74) and finally 3), the specimens presents the "spine-like" retromarginal teeth mentioned in the original description (Petrunkevitch, 1930). It is important to note that Petrunkevitch described the type of $G$. gloriae as having only one retromarginal teeth (Ret2). Nevertheless that condition was not observed in any of the Glenognatha here examined, probably the Ret 1 and the Ret 3 were lost in that specimen.

Additional material examined $(\mathbf{N}=9)$ : New records: PUERTO RICO: Bosque Estatal

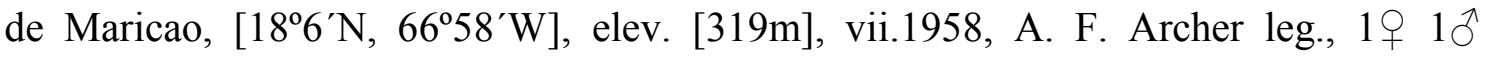
(AMNH); [Parque Nacional el Yunque], Baño de Oro trail 1-2 Km West of Rd. 191,

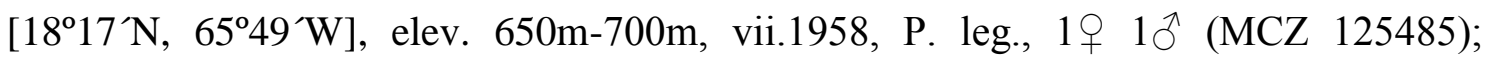
Cordillera Central, Area recreo Doña Juana N. of Villalba, [18 $\left.9^{`} \mathrm{~N}, 66^{\circ} 29^{`} \mathrm{~W}\right]$, elev. 1000m, 26.iii.1986, H. L. Levi leg., 1 q ${ }^{\Uparrow}$ (MCZ 125482). 


\section{Glenognatha sp. nov. 11}

(Figs. 269-274, 306)

Type material: male holotype from Nogales, Santa Cruz Co., Arizona, United States of America, [3120`N,11056`W], elev. [1183m], 1.ix.1991, R. L. Edwards leg., deposited in USNM ENT 00879001; paratypes: $2 \hat{\jmath} 1 \%$ from the type locality, same data as the

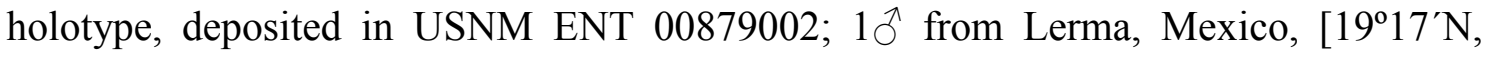
9943 W], elev. [2400m], 25.vi.1941, A. M. \& L. I. Davis leg., deposited in AMNH.

Diagnosis: males of $G$. sp. nov. 11 resemble those of G. emertoni, G. minuta and G. sp. nov. 7 by the enlarged Ret2 and Ret3 (Fig. 271B). It can be distinguished from the latter three species by the nearly $180^{\circ}$ curved tip of the embolus (Fig. 272C), and the strongly curved Ret2 (Fig. 271B). Females can be distinguished from those of G. emertoni and G. minuta by the absence of spermathecae (Fig. 274) and from those of G. sp. nov. 7 by the Prt1 base not enlarged (Fig. 271D).

Description: Male (Holotype, USNM): habitus as in figure 269. Carapace dark brown, darker along furrows. Sternum dark brown. Abdomen dorsally with two diffused longitudinal black bands in the anterior region and thin transversal black bands in its posterior region. Silver guanine spots scattered in dorsal and lateral surfaces. Venter with a median black band surrounded by silver guanine spots. Tracheal spiracle wellsclerotized. Legs yellow-brownish. Total length 3.95. Cephalothorax 1.80 long, 1.35 wide, 0.90 high. Abdomen 2.10 long, 1.65 wide, 1.55 high. Sternum 0.90 long, 0.87 wide. Eye diameters and interdistances: AME 0.09. ALE 0.10. PME 0.10. PLE 0.07. AME-AME 0.1, AME-ALE 0.20, PME-PME 0.16, PME-PLE 0.20, ALE-PLE 0.01. Clypeus height $2.10 \times$ AME diameter. Chelicerae dark brown. Three promarginal and five retromarginal teeth (Figs. 271A-C). Ret2 strongly curved (Fig. 271B). Leg measurements: I: femur 2.10 , patella 0.65 , tibia 2.02 , metatarsus 1.55 , tarsus 0.82 , total 7.15; II: 1.90, 0.62, 1.80, 1.60, 0.82; III: 1.35, 0.47, 1.00, 0.97, 0.55, 4.35; IV: 1.77, 0.47, 1.50, 1.30, 0.62, 5.67. DTS 0.45. DTE 0.90. Palp as in figures 272-273. Conductor and cymbium slender (Figs. 272A-C, 273A). Embolus tip nearly $180^{\circ}$ curved (Fig. 272C).

Female (Paratype, USNM 00879002): coloration and general habitus as in male (Fig. 270). Cephalothorax, abdomen and legs clearer than the male. Abdomen without clearly 
defined black bands. Silver guanine spots uniformly scattered in dorsal and lateral surfaces. Venter with a median diffused dark band clearer towards the petiole and surrounded by silver guanine spots. Tracheal spiracle well-sclerotized. Total length 4.25. Cephalothorax 1.67 long, 1.42 wide, 1.12 high. Abdomen 2.50 long, 2.02 wide, 1.87 high. Sternum 0.90 long, 0.90 wide. Eye diameters and interdistances: AME 0.09. ALE 0.10. PME 0.10. PLE 0.08. AME-AME 0.07, AME-ALE 0.17, PME-PME 0.13, PME-PLE 0.17, ALE-PLE 0.01. Clypeus height $1.90 \times$ AME diameter. Chelicerae brown. Three promarginal and four retromarginal teeth. (Figs. 271D-F). Leg measurements: I: femur 1.85 , patella 0.62 , tibia 1.77 , metatarsus 1.50 , tarsus 0.81 , total 6.56; II: 1.77, 0.57, 1.65, 1.42, 0.78, 6.2; III: 1.25, 0.42, 0.87, 0.82, 0.50, 3.87; IV: 1.70, $0.40,1.27,1.22,0.60,5.20$. DTS 0.47. DTE 0.56. Internal genitalia without spermathecae and copulatory ducts (Fig. 274).

Distribution: known from Arizona state in United States of America and Mexico state in Mexico (Fig. 306).

\section{Glenognatha sp. nov. 7}

(Figs. 275-281, 312)

Type material: male holotype from Parque Nacional da Serra dos Órgãos, Teresópolis,

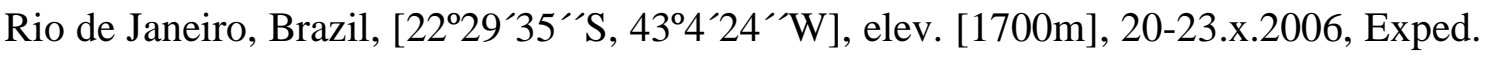
Arachné leg., deposited in MNRJ 6399; paratypes: $3 ð 392$ immature with the same data of the holotype, deposited in MNRJ 6598; $1 \widehat{\text { }}$ from Túnel do Garrafão, Alagoa, Minas Gerais, Brazil, $22^{\circ} 11^{\prime} \mathrm{S}, 4^{\circ} 44^{\prime} \mathrm{W}$, elev. [1340m], 31.ix.2008, L.F.O. Bernardi leg., deposited in UFMG 8790.

Diagnosis: males of G. sp. nov. 7 resemble those of G. emertoni, G. minuta and G. sp. nov. 11 by the enlarged Ret2 and Ret3 (Fig. 277B). It can be distinguished from $G$. emertoni by the absence of an antero-marginal projection on the cheliceral basal segment (Fig. 277A); distinguished from G. minuta by the smaller size of the Ret3 relative to Ret2 (Fig. 277B) and from G. sp. nov. 11 by the slightly curved Ret2 (Fig. 277B). Females can be distinguished from all other Glenognatha species by the enlarged base of the Prt1 (Fig. 277D). 
Description: Male (Holotype): habitus as in figure 275. Carapace pale brown, darker along furrows. Sternum brown, darker on sides. Abdomen oval, dorsally with irregular pattern of two longitudinal black bands on sides that join medially at the posterior region forming thin transverse bands. Silver guanine spots scattered in two median longitudinal bands and on lateral surfaces. Venter with a pale black median band that ramifies towards the lateral surface from the tracheal spiracle. Silver guanine spots scattered in two lighter longitudinal bands between the tracheal spiracle and the epigastric furrow. Legs yellow-brownish. Tracheal spiracle well-sclerotized. Total length 3.75. Cephalothorax 1.75 long, 1.35 wide, 0.95 high. Abdomen 2.30 long, 1.80 wide, 1.80 high. Sternum 0.90 long, 0.90 wide. Eye diameters and interdistances: AME 0.12. ALE 0.10. PME 0.15. PLE 0.12. AME-AME 0.09, AME-ALE 0.12, PME-PME 0.15, PME-PLE 0.13, ALE-PLE 0.01. Clypeus height $1.80 \times$ AME diameter. Chelicerae with the same coloration of the cephalothorax and anteriorly projected (Fig. 275B). Three promarginal and four retromarginal teeth. (Figs. 277A-C). Cheliceral fang curved (Fig. 277C). Leg measurements: I: femur 2.40, patella 0.65, tibia 2.50, metatarsus 2.20, tarsus 1.10 , total 8.85 ; II: $2.40,0.65,2.45,2.00,1.00$, 8.50; III: $1.60,0.40,1.25,1.15$, 0.57, 4.97; IV: $1.80,0.45,1.60,1.40,0.62,5.87$. DTS 0.32. DTE 0.70. Palp as in figures 278-279. Paracymbium with distal and basal portions subequal (Fig. 278D). Embolus long with a nearly $90^{\circ}$ curved tip (Figs. 278C, 279E).

Female (MNRJ 6400): coloration and general habitus as in male (Fig. 276). Abdomen with four dusky longitudinal bands. Pale patch anterior to the gonopore. Tracheal spiracle well-sclerotized. Total length 3.85. Cephalothorax 1.75 long, 1.50 wide, 1.15 high. Abdomen 2.35 long, 2.00 wide, 1.85 high. Sternum 1.00 long, 0.95 wide. Eye diameters and interdistances: AME 0.12. ALE 0.12. PME 0.15. PLE 0.13. AME-AME 0.11, AME-ALE 0.16, PME-PME 0.17, PME-PLE 0.16, ALE-PLE 0.01. Clypeus height $1.70 \times$ AME diameter. Chelicerae with the same coloration of the cephalothorax. Three promarginal and four retromarginal teeth (Figs. 277D-F). Ret1 with a basal enlargement (Fig. 277D). Leg measurements: I: femur 2.80, patella 0.75, tibia 2.75, metatarsus 2.25 , tarsus 1.10 , total 9.65 ; II: $2.50,0.65,2.37,2.10,1.05,8.67$; III: 1.60 , 0.50, 1.20, 1.25, 0.70, 5.25; IV: 2.00, 0.40, 1.95, 1.50, 0.75, 6.60. DTS 0.27. DTE 0.25. Tracheal system as in figure 281. Spinnerets as in figures 298C, 300B, 302C. Internal genitalia without spermathecae (Fig. 280). Uterus externus sac rectangular (Fig. 280). 
Variation: Male total length 3.2-3.7 ( $N=5$; average 3.5), cephalothorax length 1.5-1.7 $(N=5$; average 1.6), femur I length 2.2-2.5 $(N=5$; average 2.3). Female total length 3.7-4.5 ( $N=5$; average 3.9), cephalothorax length 1.6-2.0 $(N=5$; average 1.8), femur I length 2.0-2.8 $(N=5$; average 2.4).

Distribution: known from São Paulo, Rio de Janeiro and Minas Gerais states in Brazil (Fig. 312).

Additional material examined (N = 3): BRAZIL: Minas Gerais: Bocaina de Minas,

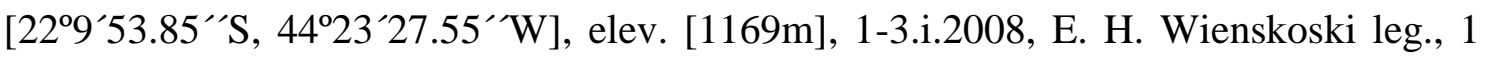
immature (MNRJ 5034). Rio de Janeiro: Teresópolis, Parque Nacional da Serra dos

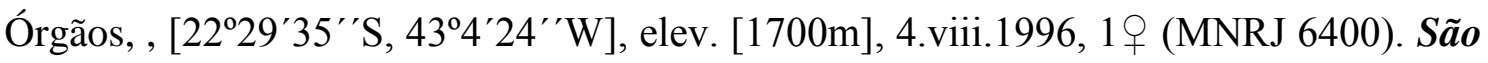

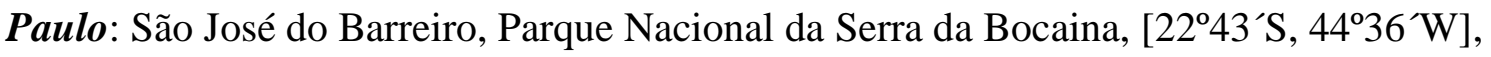
elev. [900m], 28.iv -3.v.2002, Equipe Biota leg., 1 ( (IBSP 68554).

Glenognatha minuta Banks, 1898

(Figs. 282-289, 306)

Glenognatha minuta Banks, 1898: 218, pl. 15, fig. 15. (male syntype from Baja California, San Jose del Cabo, Mexico, Eisen \& Vaslit leg., deposited in MCZ 125505, examined); Hormiga \& Döbel, 1990: 202, figs. 25-30; Platnick, 2013.

Glenognatha centralis Chamberlin, 1925: 216 (male holotype from Panama, R. V. Chamberlin leg., deposited in MCZ 125347, examined). New synonymy.

Synonymy justification: male holotype of G. centralis (MCZ 125347) (collected from the stomach of a toad, Bufo sp.) was carefully examined. I conclude that the specimen is a subadult male due to the undeveloped male palp sclerites. This conclusion was supported by the finding of a subadult male of G. foxi (from Arizona, AMNH), caught in the molting process, that exhibits a similar palp morphology. After comparison with numerous Glenognatha specimens collected in Panama, I concluded that the morphology of the chelicerae in $G$. centralis is too similar with the diagnostic morphology found in G. minuta specimens; which is characterized by enlarged Ret2 and Ret3 (Figs. 284A-C). The chelicerae orientation and the size of the tegulum, cited as diagnostic for G. centralis by Hormiga \& Döbel (1990), are not sufficient to maintain a separate species, as these characters can vary in G. minuta. The unusual shape of the 
conductor, cited by Hormiga \& Döbel (1990) also as a diagnostic character, can be an artifact of the developmental stage of the specimen, the preservation and the digestive process suffered.

Diagnosis: males of G. minuta can be easily distinguished from all other Glenognatha species by the morphology of the chelicerae, which has a small Ret1 and prominent Ret2 and Ret3, the latter being bigger (Figs. 284A-C). Females can be distinguished from all other Glenognatha (except G. emertoni) by the presence of an anterior cheliceral bulge (Figs. 284D, F); distinguished from G. emertoni by the well-developed spermathecae (Fig. 287) and the oval-shaped chelicerae in mesal view (Fig. 284F).

Description: Male (Mexico, Jalisco, Atenquique, AMNH): habitus as in figure 282. Carapace brown, darker along furrows. Sternum brown with darker edges. Abdomen oval, dorsally with diffused irregular lateral bands. Silver guanine spots scattered in dorsal and lateral surfaces. Venter pale greyish with scattered silver guanine spots. Tracheal spiracle well-sclerotized. Legs yellow-brownish. Total length 2.37. Cephalothorax 1.10 long, 0.82 wide, 0.62 high. Abdomen 1.30 long, 1.12 wide, 1.07 high. Sternum 0.55 long, 0.60 wide. Eye diameters and interdistances: AME 0.09. ALE 0.07. PME 0.08. PLE 0.08. AME-AME 0.06, AME-ALE 0.08, PME-PME 0.075, PMEPLE 0.075, ALE-PLE 0.01. Clypeus height $1.8 \times$ AME diameter. Chelicerae brown. Three promarginal and four retromarginal teeth (Figs. 284A-C). Ret 2 and Ret 3 enlarged (Fig. 284B). Leg measurements: I: femur 1.37, patella 0.35, tibia 1.25, metatarsus 1.15, tarsus 0.65 , total 4.77 ; II: $1.30,0.32,1.25,1.05,0.65,4.57$; III: $0.87,0.25,0.62,0.65$, 0.40, 2.80; IV: $1.08,0.25,0.87,0.85,0.47,3.53$. DTS 0.20. DTE 0.50. Palp as in figures 285-286, 289. Distal end of the conductor lamina sharp (Fig. 286B-C).

Female (Mexico, Yucatán, $\boldsymbol{A M N H}$ ): coloration and general habitus as in male (Fig. 283). Abdomen with silver guanine spots arranged in two longitudinal bands that extends to the lateral surfaces. Venter with a median diffused dark band. Total length 2.95. Cephalothorax 1.27 long, 0.92 wide, 0.85 high. Abdomen 1.75 long, 1.57 wide, 1.52 high. Sternum 0.17 long, 0.21 wide. Eye diameters and interdistances: AME 0.09. ALE 0.07. PME 0.08. PLE 0.07. AME-AME 0.07, AME-ALE 0.12, PME-PME 0.13, PME-PLE 0.13, ALE-PLE 0.01. Clypeus height $1.60 \times$ AME diameter. Chelicerae brown. Cheliceral basal segment with antero-marginal projection (Fig. 284D) and 
anterior bulge (Figs. 284D, F). Three promarginal and four retromarginal teeth. (Figs. 284D-F). Leg measurements: I: femur 1.57, patella 0.40, tibia 1.47, metatarsus 1.27, tarsus 0.67 , total 5.40; II: 1.47, 0.47, 1.30, 1.20, 0.65, 5.10; III: 0.95, 0.30, 0.65, 0.70, 0.37, 2.97; IV: $1.37,0.30,1.05,0.80,0.50,4.02$. DTS 0.08. DTE 0.14. Tracheal system as in figure 288. Internal genitalia with a well-developed spermathecae (Fig. 287). Uterus externus sac with a median tubiform projection (Figs. 287A-B).

Variation: male total length 2.1-2.6 $(N=10$; average 2.3), cephalothorax length 1.0-1.2 $(N=10$; average 1.1), femur I length 1.3-1.6 $(N=10$; average 1.5). Female total length 2.2-3.5 $(N=10$; average 2.9), cephalothorax length 1.0-1.7 $(N=10$; average 1.32), femur I length 1.2-2.0 $(N=10$; average 1.5). In specimens from Panama the cheliceral anterior bulge is more sharpened.

Distribution: known in Mexico, Guatemala, Costa Rica, Panama and Cuba (Fig. 306).

Additional material examined $(\mathbf{N}=30)$ : CUBA: Cienfuegos: Soledad, [Jardín

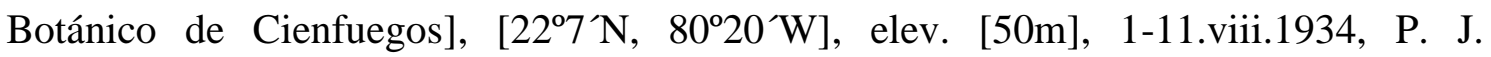
Darlington leg., $1 \delta$ (MZC 125505).

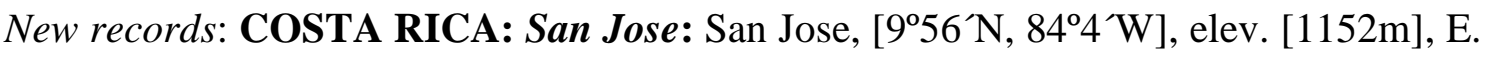

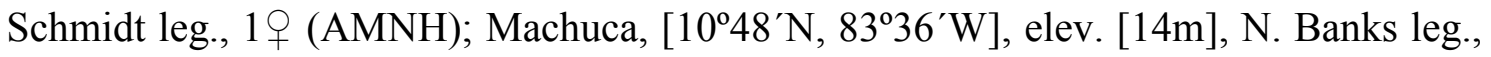

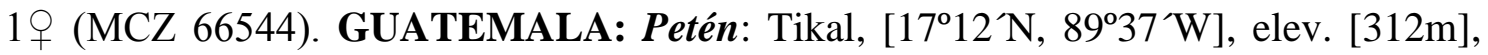
1931, Dice \& Bartlett leg., 1 (MZC 125478). MEXICO: Jalisco: Atenquique,

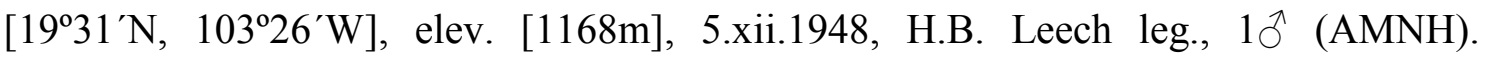

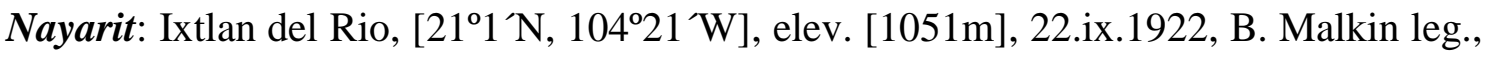

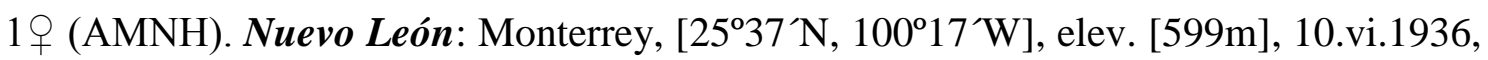

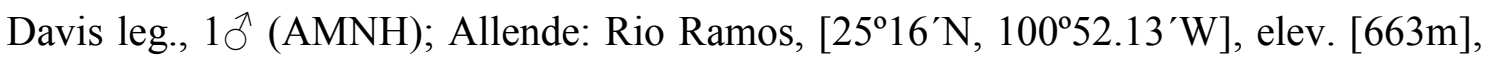
18.viii.1954, F. N. Young leg., 1ठ (AMNH). San Luis Potosí: Tamazunchale,

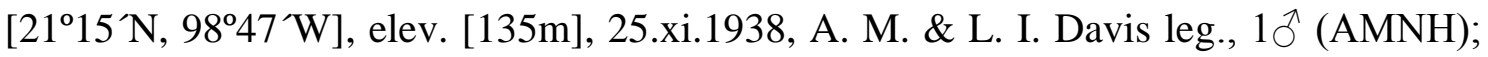

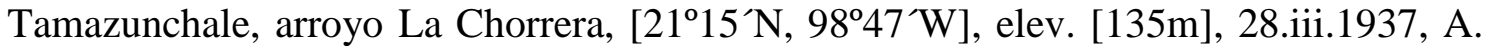
M. \& L. I. Davis leg., $1 \delta^{\Uparrow}(\mathrm{AMNH}) ; 10 \mathrm{mi}$. N.E. Xilitla, $21^{\circ} 27^{\prime} \mathrm{N}, 98^{\circ} 55^{\prime} \mathrm{W}$, elev. [132m], 19.iv.1963, W. J. Gertsch \& W. Ivie leg., $2 \widehat{O} 1$ immature (AMNH). Sinaloa: Piaxtla (River), 2350`N, 106² ${ }^{\circ}$ W, elev. [33m], 16.v.1963, W. J. Gertsch \& W. Ivie leg., 1 immature (AMNH) . Sonora: Hermosillo, [295 N, 11056`W], elev. [235m], 916.vii.1953, B. Malkin leg., 1 (AMNH). Yucatán: Chichen Itza, Cenote Stolc., 


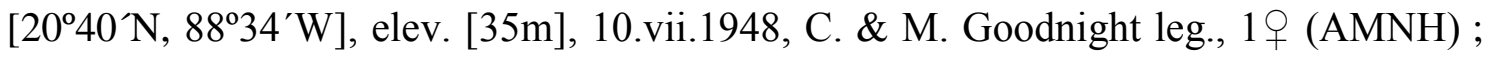
26.v.1980, J. Coddington leg., $3 \uparrow 1 \bigcirc$ (USNM); $1 \uparrow$ (USNM). PANAMA: Chiriquí: El

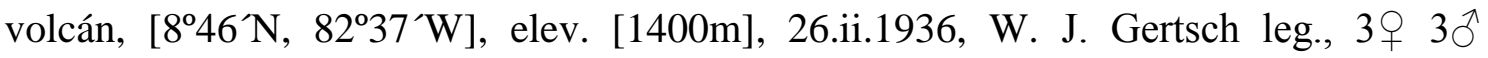

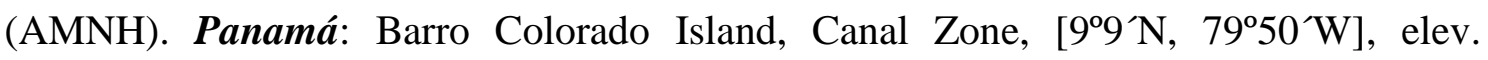
[150m], vi.1950, A. M. Chickering leg., 1 (MCZ 125496); Canal Zone, Forest reserve,

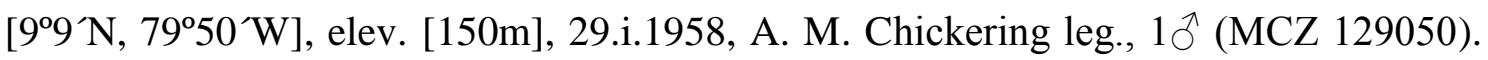

REPUBLICA DOMINICANA: La Romana: Guaymate (14 Km N), [18 $41^{\prime} \mathrm{N}$, 6858`W], elev. [264m], 16.ix.1984, R. Faitoute leg., 1 ㅇ (USNM).

\section{Glenognatha emertoni Simon, 1887}

(Figs. 290-296, 298E, 300D, 302E, 304)

Glenognatha emertoni Simon, 1887: 194 (male syntypes from Arizona, United States of America, deposited in MNHN, examined); Simon, 1894: 717, figs. 790-791; Banks, 1913: 180, pl. 2, fig. 22; Levi, 1980: 66, figs. 255-271; Platnick, 2013.

Diagnosis: Males of G. emertoni are distinguished from the remaining Glenognatha by the presence of an antero-marginal projection in the cheliceral basal segment (Fig. 292A). Females resemble those of G. minuta in having an anterior cheliceral bulge (Fig. 292F), but differ by the reduced size of the spermathecae in relation with the uterus externus (Fig. 295) and the apical position of the cheliceral bulge (Fig. 292F).

Description: male and female described by Levi (1980), additional data are presented here.

Male (USA, Arizona, AMNH): habitus as in figure 290. Tracheal spiracle wellsclerotized. Total length 4.25. Cephalothorax 2.07 long, 1.57 wide, 1.15 high. Abdomen 2.32 long, 1.90 wide, 1.80 high. Sternum 1.00 long, 1.00 wide. Eye diameters and interdistances: AME 0.10, ALE 0.07, PME 0.12, PLE 0.09. AME-AME 0.1, AME-ALE 0.18, PME-PME 0.18, PME-PLE 0.19, ALE-PLE 0.01. Clypeus height $2.30 \times$ AME diameter. Three promarginal and four retromarginal teeth (Fig. 292A-C). Leg measurements: I: femur 2.80 , patella 0.75 , tibia 2.92 , metatarsus 2.57 , tarsus 0.82 , total 9.87; II: 2.67, 0.75, 2.80, 2.40, 1.12, 9.75; III: 1.65, 0.50, 1.47, 1.50, 0.72, 5.85; IV: 2.27, 0.55, 2.00, 1.90, 0.90, 7.65. DTS 0.27. DTE 0.62. Palp as in figures 293-294. Conductor retrolateral apophysis rounded (Figs. 294B-C). Embolus with a nearly $90^{\circ}$ curved tip (Fig. 293C). 
Female (USA, Arizona, AMNH): habitus as in figure 291. Tracheal spiracle wellsclerotized. Total length 5.05. Cephalothorax 2.45 long, 1.92 wide, 1.55 high. Abdomen 2.90 long, 2.35 wide, 2.30 high. Sternum 1.32 long, 1.20 wide. Eye diameters and interdistances: AME 0.10, ALE 0.11, PME 0.13, PLE 0.10. AME-AME 0.15, AMEALE 0.22, PME-PME 0.24, PME-PLE 0.27, ALE-PLE 0.01. Clypeus height $2.9 \times$ AME diameter. Three promarginal and four retromarginal teeth (Figs. 292D-F). Leg measurements: I: femur 3.00, patella 0.70 , tibia 3.25 , metatarsus 2.77 , tarsus 1.30 , total 11.07; II: 2.95, 0.90, 2.92, 2.50, 1.22, 10.5; III: 2.17, 0.62, 1.62, 1.65, 0.85, 6.92; IV: 2.65, 0.67, 2.17, 2.02, 0.92, 8.45. DTS 0.37. DTE 0.62. Tracheal system as in figure 296. Spinnerets as in figures 298E, 300D, 302E. Internal genitalia with strongly reduced spermathecae and copulatory ducts (Figs. 295).

Variation: male total length 3.8-5.2 $(N=6$; average 4.6), cephalothorax length 1.8-2.3 $(N=6$; average 2.1), femur I length 2.8-3.2 $(N=6$; average 2.9). Female total length 4.0-5.1 $(N=10$; average 4.5), cephalothorax length 1.8-2.6 $(N=10$; average 2.1), femur I length 2.4-3.25 $(N=10$; average 2.7).

Distribution: known in Arizona and New Mexico states in United States of America (Fig. 304).

\section{Additional material examined (N = 24): UNITED STATES OF AMERICA: Arizona:}

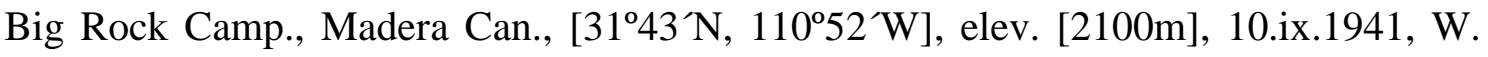

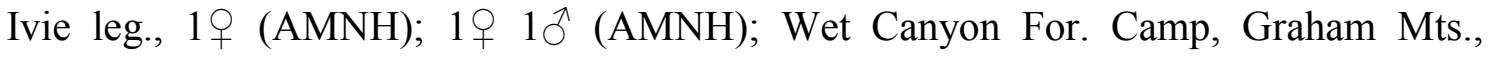
[32 $\left.42^{\prime} \mathrm{N}, 109^{\circ} 52^{\prime} \mathrm{W}\right]$, elev. [2000m], 13.ix.1952, B. Malkin leg., 1ठ(AMNH);

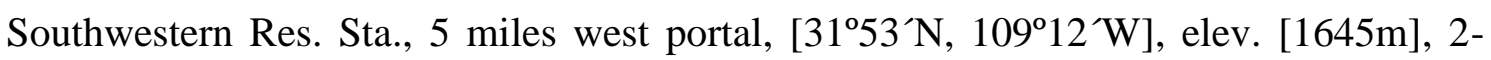
19.v.1956, M. Statham leg., 2 q 1 §̂ (AMNH); 6-20.vii.1955, W. J. Gertsch leg., $3 q$

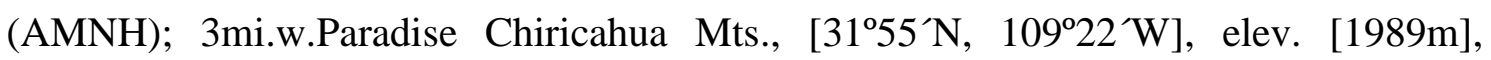
9.ix.1950, W. J. Gertsch leg., 19 (AMNH); SWRS 5mi. W. Portal Cochise County,

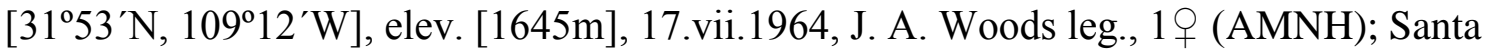

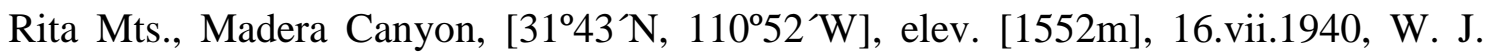

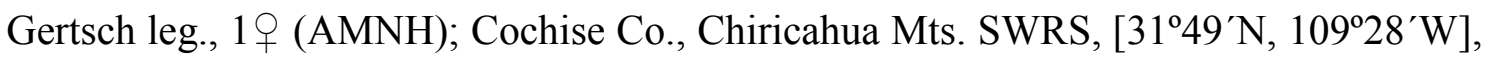
elev. [1700m], 14.vii.1966, J. Beatty leg., (under rock in dry stream), 2 q (MCZ 64709);

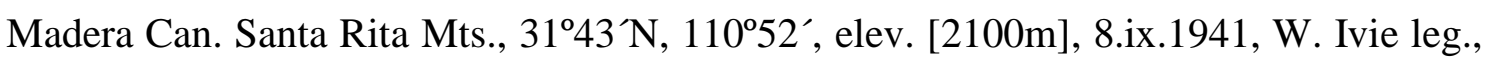

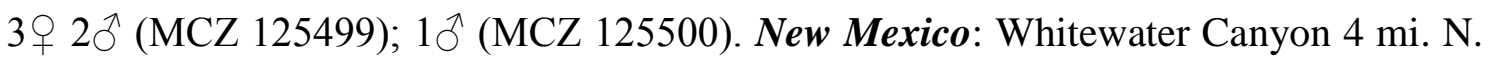




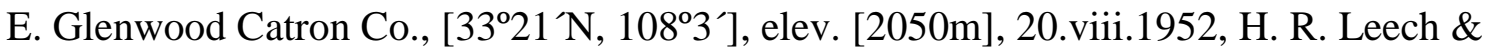
J. W. Green leg., $1 \overbrace{}^{\Uparrow}(\mathrm{AMNH})$.

\section{Species inquerendae}

Because crucial aspects for identification as the morphology of embolus, conductor and female genitalia are not documented adequately in the original descriptions, only one sex is described and the holotypes are lost, the following species are considered species inquerendae:

Glenognatha caporiaccoi Platnick, 1993

Glenognatha chamberlini Caporiacco, 1947: 23; Caporiacco, 1948: 644, fig. 46 (male holotype from Tibicuri-Cuyaha, Demerara, Guiana, deposited in Muzeo di Zoologia di Specola, Firenze, lost). Preoccupied by Hivaoa chamberlini Berland, 1942.

Glenognatha caporiaccoi: Platnick, 1993: 373 (replacement name); Platnick, 2013.

Glenognatha argenteoguttata (Berland, 1935)

Hivaoa argenteoguttata Berland, 1935: 51, figs. 16-19 (male holotype from Matuuna, Hivaoa, Marquesas Islands, 2.iii.1930, Munford et Adamson leg., deposited in Bernice Bishop Museum, Honolulu, lost).

Glenognatha argenteoguttata: Levi 1980: 64; Platnick, 2013.

Glenognatha chamberlini (Berland, 1942)

Hivaoa chamberlini Berland, 1942: 18, figs. 7e-g (female holotype from Rapa, Austral Islands, vii.1934, deposited in Bernice Bishop Museum, Honolulu, lost)

Glenognatha chamberlini: Levi 1980: 64; Platnick, 2013.

Glenognatha nigromaculata (Berland, 1933)

Dyschiriognatha nigrimaculata Berland, 1933: 64, figs. 46-48 (male holotype from Ooumu, Nukuhiva, Marquesas Islands, 13.xi.1929, Munford et Adamson leg., deposited in Bernice Bishop Museum, Honolulu, lost).

Hivaoa nigromaculata: Berland, 1935: 36, 41, 50.

Glenognatha nigromaculata: Levi 1980: 64; Platnick, 2013. 
Glenognatha phalangiops (Berland, 1933)

Hivaoa phalangiops Berland, 1942: 18, figs. 7a-d (female holotype from Mt. Perahu, Rapa, Austral Islands, 21.vii.1934, deposited in Bernice Bishop Museum, Honolulu, lost).

Glenognatha phalangiops: Levi 1980: 64; Platnick, 2013. 


\section{Conclusions}

- Glenognatha is a monophyletic genus supported by eight unambiguous synapomorphies, all free of homoplasy: 1-shaped paracymbium, setae distributed only in the distal portion of the paracymbium, conductor without basal apophysis, conductor retrolateral apophysis prolaterally folded, embolus with medial groove (char. 32[1]), male Ret1 posteriorly displaced (char. 66), medial tracheal trunks branched (char. 81[1]) and large relative to lateral tracheae (char. 82 [1]).

- The plesiomorphic condition in Glenognatha corresponds to a short distal portion of the paracymbium and an embolic groove limited to the inner surface of the embolus. Spermathecae have been lost independently in several occasions within Glenognatha.

- Considering the geographic distribution of Glenognatha, the unknown females in some species and the high probability of finding undescribed species, future addition of taxa and characters might produce modifications of the relationships described herein and improve the internal support values.

- The following taxa are newly synonymized: Dyschiriognatha montana Simon, 1897, Glenognatha mira Bryant, 1945 and Glenognatha maelfaiti Baert, 1987 with Glenognatha argyrostilba (O. P.-Cambridge, 1876) and Glenognatha centralis Chamberlin, 1925 with Glenognatha minuta Banks, 1898.

- Three new combinations are proposed in congruence with the phylogenetic results: Glenognatha argyrostilba (O. P.-Cambridge, 1876), Glenognatha dentata (Zhu \& Wen, 1978) and Glenognatha tangi (Zhu, Song \& Zhang, 2003), all previously included in Dyschiriognatha.

- Eleven species are newly described: $G$. sp. nov. $1, G$. sp. nov. 3, G. sp. nov. 4 and $G$. sp. nov. 7 from southeast Brazil, G. sp. nov. 6, G. sp. nov. 9 and G. sp. nov. 10 from the Amazonian region, G. sp. nov. 2, G. sp. nov. 5 and $G$. sp. nov. 8 from northern Andes and G. sp nov. 11 from central Mexico and Southern United States.

- The following taxa are considered species inquerendae: Glenognatha caporiaccoi Platnick, 1993; Glenognatha argenteoguttata (Berland, 1935), Glenognatha chamberlini (Berland, 1942), Glenognatha nigromaculata (Berland, 1933), and Glenognatha phalangiops (Berland, 1933). 


\section{References}

Agnarsson, I. 2004. Morphological phylogeny of cobweb spiders and their relatives (Araneae, Araneoidea, Theridiidae). Zoological Journal of the Linnean Society 14: 447-626.

Álvarez-Padilla, F. 2007. Systematics of the spider genus Metabus O. P.-Cambridge, 1899 (Araneoidea: Tetragnathidae) with additions to the tetragnathid fauna of Chile and comments on the phylogeny of Tetragnathidae. Zoological Journal of the Linnean Society 150: 285-335.

Álvarez-Padilla, F. \& G. Hormiga. 2008. A protocol for digesting internal soft tissues and mounting spiders for scanning electron microscopy. Journal of Arachnology 35: 538-542.

Álvarez-Padilla, F., D. Dimitrov, G. Giribet \& G. Hormiga. 2009. Phylogenetic relationships of the spider family Tetragnathidae (Araneae, Araneoidea) based on morphological and DNA sequence data. Cladistics 25: 109-146.

Álvarez-Padilla, F. \& G. Hormiga. 2011. Morphological and phylogenetic atlas of the orb-weaving spider family Tetragnathidae (Araneae: Araneoidea). Zoological Journal of the Linnean Society 162: 713-879.

Armendano, A. \& A. González. 2010. Comunidad de arañas (Arachnida, Araneae) del cultivo de alfalfa (Medicago sativa) en Buenos Aires, Argentina. Revista de Biología Tropical 58: 757-767.

Armendano, A. \& A. González. 2011. Spider fauna associated with wheat crops and adjacent habitats in Buenos Aires, Argentina. Revista Mexicana de Biodiversidad 82: 1176-1182.

Arnedo, M. A., G. Hormiga \& N. Scharff. 2009. Higher-level phylogenetics of linyphiid spiders (Araneae, Linyphiidae) based onmorphological and molecular evidence. Cladistics 25: 231-262. 
Baert, L. 1987. Spiders of the Galápagos Islands. Part IV. Miscellaneous families II. Bulletin de l'Institut Royal des Sciences Naturelles de Belgique 57: 141-155.

Baert, L., K. Desender \& J-P. Maelfait. 1991. Spider communities of Isla Santa Cruz (Galapagos, Ecuador). Journal of Biogeography 18: 333-340.

Balfour, R. A. \& A. L. Rypstra. 1998. The influence of habitat structure on spider density in a no-till soybean agroecosystem. The Journal of Arachnology 26: 221226.

Banks, N. 1896. New North American spiders and mites. Transactions of the American entomological Society 23: 57-77.

Banks, N. 1898. Arachnida from Baja California and other parts of Mexico. Proceedings of the California Academy of Sciences 1: 205-308.

Banks, N. 1904. The Arachnida of Florida. Proceedings of the Academy of natural Sciences of Philadelphia. 56: 120-147.

Banks, N. 1913. Notes on the types of some American spiders in European collections. Proceedings of the Academy of natural Sciences of Philadelphia. 65: 177-188.

Banks, N. 1929. Spiders from Panama. Bulletin of the museum of comparative zoology 69: 53-96.

Barrion, A. T. \& J. A. Litsinger. Riceland Spiders of South and Southeast Asia. CAB International, Wallingford, UK, xix $+700 \mathrm{pp}$.

Barrows, W. M. 1919. The taxonomic position of Mysmena bulbifera (Glenognatha bulbifera) Banks, with some observations of its habits. Ohio Journal of Science 19: 210-212.

Benoit, P. L. G. 1977. Fam. Tetragnathidae. In : La faune terrestre de l'île de SaiteHélène IV. Annales Musée Royal de l'Afrique Centrale 220: 156-162. 
Berland, L. 1929. Araignées (Araneida). In: Insects of Samoa and other Samoan terrestrial Arthropoda. London, 8: 35-78.

Berland, L. 1933. Araign. des Iles Marquises. Araignées des Iles Marquises. Bernice P. Bishop Museum Bulletin 114: 39-70.

Berland, L. 1935. Nouvelles araignées marquisiennes. Bernice P. Bishop Museum Bulletin 142: 31-63.

Berland, L. 1942. Polynesian spiders. Occasional Papers of Bernice Pauahi Bishop Museum 17(1): 1-24.

Bertkau, P. 1883. Über die Gattung Argenna Thor. und einige anderen Dictyniden. Archiv Naturgeschichte. 49: 374-382.

Birkhofer, K., S. Scheu \& D. A. Wise. 2007. Small-scale spatial pattern of web-building spiders (Araneae) in alfalfa: relationship to disturbance from cutting, prey availability, and intraguild interactions. Environmental Entomology 36: 801-810.

de Bivort BL, Clouse RM, Giribet G. 2010. A morphometrics-based phylogeny of the temperate Gondwanan mite harvestmen (Opiliones, Cyphophthalmi, Petallidae). Journal of Zoological Systematics and Evolutionary Research 48: 294-309.

Bosmans, R. \& J. Bosselaers. 1994. Spiders of the genera Pachygnatha, Dyschiriognatha and Glenognatha (Araneae, Tetragnathidae), with a revision of the Afrotropical species. Zoologica Scripta 23(4): 325-352.

Breene, R. G., D. A. Dean, M. Nyffeler \& G. B. Edwards. 1993. Biology, Predation Ecology, and Significance of Spiders in Texas Cotton Ecosystems with a Key to Species. Texas Agriculture Experiment Station, College Station, 115 pp.

Bremer K. 1988. The limits of aminoacid sequence data in angiosperm phylogenetic reconstruction. Evolution 42: 795-803. 
Bremer K. 1995. Branch support and tree stability. Cladistics 10: 295-304.

Bryant, E. B. 1945. The Argiopidae of Hispaniola. Bulletin of the Museum of Comparative Zoology 95: 357-422.

Cambridge, O. P. 1876. Catalogue of a collection of spiders made in Egypt, with descriptions of new species and characters of a new genus. Proceedings of the Zoological Society of London: 541-630.

Cambridge, O. P. 1896. Arachnida. Araneida. In: Biologia Centrali-Americana, Zoology. London, 1: 161-224.

Caporiacco, L. di. 1947. Diagnosi preliminari de specie nuove di aracnidi della Guiana Brittanica raccolte dai professori Beccari e Romiti. Monitore zoologico italiano 56: $20-34$.

Caporiacco, L. di. 1948. Arachnida of British Guiana collected in 1931 and 1936 by Professors Beccari and Romiti. Proceedings of the Zoological Society of London 118: 607-747.

Chamberlin, R. V. 1925. Diagnoses of new American Arachnida. Bulletin of the Museum of Comparative Zoology 67: 209-248.

Chamberlin, R. V. \& W. Ivie. 1936. New spiders from Mexico and Panama. Bulletin of the University of Utah 27(5): 1-103.

Chapman, E. G., J. M. Schmidt, K. D. Welch, J. D. Harwood. 2013. Molecular evidence for dietary selectivity and pest suppression potential in an epigeal spider community in winter wheat. Biological Control 65: 72-86.

Chen, X. E. \& J. C. Gao. 1990. The Sichuan farmland spiders in China. Sichuan Sci. Tech. Publ. House, Chengdu, 226 pp. 
Coddington. J.A. 1983. A Temporary Slide Mount Allowing Precise Manipulation of Small Structures. In: O. Kraus, (Ed.). Taxonomy, Biology, and Ecology of Araneae and Myriapoda. Verhandlungen des Natumissenschaftlichen Vereins in Hamburg, new series. 26:291-292.

Coddington, J. A. 1990. Ontogeny and homology in the male palpus of orb-weaving spiders and their relatives, with comments on phylogeny (Araneoclada: Araneoidea, Deinopoidea). Smithsonian Contributions to Zoology 496: 1-52.

Crosby, C. R. \& S. C. Bishop. 1928. Araneae. In: A list of the insects of New York. Memoirs of the Cornell University Agricultural Experiment Station 101: 10341074.

Crosby, C. R. \& S. C. Bishop. 1933. American spiders: Erigonae, males with cephalic pits. Annals of the Entomological Society of America. 26: 105-172.

Danielson-François, A. M. 2006. Natural History of Glenognatha emertoni (Araneae, Tetragnathidae): mating behavior and sperm release in an haplogyne. The Journal of Arachnology 34:387-398.

Daza, J. D., V. Abdala, J. S. Arias, D. García-López \& P. Ortiz. 2012. Cladistic Analysis of Iguania and a Fossil Lizard from the Late Pliocene of Northwestern Argentina. Journal of Herpetology 46:104-119.

Dean, D. A. \& W. L. Sterling. 1985. Size and phenology of ballooning spiders at two locations in eastern Texas. Journal of Arachnology 13: 111-120.

Dimitrov, D., F. Alvarez-Padilla \& G. Hormiga. 2007. The female genitalic morphology of the orb weaving spider genus Agriognatha (Araneae, Tetragnathidae). Journal of Morphology 268: 758-770.

Dimitrov, D. \& G. Hormiga. 2009. Revision and cladistic analysis of the orbweaving spider genus Cyrtognatha Keyserling, 1881 (Araneae, Tetragnathidae). Bulletin of the American Museum of Natural History 317: 1-139. 
Dimitrov, D. \& G. Hormiga. 2011. An extraordinary new genus of spiders from Western Australia with an expanded hypothesis on the phylogeny of Tetragnathidae (Araneae). Zoological Journal of the Linnean Society 161: 735768.

Döbel, H. T., R. F. Denno \& J. A. Coddington. 1990. Spider (Araneae) community structure in an intertidial salt marsh: effects of vegetation structure and tidal flooding. Environmental Entomology 19: 1356-1370.

Draney, M. L. 1997. Ground-layer spiders (Araneae) of a Georgia piedmont floodplain agroecosystem: species list, phenology and habitat selection. The Journal of Arachnology 25: 333-351.

Eberhard, W. G. 1976. Photography of orb webs in the field . Bulletin of the British Arachnological Society 3: 200-204.

Eberhard, W. G. 1982. Behavioral characters for the higher classification of orbweaving spiders. Evolution 36: 1067-1095.

Eberhard, W. G. 2010. Possible functional significance of spigot placement on the spinnerets of spiders. The Journal of Arachnology 38:407-414.

Eberhard, W. G \& B. A. Huber. 2010. Spider genitalia: precise maneuvers with a numb structure in a complex lock. Pp. 249-284. In: J. L. Leonard \& A. Córdoba-Aguilar (eds.) Evolution of primary sexual characters in animals. Oxford University Press, Oxford, U.K

Edwards, R. L. \& C. W. Senske. 2001. Observations on the breeding behavior of the spider Glenognatha heleios (Araneae: Tetragnathidae). Entomological News 112: 71-72.

Frick, H. \& N. Scharff. 2013. Phantoms of Gondwana? - phylogeny of the spider subfamily Mynogleninae (Araneae: Linyphiidae). Cladistics DOI: 10.1111/ cla. 12025 . 
Gavish-Regev, E., G. Hormiga \& N. Scharff. 2013. Pedipalp sclerite homologies and phylogenetic placement of the spider genus Stemonyphantes (Linyphiidae, Araneae) and its implications for linyphiid phylogeny. Invertebrate Systematics 27: $38-52$.

Gillespie, R. G. H. B. Croom \& G. L. Hasty. 1997. Phylogenetic Relationships and Adaptive Shifts among Major Clades of Tetragnatha Spiders (Araneae: Tetragnathidae) in Hawaii. Pacific Science: 51: 380-394.

Gillespie, R. G. 2002. Hawaiian spiders of the genus Tetragnatha: IV new, small species in the spiny leg clade. Journal of Arachnology 30: 159-172.

Gillespie, R. G., E. M. Claridge \& S. L. Goodcare. 2008. Biogeography of the fauna of French Polynesia: diversification within and between a series of hot spot archipelagos. Philosophical Transactions of the Royal Society B 363: 3335-3346.

Giribet, G. 2003. Stability in Phylogenetic Formulations and Its Relationship to Nodal Support. Systematic Biology 52: 554-564.

Goloboff, P. A., J. S. Farris, M. Källesjö, B. Oxelman, M. J. Ramírez \& C. A. Szumik. 2003. Improvements to resampling measures of group support. Cladistics 19: 324332.

Goloboff, P. A., J. M. Carpenter, J. S. Arias \& D. R. Miranda Esquivel. 2008. Weighting against homoplasy improves phylogenetic analysis of morphological data sets. Cladistics 24: 1-16.

Goloboff, P. A, J. S. Farris \& K. C. Nixon. 2008. TNT, a free program for phylogenetic analysis. Cladistics 24: 774-786.

Griswold, C.E., J.A. Coddington, G. Hormiga \& N. Scharff. 1998. Phylogeny of the orb-web building spiders (Araneae, Orbiculariae: Deinopoidea, Araneoidea). Zoological Journal of the Linnaean Society 123: 1-99. 
Griswold, C.E., M. J. Ramírez, J. A. Coddington \& N. I. Platnick. 2005. Atlas of phylogenetic data for Entelegyne spiders (Araneae: Araneomorphae: Entelegynae) with comments on their phylogeny. Proceeding of the Californian Academy of Sciences 56:1-324.

Hormiga, G. 1994. A revision and cladistics analysis of the spider family Pimoidae (Araneae: Araneoidea). Smithsonian Contributions to Zoology 549: 1-105.

Hormiga, G. 2000. Higher level phylogenetics of erigonine spiders (Araneae, Linyphiidae, Erigoninae). Smithsonian Contributions to Zoology 609: 1-160.

Hormiga, G. 2002. Orsonwelles, a new genus of giant linyphiid spiders (Araneae) from the Hawaiian islands. Invertebrate Systematics 16: 369-448.

Hormiga, G. \& H. G. Döbel. 1990. A new Glenognatha (Araneae, Tetragnathidae) from New Jersey, with redescriptions of $G$. centralis and $G$. minuta. Journal of Arachnology18: 195-204.

Hormiga, G., W. G. Eberhard \& J. A. Coddington. 1995. Web-construction behaviour in Australian Phonognatha and the phylogeny of nephiline and tetragnathid spiders (Araneae: Tetragnathidae). Australian Journal of Zoology 43: 313-364.

Hormiga, G., F. Alvarez-Padilla \& S.P. Benjamin. 2007. First records of extant Hispaniolan spiders of the families Mysmenidae, Symphytognathidae, and Ochyroceratidae (Araneae), including a new species of Ochyrocera. American Museum Novitates 3577: 1-21.

Holm, A. 1969. A new tetragnathid species from the island St-Helena (Araneae). Revue De Zoologie et de Botanique Africaines 80: 62-66.

Hu, J. L. 1984. The Chinese spiders collected from the fields and the forests. Tianjin Press of Science and Techniques, $482 \mathrm{pp}$. 
Huber, B. A \& A. Senglet. 1997. Copulation with contralateral insertion in entelegyne spiders (Araneae: Entelegynae: Tetragnathidae). Netherlands Journal of Zoology 47: 99-102.

Izquierdo, M. A. \& M. J. Ramírez. 2008. Two new spider species of the genera Aysenia and Aysenoides from southern Chile and Argentina: description and phylogenetic relationships (Araneae: Anyphaenidae, Amaurobioidinae). Zootaxa: 1861: 29-43.

Kaston, B. J. 1945. New Micryphantidae and Dictynidae with notes on other spiders. American Museum Novitates 1292: 1-14.

Kaston, B. J. 1948. Spiders of Connecticut. Bulletin - State Geological and Natural History Survey of Connecticut 70: 1-874.

Keyserling, E. 1881. Neue Spinnen aus Amerika. III. Verhandlungen der ZoologischBotanischen Gesellschaft in Wien 31: 269-314.

Keyserling, E. 1883. Neue Spinnen aus Amerika. IV. Verhandlungen der ZoologischBotanischen Gesellschaft in Wien 32: 195-226.

Kuntner M, J. A Coddington \& G. Hormiga. 2008. Phylogeny of extant nephilid orbweaving spiders (Araneae, Nephilidae): testing morphological and ethological homologies. Cladistics 24: 147-217.

Latreille P, A. 1804. Histoire naturelle générale et particulière des Crustacés et des Insectes. Paris 7: 144-305.

Levi, H.W. 1980. The orb-weaver genus Mecynogea, the subfamily Metinae and the genera Pachygnatha, Glenognatha and Azilia of the subfamily Tetragnathinae north of Mexico (Araneae: Araneidae). Bulletin of the Museum of Comparative Zoology 149: 1-75. 
Levi, H. W. 1986. The Neotropical orb-weaver genera Chrysometa and Homalometa (Araneae: Tetragnathidae). Bulletin of the Museum of Comparative Zoology 151: 91-215.

Levi, H. W. 2005. Tetragnathidae, p. 232-234. In: D. Ubick, P. Paquin, P. E. Cushing \& V. Roth (eds.). Spiders of North America: an identification manual. American Arachnological Society. 377p.

Lopardo, L. 2005. Phylogenetic revision of the genus Negayan (Araneae, Anyphaenidae, Amaurobioidinae). Zoologica Scripta, 34: 245-277.

Lopardo, L., G. Giribet \& G. Hormiga. 2011. Morphology to the rescue: molecular data and the signal of morphological characters in combined phylogenetic analyses - a case study from mysmenid spiders (Araneae, Mysmenidae), with comments on the evolution of web architecture. Cladistics 27: 278-330.

Maddison, W. P. \& D. R. Maddison. 2011. Mesquite: A modular system for evolutionary analysis, version 2.75. Available at: http://mesquiteproject.org

Magalhães, I. L. F. \& A. J. Santos. 2012. Phylogenetic analysis of Micrathena and Chaetacis spiders (Araneae: Araneidae) reveals multiple origins of extreme sexual size dimorphism and long abdominal spines. Zoological Journal of the Linnean Society 166: 15-53.

Marples, B. J. 1955. Spiders from western Samoa. Journal of the Linnean Society of London, Zoology 42: 453-504.

McCook, H. C. 1894. American spiders and their spinningwork. Philadelphia, 3: 1-285.

Mello-Leitão, C. F. de. 1944. Arañas de la provincia de Buenos Aires. Revista del Museo de La Plata (N.S., Zool.) 3: 311-393. 
Mendes, A. C. 2011. Phylogeny and taxonomic revision of Heteropachylinae (Opiliones: Laniatores: Gonyleptidae). Zoological Journal of the Linnean Society 163: 437-483.

Miller, J. A. \& G. Hormiga. 2004. Clade stability and the addition of data: a case study from erigonine spiders (Araneae: Linyphiidae, Erigoninae). Cladistics 20: 385442.

Mirande, J. M. Weighted parsimony phylogeny of the family Characidae (Teleostei: Characiformes). Cladistics 25 574-613.

Moon, M-J. 2012. Organization of the spinnerets and spigots in the orb web spider, Argiope bruennichi (Araneae: Araneidae). Entomological Research 42: 85-93.

Nixon, K. 2002. Winclada. Version 10.00.08. Avalaible at http://www.cladistics.com

Ojanguren-Affilastro, A. A. \& M. J. Ramírez. 2009. Phylogenetic analysis of the scorpion genus Brachistosternus (Arachnida, Scorpiones, Bothriuridae). Zoologica Scripta 38: 183-198.

Okuma, C. 1987. A revision of the australasian species of the genus Tetragnatha (Araneae, Tetragnathidae). Esakia 25: 37-96.

Okuma, C. 1991. A new record of Japanese spider Dyschiriognatha dentata Chu et Wen. Heptathela 5: 15-18.

Okuma, C., N. Q. Kamal, Y. Hirashima, M. Z. Alam \& K. Ogata. 1993. Illustrated Monograph of the Rice Field Spiders of Bangladesh. Institute of Postgraduate Studies in Agriculture (Salna, Gazipur, Bangladesh). Japan International Cooperation Agency Project Publication 1, 93 pp.

Opell B, D. 1979. Revision of the genera and tropical American species of the spider family Uloboridae. Bulletin of the Museum of Comparative Zoology 148: 443549. 
Opell, B.D., 1990. The relationship of book lung and tracheal systems in the spider family Uloboridae. Journal of Morphology. 206, 211-216.

Pavesi, P. 1895. Esplorazione del Guiba e dei suoi affluenti compiuta dal Cap. Bottego. XVIII. Aracnidi. Annali del Museo Civico di Storia Naturale di Genova 35: 491537.

Petrunkevitch, A. 1925a. Arachnida from Panama. Transactions of the Connecticut Academy of Arts and Sciences. 27: 51-248.

Petrunkevitch, A. 1925b. New Erigoninae from Tennessee. Journal of the New York entomological Society 33: 170-176.

Petrunkevitch, A. 1930. The spiders of Porto Rico. Part two. Transactions of the Connecticut Academy of Arts and Sciences 30: 159-356.

Platnick, N. I. 1993. Advances in spider taxonomy 1988-1991, with synonymies and transfers 1940-1980. New York, 846 pp.

Platnick, N. I. 2013. The world spider catalog, version 14.0. American Museum of Natural History, online at http://research.amnh.org/entomology/ spiders/catalog/ index.html. DOI: 10.5531/db.iz.0001.

Ramírez, M. J. 1996. Descripción de la hembra de Glenognatha lacteovittata (MelloLeitão), nueva combinación (Araneae, Tetargnathidae). Physis (Buenos Aires) 52: 35-37.

Ramírez, M. J. 2003. The spider subfamily amaurobioidinae (Araneae: Anyphaenidae): A phylogenetic revision at generic level. Bulletin of the museum of natural history. 277: 1-262.

Saaristo, M. I. 2003. Tetragnathid spiders of Seychelles (Araneae, Tetragnathidae). Phelsuma 11: 13-28. 
Saaristo, M. I. 2010. Araneae. In: Gerlach, J. \& Y. Marusik (eds.), Arachnida and Myriapoda of the Seychelles islands. Siri Scientific Press, Manchester UK, pp. 8306.

Sereno, P. C. 2007. Logical basis for morphological characters in phylogenetics. Cladistics 23: 565-587.

Scharaschkin T. \& J. A. Doyle. 2006. Character evolution in Anaxagorea (Annonaceae). The American Journal of Botany 93: 36-54.

Simon, E. 1887. Observation sur divers arachnides: synonymies et descriptions. Annales de la Société entomologique de France. Serie 6. Tomo 7: 158-159, 167, 175-176, 186-187, 193-195.

Simon, E. 1893. Arachnides de l'archipel Malais. Revue suisse de Zoologie. 1: 319-328.

Simon, E., 1894. Histoire Naturelle Des Araignées 1. Librairie Enciclopédique de Roret, Paris.

Simon, E. 1895. Etudes arachnologiques. 26e. XLI. Descriptions d'espèces et de genres nouveaux de l'ordre des Araneae. Annales de la Société Entomologique de France 64: 131-160.

Simon, E. 1897. On the spiders of the island of St Vincent. III. Proceedings of the zoological Society, London 1897: 860-890.

Simon, E. 1900. Arachnida. In Fauna Hawaiiensis, or the zoology of the Sandwich Isles: being results of the explorations instituted by the Royal Society of London promoting natural knowledge and the British Association for the Advancement of Science. London, 2: 443-519.

Song, D. X., M. S. Zhu \& J. Chen. 1999. The Spiders of China. Hebei Sci. Technol. Publ. House, Shijiazhuang, 640 pp. 
Soto, M. E. \& M. J. Ramírez. 2012. Revision and phylogenetic analysis of the spider genus Philisca Simon (Araneae: Anyphaenidae, Amaurobioidinae). Zootaxa: 3443: 1-65.

Statsoft, Inc. 2004. Statistica (data analysis software system), V.7. http://www.statsoft.com.

Sundevall, C. J. 1823. Specimen academicum genera araneidum Sueciae exhibens. Lundae, 1-22.

Tanikawa, A. 2007. An identification guide to the Japanese spiders of the families Araneidae, Nephilidae and Tetragnathidae. Arachnological Society of Japan, 121 pp.

Tanikawa, A. 2009. Hersiliidae. Nephilidae, Tetragnathidae, Araneidae. In: Ono, H. (eds.), The Spiders of Japan with keys to the families and genera and illustrations of the species. Tokai University Press, Kanagawa, pp. 149, 403-463.

Uhl, G. 1994. Genital morphology and sperm storage in Pholcus phalangioides (Fuesslin, 1775) (Pholcidae; Araneae). Acta Zoologica 75: 1-12.

Uhl, G. 2000. Two distinctly different sperm storage organs in female Dysdera erythrina (Araneae: Dysderidae). Arthropod Structure \& Development 29: 163-169.

Vega, A. S, G. H. Rua, L. T. Fabbri \& Z. E. Rúgolo de Agrasar. 2009. A MorphologyBased Cladistic Analysis of Digitaria (Poaceae, Panicoideae, Paniceae). Systematic Botany, 34: 312-323.

Vellard, J. 1926. Contribution à l'étude des araignées. Nouvelles espèces d'Argiopidae. Memórias do Instituto Butantan 3: 327-331.

Walckenaer, C. A. 1805. Tableau des aranéides ou caractères essentiels des tribus, genres, familles et races que renferme le genre Aranea de Linné, avec la désignation des espèces comprises dans chacune de ces divisions. Paris, 88 pp. 
Werenkraut, V. \& M. J. Ramírez2009. A revision and phylogenetic analysis of the spider genus Coptoprepes Simon (Araneae: Anyphaenidae, Amaurobioidinae). Zootaxa 2212: 1-40.

Young, O. P. \& G. B. Edwards. 1990. Spiders in United States field crops and their potential effect on crop pests. Journal of Arachnology 18: 1-27.

Zhu, C. D. \& Z. G. Wen. 1978. A new species of spider of the genus Dyschiriognatha (Araneae: Tetragnathidae) from China Journal of Jilin University-Medicine Edition 3: 16-18.

Zhu, M. S., D. X. Song \& J. X. Zhang. Fauna Sinica: Invertebrata Vol. 35: Arachnida: Araneae: Tetragnathidae. Science Press, Beijing, vii +418 pp. 


\section{Appendix}

\subsection{List of specimens examined for character scoring.}

\section{Outgroup}

\section{Metainae}

Chrysometa alajuela Levi, 1986

Characters coded after examination of figures and descriptions of Álvarez-Padilla \& Hormiga (2011).

\section{Tetragnathinae}

Cyrtognatha espanola Bryant, 1945)

Characters coded after examination of figures and descriptions of Álvarez-Padilla \& Hormiga (2011) and Dimitrov \& Hormiga (2009).

Tetragnatha versicolor Walckenaer, 1841

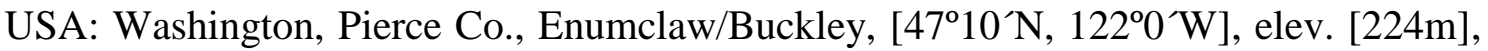

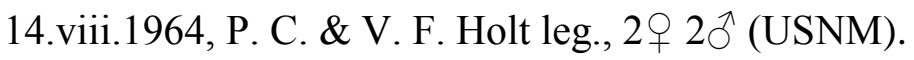

Pachygnatha autumnalis Marx, 1884

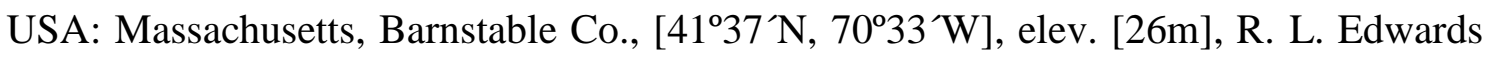
leg., 3 + $3 \hat{\jmath}$ (USNM).

Pachygnatha clercki Sundevall, 1823

USA: Alaska, Eagle river flats nr. Anchorage, [6113 $\left.{ }^{\prime} \mathrm{N}, 149^{\circ} 53^{\prime} \mathrm{W}\right]$, elev. [19m], F. S.

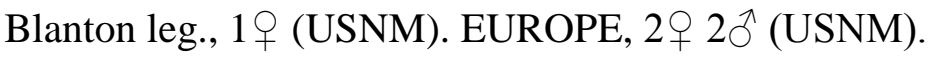

\section{Dyschiriognatha dentata}

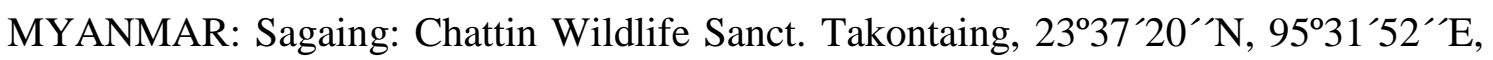
elev. [179m], 7-12.x1998, Coddington \& Baptista leg., 6 ㅇ $3 ð$ (USNM); JAPAN: Okinawa: [26 ${ }^{\circ}{ }^{`} \mathrm{~N}, 1^{2} 7^{\circ} 53^{`} \mathrm{E}$ ], elev. [15m], 22.vii.1945, C. T. Parsons \& F. G. Werner leg., $1{ }^{\Uparrow}(\mathrm{MCZ} 125497)$.

Dyschiriognatha tangi

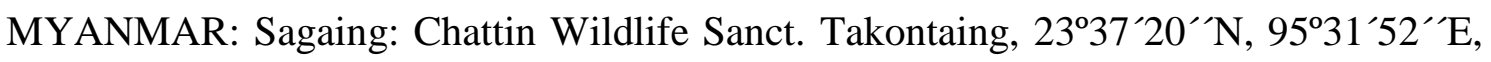
elev. [179m], 7-12.x1998, Coddington \& Baptista leg., 1 ㅇ $3 \hat{\jmath}$ (USNM). 


\section{Ingroup}

Glenognatha argyrostilba (O. P.-Cambridge, 1876)

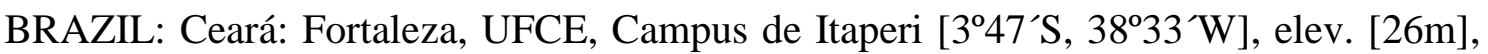
8.vii.2011, R. Azevedo leg., 19 (UFCE 852); 19 (UFCE 841). ECUADOR:

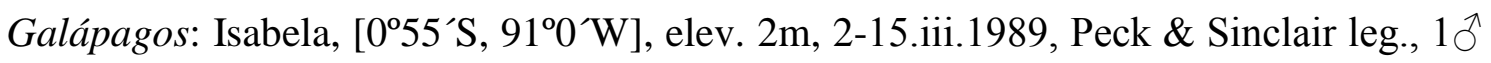
$\left.(\mathrm{AMNH}) ; 0^{\circ} 16^{\prime} \mathrm{S}, 90^{\circ} 41^{\prime} \mathrm{W}\right]$, elev. [300m], 10.iv.1982, Y. Lubin leg., 2 + (MCZ 125476). NIGERIA : Kabba: Kabba, : [750`N, 6º`E], elev. [458m], 19-23.ii.1949, B. Malkin leg., $2 \bigcirc 2 \lesssim(\mathrm{CAS})$.

Glenognatha australis (Keyserling, 1883)

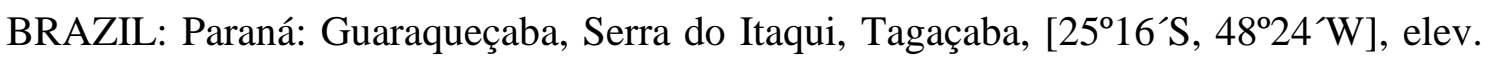
[51m], 15-22.ix.2007, G. P. Perroni leg., $1 \delta^{\Uparrow}$ (IBSP 86529). Rio Grande do Sul: Estaçao

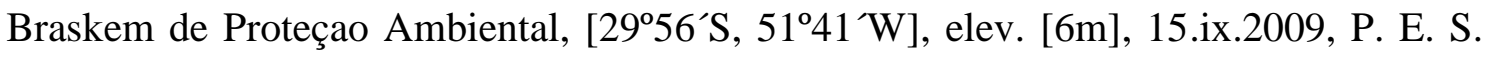
Rodrigues leg., $1 \uparrow 1 ð$ (MCN 46671); 14.vi.2006, R. Ott leg., 19 1§ (MCN 42102); 15.ix.2009, P. E. S. Rodrigues leg., 1 + $1 \overbrace{}^{\lambda}$ (MCN 46671). Rio Pardo, 2958`S, 52 $21^{\prime}$ W, elev. [134m], 6.vii.1966, A. Lise leg., 24 ๆ 18 ภ 2 immature (MCZ 125524). São Paulo: Jundiaí, Serra do Japi, [23¹4’S, 4655’W], elev. [966m], 8-10.xii.1997, A. J. Santos leg., 19 (IBSP 36559). PERU: [Piura]: Quebrada Mogollón, [4³2’S, 81`W], elev. [700m], 24.ix.1939, HEF \& DLF leg., 19 1 ${ }^{\circledR}$ (CAS 9039650).

Glenognatha emertoni Simon, 1887

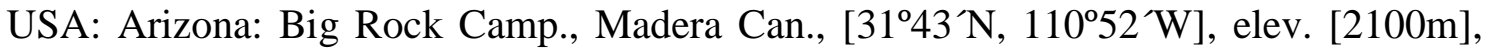
10.ix.1941, W. Ivie leg., $1 q(\mathrm{AMNH}) ; 1 q 1 ð$ (AMNH); Southwestern Res. Sta., 5 miles west portal, [31 ${ }^{\circ} 53^{\prime} \mathrm{N}, 1^{\circ} 19^{\circ} 2^{\prime} \mathrm{W}$ ], elev. [1645m], 2-19.v.1956, M. Statham leg.,

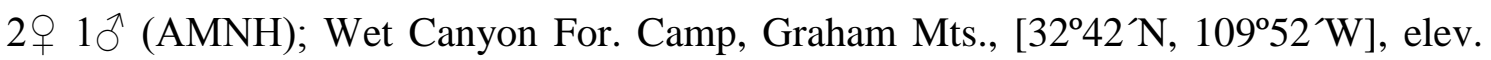
[2000m], 13.ix.1952, B. Malkin leg., 1ðึ (AMNH); 3mi.w.Paradise Chiricahua Mts., [31 $\left.55^{\prime} \mathrm{N}, 1^{\circ} 9^{\circ} 22^{\prime} \mathrm{W}\right]$, elev. [1989m], 9.ix.1950, W. J. Gertsch leg., 1 ( $(\mathrm{AMNH})$.

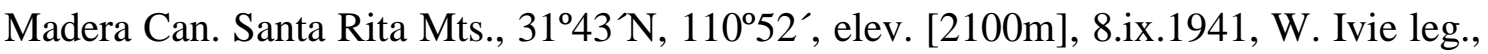
$3 \uparrow 2 ð$ (MCZ 125499). New Mexico: Whitewater Canyon 4 mi. N. E. Glenwood Catron

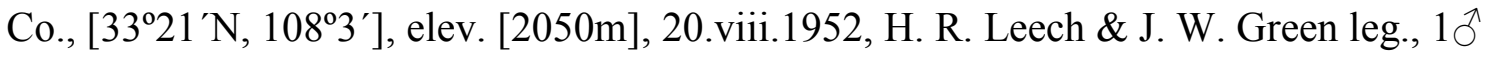
(AMNH). 
Glenognatha foxi (McCook, 1894)

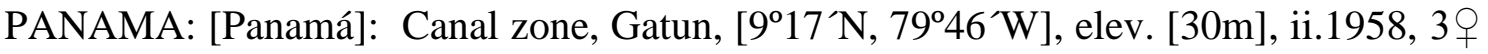

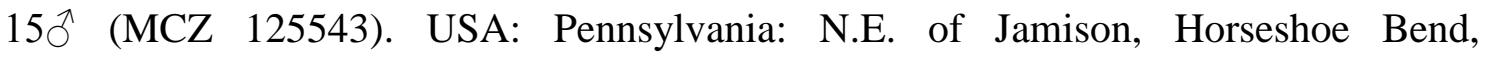

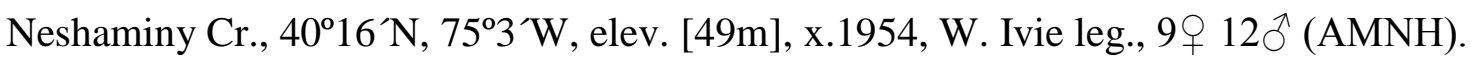

Glenognatha gaujoni Simon, 1895

BRAZIL: Acre: Marechal Thaumaturgo, Parque Nacional da Serra do Divisor, [8 $8^{\circ} 17^{\prime} \mathrm{S}$,

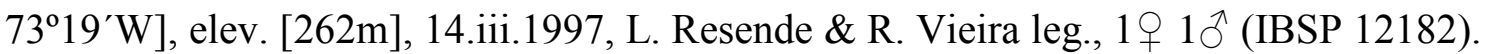
Amazonas: Manaus, Reserva Florestal Adolpho Ducke, [259`S, 5956`W], elev. [90m], vii.1999, A. J. Santos leg., $192{ }^{\Uparrow}$ (IBSP 26965); Reserva do PDBFF, [2²9`S, 60²`W], elev. [115m], ii-ix.2001, E. M. Venticinque leg., $1 q$ (IBSP 55545). PERU: Cuzco: Cashiriari, Rio Camisea, $11^{\circ} 52^{\prime} \mathrm{S}, 72^{\circ} 39^{\prime} \mathrm{W}$, elev. 690m, xii.1997, S. Córdoba leg., $1 \overbrace{}^{\wedge}$

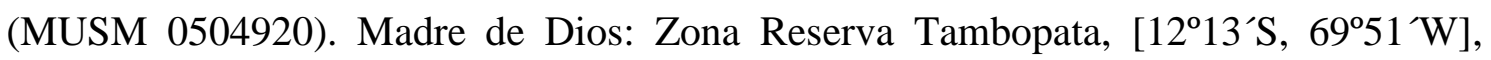
elev. 290m, 10-11.v.1991, D. Silva leg., 7 $7 ð$ (MUSM 0504927).

Glenognatha globosa (Petrunkevitch, 1925)

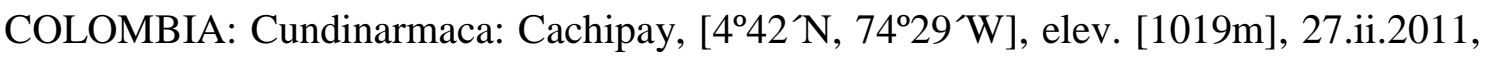
J. Cabra leg., 69 오 5 immature (ICN 5991). PANAMA: Chiriquí: Boquete, [846N,

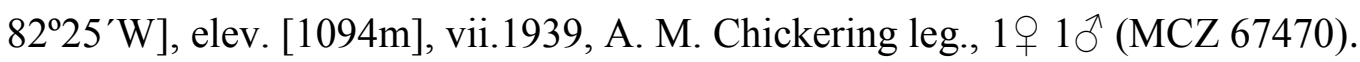

Glenognatha gloriae (Petrunkevitch, 1930)

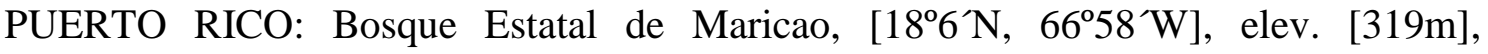
vii.1958, A. F. Archer leg., $1 q 1 \delta$ (AMNH); [Parque Nacional el Yunque], Baño de

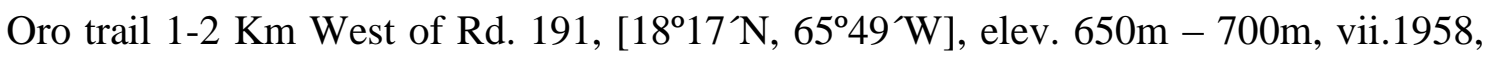
P. leg., $191 \delta^{\Uparrow}$ (MCZ 125485); Cordillera Central, Area recreo Doña Juana N. of

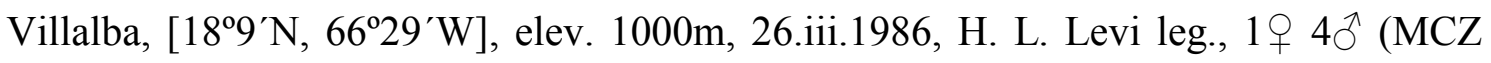
125482).

Glenognatha heleios Hormiga, 1990

USA: New Jersey: Oceanville, [39²8`N, 74²7`W], elev. [12m], 26.ix.1949, P. F.

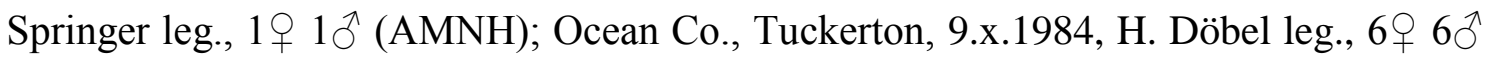
(USNM). 
Glenognatha hirsutissima (Berland, 1935)

FRENCH POLYNESIA: Marquesas Islands: Nuku Hiva, Tekao Ridge, 851'39”S, 140¹0`24”W, elev. 400m, 21.x.1999, D. A. \& J. T. Polhemus leg., $2 \overbrace{}^{`} 1$ (USNM).

Glenognatha iviei Levi, 1980

USA: Mississippi, Gulfport, 18.viii.1922, W. Ivie leg. 1ð (holotype) (AMNH).

Glenognatha lacteovittata (Mello-Leitão, 1944)

BRAZIL: Rio de Janeiro: Bom Jesus do Itabapuana, São José do Calçado, Rio Itabapuana, Usina Hidrelétrica de Rosal, [2058`S, 4143`W, elev. [605m], xi.1999, I. Knysak leg., $2 \propto 3 \hat{\jmath}$ (IBSP 26460). Santa Catarina: Bombinhas, Arie Costeira de Zimbros, [27오 $\left.{ }^{\circ}, 48^{\circ} 31^{\prime} \mathrm{W}\right]$, elev. [74m], 4.vii.2011, A. L. Trivia \& L. Malta leg., 1 \% (IBSP 163680); São Cristovão do Sul, Monte Alegre, [27ำ $\left.15^{\prime} \mathrm{S}, 50^{\circ} 26^{\prime} \mathrm{W}\right]$, elev.

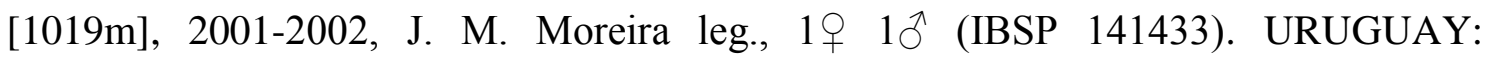
[Montevideo]: Montevideo, Ruta 1 Km 12, [3440’S, 56²9`W], elev. [3m], 2001, M. A. Benamu leg., $191 \delta^{\Uparrow}$ (IBSP 44084). PERU: Madre de Dios: Zona Reservada Pakitza, $11^{\circ} 56^{\prime} \mathrm{S}, 71^{\circ} 17^{\prime} \mathrm{W}$, elev. 356m, 18.viii.1992, D. Silva leg., $397{ }^{\Uparrow}$ (MUSM 0504907).

Glenognatha minuta Banks, 1898

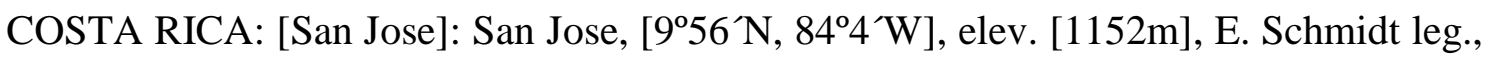
$1 q(\mathrm{AMNH})$; MEXICO: Nayarit: Ixtlan del Rio, [21 $\left.{ }^{\circ} 1^{\prime} \mathrm{N}, 104^{\circ} 21^{\prime} \mathrm{W}\right]$, elev. [1051m], 22.ix.1922, B. Malkin leg., 1 ( $(\mathrm{AMNH})$. San Luis Potosí: 10 mi. N.E. Xilitla, $21^{\circ} 27^{\prime} \mathrm{N}$, 98 $55^{\prime}$ W, elev. [132m], 19.iv.1963, W. J. Gertsch \& W. Ivie leg., $2{ }^{\lambda} 1$ immature

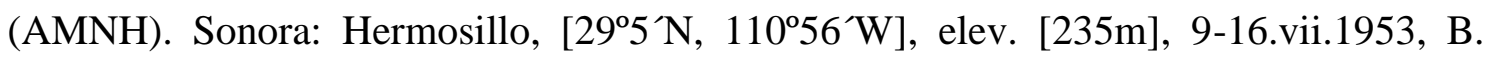
Malkin leg., 19 (AMNH). PANAMA: [Chiriquí]: El volcán, [84ำ $\mathrm{N}, 82^{\circ} 37^{\prime} \mathrm{W}$ ], elev. [1400m], 26.ii.1936, W. J. Gertsch leg., $3 q 3 \curvearrowright$ (AMNH).

Glenognatha smilodon Bosmans \& Bosselaers, 1994

CAMEROON, Poli Mountains, Pic Hosseré Vokré, 17.iv.1983, elev. 900m, Bosmans R. \& Van Stelle leg., ठ̊ (holotype) (MRAC 202710).

Glenognatha spherella Chamberlin \& Ivie, 1936

COSTA RICA: [San José]: San José, [956№ 84`W], elev. [1152m], E. Schmidt leg., $7 \nmid 3{ }^{\Uparrow}$ (AMNH). MEXICO: Michoacán: Garnica Pass (summit, 9300ft), 19²0^N, 
$100^{\circ} 55^{\prime} \mathrm{W}$ ], elev. [2332m], 8.v.1963, W. J. Gertsch \& W. Ivie leg., 12 \% $23 \overbrace{}^{\Uparrow} 9$

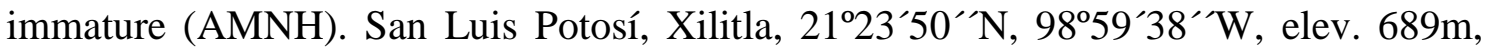
27-31.viii.2011, FAPLAB leg., $1 q 3 \hat{\jmath}$ (CAS 9046859); 14-18.xi.2011, FAPLAB leg., 1ठ (CAS 9046858) ; $3 ð$ (CAS 9046857). PANAMA: [Panamá]: Barro Colorado, Canal

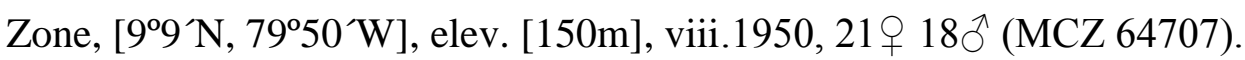

Glenognatha sp. nov. 1

BRAZIL: Paraná: Antonina, Reserva Natural da Cachoeira, [2524`2,4”S,

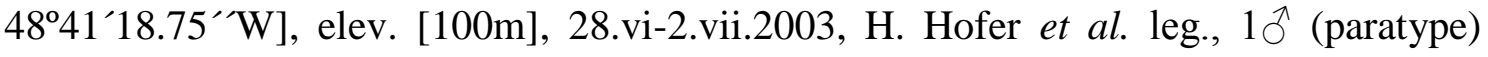
(IBSP 131182). Rio Grande do Sul: Triunfo, Estaçao Braskem de Proteçao Ambiental,

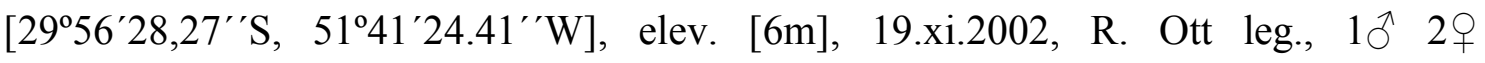
(paratype) (MCN 34952). São Paulo: Salesópolis, Barragem do Rio Paraitinga,

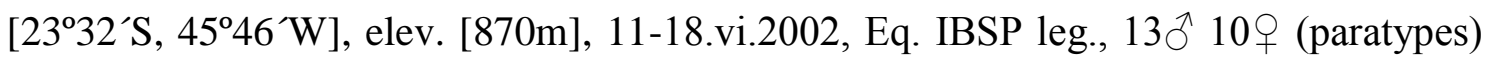
(IBSP 147861); 18-25.vi.2002, $3 ð 5$ (IBSP 147809).

Glenognatha sp. nov. 2

COLOMBIA: Valle Del Cauca: Dagua, Alto Queremal, Hacienda San Pedro, $3^{\circ} 29^{\prime} 5.42^{\prime \prime} \mathrm{N}, 76^{\circ} 42^{\prime} \mathrm{W}$, elev. 1800m, 21-26.xi.2009, J. Cabra leg., $12 \bigcirc^{\Uparrow} 9$ (paratypes) (ICN 5982). COSTA RICA: Punta Arenas/Alajuelas: Monte Verde Biological Reserve,

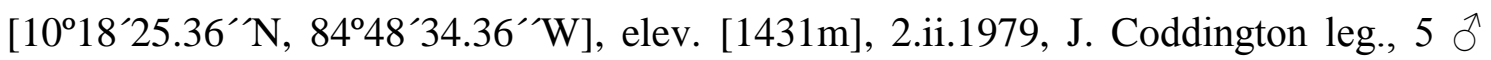
(MCZ 125511).

Glenognatha sp. nov. 3

BRAZIL: Rio Grande do Sul: São Francisco de Paula, Potreiro Velho, [29²8’S,

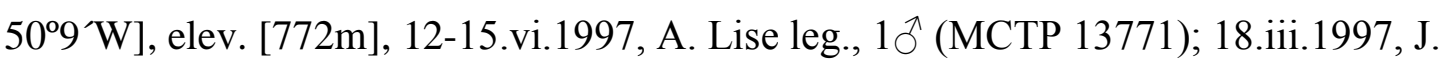
Ketterl leg., 1 (MCTP 17493). São Paulo: São José do Barreiro, Parque Nacional da Serra da Bocaina, [22 $43^{\circ} \mathrm{S}, 44^{\circ} 36^{\prime} \mathrm{W}$ ], elev. [900m], 28.iv - 3.v.2002, Equipe Biota leg., Pitfall, $4 q$ (IBSP 68552); $101 q$ (IBSP 57091); 1ð (IBSP 57078).

Glenognatha sp. nov. 4

BRAZL: São Paulo: Estação Biológica Boracéia, Salesópolis, São Paulo, Brazil, 2339`20.6”S,4553`28.6”W, elev. [900m], 1.iv.2001, S.T.P. Amarante e Equipe leg.,

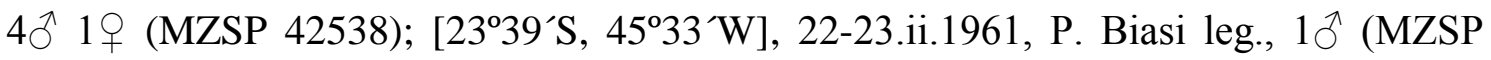

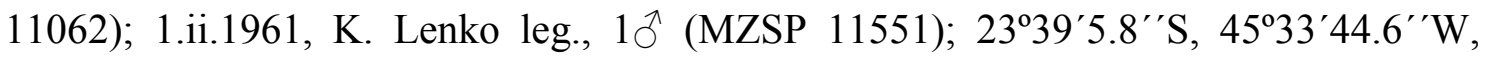


1.iv.2001, 1 q (MZSP 39047); Trilha dos Pilões, 2339¹.9”'S, 4553’36”W, 4.iv.2001, (varredura), $2 \hat{\jmath} 1$ (

Glenognatha sp. nov. 5

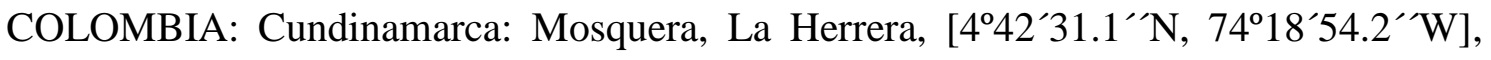
elev. 2600m, 9.ii.2002, J. Martinez, C. Niño, A. Castañeda, G. Mora leg., $43 \widehat{\bigcirc} 4 q 8$ immature (ICN 1973);

Glenognatha sp. nov. 6

COLOMBIA: Vaupés: Taraira, Lago Taraira. Estación Biológica Caparu, [1%`S,

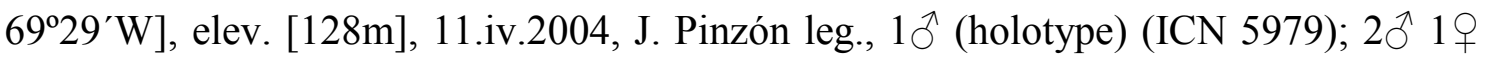
(paratypes) (ICN 5980); PERU: Loreto: P.N. Pacaya-Samiria Pithecia, 56’ S, 74º50 W, elev. 100m, 16.viii.1989, D. Silva leg., 1 त 3 क 6 immature (MUSM 0504921). VENEZUELA: [Amazonas]: Igarapé forest of upper Rio Yaciba, [1 ${ }^{\circ} 14^{\prime} \mathrm{N}, 66^{\circ} 44^{`} \mathrm{~W}$ ], elev. [109m], 7.xii.1953, W. M. Beebe leg., $1 \overbrace{}^{\Uparrow} 1$ (AMNH).

Glenognatha sp. nov. 7

BRAZIL: Rio de Janeiro: Teresópolis, Parque Nacional da Serra dos Órgãos,

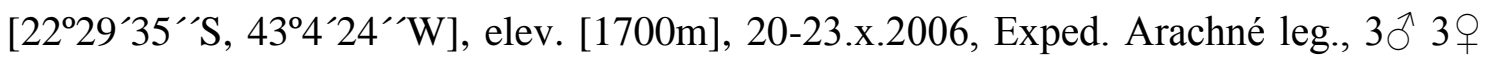
(paratypes) (MNRJ 6598); 4.viii.1996, 1 q (MNRJ 6400). São Paulo: São José do Barreiro, Parque Nacional da Serra da Bocaina, [22 $43^{\prime} \mathrm{S}, 4^{\circ} 36^{\prime} \mathrm{W}$ ], elev. [900m], 28.iv -3.v.2002, Equipe Biota leg., 1 (IBSP 68554).

Glenognatha sp. nov. 8

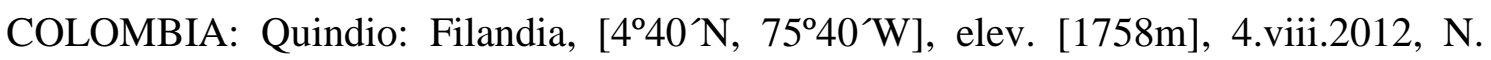
Hazzi leg., 6 ㅇ 1ð (ICN 5998)Valle Del Cauca: Dagua, Alto Queremal, Hacienda San Pedro, $3^{\circ} 29^{\prime} \mathrm{N}, 76^{\circ} 42^{\prime} \mathrm{W}$, elev. 1800m, 21.ix.2009, J. Cabra leg., $4 \bigcirc^{\Uparrow} 19+$ (paratypes) (ICN 5986).

Glenognatha sp. nov. 9

BRAZIL: Amazonas: Manaus, Lago Janauari, 3²0’S, 60¹7`W, elev. [12m], vi.1987vii.1988, J. Adis et al. leg., $1 \sigma^{\Uparrow}$ (holotype) (IBSP 120209); $1 \hat{\sigma}$ (paratype) (IBSP 120266); 1 § (IBSP 120166); 1 ภ (IBSP 120220). 
Glenognatha sp. nov. 10

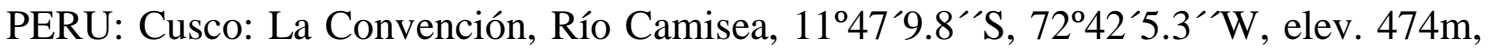

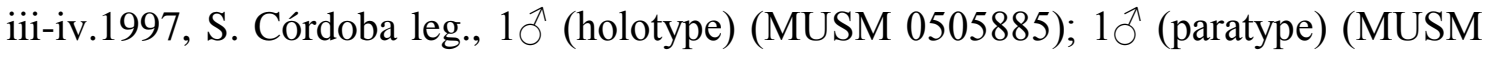

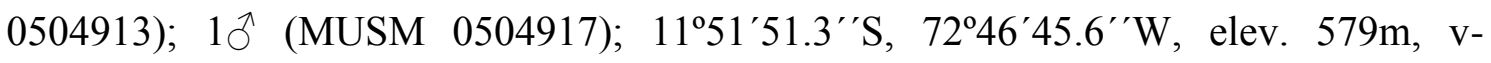
vi.1997, S. Córdoba leg., $1 \overbrace{}^{\Uparrow}$ (MUSM 0504905)

Glenognatha sp. nov. 11

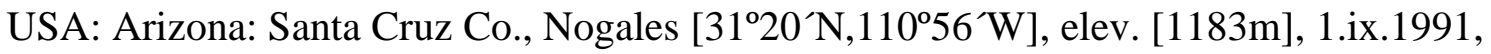

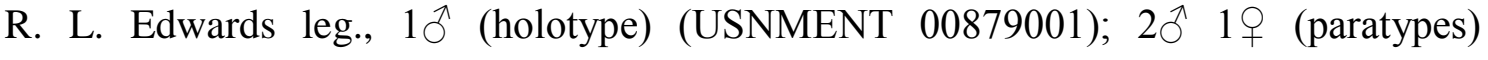

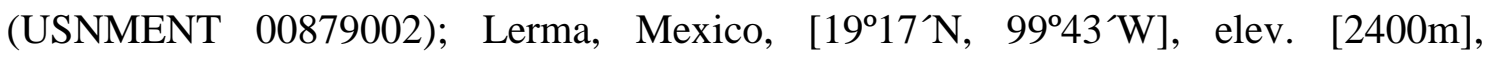
25.vi.1941, A. M. \& L. I. Davis leg. 1ठ (paratype) (AMNH). 


\subsection{Data matrix}

\begin{tabular}{|c|c|c|c|c|c|c|c|c|c|c|c|c|c|c|c|c|c|c|c|c|c|c|c|c|c|c|c|c|c|c|c|c|c|c|c|c|}
\hline \multirow[t]{2}{*}{ Taxa } & \multicolumn{12}{|c|}{ Spigots } & \multicolumn{24}{|c|}{ Male papal morphology } \\
\hline & 1 & 2 & 3 & 4 & 5 & 6 & 7 & 8 & 9 & 10 & 11 & 12 & 13 & 14 & 15 & 16 & 17 & 18 & 19 & 20 & 21 & 22 & 23 & 24 & 25 & 26 & 27 & 28 & 29 & 30 & 31 & 32 & 33 & 34 & 35 & 36 \\
\hline Ch. alajuela & 0 & 0 & 2 & 0 & 0 & 0 & 0 & 0 & 0 & 1 & 0 & 0 & 0 & 1 & 0 & - & 1 & 2 & - & 0 & - & 0 & 0 & 0 & 0 & 1 & - & - & 0 & 0 & 0 & 0 & - & 0 & 1 & 0 \\
\hline C. espanola & 0 & 0 & 2 & 0 & 0 & 0 & 1 & 0 & 0 & 1 & 1 & 1 & 1 & 0 & 1 & - & 0 & 1 & 0 & 0 & - & 0 & 0 & 0 & 0 & 0 & 0 & 1 & 0 & 0 & 0 & 0 & - & 0 & 1 & 0 \\
\hline T. versicolor & 0 & 0 & 2 & 0 & 0 & 0 & 0 & 0 & 1 & 1 & 0 & 0 & 1 & 0 & 1 & - & 1 & 1 & 0 & 1 & 0 & 0 & 0 & 0 & 0 & 0 & 0 & 0 & 0 & 0 & 0 & 0 & - & 0 & 1 & 1 \\
\hline P. autumnalis & 1 & 1 & 1 & 1 & 1 & 0 & 0 & 0 & 0 & 0 & 1 & 1 & 1 & 0 & 1 & - & 1 & 1 & 0 & 1 & 0 & 0 & 0 & 0 & 0 & 0 & 0 & 0 & 0 & 0 & 0 & 0 & - & 0 & 0 & 0 \\
\hline P. clercki & 1 & 1 & $?$ & 1 & 1 & 0 & 0 & 0 & 0 & 0 & 1 & 1 & 1 & 0 & 1 & - & 1 & 1 & 0 & 1 & 0 & 0 & 0 & 0 & 0 & 0 & 0 & 0 & 0 & 0 & 0 & 0 & - & 0 & 0 & 1 \\
\hline D. dentata & 0 & 1 & 1 & 0 & 0 & 1 & 0 & 1 & 0 & 0 & 1 & 1 & 1 & 0 & 2 & 1 & 1 & 0 & 1 & 1 & 1 & 2 & 1 & 0 & 0 & 0 & 0 & 0 & 0 & 0 & 2 & 1 & 1 & 0 & 0 & 0 \\
\hline D. tangi & 0 & 1 & 1 & 0 & 0 & 1 & 0 & 1 & 0 & 0 & 1 & 1 & 1 & 0 & 2 & 1 & 1 & 0 & 1 & 1 & 1 & 0 & 0 & 0 & 0 & 0 & 1 & 0 & 0 & 0 & 0 & 1 & 1 & 0 & 0 & 0 \\
\hline G. argyrostilba & 0 & 1 & 1 & 0 & 0 & 1 & 0 & 1 & 0 & 0 & 1 & 1 & 1 & 0 & 2 & 1 & 0 & 0 & 1 & 1 & 1 & 0 & 0 & 0 & 0 & 0 & 0 & 0 & 0 & 0 & 0 & 1 & 1 & 0 & 0 & 0 \\
\hline G. australis & 0 & 1 & 1 & 0 & 0 & 1 & 0 & 1 & 0 & 0 & 1 & 1 & 1 & 0 & 2 & 1 & 0 & 0 & 1 & 1 & 1 & 0 & 0 & 1 & 0 & 0 & 1 & 0 & 0 & 0 & 0 & 1 & 1 & 0 & 0 & 0 \\
\hline G. emertoni & 0 & 1 & 1 & 0 & 0 & 1 & 0 & 1 & 0 & 0 & 1 & 1 & 1 & 0 & 2 & 1 & 0 & 0 & 1 & 1 & 1 & 0 & 0 & 1 & 0 & 0 & 0 & 0 & 0 & 0 & 0 & 1 & 1 & 0 & 0 & 1 \\
\hline G. foxi & 0 & 1 & 1 & 0 & 0 & 1 & 0 & 1 & 0 & 0 & 1 & 1 & 1 & 0 & 2 & 1 & 0 & 0 & 1 & 1 & 1 & 0 & 0 & 1 & 0 & 0 & 0 & 1 & 0 & 0 & 0 & 1 & 1 & 0 & 0 & 0 \\
\hline G. gaujoni & 0 & 1 & 1 & 0 & 0 & 1 & 0 & 1 & 0 & 0 & 1 & 1 & 1 & 0 & 2 & 1 & 0 & 0 & 1 & 1 & 1 & 0 & 0 & 0 & 0 & 0 & 0 & 0 & 0 & 1 & 1 & 1 & 1 & 0 & 0 & 1 \\
\hline G. globosa & 0 & 1 & 1 & 0 & 0 & 1 & 0 & 1 & 0 & 0 & 1 & 1 & 1 & 0 & 2 & 1 & 0 & 0 & 1 & 1 & 1 & 0 & 0 & 0 & 0 & 0 & 1 & 0 & 0 & 1 & 0 & 1 & 1 & 0 & 0 & 1 \\
\hline G. gloriae & 0 & 1 & 1 & 0 & 0 & 1 & 0 & 1 & 0 & 0 & 1 & 1 & 1 & 0 & 2 & 1 & 0 & 0 & 1 & 1 & 1 & 0 & 0 & 1 & 0 & 0 & 0 & 0 & 1 & 0 & 0 & 1 & 1 & 0 & 0 & 0 \\
\hline G. heleios & 0 & 1 & 1 & 0 & 0 & 1 & 0 & 1 & 0 & 0 & 1 & 1 & 1 & 0 & 2 & 1 & 1 & 0 & 1 & 1 & 1 & 0 & 0 & 1 & 0 & 0 & 0 & 1 & 0 & 0 & 0 & 1 & 1 & 0 & 0 & 0 \\
\hline G. hirsutissima & 0 & 1 & 1 & 0 & 0 & 1 & 0 & 1 & 0 & 0 & 1 & 1 & 1 & 0 & 2 & 1 & 0 & 0 & 1 & 1 & 1 & 0 & 0 & 1 & 0 & 0 & 0 & 1 & 0 & 0 & 0 & 1 & 1 & 0 & 0 & 0 \\
\hline G. iviei & $?$ & $?$ & $?$ & $?$ & $?$ & $?$ & 0 & $?$ & 0 & $?$ & $?$ & 1 & 1 & 0 & 2 & 1 & 1 & 0 & 1 & 1 & 1 & 0 & 0 & 1 & 0 & 0 & 0 & 0 & 0 & 0 & 0 & 1 & 1 & 0 & 0 & 0 \\
\hline G. lacteovittata & 0 & 1 & 0 & 0 & 0 & 0 & 0 & 0 & 0 & 0 & 1 & 1 & 1 & 0 & 2 & 0 & 0 & 0 & 1 & 1 & 1 & 0 & 0 & 0 & 1 & 0 & 0 & 0 & 2 & 0 & 2 & 1 & 0 & 0 & 0 & 0 \\
\hline G. minuta & 0 & 1 & 1 & 0 & 0 & 1 & 0 & 1 & 0 & 0 & 1 & 1 & 1 & 0 & 2 & 1 & 0 & 0 & 1 & 1 & 1 & 0 & 0 & 1 & 0 & 0 & 0 & 0 & 0 & 0 & 0 & 1 & 1 & 0 & 0 & 0 \\
\hline G. smilodon & $?$ & $?$ & $?$ & $?$ & $?$ & $?$ & 0 & $?$ & 0 & $?$ & $?$ & 1 & 1 & 0 & 2 & 1 & 0 & 0 & 1 & 1 & 1 & 0 & 0 & 1 & 1 & 0 & 0 & 0 & 0 & 0 & 0 & 1 & 1 & 1 & 0 & 0 \\
\hline G. spherella & 0 & 1 & 1 & 0 & 0 & 1 & 0 & 1 & 0 & 0 & 1 & 1 & 1 & 0 & 2 & 1 & 0 & 0 & 1 & 1 & 1 & 0 & 0 & 1 & 0 & 0 & 0 & 1 & 1 & 0 & 0 & 1 & 1 & 0 & 0 & 0 \\
\hline$G$. sp. nov. 1 & 0 & 1 & 1 & 0 & 0 & 1 & 0 & 1 & 0 & 0 & 1 & 1 & 1 & 0 & 2 & 1 & 0 & 0 & 1 & 1 & 1 & 0 & 0 & 1 & 0 & 0 & 0 & 1 & 0 & 0 & 0 & 1 & 1 & 0 & 0 & 0 \\
\hline G. sp. nov. 2 & 0 & 1 & 1 & 0 & 0 & 1 & 0 & 1 & 0 & 0 & 1 & 1 & 1 & 0 & 2 & 1 & 0 & 0 & 1 & 1 & 1 & 0 & 0 & 0 & 0 & 0 & 0 & 0 & 0 & 1 & 0 & 1 & 1 & 0 & 0 & 0 \\
\hline$G$. sp. nov. 3 & 0 & 1 & 1 & 0 & 0 & 1 & 0 & 1 & 0 & 0 & 1 & 1 & 1 & 0 & 2 & 1 & 1 & 0 & 1 & 1 & 1 & 0 & 0 & 1 & 0 & 0 & 0 & 0 & 0 & 0 & 0 & 1 & 1 & 0 & 0 & 0 \\
\hline G. sp. nov. 4 & 0 & 1 & 0 & 0 & 0 & 0 & 0 & 0 & 0 & 0 & 1 & 1 & 1 & 0 & 2 & 0 & 0 & 0 & 1 & 1 & 1 & 0 & 0 & 0 & 0 & 0 & 0 & 0 & 0 & 0 & 0 & 1 & 0 & 0 & 0 & 0 \\
\hline$G$. sp. nov. 5 & 0 & 1 & 0 & 0 & 0 & 0 & 0 & 0 & 0 & 0 & 1 & 1 & 1 & 0 & 2 & 0 & 0 & 0 & 1 & 1 & 1 & 0 & 0 & 0 & 1 & 0 & 1 & 0 & 0 & 0 & 2 & 1 & 0 & 0 & 0 & 0 \\
\hline G. sp. nov. 6 & 0 & 1 & 1 & 0 & 0 & 1 & 0 & 1 & 0 & 0 & 1 & 1 & 1 & 0 & 2 & 1 & 0 & 0 & 1 & 1 & 1 & 1 & 0 & 1 & 0 & 0 & 1 & 0 & 0 & 0 & 0 & 1 & 1 & 0 & 0 & 0 \\
\hline G. sp. nov. 7 & 0 & 1 & 1 & 0 & 0 & 1 & 0 & 1 & 0 & 0 & 1 & 1 & 1 & 0 & 2 & 1 & 0 & 0 & 1 & 1 & 1 & 0 & 0 & 1 & 0 & 0 & 0 & 0 & 0 & 0 & 0 & 1 & 1 & 0 & 0 & 1 \\
\hline G. sp. nov. 8 & 0 & 1 & 1 & 0 & 0 & 1 & 0 & 1 & 0 & 0 & 1 & 1 & 1 & 0 & 2 & 1 & 0 & 0 & 1 & 1 & 1 & 0 & 0 & 0 & 0 & 0 & 1 & 0 & 0 & 1 & 0 & 1 & 1 & 0 & 0 & 1 \\
\hline G. sp. nov. 9 & 0 & $?$ & 1 & $?$ & $?$ & 1 & 0 & 1 & 0 & 0 & 1 & 1 & 1 & 0 & 2 & 1 & 0 & 0 & 1 & 1 & 1 & 0 & 1 & 1 & 0 & 0 & 0 & 0 & 0 & 0 & 0 & 1 & 1 & 0 & 0 & 0 \\
\hline G. sp. nov. 10 & 0 & $?$ & 1 & $?$ & $?$ & 1 & 0 & 1 & 0 & 0 & 1 & 1 & 1 & 0 & 2 & 1 & 0 & 0 & 1 & 1 & 1 & 0 & 0 & 1 & 0 & 0 & 0 & 0 & 0 & 0 & 0 & 1 & 1 & 0 & 0 & 0 \\
\hline G. sp. nov. 11 & 0 & 1 & 1 & 0 & 0 & 1 & 0 & 1 & 0 & 0 & 1 & 1 & 1 & 0 & 2 & 1 & 0 & 0 & 1 & 1 & 1 & 0 & 0 & 1 & 0 & 0 & 1 & 0 & 0 & 0 & 0 & 1 & 1 & 0 & 0 & 0 \\
\hline
\end{tabular}




\begin{tabular}{|c|c|c|c|c|c|c|c|c|c|c|c|c|c|c|c|c|c|c|c|c|c|c|c|c|c|c|c|c|c|c|c|c|}
\hline \multirow[t]{2}{*}{ Taxa } & \multicolumn{11}{|c|}{ Female genitalia } & \multicolumn{21}{|c|}{ Cephalothorax, chelicerae and legs } \\
\hline & 37 & 38 & 39 & 40 & 41 & 42 & 43 & 44 & 45 & 46 & 47 & 48 & 49 & 50 & 51 & 52 & 53 & 54 & 55 & 56 & 57 & 58 & 59 & 60 & 61 & 62 & 63 & 64 & 65 & 66 & 67 & 68 \\
\hline Ch. alajuela & 0 & 0 & 1 & 0 & 0 & 2 & 0 & - & - & - & 0 & 1 & 1 & 0 & 0 & 0 & 0 & 0 & 0 & 0 & - & 0 & 0 & 0 & 0 & 0 & 0 & 0 & 0 & 0 & 0 & 0 \\
\hline C. espanola & 1 & 0 & - & 0 & 0 & 0 & 1 & 0 & - & 0 & 1 & 1 & 0 & 0 & 0 & 0 & 0 & 0 & 0 & 1 & 0 & 1 & 0 & 0 & 0 & 0 & 0 & 0 & 0 & 0 & 1 & 0 \\
\hline T. versicolor & 1 & 0 & 1 & 1 & 0 & 0 & 1 & 0 & - & 0 & 1 & 0 & 1 & 0 & 0 & 0 & 1 & 0 & 1 & 1 & 1 & 0 & 0 & 1 & 0 & 0 & 0 & 0 & 0 & 0 & 0 & 0 \\
\hline P. autumnalis & 1 & 0 & 0 & 1 & 0 & 0 & 1 & 0 & - & 0 & 0 & 1 & 1 & 1 & 0 & 1 & 1 & 0 & 0 & 0 & - & 1 & 0 & 0 & 0 & 0 & 0 & 0 & 0 & 0 & 1 & 0 \\
\hline P. clercki & 1 & 0 & 0 & 1 & 0 & 0 & 1 & 0 & - & 1 & 0 & 1 & 1 & 1 & 0 & 1 & 1 & 0 & 0 & 1 & 1 & 1 & 0 & 0 & 0 & 0 & 0 & 0 & 0 & 0 & 1 & 0 \\
\hline D. dentata & 1 & 0 & 0 & 0 & 1 & 1 & 1 & 0 & - & 1 & 0 & 1 & 2 & 1 & 1 & 1 & 1 & 1 & 0 & 0 & - & 1 & 0 & 0 & 0 & 0 & 0 & 1 & 0 & 1 & 1 & 0 \\
\hline D. tangi & 1 & 0 & 0 & 0 & 1 & 1 & 1 & 0 & - & 0 & 0 & 1 & 1 & 1 & 1 & 0 & 1 & 0 & 0 & 0 & - & 1 & 0 & 0 & 0 & 0 & 0 & 0 & 0 & 1 & 1 & 0 \\
\hline G. argyrostilba & 1 & 0 & 0 & 0 & 1 & 1 & 1 & 0 & - & 0 & 0 & 1 & 1 & 1 & 1 & 1 & 1 & 1 & 0 & 0 & - & 1 & 0 & 0 & 0 & 0 & 0 & 0 & 0 & 1 & 1 & 0 \\
\hline G. australis & 1 & 0 & 0 & 0 & 1 & 1 & 1 & 2 & 0 & 0 & 0 & 1 & 1 & 0 & 0 & 0 & 0 & 0 & 0 & 0 & - & 0 & 0 & 0 & 0 & 0 & 1 & 1 & 0 & 1 & 0 & 0 \\
\hline G. emertoni & 1 & 0 & 0 & 0 & 1 & 1 & 1 & 1 & - & 0 & 0 & 1 & 1 & 0 & 0 & 0 & 0 & 0 & 1 & 0 & - & 0 & 0 & 1 & 0 & 0 & 1 & 1 & 1 & 0 & 0 & 0 \\
\hline G. foxi & 1 & 0 & 0 & 0 & 1 & 1 & 1 & 0 & - & 0 & 0 & 1 & 1 & 0 & 0 & 0 & 0 & 0 & 0 & 1 & 0 & 1 & 0 & 0 & 0 & 0 & 0 & 0 & 0 & 1 & 0 & 0 \\
\hline G. gaujoni & 1 & 1 & - & - & - & - & 1 & 2 & 1 & 0 & 0 & 1 & 1 & 0 & 0 & 0 & 1 & 0 & 1 & 0 & - & 1 & 0 & 0 & 0 & 0 & 0 & 0 & 0 & 1 & 0 & 1 \\
\hline G. globosa & 1 & 1 & - & - & - & - & 1 & 2 & 1 & 0 & 0 & 1 & 1 & 0 & 0 & 0 & 1 & 0 & 1 & 0 & - & 0 & 0 & 0 & 0 & 0 & 0 & 0 & 0 & 1 & 0 & 1 \\
\hline G. gloriae & 1 & 0 & 0 & 0 & 1 & 1 & 1 & 1 & - & 0 & 0 & 1 & 2 & 0 & 0 & 0 & 0 & 0 & 0 & 0 & - & 0 & 0 & 0 & 0 & 0 & 1 & 1 & 0 & 1 & 1 & 0 \\
\hline G. heleios & 1 & 0 & 0 & 0 & 1 & 1 & 1 & 0 & - & 0 & 0 & 1 & 1 & 0 & 0 & 0 & 0 & 0 & 0 & 1 & 0 & 1 & 0 & 0 & 1 & 1 & 0 & 0 & 0 & 0 & 0 & 0 \\
\hline G. hirsutiss & 1 & 0 & 0 & 0 & 1 & 1 & 1 & 0 & - & 0 & 0 & 1 & 2 & 0 & 0 & 0 & 0 & 0 & 0 & 0 & - & 0 & 1 & 0 & 0 & 0 & 0 & 0 & 0 & 0 & - & 0 \\
\hline G. iviei & ? & $?$ & $?$ & $?$ & $?$ & $?$ & $?$ & ? & $?$ & $?$ & $?$ & $?$ & 1 & 0 & 0 & 0 & 0 & 0 & 0 & 1 & 0 & 1 & 0 & 0 & 1 & 2 & 0 & 0 & 0 & 0 & 0 & $?$ \\
\hline G. lacteovittata & 1 & 1 & - & - & - & - & 1 & 1 & - & 0 & 0 & 1 & 1 & 0 & 0 & 0 & 0 & 0 & 0 & 0 & - & 1 & 0 & 0 & 0 & 0 & 0 & 0 & 0 & 1 & 0 & 0 \\
\hline G. minuta & 1 & 0 & 0 & 0 & 1 & 1 & 1 & 1 & - & 0 & 0 & 1 & 1 & 0 & 0 & 0 & 0 & 0 & 1 & 0 & - & 0 & 0 & 0 & 0 & 0 & 1 & 0 & 1 & 0 & 0 & 0 \\
\hline G. smilodon & ? & $?$ & $?$ & $?$ & $?$ & $?$ & $?$ & $?$ & $?$ & $?$ & $?$ & $?$ & 1 & 0 & 0 & 0 & 0 & 0 & 0 & 0 & - & 1 & 0 & 0 & 1 & 1 & 0 & 0 & 0 & 1 & 0 & $?$ \\
\hline G. spherella & 1 & 0 & 0 & 0 & 1 & 1 & 1 & 1 & - & 0 & 0 & 1 & 1 & 0 & 0 & 0 & 0 & 0 & 0 & 0 & - & 0 & 0 & 0 & 0 & 0 & 1 & 1 & 0 & 1 & 1 & 0 \\
\hline$G$. sp. nov. 1 & 1 & 0 & 0 & 0 & 1 & 1 & 1 & 2 & 0 & 0 & 0 & 1 & 1 & 0 & 0 & 0 & 0 & 0 & 1 & 0 & - & 0 & 0 & 0 & 0 & 0 & 0 & 0 & 0 & 1 & 0 & 0 \\
\hline G. sp. nov. 2 & 1 & 0 & 0 & 0 & 1 & 1 & 1 & 1 & - & 0 & 0 & 1 & 1 & 0 & 0 & 0 & 0 & 0 & 1 & 0 & - & 1 & 0 & 0 & 0 & 0 & 0 & 0 & 0 & 1 & 0 & 0 \\
\hline G. sp. nov. 3 & 1 & 0 & 0 & 0 & 1 & 1 & 1 & 2 & 0 & 0 & 0 & 1 & 1 & 0 & 0 & 0 & 0 & 0 & 0 & 1 & 0 & 1 & 0 & 0 & 1 & 0 & 0 & 0 & 0 & 1 & 0 & 0 \\
\hline$G$ sp. nov. 4 & 1 & 1 & - & - & - & - & 1 & 1 & - & 0 & 0 & 1 & 2 & 0 & 0 & 0 & 0 & 0 & 0 & 0 & - & 1 & 0 & 0 & 0 & 0 & 0 & 0 & 0 & 1 & 0 & 0 \\
\hline$G$. sp. nov. 5 & 1 & 1 & - & - & - & - & 1 & 1 & - & 0 & 0 & 1 & 1 & 0 & 0 & 0 & 0 & 0 & 0 & 0 & - & 1 & 0 & 0 & 0 & 0 & 0 & 0 & 0 & 1 & 0 & 0 \\
\hline G. sp. nov. 6 & 1 & 0 & 0 & 0 & 1 & 1 & 1 & 2 & 0 & 0 & 0 & 1 & 1 & 0 & 0 & 0 & 0 & 0 & 1 & 1 & 1 & 1 & 0 & 0 & 1 & 0 & 0 & 0 & 0 & 1 & 1 & 0 \\
\hline G. sp. nov. 7 & 1 & 1 & - & - & - & - & 1 & 1 & - & 0 & 0 & 1 & 1 & 0 & 0 & 0 & 0 & 0 & 1 & 0 & - & 0 & 0 & 0 & 0 & 0 & 1 & 1 & 1 & 1 & 0 & 0 \\
\hline G. sp. nov. 8 & 1 & 1 & - & - & - & - & 1 & 2 & 1 & 0 & 0 & 1 & 1 & 0 & 0 & 0 & 1 & 0 & 1 & 0 & - & 0 & 0 & 0 & 0 & 0 & 0 & 0 & 0 & 1 & 0 & 0 \\
\hline G. sp. nov. 9 & $?$ & $?$ & $?$ & $?$ & $?$ & $?$ & $?$ & $?$ & $?$ & $?$ & $?$ & $?$ & 1 & 0 & 0 & 0 & 0 & 0 & 0 & 1 & 0 & 0 & 0 & 0 & 0 & 0 & 0 & 0 & 0 & 1 & 0 & $?$ \\
\hline G. sp. nov. 10 & $?$ & $?$ & $?$ & $?$ & $?$ & $?$ & $?$ & $?$ & $?$ & $?$ & $?$ & $?$ & 1 & 0 & 0 & 0 & 0 & 0 & 0 & 1 & 0 & 0 & 0 & 0 & 0 & 0 & 0 & 0 & 0 & 1 & 0 & $?$ \\
\hline G. sp. nov. 11 & 1 & 1 & - & - & - & - & 1 & 1 & - & 0 & 0 & 1 & 1 & 0 & 0 & 0 & 0 & 0 & 1 & 0 & - & 0 & 0 & 0 & 0 & 0 & 1 & 1 & 1 & 1 & 1 & 0 \\
\hline
\end{tabular}




\begin{tabular}{|c|c|c|c|c|c|c|c|c|c|c|c|c|c|c|}
\hline \multirow[t]{2}{*}{ Taxa } & \multicolumn{11}{|c|}{ Cephalothorax, chelicerae and legs } & \multicolumn{3}{|c|}{ Abdomen } \\
\hline & 69 & 70 & 71 & 72 & 73 & 74 & 75 & 76 & 77 & 78 & 79 & 80 & 81 & 82 \\
\hline Ch. alajuela & 0 & 0 & 0 & 0 & 0 & - & 0 & 0 & 0 & - & 0 & 0 & 0 & 0 \\
\hline C. espanola & 0 & 0 & 0 & 0 & 0 & - & 0 & 0 & 1 & 1 & 0 & 0 & 0 & 0 \\
\hline T. versicolor & 0 & 0 & 0 & 1 & 0 & - & 0 & 1 & 1 & 0 & 0 & 0 & 0 & 0 \\
\hline P. autumnalis & 0 & 0 & 0 & 0 & 0 & - & 0 & 0 & 1 & 0 & 0 & 1 & 0 & 0 \\
\hline P. clercki & 0 & 0 & 0 & 0 & 0 & - & 0 & 0 & 1 & 0 & 0 & 1 & 0 & 0 \\
\hline D. dentata & 0 & 0 & 0 & 0 & 0 & - & 0 & 0 & 1 & 0 & 0 & 1 & 1 & 1 \\
\hline D. tangi & 0 & 0 & 0 & 0 & 0 & - & 0 & 0 & 1 & 0 & 0 & 1 & 1 & 1 \\
\hline G. argyrostilba & 0 & 0 & 0 & 0 & 0 & - & 0 & 0 & 1 & 0 & 0 & 1 & 1 & 1 \\
\hline G. australis & 0 & 0 & 0 & 0 & 0 & - & 0 & 0 & 1 & 0 & 0 & 1 & 1 & 1 \\
\hline G. emertoni & 0 & 0 & 1 & 1 & 0 & - & 0 & 0 & 1 & 0 & 0 & 1 & 1 & 1 \\
\hline G. foxi & 1 & 0 & 0 & 0 & 0 & - & 0 & 0 & 1 & 0 & 0 & 1 & 1 & 1 \\
\hline G. gaujoni & 1 & 1 & 0 & 0 & 0 & - & 0 & 0 & 1 & 0 & 0 & 1 & 1 & 1 \\
\hline G. globosa & 0 & 0 & 0 & 0 & 0 & - & 0 & 1 & 1 & 0 & 0 & 1 & 1 & 1 \\
\hline G. gloriae & 0 & 0 & 0 & 0 & 0 & - & 0 & 0 & 1 & 0 & 0 & 1 & 1 & 1 \\
\hline G. heleios & 0 & 0 & 0 & 0 & 0 & - & 0 & 0 & 1 & 0 & 0 & 1 & 1 & 1 \\
\hline G. hirsutissima & 0 & 0 & 0 & 0 & 0 & - & 0 & 0 & 1 & 0 & 1 & 1 & 1 & 1 \\
\hline G. iviei & $?$ & $?$ & $?$ & $?$ & $?$ & $?$ & $?$ & 0 & 1 & 0 & 0 & $?$ & $?$ & $?$ \\
\hline G. lacteovittata & 0 & 0 & 0 & 0 & 1 & 1 & 0 & 0 & 1 & 0 & 0 & 1 & 1 & 1 \\
\hline G. minuta & 0 & 0 & 1 & 1 & 0 & - & 0 & 0 & 1 & 0 & 0 & 1 & 1 & 1 \\
\hline G. smilodon & $?$ & $?$ & $?$ & $?$ & $?$ & $?$ & $?$ & 0 & 1 & 0 & 0 & $?$ & $?$ & $?$ \\
\hline G. spherella & 0 & 0 & 0 & 0 & 1 & 0 & 0 & 0 & 1 & 0 & 0 & 1 & 1 & 1 \\
\hline G. sp. nov. 1 & 0 & 0 & 0 & 0 & 0 & - & 0 & 0 & 1 & 0 & 0 & 1 & 1 & 1 \\
\hline G. sp. nov. 2 & 0 & 0 & 0 & 0 & 0 & - & 0 & 0 & 1 & 0 & 0 & 1 & 1 & 1 \\
\hline G. sp. nov. 3 & 0 & 0 & 0 & 0 & 0 & - & 0 & 0 & 1 & 0 & 0 & 1 & 1 & 1 \\
\hline$G$. sp. nov. 4 & 0 & 0 & 0 & 0 & 1 & 0 & 0 & 0 & 1 & 0 & 0 & 1 & 1 & 1 \\
\hline G. sp. nov. 5 & 0 & 0 & 0 & 0 & 1 & 0 & 0 & 0 & 1 & 0 & 0 & 1 & 1 & 1 \\
\hline G. sp. nov. 6 & 0 & 0 & 0 & 0 & 0 & - & 0 & 0 & 1 & 0 & 0 & 1 & 1 & 1 \\
\hline G. sp. nov. 7 & 0 & 0 & 0 & 0 & 0 & - & 1 & 0 & 1 & 0 & 0 & 1 & 1 & 1 \\
\hline G. sp. nov. 8 & 0 & 1 & 0 & 0 & 0 & - & 0 & 1 & 1 & 0 & 0 & 1 & 1 & 1 \\
\hline$G$. sp. nov. 9 & $?$ & $?$ & $?$ & $?$ & $?$ & $?$ & $?$ & 0 & 1 & 0 & 0 & 1 & 1 & 1 \\
\hline G. sp. nov. 10 & $?$ & $?$ & $?$ & $?$ & $?$ & $?$ & $?$ & 0 & 1 & 0 & 0 & 1 & 1 & 1 \\
\hline G. sp. nov. 11 & 0 & 0 & 0 & 0 & 0 & - & 0 & 0 & 1 & 0 & 0 & 1 & 1 & 1 \\
\hline
\end{tabular}



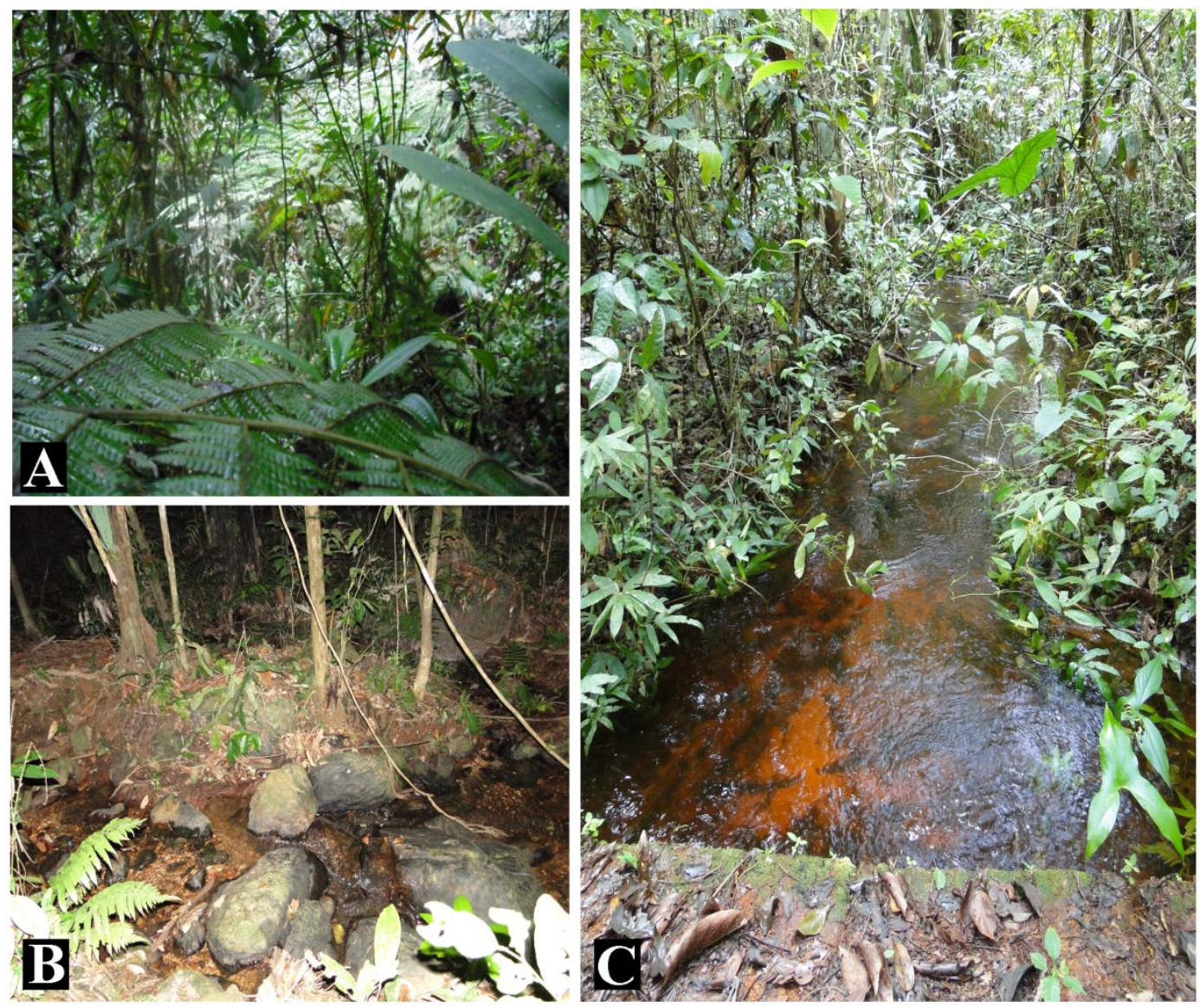

Figure 91. Habitats of Glenognatha species. A, Glenognatha sp. nov. 2 and

Glenognatha sp. nov. 8, Colombia, Valle del Cauca. B-C. Glenognatha gaujoni. B,

Brazil, Rondônia. C, Peru, Loreto. Photos 2B-C by Rafael Prezzi Indicatti.
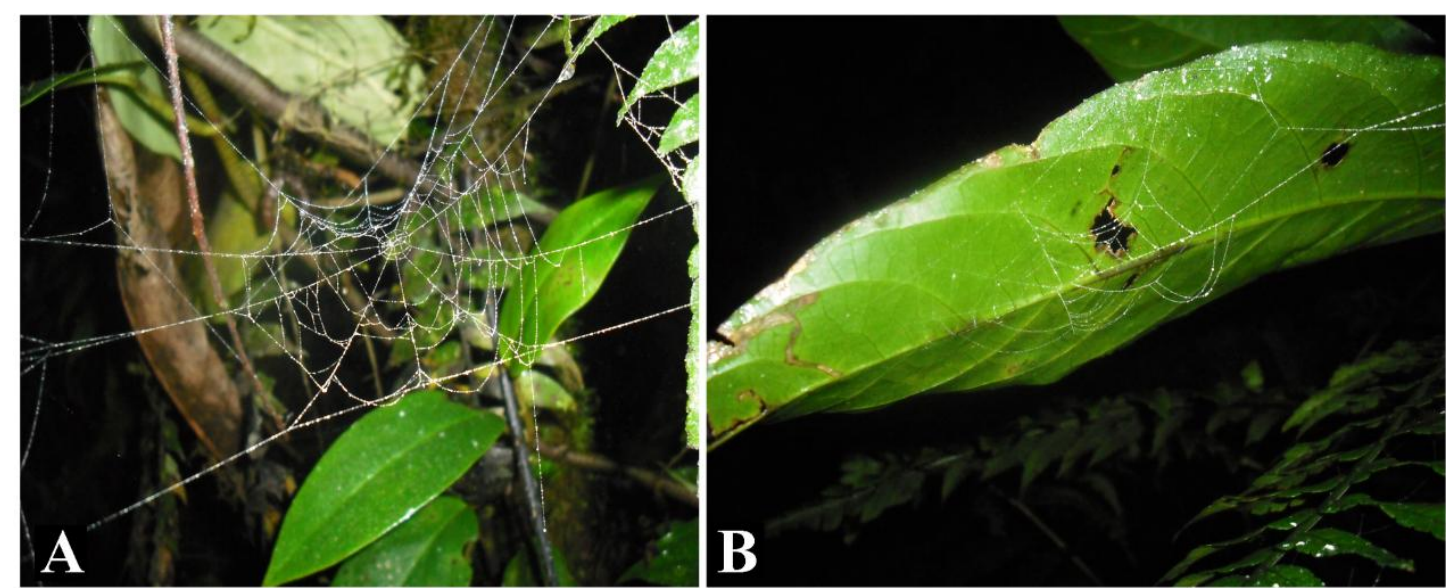

Figure 92. Webs of Glenognatha sp. nov. 2 from Colombia, Valle del Cauca. 

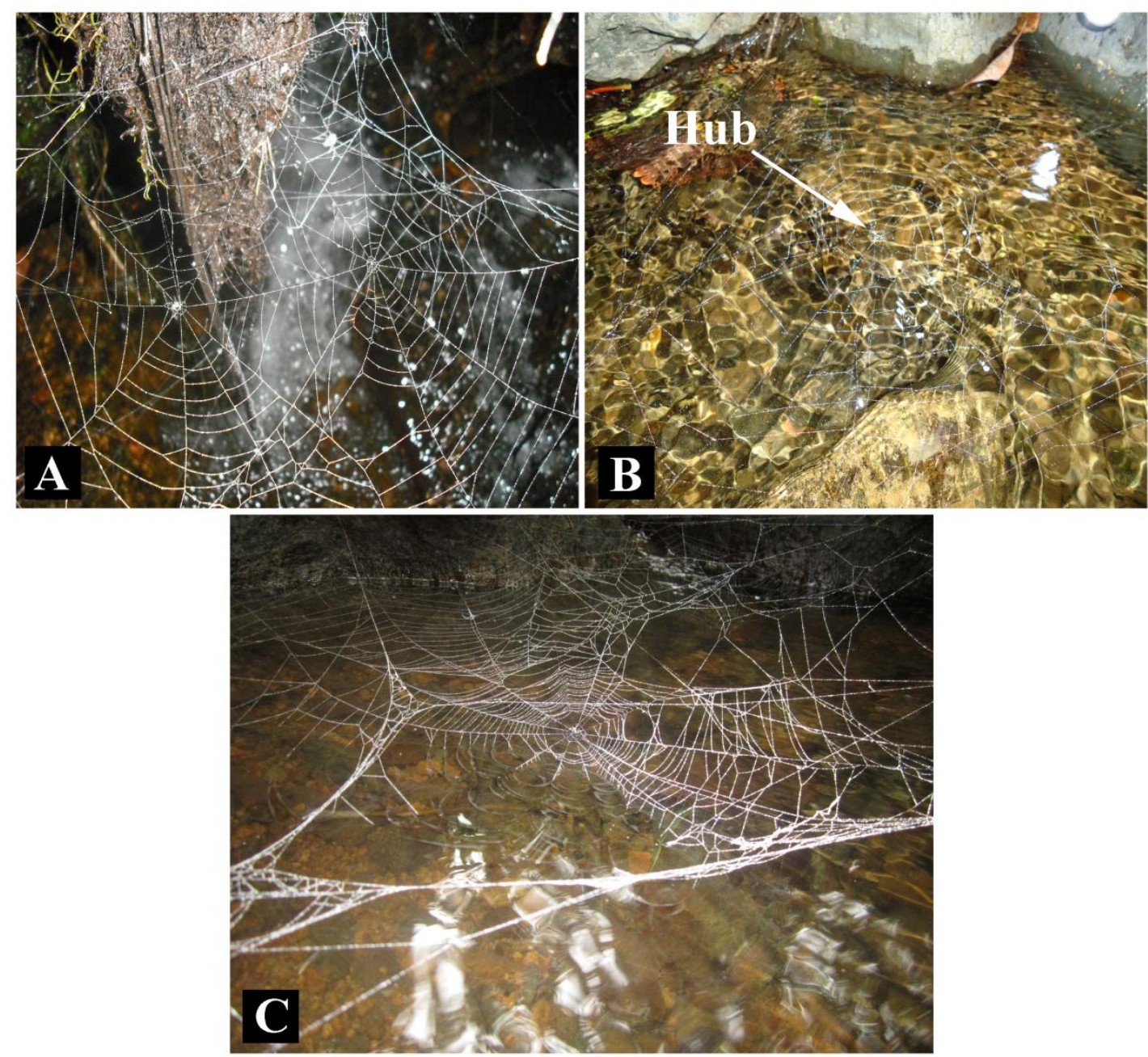

Figure 93. Webs of Glenognatha sp. nov. 8. A-B, Colombia, Valle del Cauca. C, Colombia, Nariño. Photo 2C by Nicolas Hazzi. 

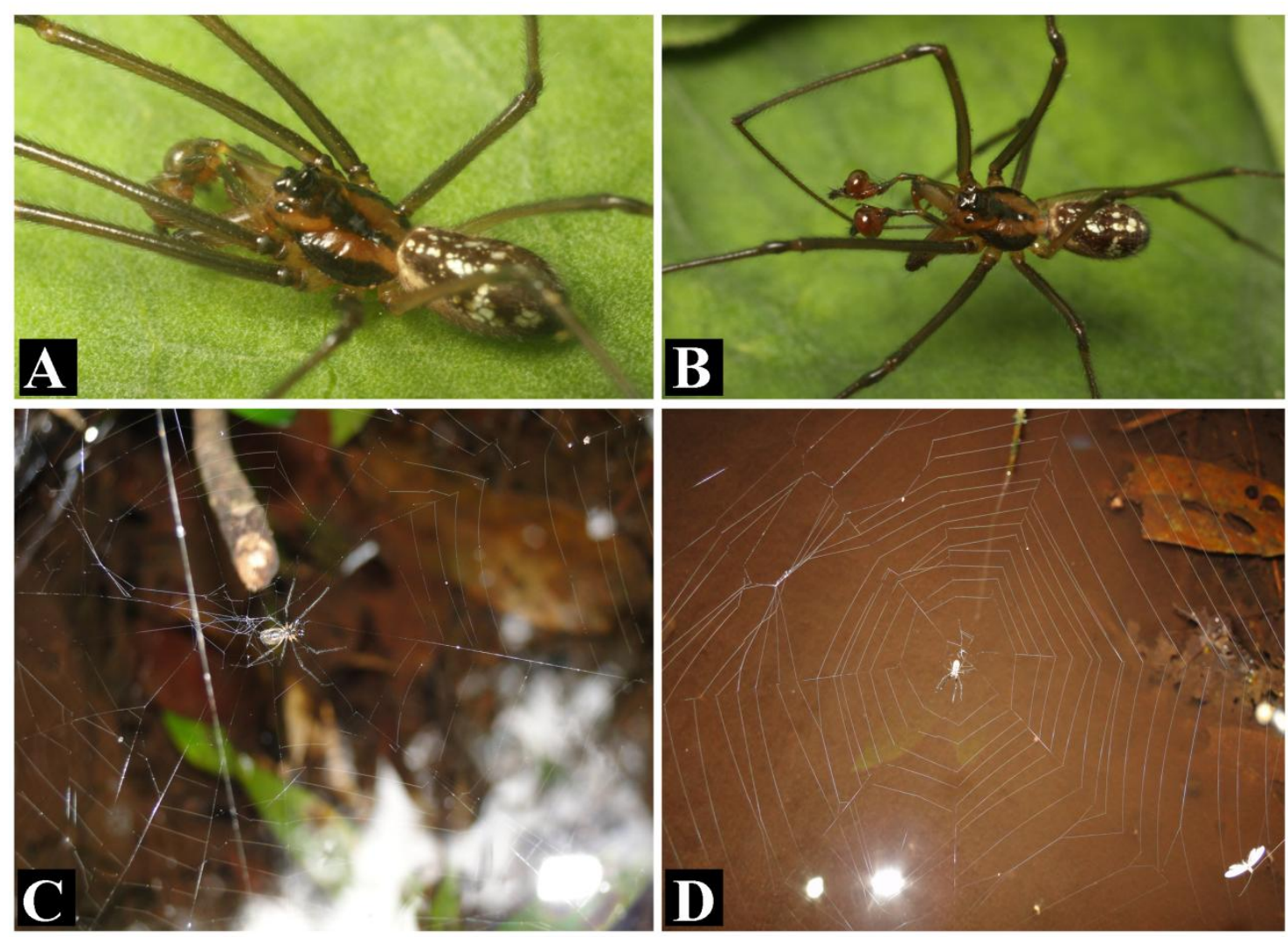

Figure 94. Webs and habitus of Glenognatha gaujoni. A-B, Brazil, Rondônia. C-D, Peru, Loreto. Photos 4A-B by Flávio Yamamoto, 4C-D by Rafael Prezzi Indicatti. 

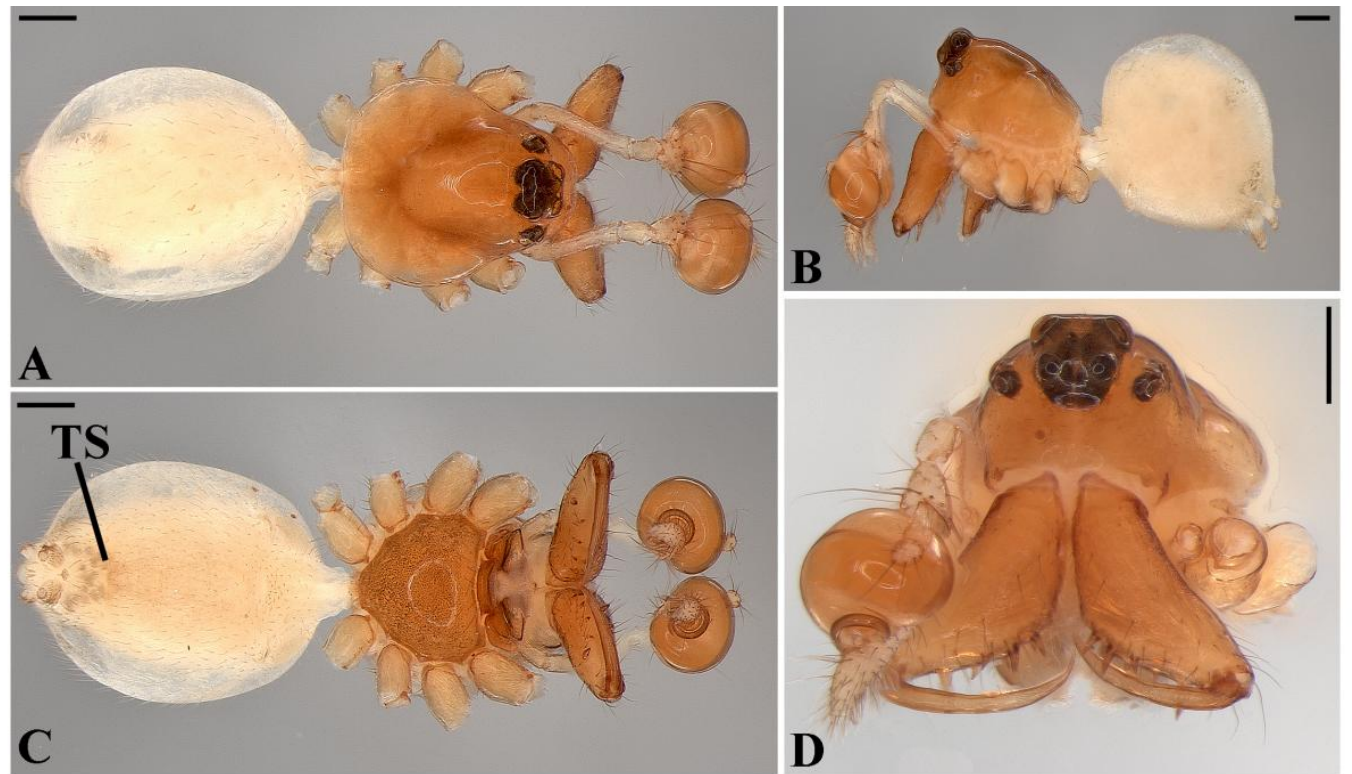

Figure 95. Glenognatha sp. nov. 4 from Brazil (MZSP 42538). Male habitus. A, dorsal. B, lateral. C, ventral. D, frontal. Scale bars, $200 \mu \mathrm{m}$. TS: tracheal spiracle.
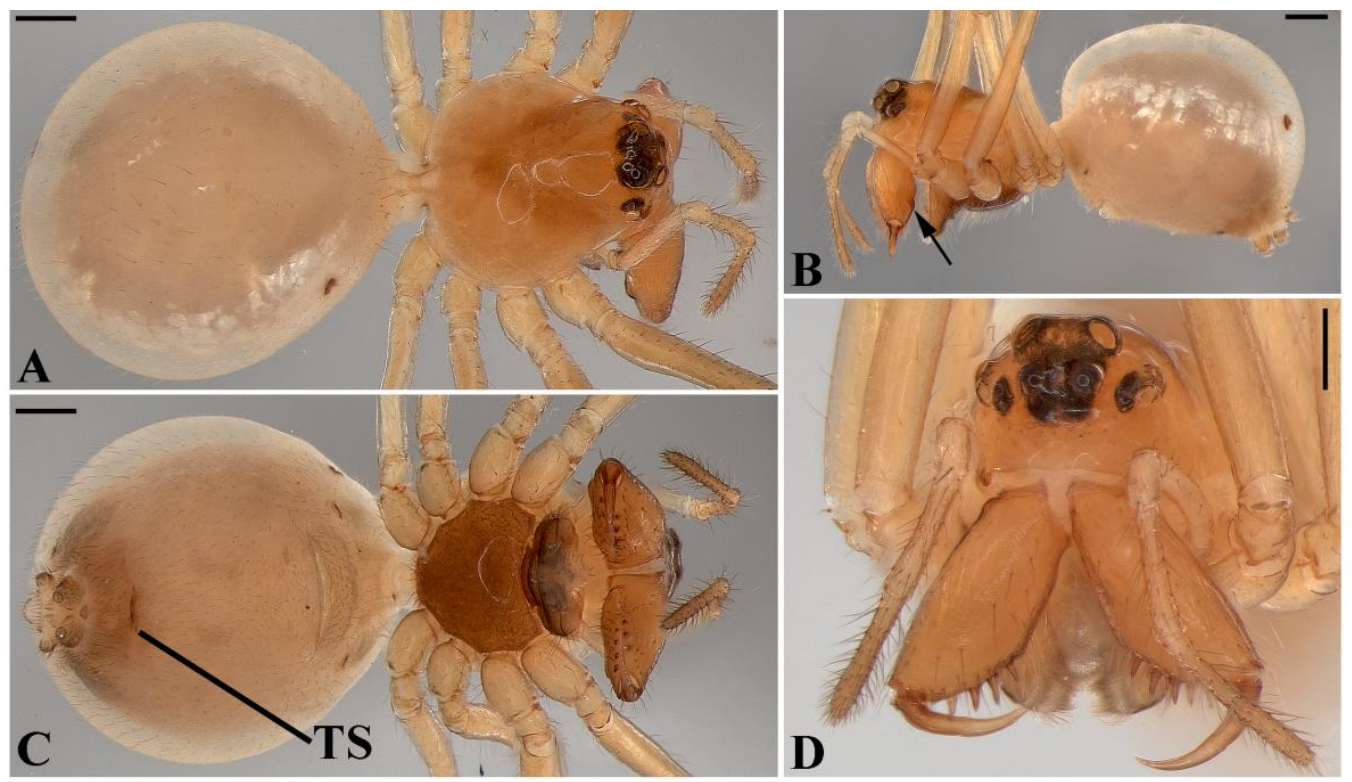

Figure 96. Glenognatha sp. nov. 4 from Brazil (MZSP 42538). Female habitus. A, dorsal. B, lateral (arrow, cheliceral bulge). C, ventral. D, frontal. Scale bars, $200 \mu \mathrm{m}$. TS: tracheal spiracle. 


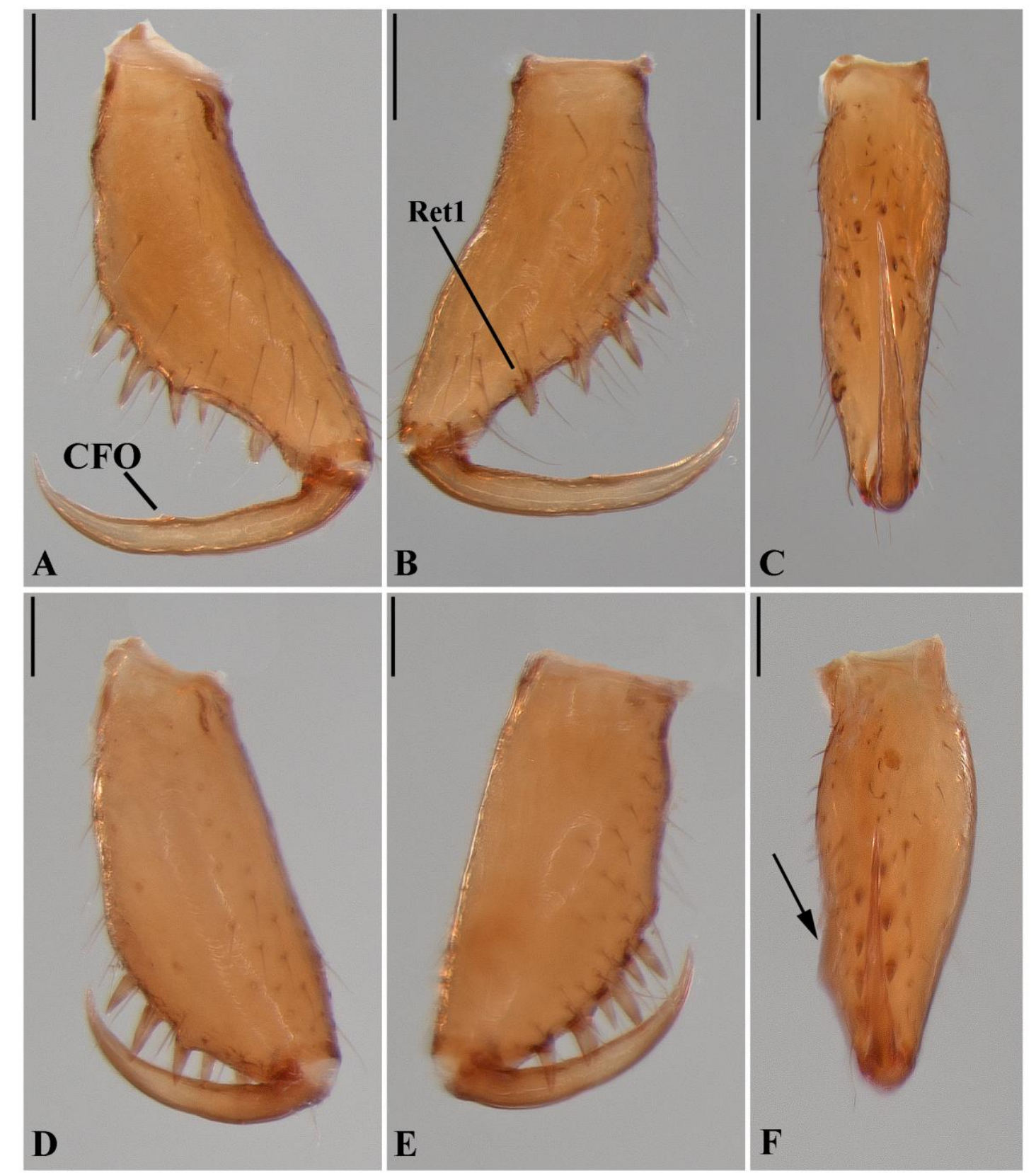

Figure 97. Glenognatha sp. nov. 4 from Brazil. A-C, Male left chelicerae (MZSP 42538). A, anterior. B, posterior. C, mesal. D-F, Female left chelicerae (MZSP 39047). D, anterior. E, posterior. F, mesal (arrow, cheliceral bulge). Scale bars, $100 \mu \mathrm{m}$. CFO: cheliceral fang outgrowth. Ret: retromarginal tooth. 


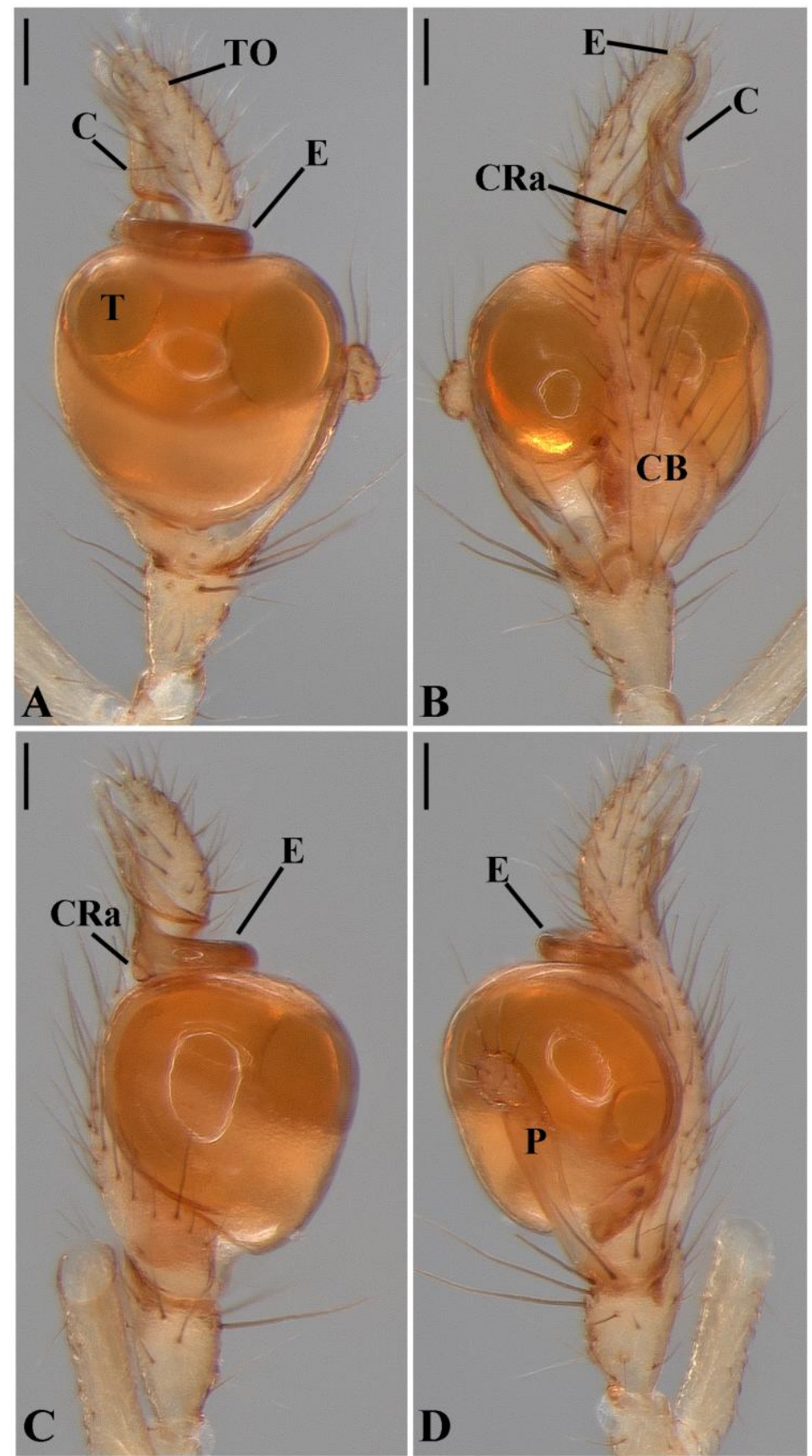

Figure 98. Glenognatha sp. nov. 4 from Brazil (MZSP 42538). Male left palp. A, ventral. B, dorsal. C, prolateral. D, retrolateral. Scale bars, $100 \mu \mathrm{m}$. C: conductor. CB: cymbium. CRa: conductor retrolateral apophysis. E: embolus. P: paracymbium. T: tegulum. TO: tarsal organ. 

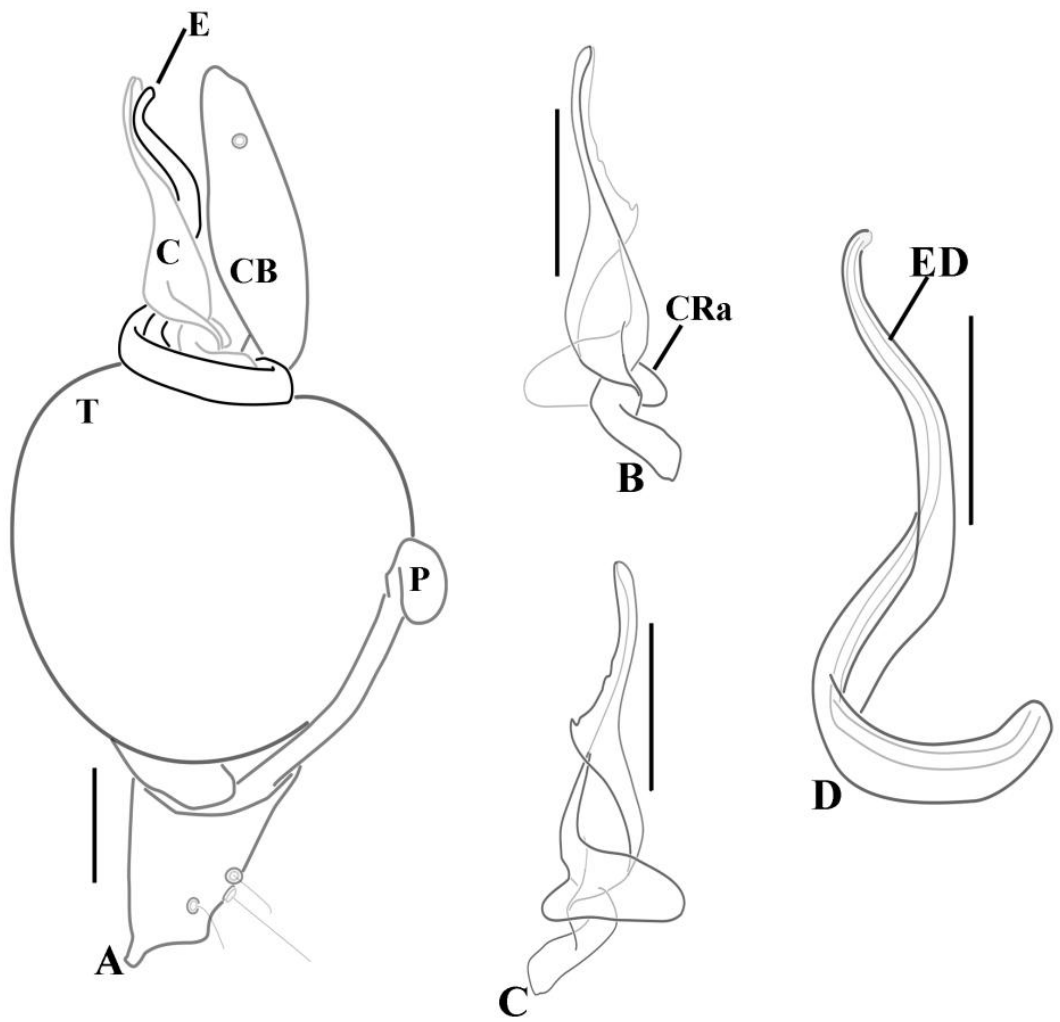

Figure 99. Glenognatha sp. nov. 4 from Brazil (MZSP 42538). Male left palp, schematic. A, ventral. B, conductor ventral. C, conductor dorsal. D, embolus ventral. Scale bars $100 \mu \mathrm{m}$. C: conductor. CB: cymbium. CRa: conductor retrolateral apophysis. E: embolus. ED: ejaculatory duct. P: paracymbium. T: tegulum.
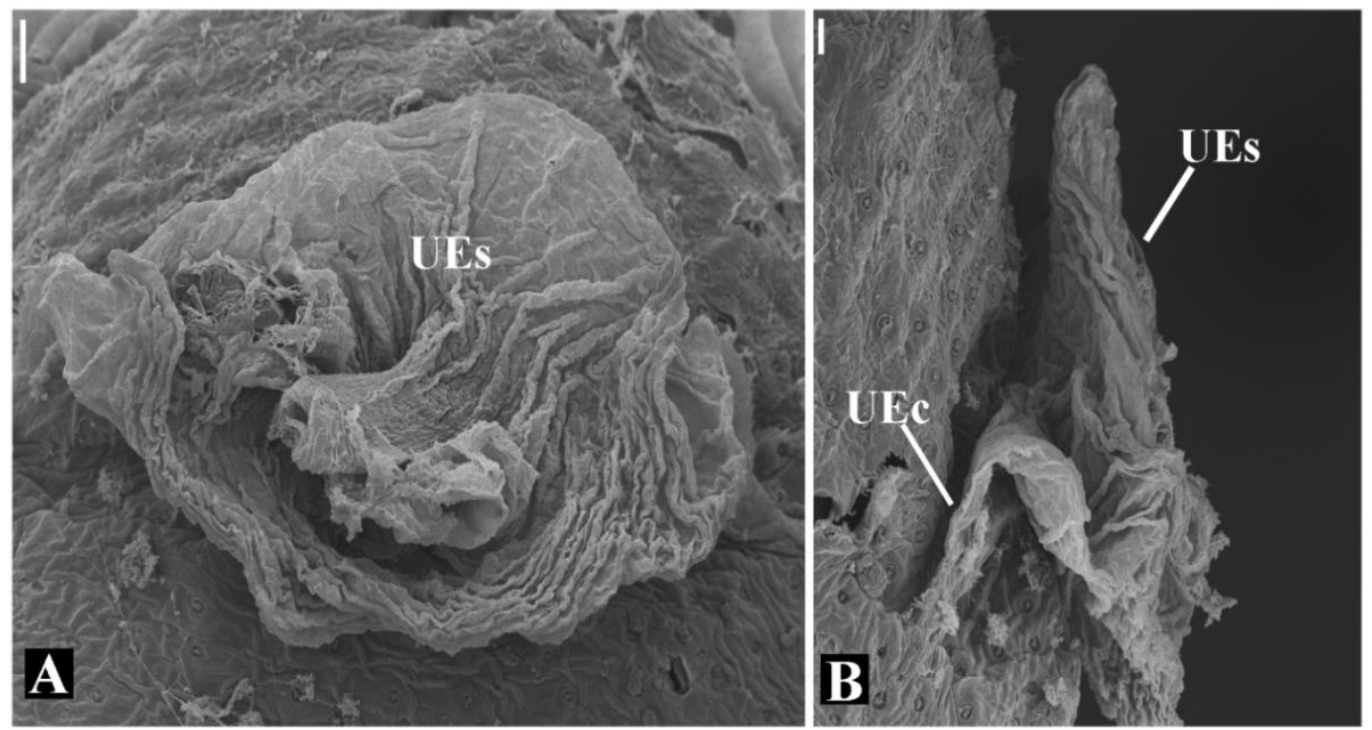

Figure 100. Glenognatha sp. nov. 4 from Brazil. Female genitalia. A, dorsal. B, lateral. Scale bars, $20 \mathrm{~mm}$. UEc: uterus externus chamber. UEs: uterus externus sac. 


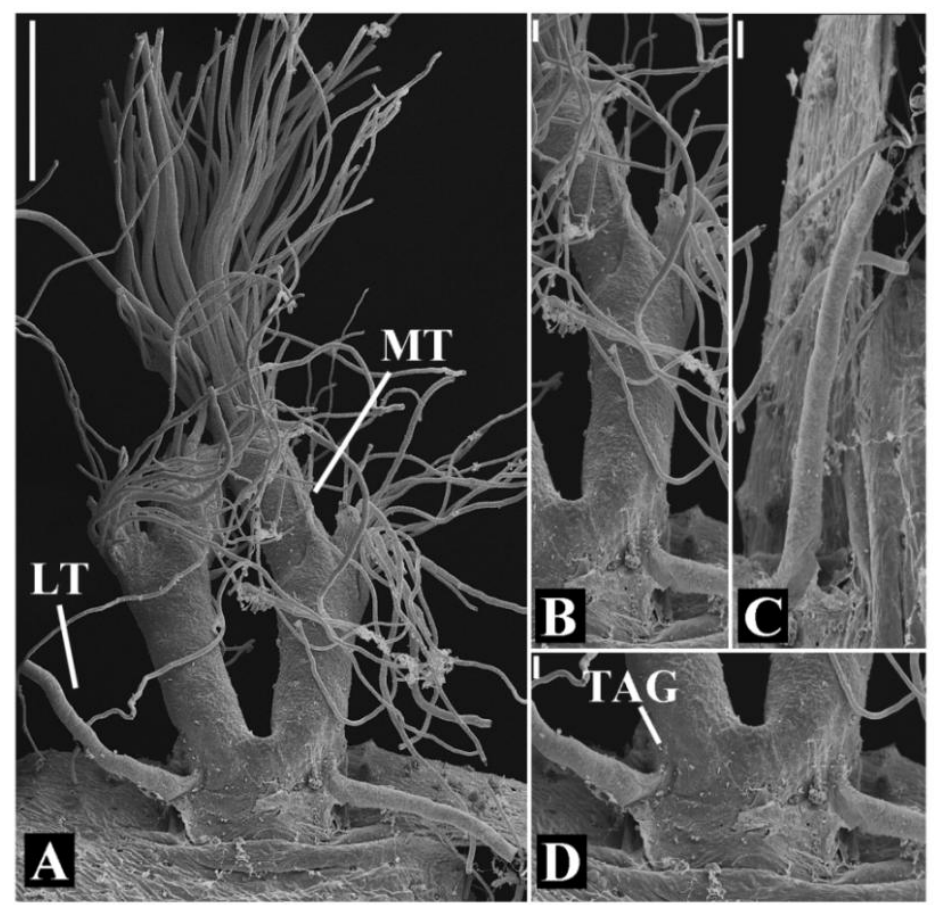

Figure 101. Glenognatha sp. nov. 4 from Brazil. Female tracheal system. A, dorsal. B, median trunk. C, lateral trunk. D, tracheal spiracle posterior view. Scale bars, $100 \mu \mathrm{m}$ (A), $10 \mu \mathrm{m}$ (B-D). LT: lateral tracheae. MT: median trunks. TAG: tracheal atrium gland.

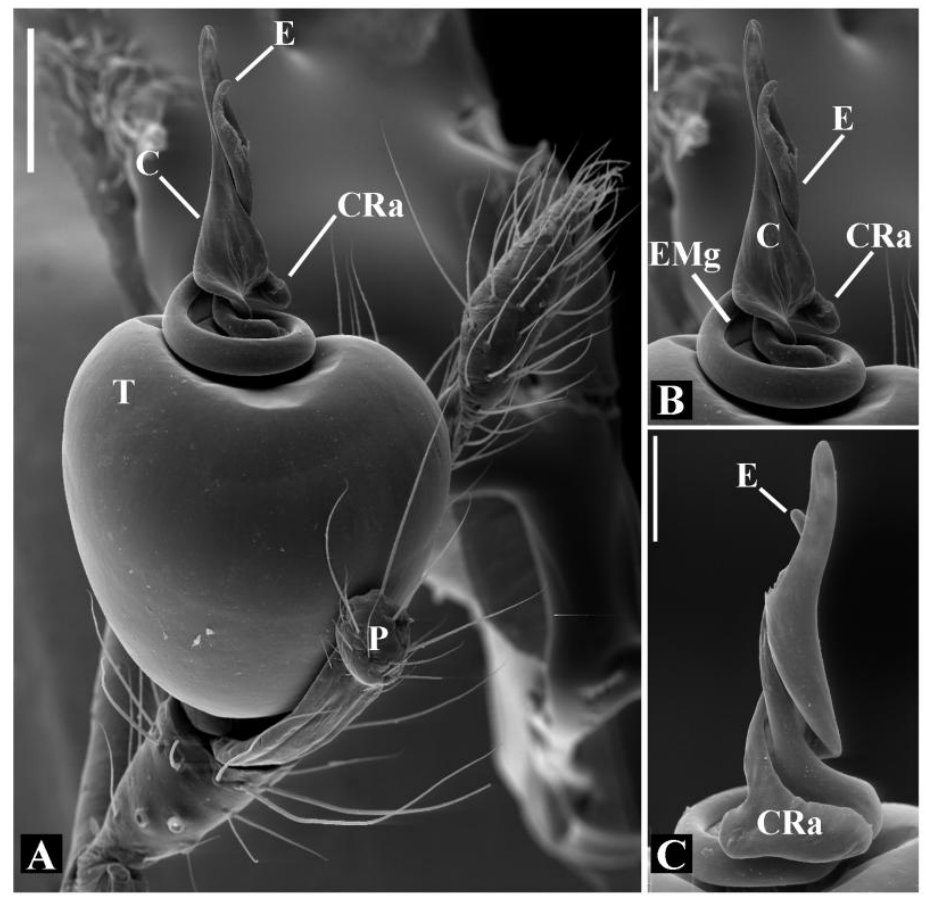

Figure 102. Glenognatha sp. nov. 4 from Brazil. Male left palp. A, ventral. B, embolus and conductor ventral. C, embolus and conductor dorsal. Scale bars $100 \mu \mathrm{m}$ (A), $50 \mu \mathrm{m}$ (B, C). C: conductor. CB: cymbium. CRa: conductor retrolateral apophysis. E: embolus. EMg: embolic medial groove. P: paracymbium. T: tegulum. 

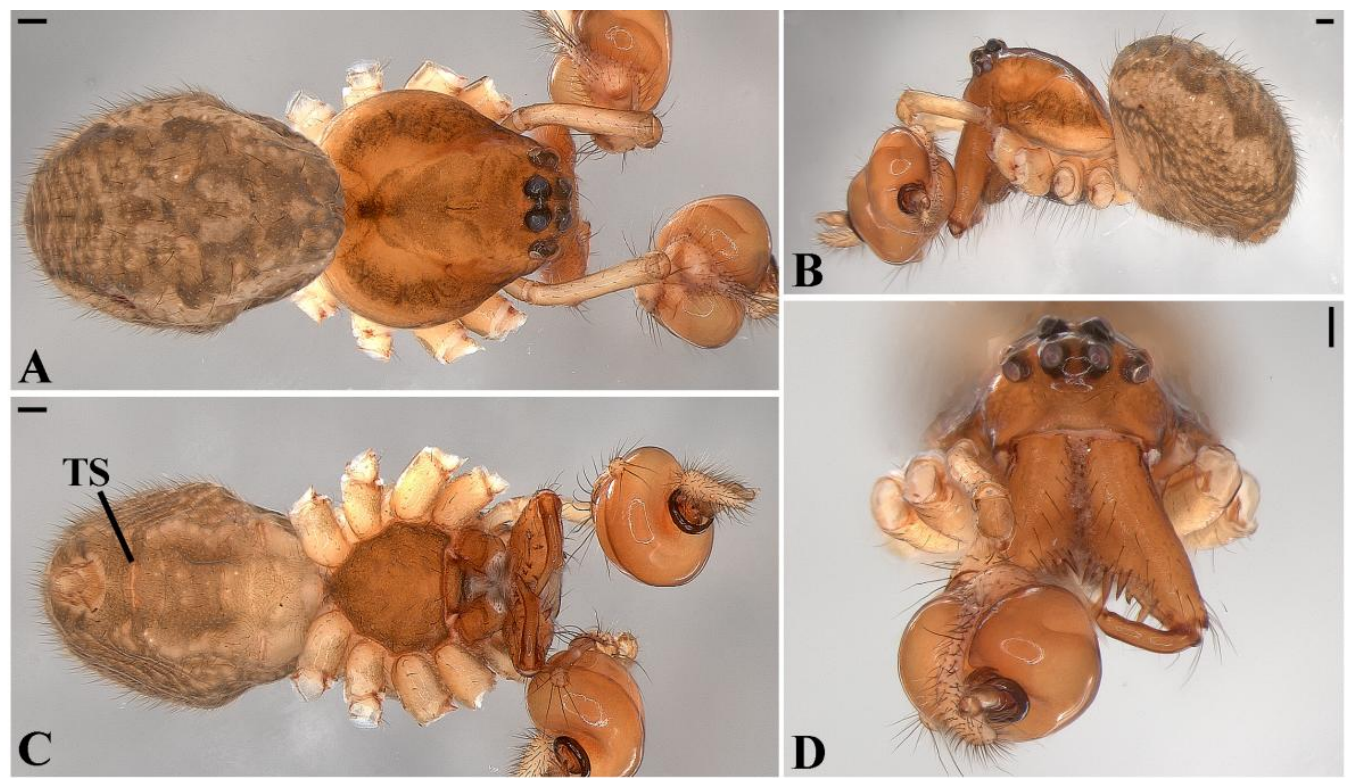

Figure 103. Glenognatha sp. nov. 5 from Colombia (ICN 1973). Male habitus. A, dorsal. B, lateral. C, ventral. D, frontal. Scale bars, $200 \mu \mathrm{m}$. TS: tracheal spiracle.
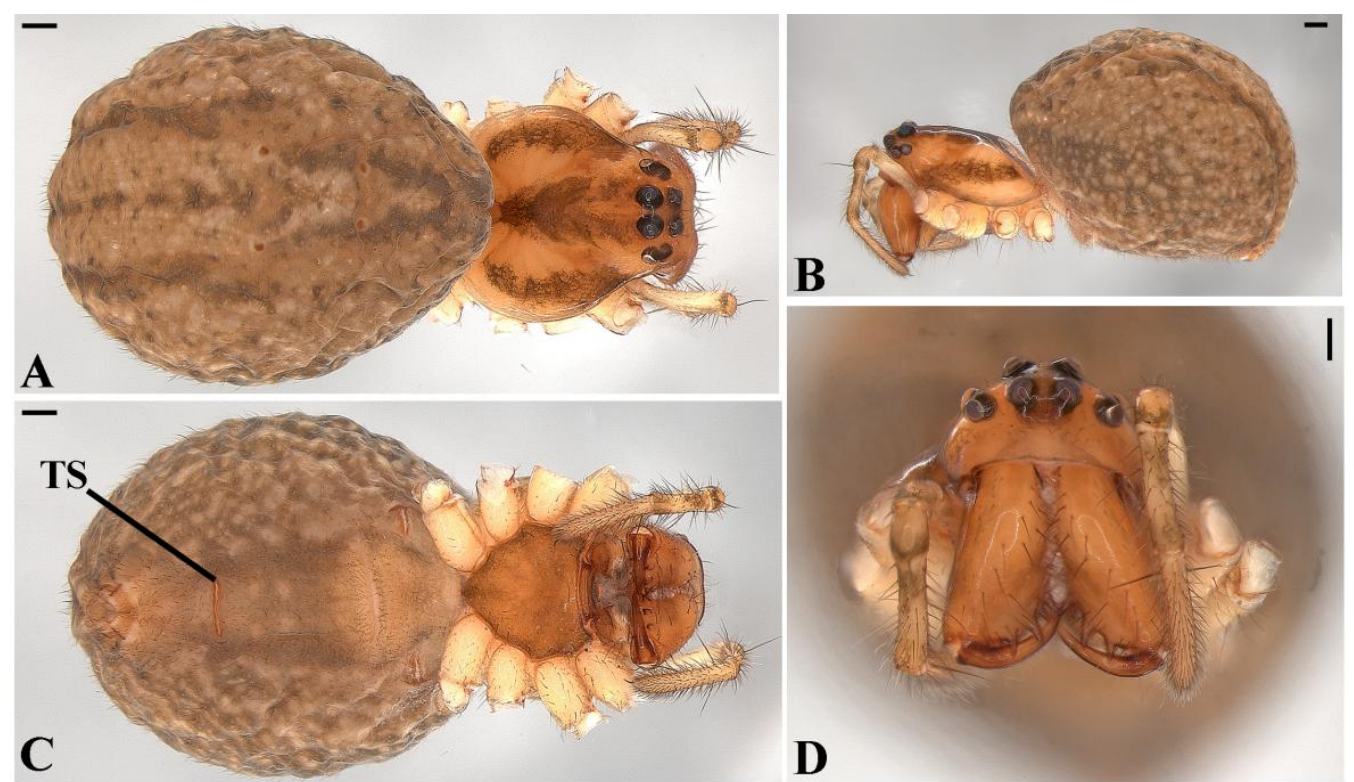

Figure 104. Glenognatha sp. nov. 5 from Colombia (ICN 1973). Female habitus. A, dorsal. B, lateral. C, ventral. D, frontal. Scale bars, $200 \mu \mathrm{m}$. TS: tracheal spiracle. 


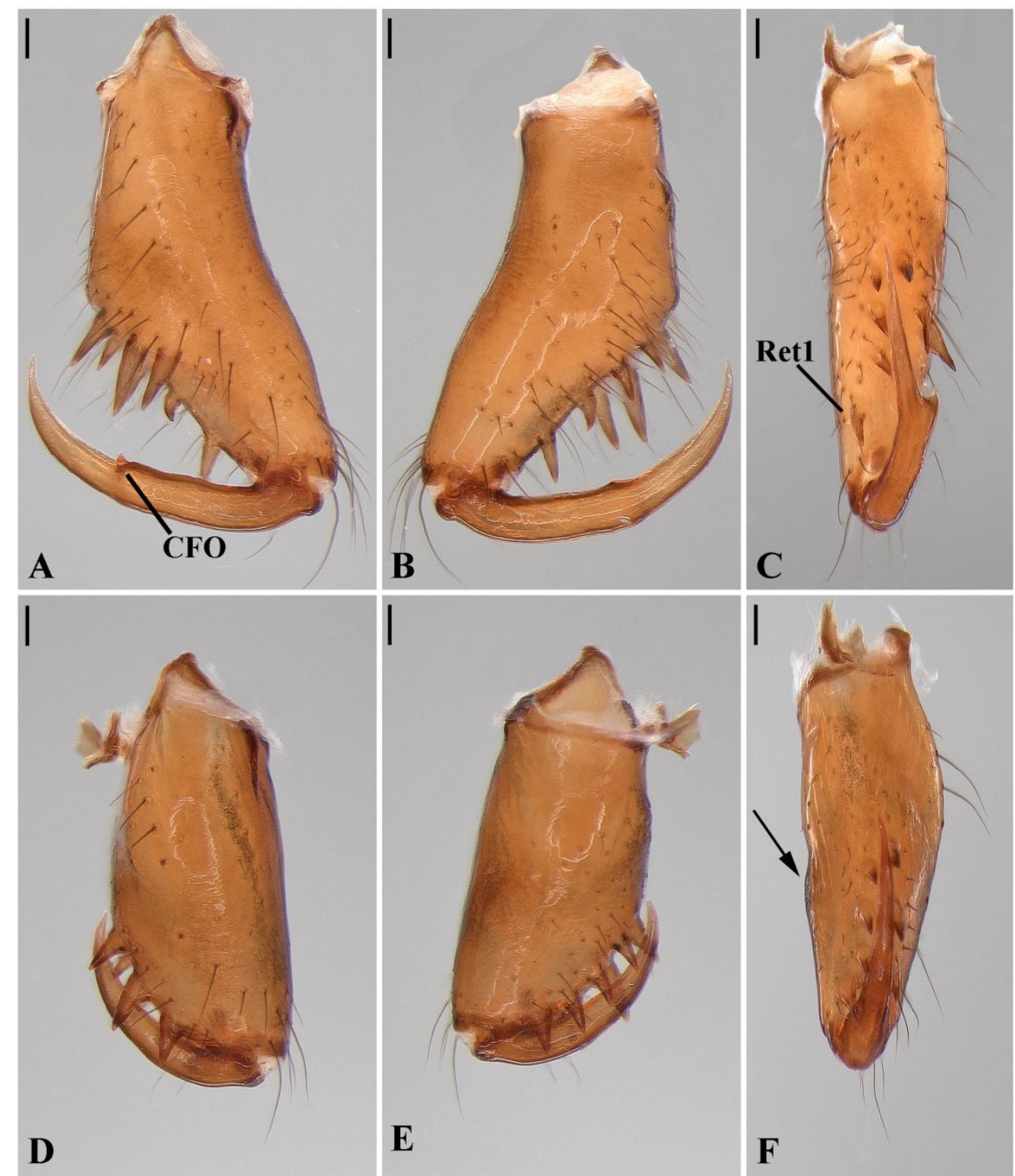

Figure 105. Glenognatha sp. nov. 5 from Colombia (ICN 2141). A-C, Male left chelicerae. A, anterior. B, posterior. C, mesal. D-F, Female left chelicerae. D, anterior. $\mathbf{E}$, posterior. F, mesal (arrow, cheliceral bulge). Scale bars, $100 \mu \mathrm{m}$. CFO: cheliceral fang outgrowth. Ret: retromarginal tooth. 


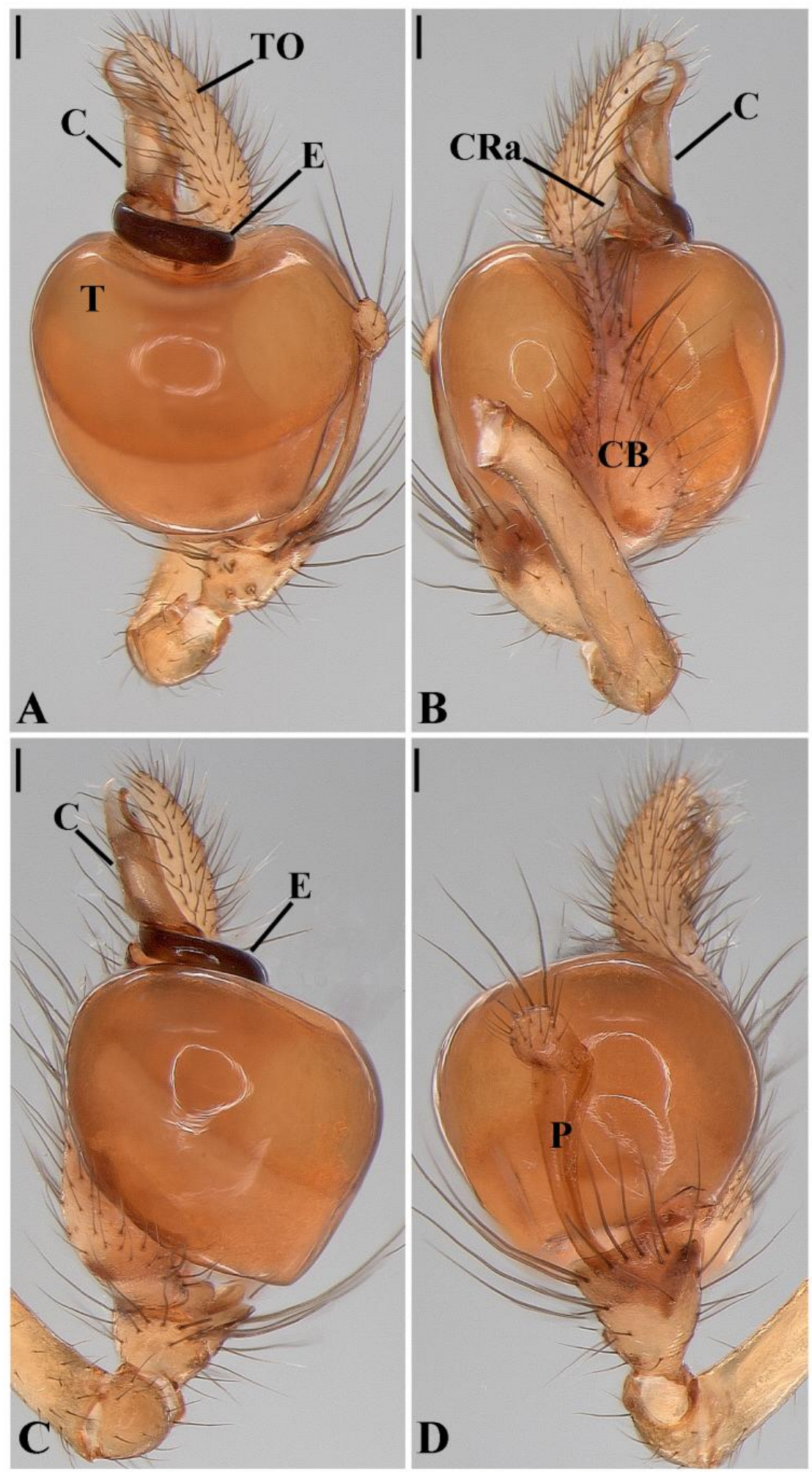

Figure 106. Glenognatha sp. nov. 5 from Colombia (ICN 1973). Male left palp. A, ventral. B, dorsal. C, prolateral. D, retrolateral. Scale bars, $100 \mu \mathrm{m}$. C: conductor. CB: cymbium. CRa: conductor retrolateral apophysis. E: embolus. P: paracymbium. T: tegulum. TO: tarsal organ. 

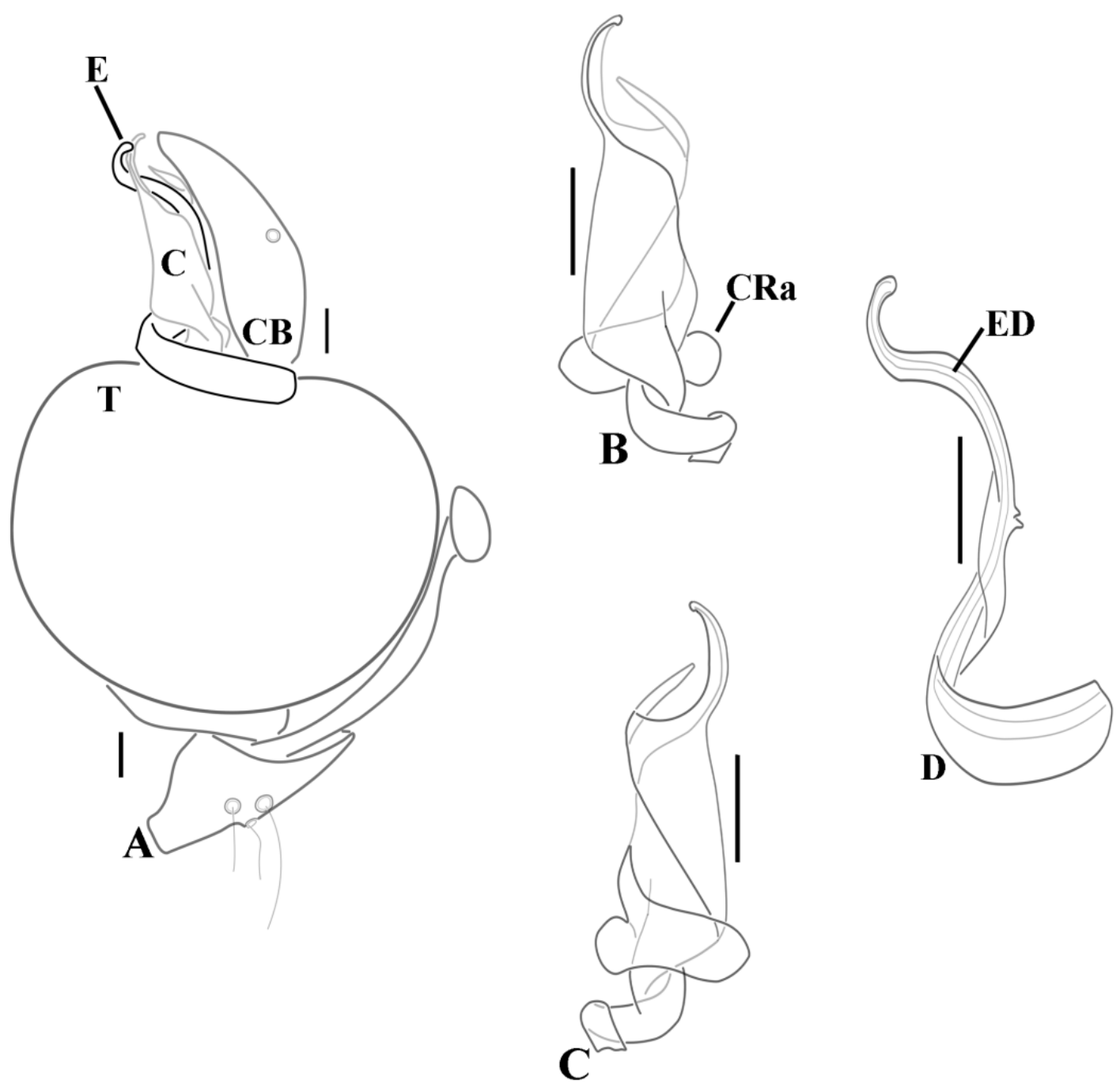

Figure 107. Glenognatha sp. nov. 5 from Colombia (Paratype ICN 2141). Male left palp, schematic. A, ventral. B, conductor ventral. C, conductor dorsal. D, embolus ventral. Scale bars $100 \mu \mathrm{m}$. C: conductor. CB: cymbium. CRa: conductor retrolateral apophysis. E: embolus. ED: ejaculatory duct. T: tegulum. 

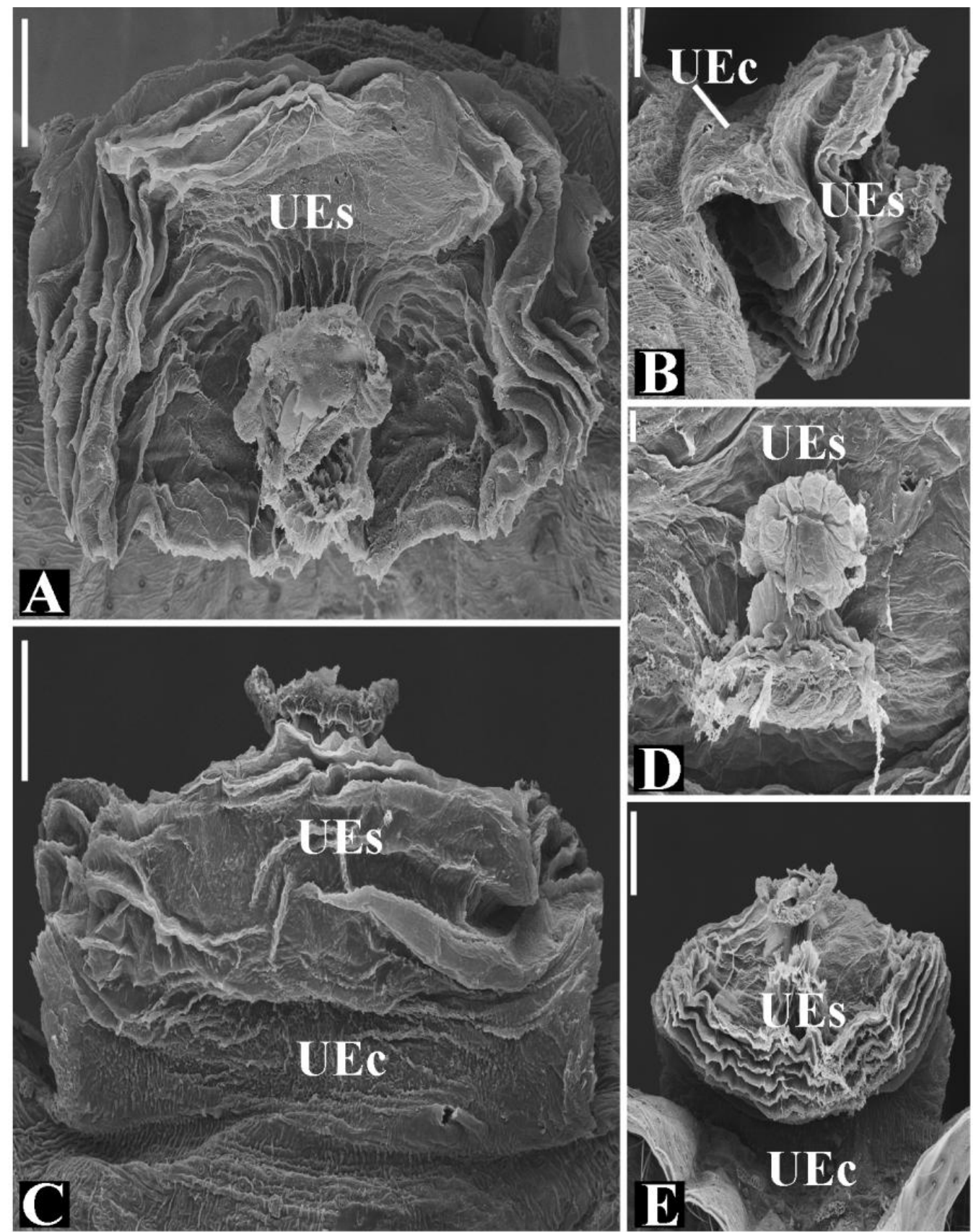

Figure 108. Glenognatha sp. nov. 5 from Colombia. Female genitalia. A, dorsal. B, lateral. C, anterior D, uterus externus cuticle. E, posterior. Scale bars, $100 \mu \mathrm{m}$ (A-D), $20 \mu \mathrm{m}$ (E). UEc: uterus externus chamber. UEs: uterus externus sac. 


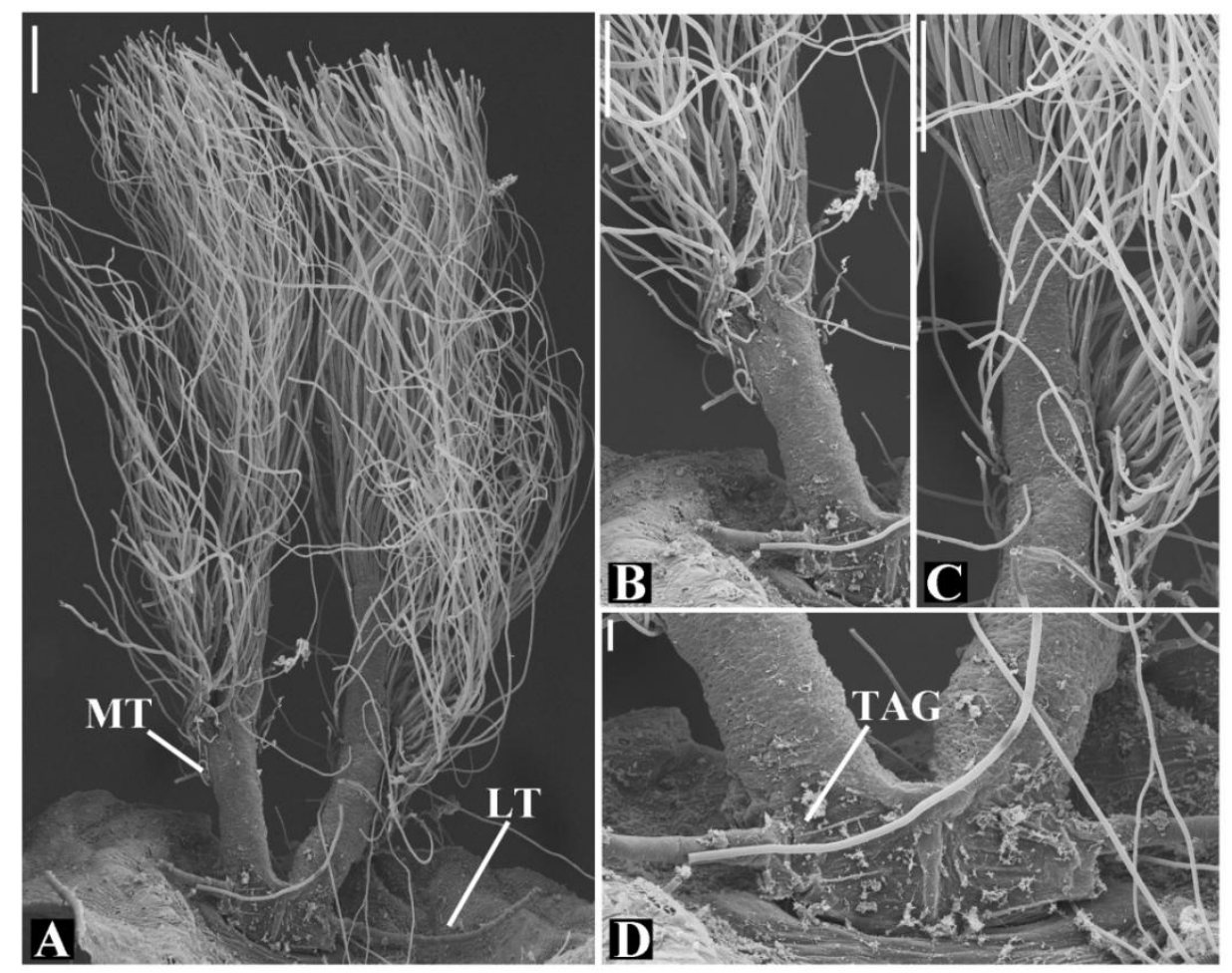

Figure 109. Glenognatha sp. nov. 5 from Colombia. Female tracheal system. A, dorsal. B, median and lateral trunks. C, median trunk distal. D, tracheal spiracle posterior view. Scale bars, $100 \mu \mathrm{m}$ (A-C), $20 \mu \mathrm{m}$ (D). LT: lateral tracheae. MT: median trunks. TAG: tracheal atrium gland.
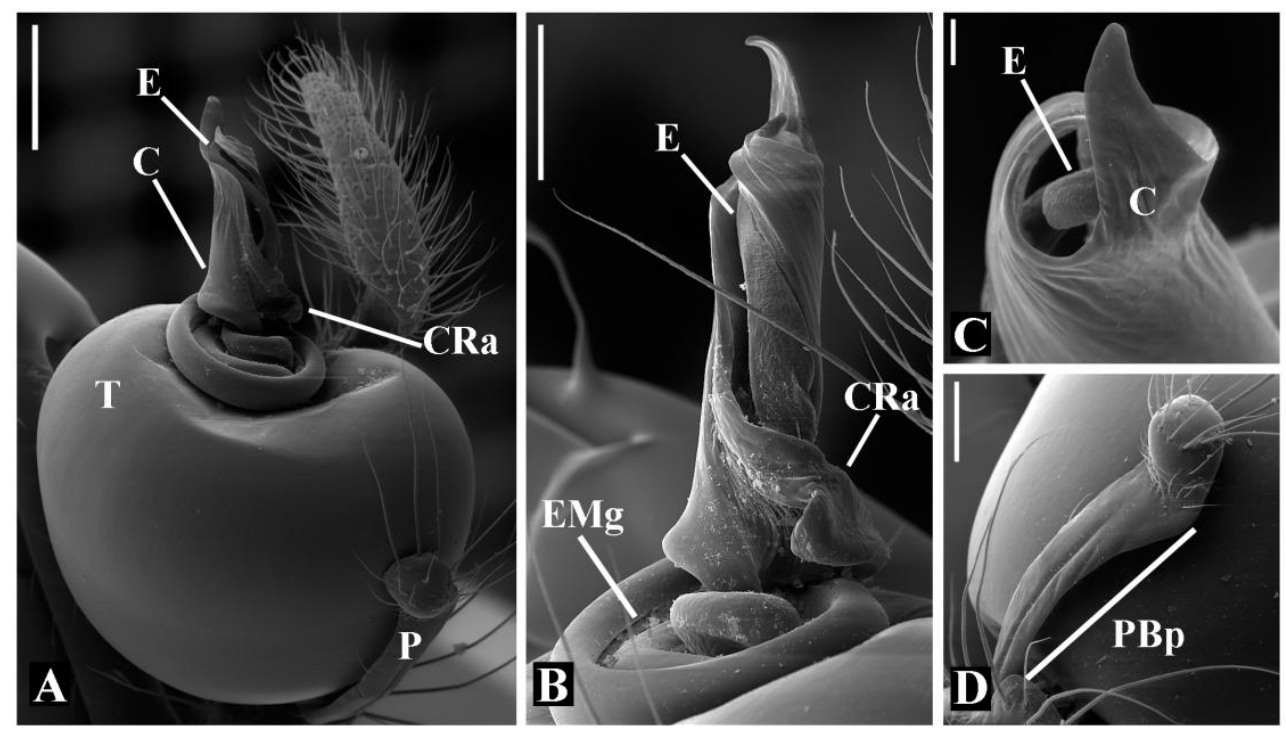

Figure 110. Glenognatha sp. nov. 5 from Colombia. Male left palp. A, ventral. B, embolus and conductor. $\mathbf{C}$, embolus and conductor tip. D, paracymbium. Scale bars $200 \mu \mathrm{m}$ (A), $100 \mu \mathrm{m}$ (B, D) $20 \mu \mathrm{m}$ (C). C: conductor. CRa: conductor retrolateral apophysis. E: embolus. EMg: embolic medial groove. P: paracymbium. PBp: paracymbium basal portion. T: tegulum. 

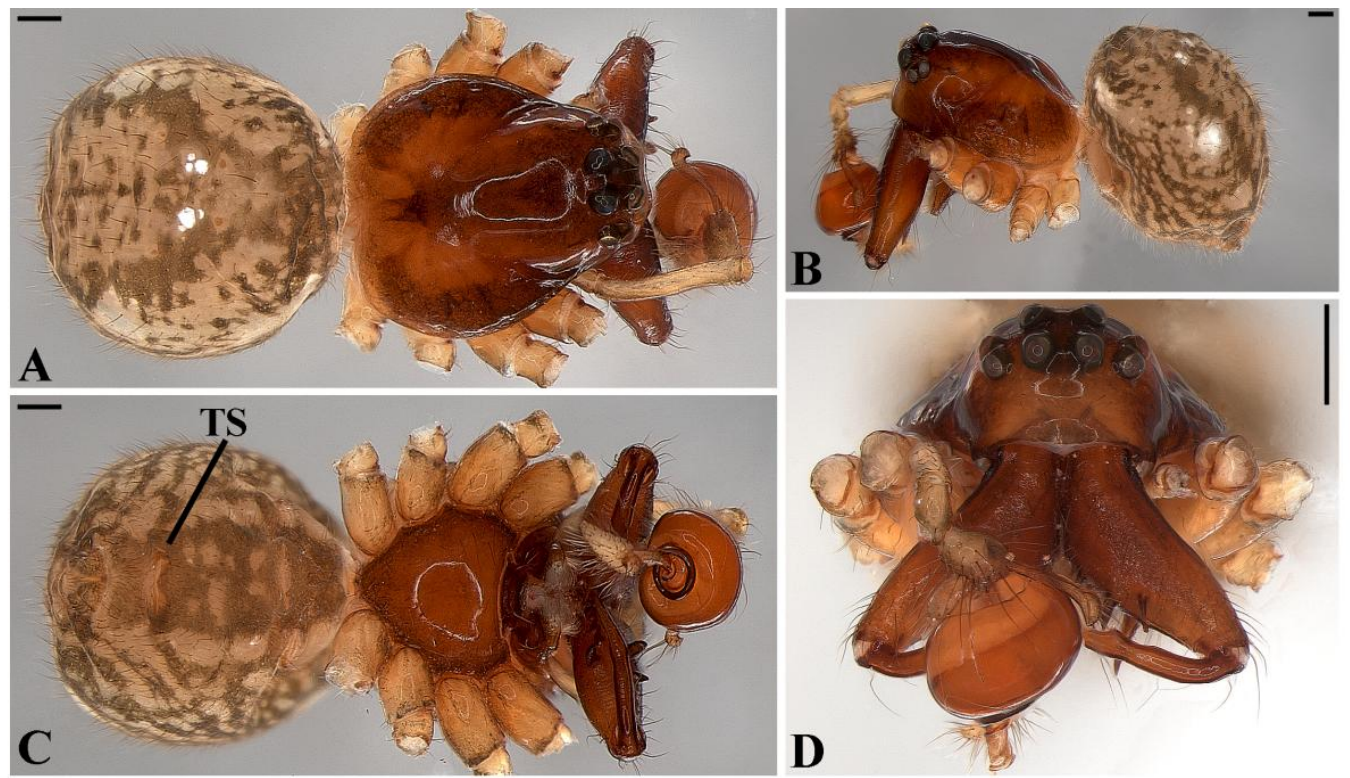

Figure 111. Glenognatha lacteovittata from Brazil (IBSP 141433). Male habitus. A, dorsal. B, lateral. C, ventral. D, frontal. Scale bars, $200 \mu \mathrm{m}$. TS: tracheal spiracle.
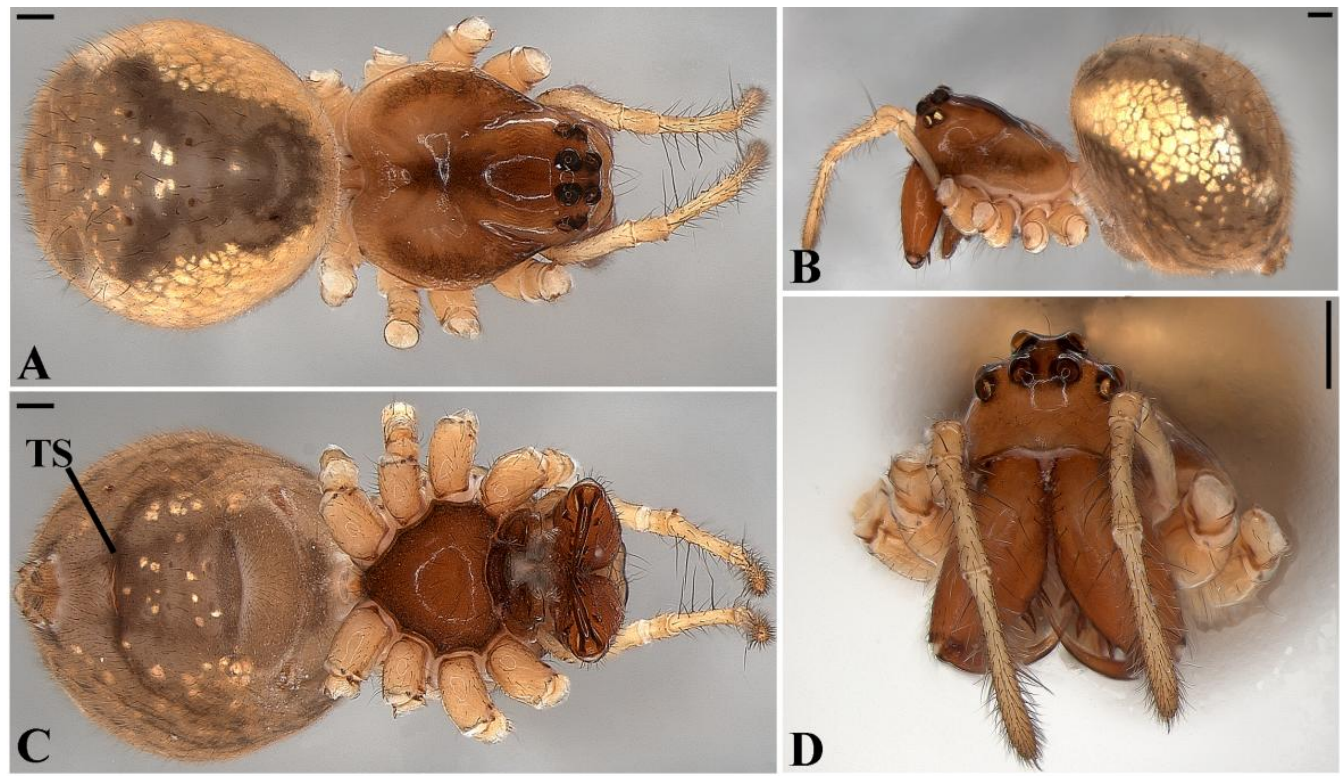

Figure 112. Glenognatha lacteovittata from Brazil (IBSP 163680). Female habitus. A, dorsal. B, lateral. C, ventral. D, frontal. Scale bars, $200 \mu \mathrm{m}$. TS: tracheal spiracle. 


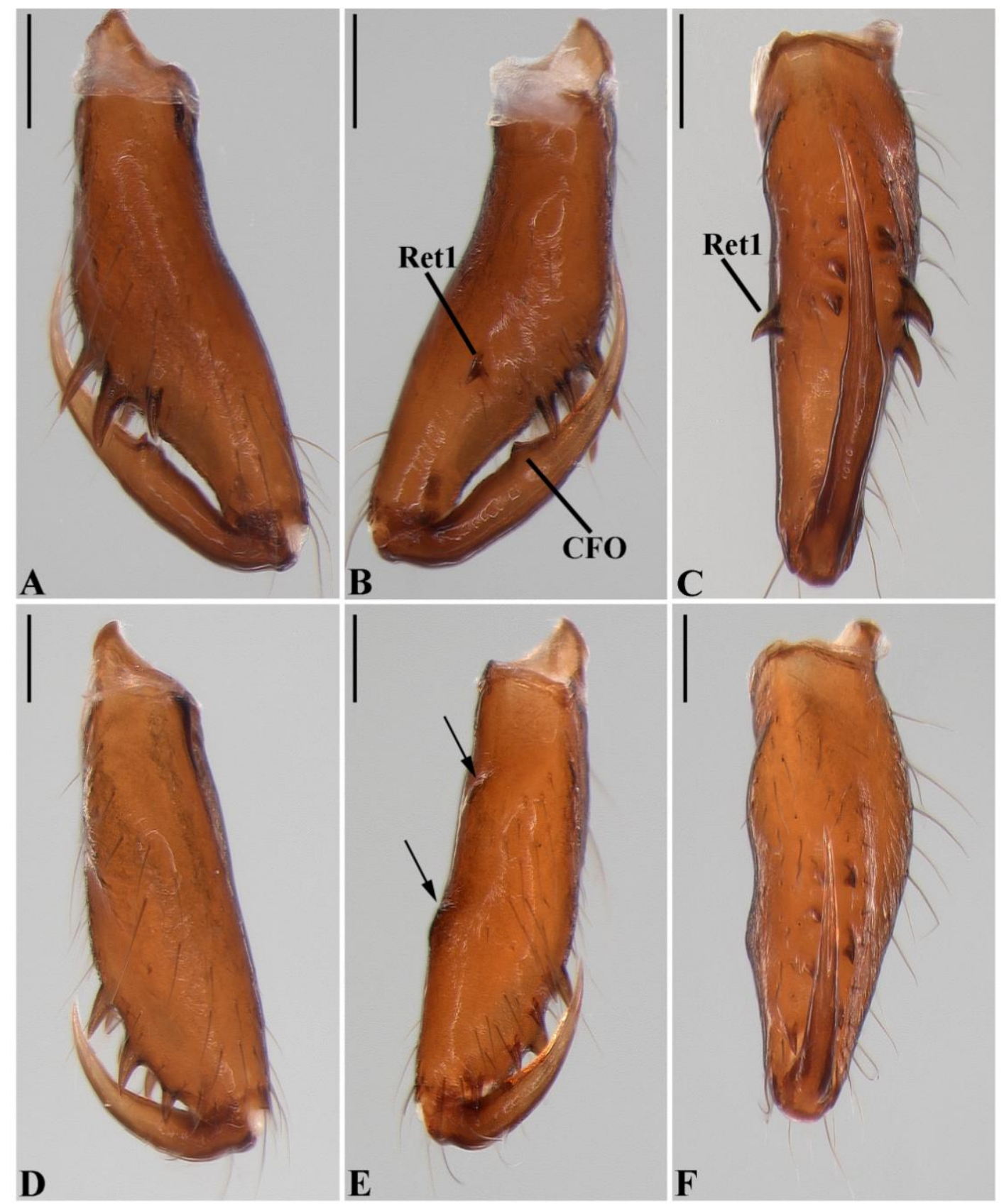

Figure 113. Glenognatha lacteovittata from Uruguay (IBSP 44084). A-C, Male left chelicerae. A, anterior. B, posterior. C, mesal. D-F, Female left chelicerae. D, anterior. E, posterior (arrows, cheliceral bulges). F, mesal. Scale bars, $100 \mu \mathrm{m}$. CFO: cheliceral fang outgrowth. Ret: retromarginal tooth. 

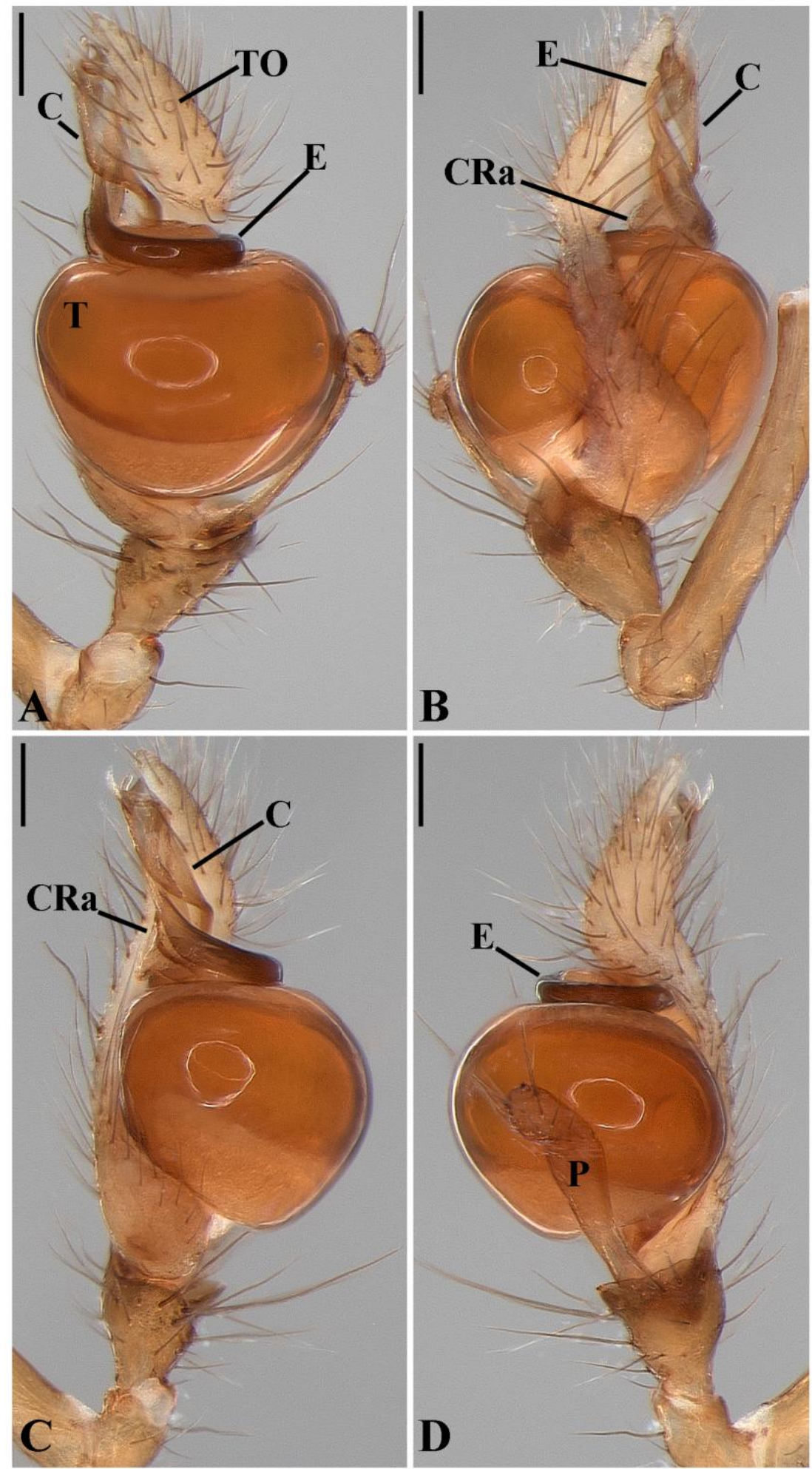

Figure 114. Glenognatha lacteovittata from Uruguay (IBSP 44084). Male left palp. A, ventral. B, dorsal. C, prolateral. D, retrolateral. Scale bars, $100 \mu \mathrm{m}$. C: conductor. CRa: conductor retrolateral apophysis. E: embolus. P: paracymbium. T: tegulum. TO: tarsal organ. 

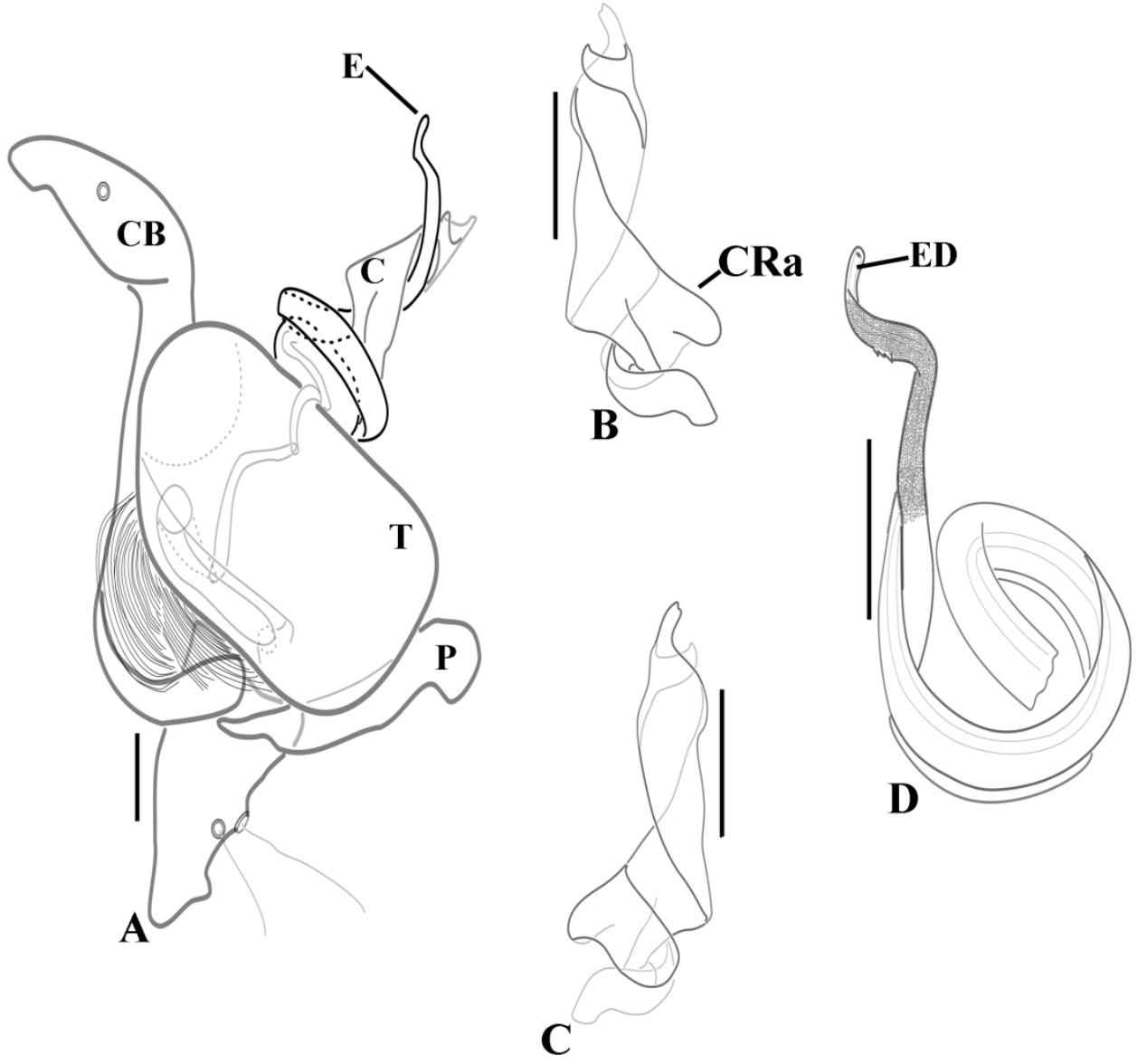

Figure 115. Glenognatha lacteovittata from Brazil (IBSP 70038). Male left palp, schematic. A, ventral. B, conductor ventral. C, conductor dorsal. D, embolus ventral. Scale bars $100 \mu \mathrm{m}$. C: conductor. CB: cymbium. CRa: conductor retrolateral apophysis. E: embolus. ED: ejaculatory duct. P: paracymbium. T: tegulum. 

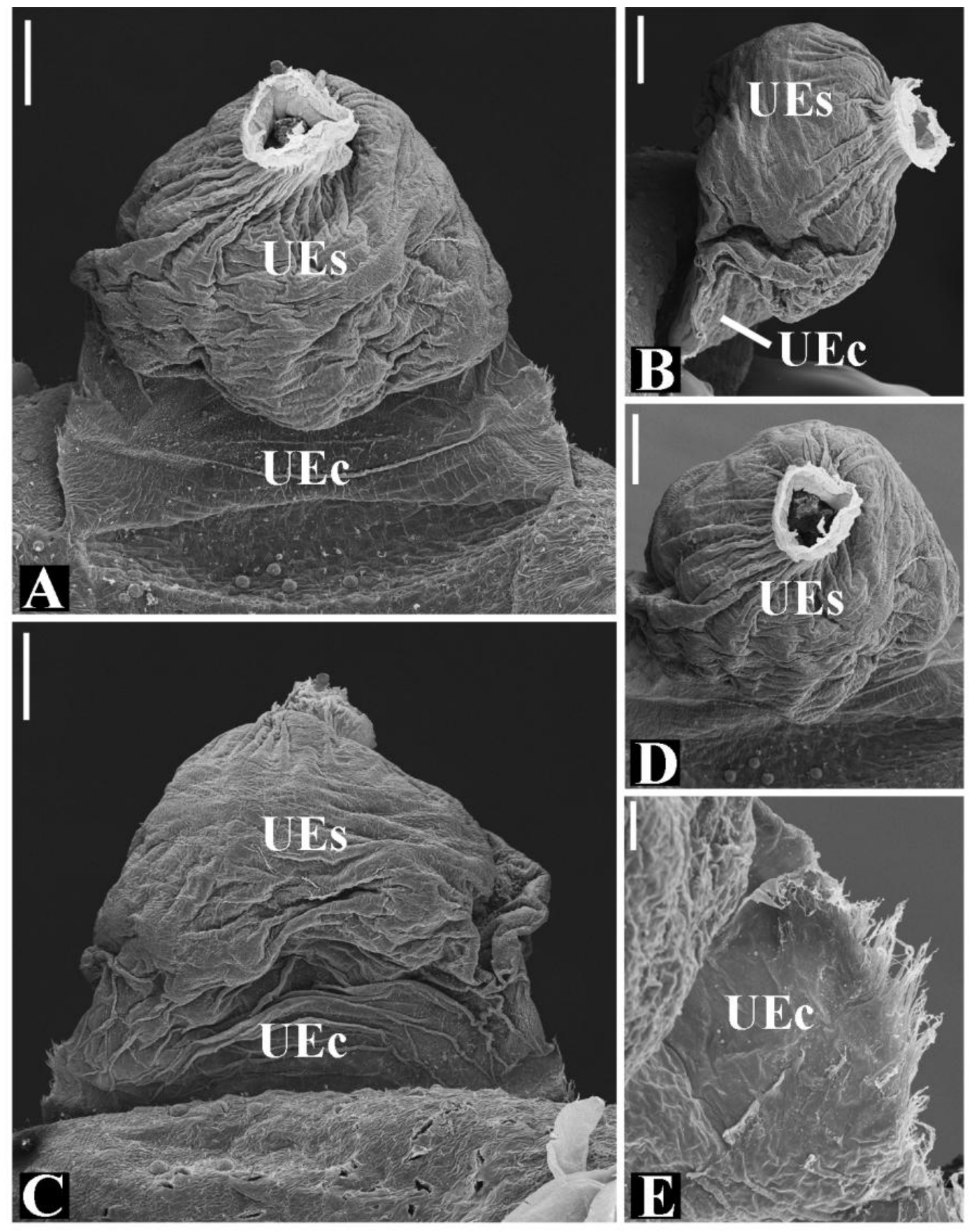

Figure 116. Glenognatha lacteovittata from Brazil. Female genitalia. A, dorsal. B, lateral. C, ventral D-E, uterus externus cuticle. Scale bars, $100 \mu \mathrm{m}$ (A-D), $10 \mu \mathrm{m}(\mathrm{E})$. UEc: uterus externus chamber. UEs: uterus externus sac. 


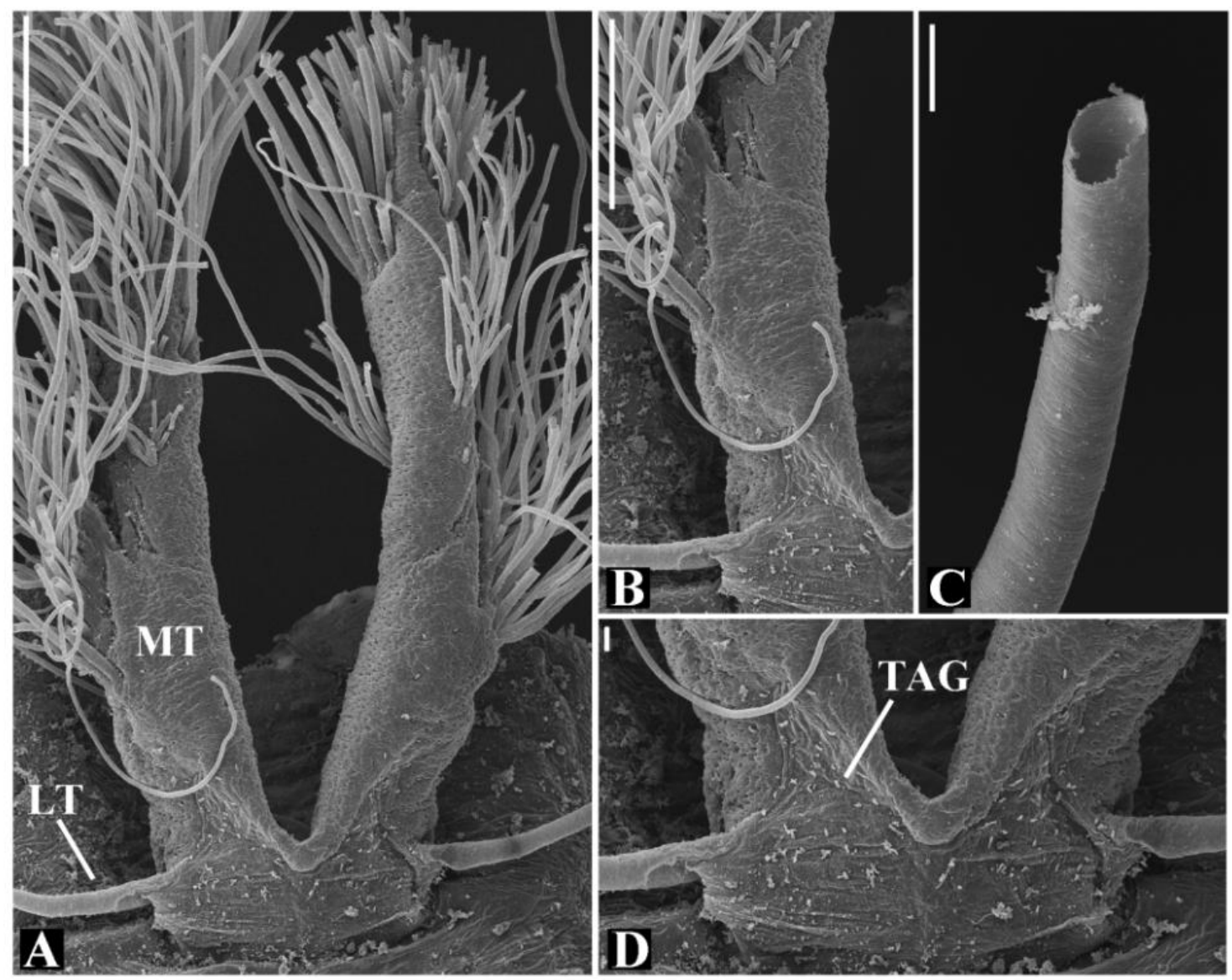

Figure 117. Glenognatha lacteovittata from Brazil. Female tracheal system. A, dorsal.

B, median trunk cuticle. C, lateral trunk cuticle. D, tracheal spiracle posterior view. Scale bars, $100 \mu \mathrm{m}$ (A-B), $10 \mu \mathrm{m}$ (C-D). LT: lateral tracheae. MT: median trunks. TAG: tracheal atrium gland. 


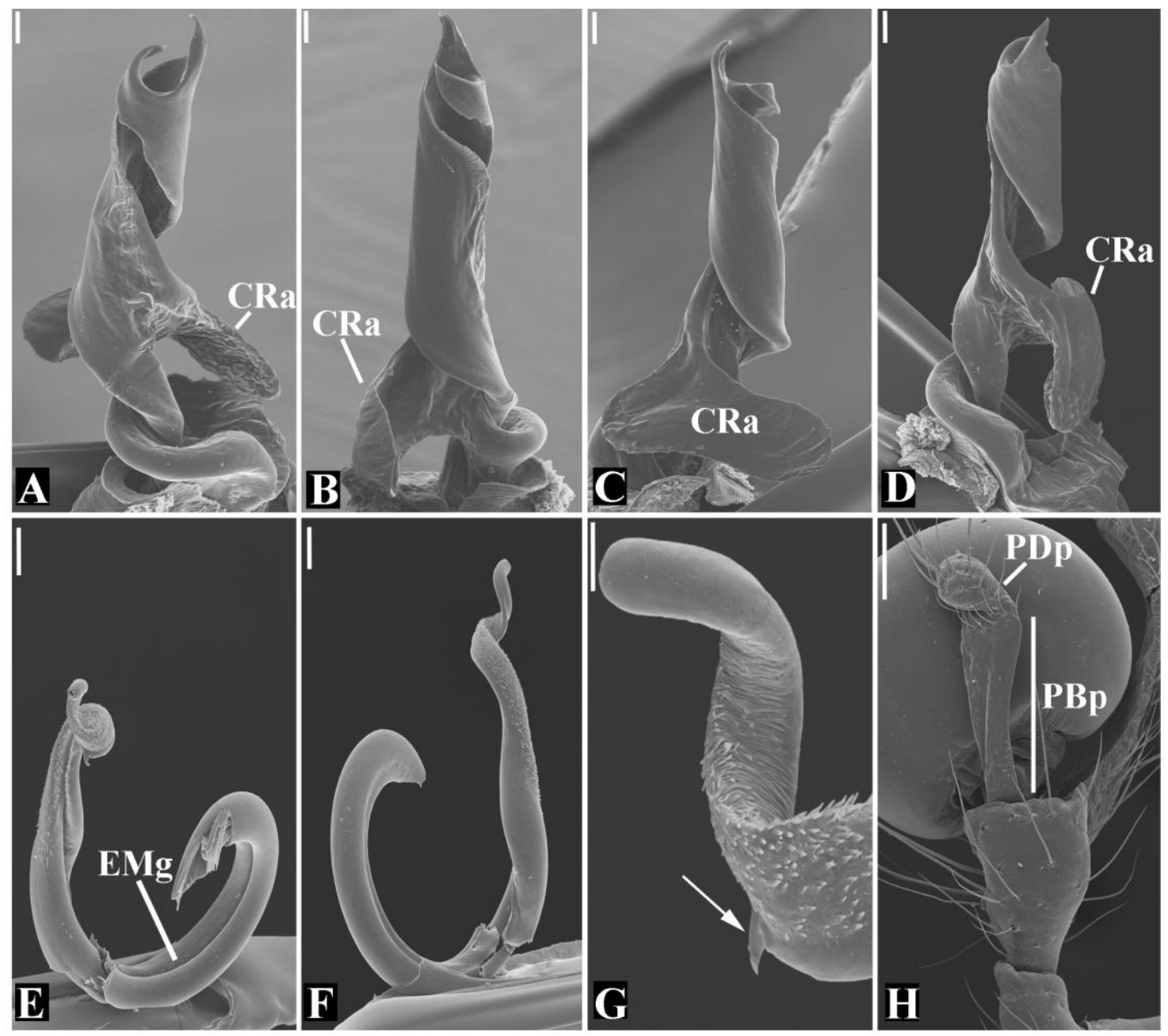

Figure 118. Glenognatha lacteovittata from Uruguay. A-D, conductor. A, ventral. B, prolateral. C, dorsal. D, retrolateral. E-G, embolus (arrow, tooth-like projection). H, paracymbium. Scale bars $20 \mu \mathrm{m}$ (A-D), (E-F) $30 \mu \mathrm{m}, 10 \mu \mathrm{m}(\mathrm{G}), 100 \mu \mathrm{m}(\mathrm{H})$. CRa: conductor retrolateral apophysis. EMg: embolic medial groove. PDP: paracymbium distal portion. PBp: paracymbium basal portion. 

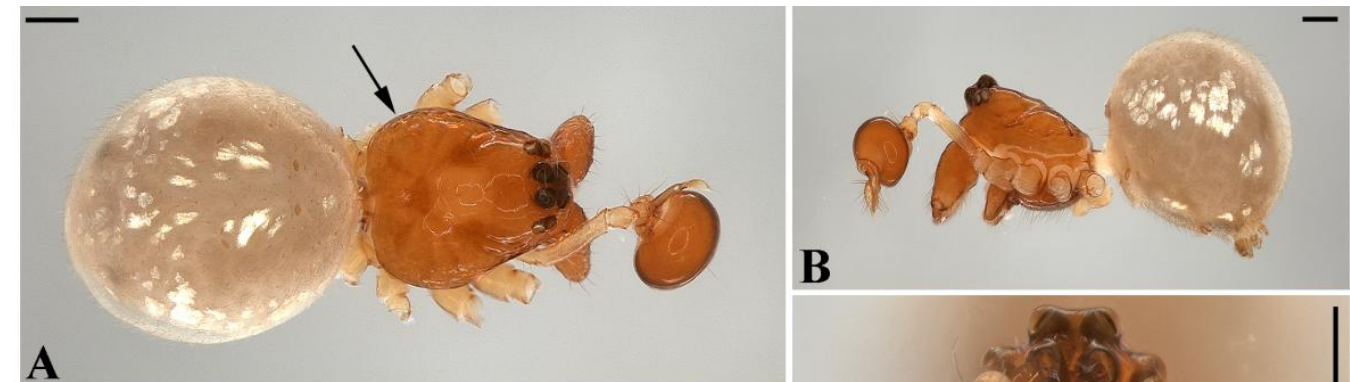

A
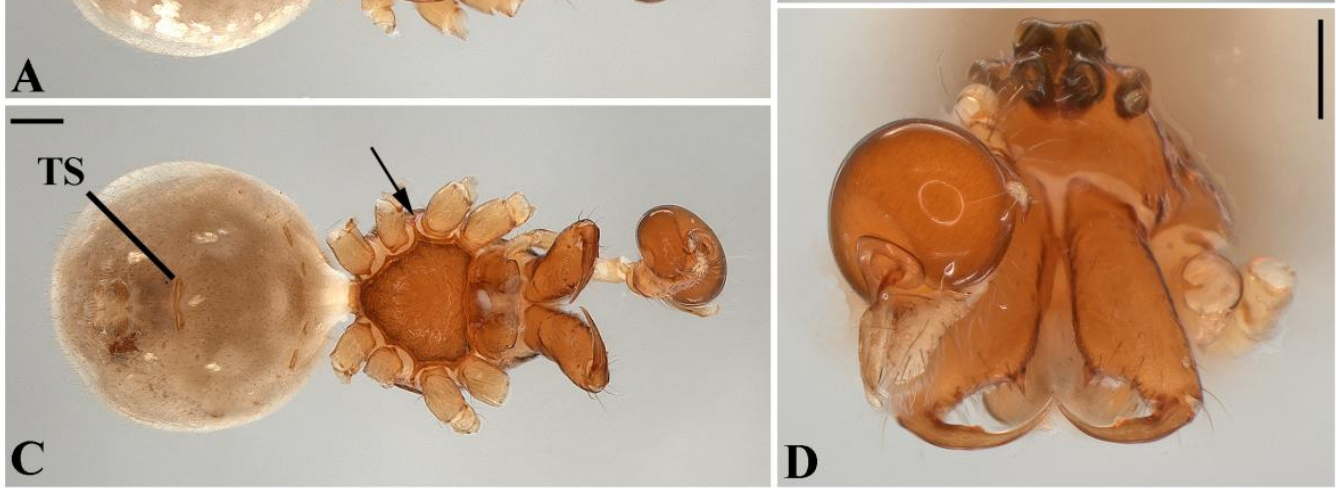

Figure 119. Glenognatha tangi from Myanmar (USNM). Male habitus. A, dorsal (arrow, lateral depressions). B, lateral. C, ventral (arrow, pleural bar). D, frontal. Scale bars, $200 \mu \mathrm{m}$. TS: tracheal spiracle.
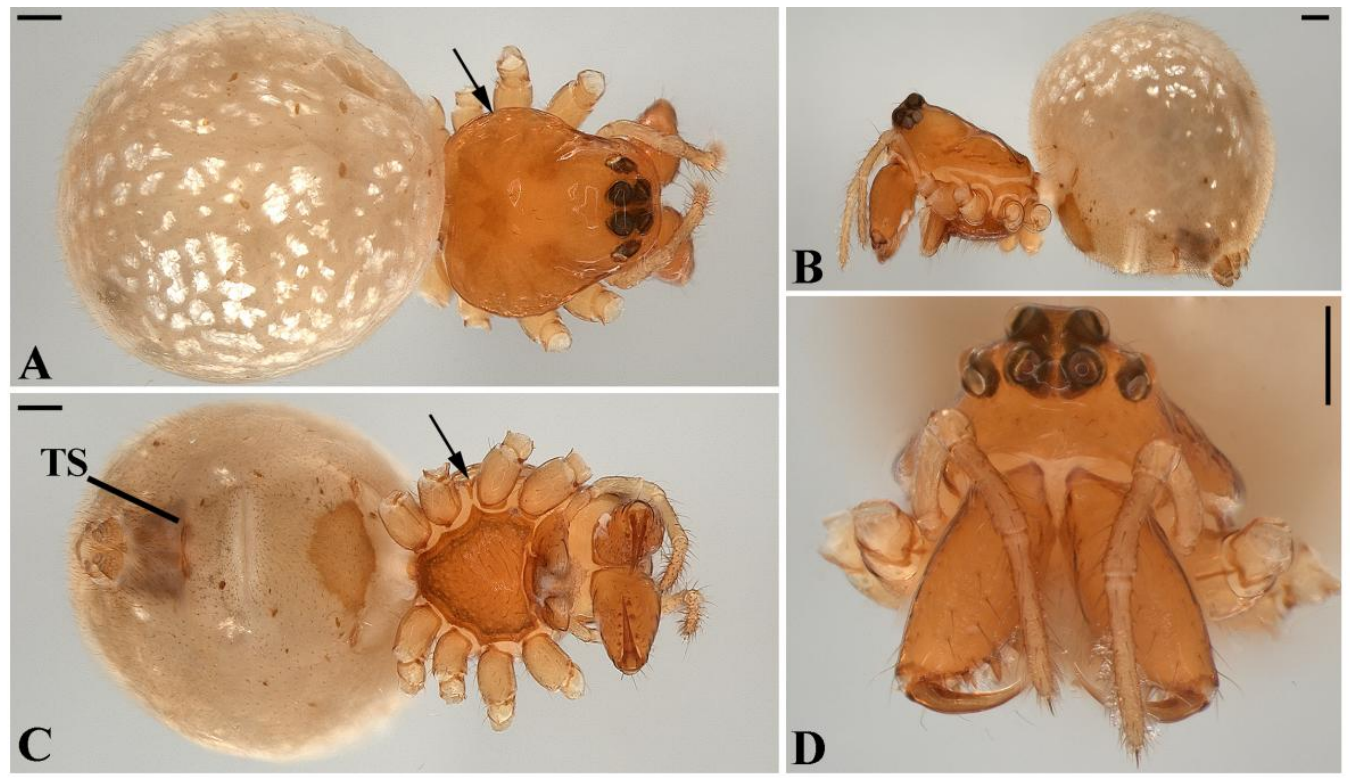

Figure 120. Glenognatha tangi from Myanmar (USNM). Female habitus. A, dorsal (arrow, lateral depressions). B, lateral. C, ventral (arrow, pleural bar). D, frontal. Scale bars, $200 \mu \mathrm{m}$. TS: tracheal spiracle. 


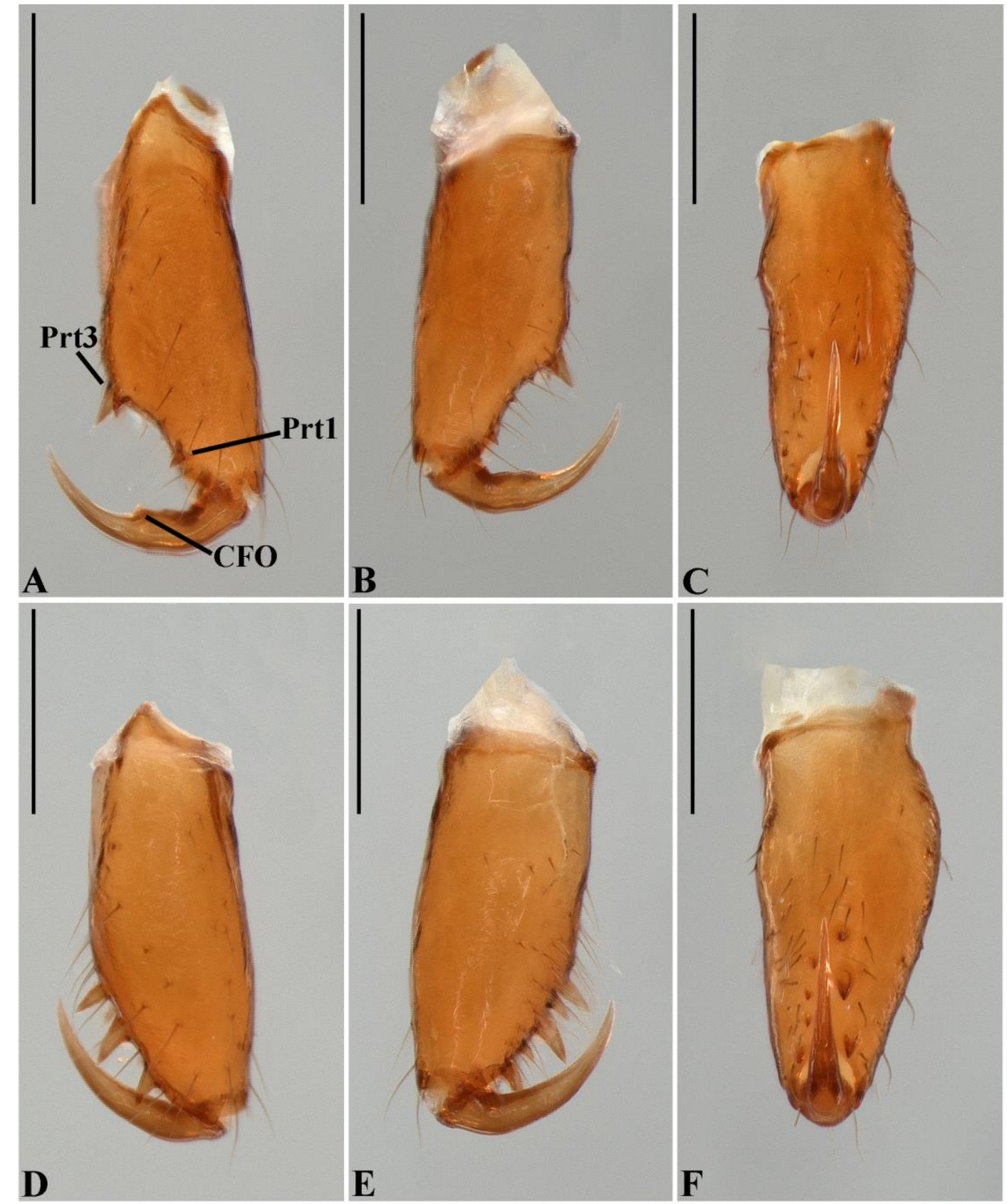

Figure 121. Glenognatha tangi from Myanmar (USNM). A-C, Male left chelicerae. A, anterior. B, posterior. C, mesal. D-F, Female left chelicerae. D, anterior. E, posterior. F, mesal. Scale bars, $100 \mu \mathrm{m}$. Prt: promarginal tooth. 


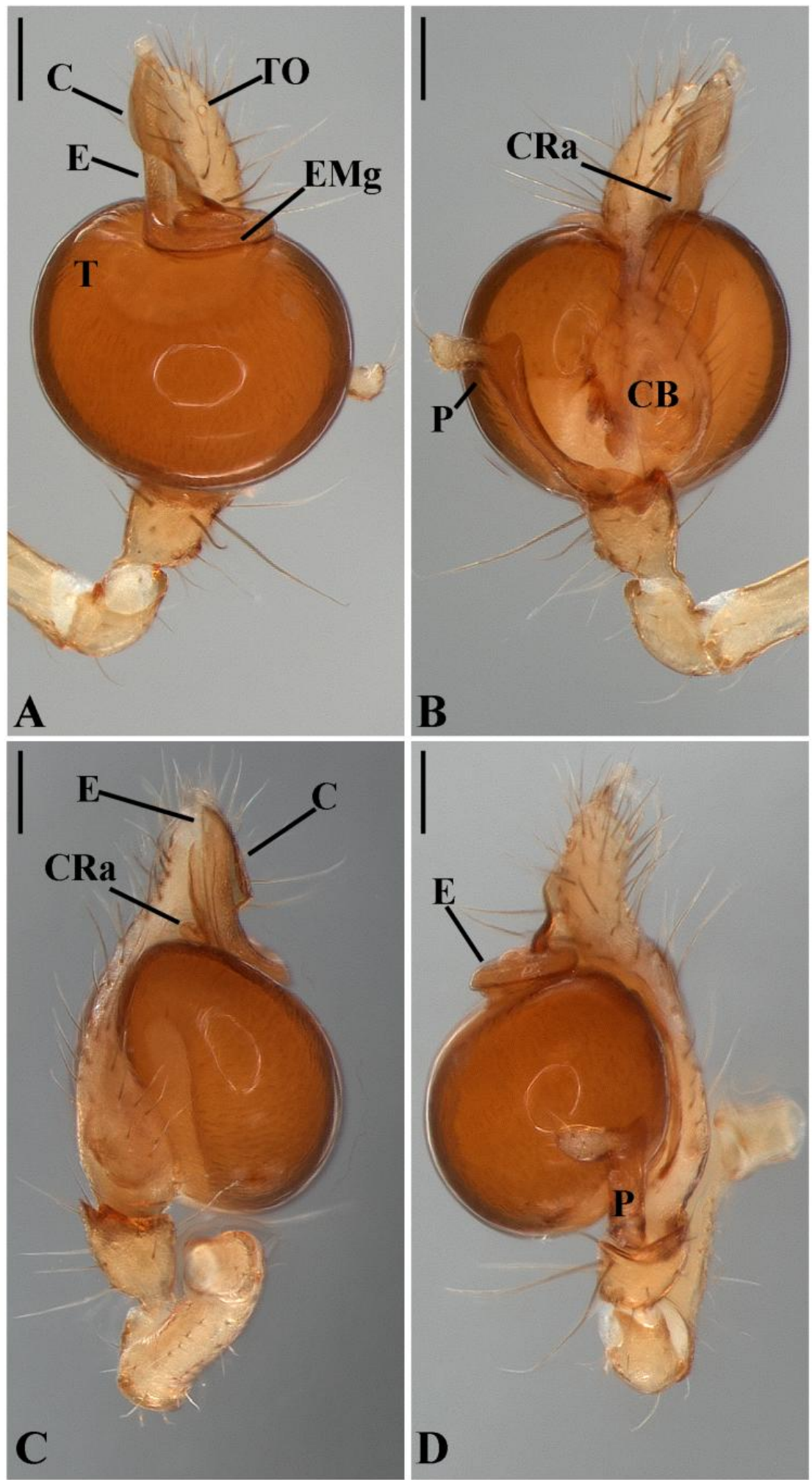

Figure 122. Glenognatha tangi from Myanmar (USNM). Male left palp. A, ventral. B, dorsal. C, prolateral. D, retrolateral. Scale bars, $100 \mu \mathrm{m}$. C: conductor. CB: cymbium. CRa: conductor retrolateral apophysis. E: embolus. EMg: embolic medial groove. P: paracymbium. T: tegulum. TO: tarsal organ. 

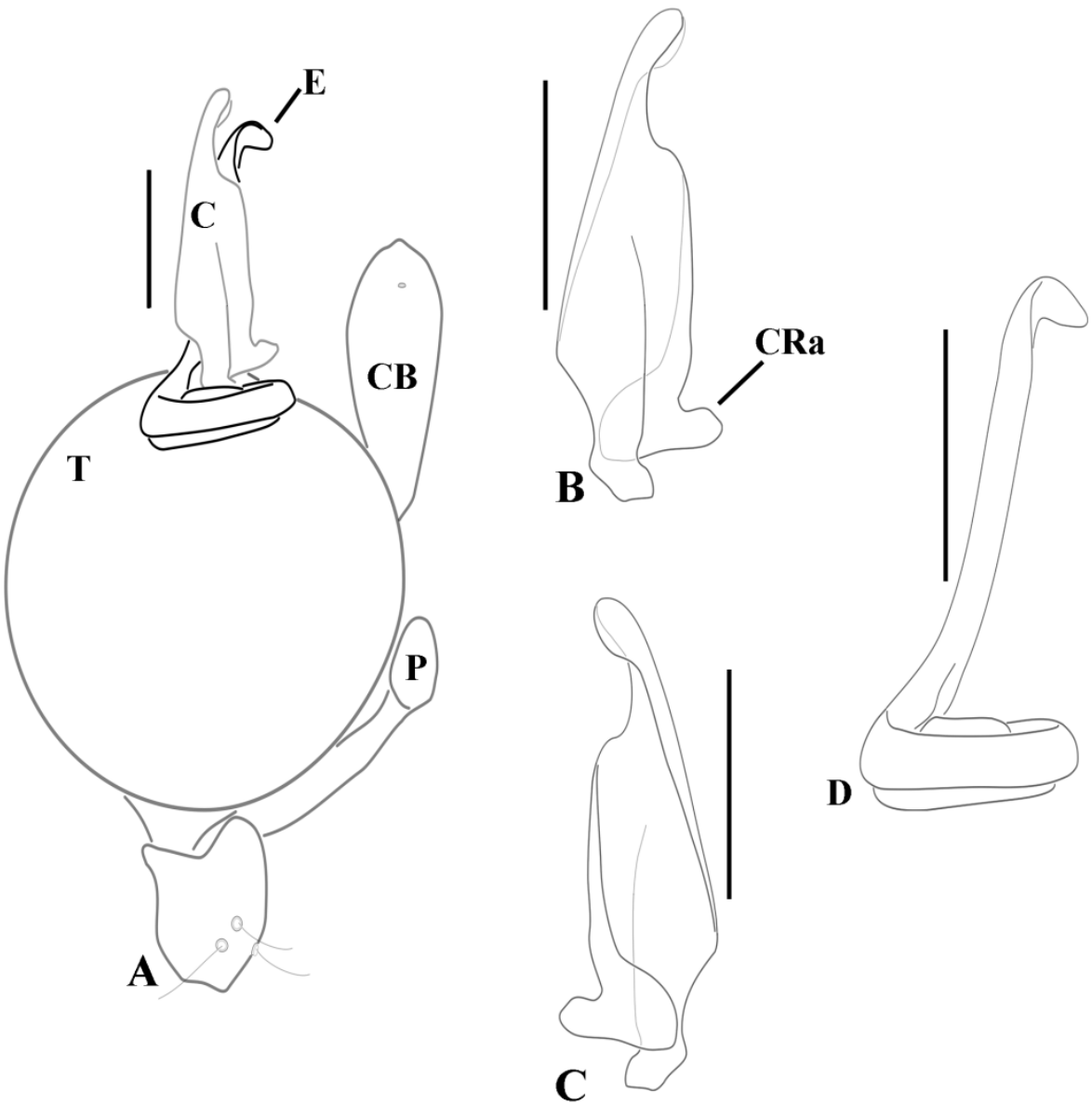

Figure 123. Glenognatha tangi from Myanmar (USNM). Male left palp, schematic. A, ventral. B, conductor ventral. C, conductor dorsal. D, embolus ventral. Scale bars 100 $\mu \mathrm{m}$. C: conductor. CB: cymbium. CRa: conductor retrolateral apophysis. E: embolus. P: paracymbium. T: tegulum.
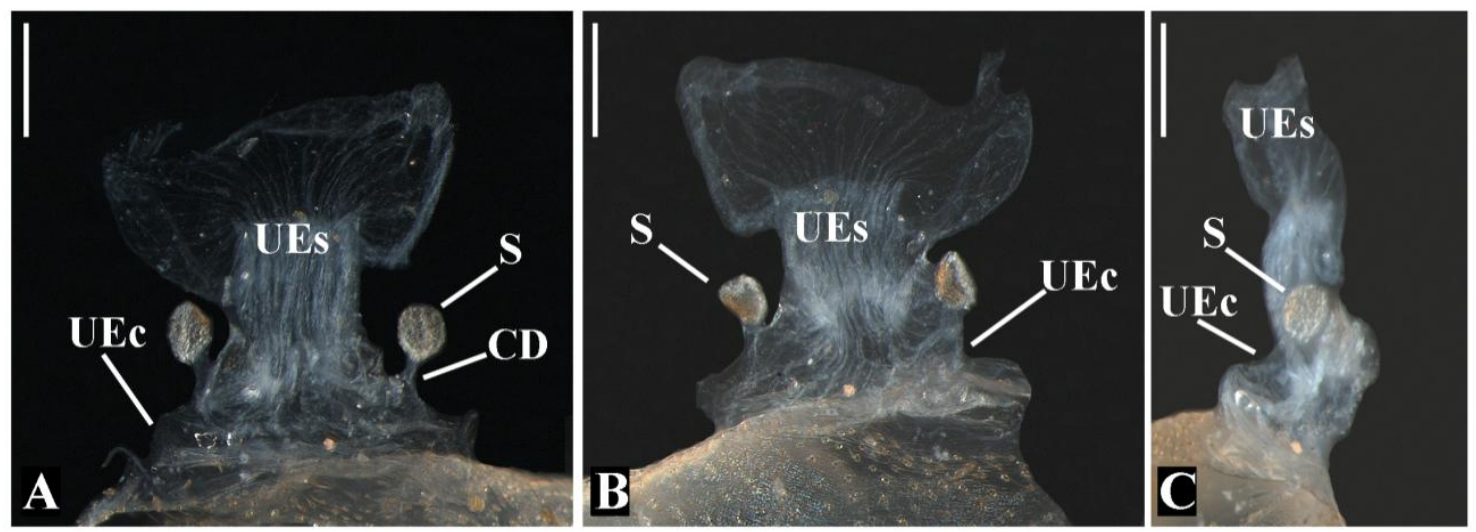

Figure 124. Glenognatha tangi from Myanmar. Female genitalia. A, dorsal. B, ventral. C, lateral. Scale bars, $100 \mu \mathrm{m}$. CD: copulatory duct. UEc: uterus externus chamber. UEs: uterus externus sac. S: spermathecae. 

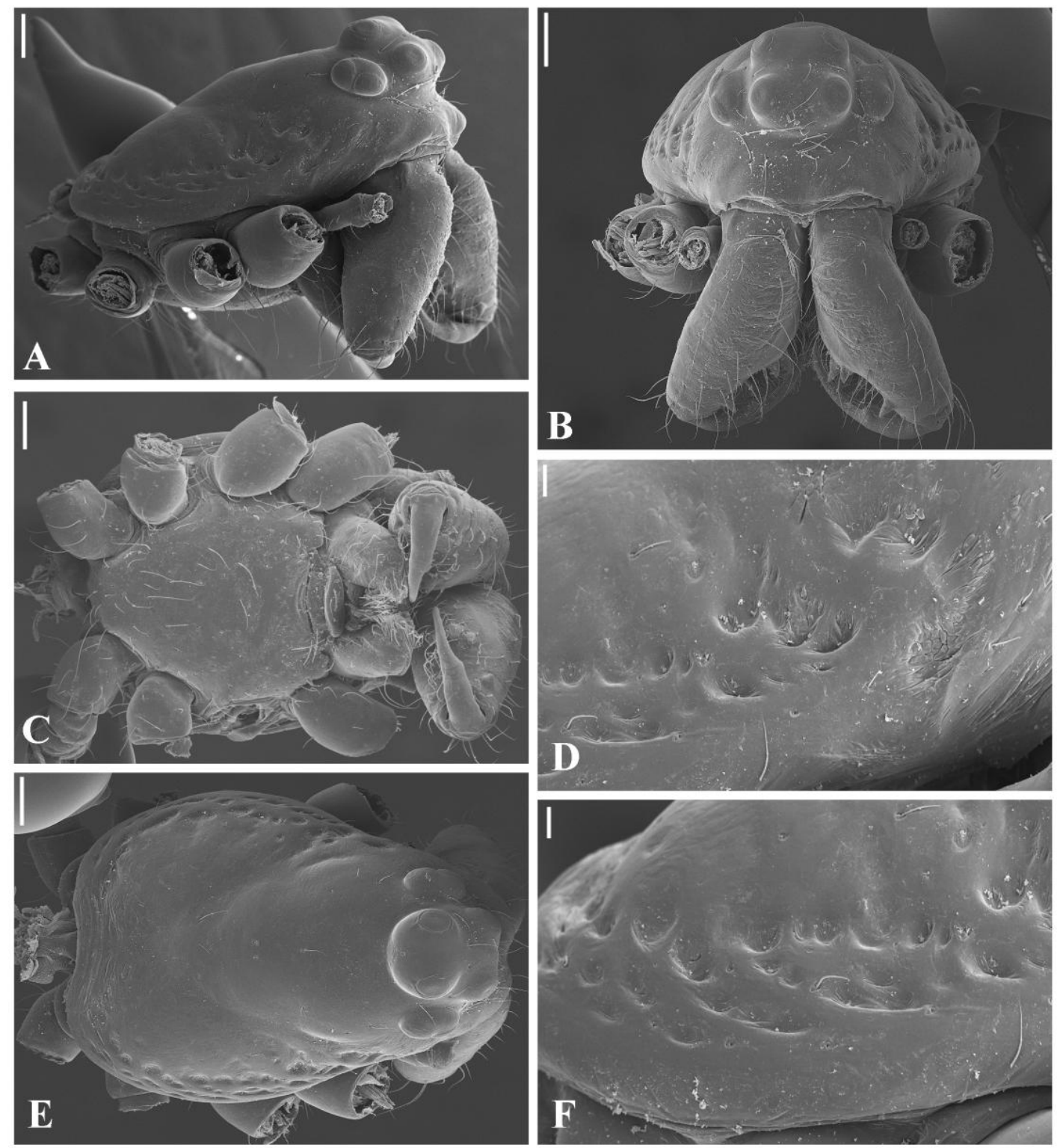

Figure 125. Glenognatha tangi from Myanmar. Female cephalothorax. A, lateral. B, frontal. C, ventral. E, dorsal. D, F carapace lateral cuticle. Scale bars $100 \mu \mathrm{m}$ (A-C, E), $20 \mu \mathrm{m}(\mathrm{D}, \mathrm{F})$. 


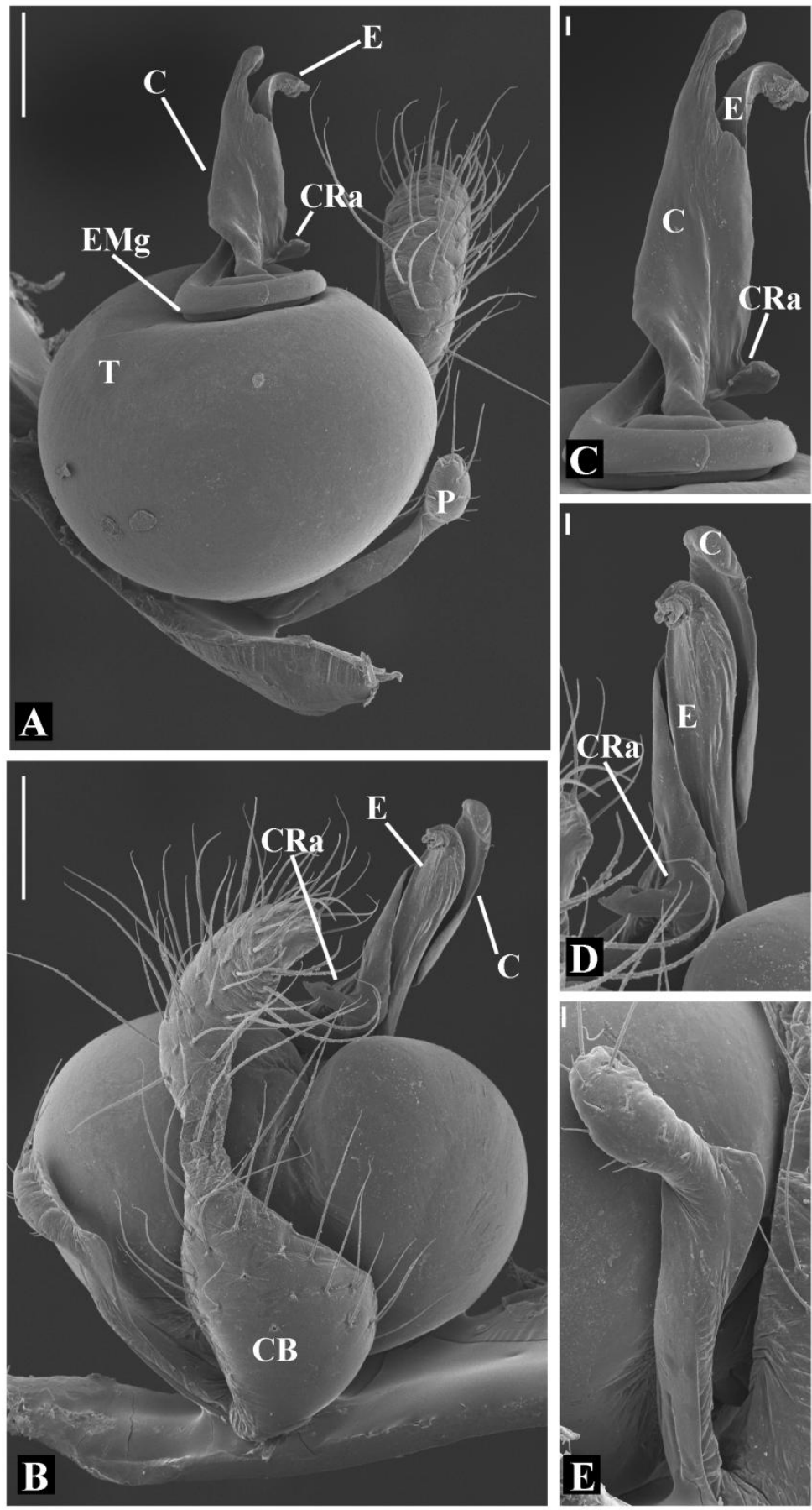

Figure 126. Glenognatha tangi from Myanmar. Male left palp. A, ventral. B, dorsal. C, embolus and conductor ventral. D, embolus and conductor dorsal. E, paracymbium. Scale bars $100 \mu \mathrm{m}$ (A-B), $10 \mu \mathrm{m}$ (C-E). C: conductor. CB: cymbium. CRa: conductor retrolateral apophysis. E: embolus. EMg: embolic medial groove. P: paracymbium. T: tegulum. 

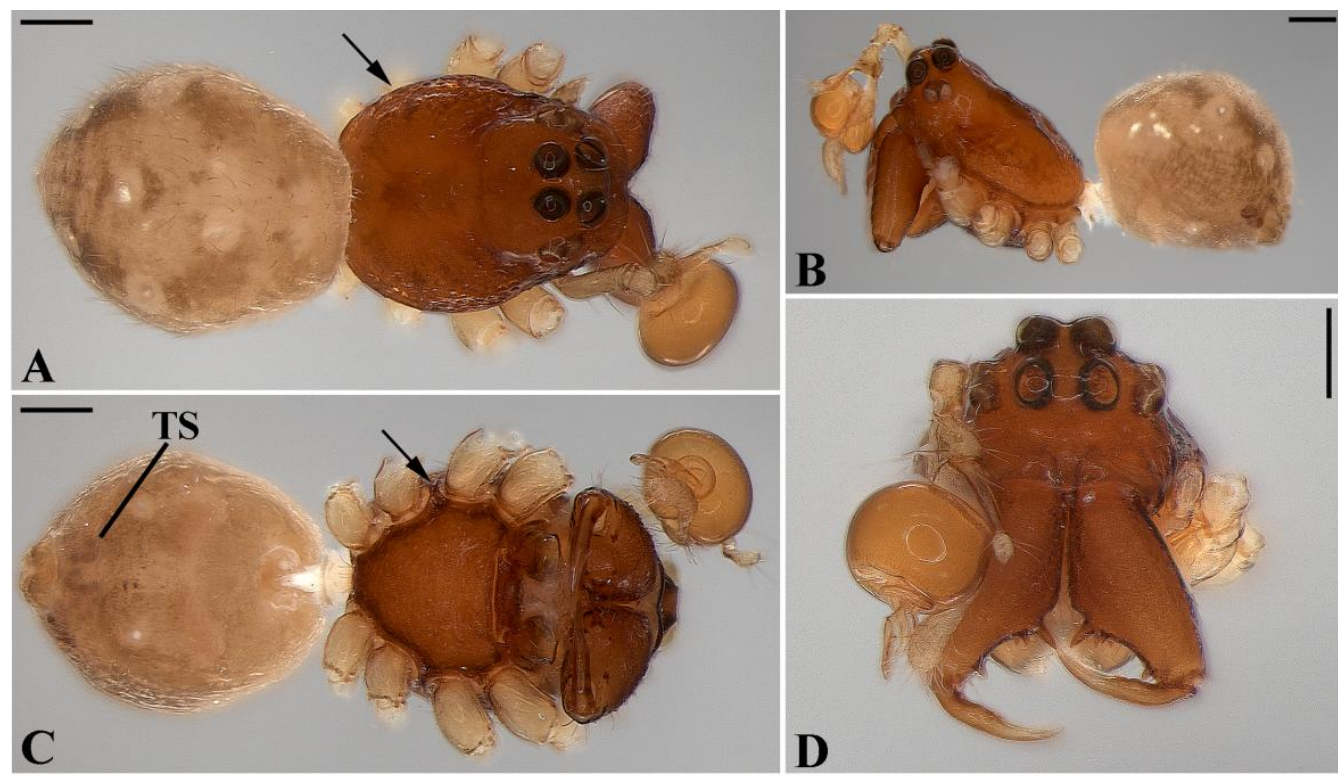

Figure 127. Glenognatha argyrostilba from Nigeria (CAS). Male habitus. A, dorsal (arrow, lateral depressions). B, lateral. C, ventral (arrow, pleural bar). D, frontal. Scale bars, $200 \mu \mathrm{m}$. TS: tracheal spiracle.
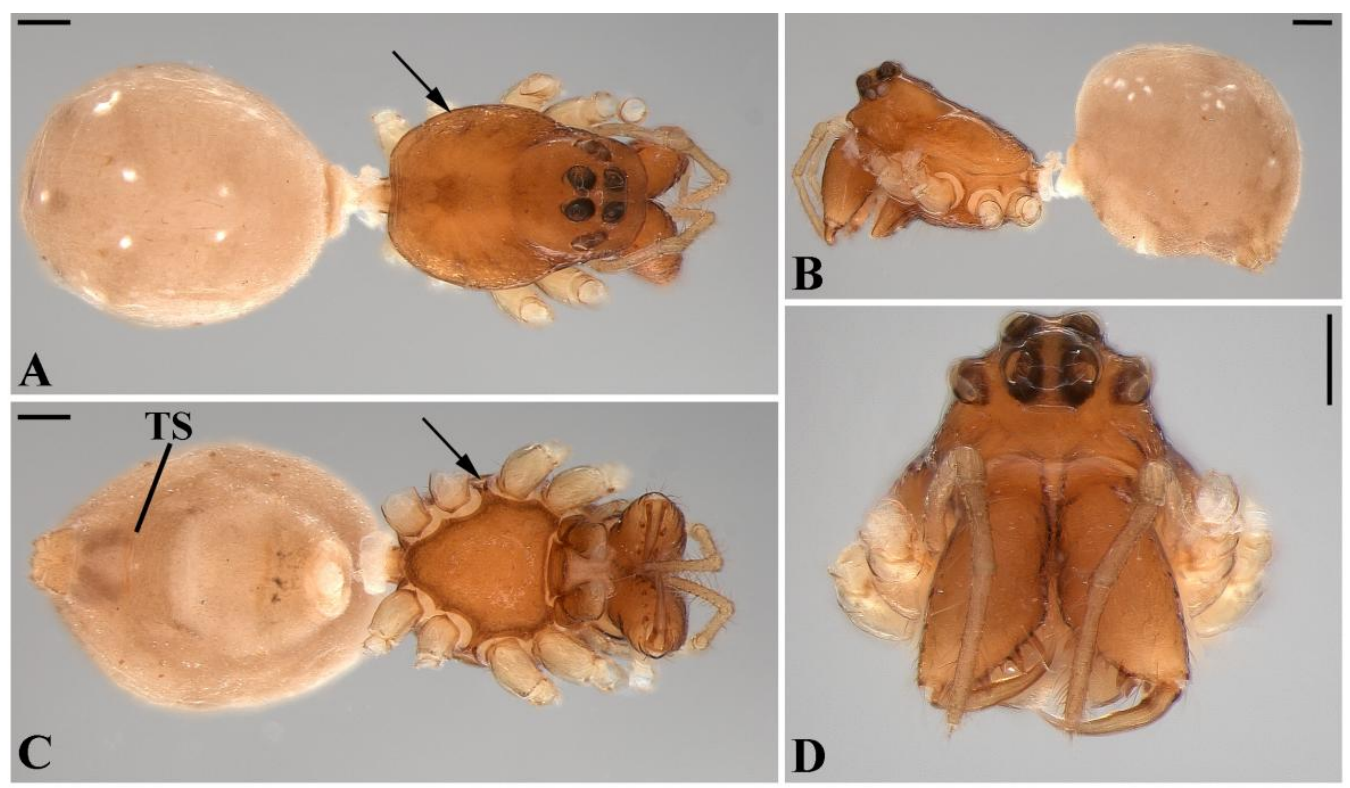

Figure 128. Glenognatha argyrostilba from Nigeria (CAS). Female habitus. A, dorsal (arrow, lateral depressions). B, lateral. C, ventral (arrow, pleural bar). D, frontal. Scale bars, $200 \mu \mathrm{m}$. TS: tracheal spiracle. 


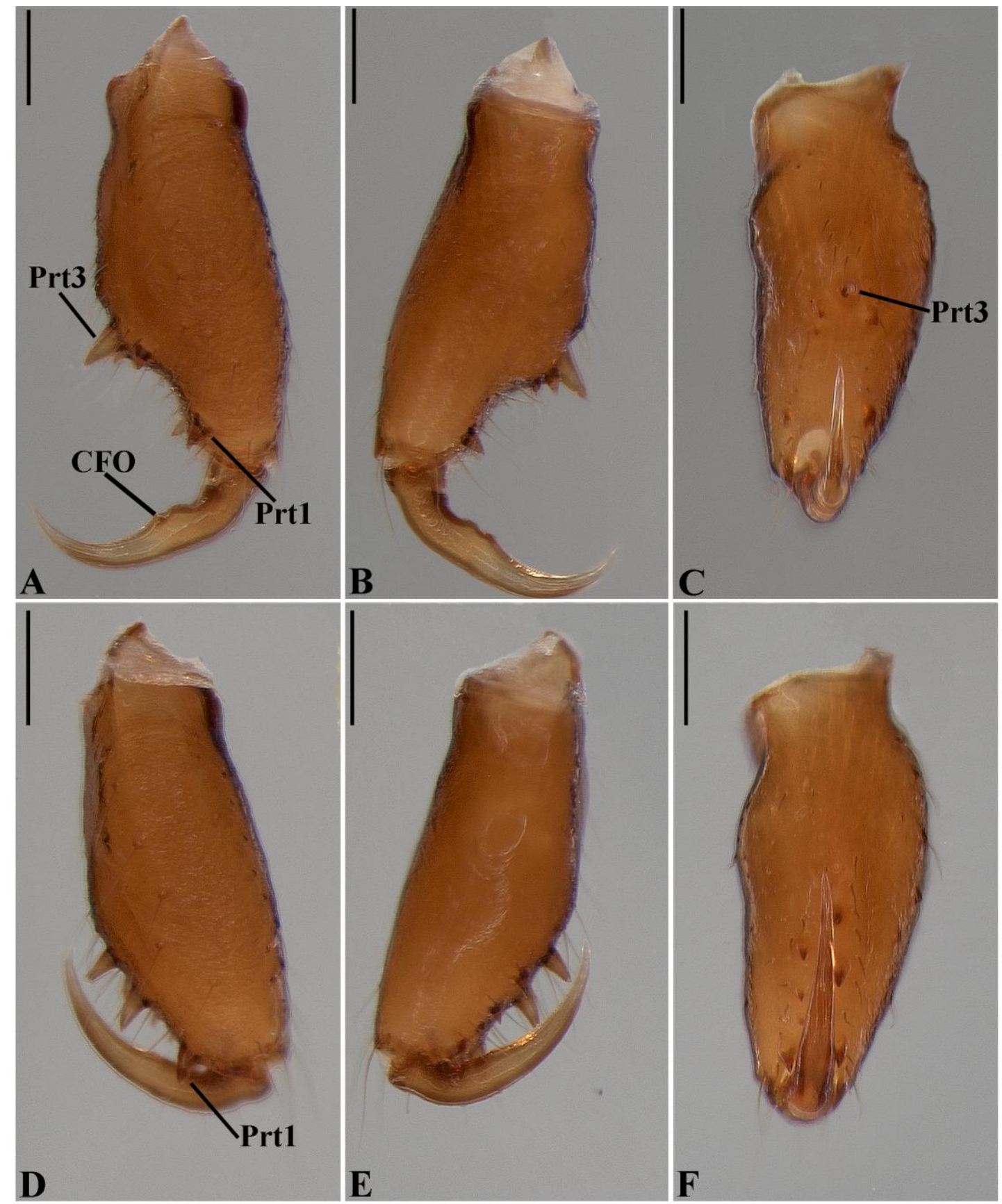

Figure 129. Glenognatha argyrostilba from Nigeria (CAS). A-C, Male left chelicerae.

A, anterior. B, posterior. C, mesal. D-F, Female left chelicerae. D, anterior. E, posterior.

F, mesal. Scale bars, $100 \mu \mathrm{m}$. Prt: promarginal tooth. CFO: cheliceral fang outgrowth. 


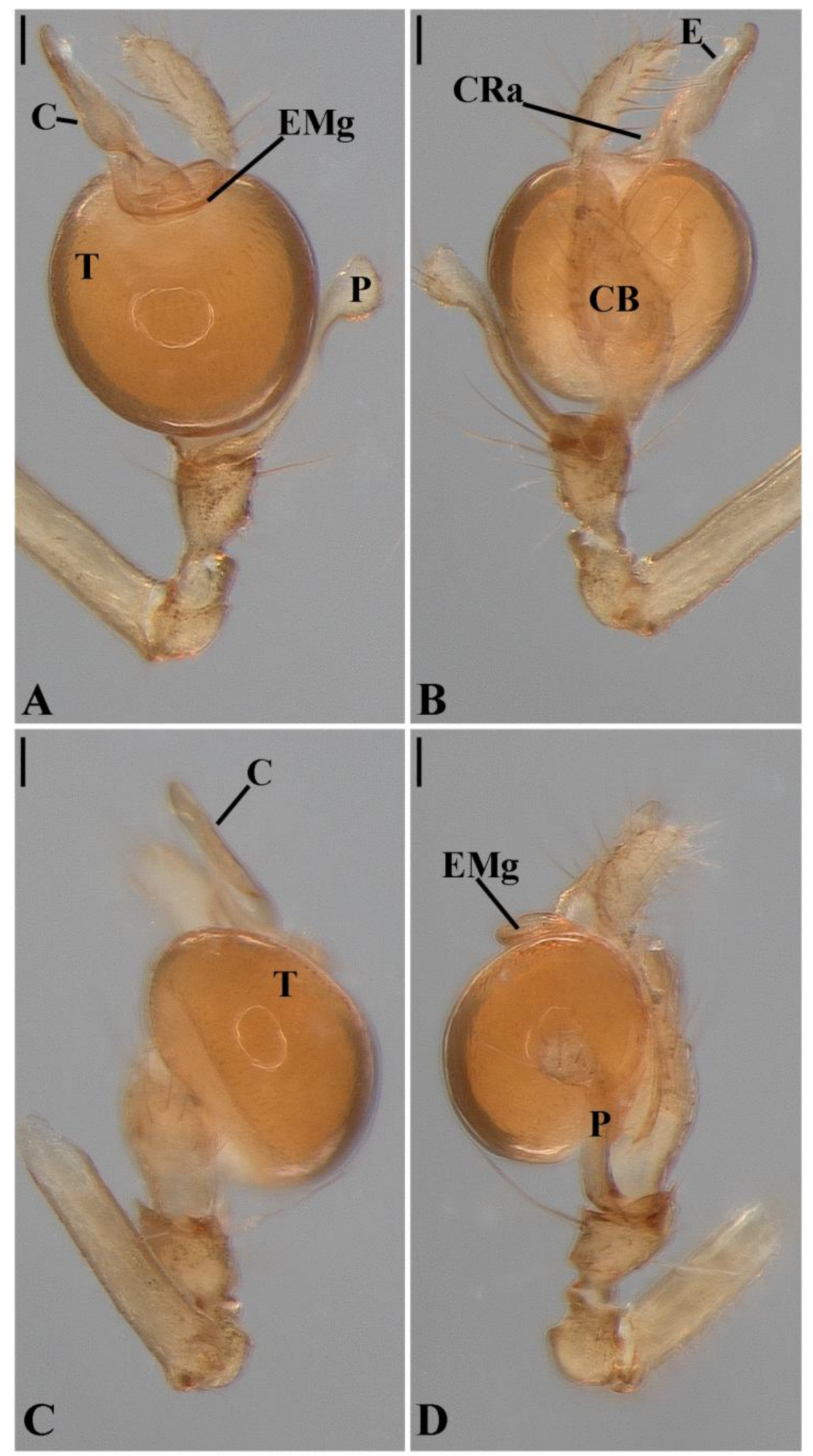

Figure 130. Glenognatha argyrostilba from Nigeria (CAS). Male left palp. A, ventral. B, dorsal. C, prolateral. D, retrolateral. Scale bars, $100 \mu \mathrm{m}$. C: conductor. CB: cymbium. CRa: conductor retrolateral apophysis. E: embolus. EMg: embolic medial groove. P: paracymbium. T: tegulum. 

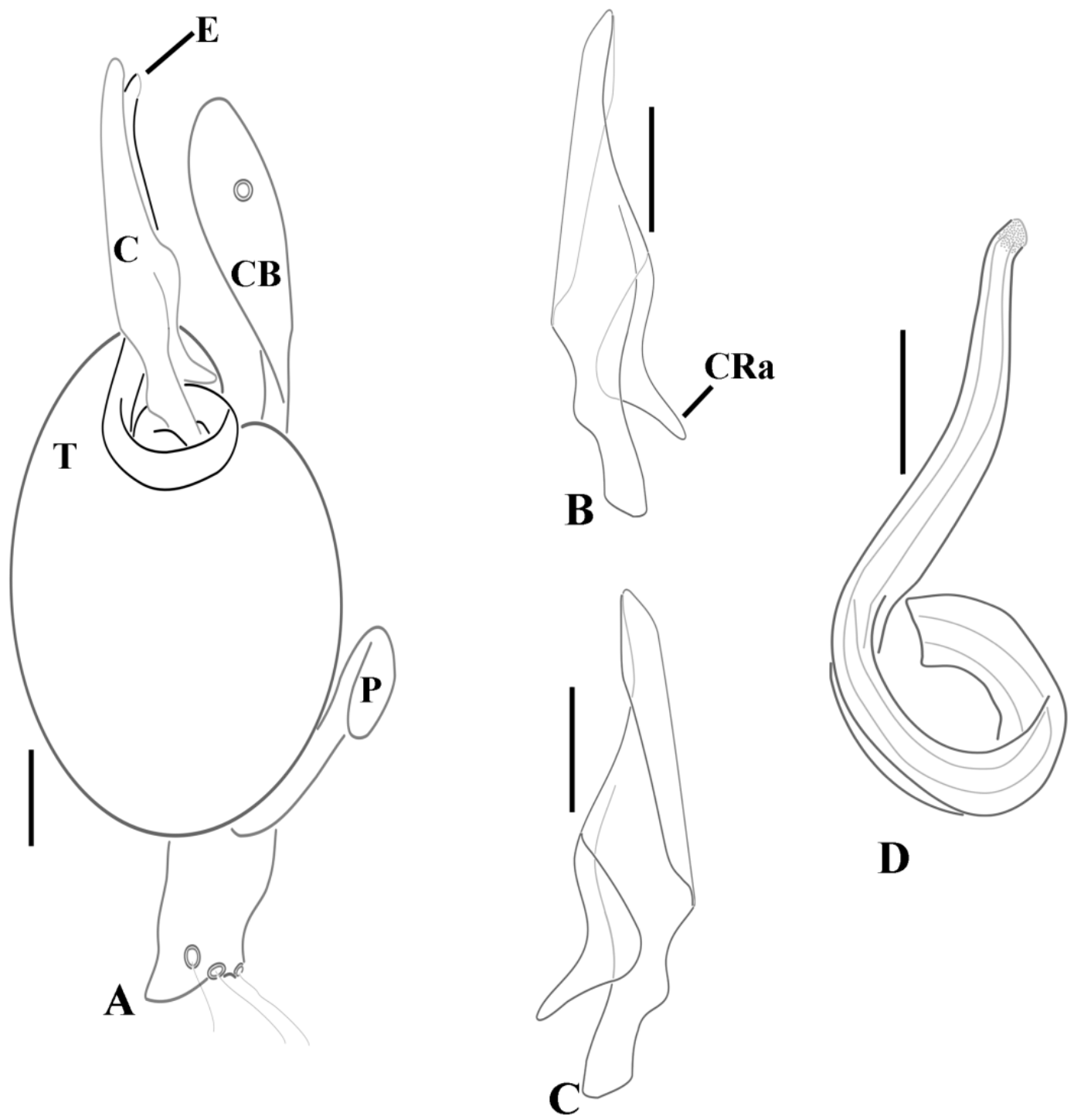

Figure 131. Glenognatha argyrostilba from Nigeria (CAS). Male left palp, schematic. A, ventral. B, conductor ventral. C, conductor dorsal. D, embolus ventral. Scale bars $100 \mu \mathrm{m}$. C: conductor. CB: cymbium. CRa: conductor retrolateral apophysis. E: embolus. P: paracymbium. T: tegulum. 

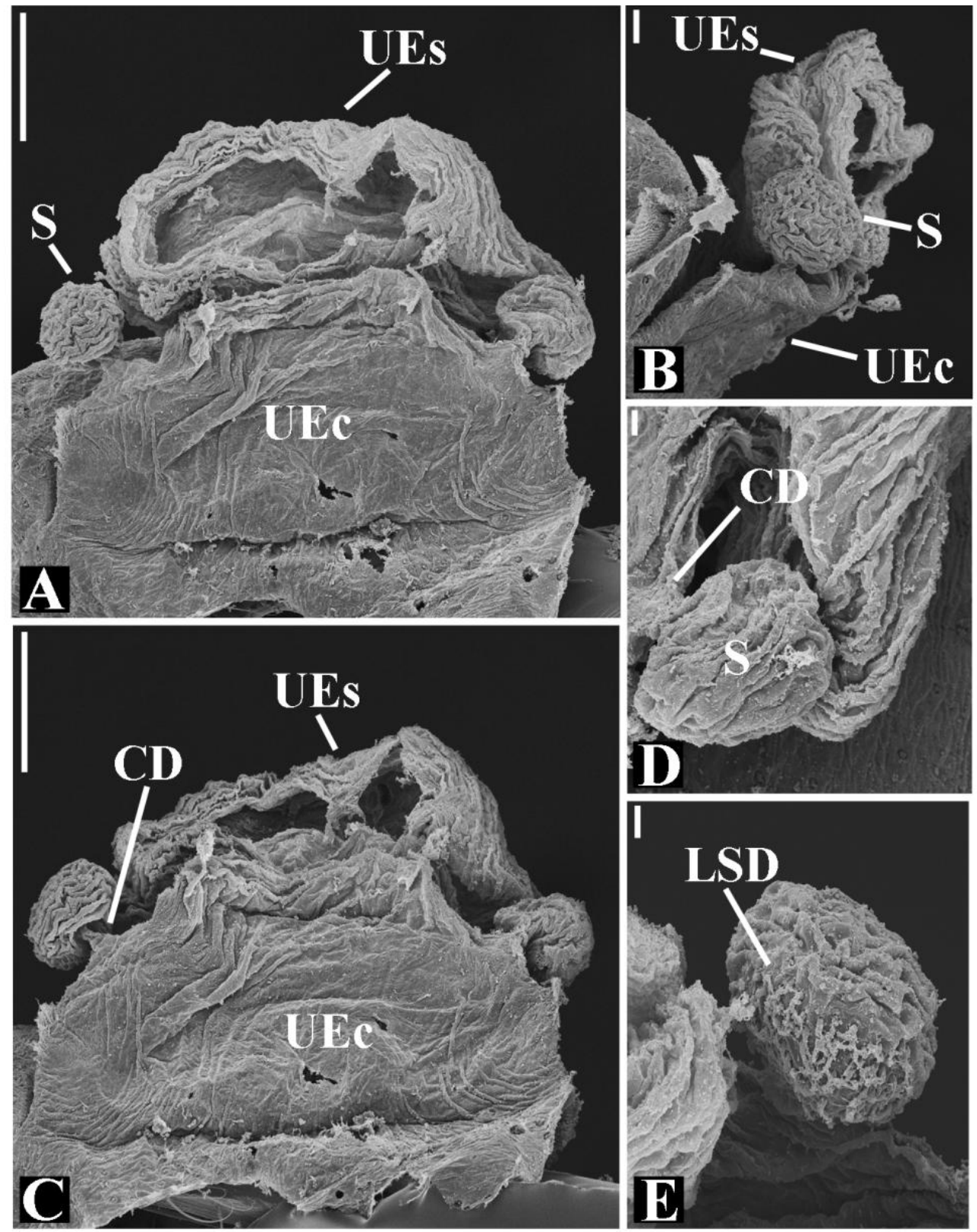

Figure 132. Glenognatha argyrostilba from Nigeria. Female genitalia. A, dorsal. B, lateral. C, ventral. D-E, spermathecae and copulatory duct. Scale bars, $100 \mu \mathrm{m}$ (A, C), $20 \mu \mathrm{m}(\mathrm{B}), 10 \mu \mathrm{m}(\mathrm{D}, \mathrm{E}) . \mathrm{CD}$ : copulatory duct. LSD: long-stem gland ducts. UEc: uterus externus chamber. UEs: uterus externus sac. S: spermathecae. 


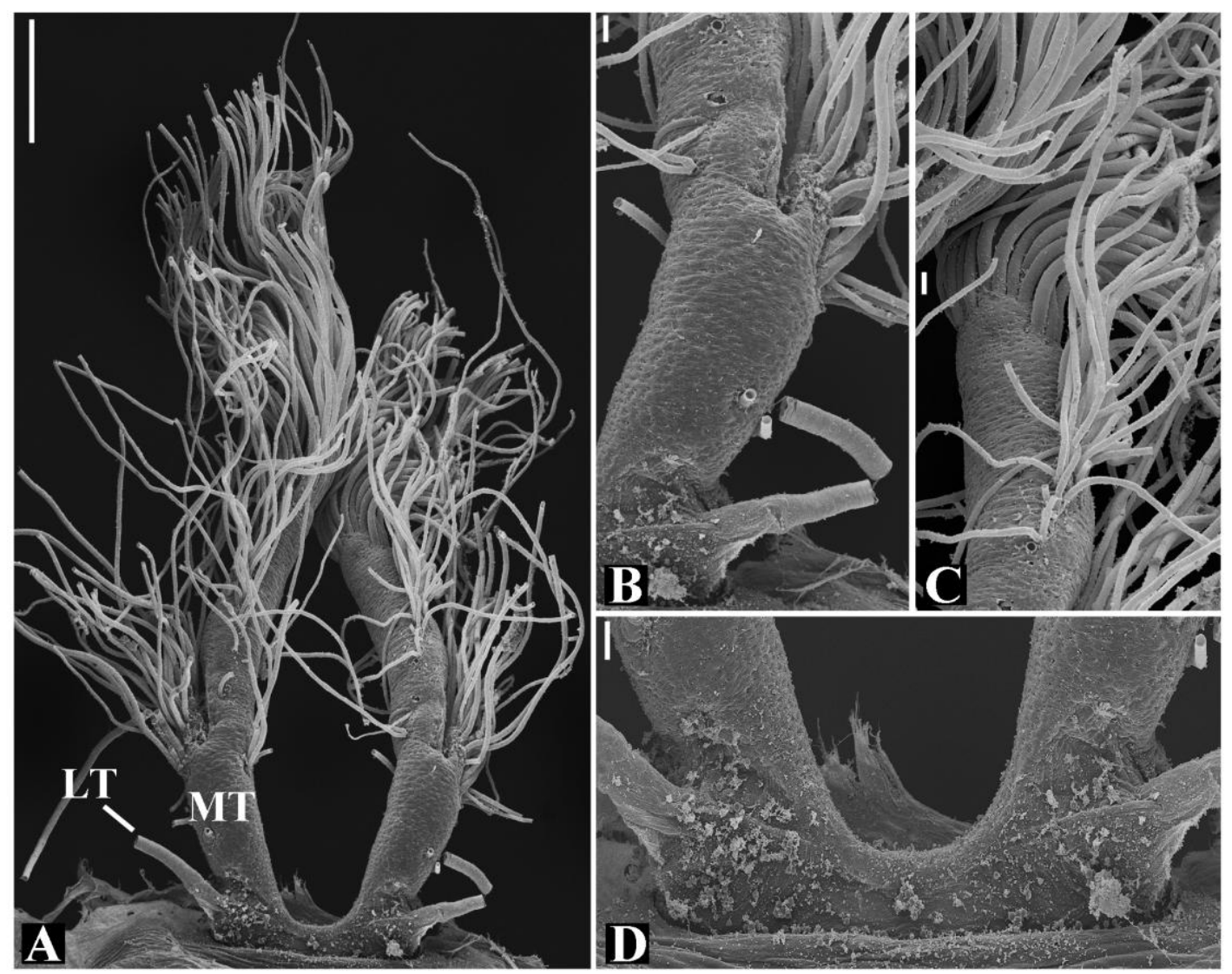

Figure 133. Glenognatha argyrostilba from Nigeria. Female tracheal system. A, dorsal. $\mathbf{B}$, median and lateral trunks. $\mathbf{C}$, median trunk distal. D, tracheal spiracle posterior view. Scale bars, $100 \mu \mathrm{m}$ (A), $10 \mu \mathrm{m}$ (B-D). LT: lateral trachea. MT: median trunks. TAG: tracheal atrium gland. 

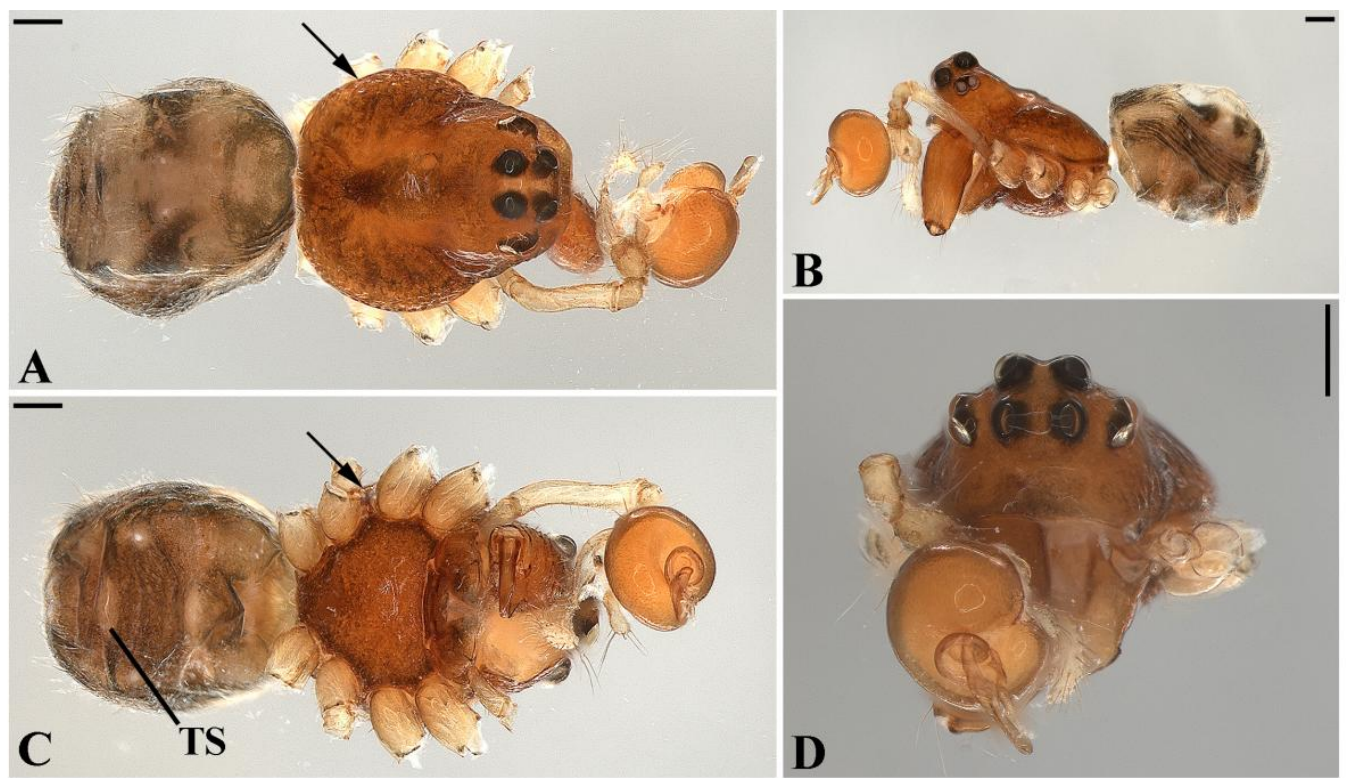

Figure 134. Glenognatha argyrostilba from Ecuador (AMNH). Male habitus. A, dorsal (arrow, lateral depressions). B, lateral. C, ventral (arrow, pleural bar). D, frontal. Scale bars, $200 \mu \mathrm{m}$. TS: tracheal spiracle.
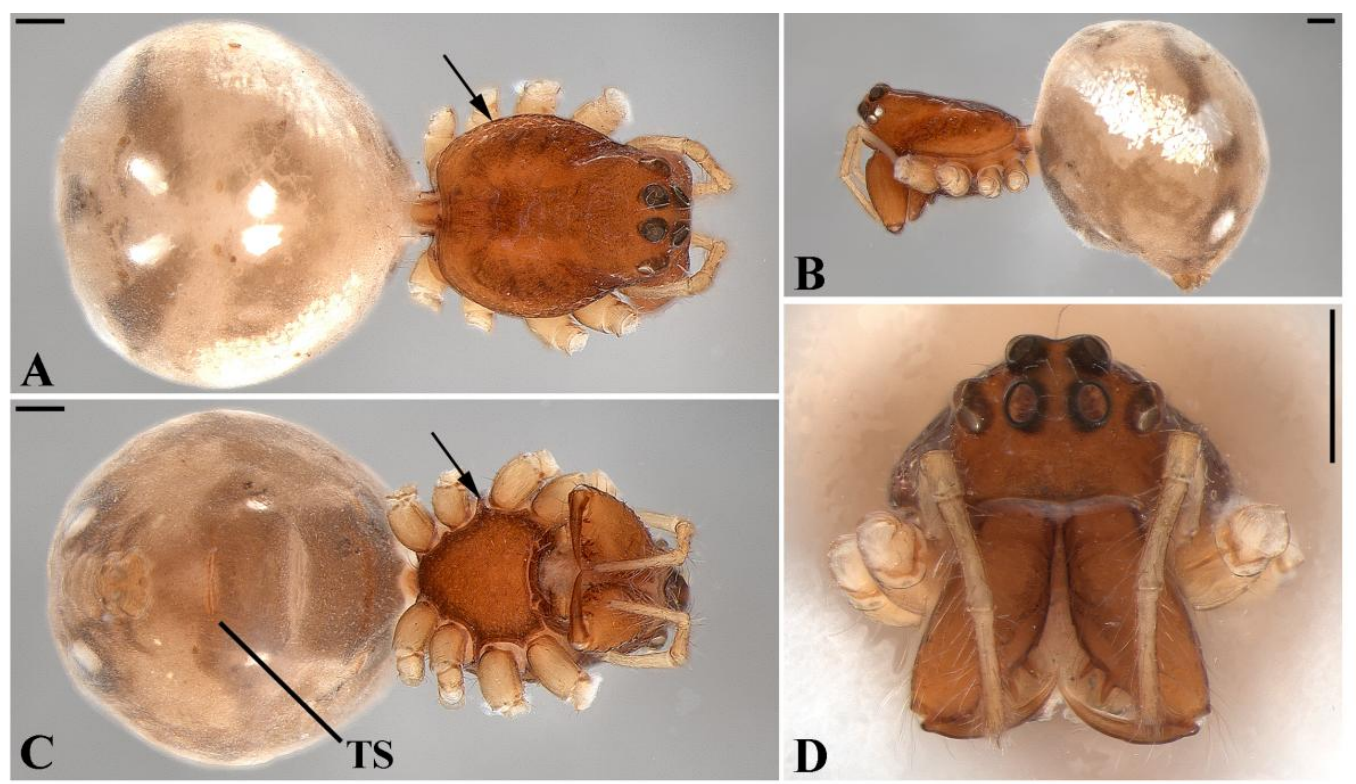

Figure 135. Glenognatha argyrostilba from Ecuador (MCZ 125476). Female habitus. A, dorsal (arrow, lateral depressions). B, lateral. C, ventral (arrow, pleural bar). D, frontal. Scale bars, $200 \mu \mathrm{m}$. TS: tracheal spiracle. 


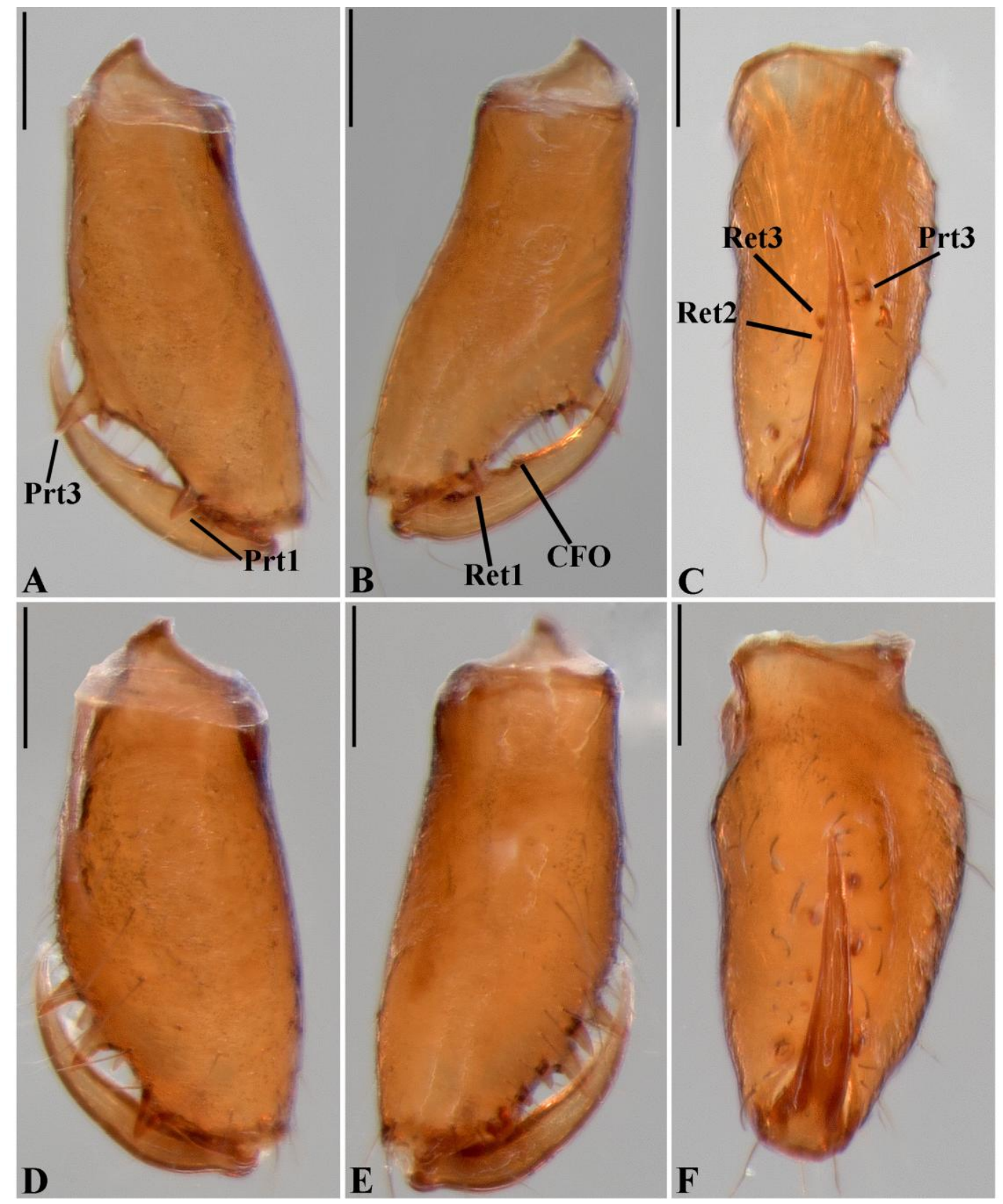

Figure 136. Glenognatha argyrostilba from Ecuador (AMNH). A-C, Male left chelicerae. A, anterior. B, posterior. C, mesal. D-F, Female left chelicerae. D, anterior. E, posterior. F, mesal. Scale bars, $100 \mu \mathrm{m}$. Prt: promarginal tooth. CFO: cheliceral fang outgrowth. Ret: retromarginal tooth. 


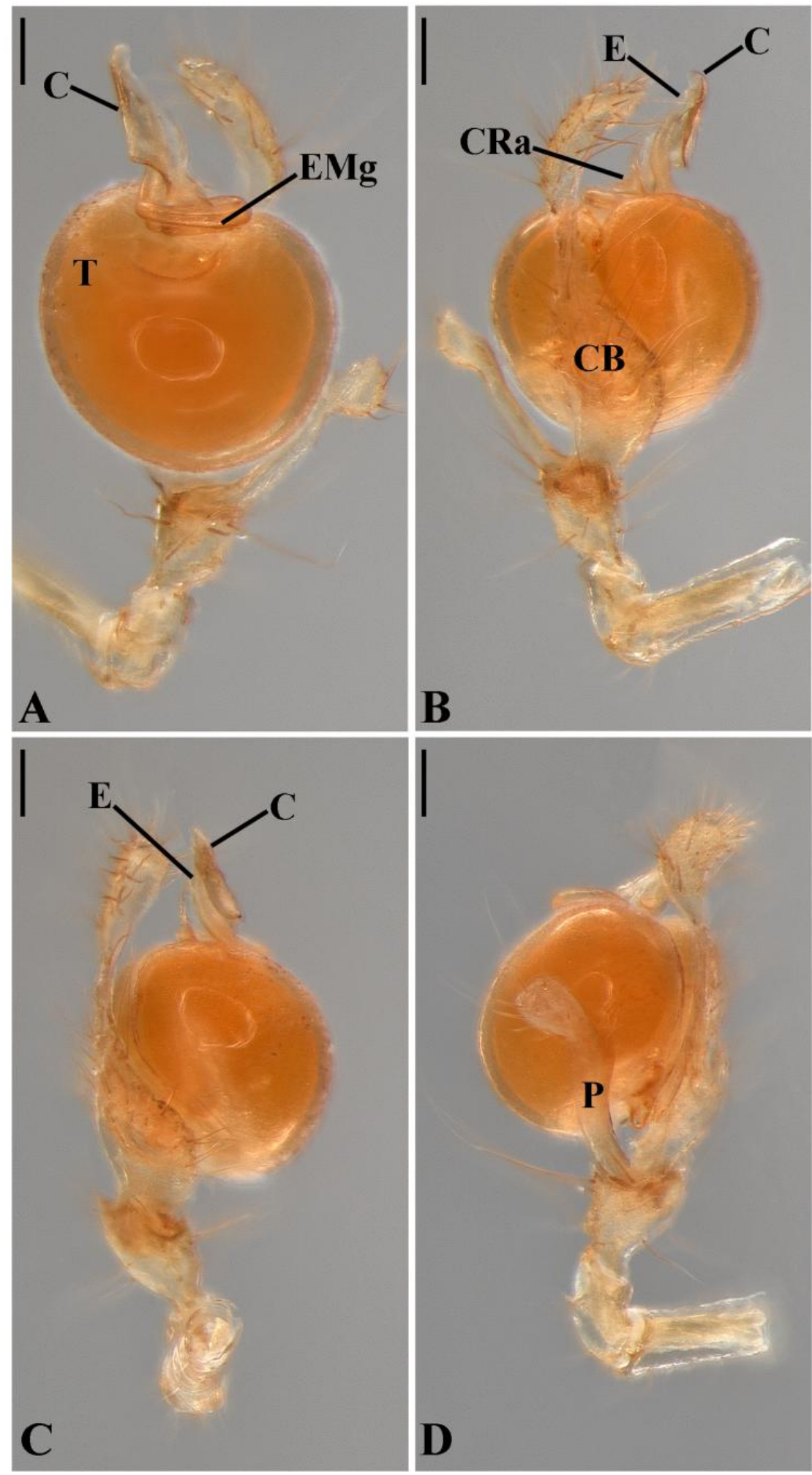

Figure 137. Glenognatha argyrostilba from Ecuador. Male left palp. A, ventral. B, dorsal. C, prolateral. D, retrolateral. Scale bars, $100 \mu \mathrm{m}$. C: conductor. CB: cymbium. CRa: conductor retrolateral apophysis. E: embolus. EMg: embolic medial groove. P: paracymbium. T: tegulum. 

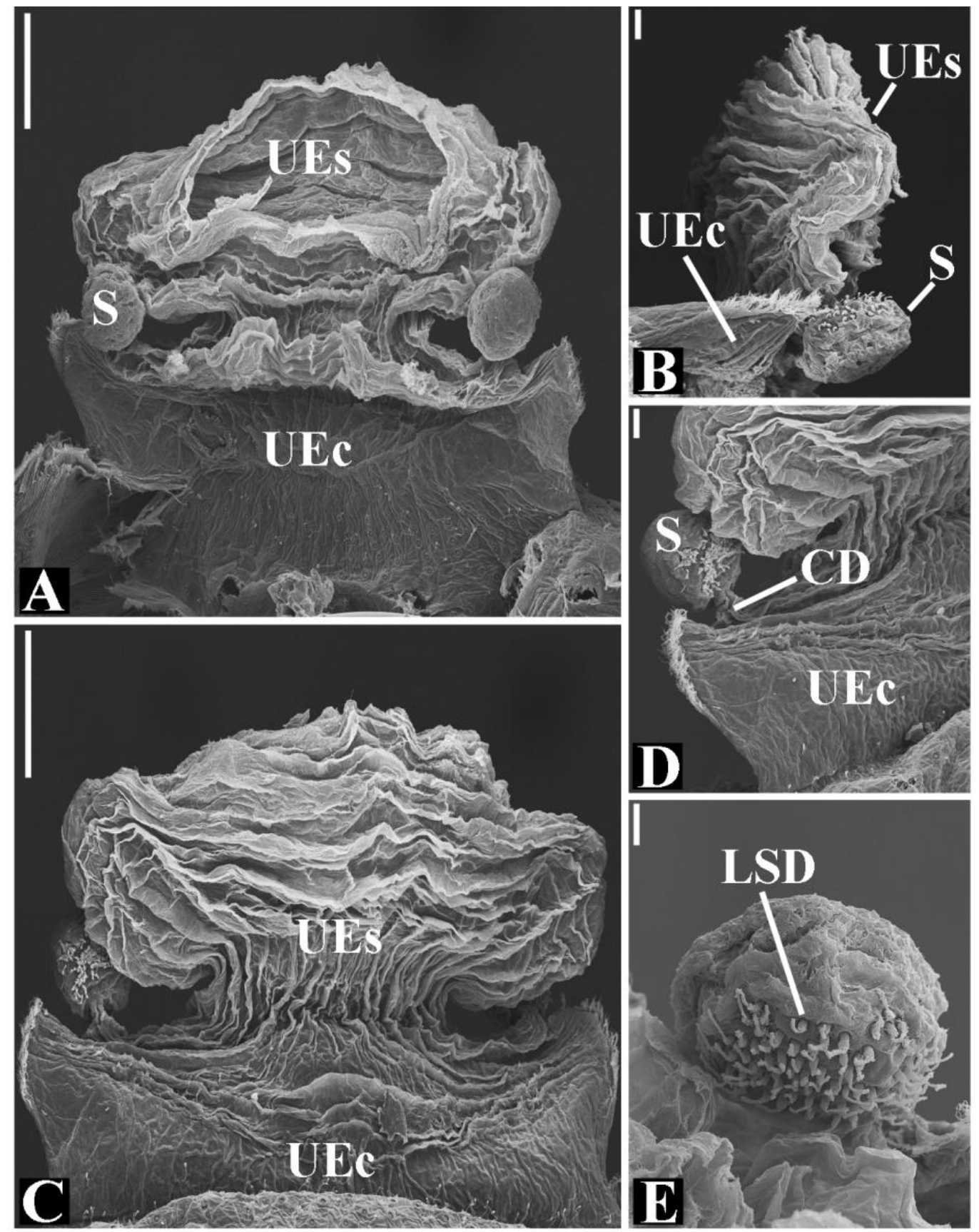

Figure 138. Glenognatha argyrostilba from Ecuador. Female genitalia. A, dorsal. B, lateral. C, ventral. D-E, spermathecae and copulatory duct. Scale bars, $100 \mu \mathrm{m}$ (A, C), $20 \mu \mathrm{m}(\mathrm{B}, \mathrm{D}), 10 \mu \mathrm{m}(\mathrm{E}) . \mathrm{CD}$ : copulatory duct. LSD: long-stem gland ducts. UEc: uterus externus chamber. UEs: uterus externus sac. S: spermathecae. 


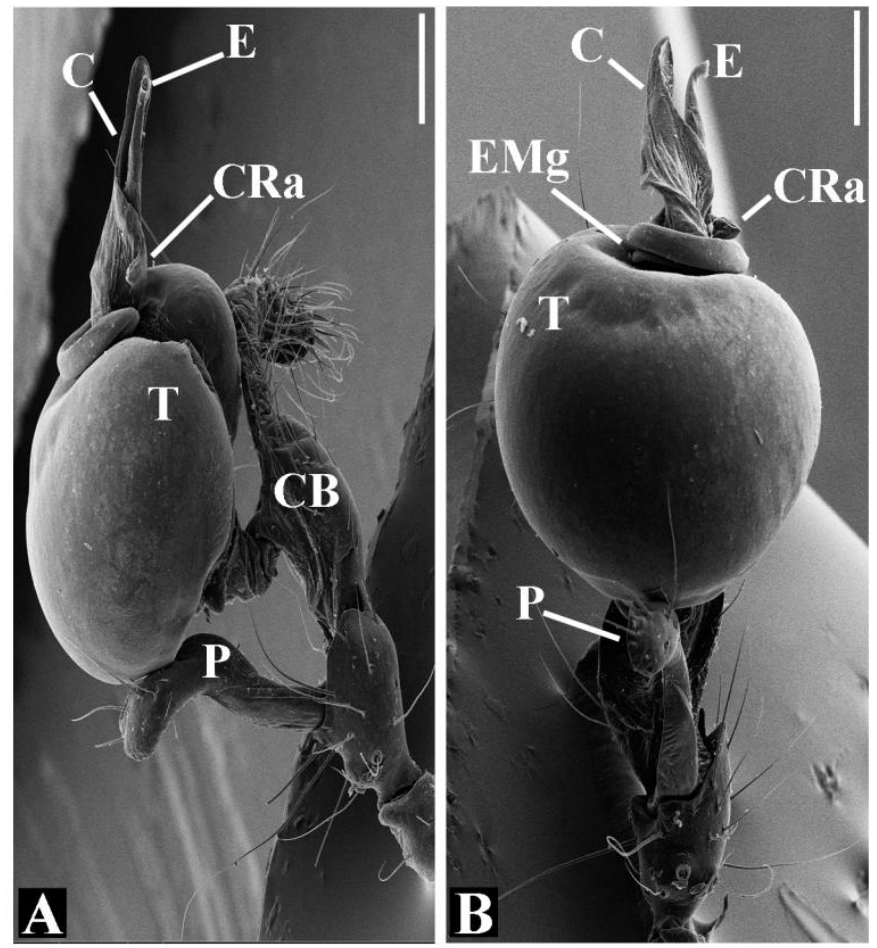

Figure 139. Glenognatha argyrostilba from Ecuador. Male left palp. A. retrolateral. B. ventral. Scale bars $100 \mu \mathrm{m}$. C: conductor. CB: cymbium. CRa: conductor retrolateral apophysis. E: embolus. EMg: embolic medial groove. P: paracymbium. T: tegulum.

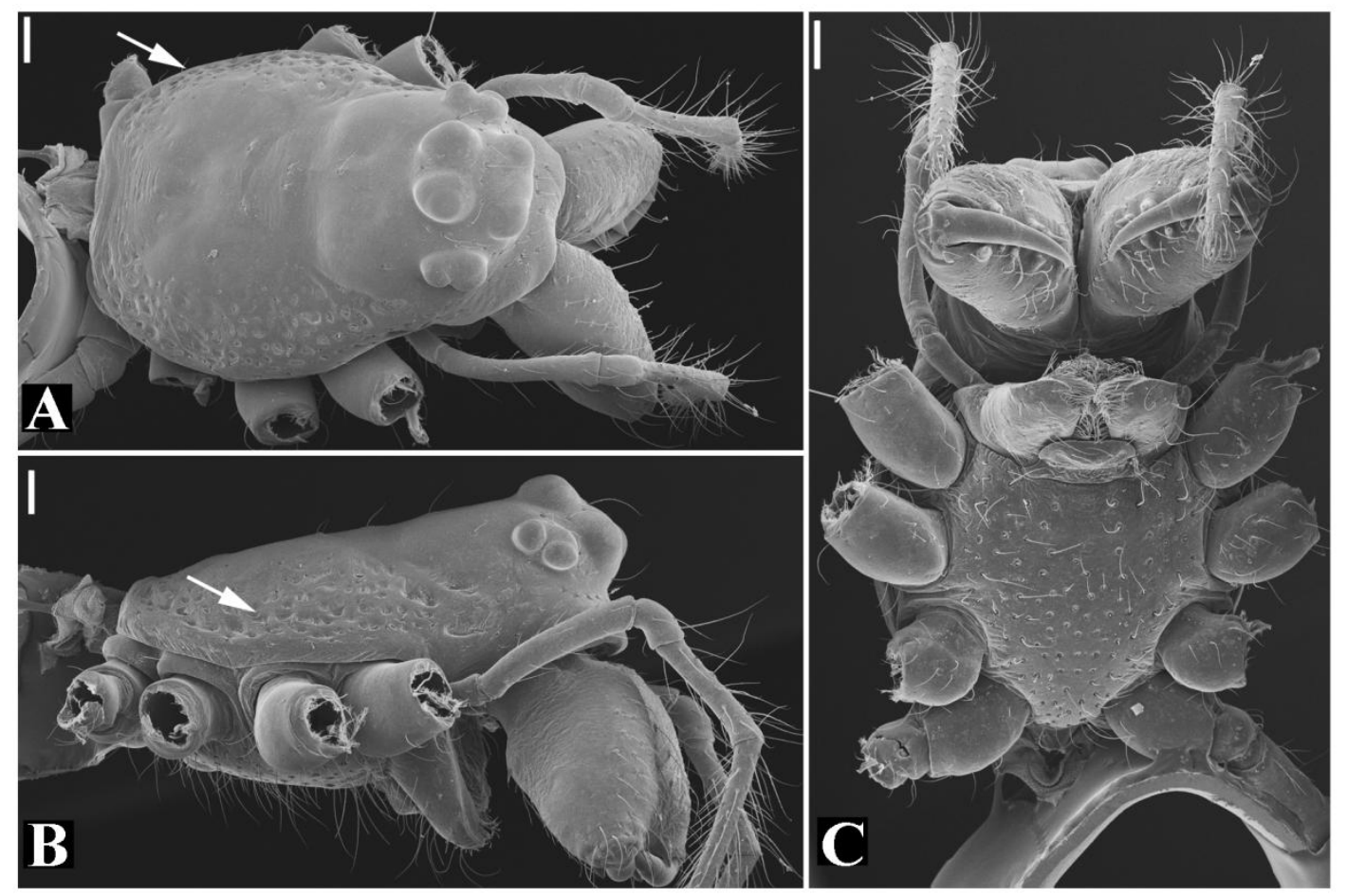

Figure 140. Glenognatha argyrostilba from Ecuador. Female cephalothorax. A, dorsal (arrow, lateral depressions). B, lateral (arrow, lateral depressions). C, ventral. Scale bars, $100 \mu \mathrm{m}$. 

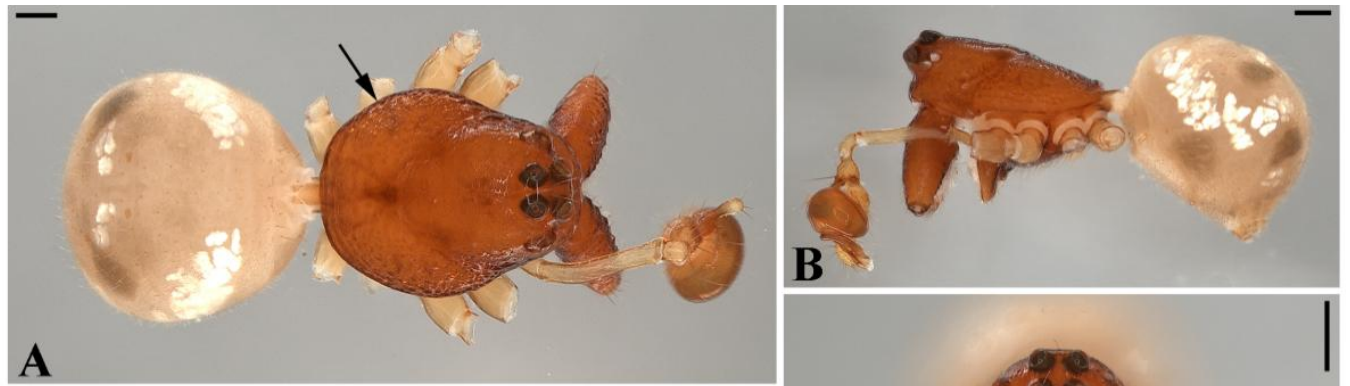

A

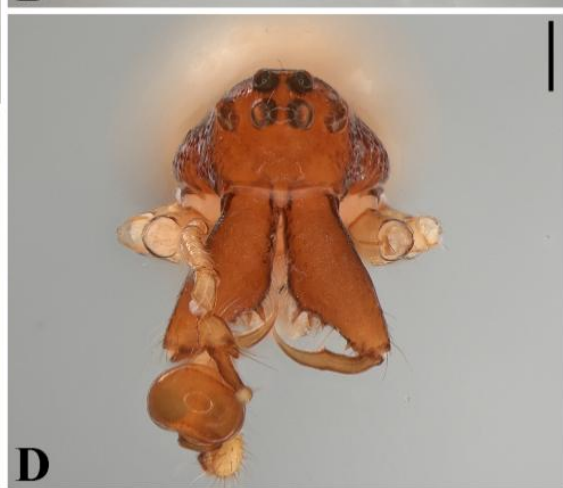

Figure 141. Glenognatha dentata from Myanmar (USNM). Male habitus. A, dorsal (arrow, lateral depressions). B, lateral. C, ventral (arrow, pleural bar). D, frontal. Scale bars, $200 \mu \mathrm{m}$. TS: tracheal spiracle.
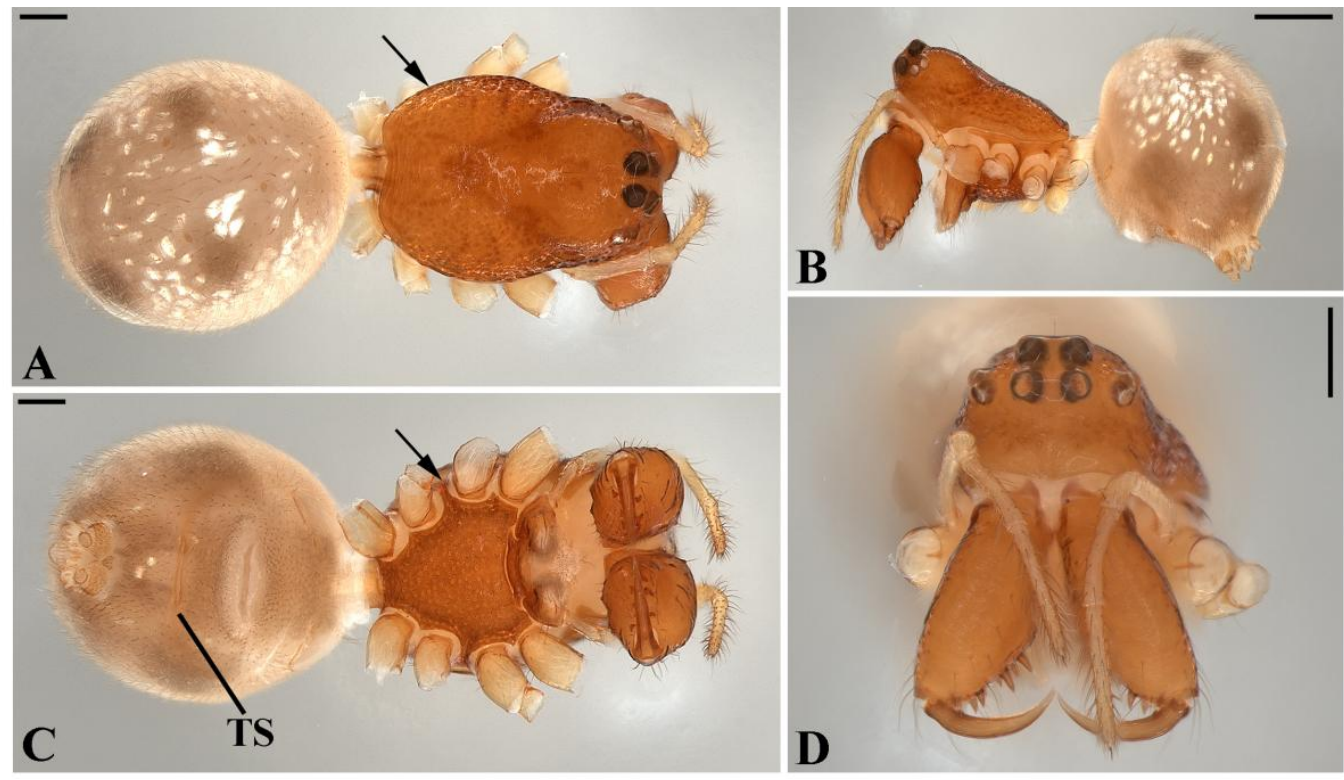

Figure 142. Glenognatha dentata from Myanmar (USNM). Female habitus. A, dorsal (arrow, lateral depressions). B, lateral. C, ventral (arrow, pleural bar). D, frontal. Scale bars, $200 \mu \mathrm{m}$. TS: tracheal spiracle. 


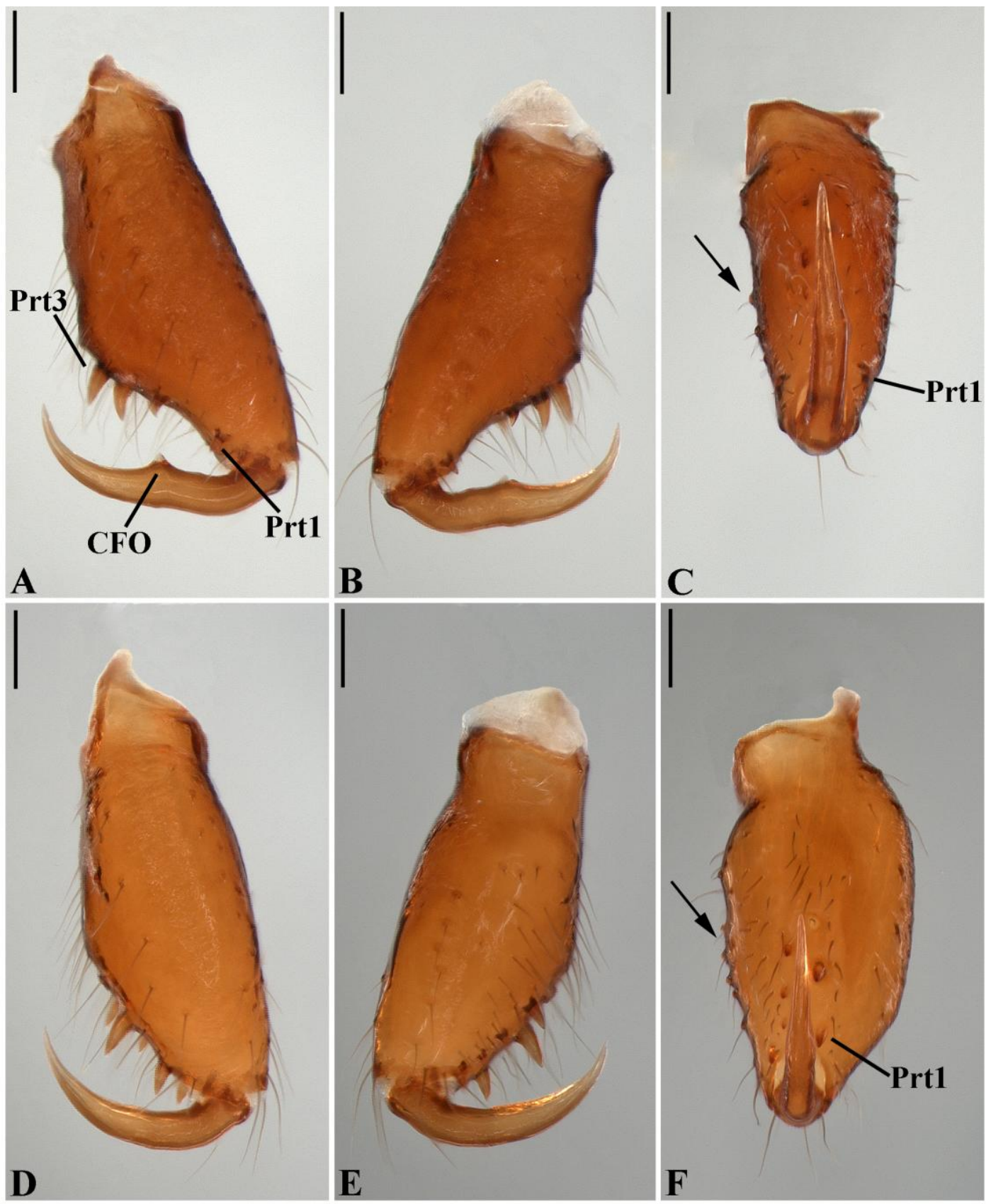

Figure 143. Glenognatha dentata from Myanmar (USNM). A-C, Male left chelicerae. A, anterior. B, posterior. C, mesal (arrow, small tubercles). D-F, Female left chelicerae. D, anterior. E, posterior. F, mesal (arrow, small tubercles). Scale bars, $100 \mu \mathrm{m}$. CFO: cheliceral fang outgrowth. Prt: promarginal tooth. 


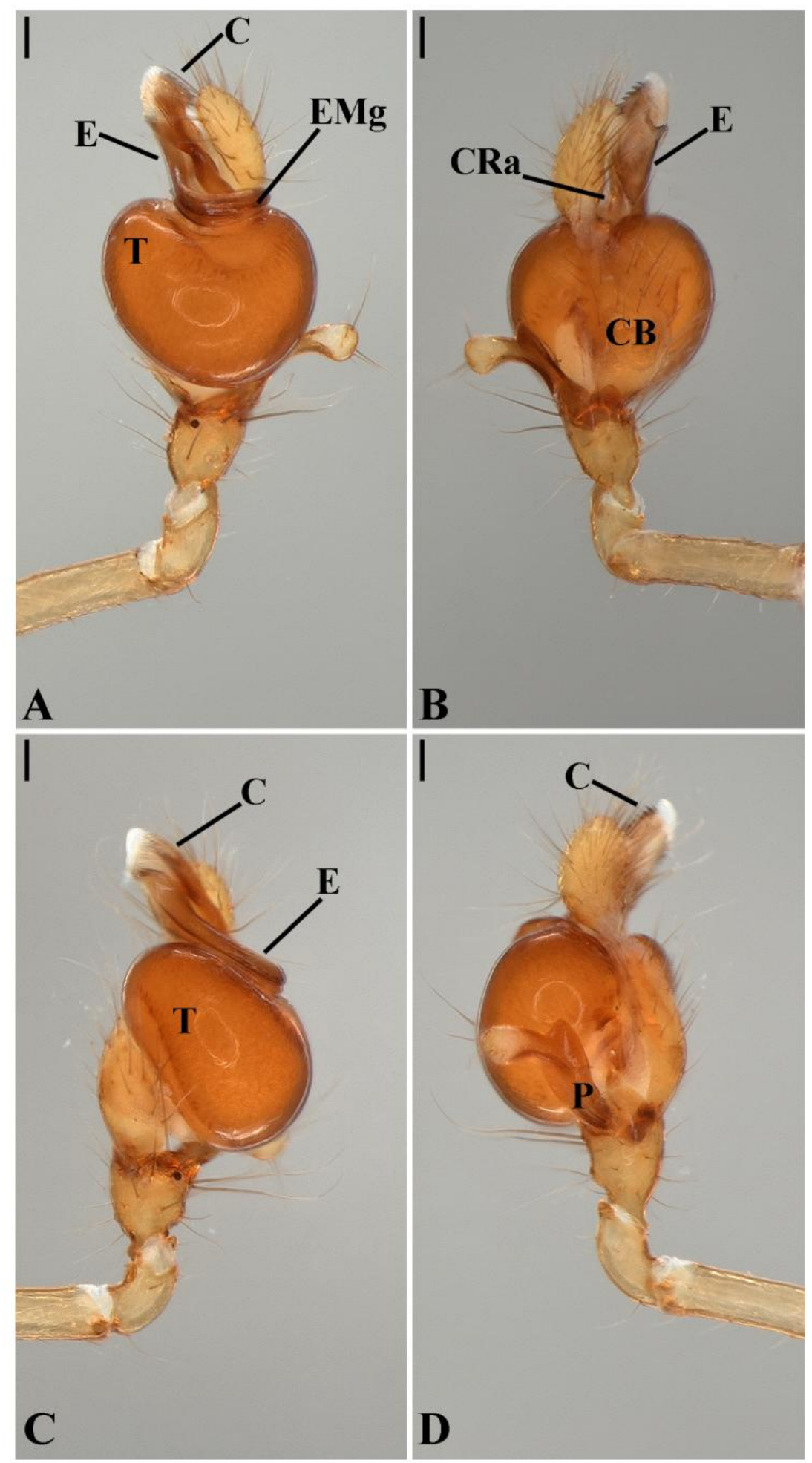

Figure 144. Glenognatha dentata from Myanmar (USNM). Male left palp. A, ventral. B, dorsal. C, prolateral. D, retrolateral. Scale bars, $100 \mu \mathrm{m}$. C: conductor. CB: cymbium. CRa: conductor retrolateral apophysis. E: embolus. EMg: embolic medial groove. P: paracymbium. T: tegulum. 

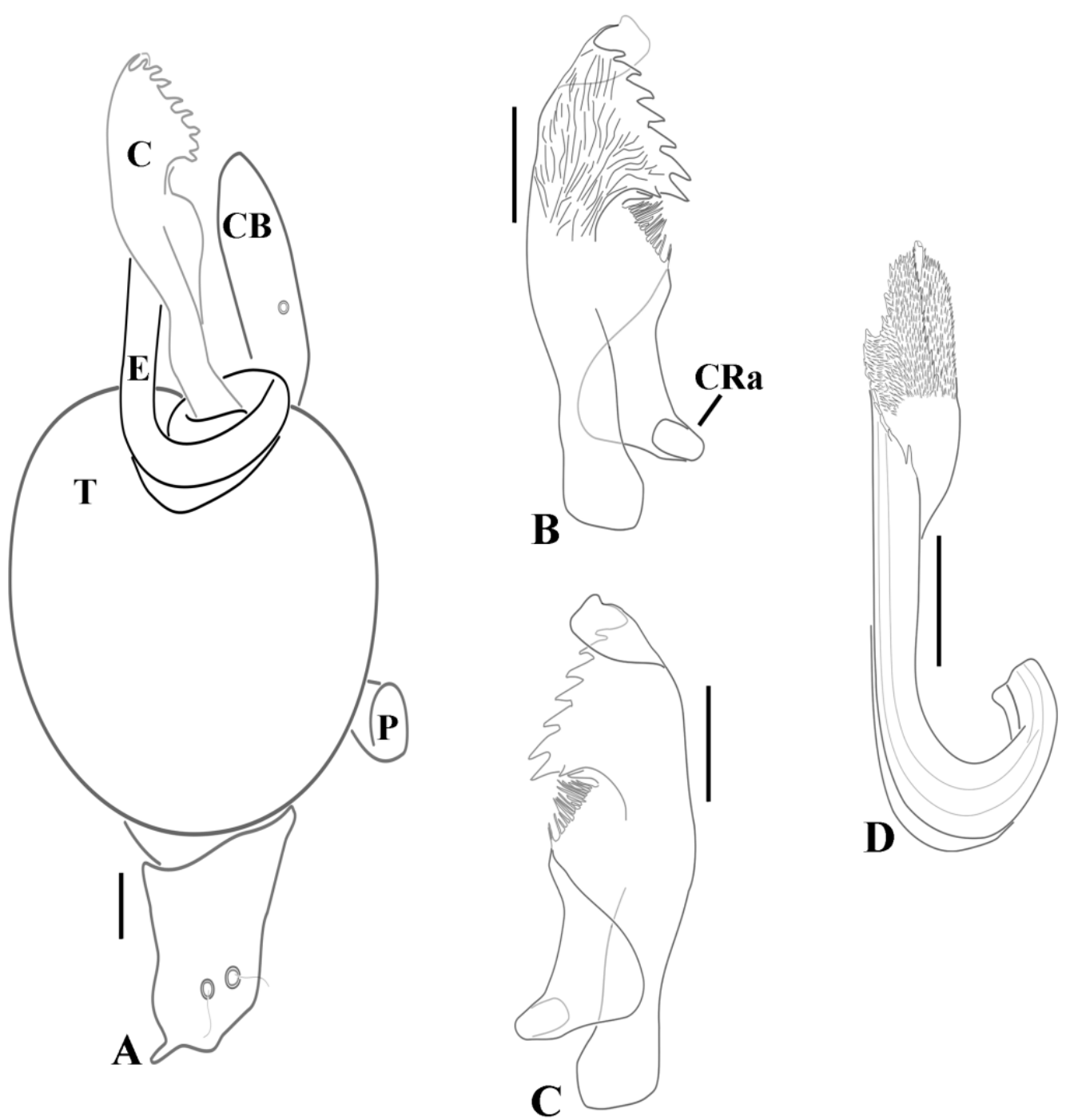

Figure 145. Glenognatha dentata from Vietnam (MCZ 84023). Male left palp, schematic. A, ventral. B, conductor ventral. C, conductor dorsal. D, embolus ventral. Scale bars $100 \mu \mathrm{m}$. C: conductor. CRa: conductor retrolateral apophysis. E: embolus. P: paracymbium. T: tegulum. 

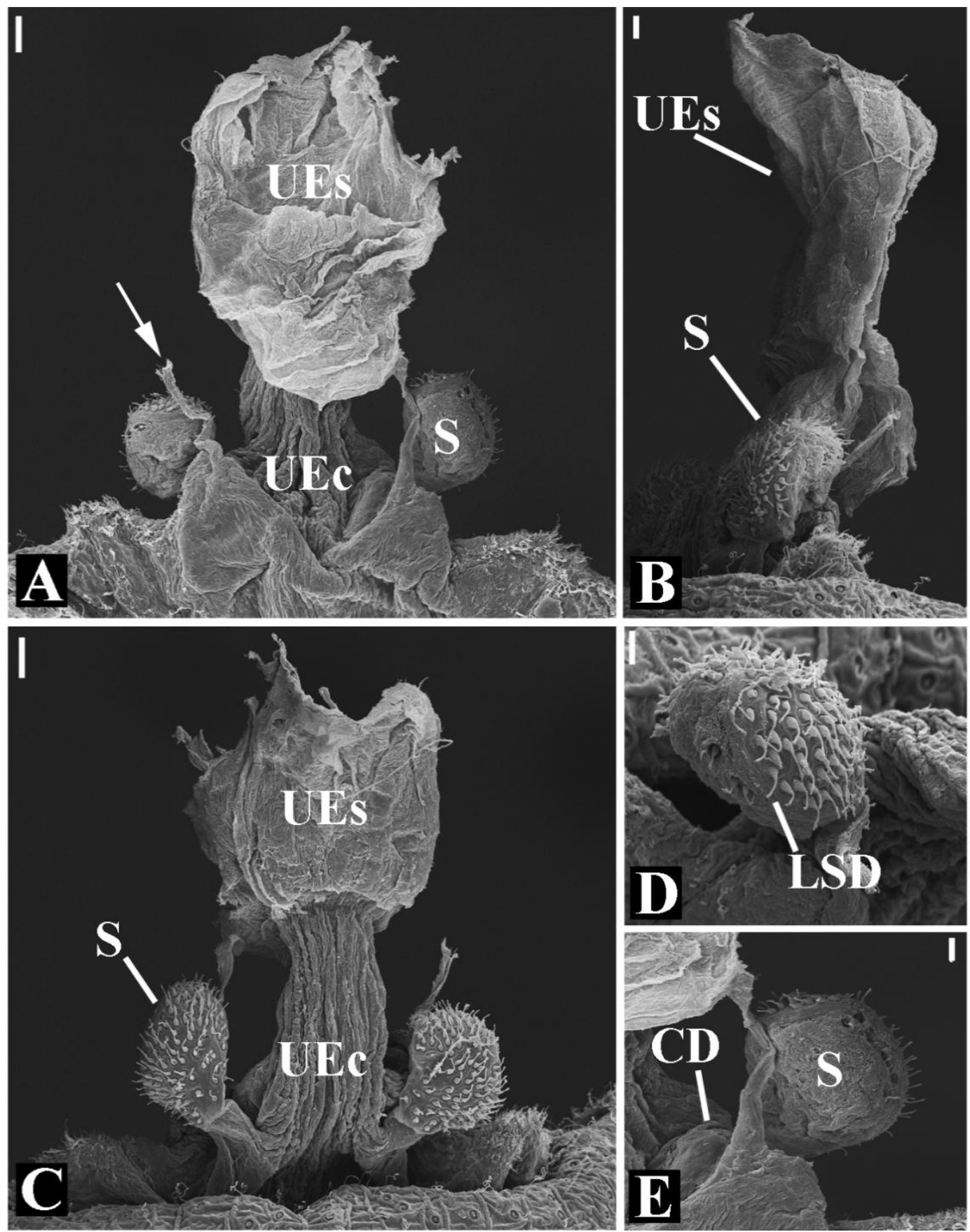

Figure 146. Glenognatha dentata from Myanmar. Female genitalia. A, dorsal (arrow, uterus externus chamber projection). B, lateral. C, ventral. D-E, spermathecae and copulatory duct. Scale bars, $20 \mu \mathrm{m}$ (A, C), $10 \mu \mathrm{m}$ (B), $10 \mu \mathrm{m}$ (D, E). CD: copulatory duct. LSD: long-stem gland ducts. UEc: uterus externus chamber. UEs: uterus externus sac. S: spermathecae. 


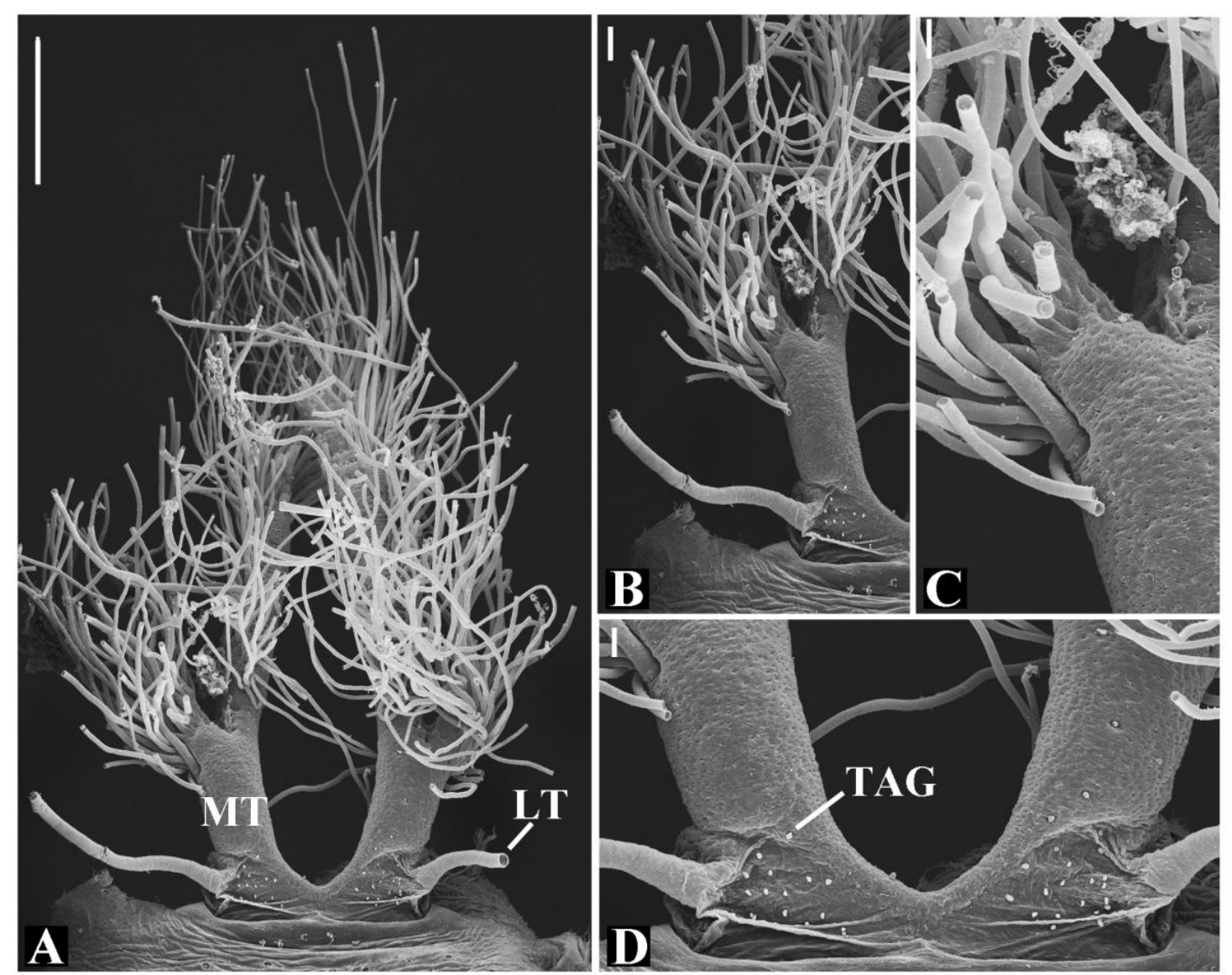

Figure 147. Glenognatha dentata from Myanmar. Female tracheal system. A, dorsal. B, median and lateral trunks. C, median trunk cuticle. D, tracheal spiracle posterior view. Scale bars, $100 \mu \mathrm{m}$ (A), $20 \mu \mathrm{m}$ (B), $10 \mu \mathrm{m}$ (C, D). LT: lateral trachea. MT: median trunks. TAG: tracheal atrium gland. 

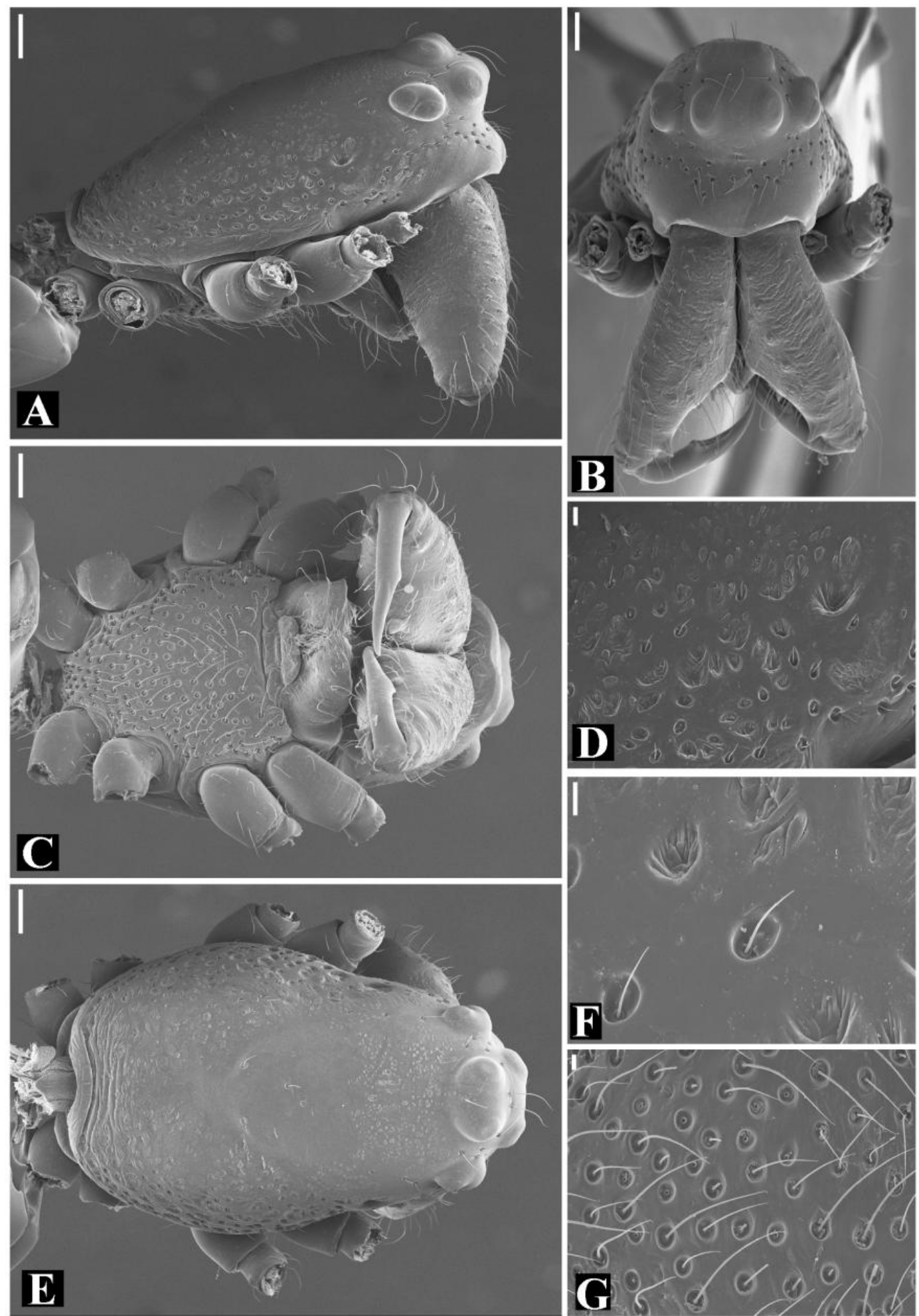

Figure 148. Glenognatha dentata from Myanmar. Female cephalothorax. A, lateral. B, frontal. C, ventral. E, dorsal. D, F, carapace lateral cuticle. G, sternum cuticle. Scale bars $100 \mu \mathrm{m}$ (A-C, E), $20 \mu \mathrm{m}$ (D), $10 \mu \mathrm{m}$ (F, G). 


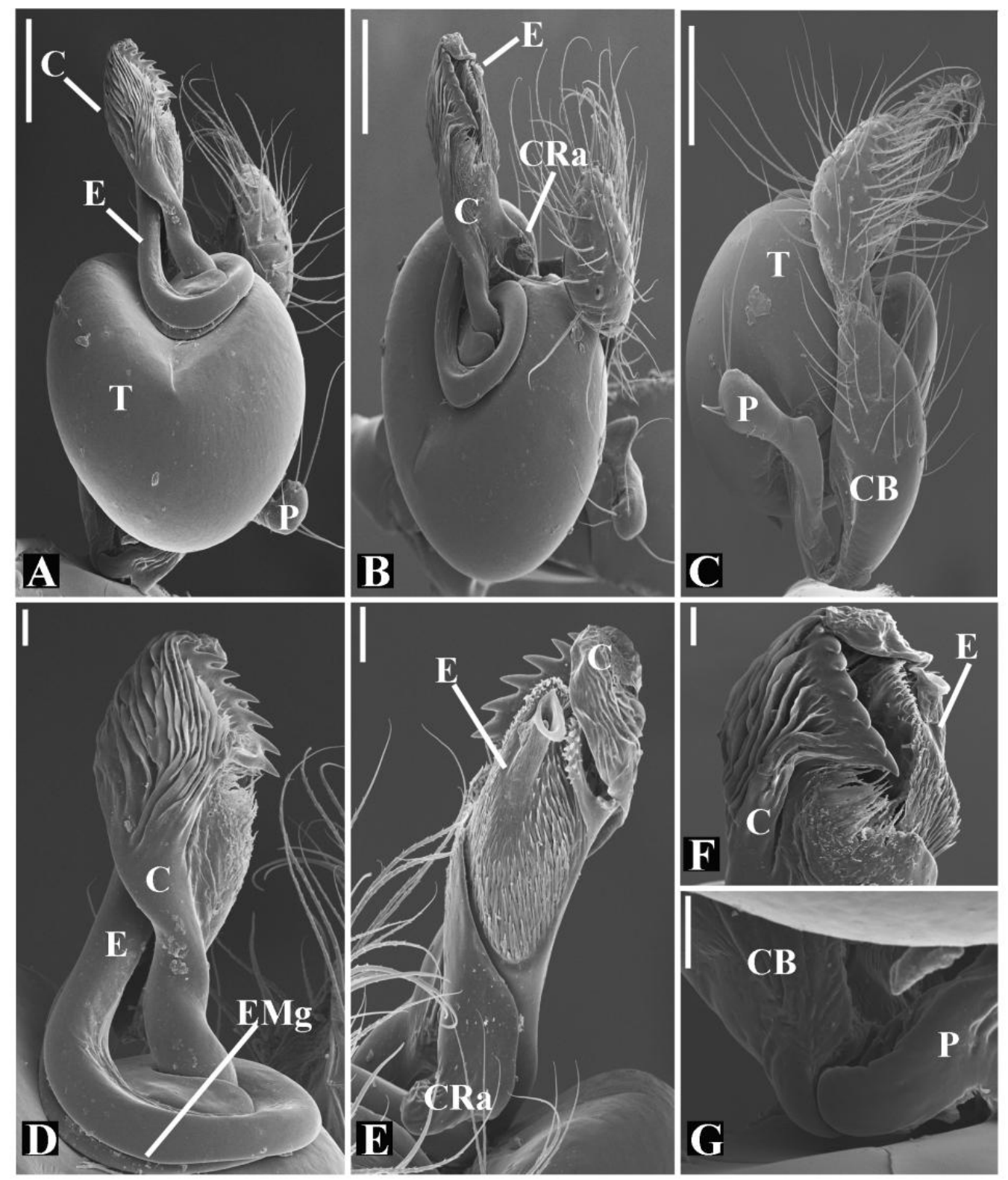

Figure 149. Glenognatha dentata from Myanmar. Male left palp. . A, ventral. B, ventroretorlateral. C, dorsoretrolateral. D, embolus and conductor ventral. E, embolus and conductor dorsal, F, embolus and conductor apical. G, cymbium/paracymbium articulation. Scale bars, $100 \mu \mathrm{m}$ (A-C), $20 \mu \mathrm{m}$ (D-G). C: conductor. CB: cymbium. CRa: conductor retrolateral apophysis. E: embolus. EMg: embolic medial groove. P: paracymbium. T: tegulum. 

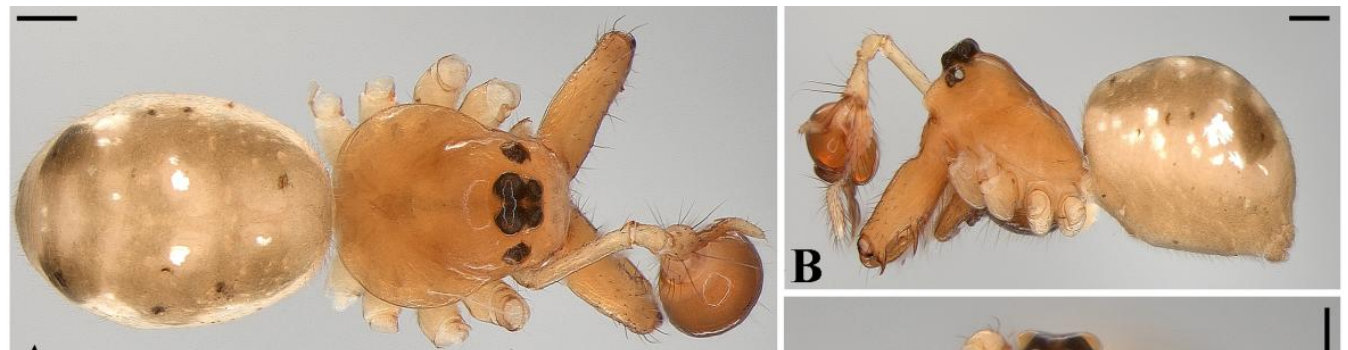

A
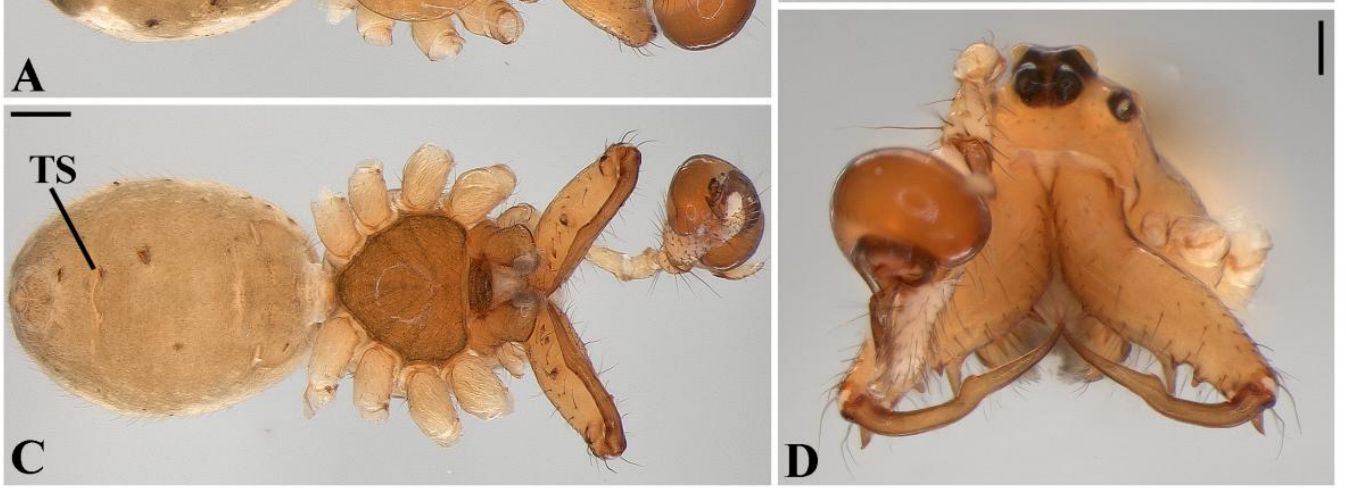

Figure 150. Glenognatha sp. nov. 2 from Colombia (ICN 5982). Male habitus. A, dorsal. B, lateral. C, ventral. D, frontal. Scale bars, $200 \mu \mathrm{m}$. TS: tracheal spiracle.
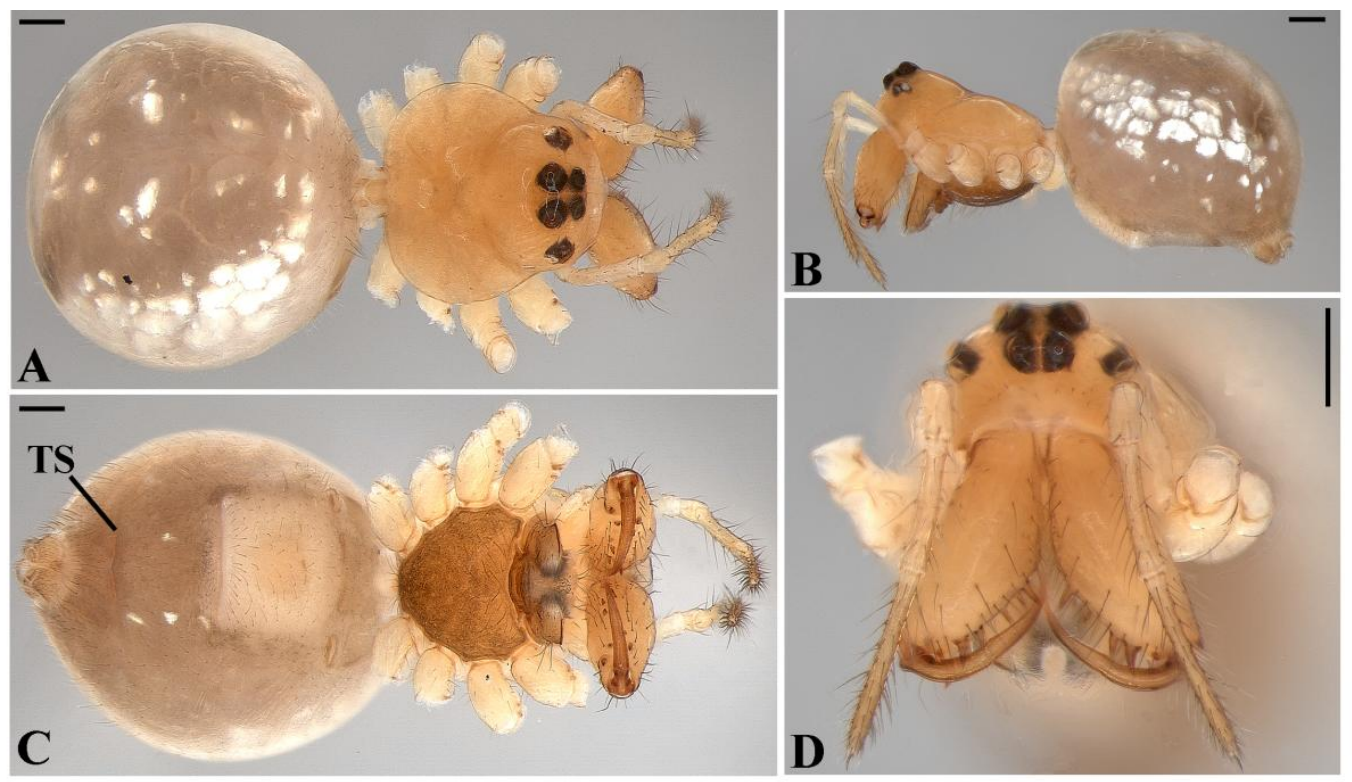

Figure 151. Glenognatha sp. nov. 2 from Colombia (ICN 5982). Female habitus. A, dorsal. B, lateral. C, ventral. D, frontal. Scale bars, $200 \mu \mathrm{m}$. TS: tracheal spiracle. 

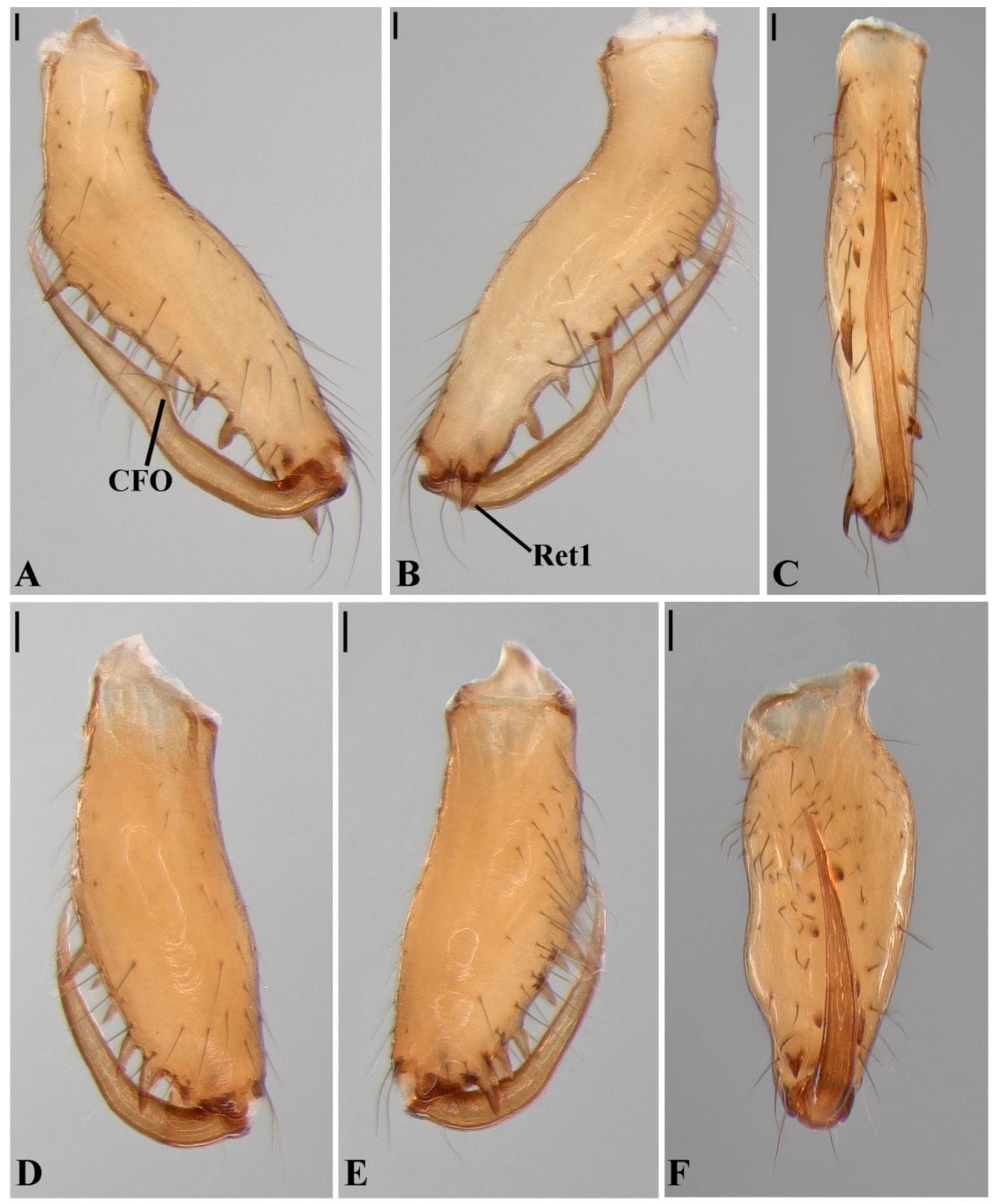

Figure 152. Glenognatha sp. nov. 2 from Colombia (ICN 5982). A-C, Male left chelicerae. A, anterior. B, posterior. C, mesal. D-F, Female left chelicerae. D, anterior. E, posterior. F, mesal. Scale bars, $100 \mu \mathrm{m}$. CFO: cheliceral fang outgrowth. Ret: retromarginal tooth. 

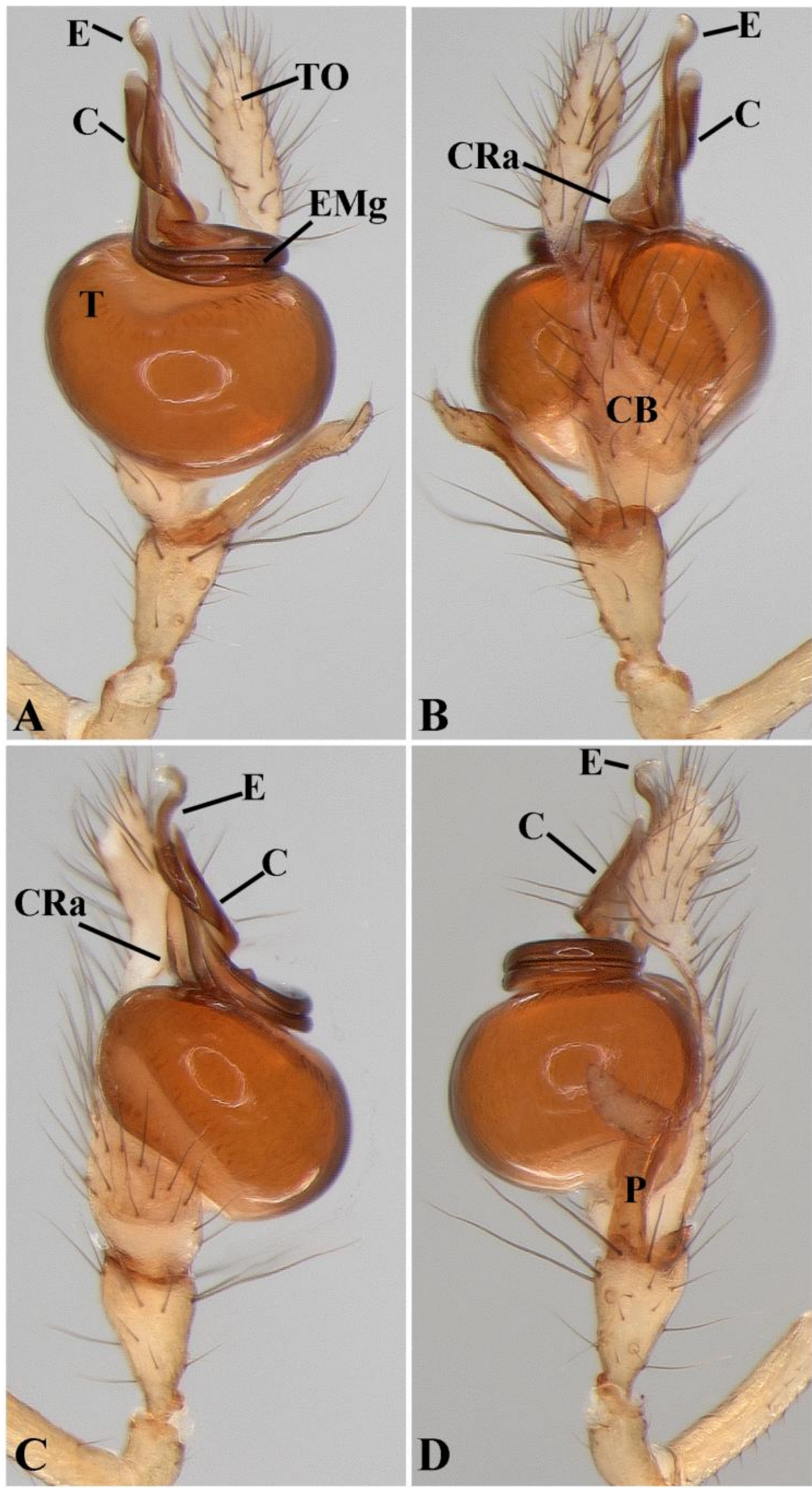

Figure 153. Glenognatha sp. nov. 2 from Colombia (ICN 5982). Male left palp. A, ventral. B, dorsal. C, prolateral. D, retrolateral. Scale bars, $100 \mu \mathrm{m}$. C: conductor. CB: cymbium. CRa: conductor retrolateral apophysis. E: embolus. EMg: embolic medial groove. P: paracymbium. T: tegulum. TO: tarsal organ. 

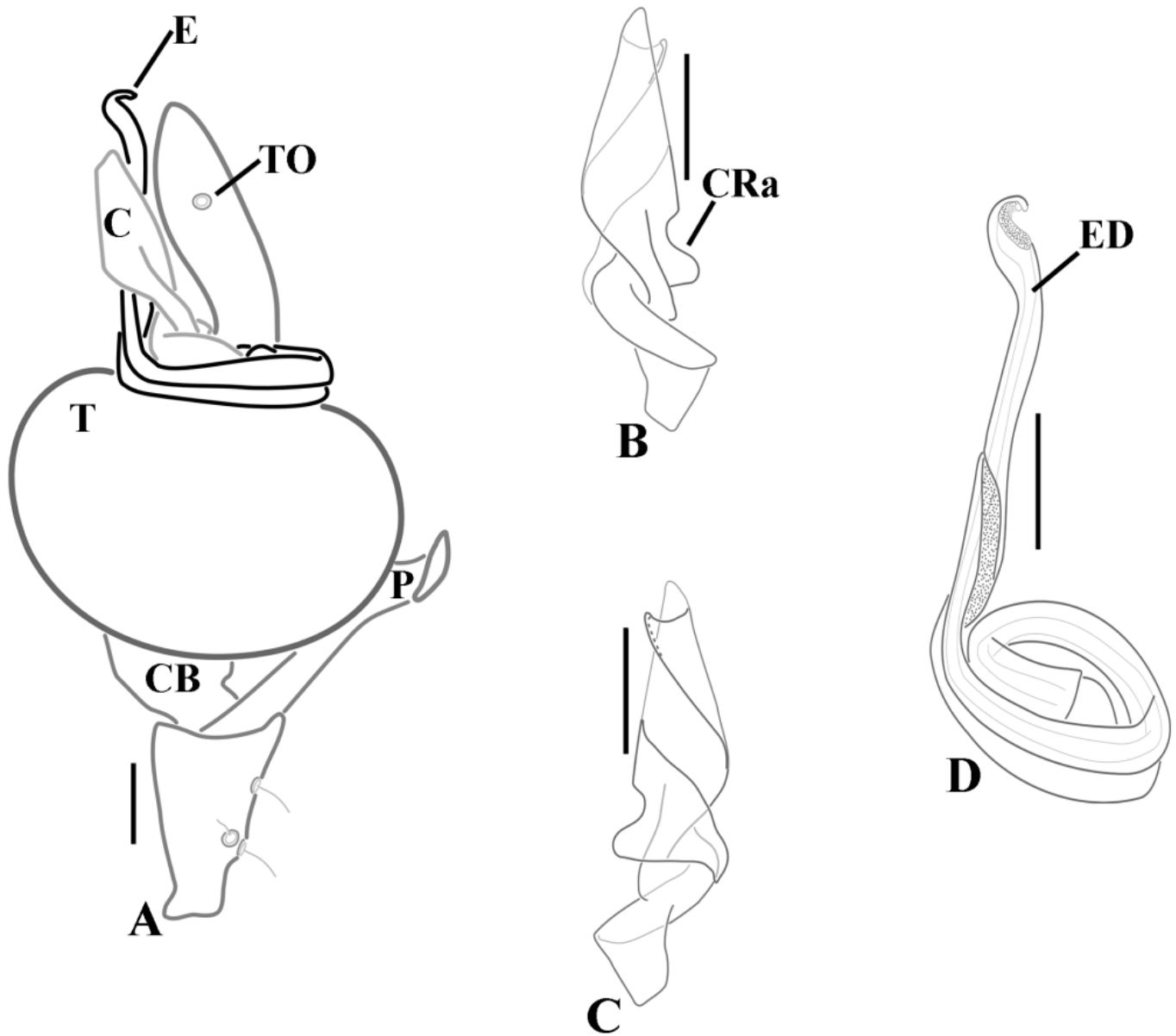

Figure 154. Glenognatha sp. nov. 2 from Colombia (ICN 5982). Male left palp, schematic. A, ventral. B, conductor ventral. C, conductor dorsal. D, embolus ventral. Scale bars $100 \mu \mathrm{m}$. C: conductor. CB: cymbium. CRa: conductor retrolateral apophysis. E: embolus. ED: ejaculatory duct. P: paracymbium. T: tegulum. TO: tarsal organ. 


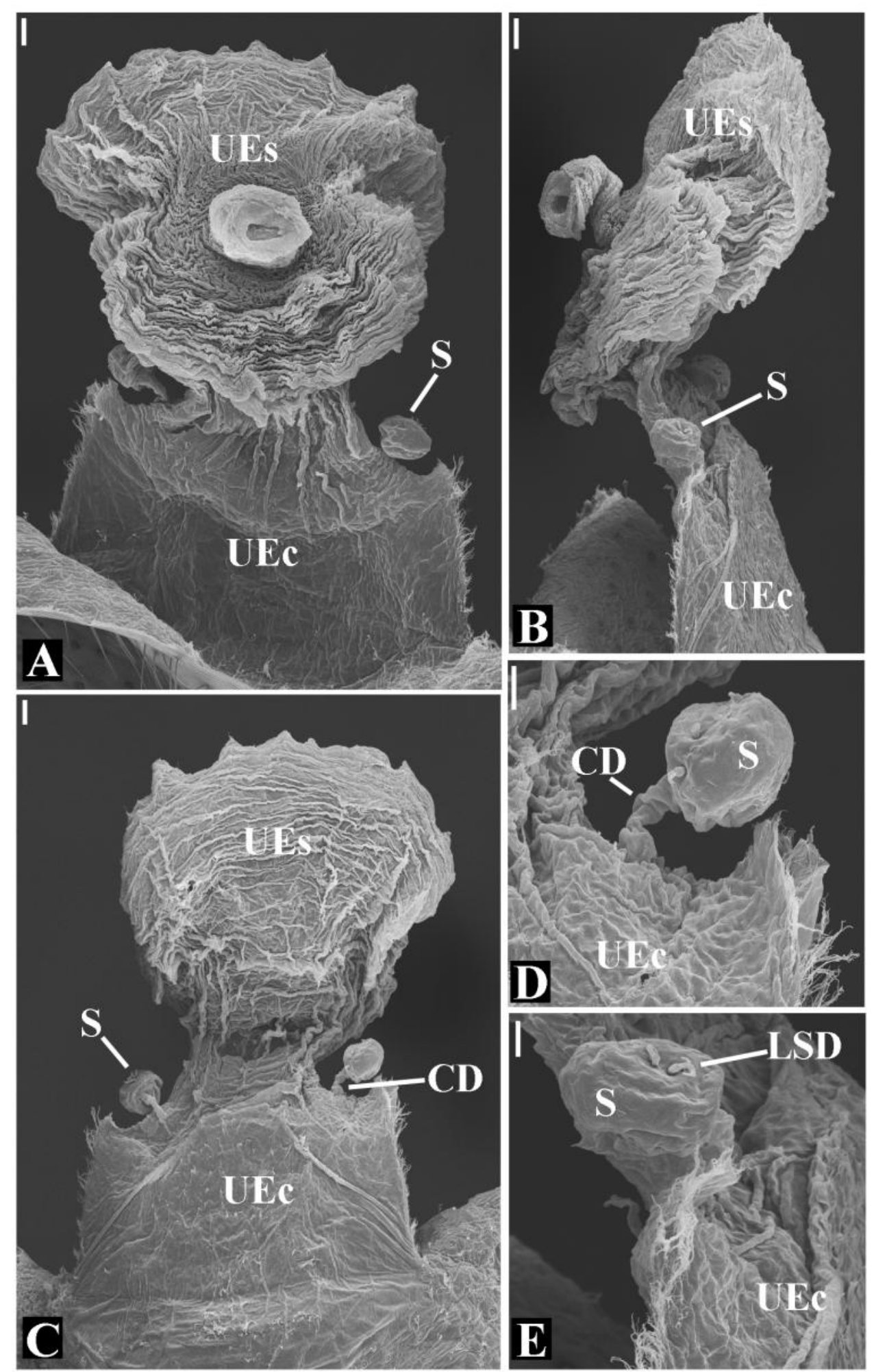

Figure 155. Glenognatha sp. nov. 2 from Colombia. Female genitalia. A, dorsal. B, lateral. C, ventral D-E, spermathecae and copulatory duct. Scale bars, $20 \mu \mathrm{m}$ (A-C), $10 \mu \mathrm{m}(\mathrm{D}, \mathrm{E})$. CD: copulatory duct. LSD: long-stem gland ducts. UEc: uterus externus chamber. UEs: uterus externus sac. S: spermathecae. 


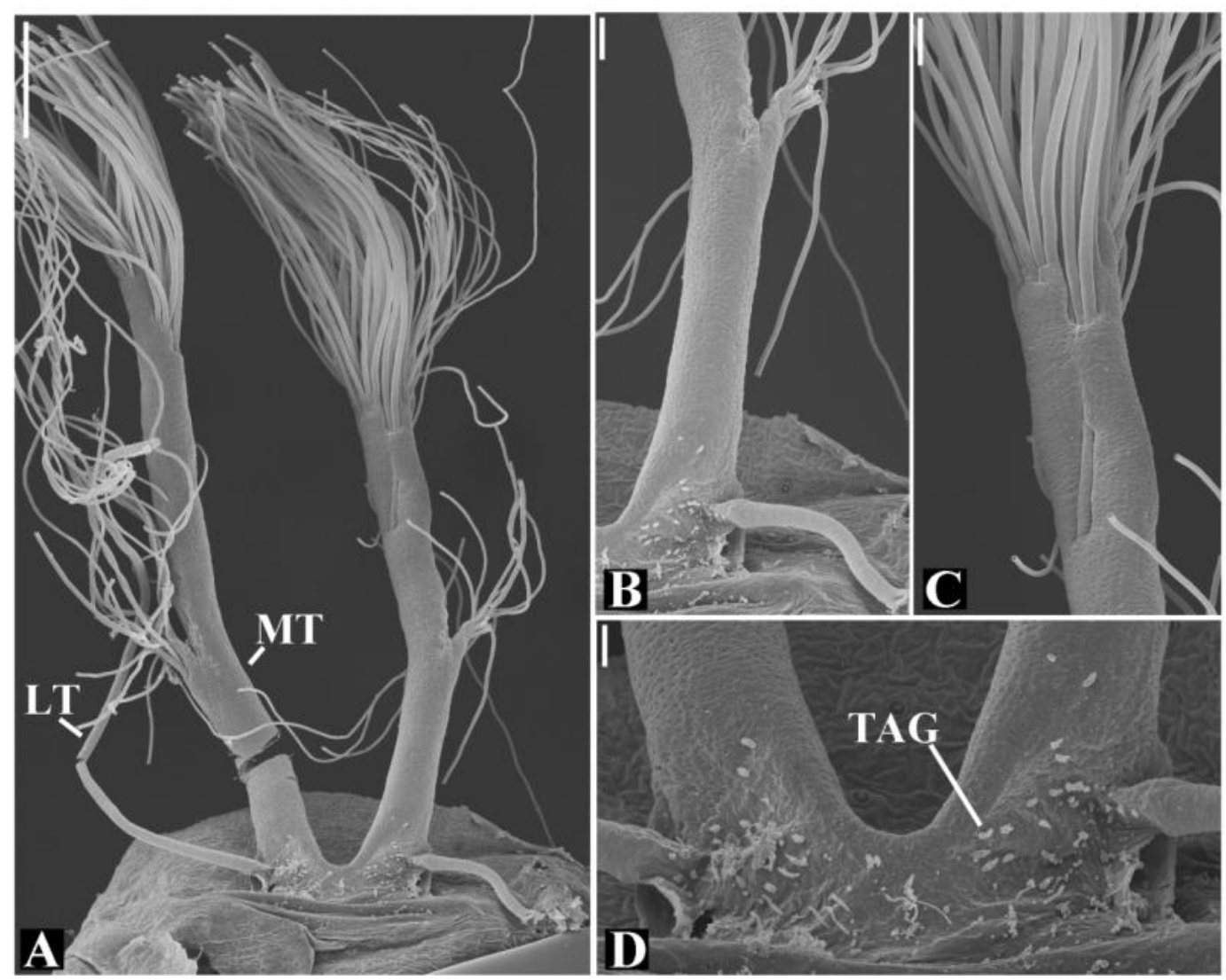

Figure 156. Glenognatha sp. nov. 2 from Colombia. Female tracheal system. A, dorsal. $\mathbf{B}$, median and lateral trunks. $\mathbf{C}$, median trunk distal. D, tracheal spiracle posterior view. Scale bars, $100 \mu \mathrm{m}$ (A), $20 \mu \mathrm{m}$ (B-D). LT: lateral trachea. MT: median trunks. TAG: tracheal atrium gland. 


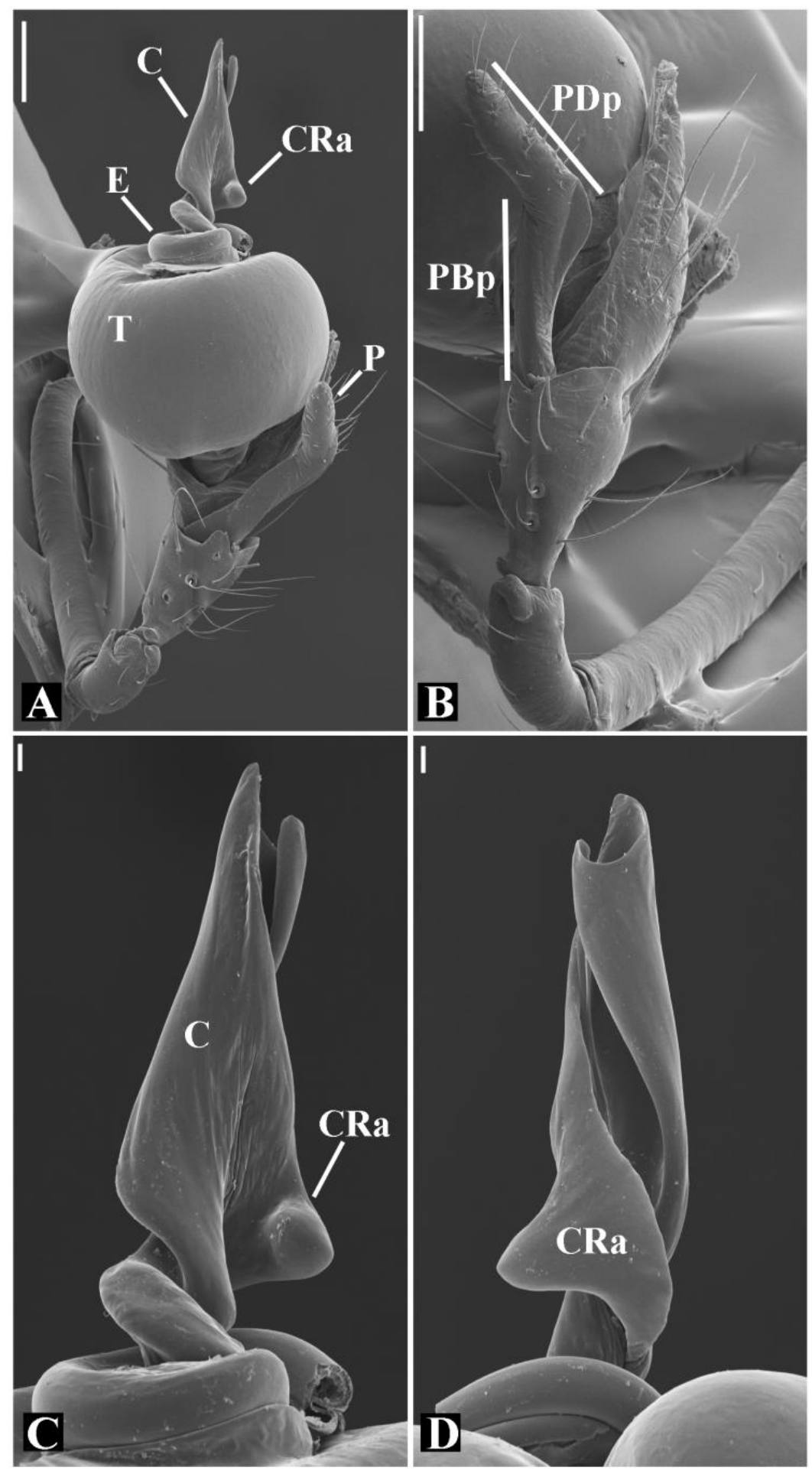

Figure 157. Glenognatha sp. nov. 2 from Colombia. Male left palp. A, ventral. B, retrolateral. C, conductor ventral. D, conductor dorsal. Scale bars $100 \mu \mathrm{m}(\mathrm{A}, \mathrm{B}) 10 \mu \mathrm{m}$ (C, D). C: conductor. CRa: conductor retrolateral apophysis. E: embolus. P: paracymbium. PBp: paracymbium basal portion. PDp: paracymbium distal portion. T: tegulum. 


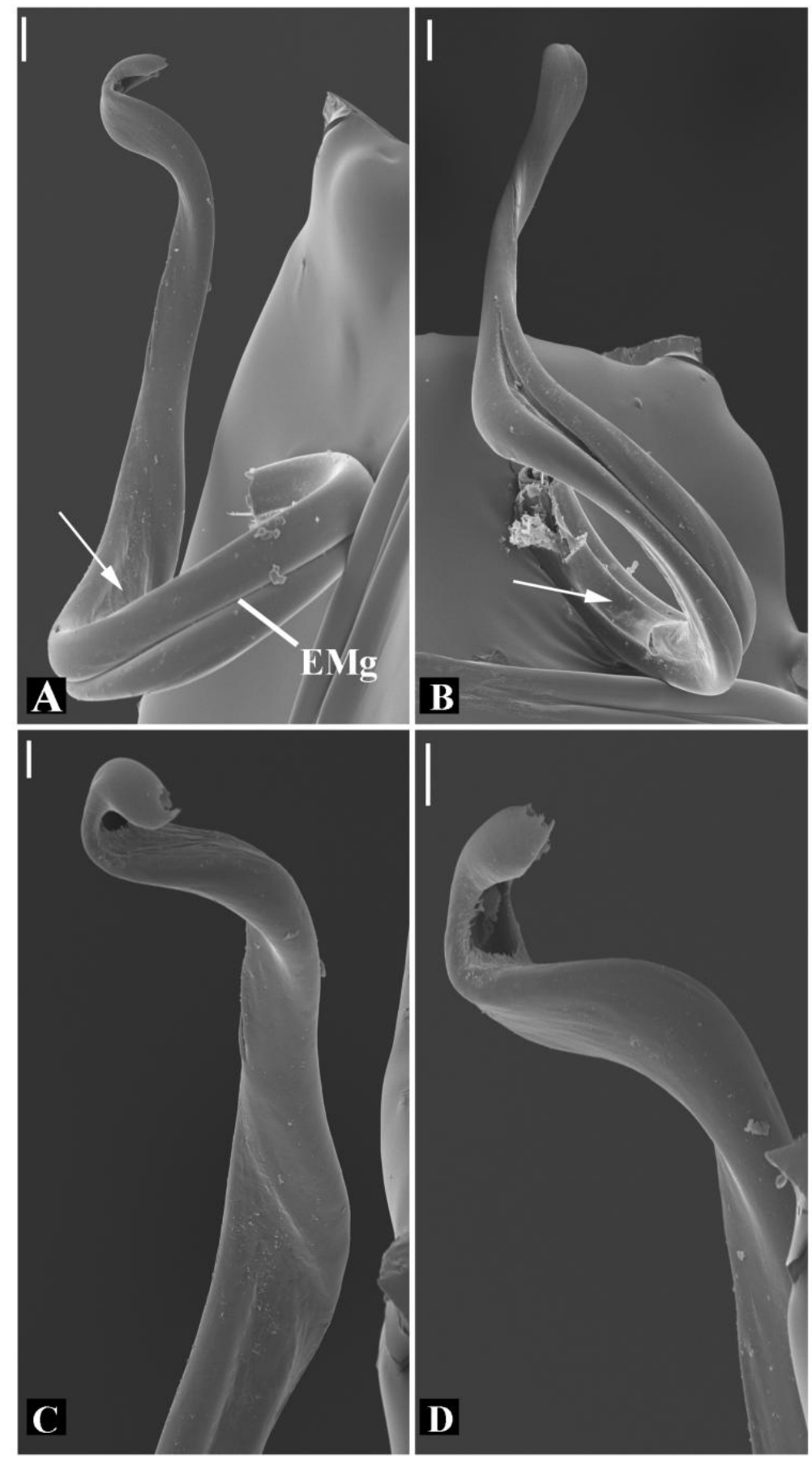

Figure 158. Glenognatha sp. nov. 2 from Colombia. Embolus. A, ventral (arrow, inner membrane). B, dorsoprolateral (arrow, inner membrane). C-D, distal. Scale bars $20 \mu \mathrm{m}$ (A,B), $10 \mu \mathrm{m}(\mathrm{C}, \mathrm{D})$. 


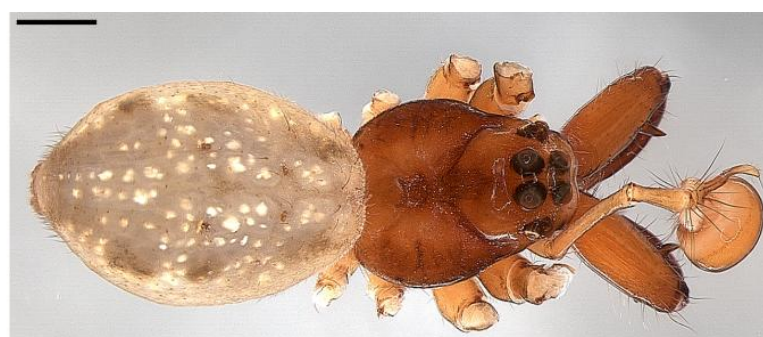

A

C

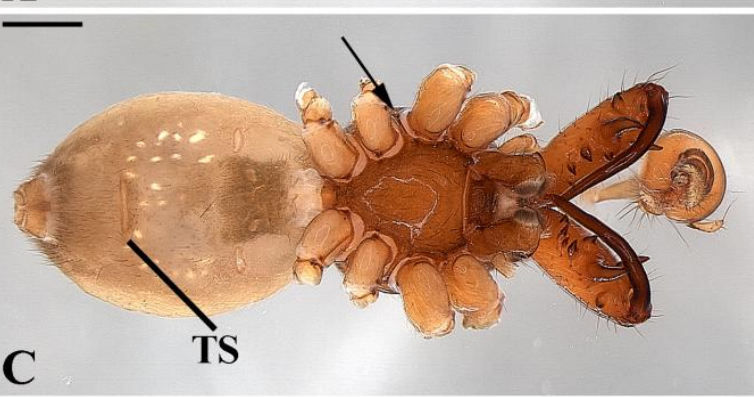

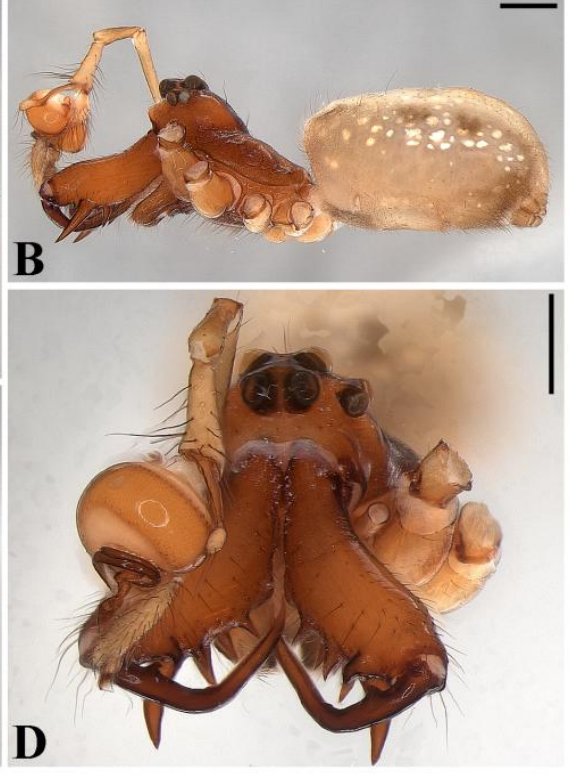

D

Figure 159. Glenognatha gaujoni from Brazil (IBSP 26965). Male habitus. A, dorsal. B, lateral. C, ventral (arrow, pleural bar). D, frontal. Scale bars, $500 \mu \mathrm{m}$. TS: tracheal spiracle.
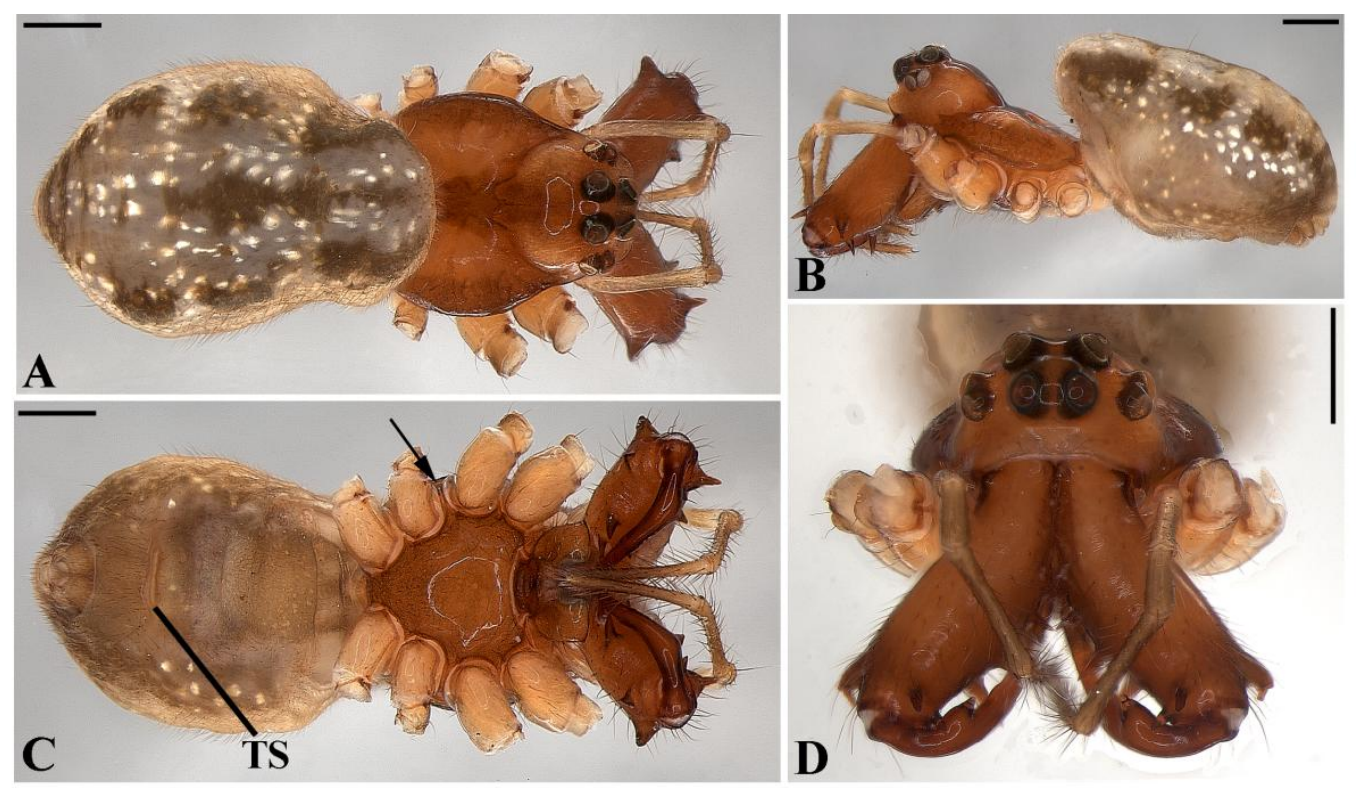

Figure 160. Glenognatha gaujoni from Brazil (IBSP 55545). Female habitus. A, dorsal.

B, lateral. C, ventral (arrow, pleural bar). D, frontal. Scale bars, $500 \mu \mathrm{m}$. TS: tracheal spiracle. 


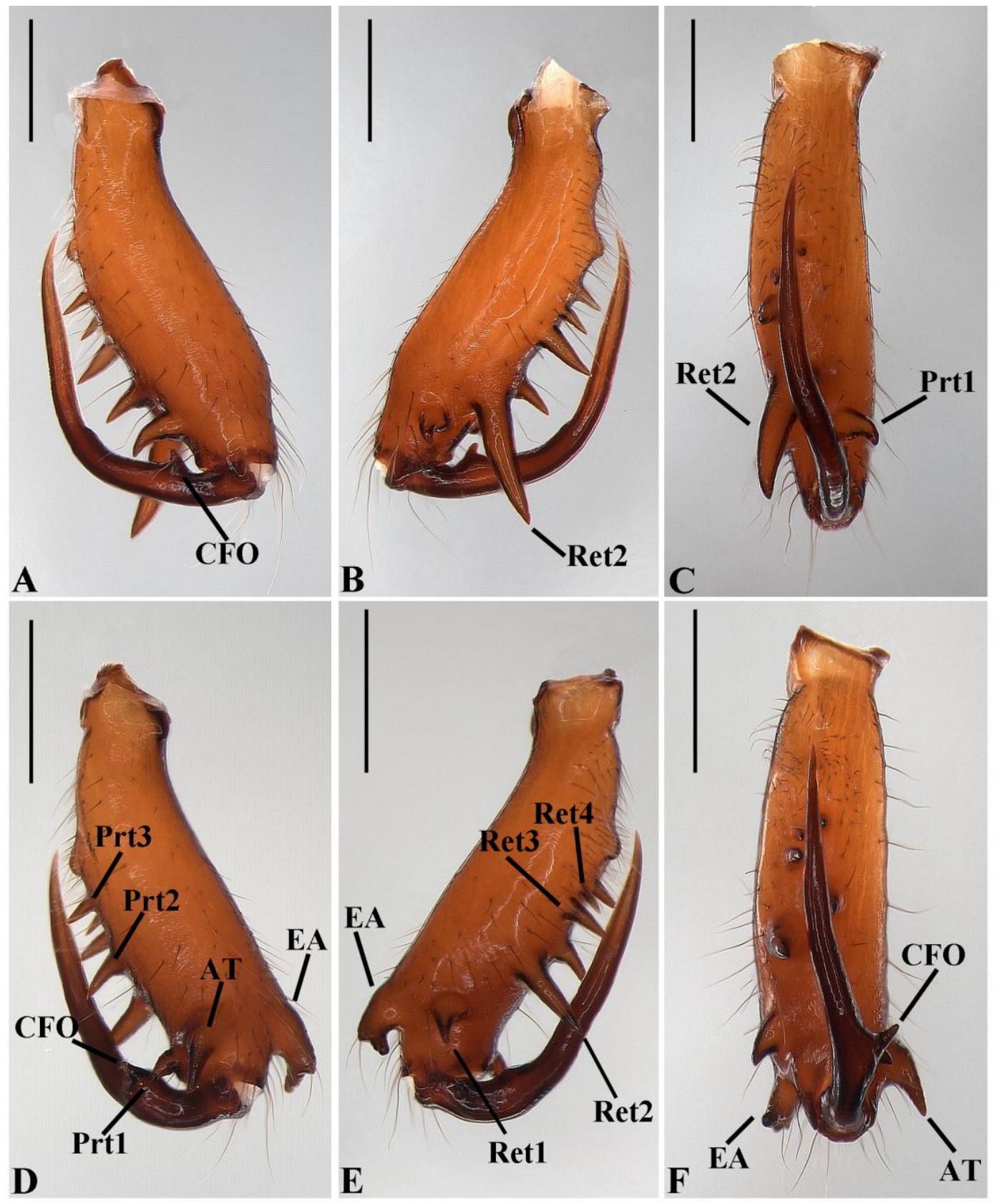

Figure 161. Glenognatha gaujoni from Brazil. A-C, Male left chelicerae (IBSP 12182). A, anterior. B, posterior. C, mesal. D-F, Female left chelicerae (IBSP 12612). D, anterior. E, posterior. F, mesal. Scale bars, $500 \mu \mathrm{m}$. AT: anterior tooth. CFO: cheliceral fang outgrowth. EA: ectal apophysis. Prt: promarginal tooth. Ret: retromarginal tooth. 

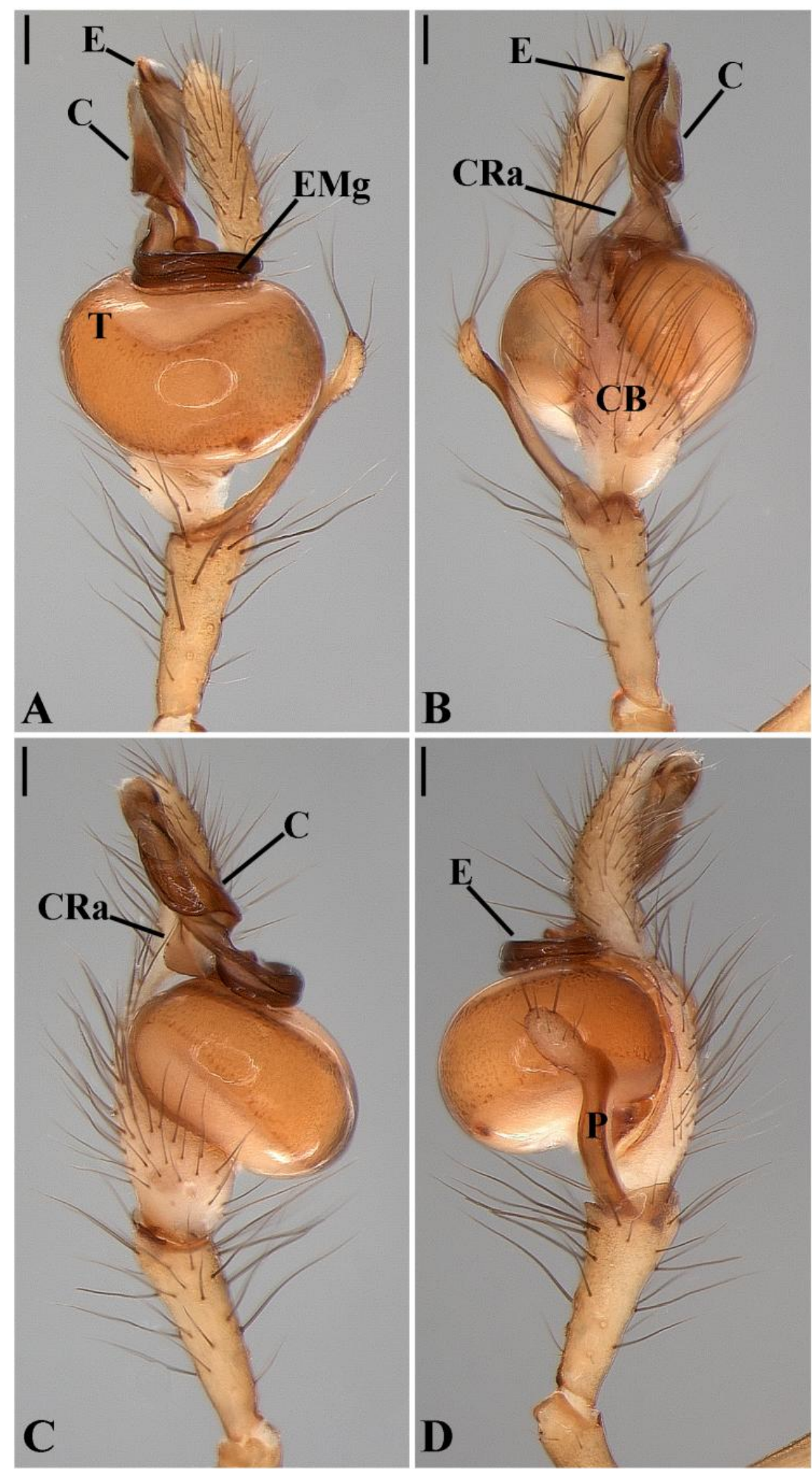

Figure 162. Glenognatha gaujoni from Brazil (IBSP 26965). Male left palp. A, ventral. B, dorsal. C, prolateral. D, retrolateral. Scale bars, $100 \mu \mathrm{m}$. C: conductor. CB: cymbium. CRa: conductor retrolateral apophysis. E: embolus. EMg: embolic medial groove. P: paracymbium. T: tegulum. 

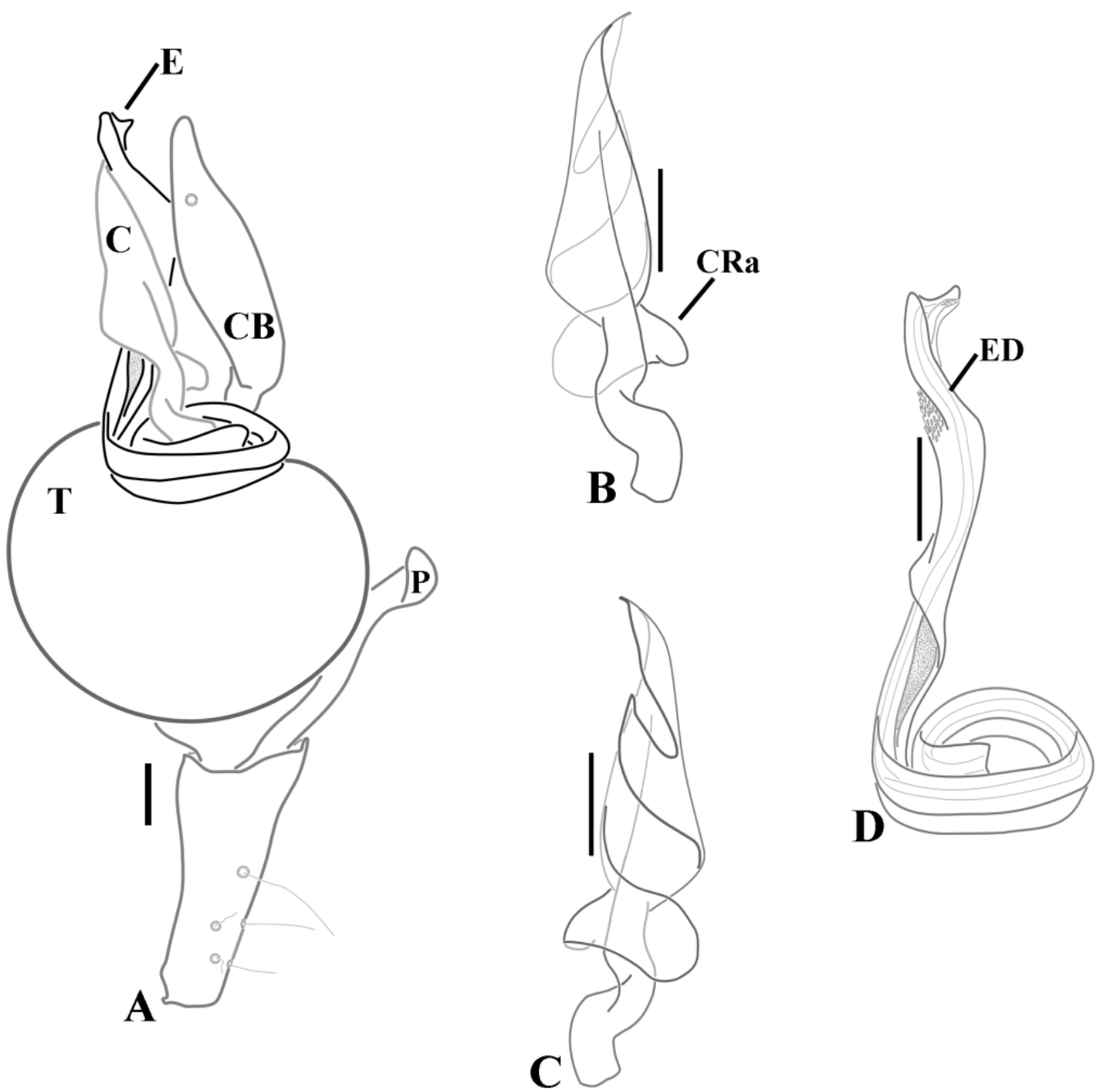

Figure 163. Glenognatha gaujoni from Peru (MUSM 0504920). Male left palp, schematic. A, ventral. B, conductor ventral. C, conductor dorsal. D, embolus ventral. Scale bars $100 \mu \mathrm{m}$. C: conductor. CB: cymbium. CRa: conductor retrolateral apophysis. E: embolus. ED: ejaculatory duct. P: paracymbium. T: tegulum. 


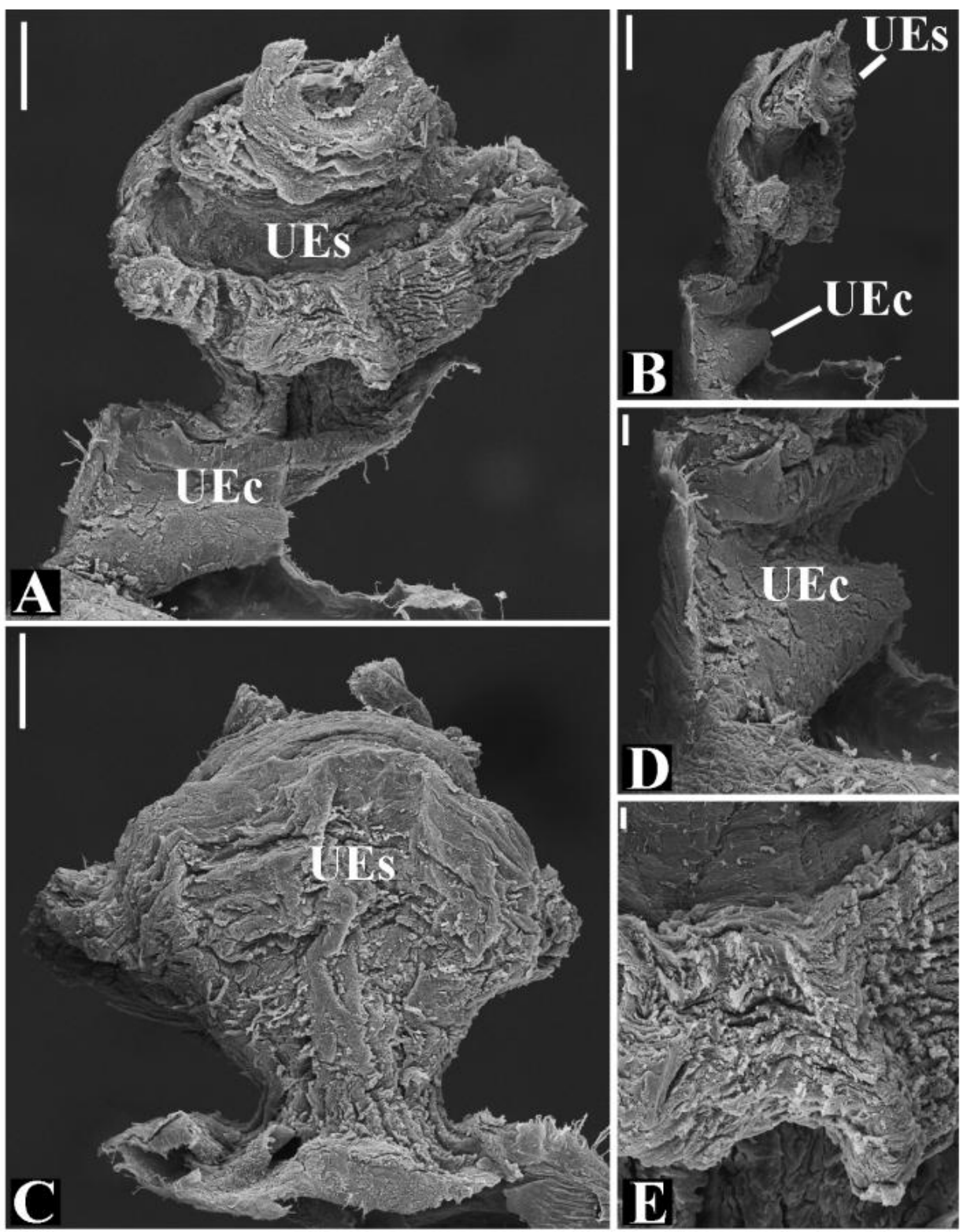

Figure 164. Glenognatha gaujoni from Peru. Female genitalia. A, dorsal. B, lateral. C, ventral. D-E, uterus externus cuticle. Scale bars, $100 \mu \mathrm{m}$ (A-C), $20 \mu \mathrm{m}$ (D), $10 \mu \mathrm{m}$ (E). UEc: uterus externus chamber. UEs: uterus externus sac. 


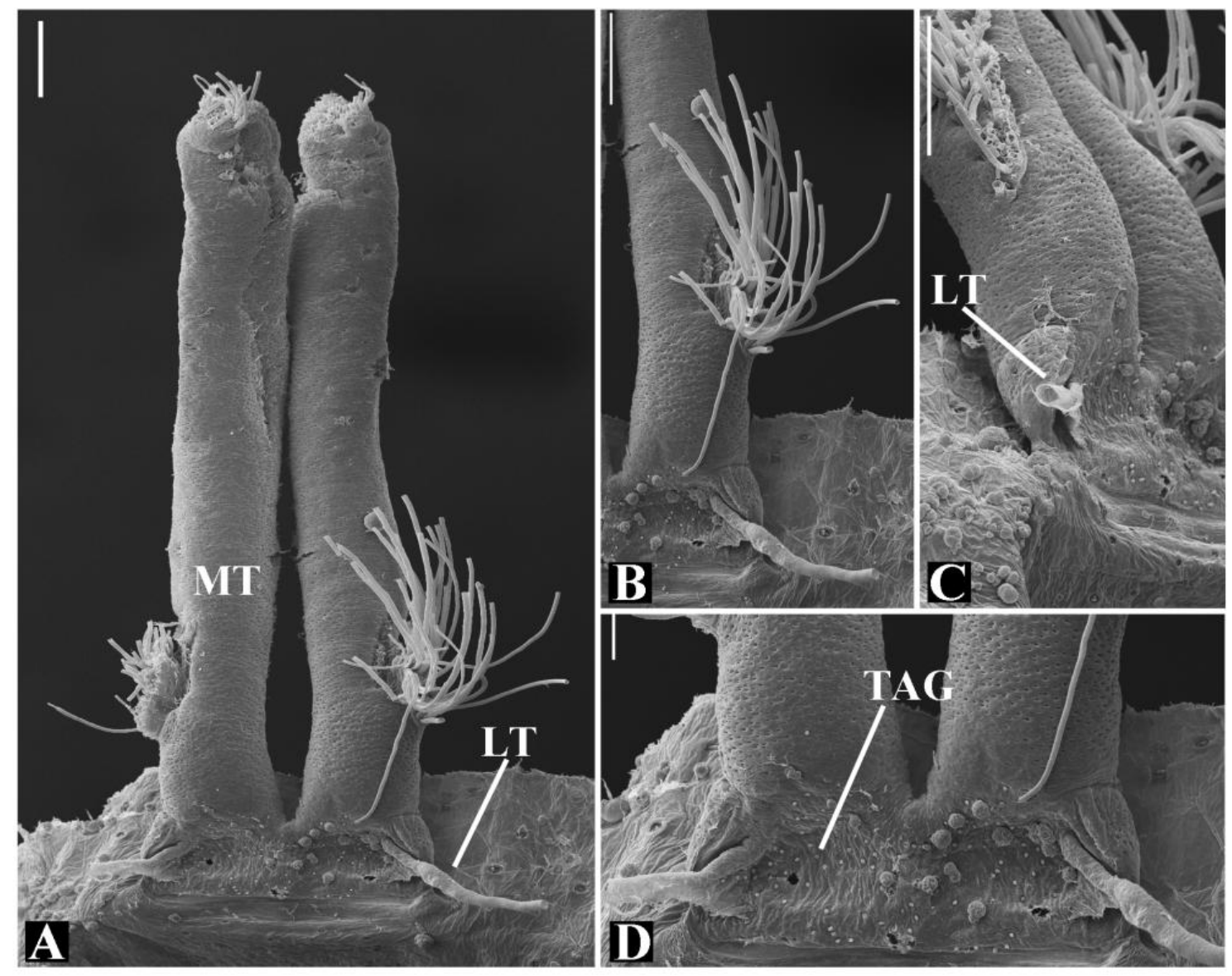

Figure 165. Glenognatha gaujoni from Brazil. Female tracheal system. A, dorsal. B-C, median and lateral trunks. D, tracheal spiracle posterior view. Scale bars, $100 \mu \mathrm{m}$ (AC), $30 \mu \mathrm{m}$ (D). LT: lateral tracheae. MT: median trunks. TAG: tracheal atrium gland. 


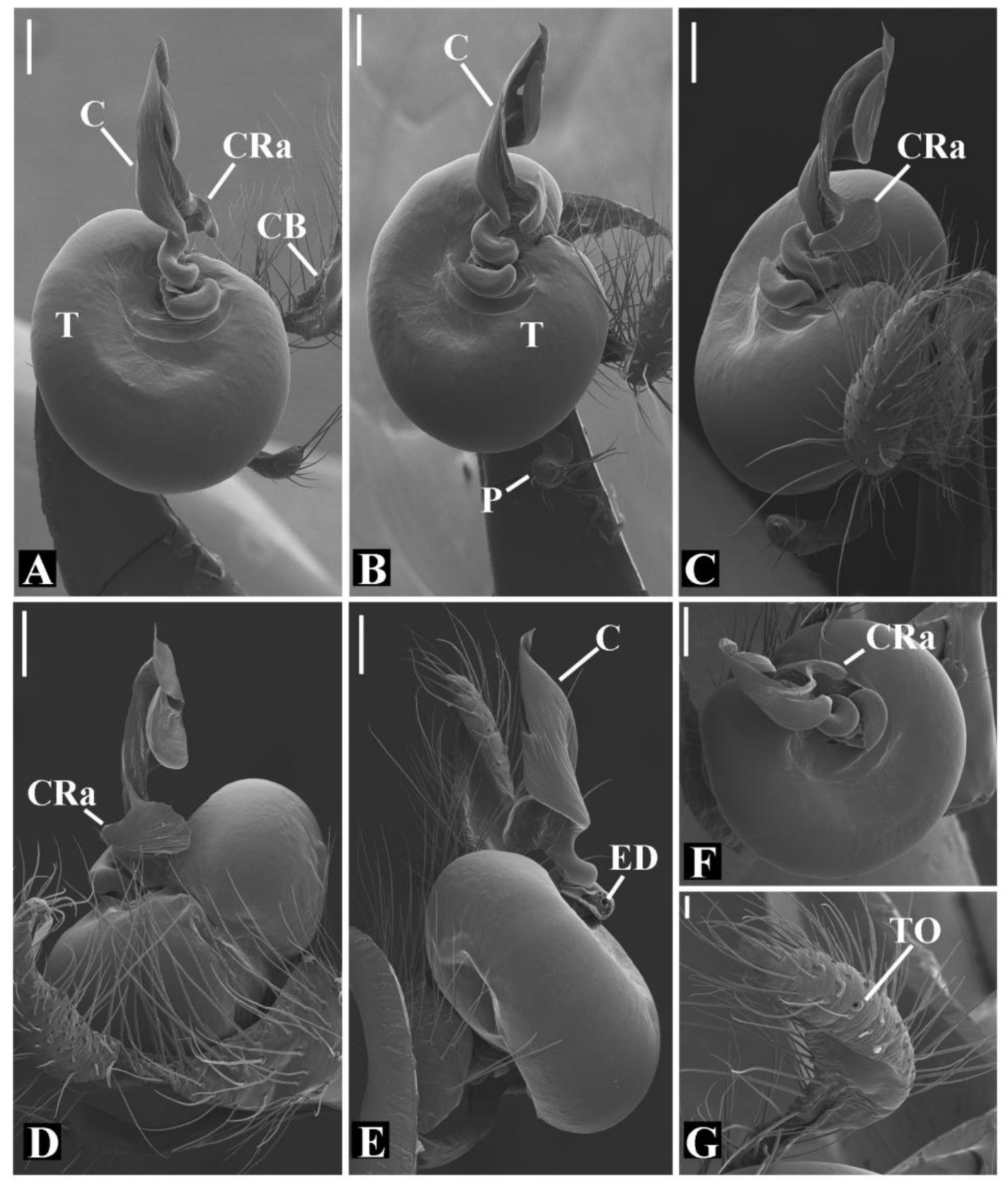

Figure 166. Glenognatha gaujoni from Brazil. Male left palp. A, ventral. B, ventroretrolateral. C, retrolateral. D, dorsal. E, prolateral. F, anterior. $\mathbf{G}$, paracymbium. Scale bars $100 \mu \mathrm{m}(\mathrm{A}-\mathrm{F}), 30 \mu \mathrm{m}(\mathrm{G})$. C: conductor. CB: cymbium. CRa: conductor retrolateral apophysis. ED: ejaculatory duct. P: paracymbium. T: tegulum. TO: tarsal organ. 


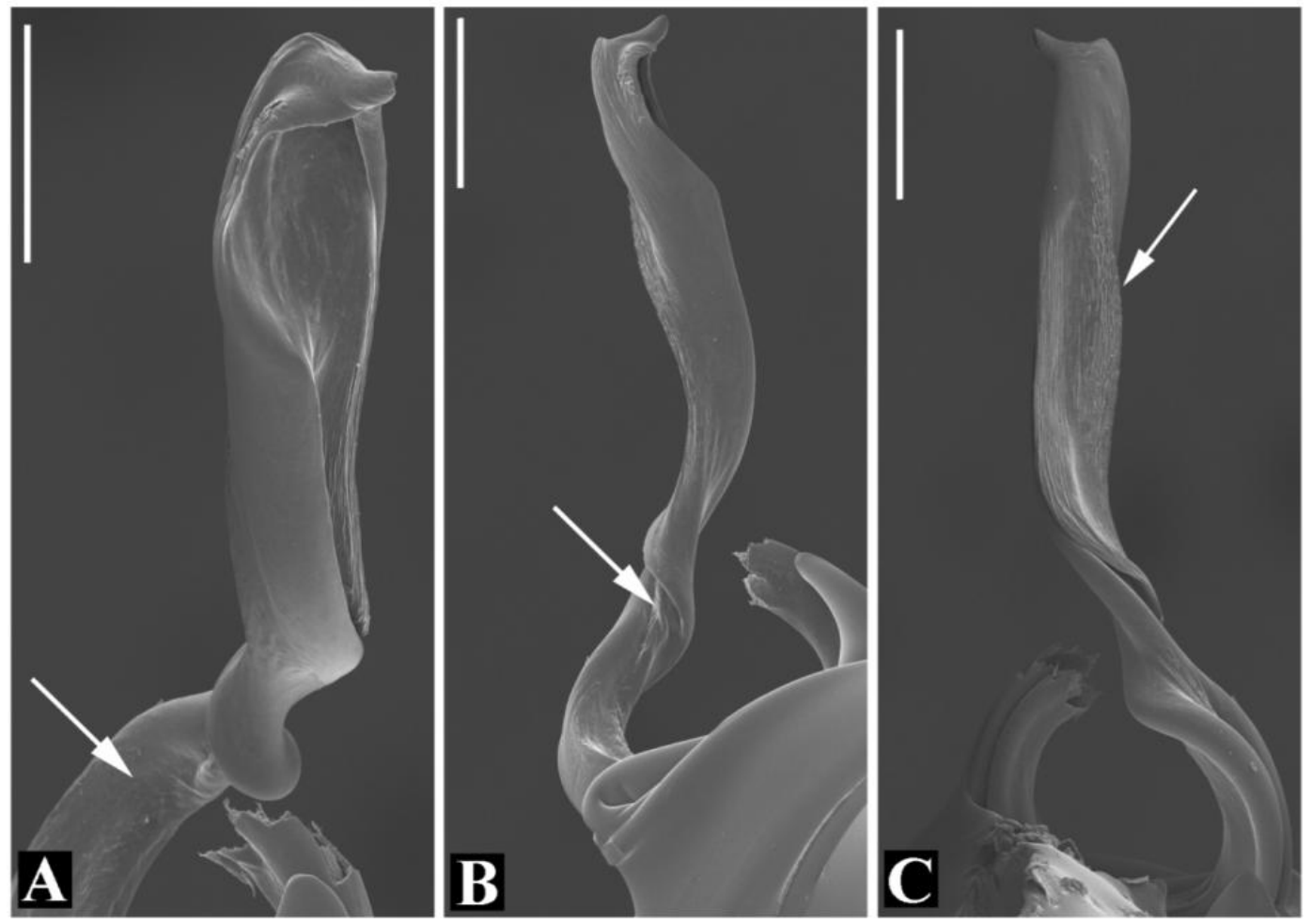

Figure 167. Glenognatha gaujoni from Brazil. A-C, Embolus. A, ventroretrolateral (arrow, inner membrane). B, ventral (arrow, inner membrane). C, dorsal (arrow, scaleshaped projections). Scale bars, $100 \mu \mathrm{m}$ (A-C). 


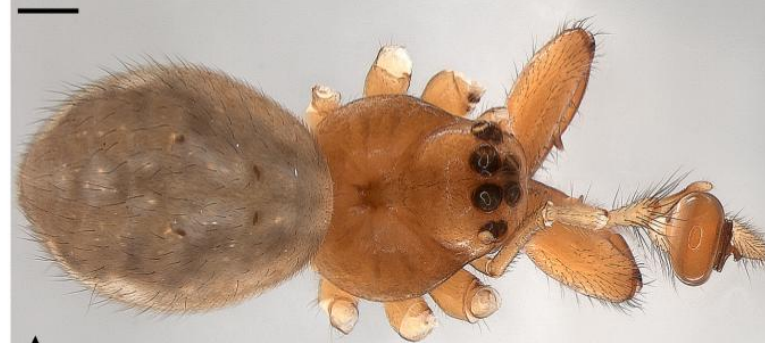

A

C

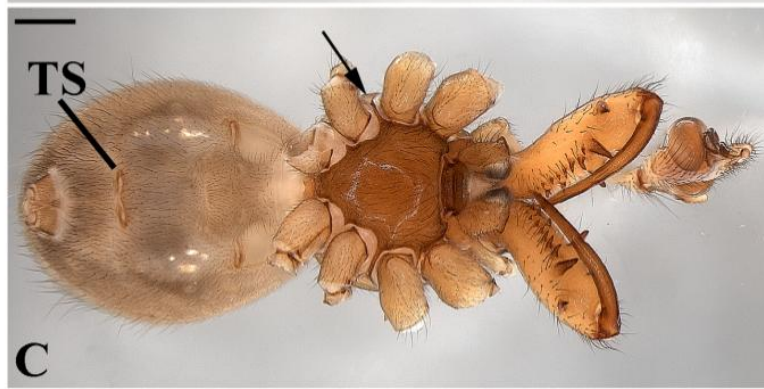

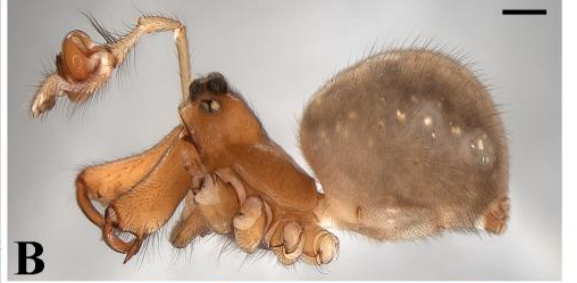

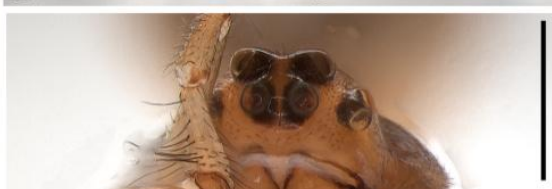

D

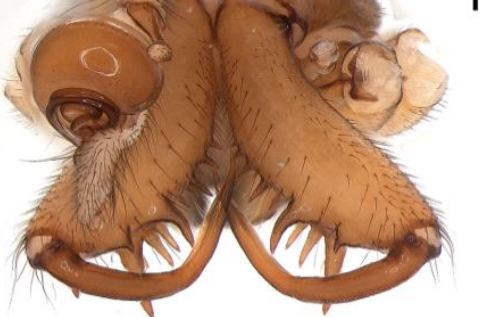

Figure 168. Glenognatha sp. nov. 8 from Colombia (ICN 5986). Male habitus. A, dorsal. B, lateral. C, ventral (arrow, pleural bar). D, frontal. Scale bars, $500 \mu \mathrm{m}$. TS: tracheal spiracle.
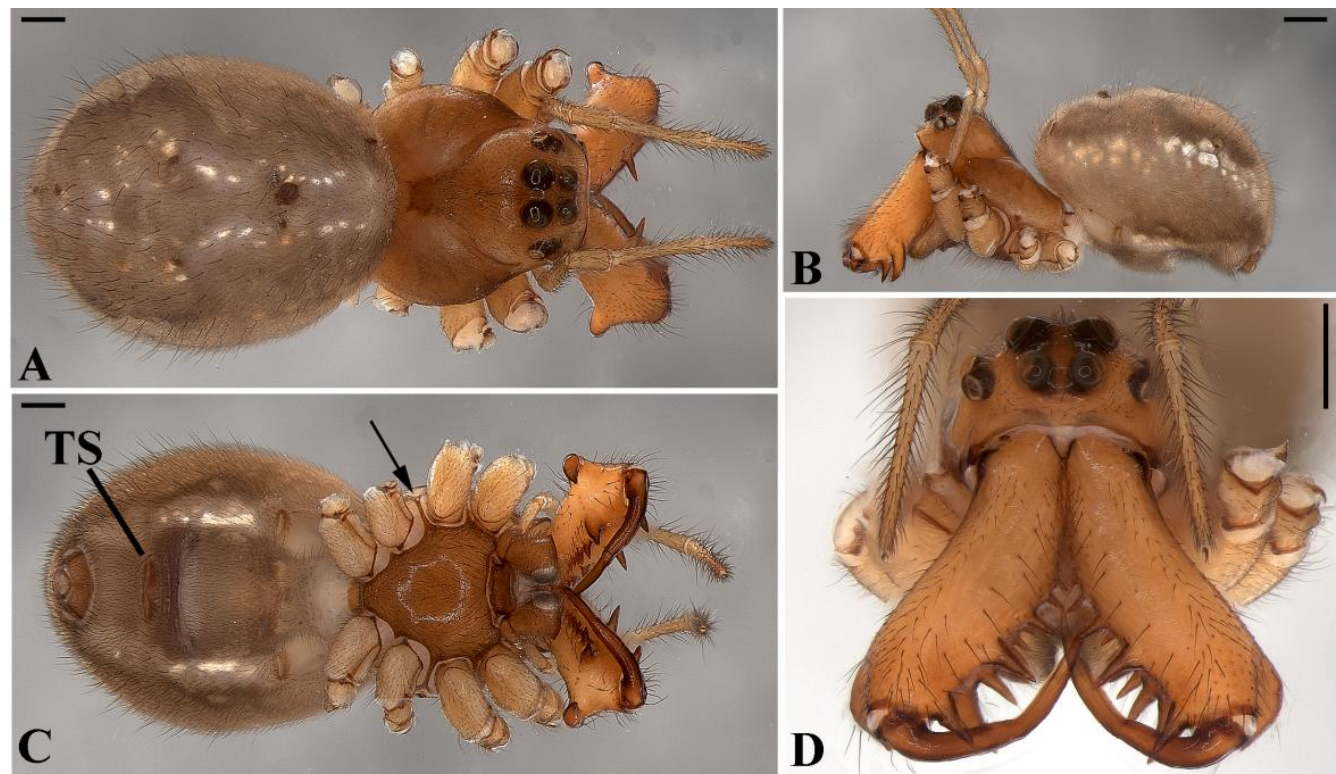

Figure 169. Glenognatha sp. nov. 8 from Colombia (ICN 5986). Female habitus. A, dorsal. B, lateral. C, ventral (arrow, pleural bar). D, frontal. Scale bars, $500 \mu \mathrm{m}$. TS: tracheal spiracle. 

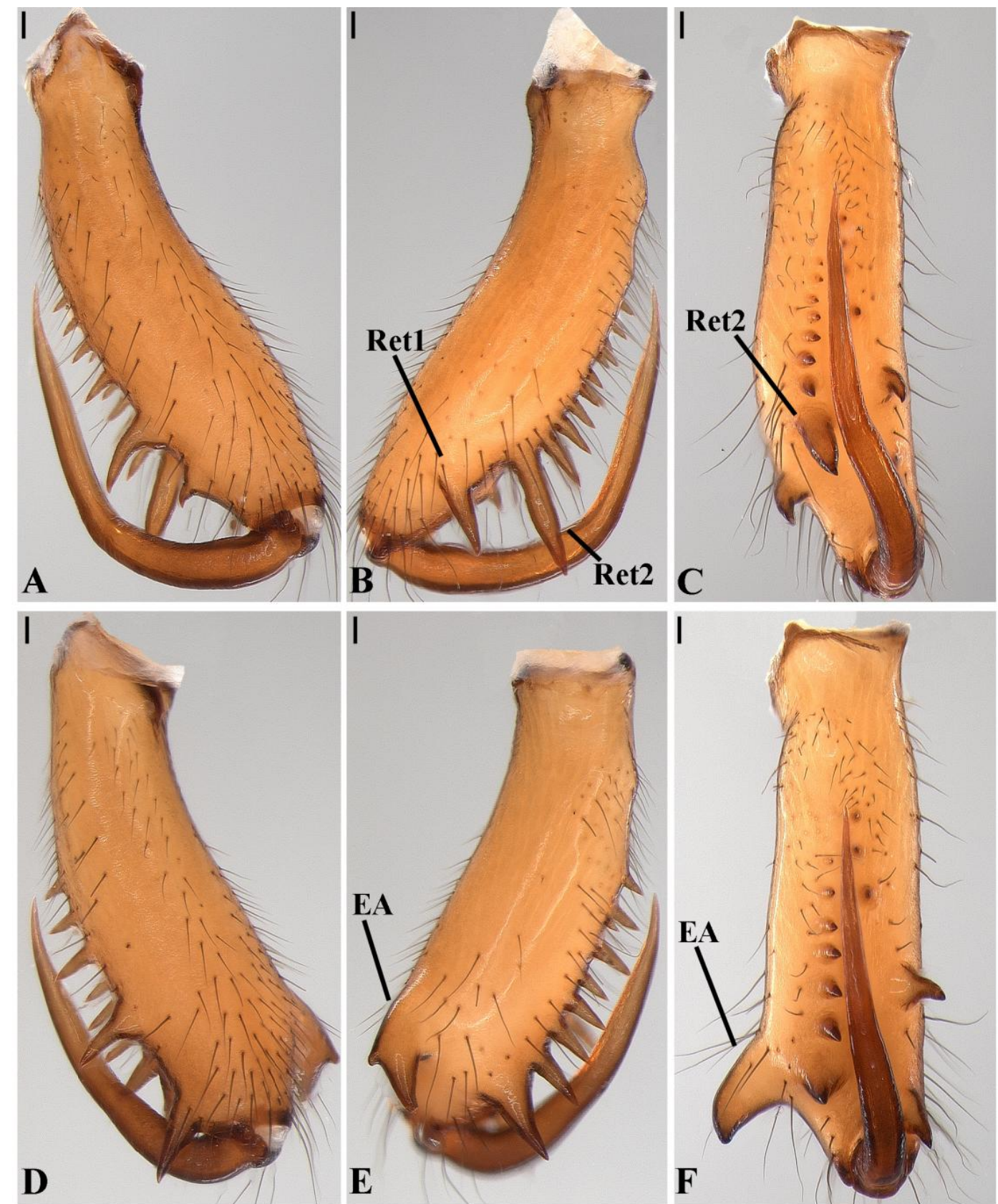

Figure 170. Glenognatha sp. nov. 8 from Colombia (ICN 5986). A-C, Male left chelicerae. A, anterior. B, posterior. C, mesal. D-F, Female left chelicerae. D, anterior. E, posterior. F, mesal. Scale bars, $100 \mu \mathrm{m}$. EA: ectal apophysis. Ret: retromarginal tooth. 


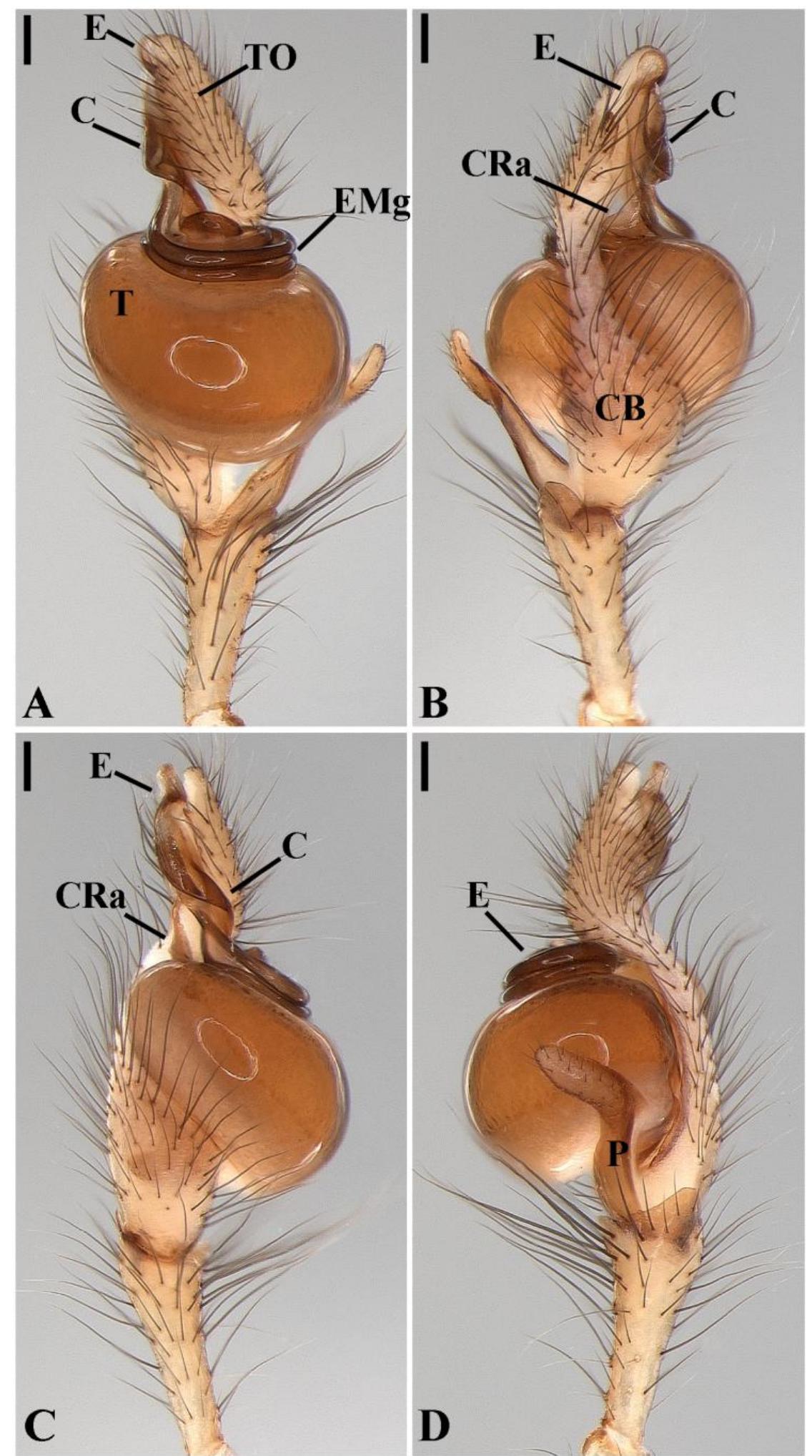

Figure 171. Glenognatha sp. nov. 8 from Colombia (ICN 5986). Male left palp. A, ventral. B, dorsal. C, prolateral. D, retrolateral. Scale bars, $100 \mu \mathrm{m}$. C: conductor. CB: cymbium. CRa: conductor retrolateral apophysis. E: embolus. EMg: embolic medial groove. P: paracymbium. T: tegulum. TO: tarsal organ. 

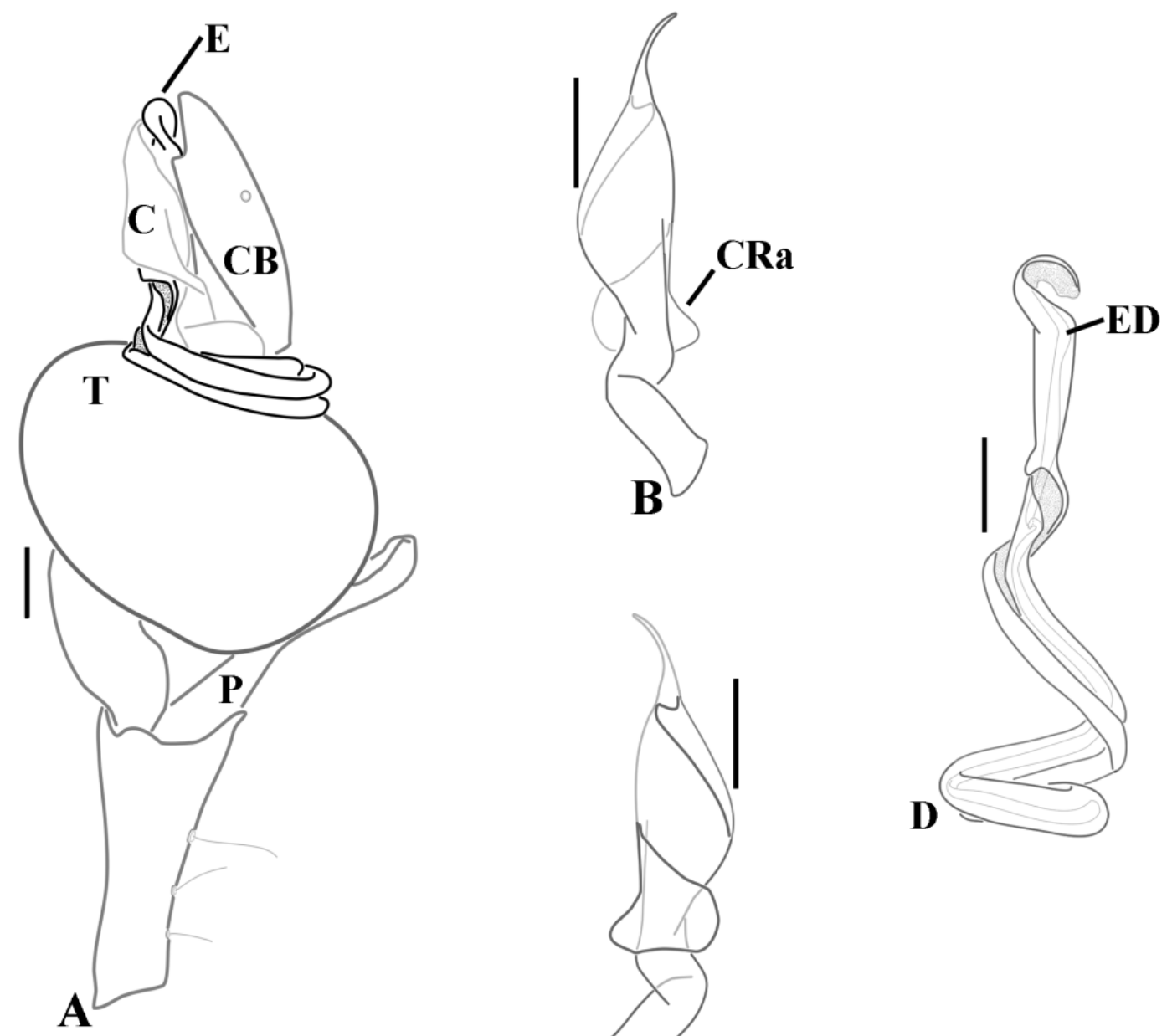

Figure 172. Glenognatha sp. nov. 8 from Colombia (ICN 5986). Male left palp, schematic. A, ventral. B, conductor ventral. C, conductor dorsal. D, embolus ventral. Scale bars $100 \mu \mathrm{m}$. C: conductor. CB: cymbium. CRa: conductor retrolateral apophysis. E: embolus. ED: ejaculatory duct. P: paracymbium. T: tegulum. 

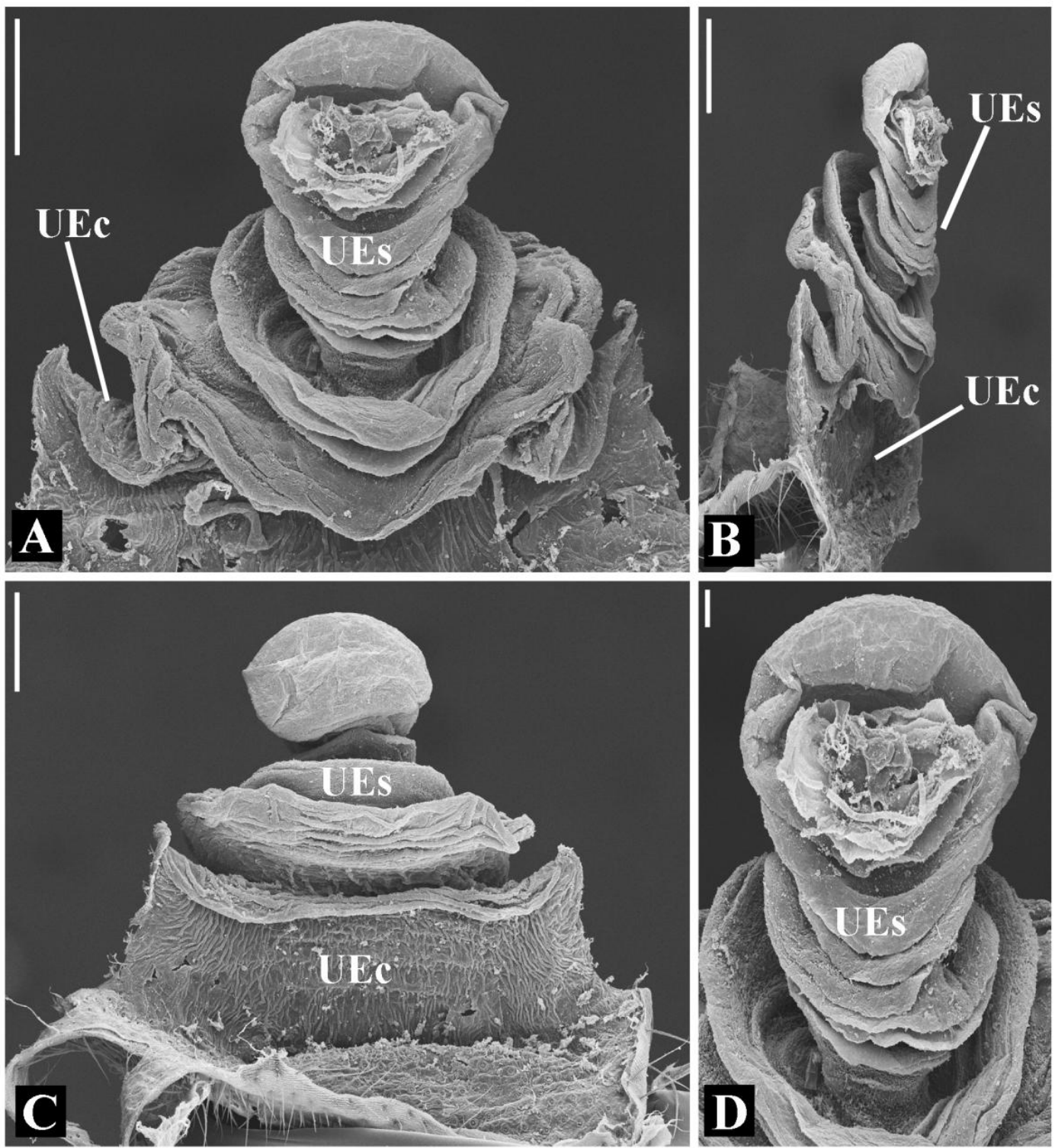

Figure 173. Glenognatha sp. nov. 8 from Colombia. Female genitalia. A, dorsal. B, lateral. C, ventral D, uterus externus cuticle. Scale bars, $100 \mu \mathrm{m}$ (A-C), $20 \mu \mathrm{m}$ (D). UEc: uterus externus chamber. UEs: uterus externus sac. 


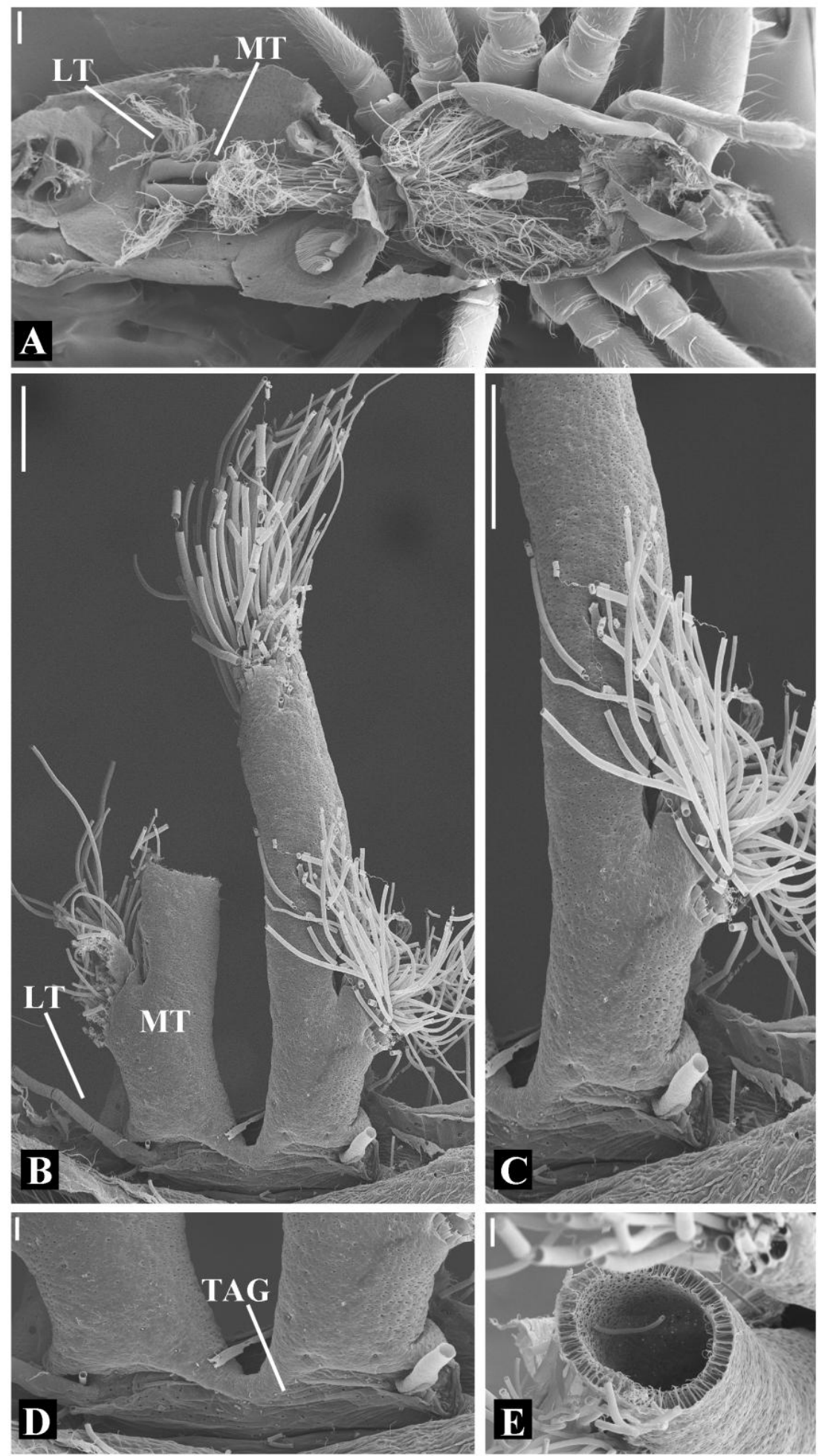

Figure 174. Glenognatha sp. nov. 8 from Colombia. Female tracheal system. A, dorsal whole montage. $\mathbf{B}$, dorsal. $\mathbf{C}$, median and lateral trunks. $\mathbf{D}$, tracheal spiracle posterior view. E, median trunk transversal cut. Scale bars, $200 \mu \mathrm{m}$ (A), $100 \mu \mathrm{m}$ (B, C), $20 \mu \mathrm{m}$ (D, E). LT: lateral tracheae. MT: median trunks. TAG: tracheal atrium gland. 

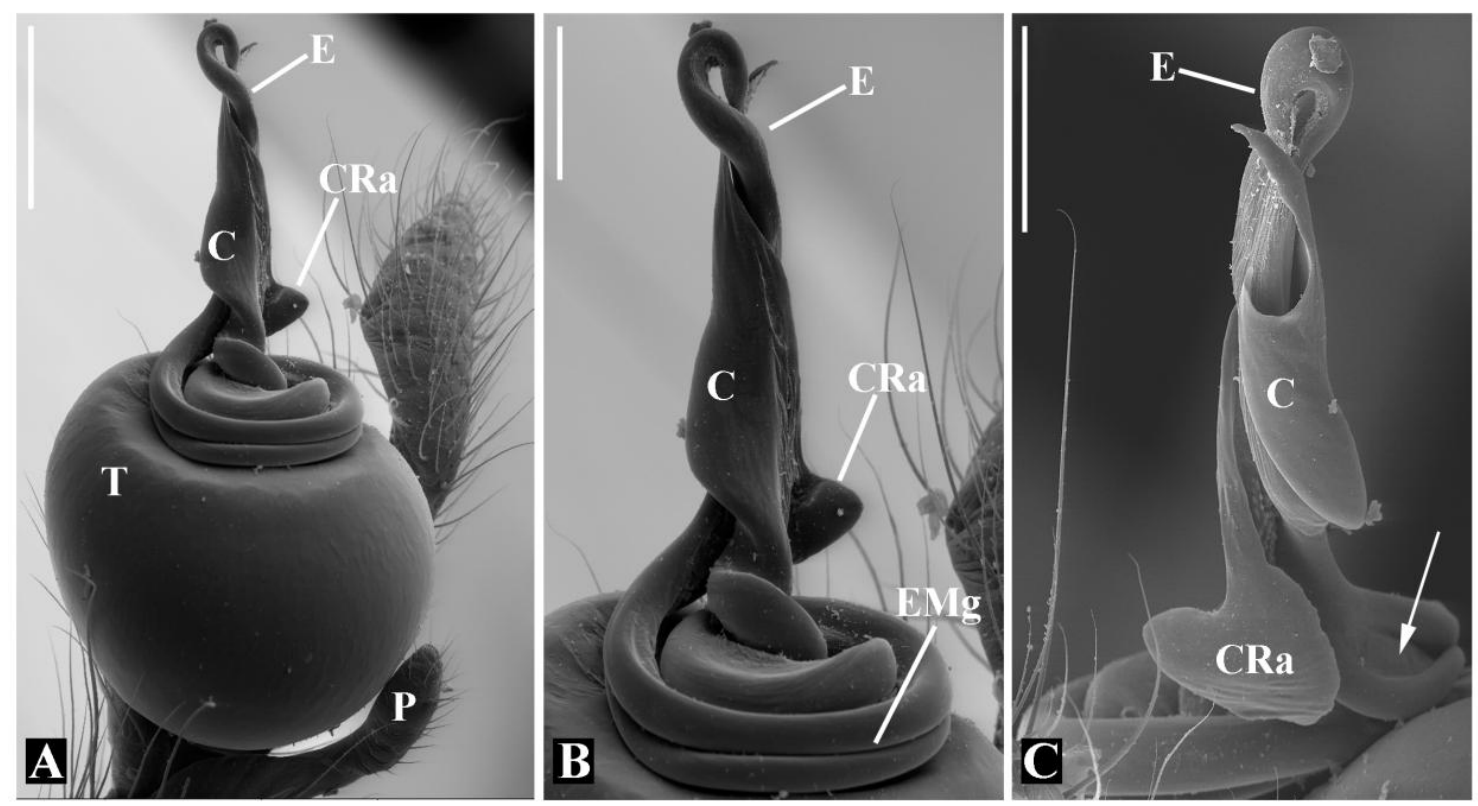

Figure 175. Glenognatha sp. nov. 8 from Colombia. Male left palp. A, ventral. B, embolus and conductor ventral. $\mathbf{C}$, embolus and conductor dorsal (arrow, inner membrane). Scale bars $200 \mu \mathrm{m}$ (A), $100 \mu \mathrm{m}$ (B, C). C: conductor. CRa: conductor retrolateral apophysis. E: embolus. EMg: embolic medial groove. P: paracymbium. T: tegulum. 

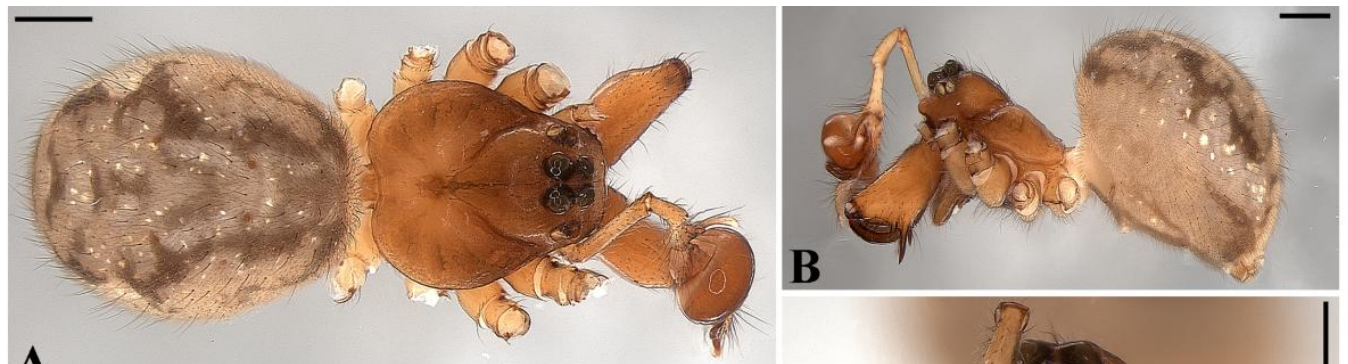

\section{A}
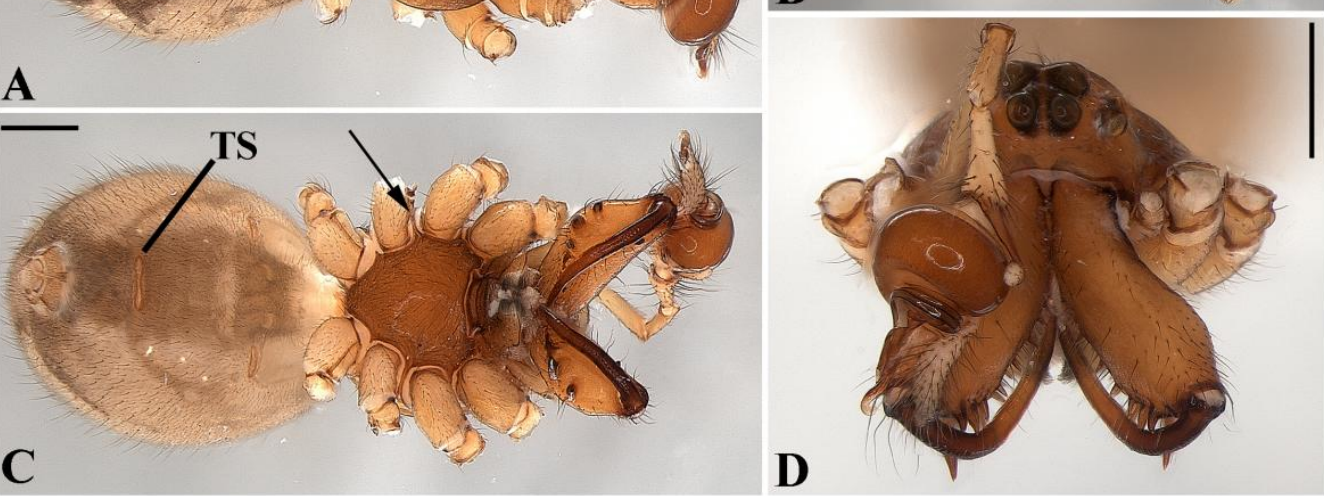

Figure 176. Glenognatha globosa from Colombia (ICN 5991). Male habitus. A, dorsal.

B, lateral. C, ventral (arrow, pleural bar). D, frontal. Scale bars, $500 \mu \mathrm{m}$. TS: tracheal spiracle.
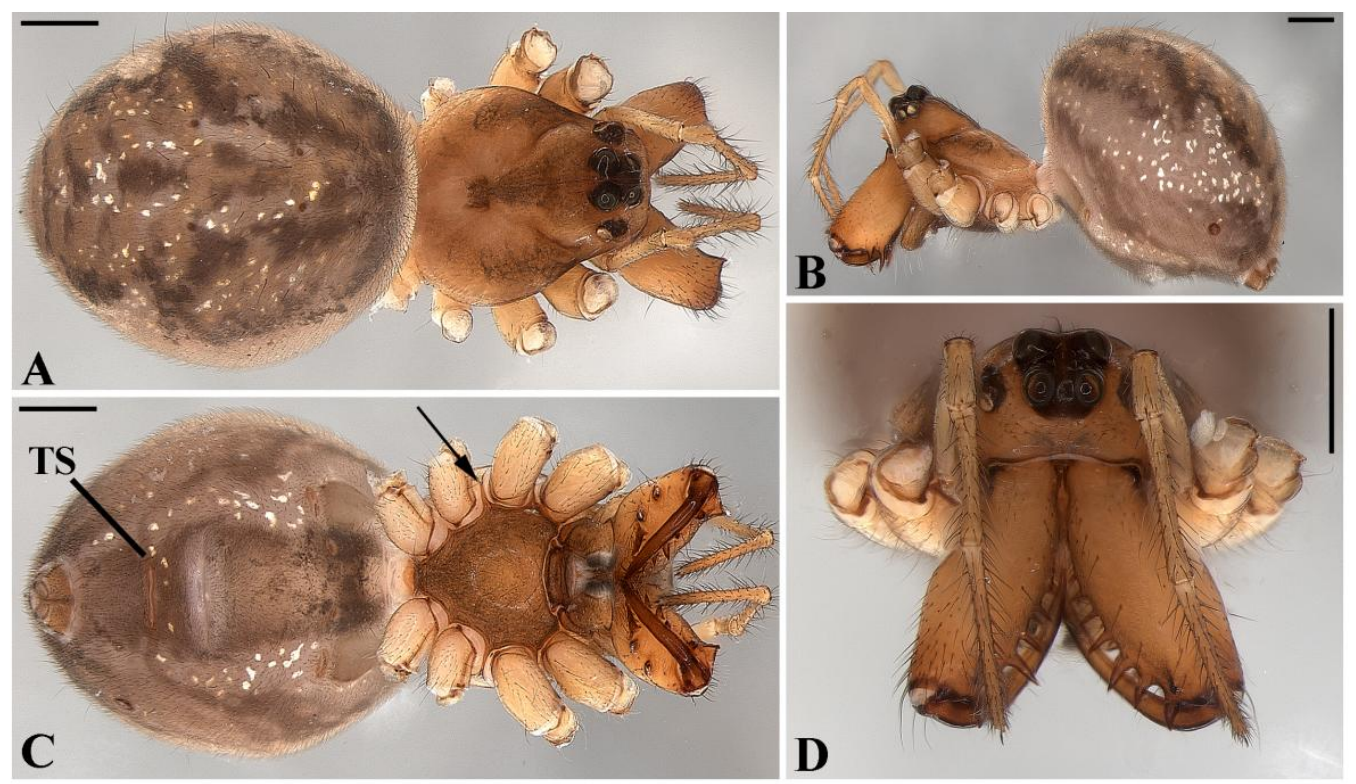

Figure 177. Glenognatha globosa from Colombia (ICN 5991). Female habitus. A, dorsal. B, lateral. C, ventral (arrow, pleural bar). D, frontal. Scale bars, $500 \mu \mathrm{m}$. TS: tracheal spiracle. 


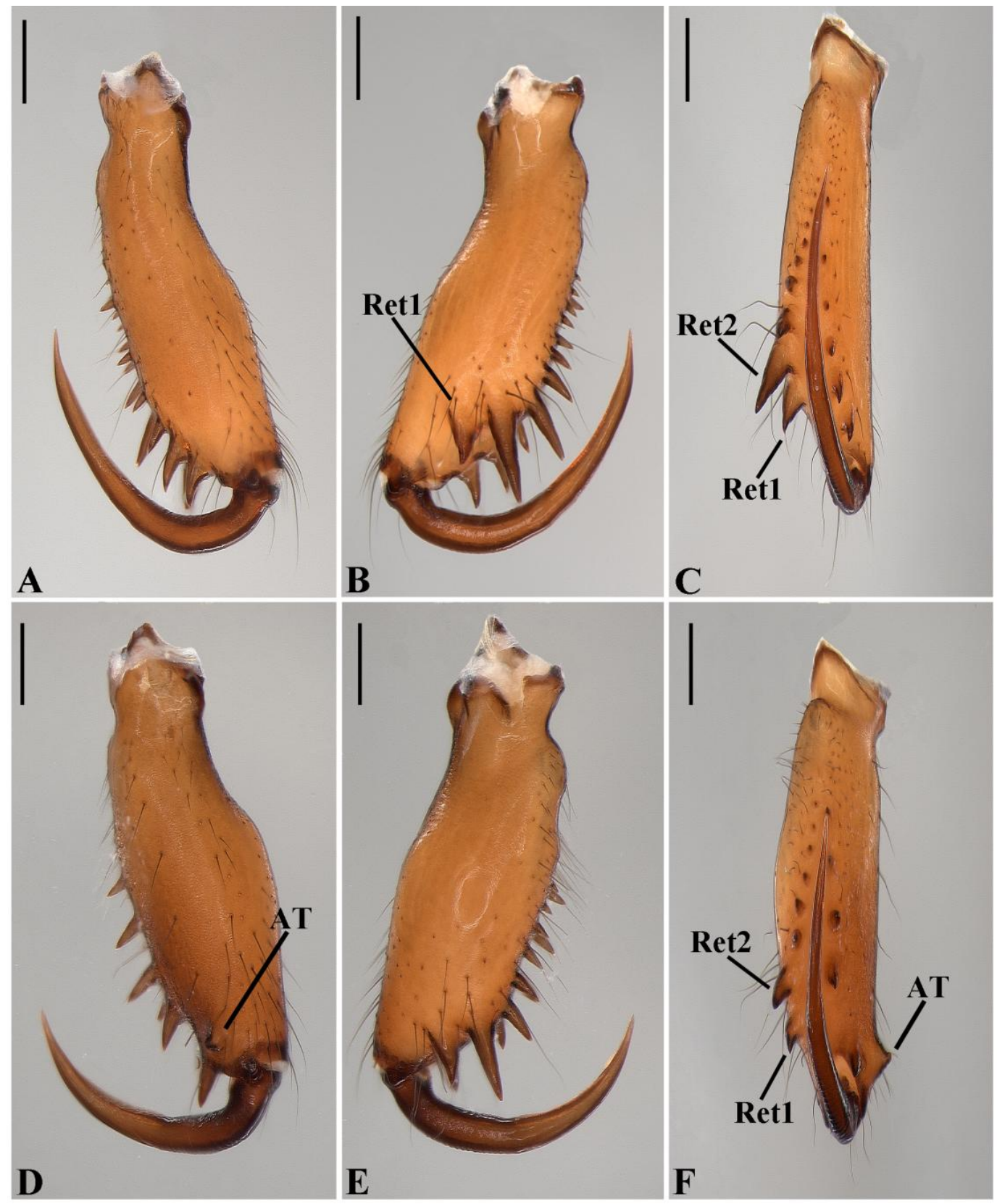

Figure 178. Glenognatha globosa from Colombia (ICN 5991). A-C, Male left chelicerae. A, anterior. B, posterior. C, mesal. D-F, Female left chelicerae. D, anterior. E, posterior. F, mesal. Scale bars, $200 \mu \mathrm{m}$. AT: anterior tooth. Ret: retromarginal tooth. 

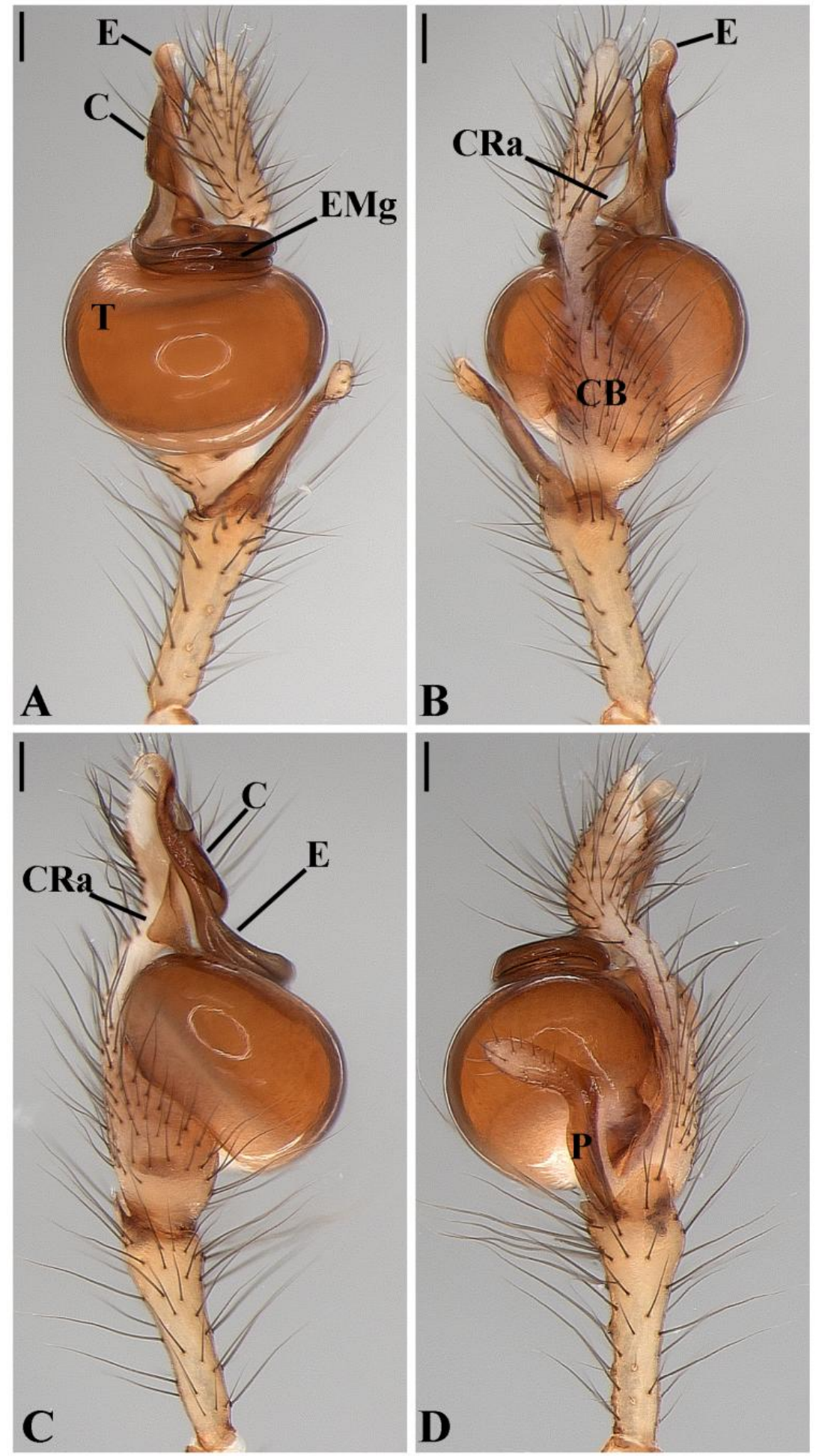

Figure 179. Glenognatha globosa from Colombia (ICN 5991). Male left palp. A, ventral. B, dorsal. C, prolateral. D, retrolateral. Scale bars, $100 \mu \mathrm{m}$. C: conductor. CB: cymbium. CRa: conductor retrolateral apophysis. E: embolus. EMg: embolic medial groove. P: paracymbium. T: tegulum. 

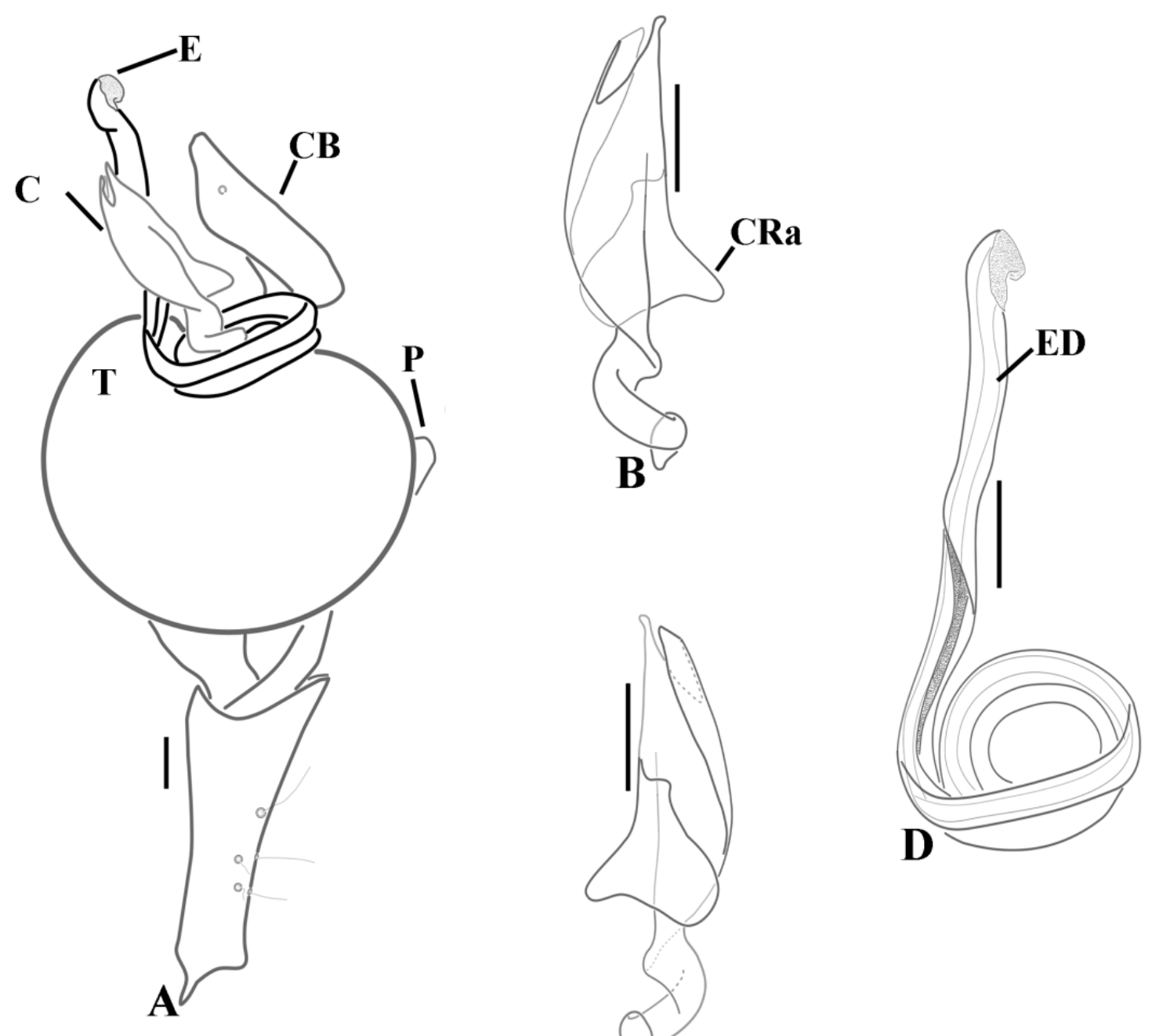

Figure 180. Glenognatha globosa from Colombia (ICN 5991). Male left palp, schematic. A, ventral. B, conductor ventral. C, conductor dorsal. D, embolus ventral. Scale bars $100 \mu \mathrm{m}$. C: conductor. CB: cymbium. CRa: conductor retrolateral apophysis. E: embolus. ED: ejaculatory duct. P: paracymbium. T: tegulum. 

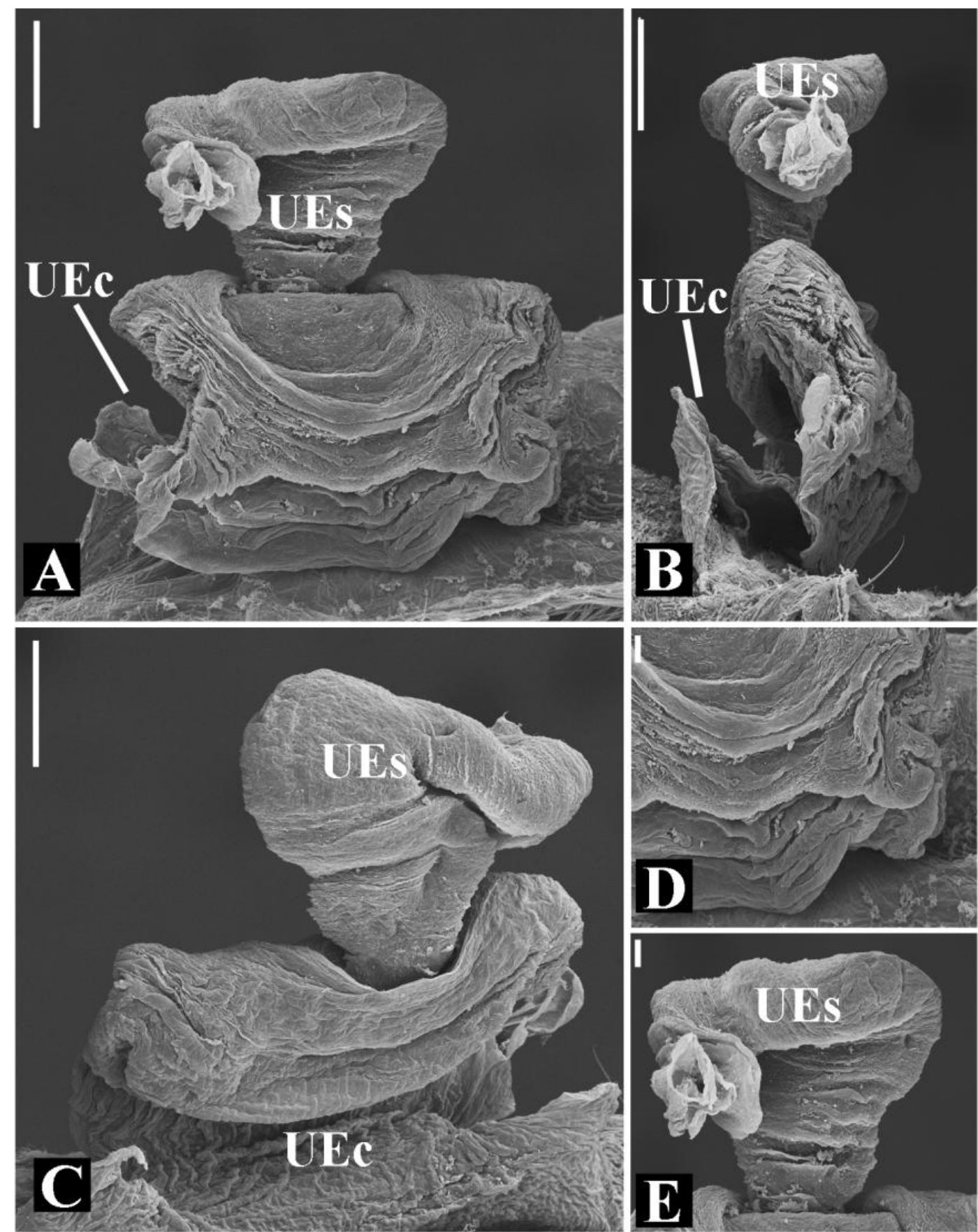

Figure 181. Glenognatha globosa from Colombia. Female genitalia. A, dorsal. B, lateral. C, ventral. D-E, uterus externus cuticle. Scale bars, $100 \mu \mathrm{m}$ (A-C), $20 \mu \mathrm{m}$ (D, E). UEc: uterus externus chamber. UEs: uterus externus sac. 


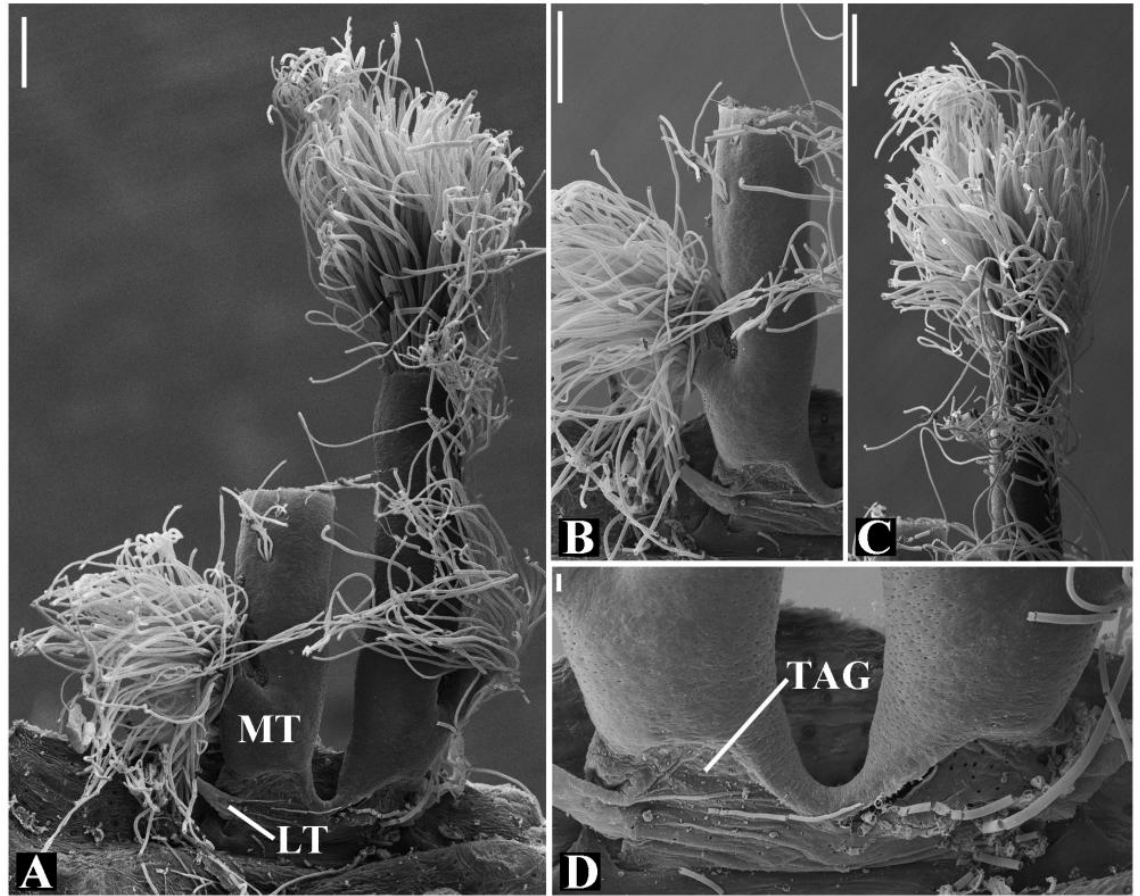

Figure 182. Glenognatha globosa from Colombia. Female tracheal system. A, dorsal. B, median and lateral trunks. C, median trunk distal. D, tracheal spiracle posterior view. Scale bars, $100 \mu \mathrm{m}$ (A-C), $10 \mu \mathrm{m}$ (D). LT: lateral tracheae. MT: median trunks. TAG: tracheal atrium gland.
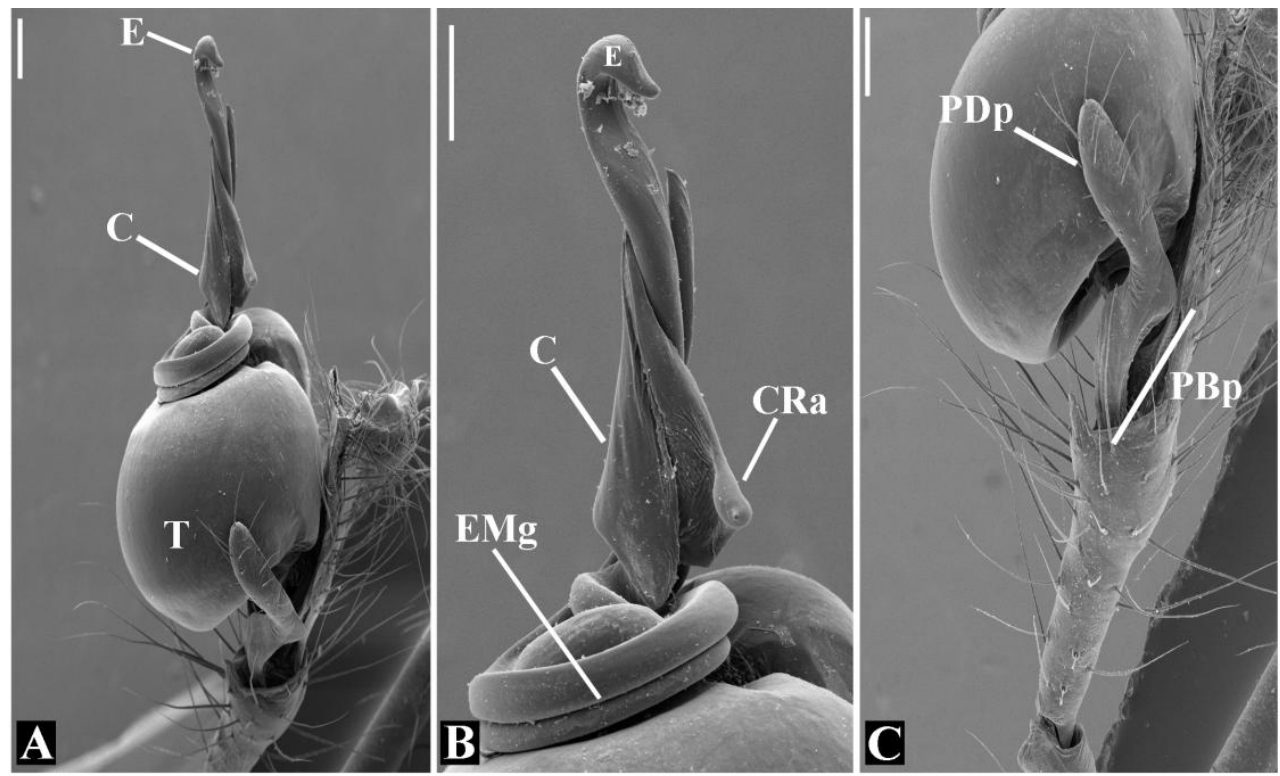

Figure 183. Glenognatha globosa from Colombia. Male left palp. A. retrolateral. B. embolus and conductor. C, paracymbium. Scale bars $100 \mu \mathrm{m}$. C: conductor. CRa: conductor retrolateral apophysis. E: embolus. EMg: embolic medial groove. PBp: paracymbium basal portion. PDp: paracymbium basal portion. T: tegulum. 

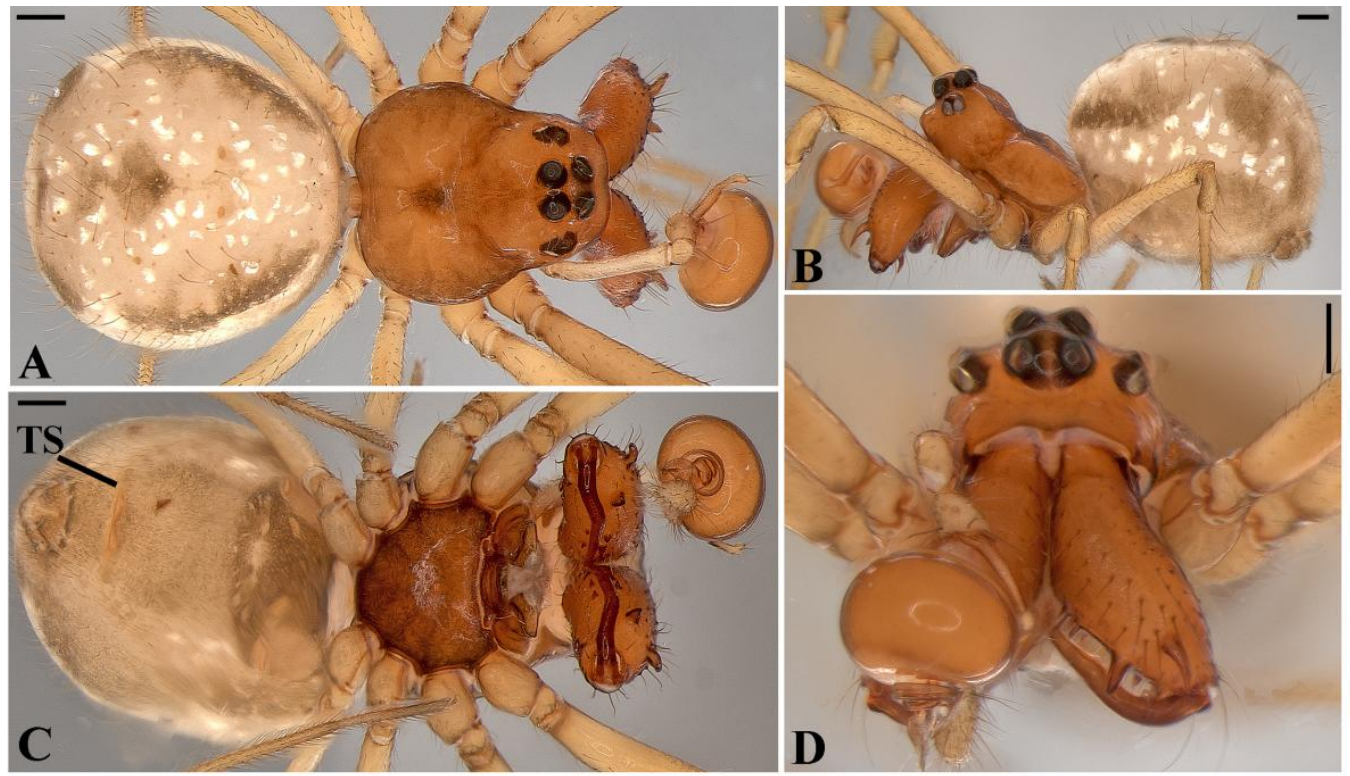

Figure 184. Glenognatha sp. nov. 6 from Colombia (Holotype, ICN 5979). Male habitus. A, dorsal. B, lateral. C, ventral. D, frontal. Scale bars, $200 \mu \mathrm{m}$. TS: tracheal spiracle.
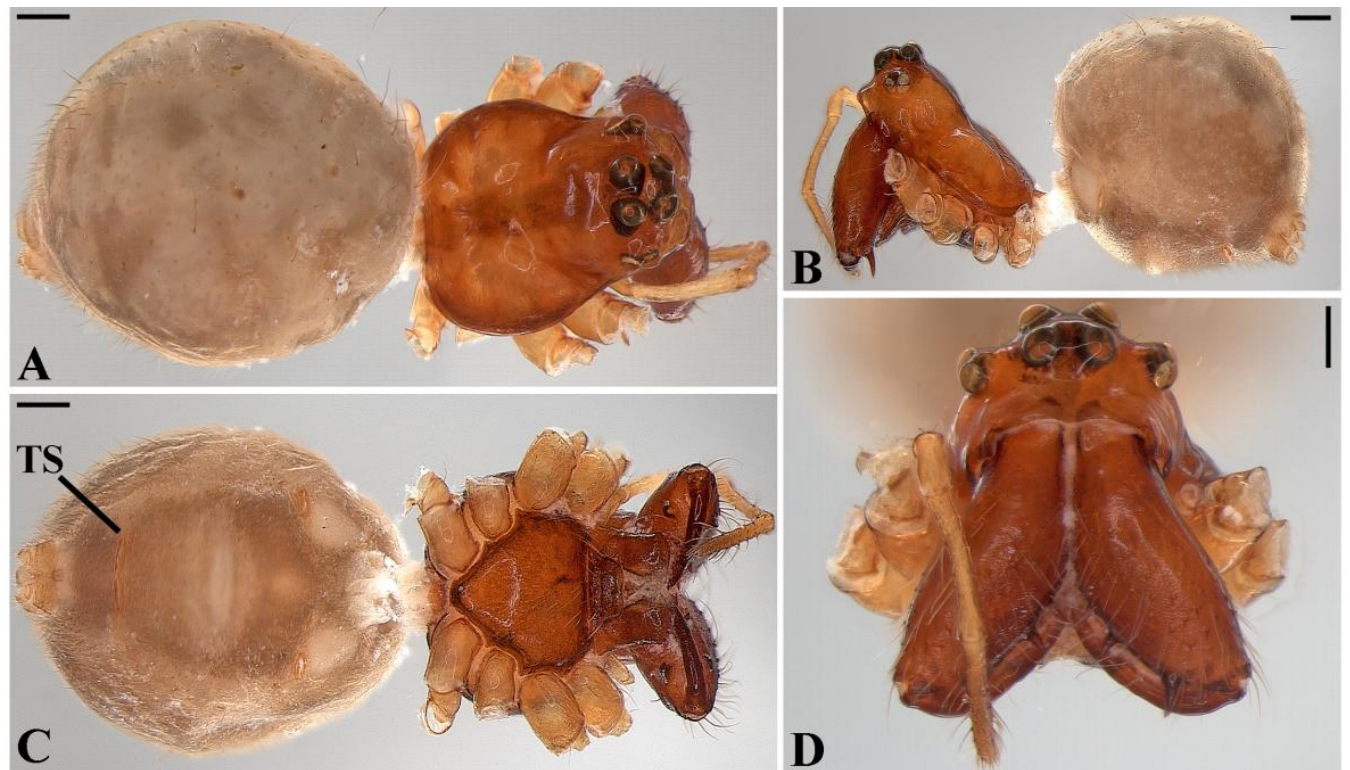

Figure 185. Glenognatha sp. nov. 6 from Venezuela (AMNH). Female habitus. A, dorsal. B, lateral. C, ventral. D, frontal. Scale bars, $200 \mu \mathrm{m}$. TS: tracheal spiracle. 


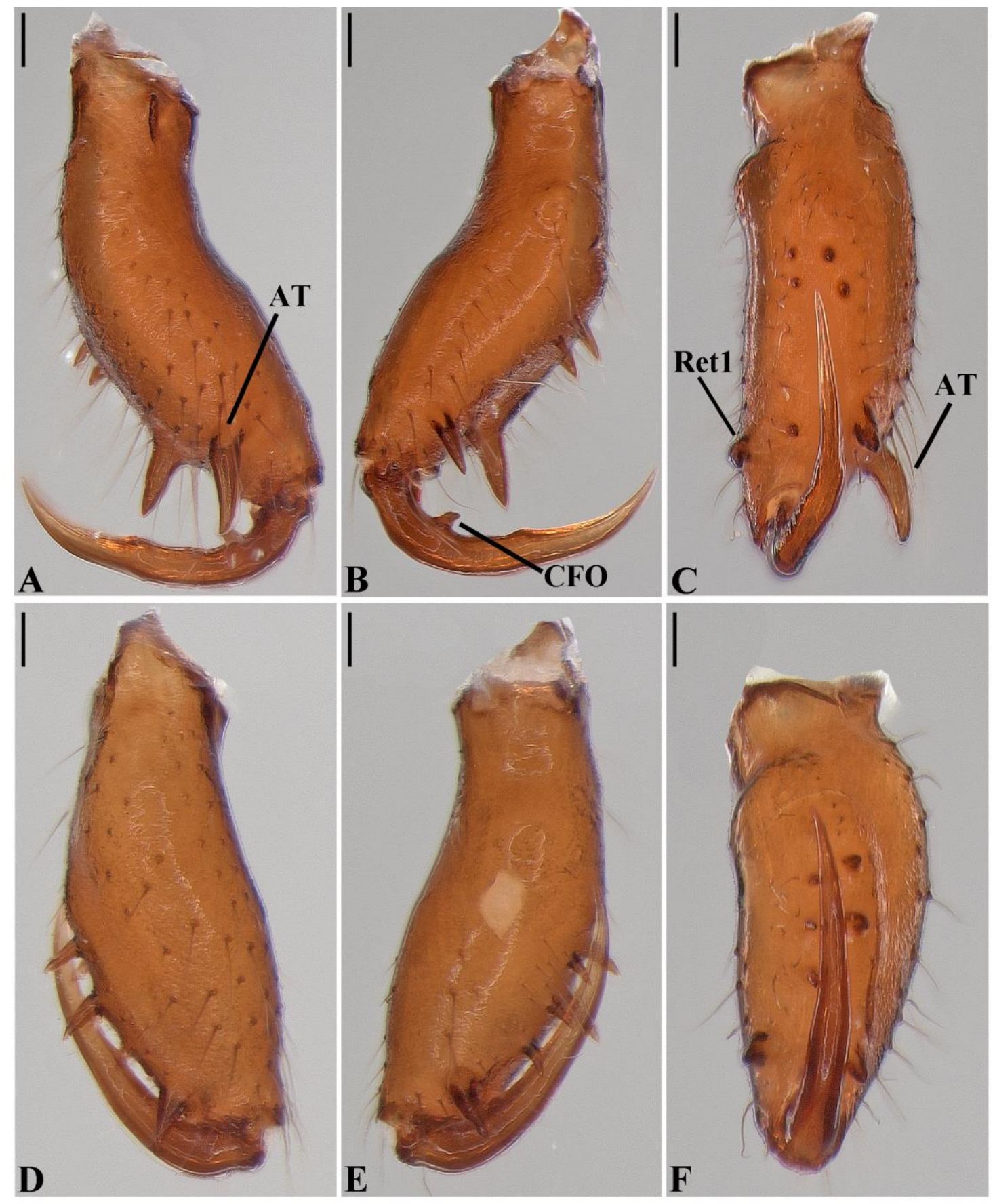

Figure 186. Glenognatha sp. nov. 6 from Colombia (Paratype ICN 5980). A-C, Male left chelicerae. A, anterior. B, posterior. C, mesal. D-F, Female left chelicerae. D, anterior. E, posterior. F, mesal. Scale bars, $100 \mu \mathrm{m}$. AT: anterior tooth. CFO: cheliceral fang outgrowth. Ret: retromarginal tooth. 


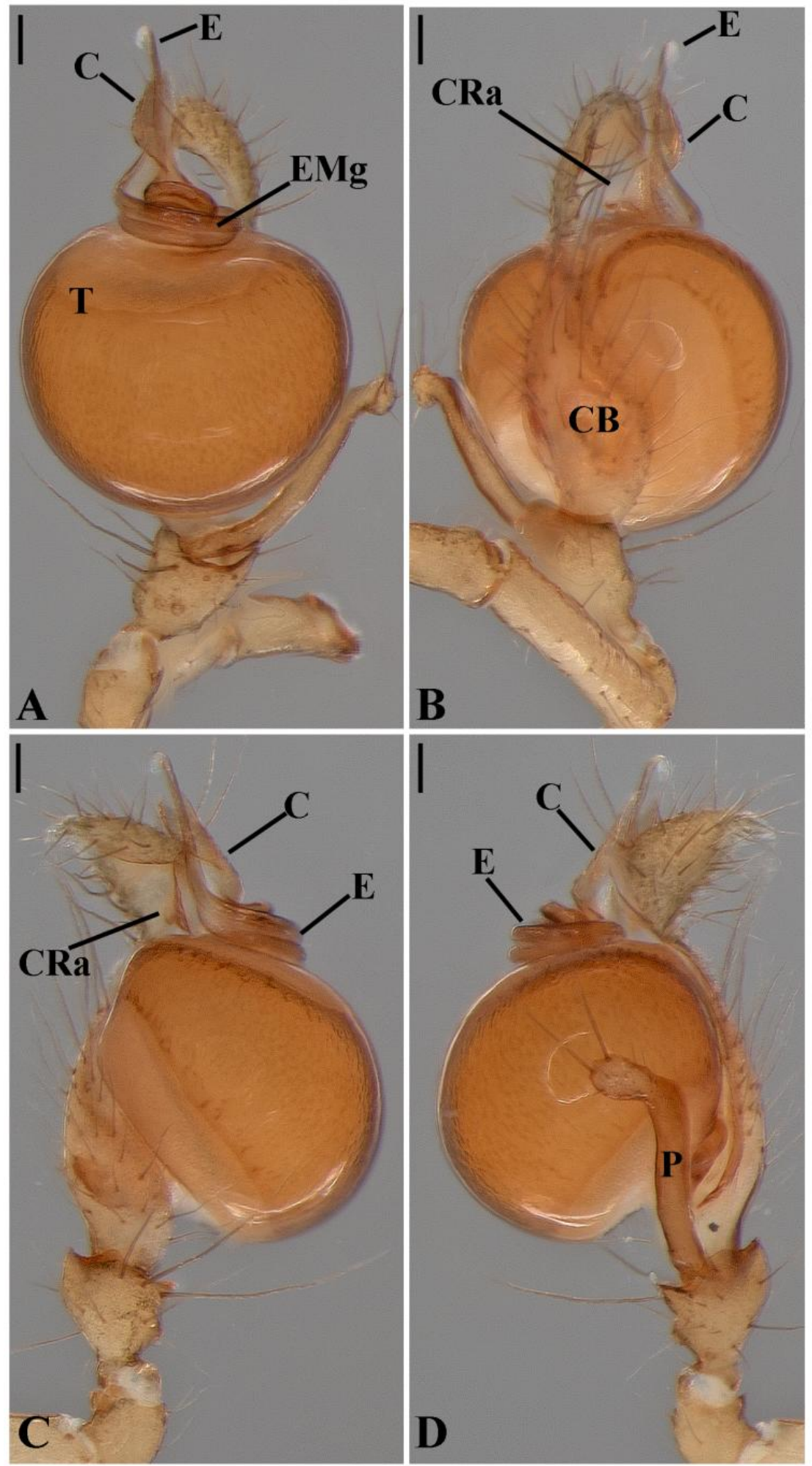

Figure 187. Glenognatha sp. nov. 6 from Colombia (Holotype ICN 5979). Male left palp A-D. A, ventral. B, dorsal. C, prolateral. D, retrolateral. Scale bars, $100 \mu \mathrm{m}$. C: conductor. CB: cymbium. CRa: conductor retrolateral apophysis. E: embolus. EMg: embolic medial groove. P: paracymbium. T: tegulum. 

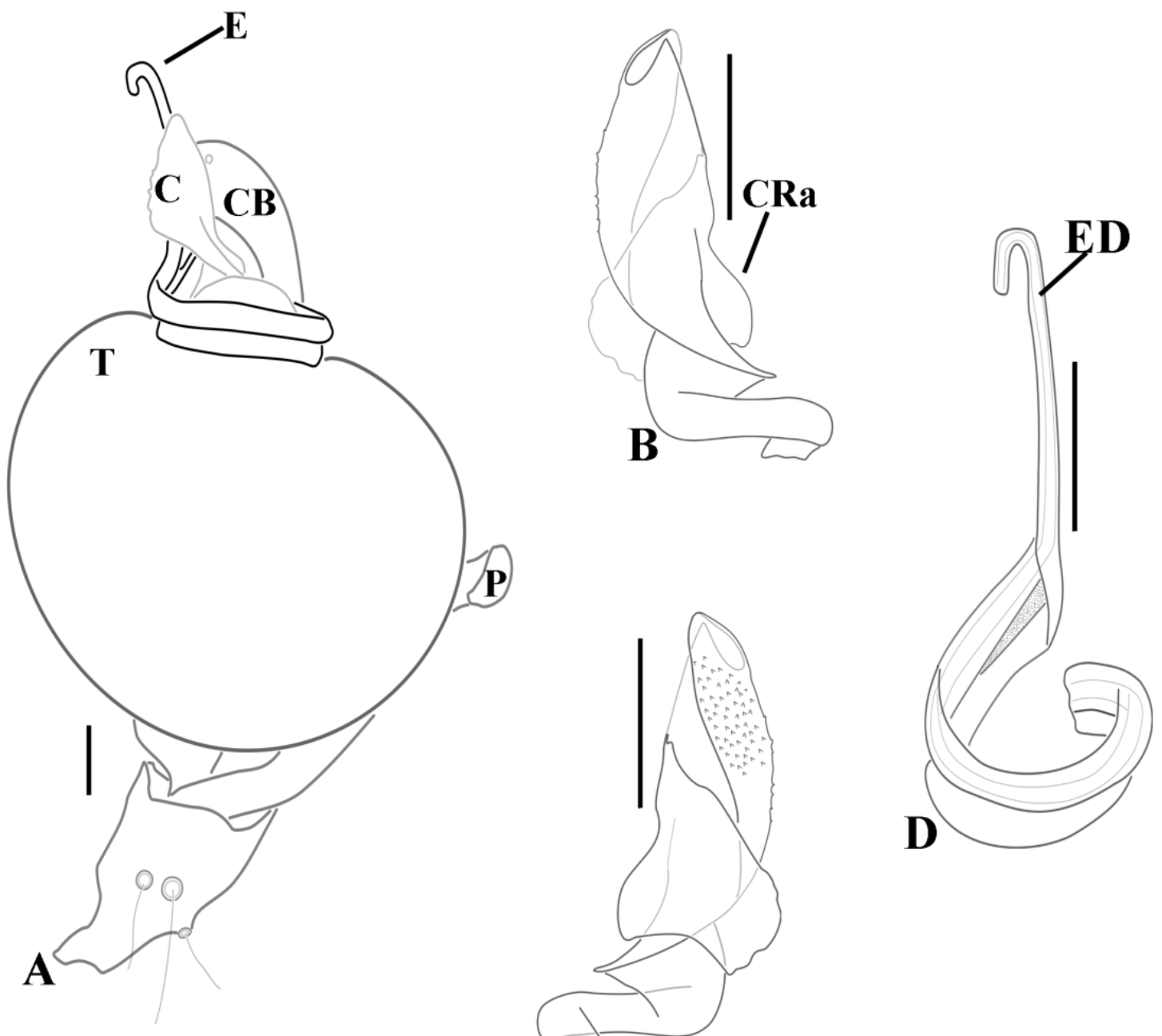

\section{C}

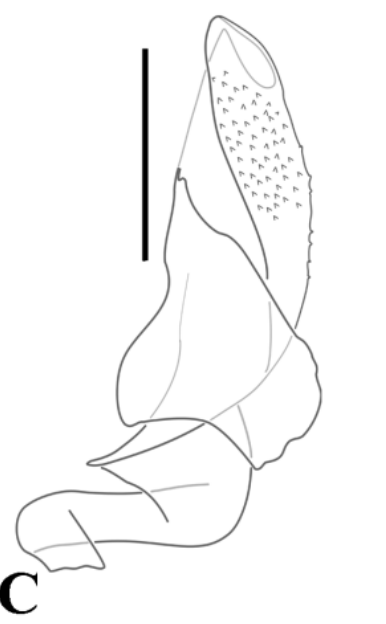

D

Figure 188. Glenognatha sp. nov. 6 from Colombia (Paratype ICN 5980). Male left palp, schematic. A, ventral. B, conductor ventral. C, conductor dorsal. D, embolus ventral. Scale bars $100 \mu \mathrm{m}$. C: conductor. CB: cymbium. CRa: conductor retrolateral apophysis. E: embolus. ED: ejaculatory duct. P: paracymbium. T: tegulum. 

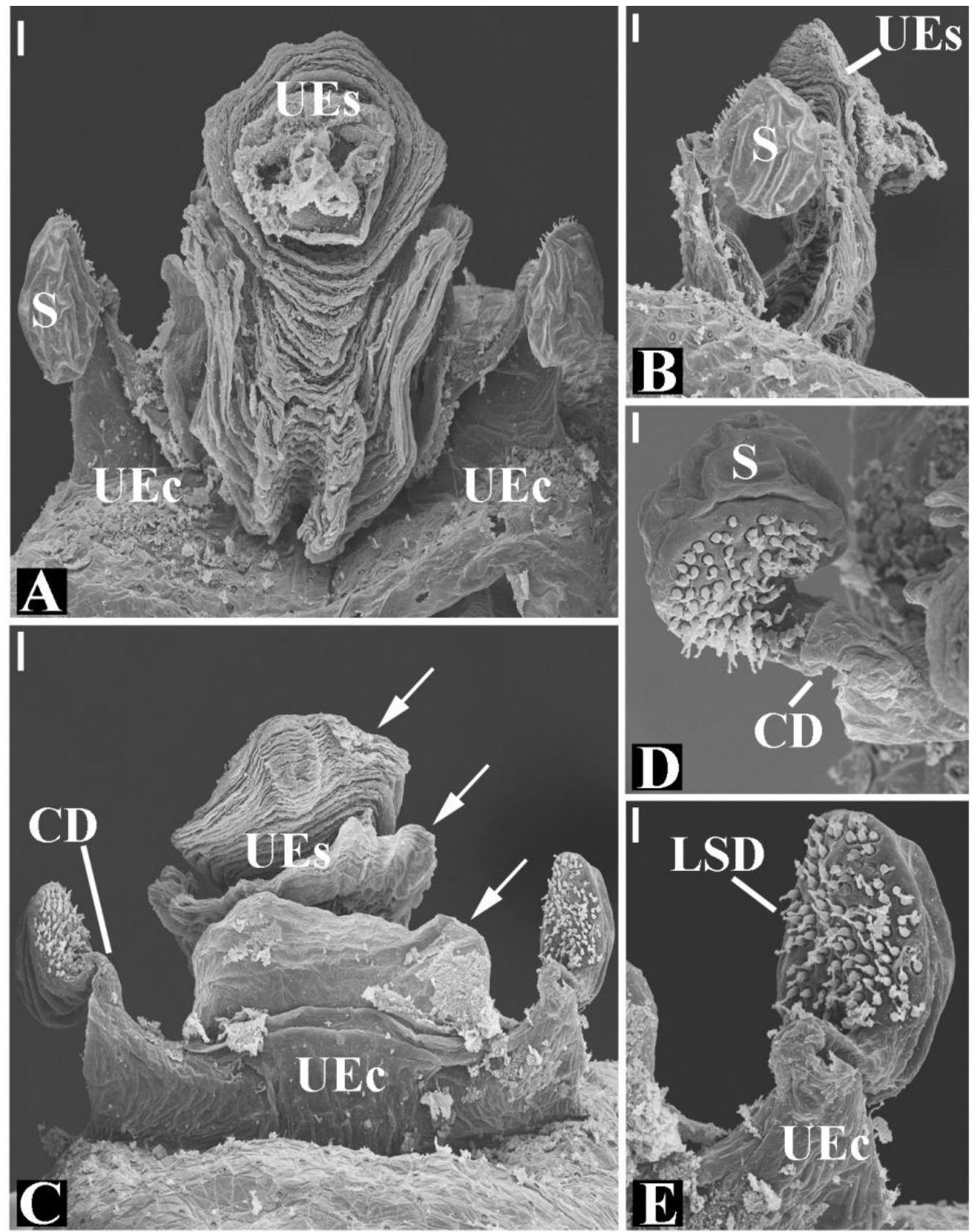

Figure 189. Glenognatha sp. nov. 6 from Colombia. Female genitalia. A, dorsal. B, lateral. C, ventral (arrows, divisions of the uterus externus sac) D-E, spermathecae and copulatory duct. Scale bars, $20 \mu \mathrm{m}$ (A-C), $10 \mu \mathrm{m}$ (D, E). CD: copulatory duct. LSD: long-stem gland ducts. UEc: uterus externus chamber. UEs: uterus externus sac. S: spermathecae. 


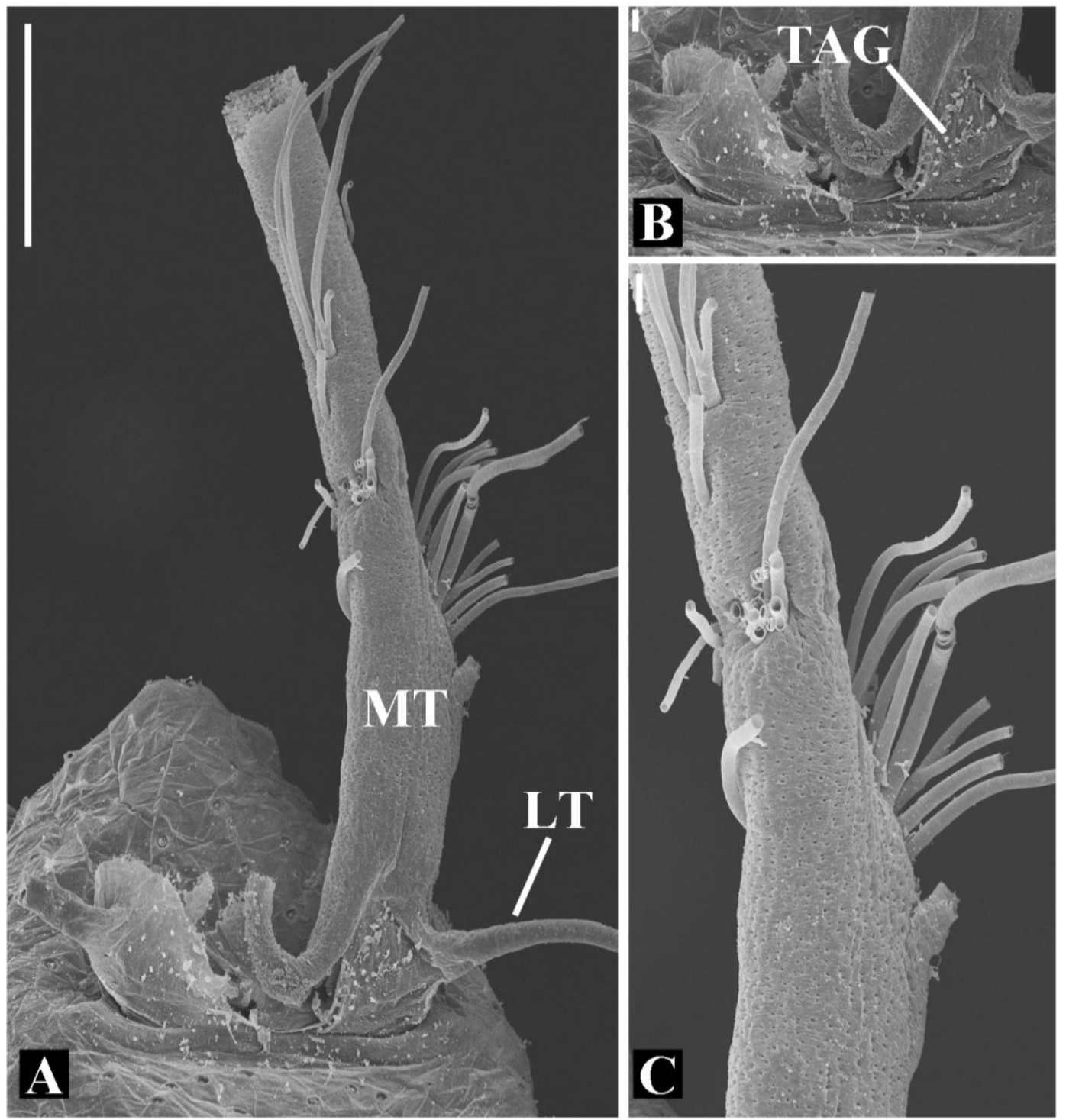

Figure 190. Glenognatha sp. nov. 6 from Colombia. Female tracheal system. A, dorsal. B, tracheal spiracle posterior view. C, median trunk cuticle. Scale bars, $100 \mu \mathrm{m}$ (A), $10 \mu \mathrm{m}(\mathrm{B}, \mathrm{C})$. LT: lateral trachea. MT: median trunks. TAG: tracheal atrium gland. 

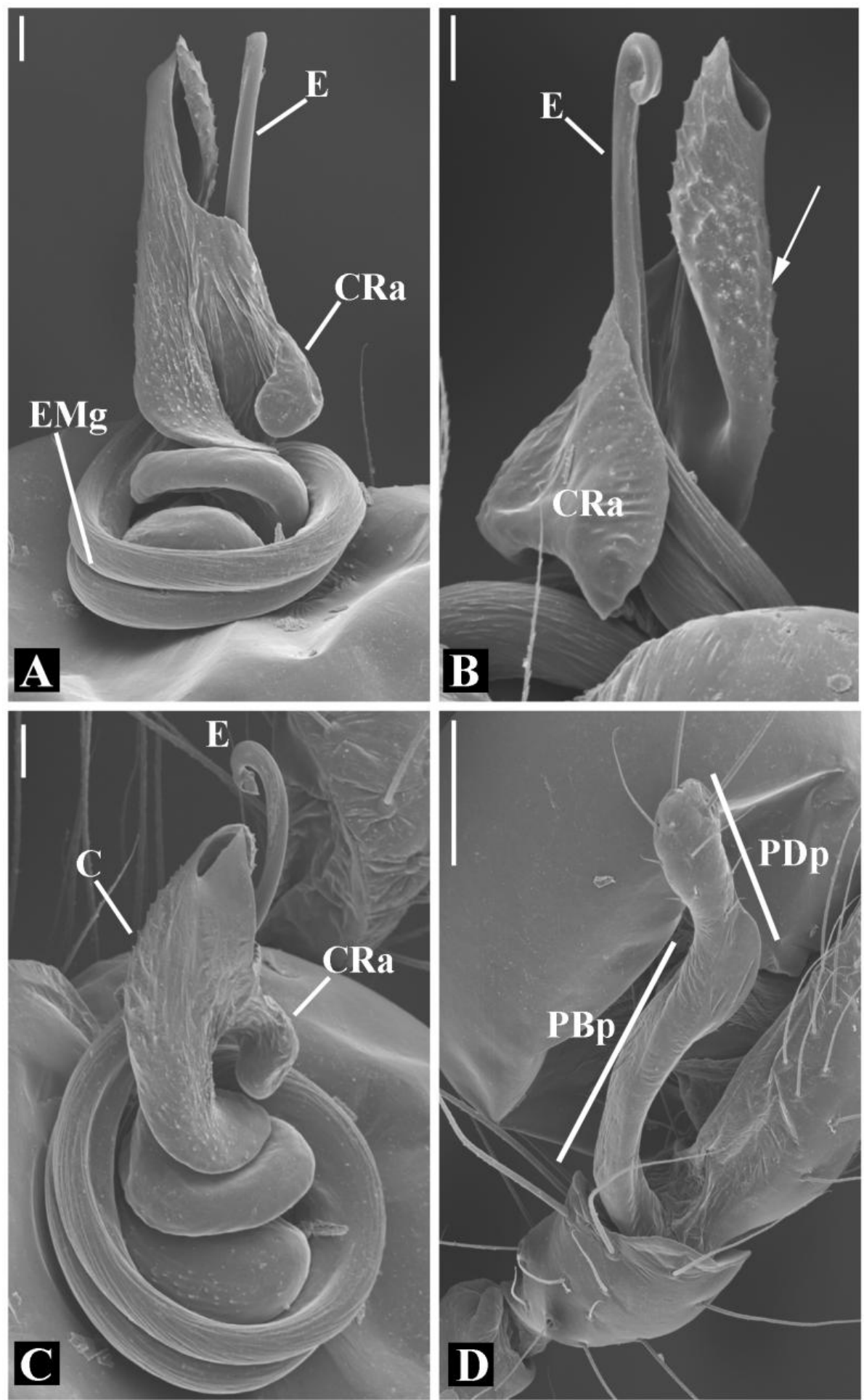

Figure 191. Glenognatha sp. nov. 6 from Peru. A-C, embolus and conductor. A, ventral. B, dorsal (arrow, pointed projections). C, anteroventral. D, paracymbium. Scale bars $20 \mu \mathrm{m}$ (A-C), $100 \mu \mathrm{m}$ (D). C: conductor. CRa: conductor retrolateral apophysis. E: embolus. EMg: embolus medial groove. PBp: paracymbium basal portion. PDp: paracymbium distal portion. 

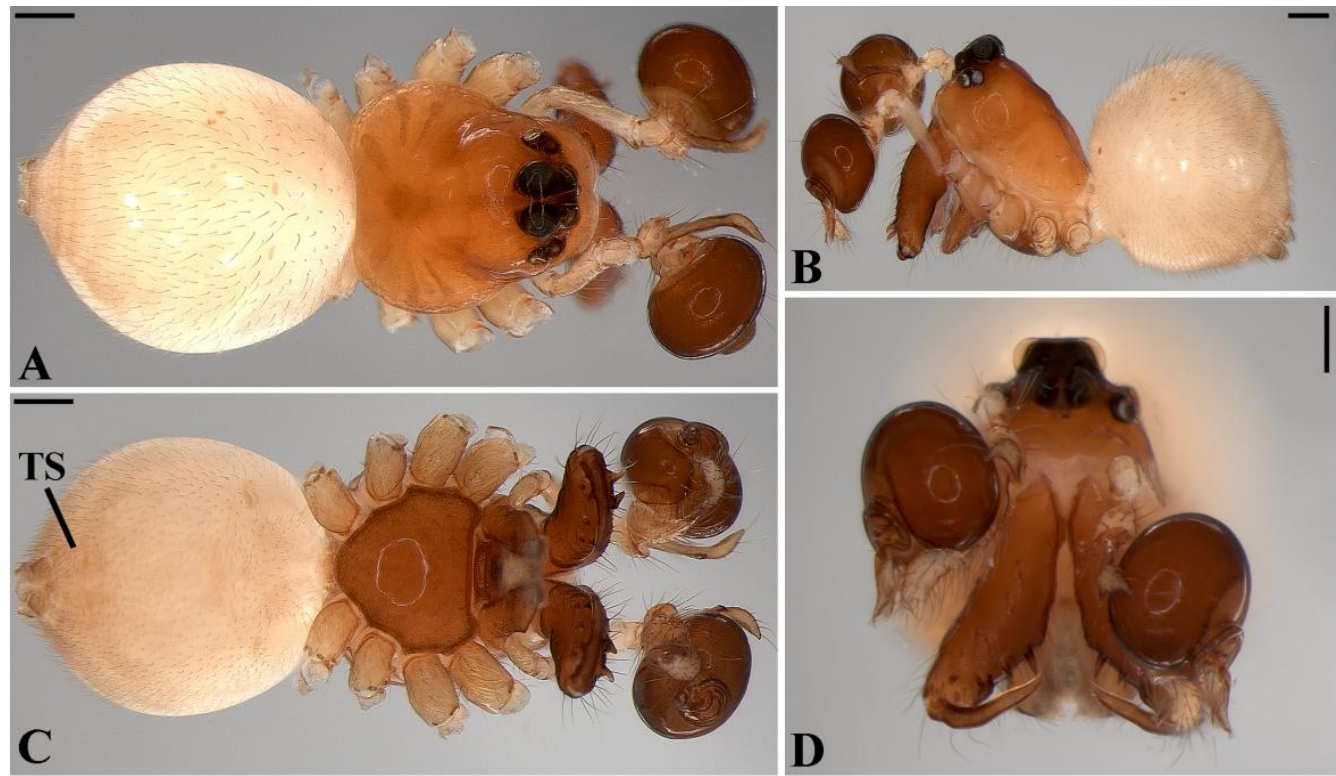

Figure 192. Glenognatha sp. nov. 3 from Brazil (MCTP 13771). Male habitus. A, dorsal. B, lateral. C, ventral. D, frontal. Scale bars, $200 \mu \mathrm{m}$. TS: tracheal spiracle.
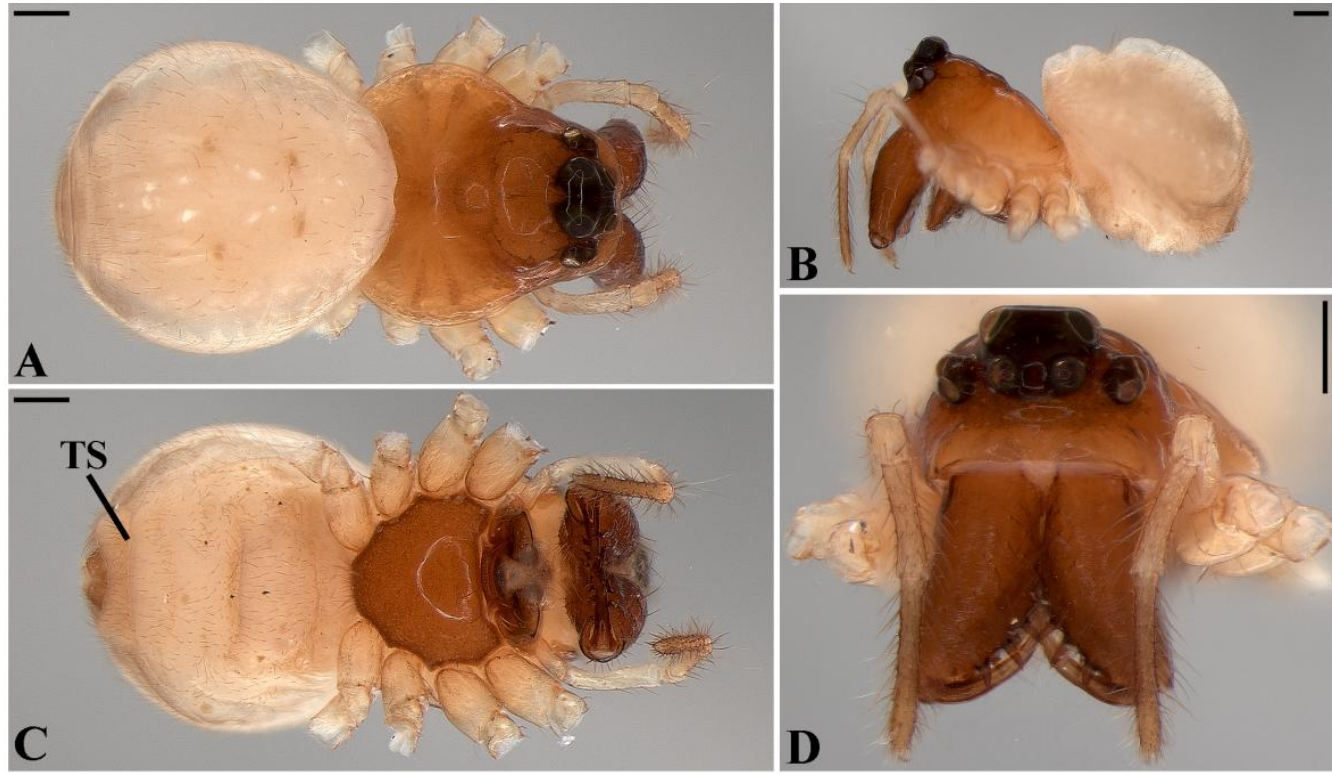

Figure 193. Glenognatha sp. nov. 3 from Brazil (MCTP 17493). Female habitus. A, dorsal. B, lateral. C, ventral. D, frontal. Scale bars, $200 \mu \mathrm{m}$. TS: tracheal spiracle. 


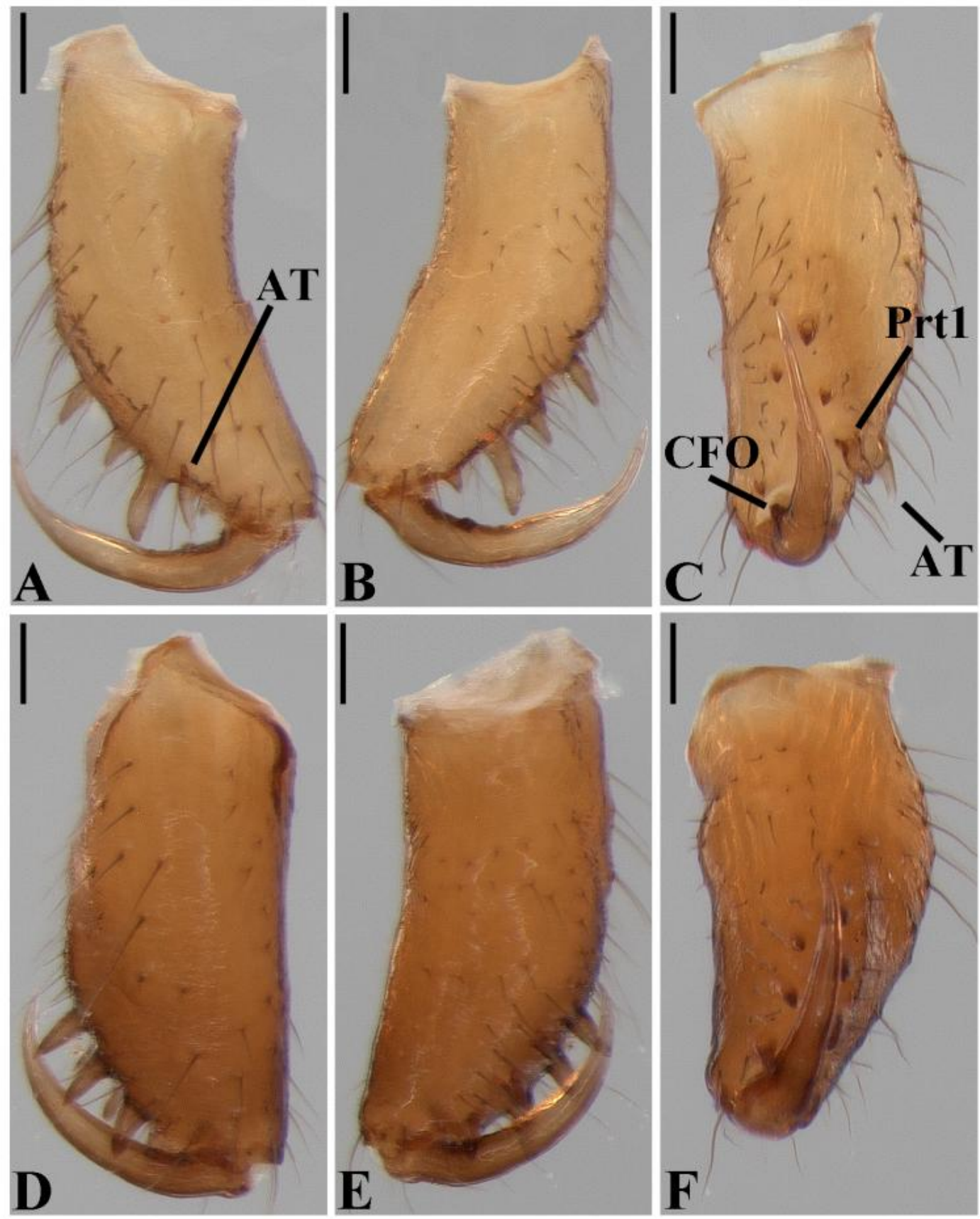

Figure 194. Glenognatha sp. nov. 3 from Brazil. A-C, Male left chelicerae (IBSP 57091). A, anterior. B, posterior. C, mesal. D-F, Female left chelicerae (IBSP 57078). D, anterior. E, posterior. F, mesal. Scale bars, $100 \mu \mathrm{m}$. AT: Anterior tooth. CFO: cheliceral fang outgrowth. Prt: promarginal tooth. 


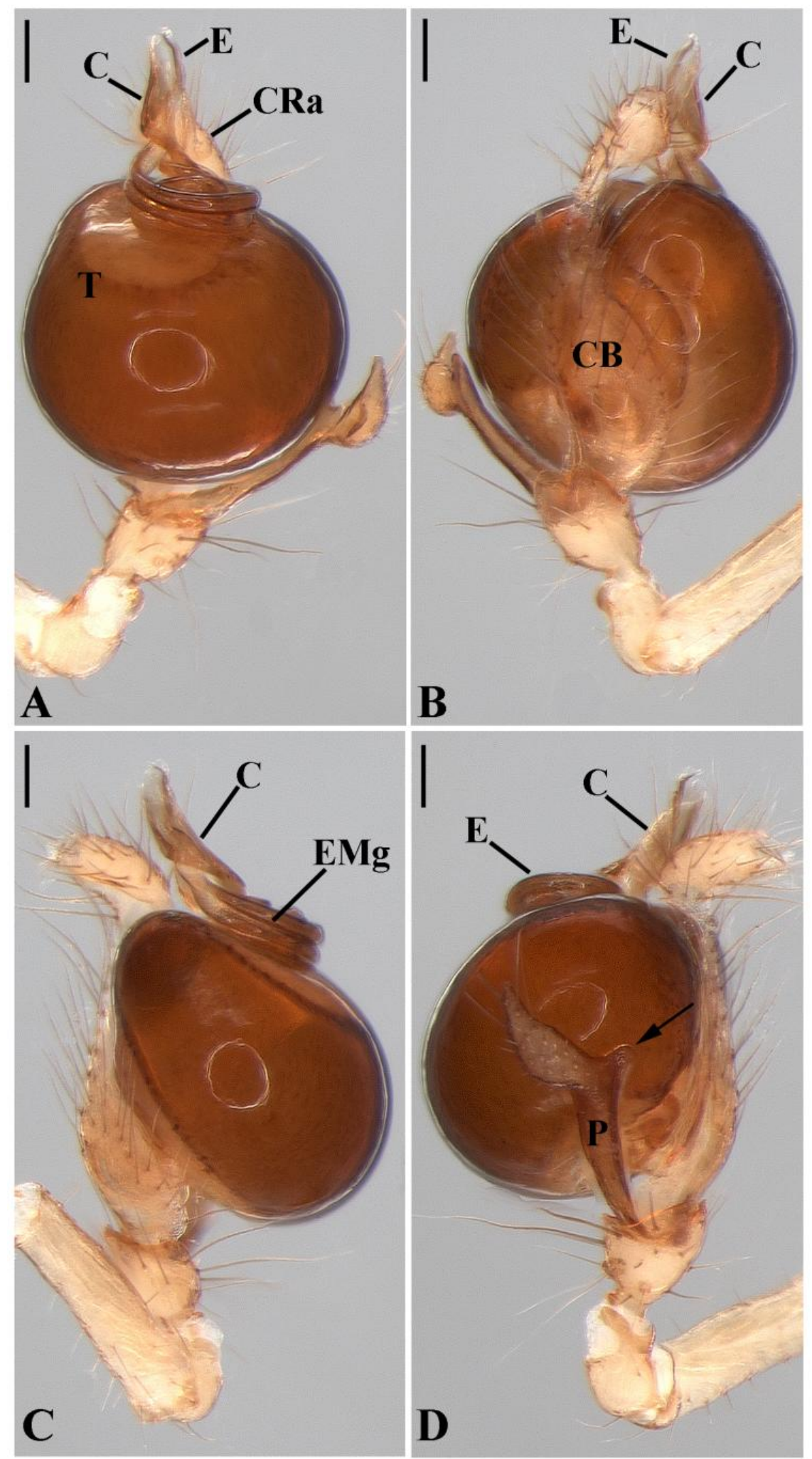

Figure 195. Glenognatha sp. nov. 3 from Brazil (MCTP 13771). Male left palp. A, ventral. B, dorsal. C, prolateral. D, retrolateral (arrow, paracymbium process). Scale bars, $100 \mu \mathrm{m}$. C: conductor. CB: cymbium. CRa: conductor retrolateral apophysis. E: embolus. EMg: embolic medial groove. P: paracymbium. T: tegulum. 

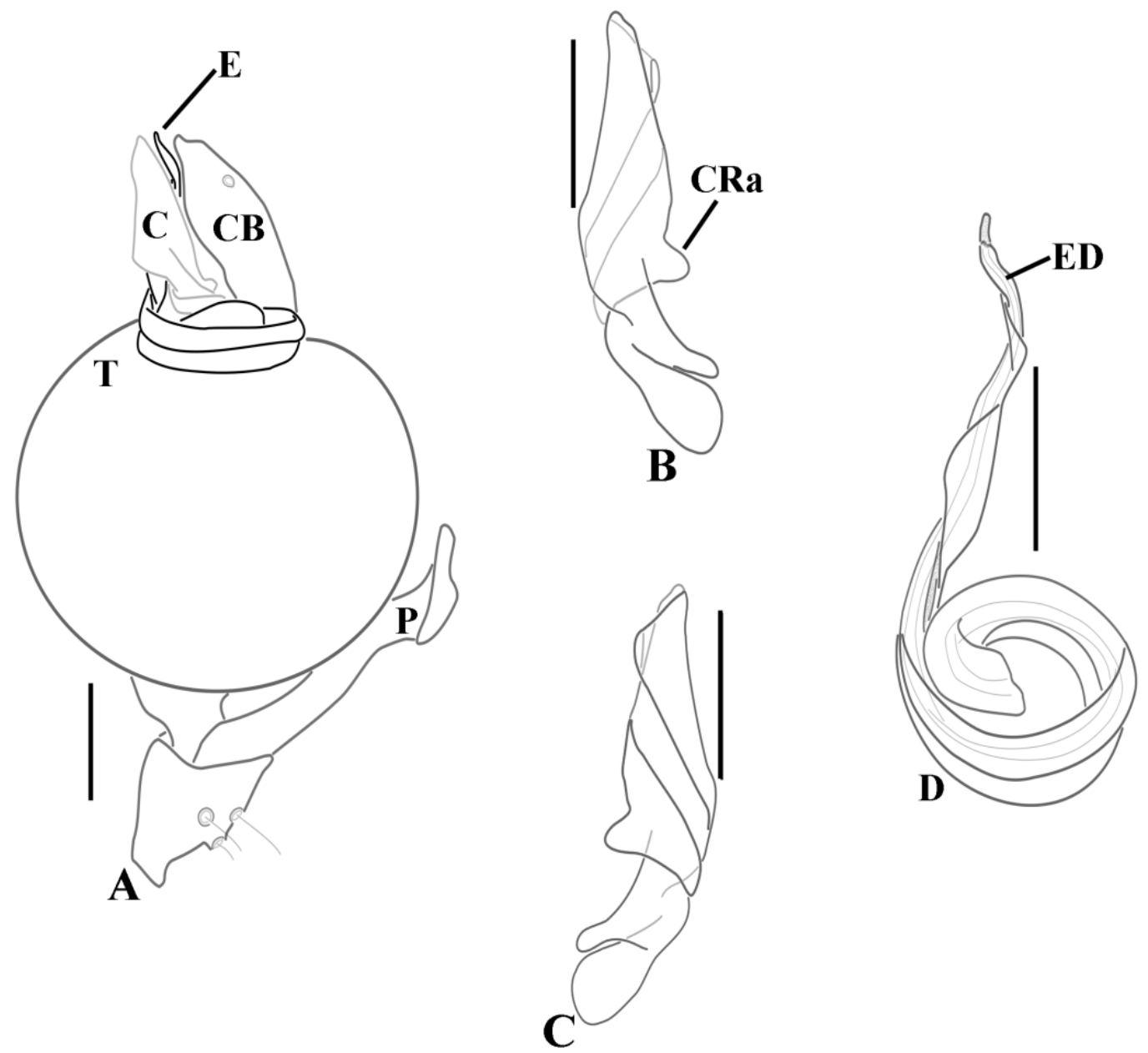

Figure 196. Glenognatha sp. nov. 3 from Brazil (IBSP 57090). Male left palp, schematic. A, ventral. B, conductor ventral. C, conductor dorsal. D, embolus ventral. Scale bars $100 \mu \mathrm{m}$. C: conductor. CB: cymbium. CRa: conductor retrolateral apophysis. E: embolus. ED: ejaculatory duct. P: paracymbium. T: tegulum. 

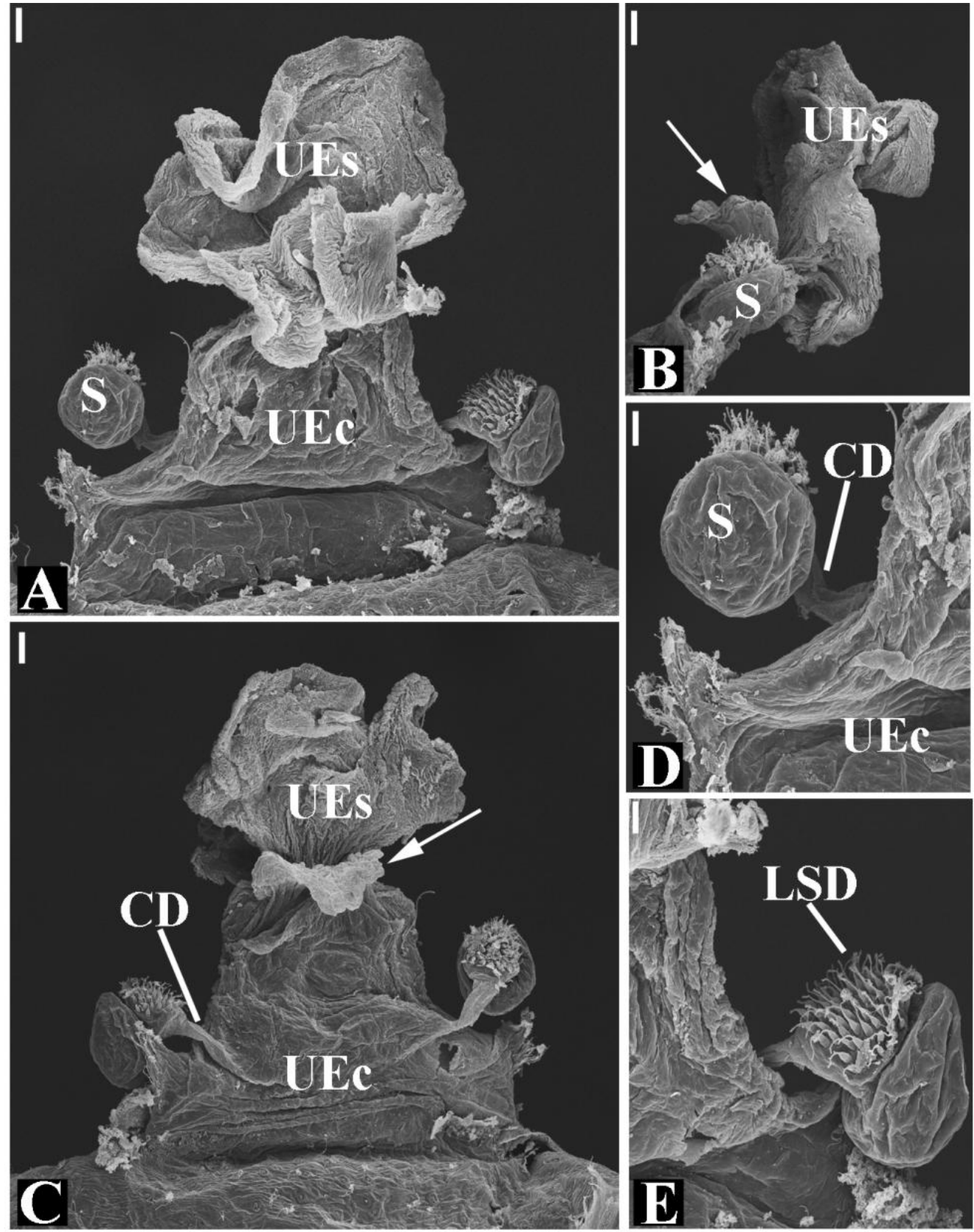

Figure 197. Glenognatha sp. nov. 3 from Brazil. Female genitalia. A, dorsal. B, lateral (arrow, disc-shaped basal region). C, ventral (arrow, disc-shaped basal region). D-E, spermathecae and copulatory duct. Scale bars, $20 \mu \mathrm{m}$ (A-C), $10 \mu \mathrm{m}$ (D, E). CD: copulatory duct. LSD: long-stem gland ducts. UEc: uterus externus chamber. UEs: uterus externus sac. S: spermathecae. 


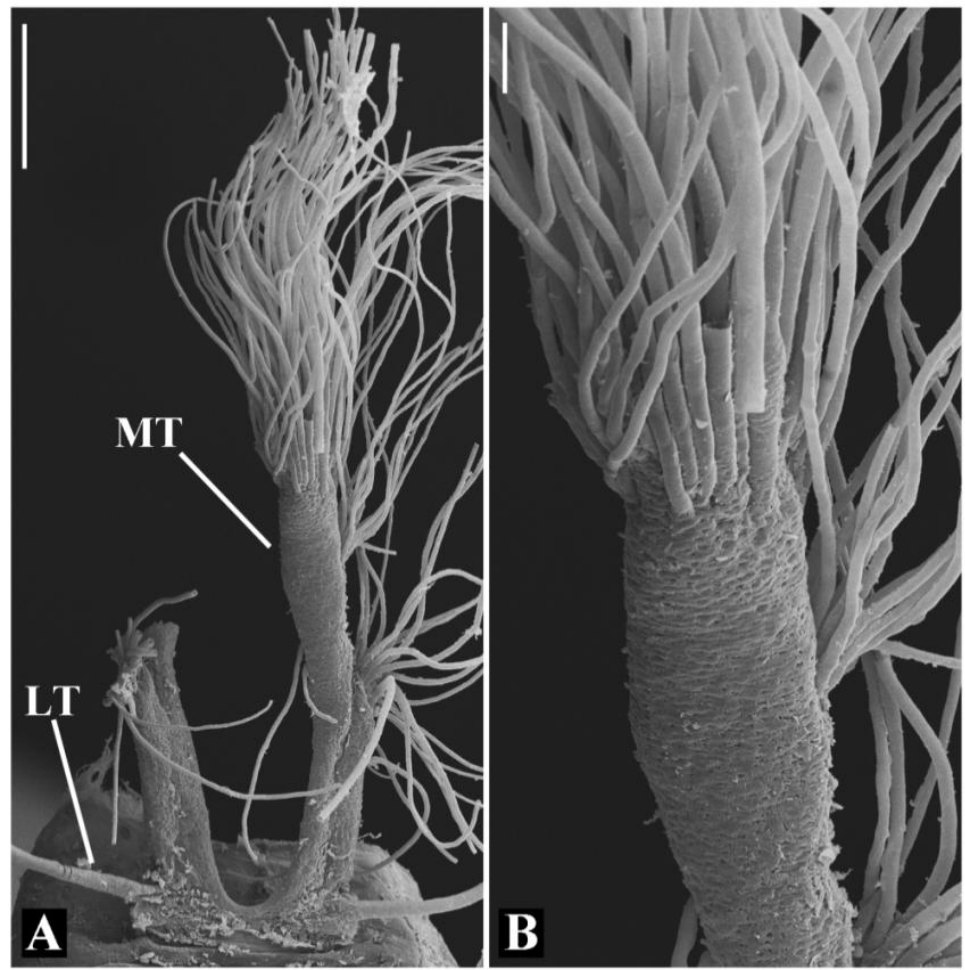

Figure 198. Glenognatha sp. nov. 3 from Brazil. Female tracheal system. A, dorsal. B, median trunk distal. Scale bars $100 \mu \mathrm{m}$ (A), $20 \mu \mathrm{m}$ (B). LT: lateral tracheae. MT: median trunks. TAG: tracheal atrium gland.
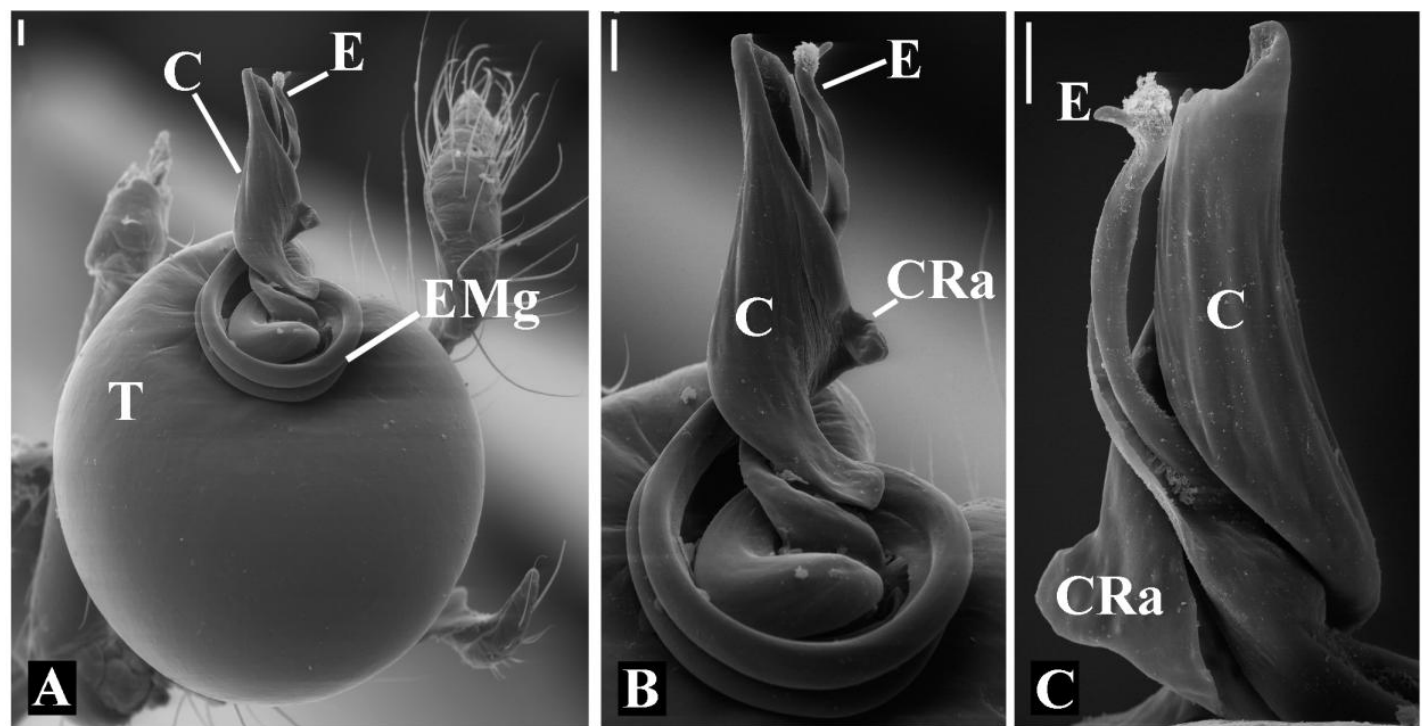

Figure 199. Glenognatha sp. nov. 3 from Brazil. Male left palp. A, ventral. B, embolus and conductor ventral. C, embolus and conductor dorsal. Scale bars $20 \mu \mathrm{m}$. C: conductor. CRa: conductor retrolateral apophysis. E: embolus. EMg: embolic medial groove. T: tegulum. 


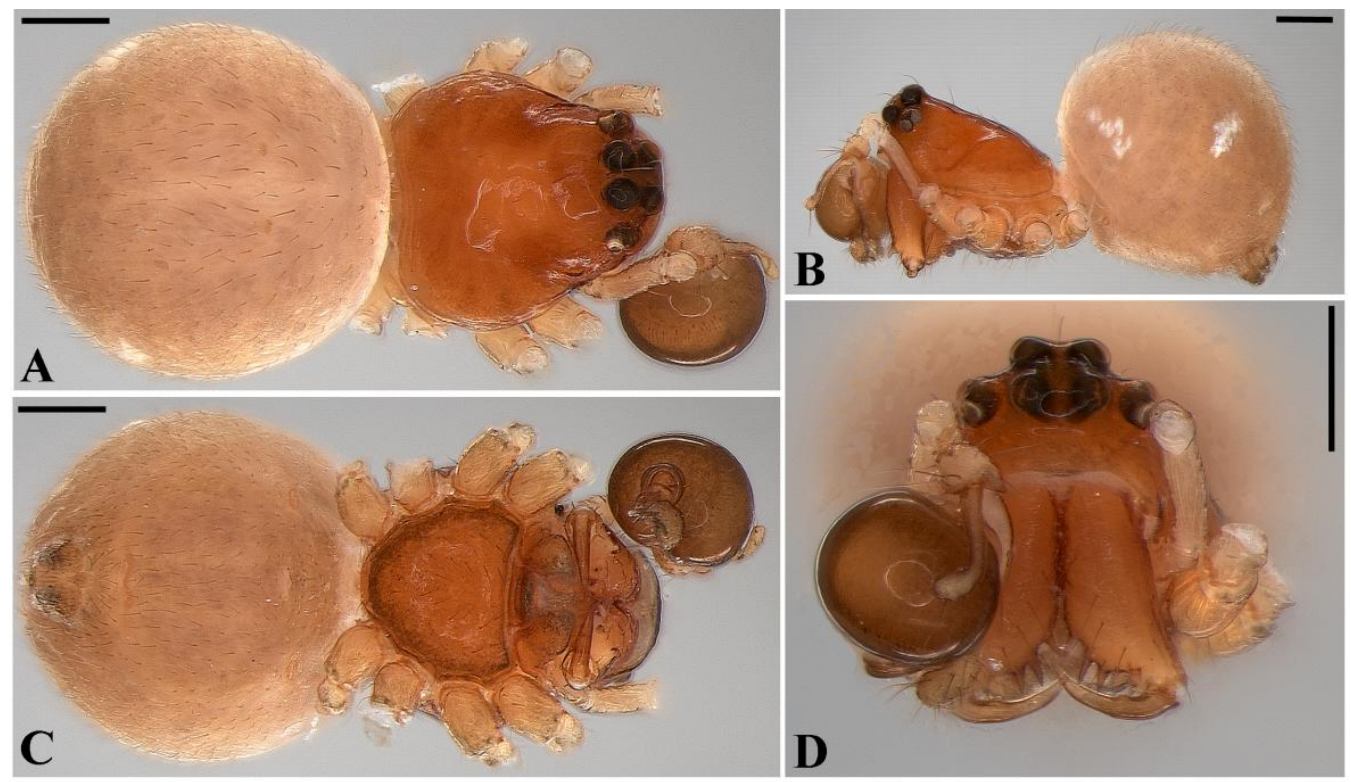

Figure 200. Glenognatha foxi from USA (AMNH). Male habitus. A, dorsal. B, lateral. C, ventral. D, frontal. Scale bars, $200 \mu \mathrm{m}$.

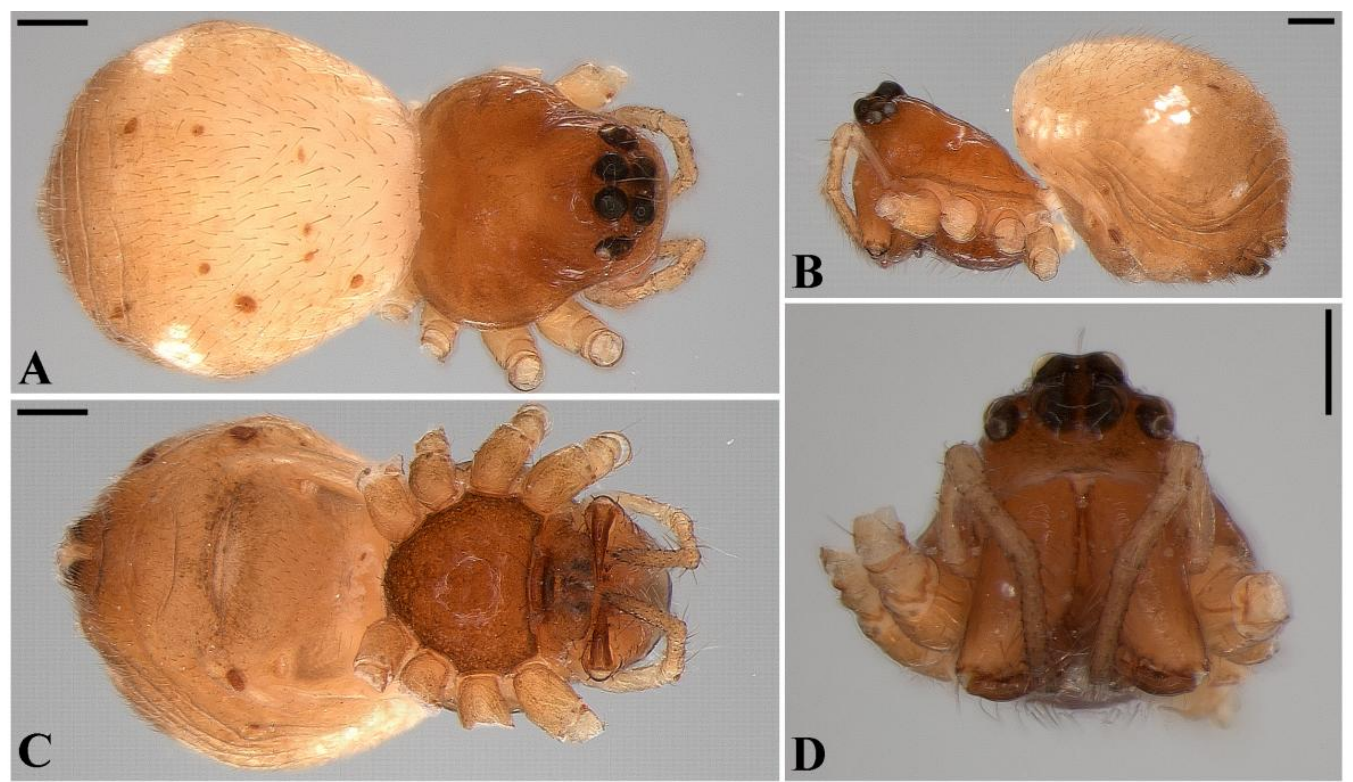

Figure 201. Glenognatha foxi from USA (AMNH). Female habitus. A, dorsal. B, lateral. C, ventral. D, frontal. Scale bars, $200 \mu \mathrm{m}$. 


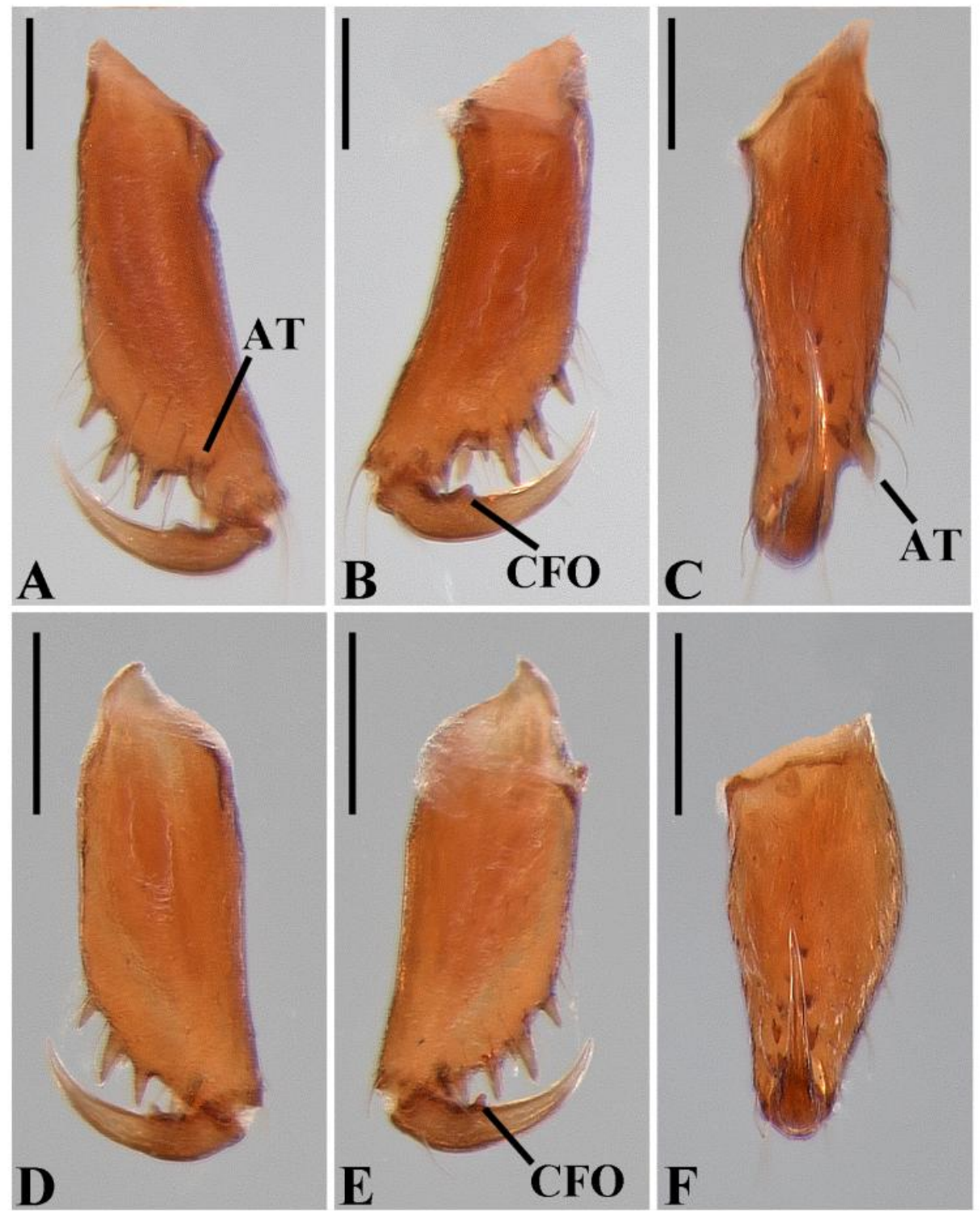

Figure 202. Glenognatha foxi from USA (AMNH). A-C, Male left chelicerae. A, anterior. B, posterior. C, mesal. D-F, Female left chelicerae. D, anterior. E, posterior. F, mesal. Scale bars, $100 \mu \mathrm{m}$. AT: anterior tooth. CFO: cheliceral fang outgrowth. 


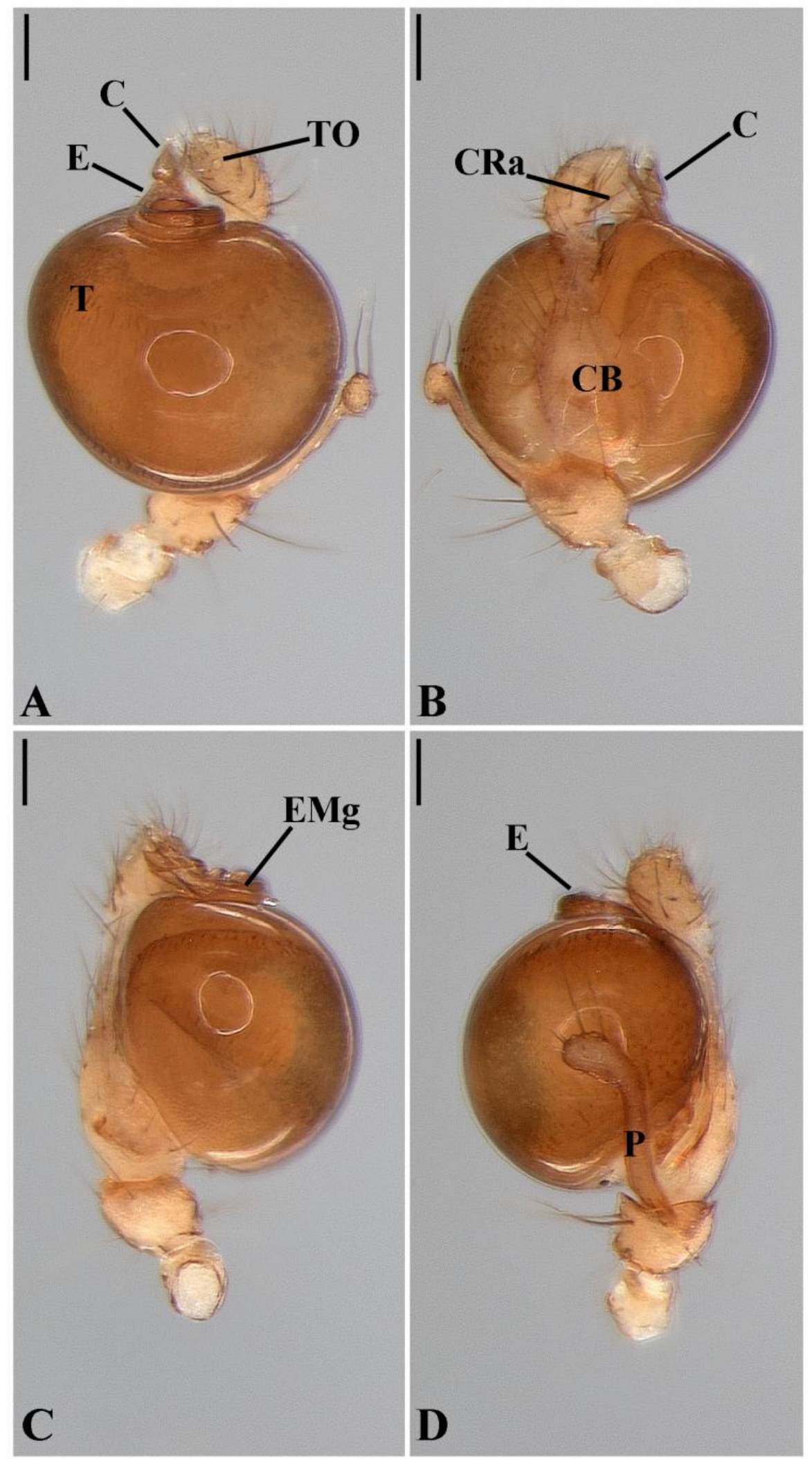

Figure 203. Glenognatha foxi from USA (AMNH). A-C, Male left chelicerae. A, anterior. B, posterior. C, mesal. D-F, Female left chelicerae. D, anterior. E, posterior. F, mesal. Scale bars, $100 \mu \mathrm{m}$. C: conductor. CB: cymbium. CRa: conductor retrolateral apophysis. E: embolus. EMg: embolic medial groove. P: paracymbium. T: tegulum. 


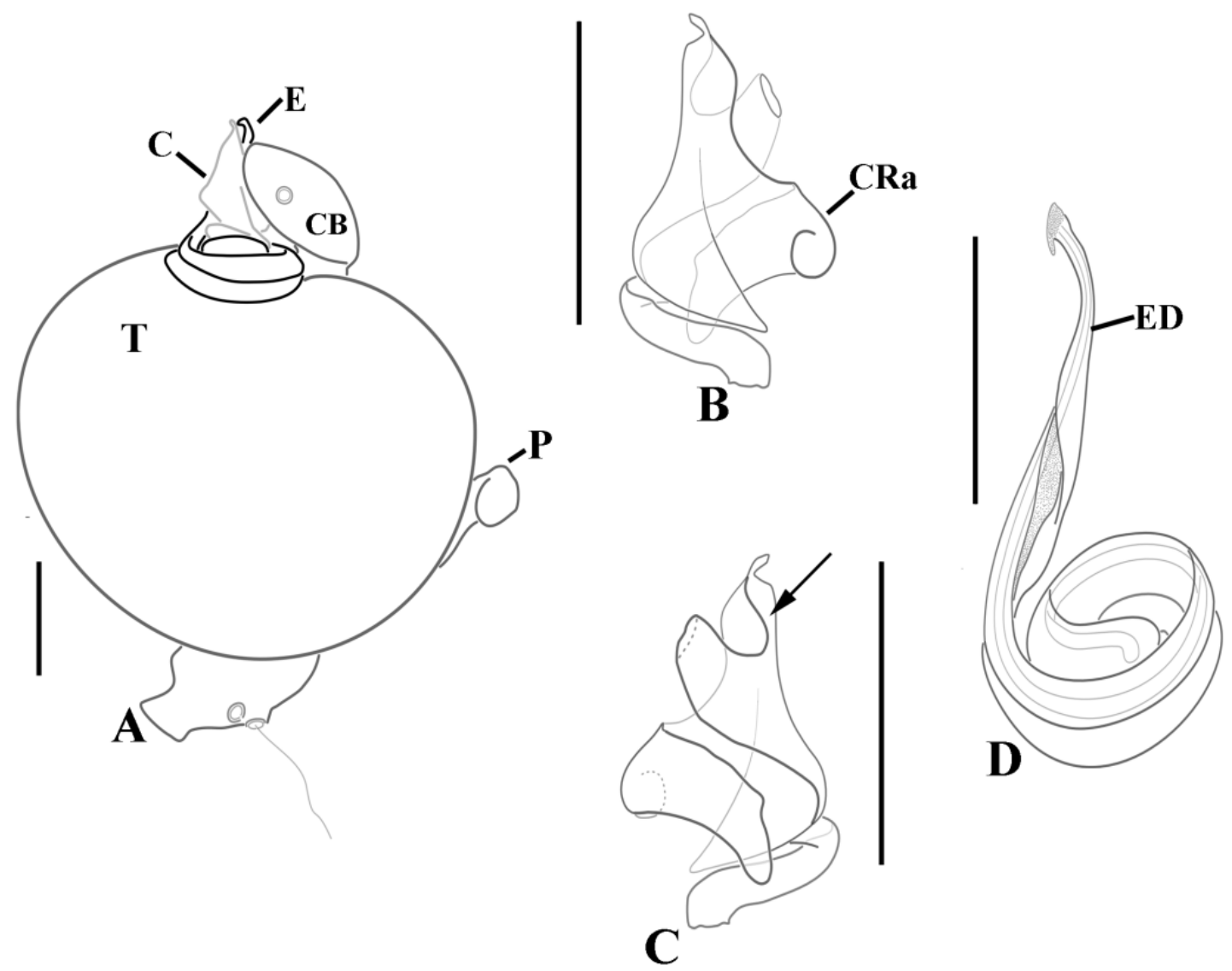

Figure 204. Glenognatha foxi from USA (AMNH). Male left palp, schematic. A, ventral. B, conductor ventral. C, conductor dorsal (arrow, excavation). D, embolus ventral. Scale bars $100 \mu \mathrm{m}$. C: conductor. CB: cymbium. CRa: conductor retrolateral apophysis. E: embolus. ED: ejaculatory duct. P: paracymbium. T: tegulum. 

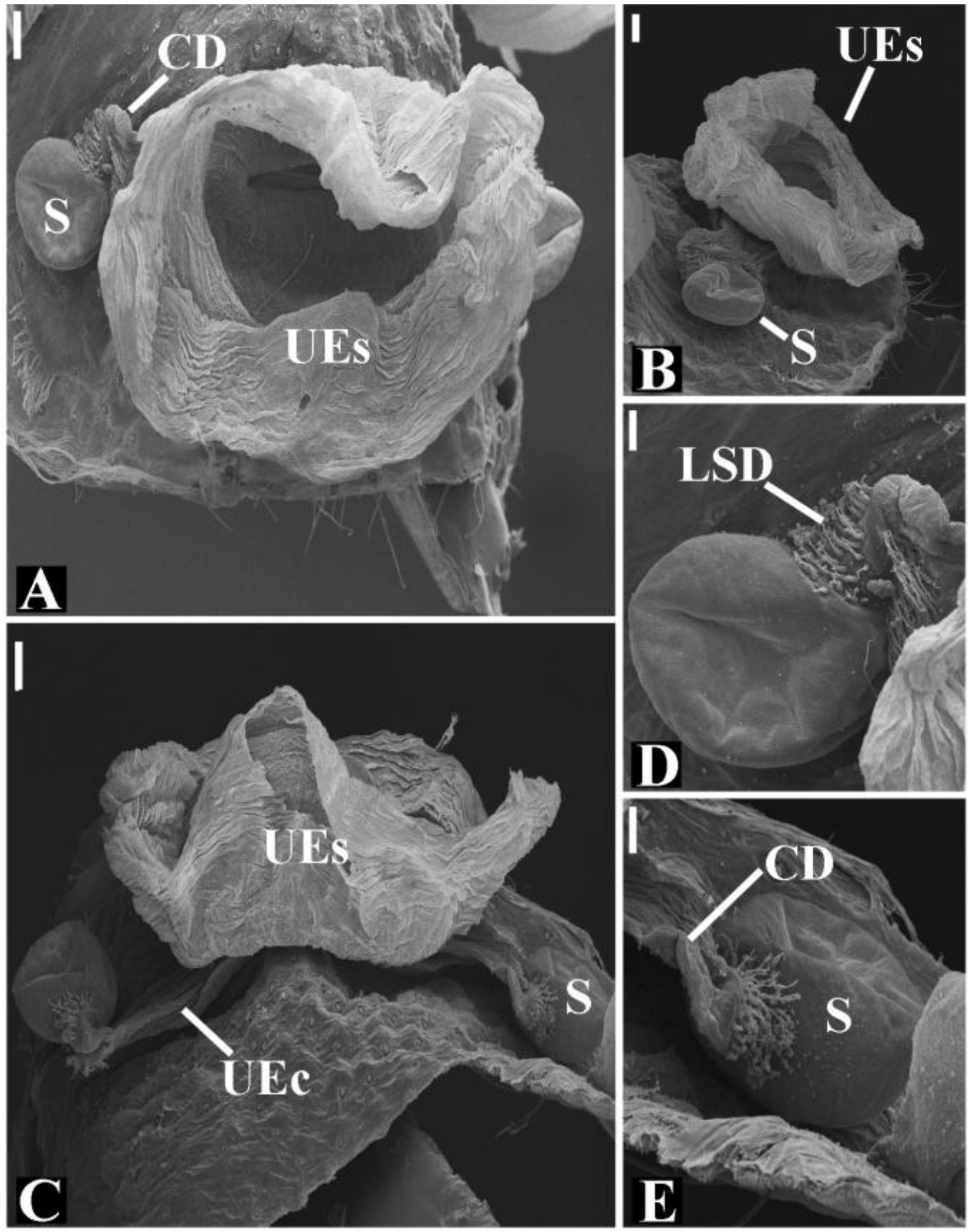

Figure 205. Glenognatha foxi from USA. Female genitalia. A, dorsal. B, lateral. C, anterior. D-E, spermathecae and copulatory duct. Scale bars, $20 \mu \mathrm{m}$ (A-C), $10 \mu \mathrm{m}$ (D, E). CD: copulatory duct. LSD: long-stem gland ducts. UEc: uterus externus chamber. UEs: uterus externus sac. S: spermathecae. 


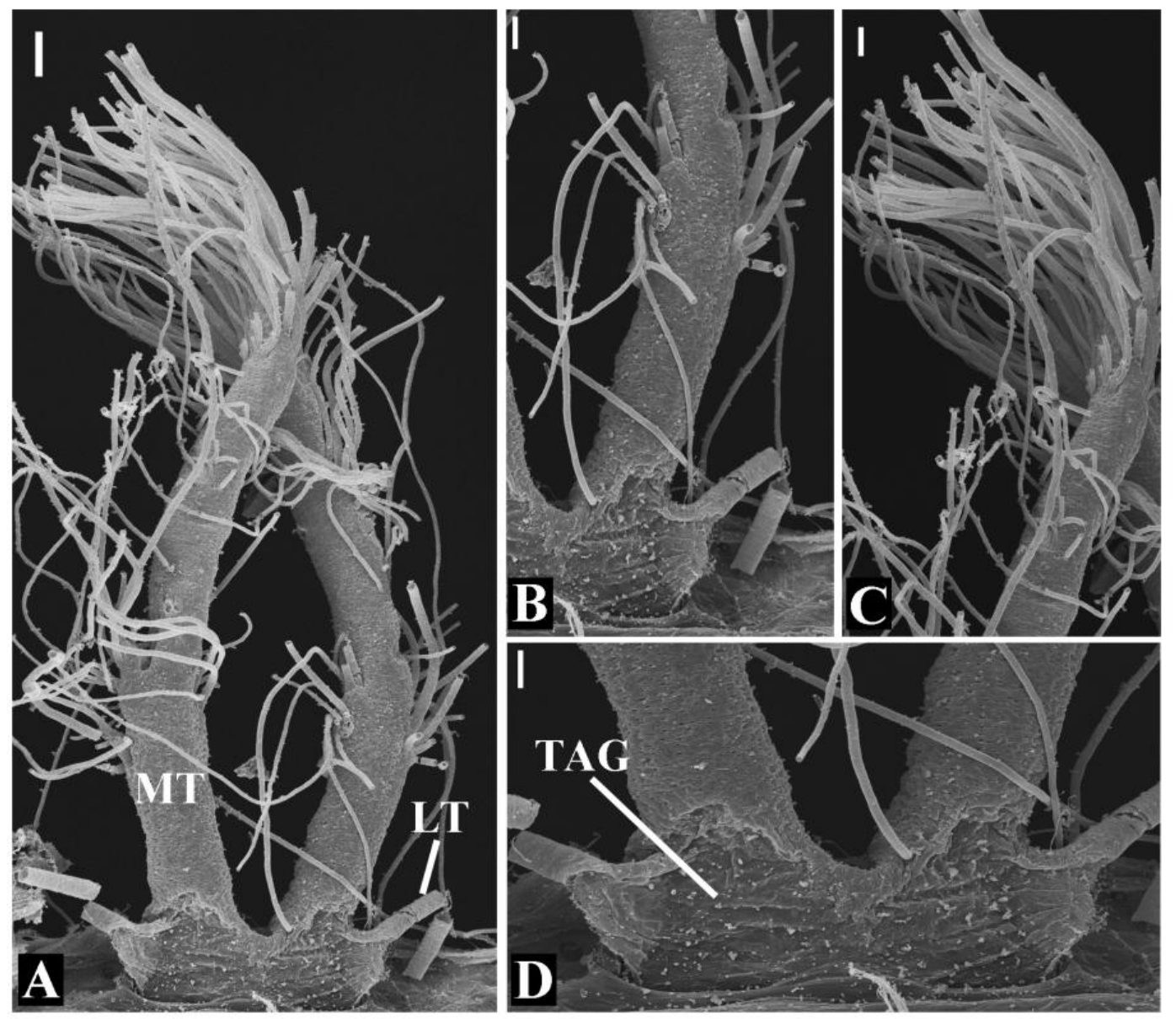

Figure 206. Glenognatha foxi from USA. Female tracheal system. A, dorsal. B, median and lateral trunks. C, median trunk distal. D, tracheal spiracle posterior view. Scale bars, $20 \mu \mathrm{m}$ (A), $10 \mu \mathrm{m}$ (B-D). LT: lateral tracheae. MT: median trunks. TAG: tracheal atrium gland. 

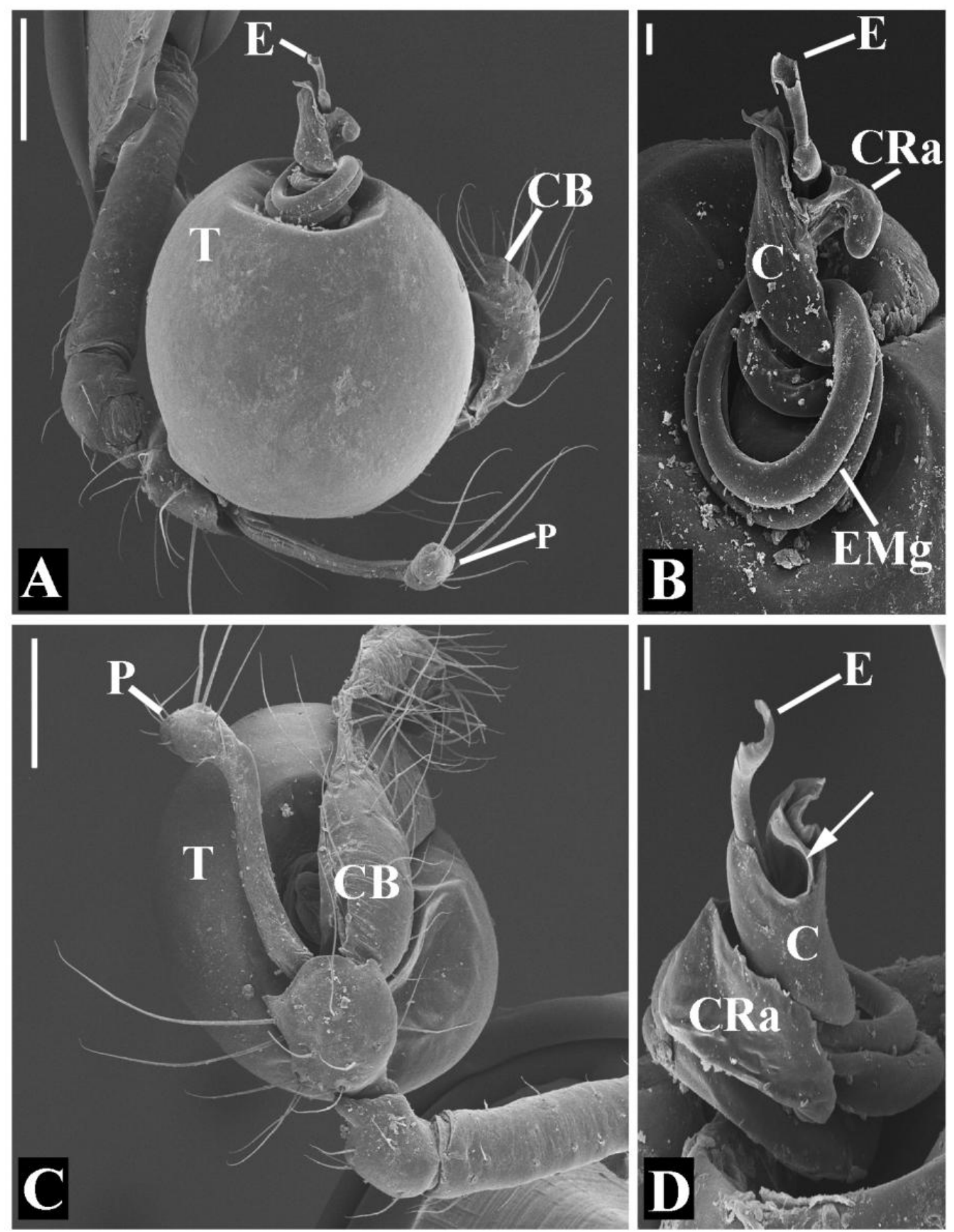

Figure 207. Glenognatha foxi from USA. A, Male left palp ventral. B, Embolus and conductor ventroretrolateral. C, Paracymbium. D, Embolus and conductor dorsal (arrow, excavation). Scale bars $100 \mu \mathrm{m}(\mathrm{A}, \mathrm{C}), 10 \mu \mathrm{m}$ (B, D). C: conductor. CB: cymbium. CRa: conductor retrolateral apophysis. E: embolus. EMg: embolic medial groove. P: paracymbium. T: tegulum. 

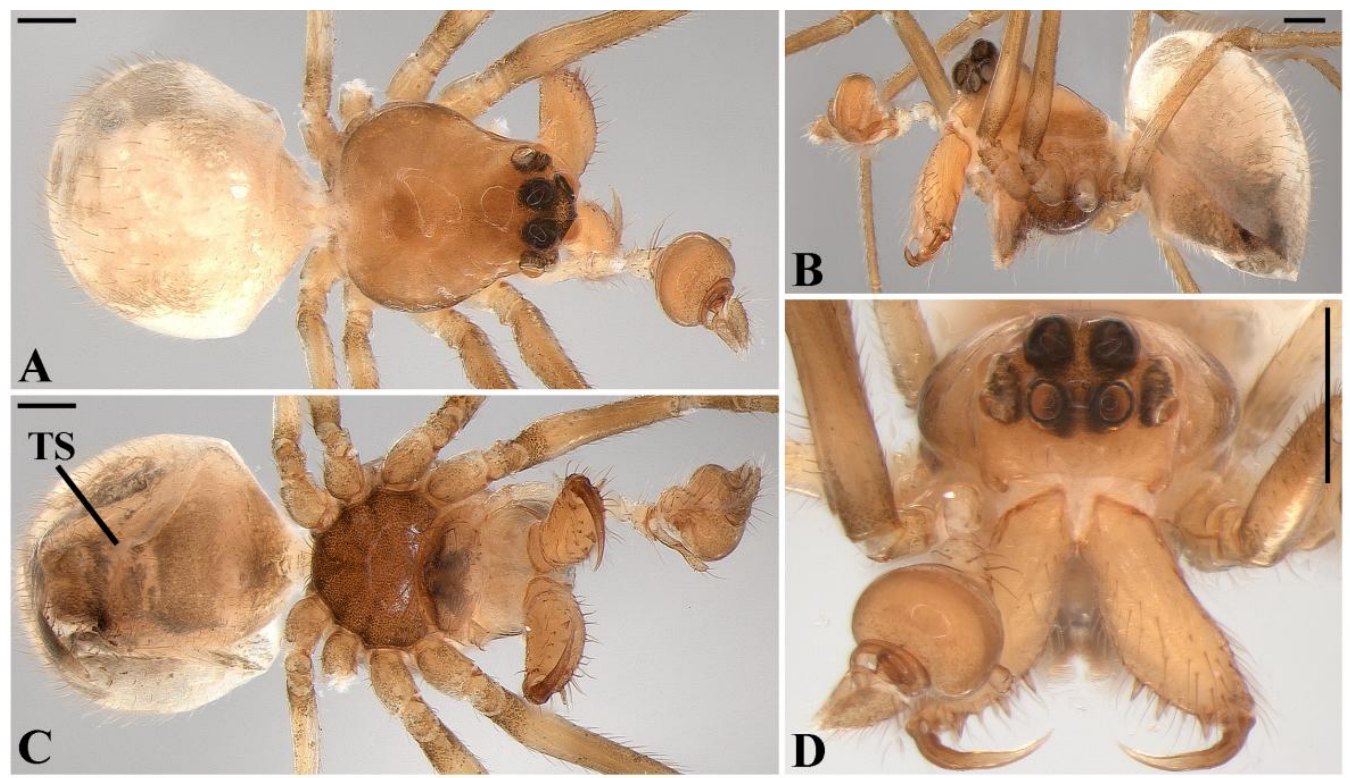

Figure 208. Glenognatha sp. nov. 9 from Brazil (Holotype IBSP 120209). Male habitus. A, dorsal. B, lateral. C, ventral. D, frontal. Scale bars, $200 \mu \mathrm{m}$. TS: tracheal spiracle.

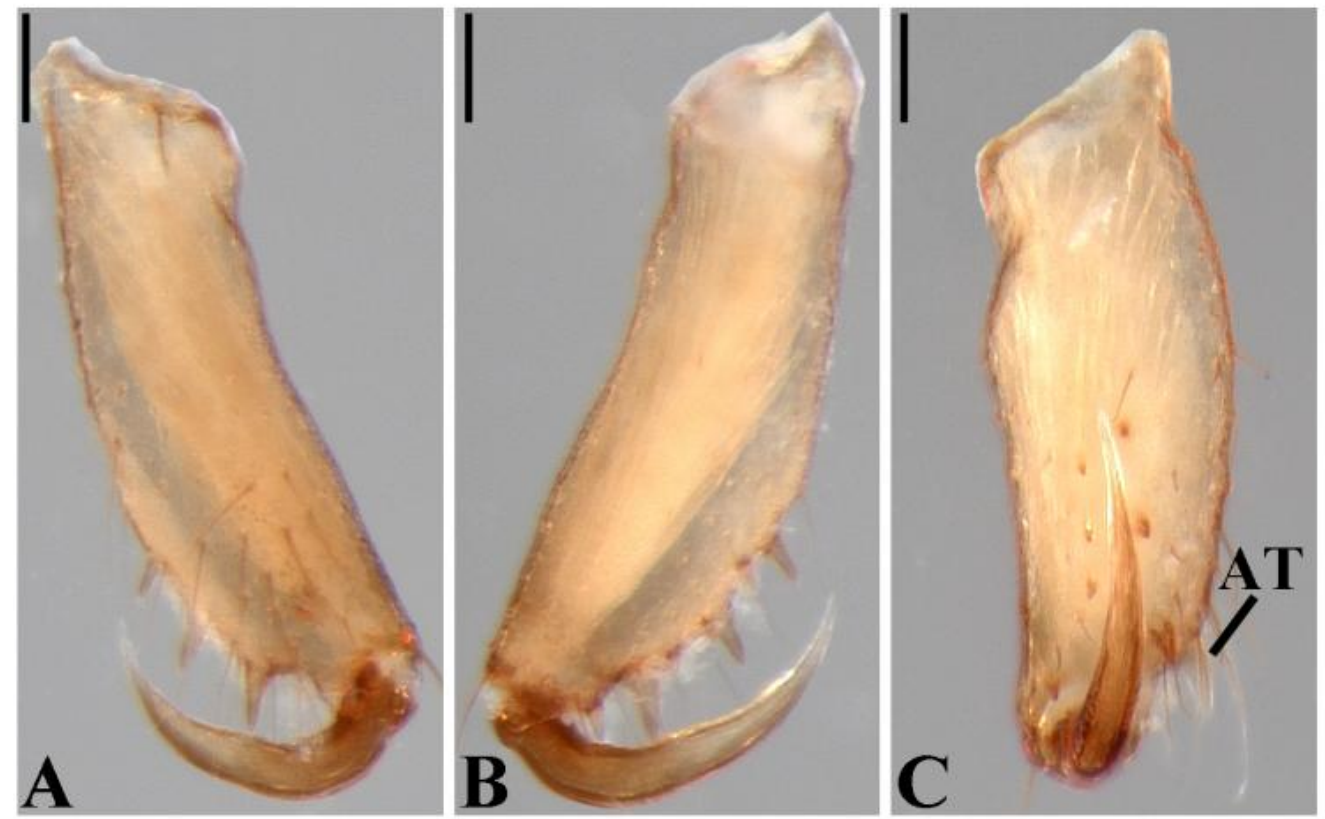

Figure 209. Glenognatha sp. nov. 9 from Brazil (IBSP120220). A-C, Male left chelicerae. A, anterior. B, posterior. C, mesal. Scale bars, $100 \mu \mathrm{m}$. AT: anterior tooth. 


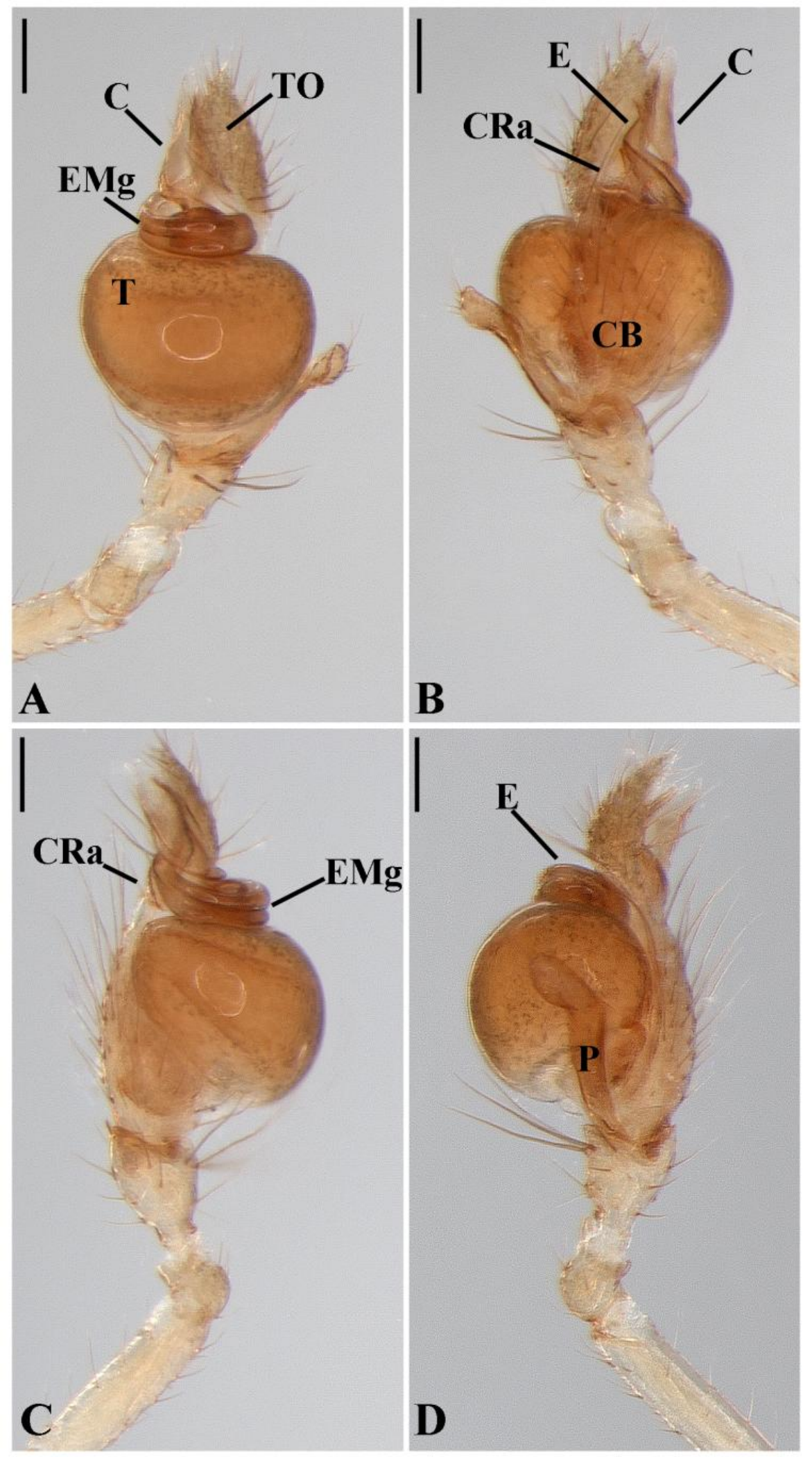

Figure 210. Glenognatha sp. nov. 9 from Brazil (Holotype IBSP 120209). Male left palp. A, ventral. B, dorsal. C, prolateral. D, retrolateral. Scale bars, $100 \mu \mathrm{m}$. C: conductor. CB: cymbium. CRa: conductor retrolateral apophysis. E: embolus. EMg: embolic medial groove. P: paracymbium. T: tegulum. TO: tarsal organ. 

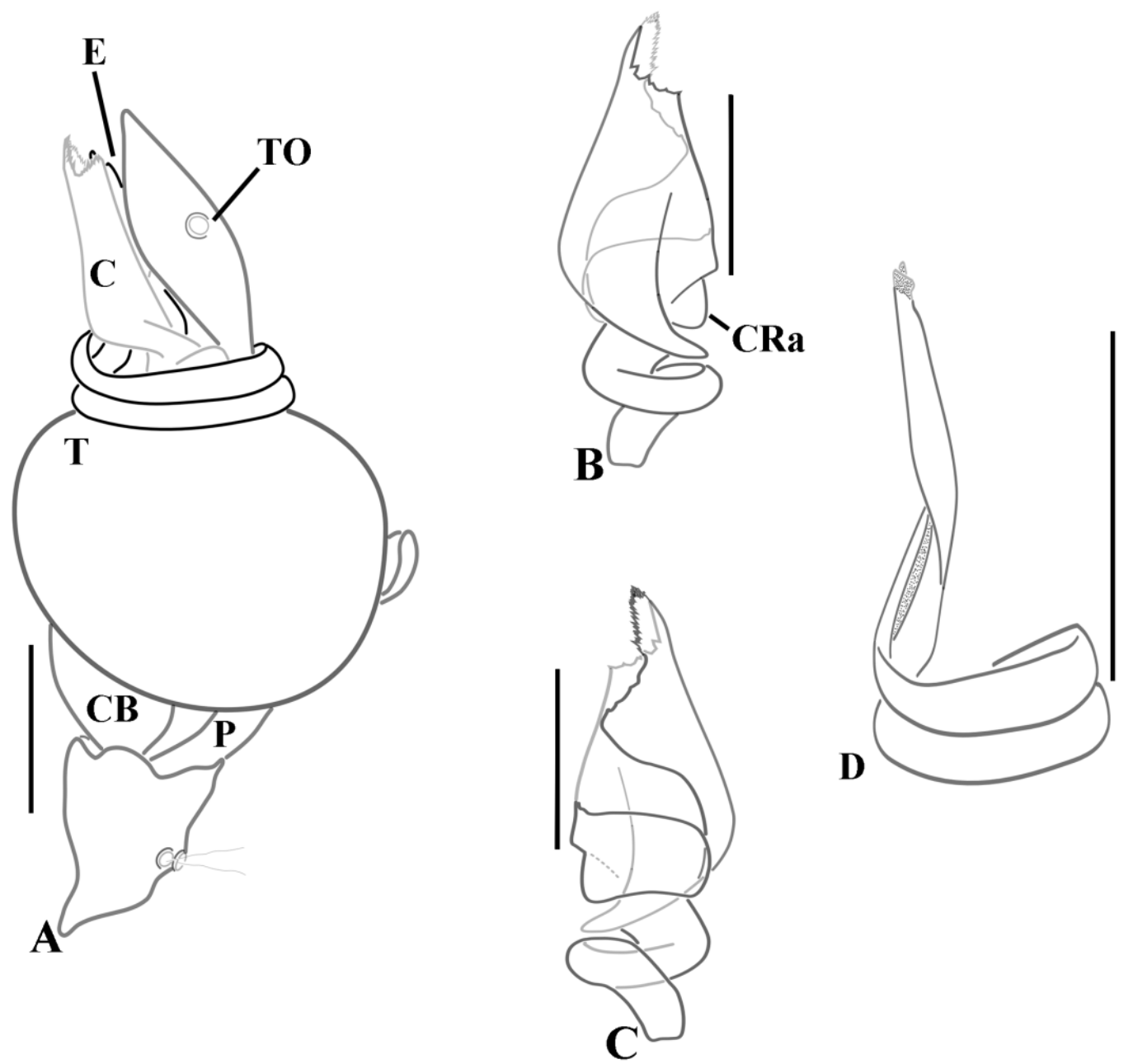

Figure 211. Glenognatha sp. nov. 9 from Brazil (IBSP 120220). Male left palp, schematic. A, ventral. B, conductor ventral. C, conductor dorsal. D, embolus ventral. Scale bars $100 \mu \mathrm{m}$. C: conductor. CB: cymbium. CRa: conductor retrolateral apophysis. E: embolus. P: paracymbium. T: tegulum. TO: tarsal organ. 


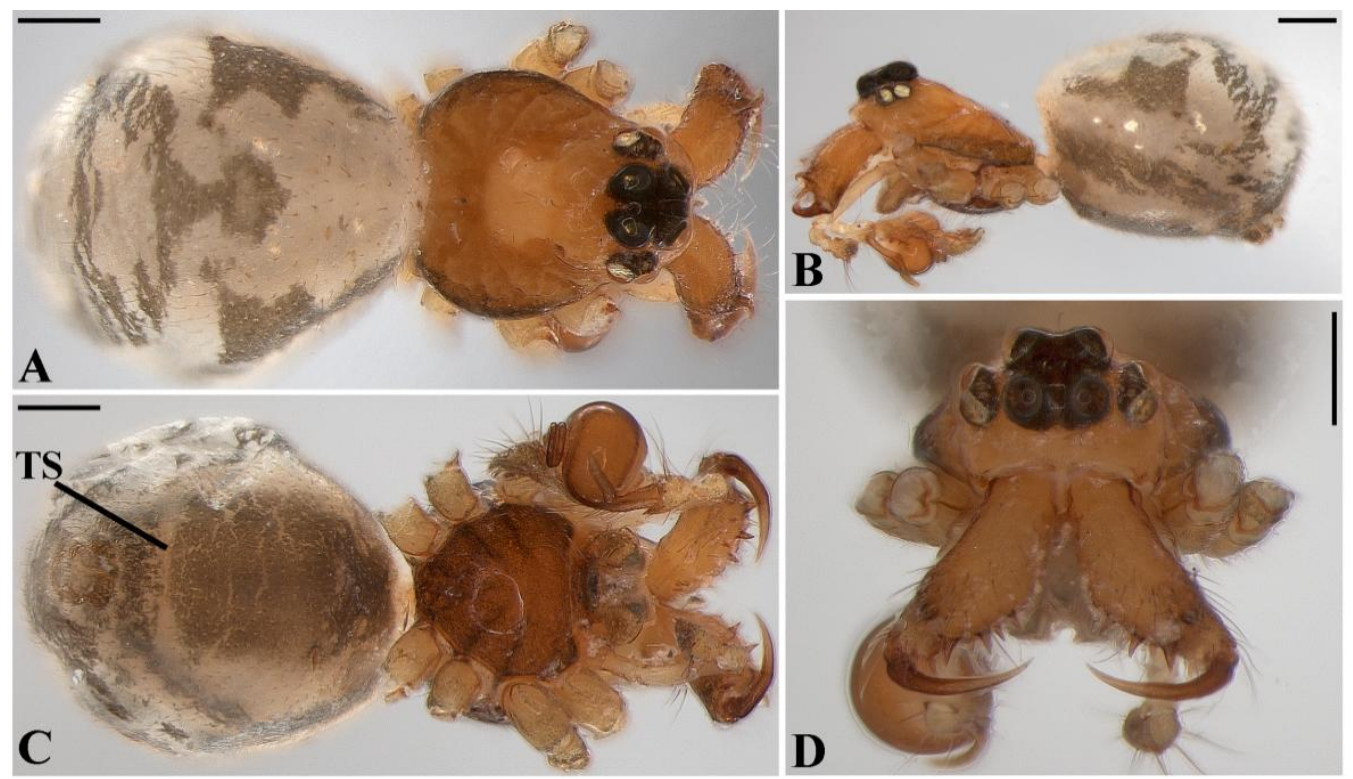

Figure 212. Glenognatha sp. nov. 10 from Peru (MUSM 054913). Male habitus. A, dorsal. B, lateral. C, ventral. D, frontal. Scale bars, $200 \mu \mathrm{m}$. TS: tracheal spiracle.
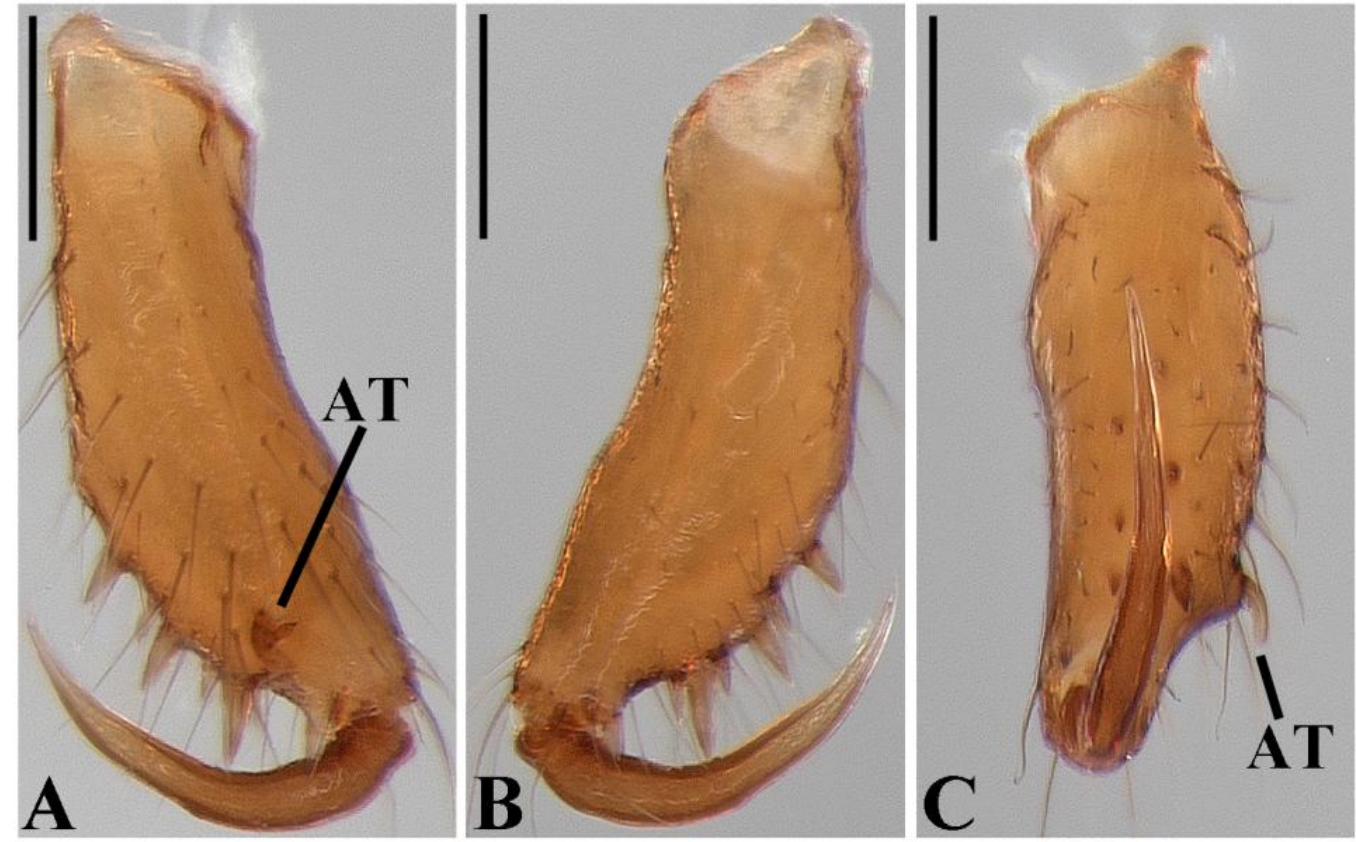

Figure 213. Glenognatha sp. nov. 10 from Peru (MUSM 0504905). A-C, Male left chelicerae. A, anterior. B, posterior. C, mesal. D-F, Female left chelicerae. D, anterior. $\mathbf{E}$, posterior. F, mesal. Scale bars, $100 \mu \mathrm{m}$. AT: anterior tooth. 


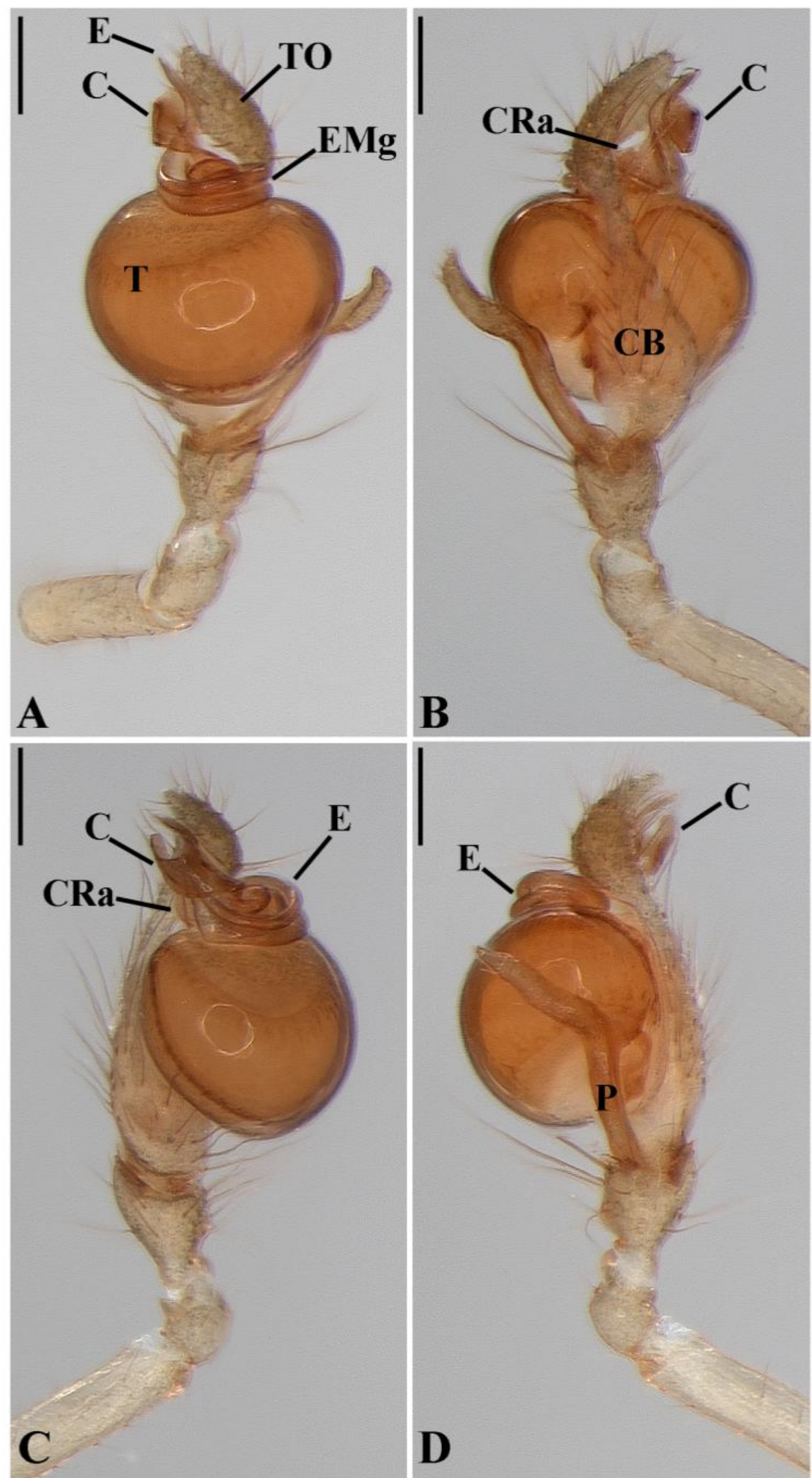

Figure 214. Glenognatha sp. nov. 10 from Peru (Holotype MUSM 050913). Male left palp. A, ventral. B, dorsal. C, prolateral. D, retrolateral. Scale bars,100 $\mu \mathrm{m}$. C: conductor. CB: cymbium. CRa: conductor retrolateral apophysis. E: embolus. EMg: embolic medial groove. P: paracymbium. T: tegulum. TO: tarsal organ. 

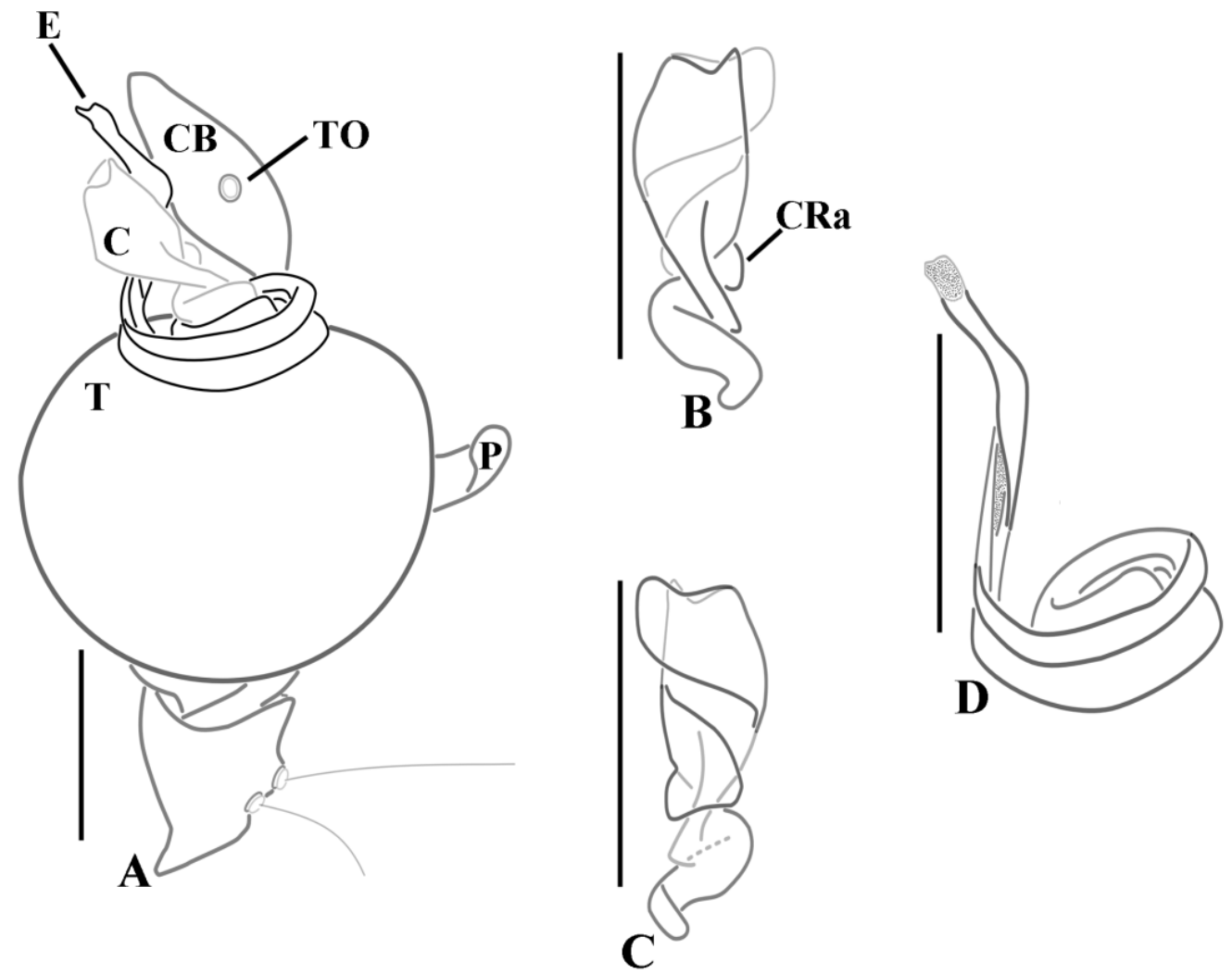

Figure 215. Glenognatha sp. nov. 10 from Peru (Holotype MUSM 050913). Male left palp, schematic. A, ventral. B, conductor ventral. C, conductor dorsal. D, embolus ventral. Scale bars $100 \mu \mathrm{m}$. C: conductor. CRa: conductor retrolateral apophysis. E: embolus. P: paracymbium. T: tegulum. TO: tarsal organ.
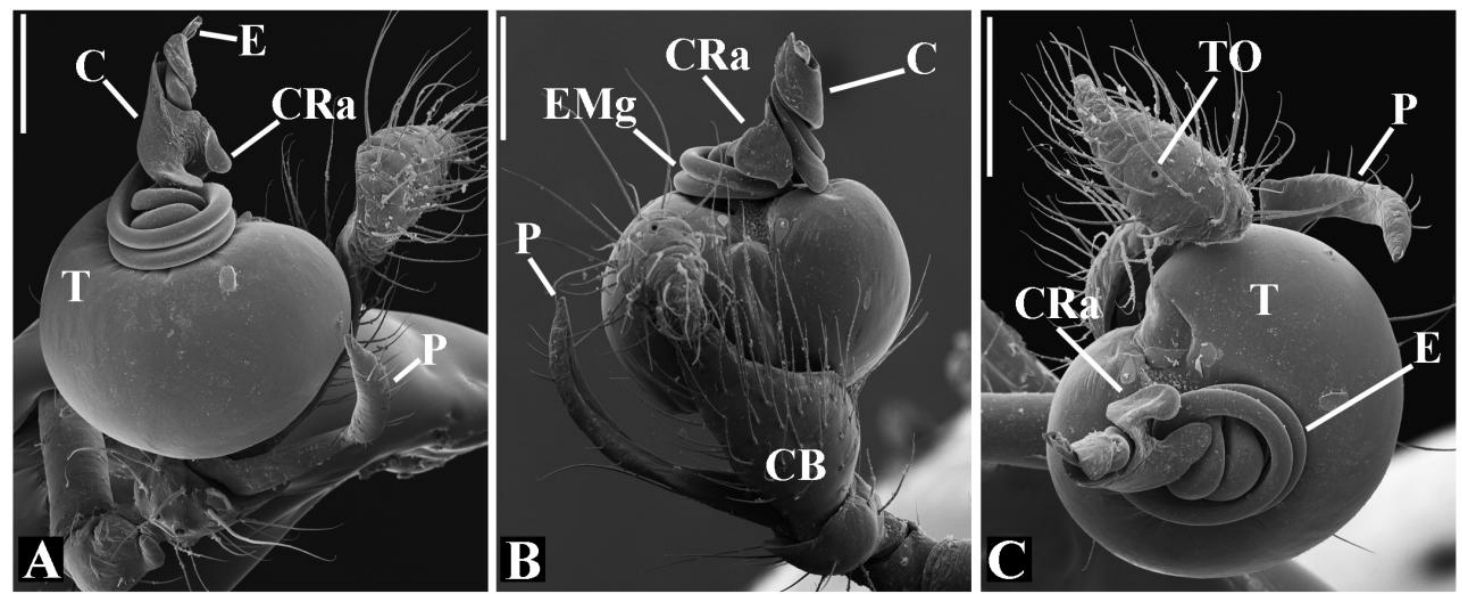

Figure 216. Glenognatha sp. nov. 10 from Peru. Male left palp. A, ventroretrolateral. B, dorsal. C, anterior. . Scale bars $100 \mu \mathrm{m}$. C: conductor. CB: cymbium. CRa: conductor retrolateral apophysis. E: embolus. EMg: embolic medial groove. P: paracymbium. T: tegulum. TO: tarsal organ. 


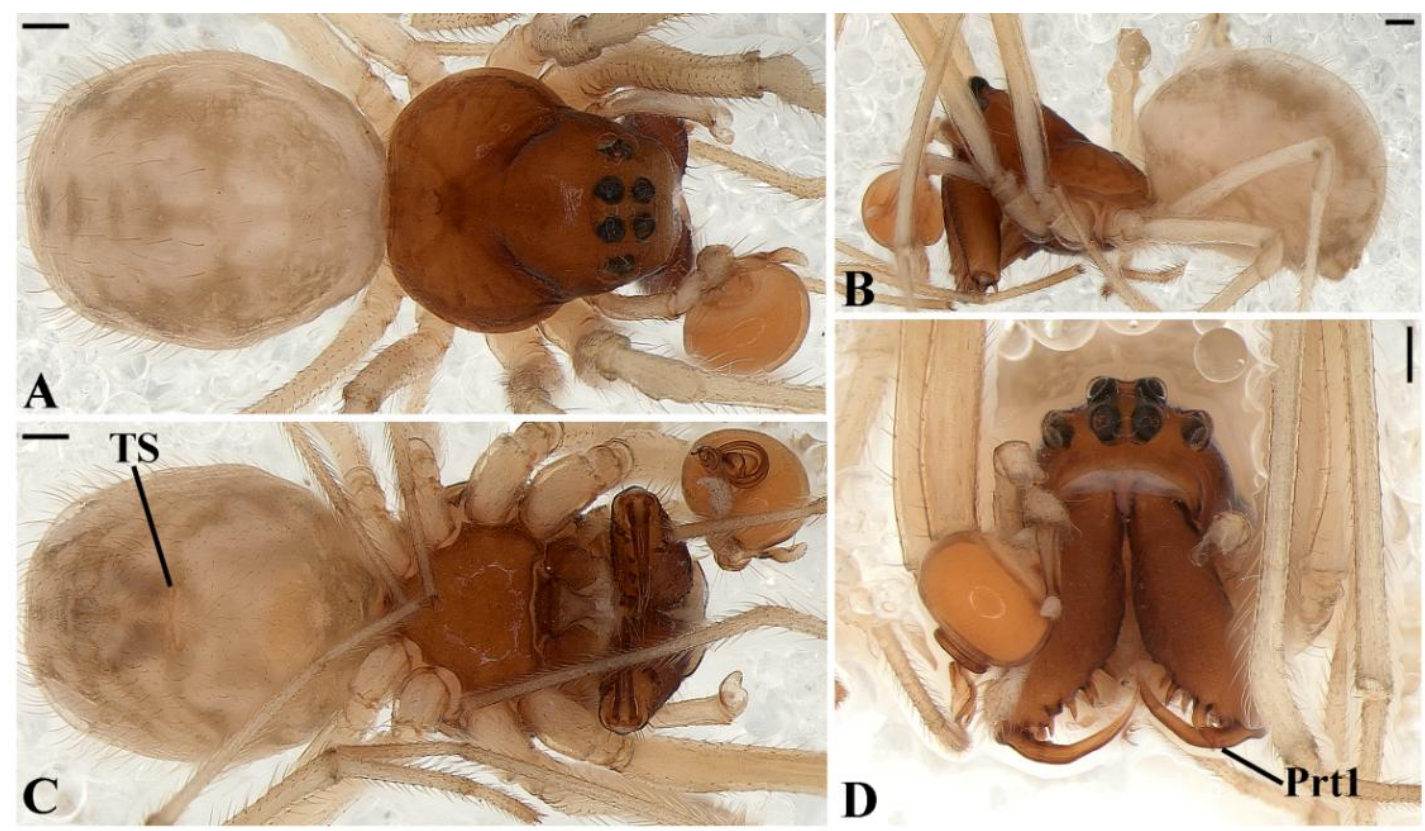

Figure 217. Glenognatha smilodon from Cameroon (Holotype MRAC 202710). Male habitus. A, dorsal. B, lateral. C, ventral. D, frontal. Scale bars, $200 \mu \mathrm{m}$. TS: tracheal spiracle. 


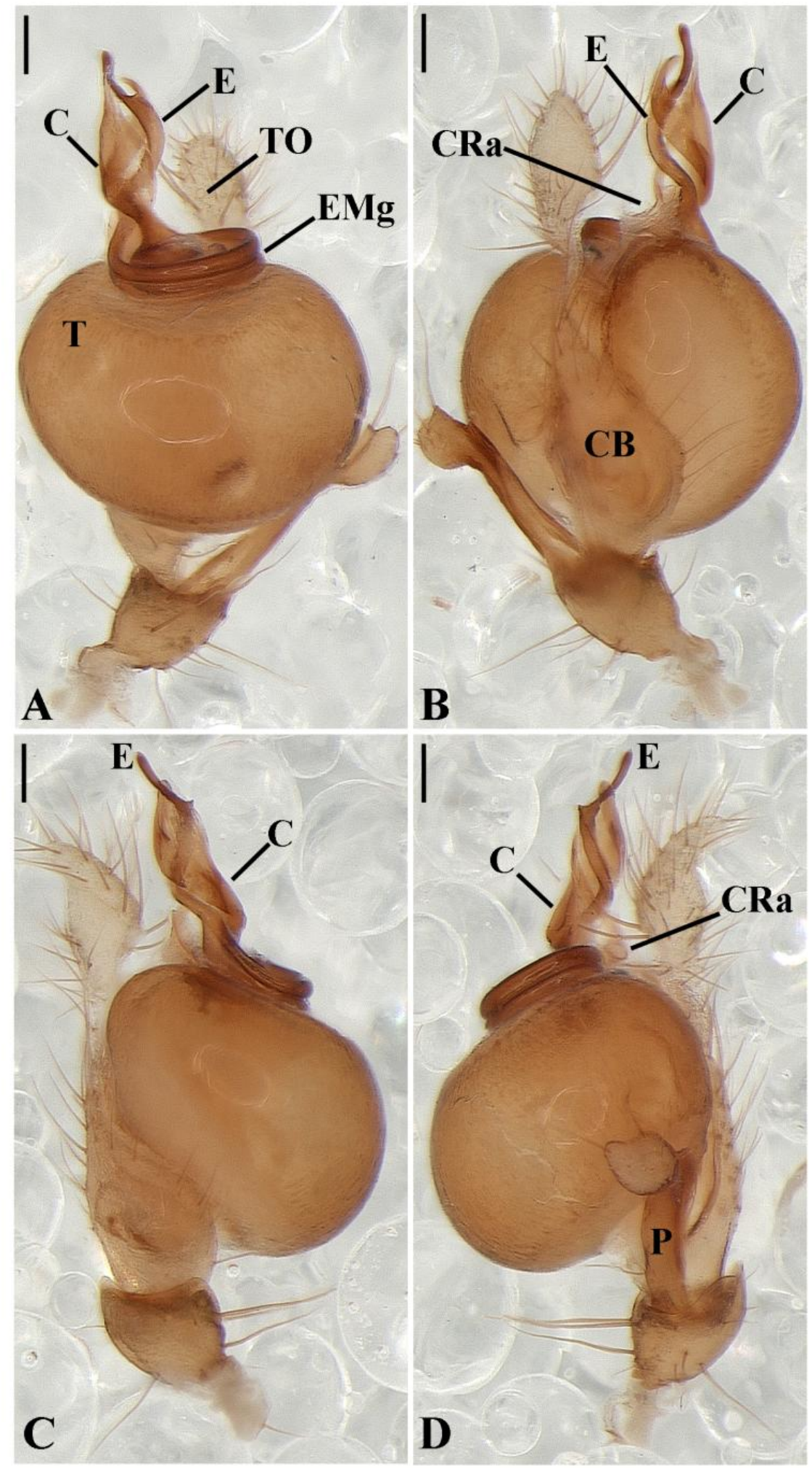

Figure 218. Glenognatha smilodon from Cameroon (Holotype MRAC 202710). Male left palp. A, ventral. B, dorsal. C, prolateral. D, retrolateral. Scale bars, $100 \mu \mathrm{m}$. C: conductor. $\mathrm{CB}$ : cymbium. CRa: conductor retrolateral apophysis. E: embolus. EMg: embolic medial groove. P: paracymbium. T: tegulum. TO: tarsal organ. 

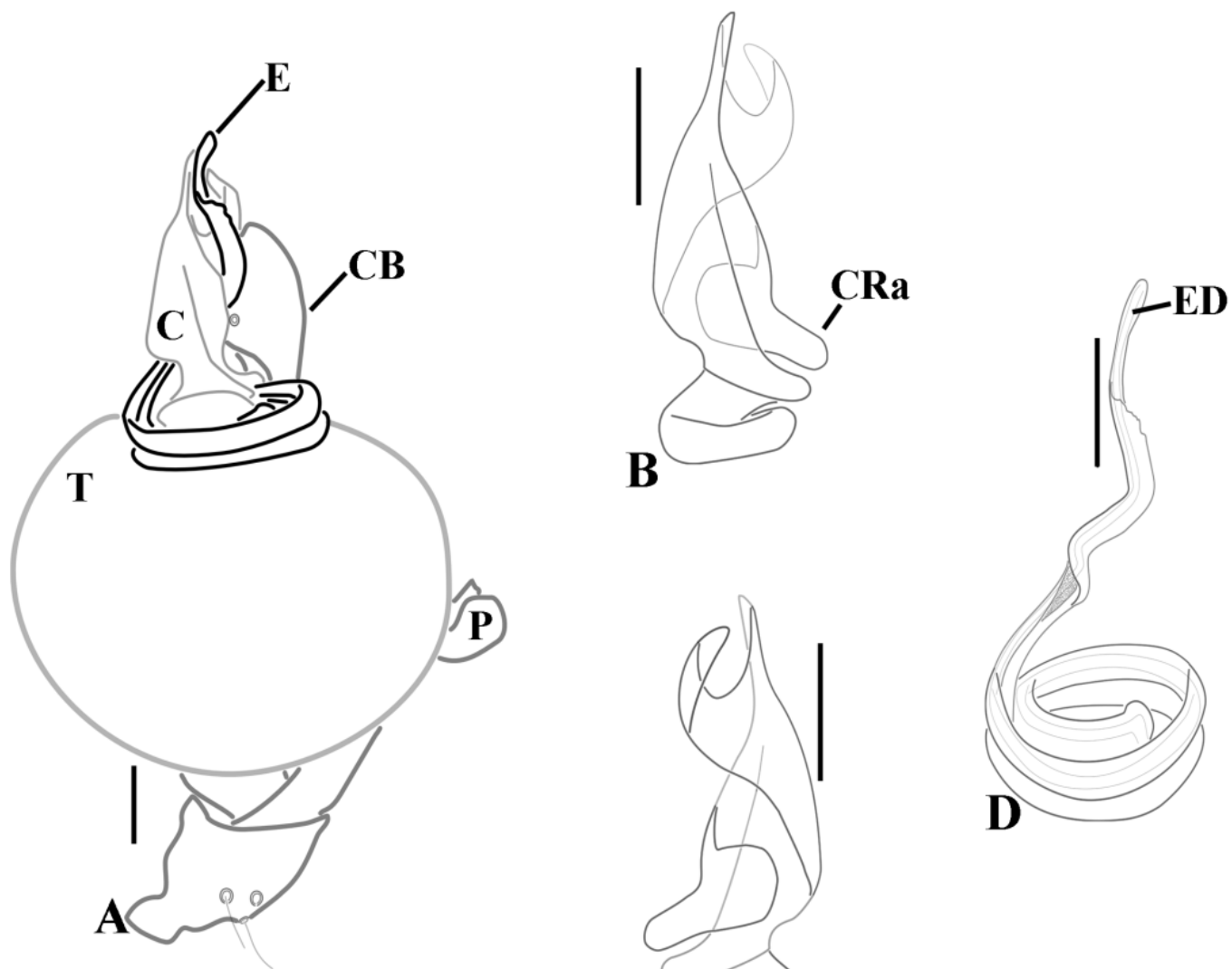

Figure 219. Glenognatha smilodon from Cameroon (Holotype MRAC 202710). Male left palp, schematic. A, ventral. B, conductor ventral. C, conductor dorsal. D, embolus ventral. Scale bars $100 \mu \mathrm{m}$. C: conductor. CB: cymbium. CRa: conductor retrolateral apophysis. E: embolus. ED: ejaculatory duct. P: paracymbium. T: tegulum. 


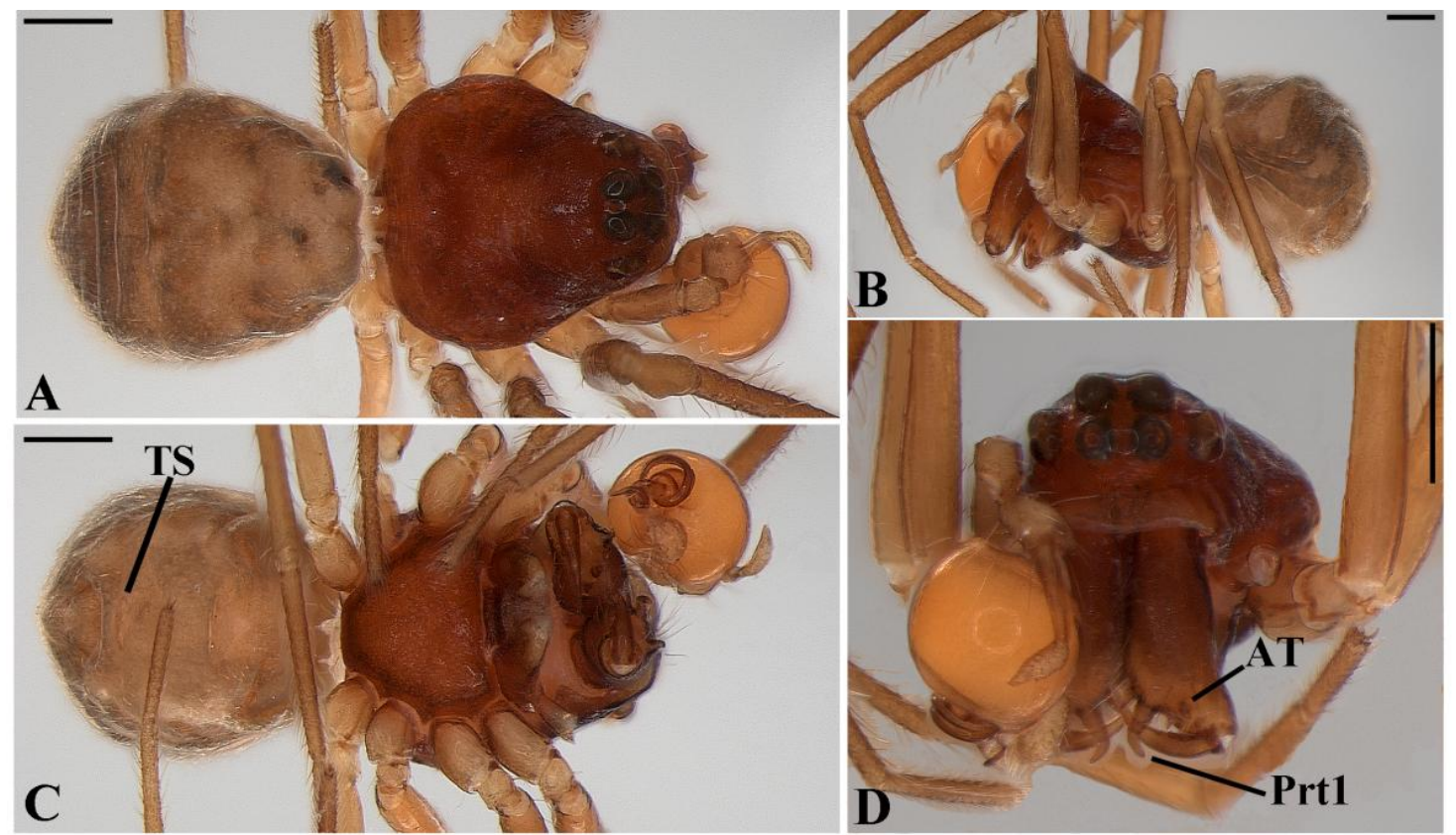

Figure 220. Glenognatha iviei from USA (Holotype AMNH). Male habitus. A, dorsal. B, lateral. C, ventral. D, frontal. Scale bars, $200 \mu \mathrm{m}$. AT: Anterior tooth. Prt: promarginal tooth. TS: tracheal spiracle.
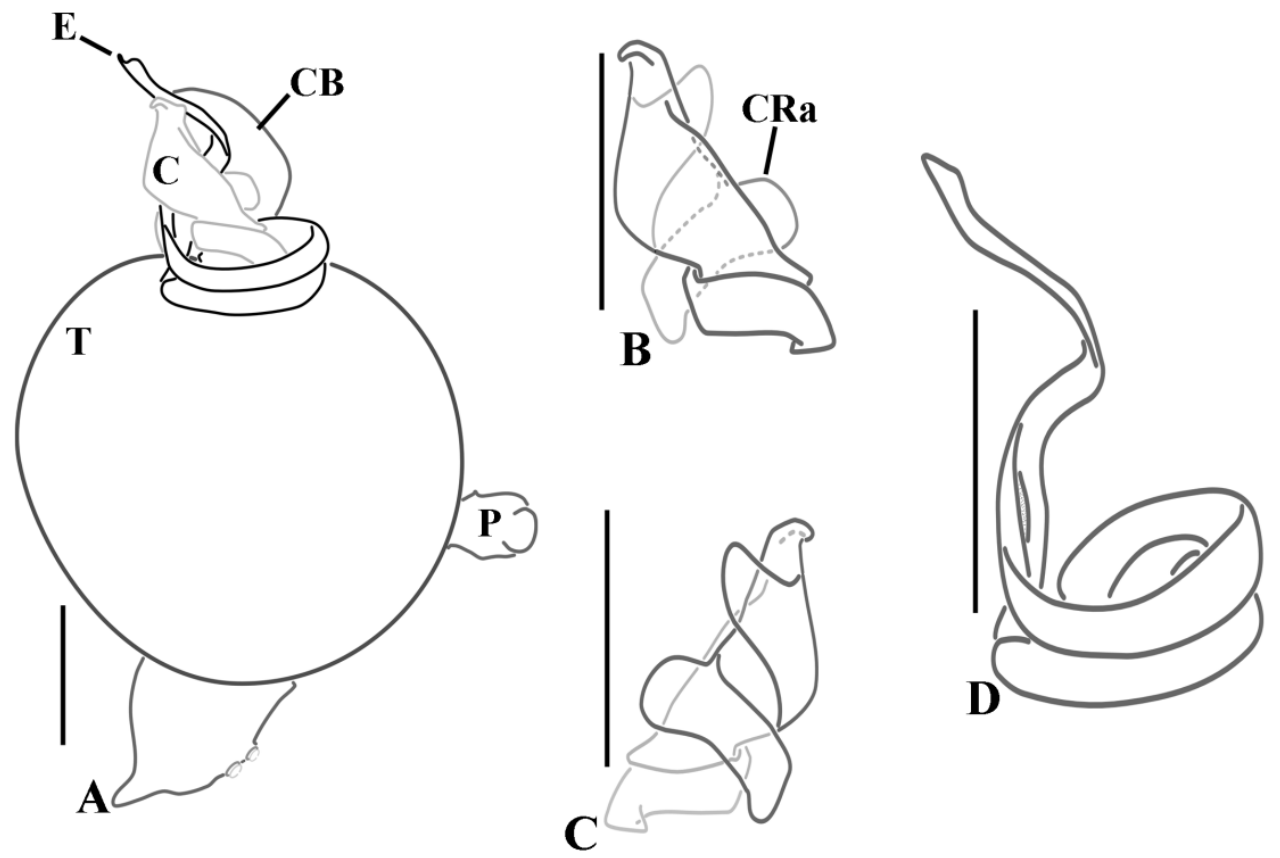

Figure 221. Glenognatha iviei from USA (Holotype AMNH). Male left palp, schematic. A, ventral. B, conductor ventral. C, conductor dorsal. D, embolus ventral. Scale bars $100 \mu \mathrm{m}$. C: conductor. CB: cymbium. CRa: conductor retrolateral apophysis. E: embolus. P: paracymbium. T: tegulum. 


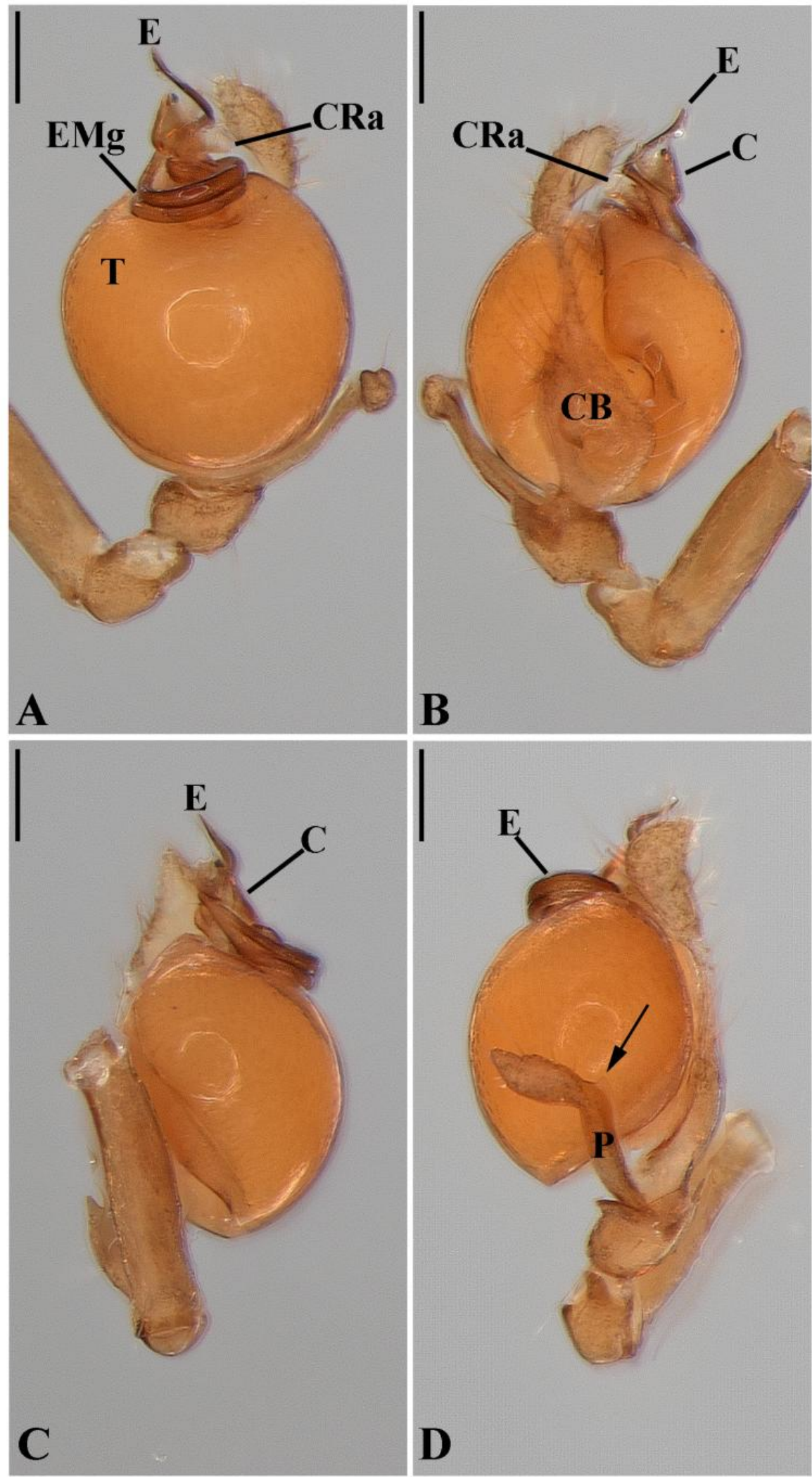

Figure 222. Glenognatha iviei from USA (Holotype AMNH). Male left palp. A, ventral. B, dorsal. C, prolateral. D, retrolateral (arrow, paracymbium process). Scale bars, $100 \mu \mathrm{m}$. C: conductor. CB: cymbium. CRa: conductor retrolateral apophysis. E: embolus. EMg: embolic medial groove. P: paracymbium. T: tegulum. 

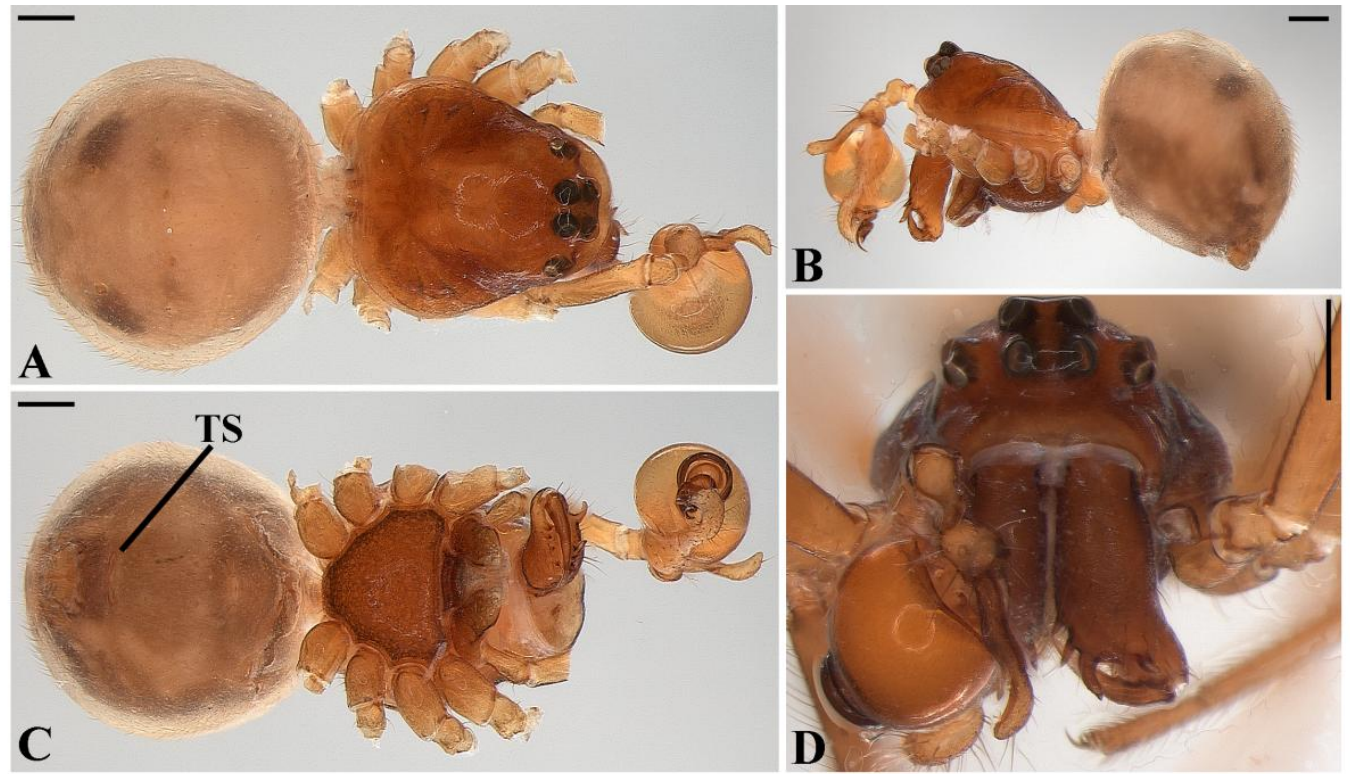

Figure 223. Glenognatha heleios from USA (AMNH). Male habitus. A, dorsal. B, lateral. C, ventral. D, frontal (Paratype). Scale bars, $200 \mu \mathrm{m}$. TS: tracheal spiracle.
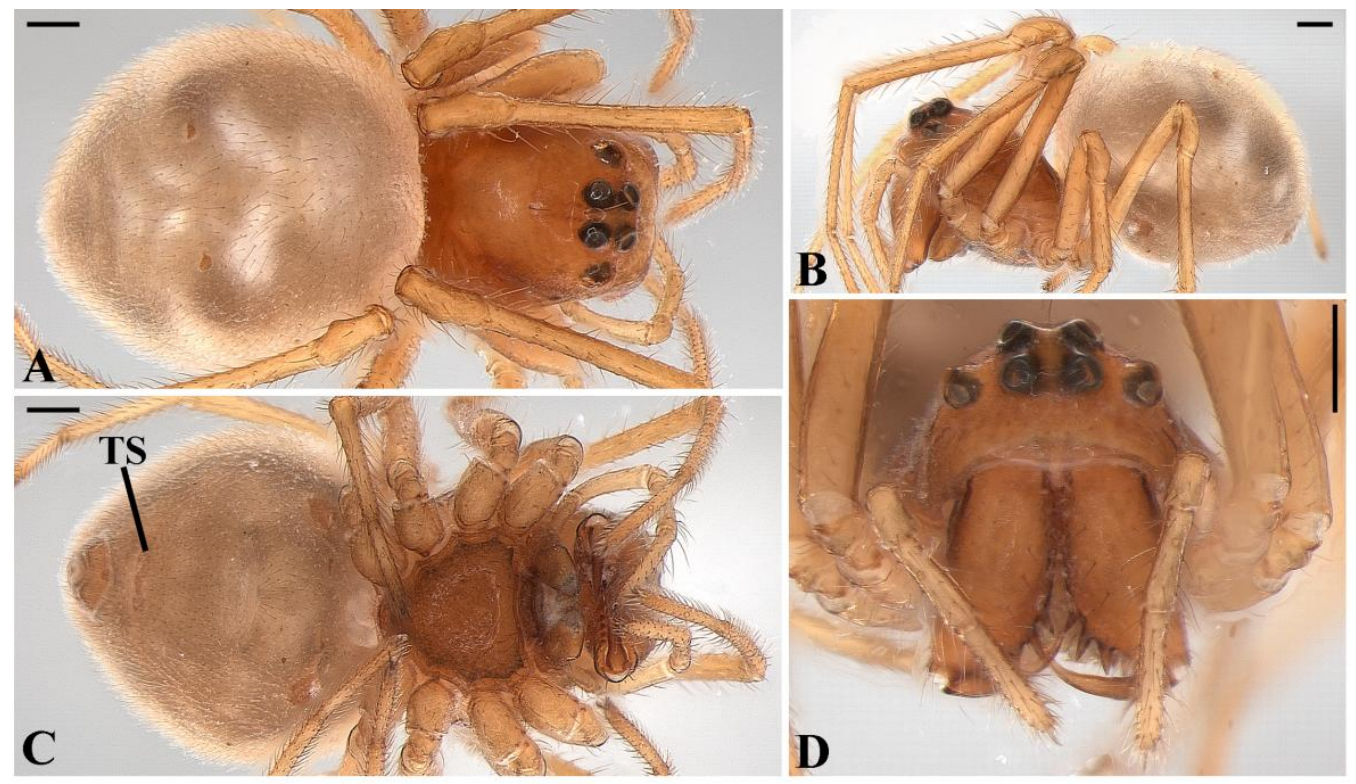

Figure 224. Glenognatha heleios from USA (Paratype AMNH). Female habitus. A, dorsal. B, lateral. C, ventral. D, frontal. Scale bars, $200 \mu \mathrm{m}$. TS: tracheal spiracle. 


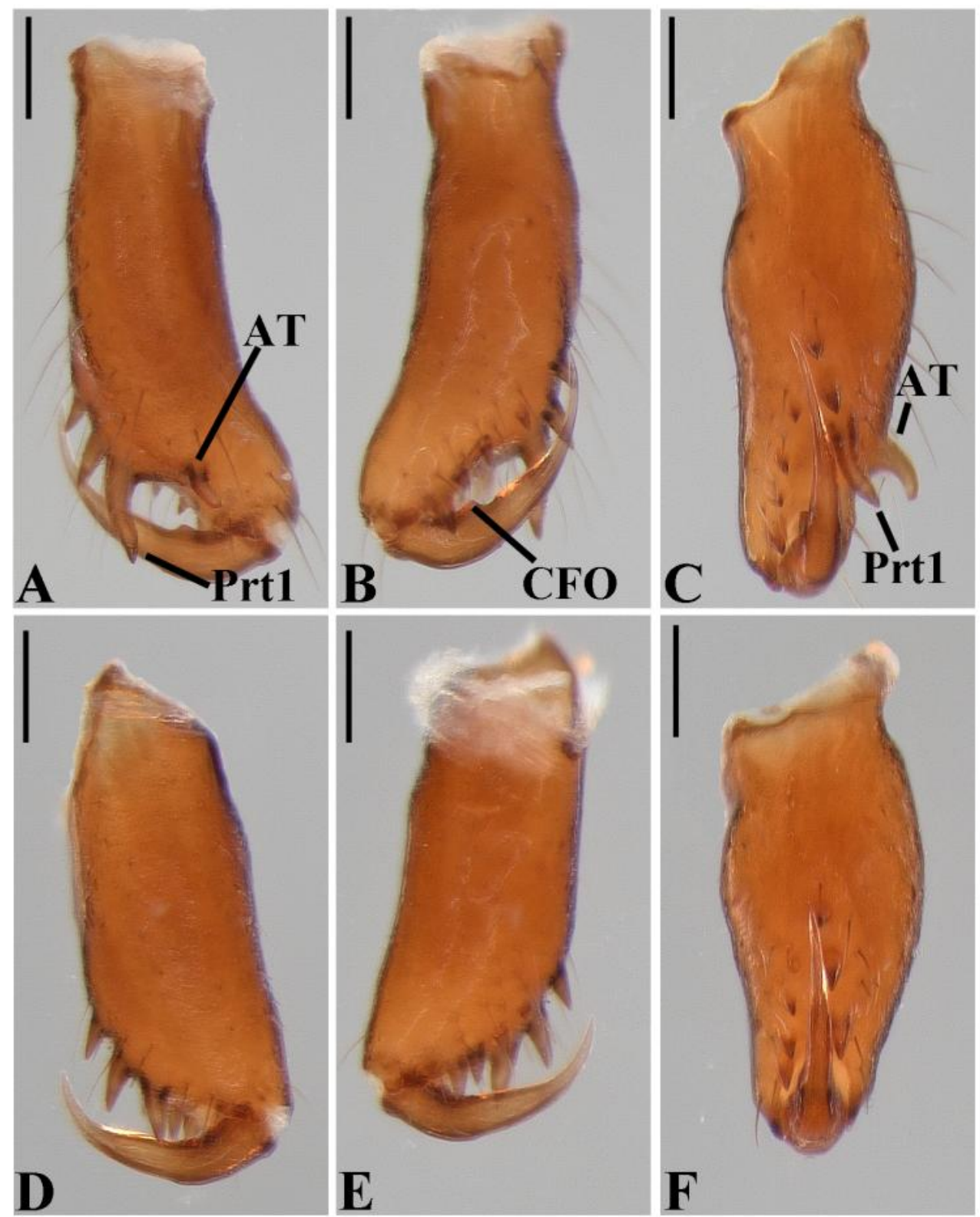

Figure 225. Glenognatha heleios from USA (AMNH). A-C, Male left chelicerae. A, anterior. B, posterior. C, mesal. D-F, Female left chelicerae. D, anterior. E, posterior. F, mesal. Scale bars, $100 \mu \mathrm{m}$. AT: anterior tooth. CFO: cheliceral fang outgrowth. Prt: promarginal tooth. 


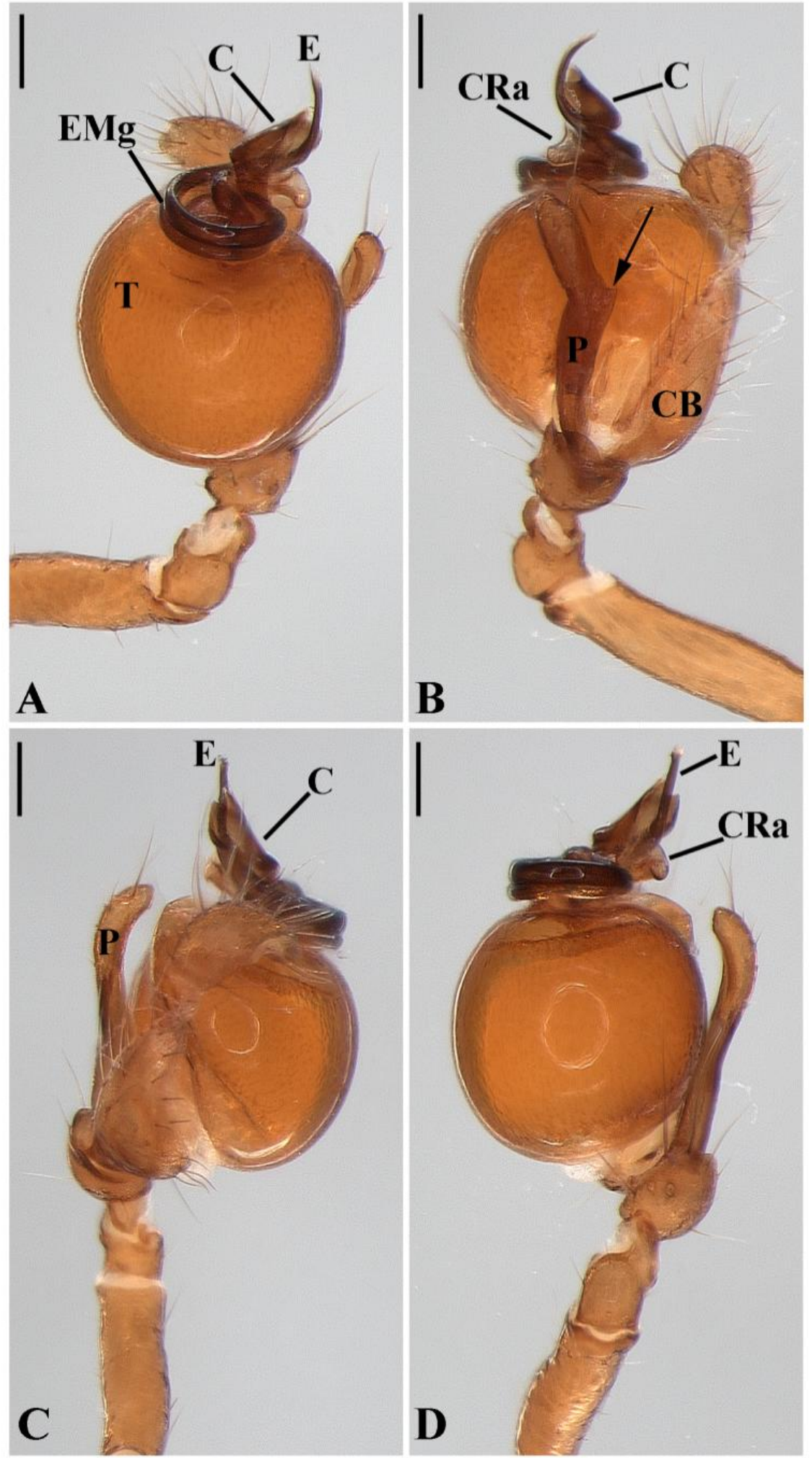

Figure 226. Glenognatha heleios from USA (Paratype AMNH). Male left palp. A, ventral. B, dorsal (arrow, paracymbium process). C, prolateral. D, retrolateral. Scale bars, $100 \mu \mathrm{m}$. C: conductor. CB: cymbium. CRa: conductor retrolateral apophysis. E: embolus. EMg: embolic medial groove. P: paracymbium. T: tegulum. 

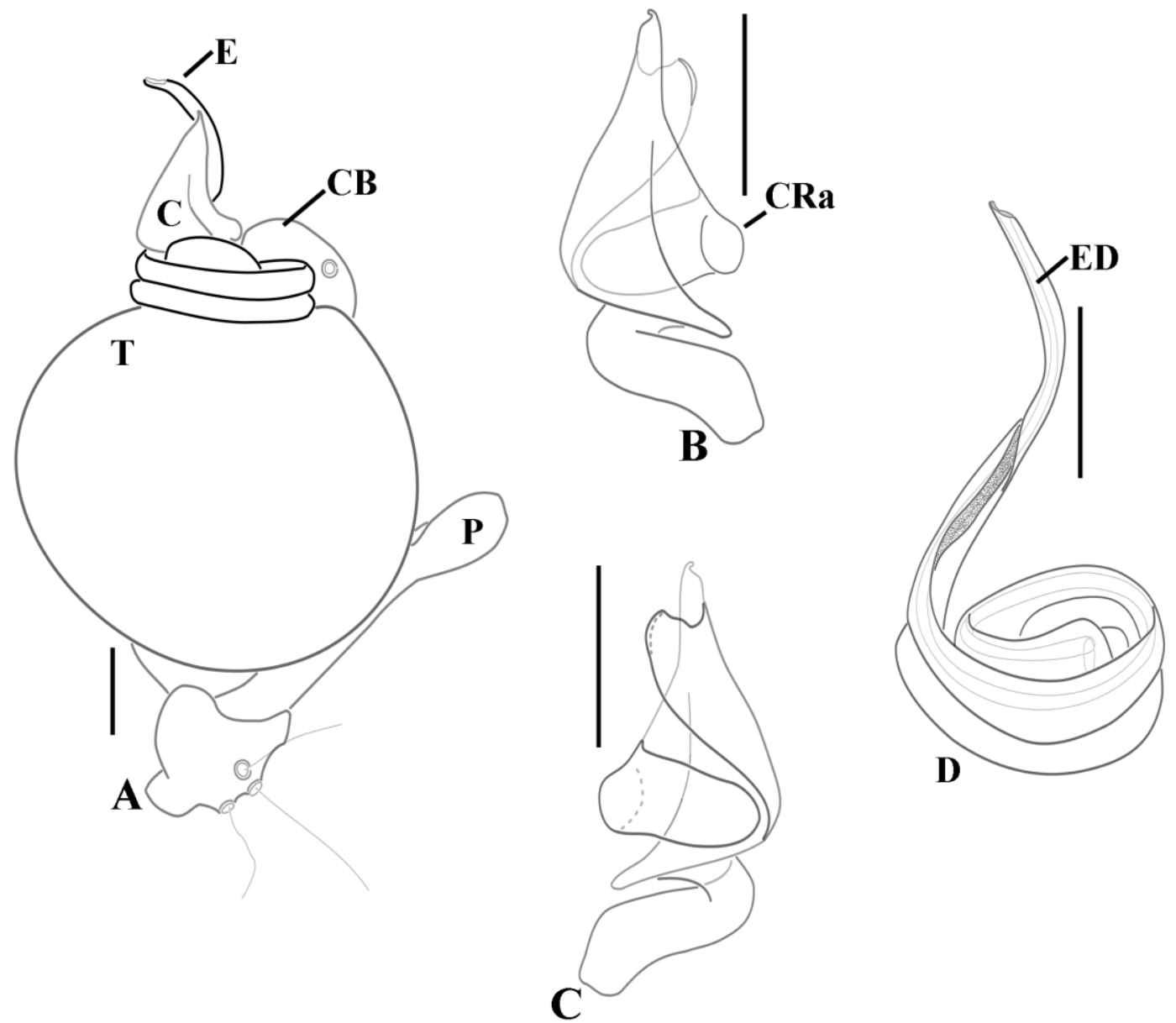

Figure 227. Glenognatha heleios from USA (Paratype AMNH). Male left palp, schematic. A, ventral. B, conductor ventral. C, conductor dorsal. D, embolus ventral. Scale bars $100 \mu \mathrm{m}$. C: conductor. CB: cymbium. CRa: conductor retrolateral apophysis. E: embolus. ED: ejaculatory duct. P: paracymbium. T: tegulum. 


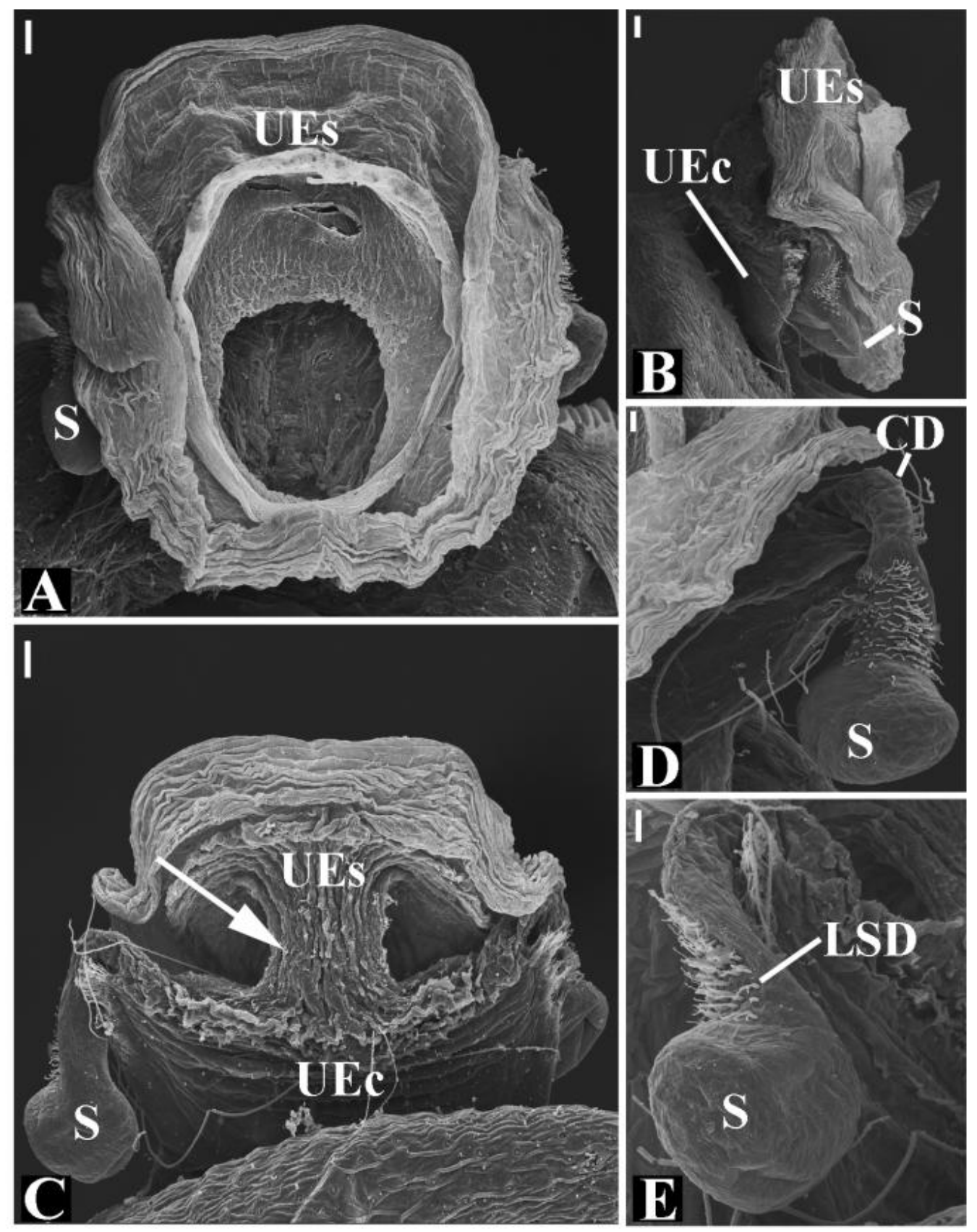

Figure 228. Glenognatha heleios from USA. Female genitalia. A, dorsal. B, lateral. C, ventral (arrow, constricted base). D-E, spermathecae and copulatory duct. Scale bars, $20 \mu \mathrm{m}$ (A-C), $10 \mu \mathrm{m}$ (D, E). CD: copulatory duct. LSD: long-stem gland ducts. UEc: uterus externus chamber. UEs: uterus externus sac. S: spermathecae. 


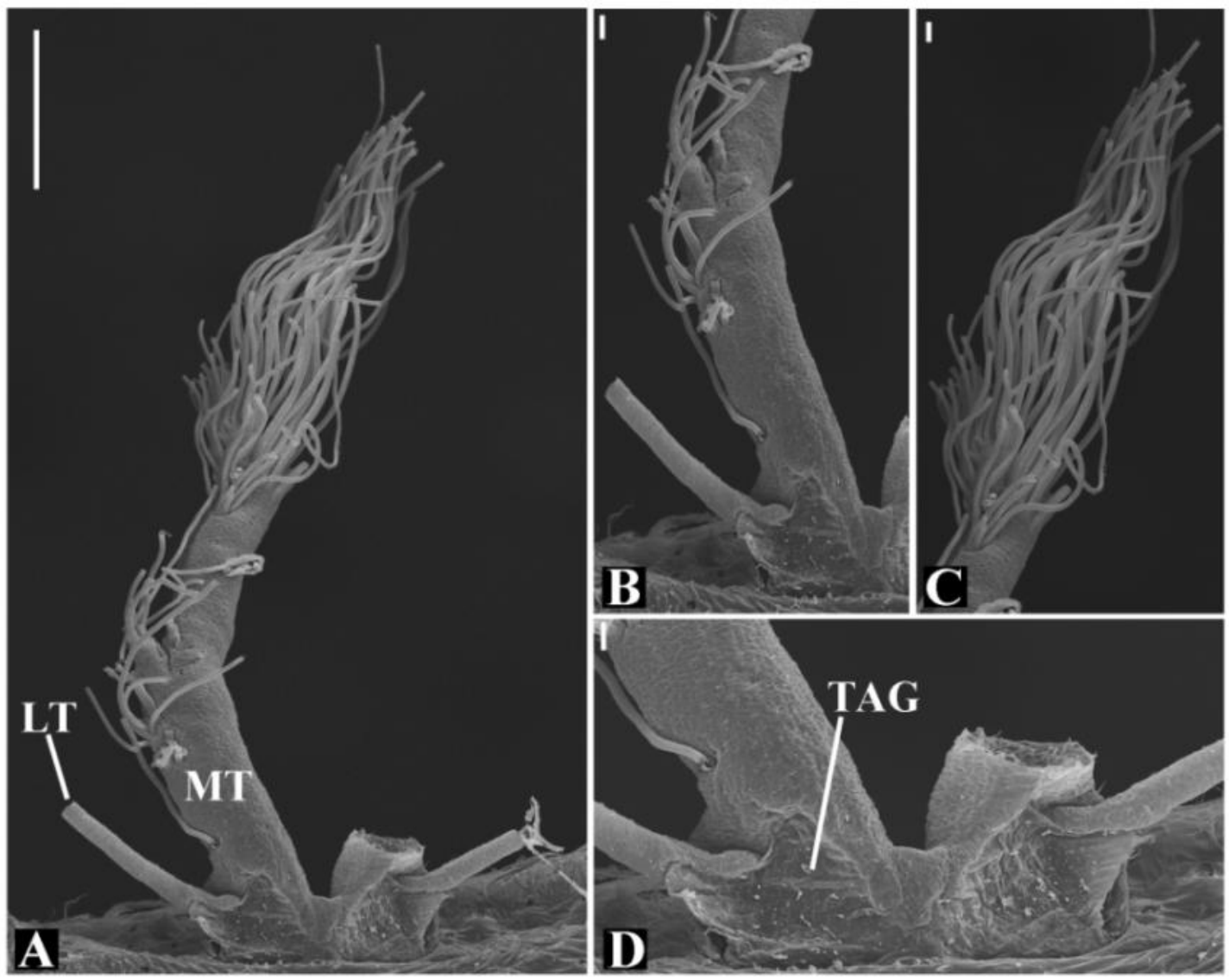

Figure 229. Glenognatha heleios from USA. Female tracheal system. A, dorsal. B, median and lateral trunks. C, median trunk distal. D, tracheal spiracle posterior view. Scale bars, $100 \mu \mathrm{m}$ (A), $10 \mu \mathrm{m}$ (B-D). LT: lateral tracheae. MT: median trunks. TAG: tracheal atrium gland. 

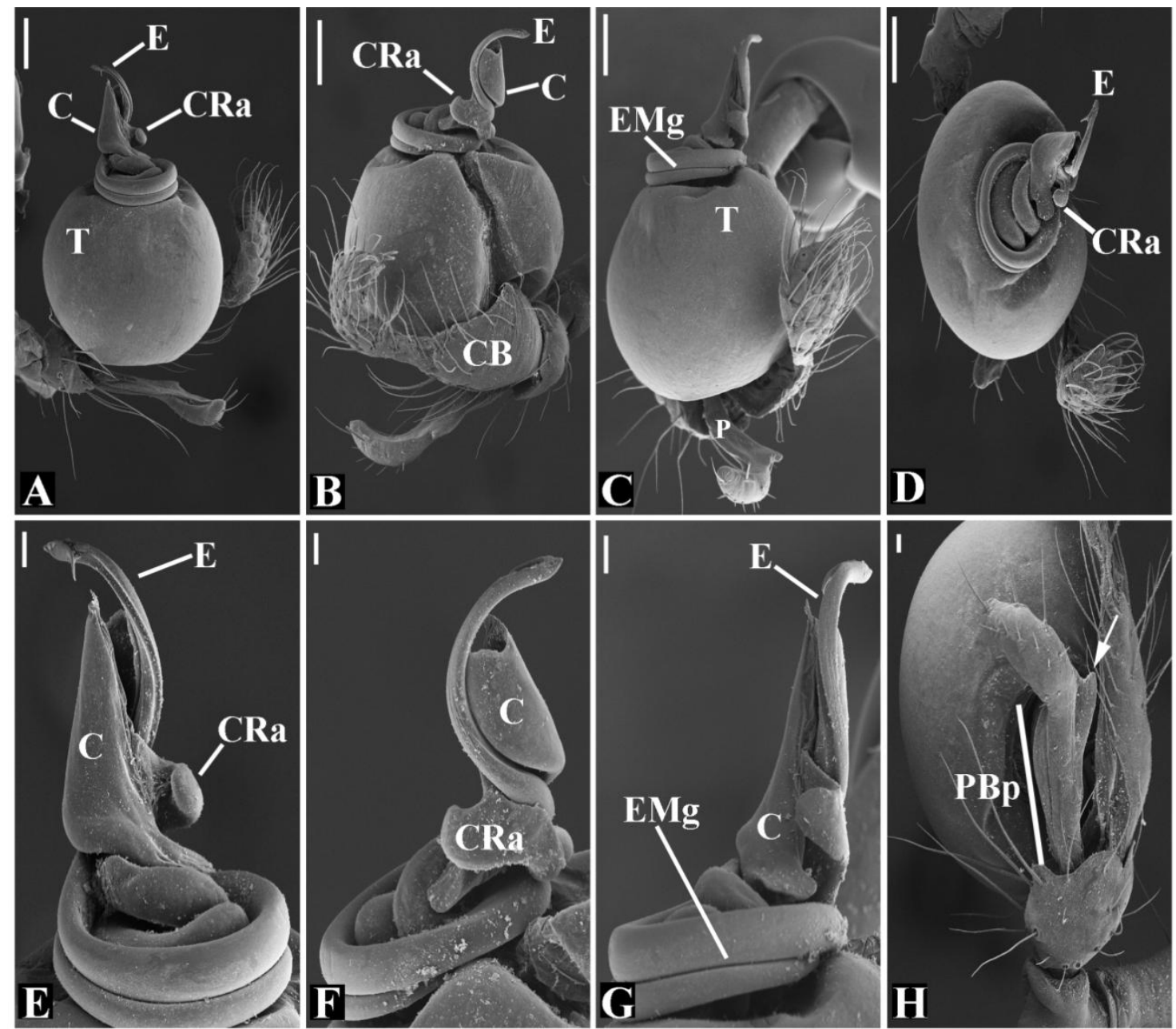

Figure 230. Glenognatha heleios from USA. A-D, Male left palp. A, ventral. B, dorsal. C, retrolateral. D, anterior. E-G, embolus and Conductor. E, ventral. F, dorsal. G, retrolateral. H, paracymbium (arrow, paracymbium process). Scale bars $100 \mu \mathrm{m}$ (A-D), $20 \mu \mathrm{m}(\mathrm{E}-\mathrm{H}) . \mathrm{C}$ : conductor. CB: cymbium. CRa: conductor retrolateral apophysis. E: embolus. EMg: embolic medial groove. PBp: paracymbium basal portion. T: tegulum. 

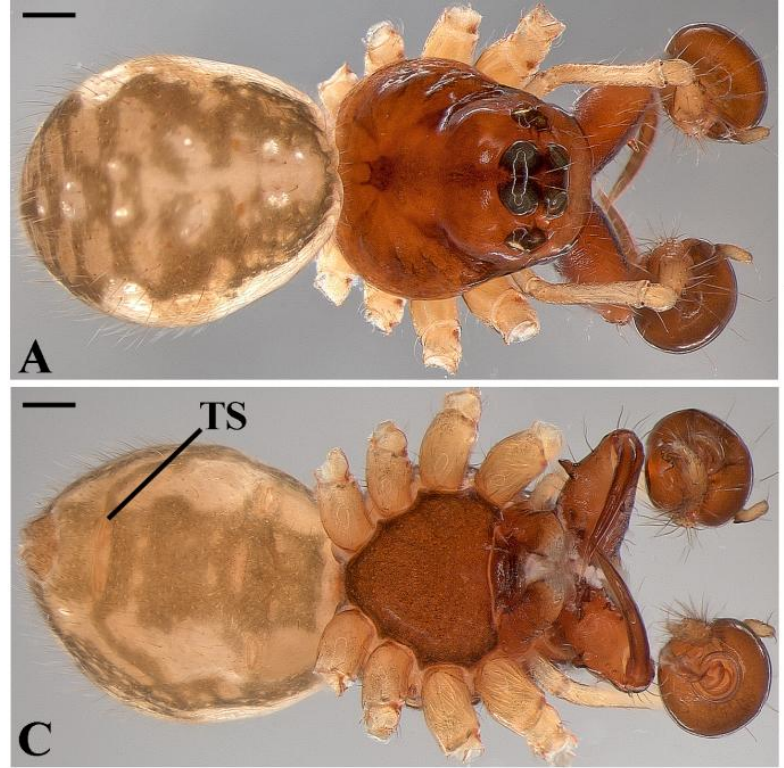
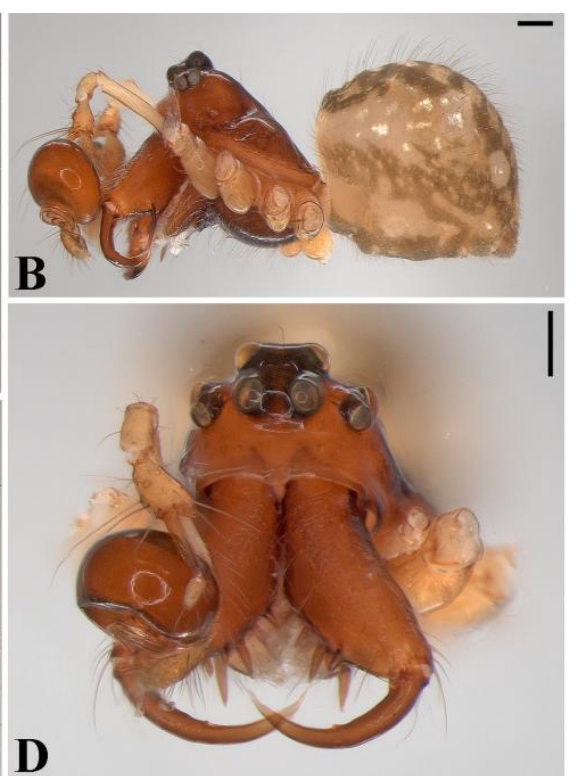

Figure 231. Glenognatha sp. nov. 1 from Brazil (IBSP 147809). Male habitus. A, dorsal. B, lateral. C, ventral. D, frontal. Scale bars, $200 \mu \mathrm{m}$. TS: tracheal spiracle.

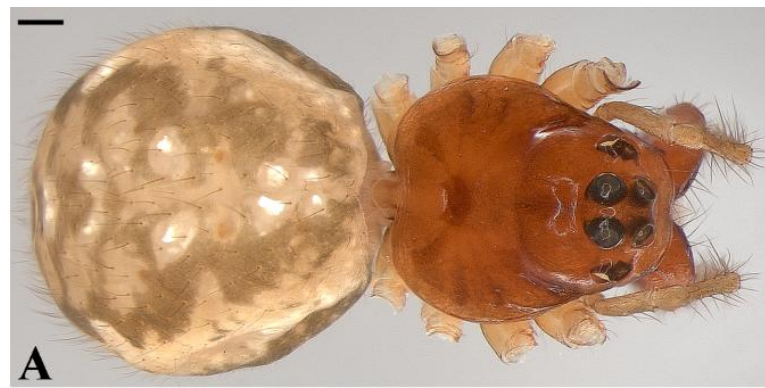

A

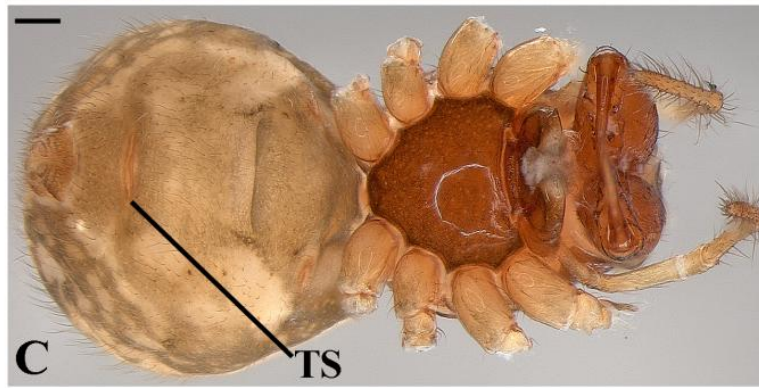

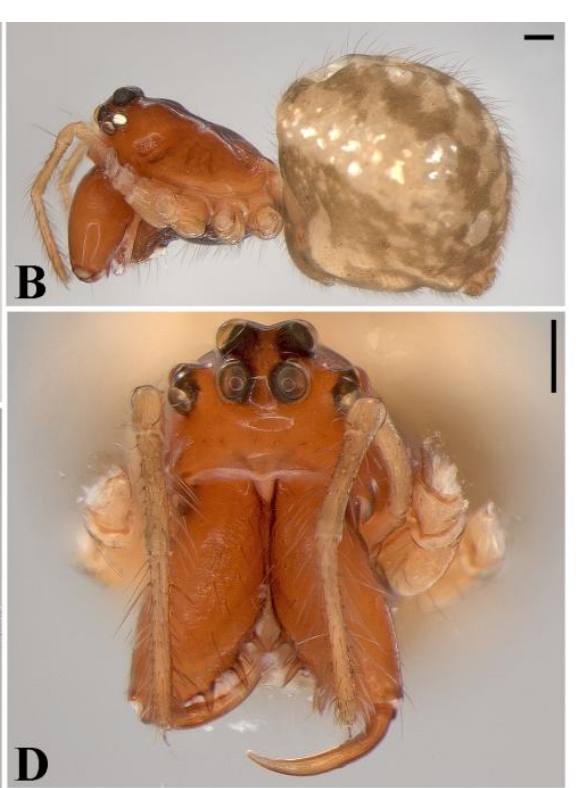

D

Figure 232. Glenognatha sp. nov. 1 from Brazil (IBSP 147809). Female habitus. A, dorsal. B, ventral. C, lateral. D, frontal. Scale bars, $200 \mu \mathrm{m}$. TS: tracheal spiracle. 


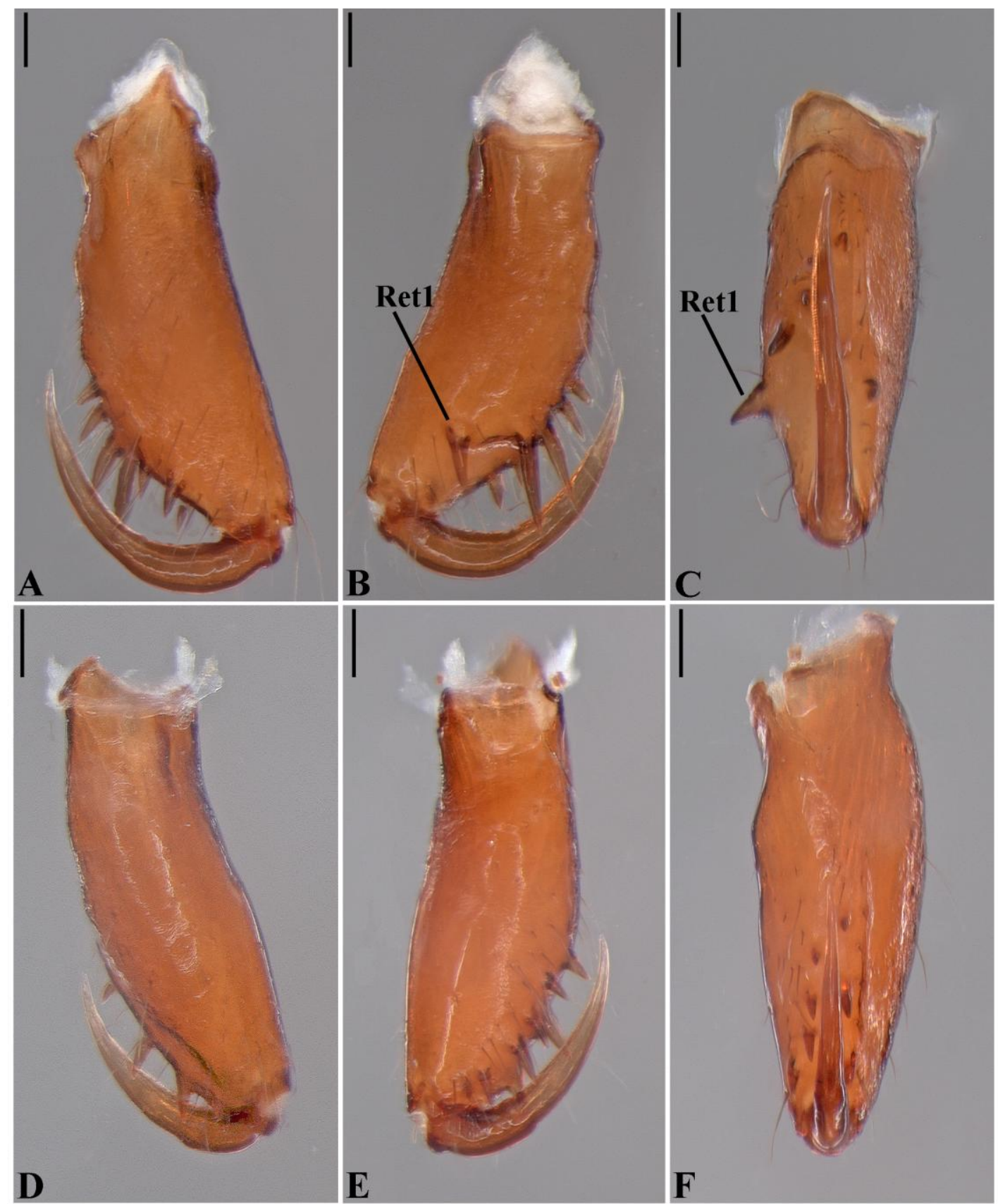

Figure 233. Glenognatha sp. nov. 1 from Brazil (IBSP 147861). A-C, Male left chelicerae. A, anterior. B, posterior. C, mesal. D-F, Female left chelicerae. D, anterior. E, posterior. F, mesal. Scale bars, $100 \mu \mathrm{m}$. Ret: retromarginal tooth. 


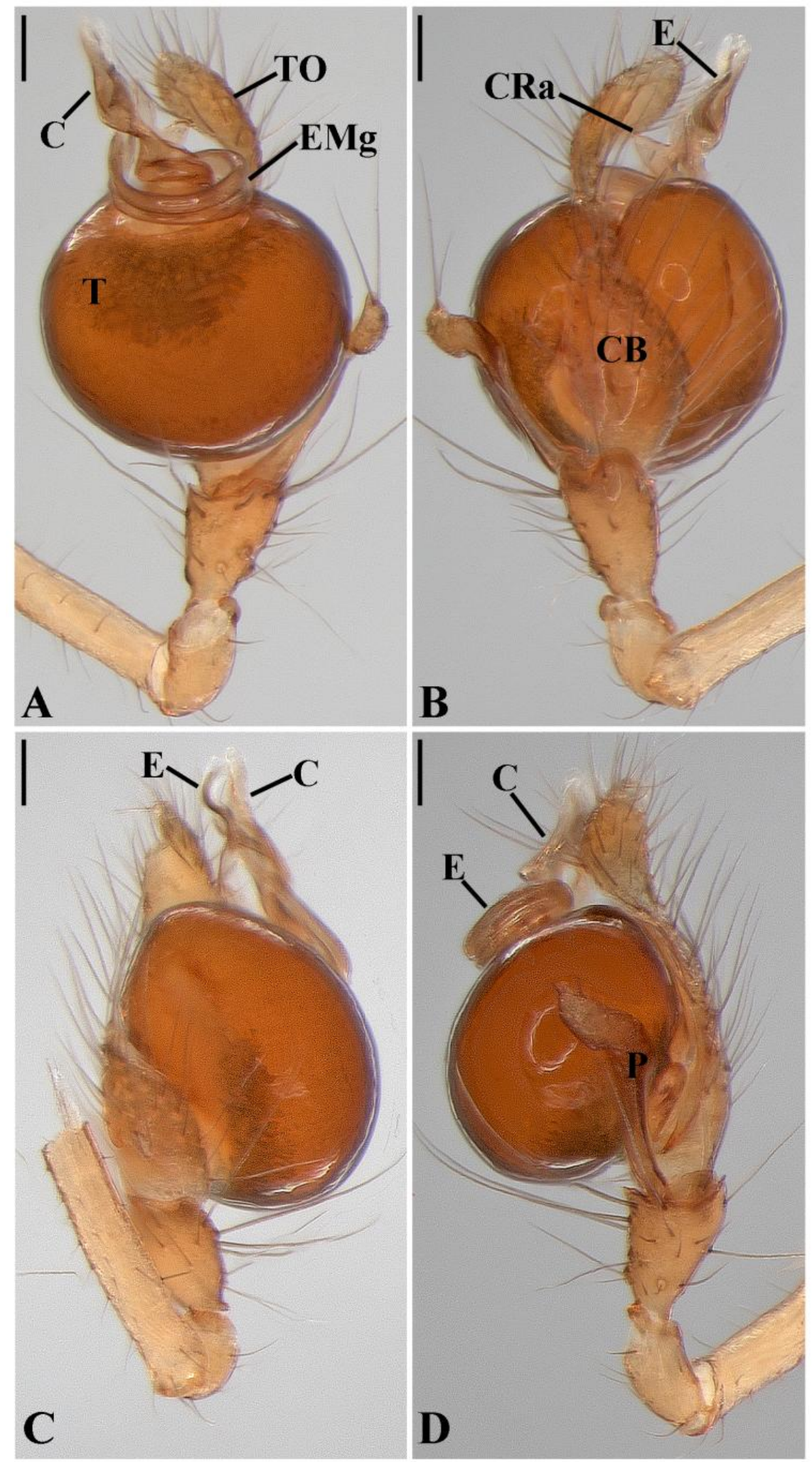

Figure 234. Glenognatha sp. nov. 1 from Brazil (IBSP 147809). Male left palp. A, ventral. B, dorsal. C, prolateral. D, retrolateral. Scale bars, $100 \mu \mathrm{m}$. C: conductor. CB: cymbium. CRa: conductor retrolateral apophysis. E: embolus. EMg: embolic medial groove. P: paracymbium. T: tegulum. TO: tarsal organ. 

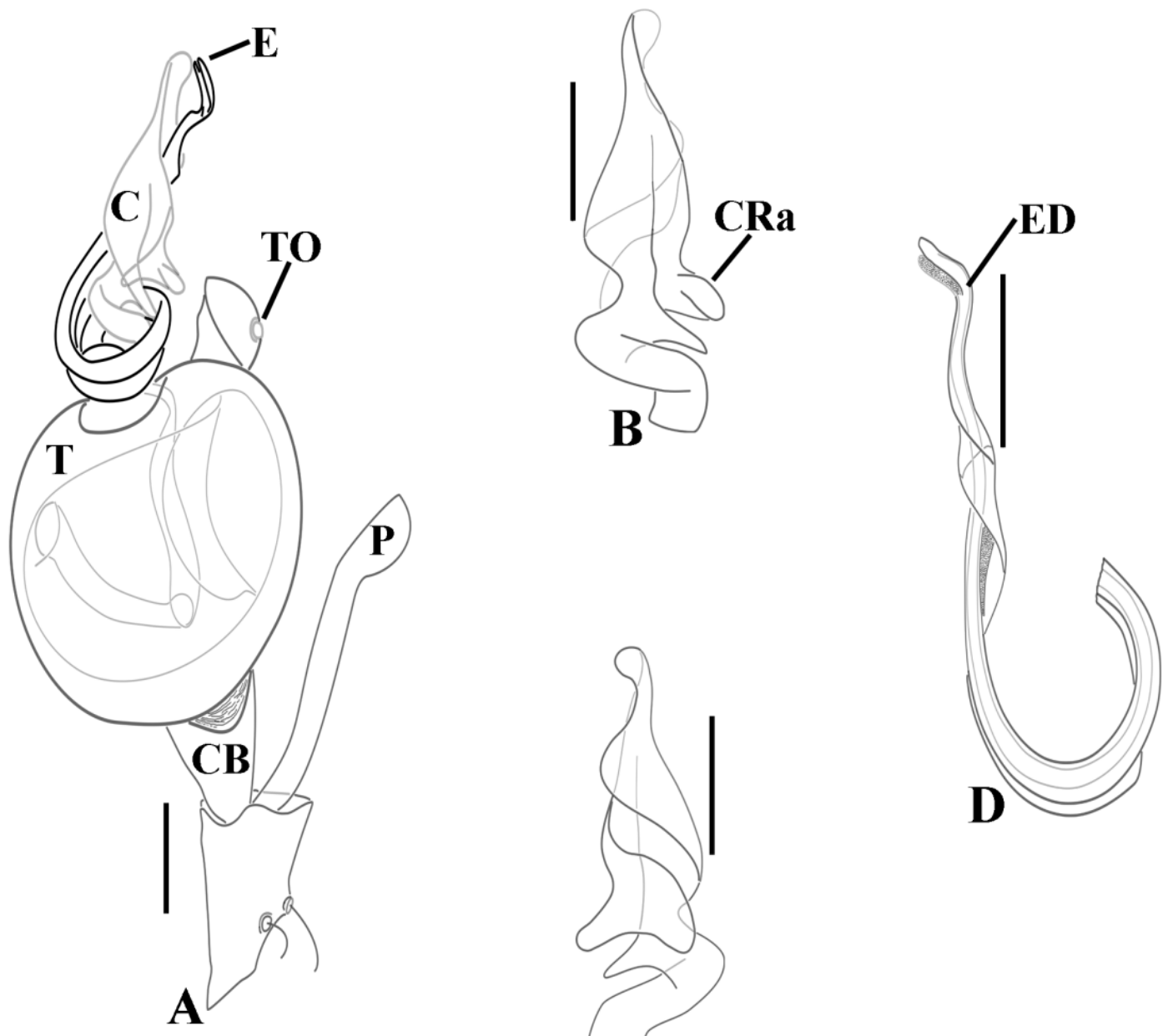

Figure 235. Glenognatha sp. nov. 1 from Brazil (Paratype IBSP 147861). Male left palp, schematic. A, ventral. B, conductor ventral. C, conductor dorsal. D, embolus ventral. Scale bars $100 \mu \mathrm{m}$. C: conductor. CB: cymbium. CRa: conductor retrolateral apophysis. E: embolus. ED: ejaculatory duct. P: paracymbium. T: tegulum. TO: tarsal organ. 


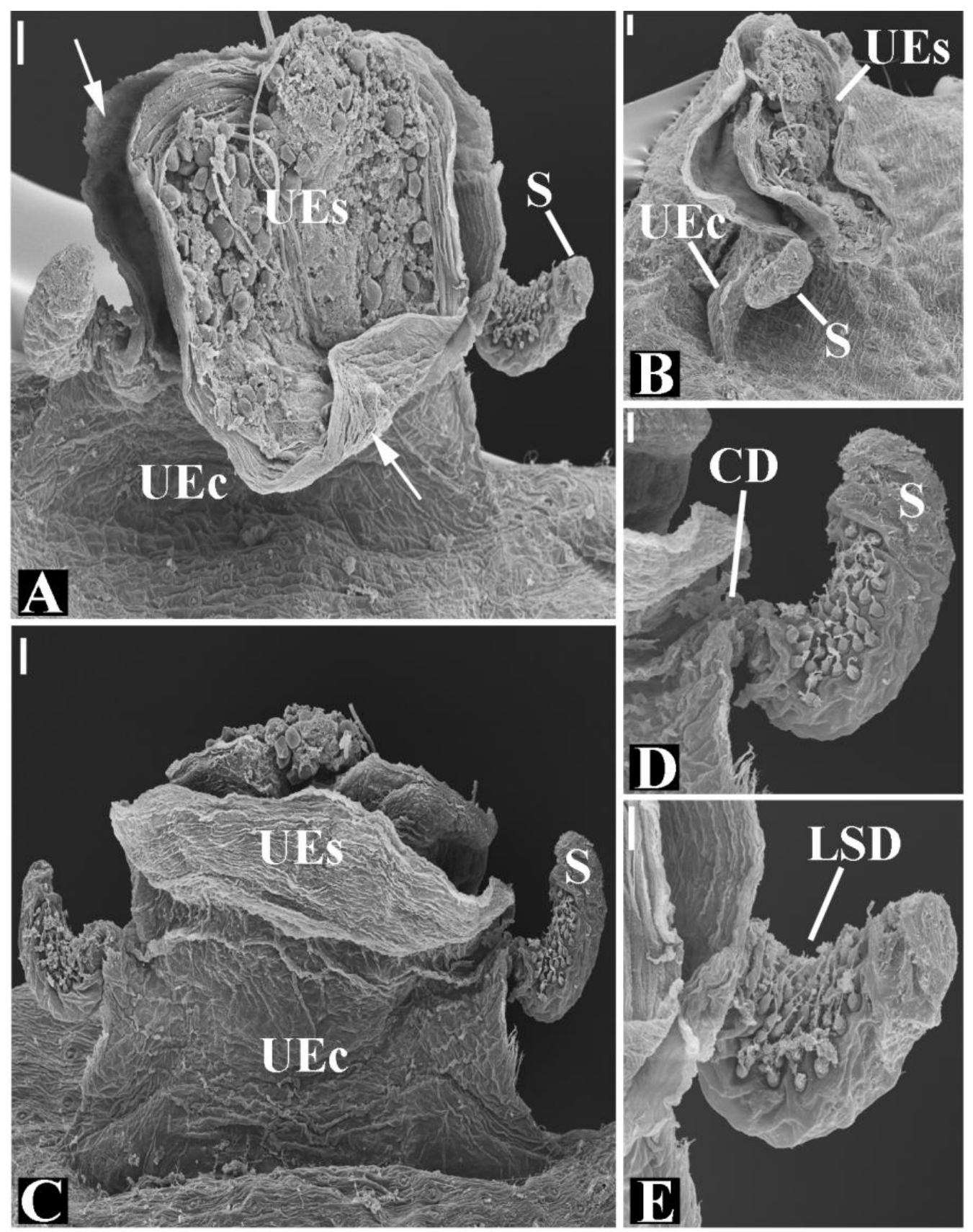

Figure 236. Glenognatha sp. nov. 1 from Brazil. Female genitalia. A, dorsal (arrows, disc-shaped portions). B, lateral. C, ventral D-E, spermathecae and copulatory duct. Scale bars, $20 \mu \mathrm{m}$ (A-C), $10 \mu \mathrm{m}$ (D-E). CD: copulatory duct. LSD: long-stem gland ducts. UEc: uterus externus chamber. UEs: uterus externus sac. S: spermathecae. 


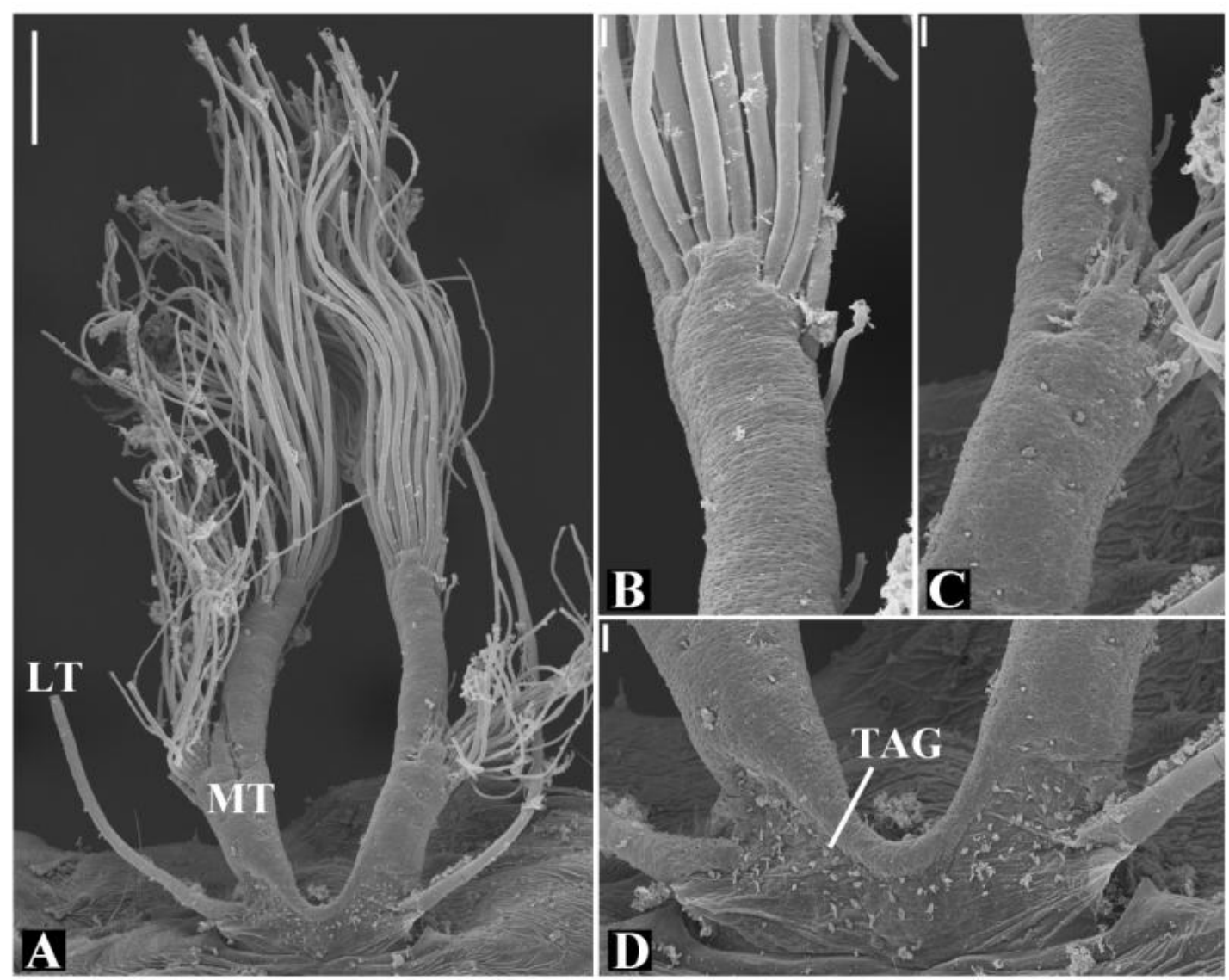

Figure 237. Glenognatha sp. nov. 1 from Brazil. Female tracheal system. A, dorsal. B, median trunk distal. $\mathbf{C}$, median trunk basal. D, tracheal spiracle posterior view. Scale bars, $100 \mu \mathrm{m}$ (A), $10 \mu \mathrm{m}$ (B-D). LT: lateral trachea. MT: median trunks. TAG: tracheal atrium gland. 


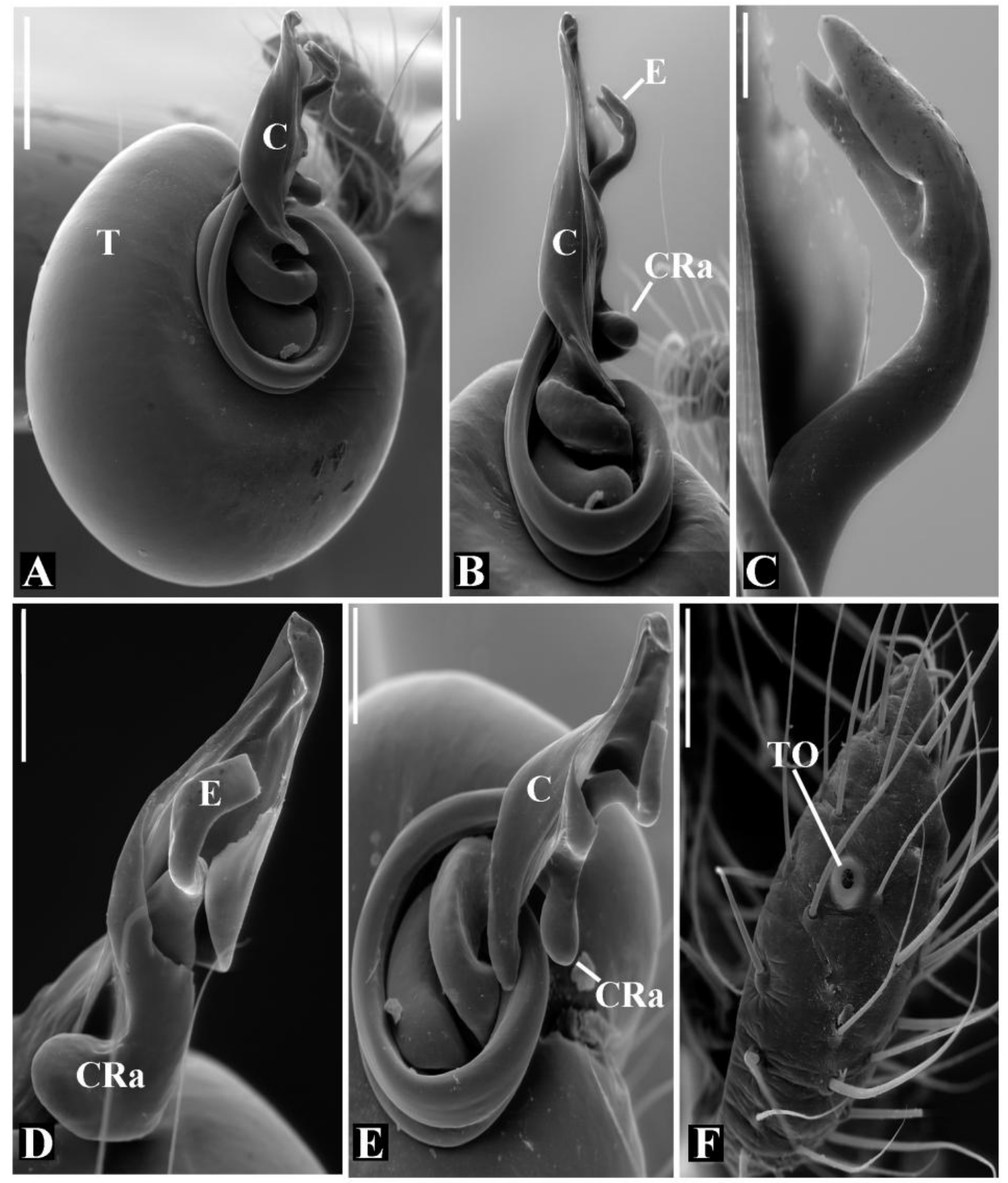

Figure 238. Glenognatha sp. nov. 1 from Brazil. Male left palp. A, ventral. B, embolus and conductor ventral. C, embolus tip. D, embolus and conductor retrolateral. E, anteroretrolateral. F, cymbium. Scale bars, $100 \mu \mathrm{m}$ (A), $50 \mu \mathrm{m}$ (B, D-F), $10 \mu \mathrm{m}$ (C). C: conductor. CRa: conductor retrolateral apophysis. E: embolus. T: tegulum. TO: tarsal organ. 

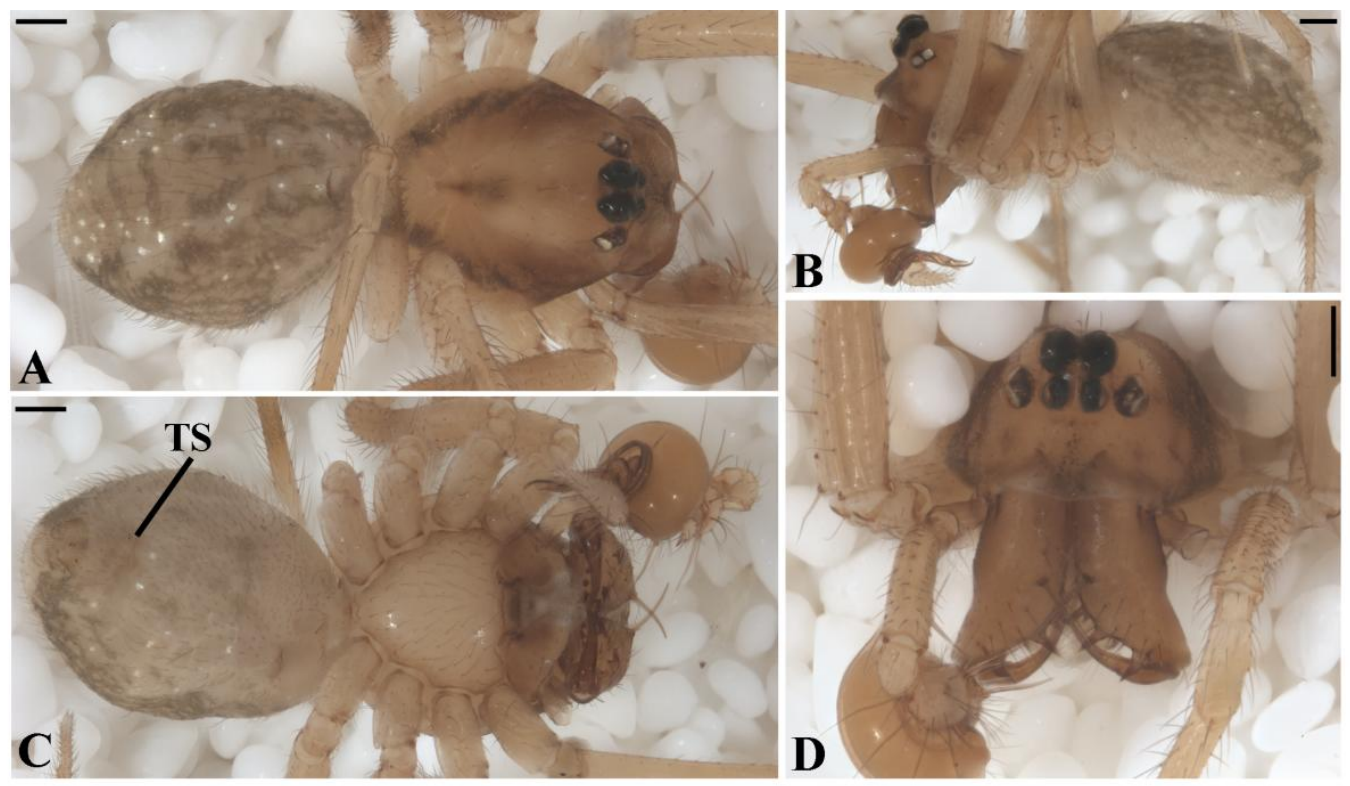

Figure 239. Glenognatha hirsutissima from Marquesas Islands (USNM). Male habitus. A, dorsal. B, lateral. C, ventral. D, frontal. Scale bars, $200 \mu \mathrm{m}$. TS: tracheal spiracle.
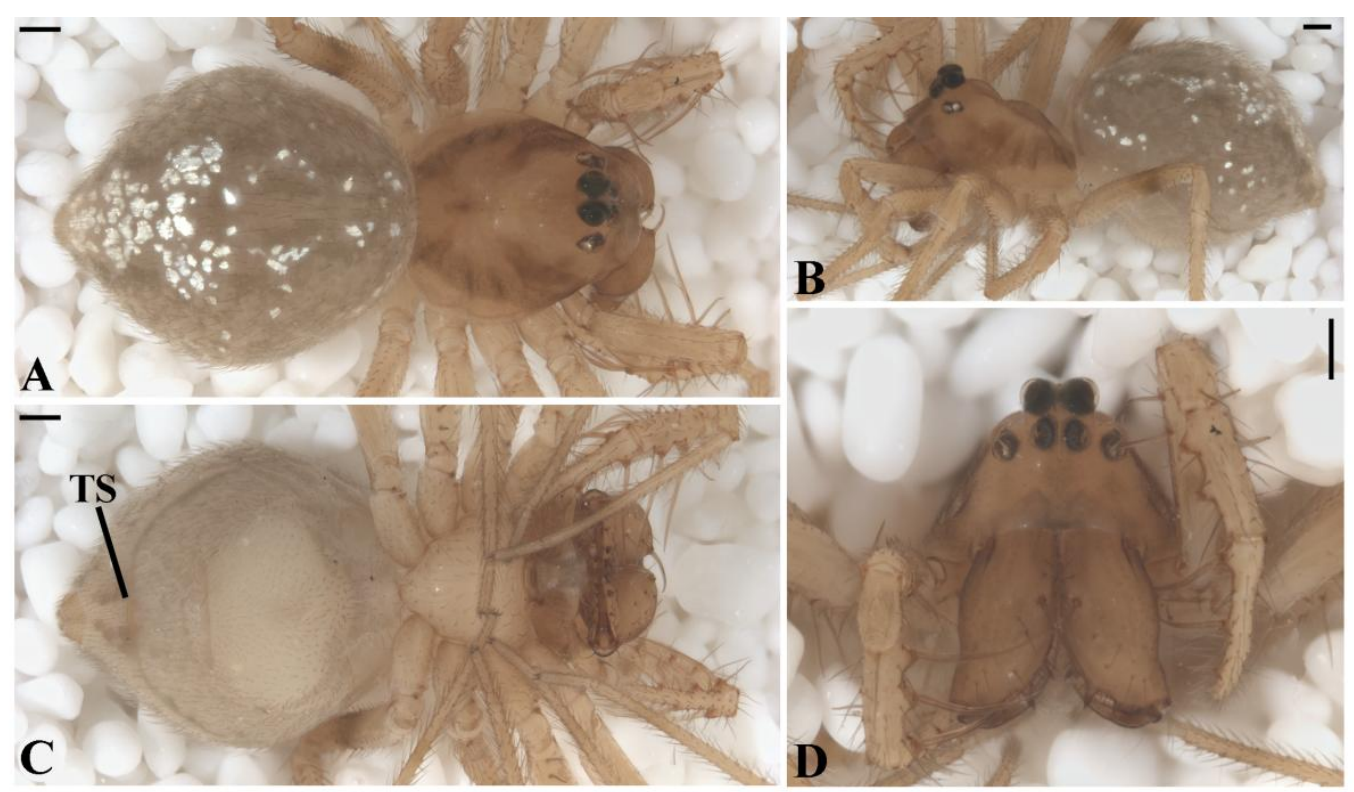

Figure 240. Glenognatha hirsutissima from Marquesas Islands (USNM). Female habitus. A, dorsal. B, lateral. C, ventral. D, frontal. Scale bars, $200 \mu \mathrm{m}$. TS: tracheal spiracle. 


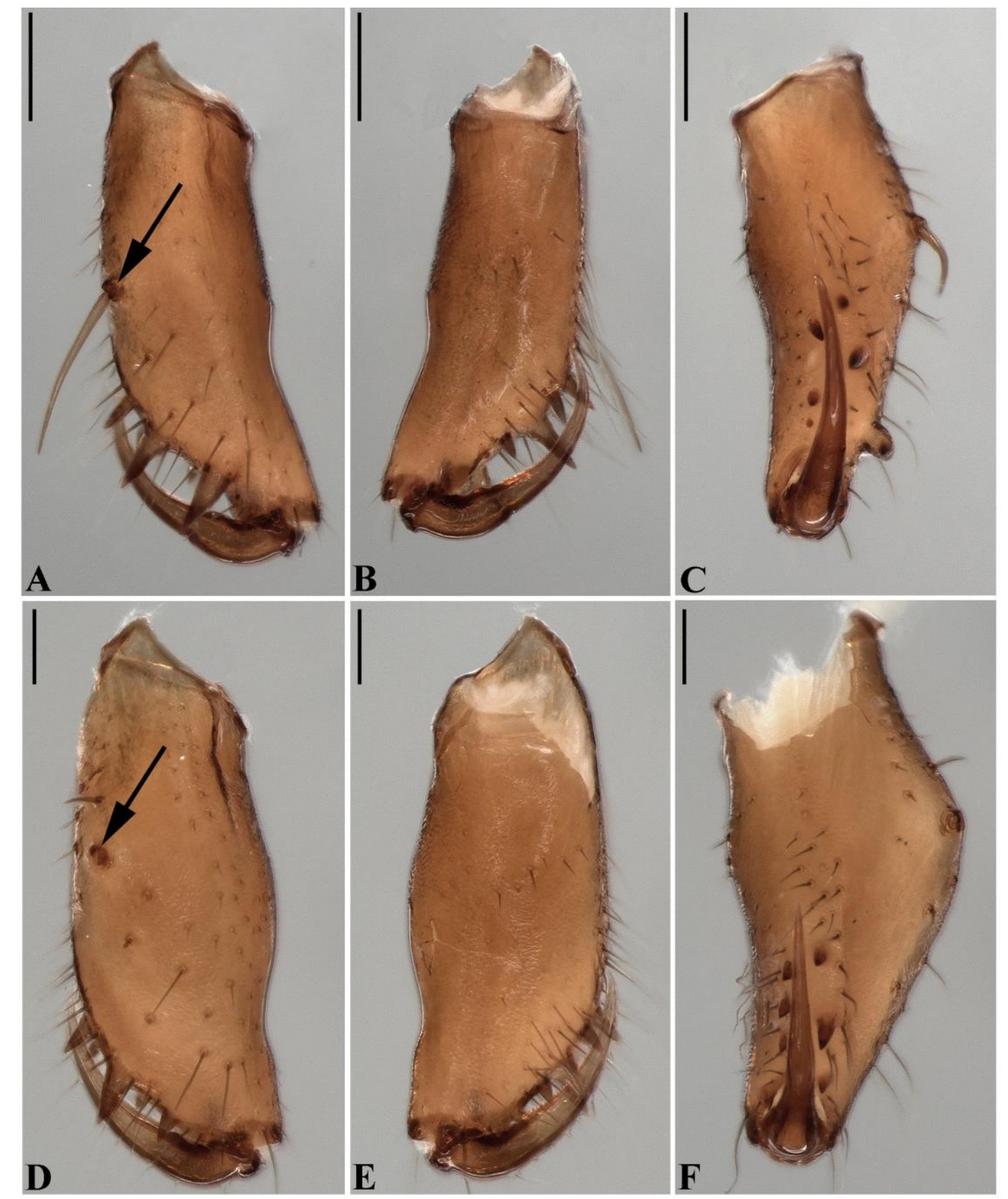

Figure 241. Glenognatha hirsutissima from Marquesas Islands (USNM). A-C, Male left chelicerae. A, anterior (arrow, anterior macrosetae). B, posterior. C, mesal. D-F, Female left chelicerae. D, anterior (arrow, anterior spine socket). E, posterior. F, mesal. Scale bars, $100 \mu \mathrm{m}$. 


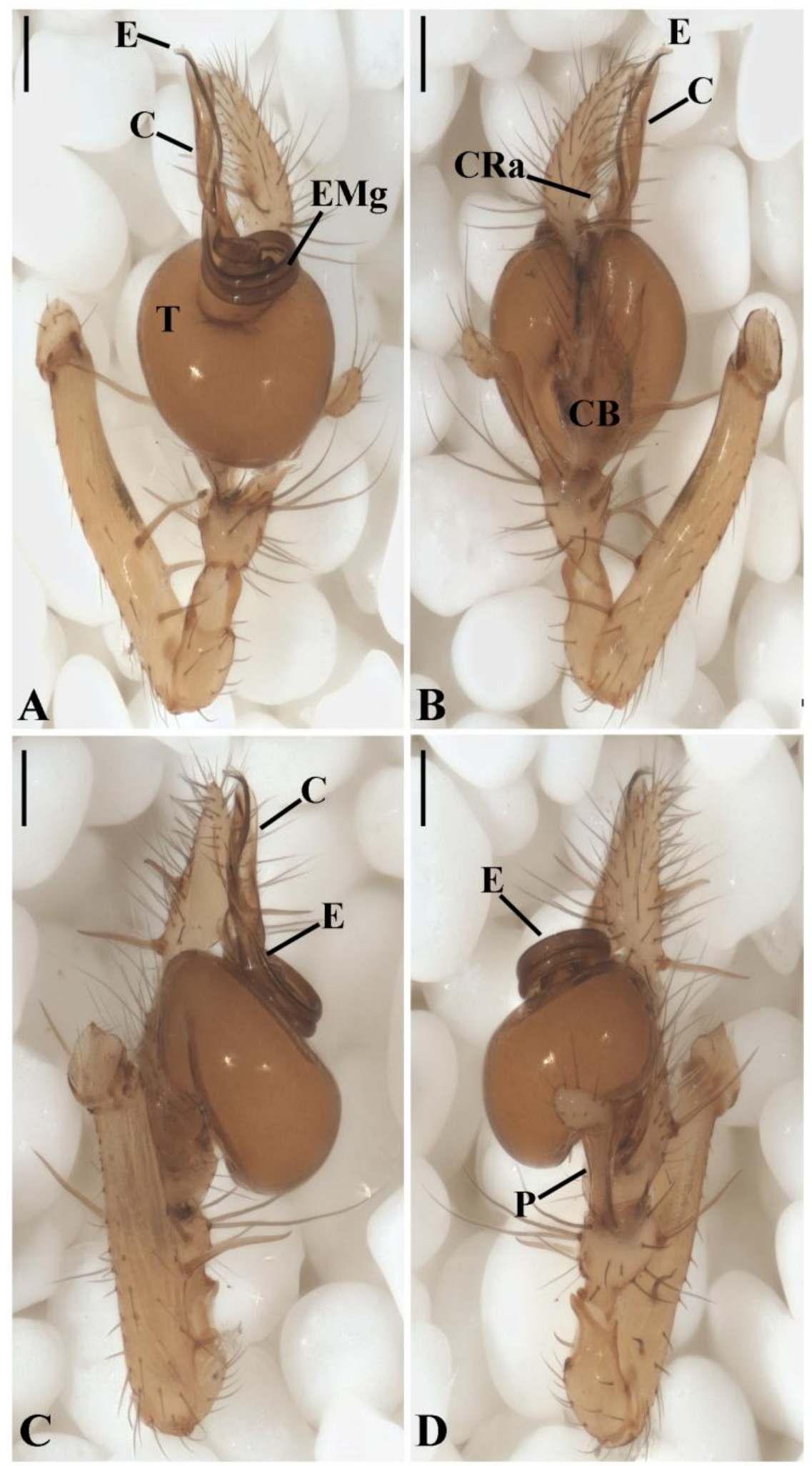

Figure 242. Glenognatha hirsutissima from Marquesas Islands (USNM). Male left palp. A, ventral. B, dorsal. C, prolateral. D, retrolateral. Scale bars, $100 \mu \mathrm{m}$. C: conductor. CB: cymbium. CRa: conductor retrolateral apophysis. E: embolus. EMg: embolic medial groove. P: paracymbium. T: tegulum. 

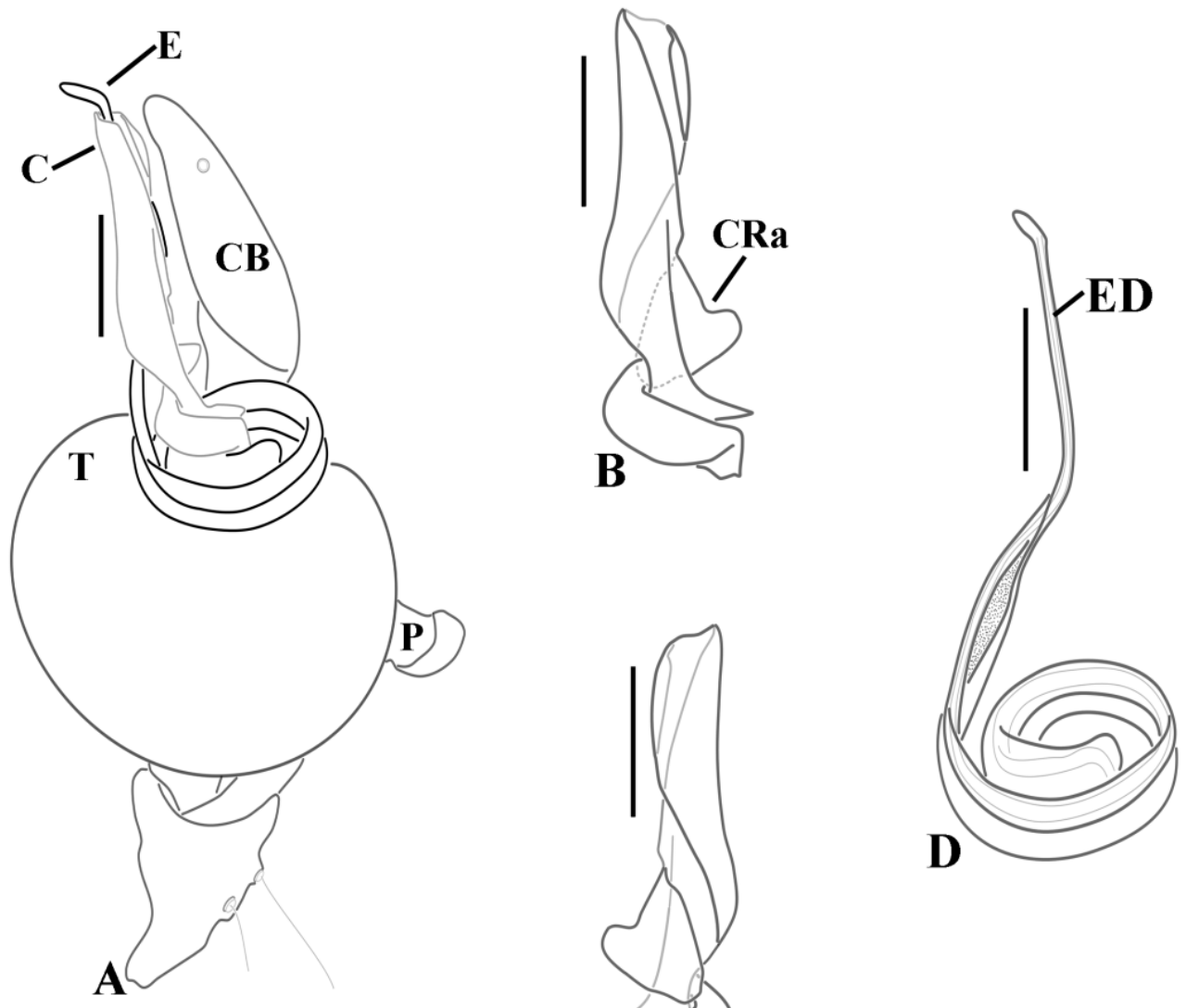

Figure 243. Glenognatha hirsutissima from Marquesas Islands (USNM). Male left palp, schematic. A, ventral. B, conductor ventral. C, conductor dorsal. D, embolus ventral. Scale bars $100 \mu \mathrm{m}$. C: conductor. CB: cymbium. CRa: conductor retrolateral apophysis. E: embolus. ED: ejaculatory duct. P: paracymbium. T: tegulum.
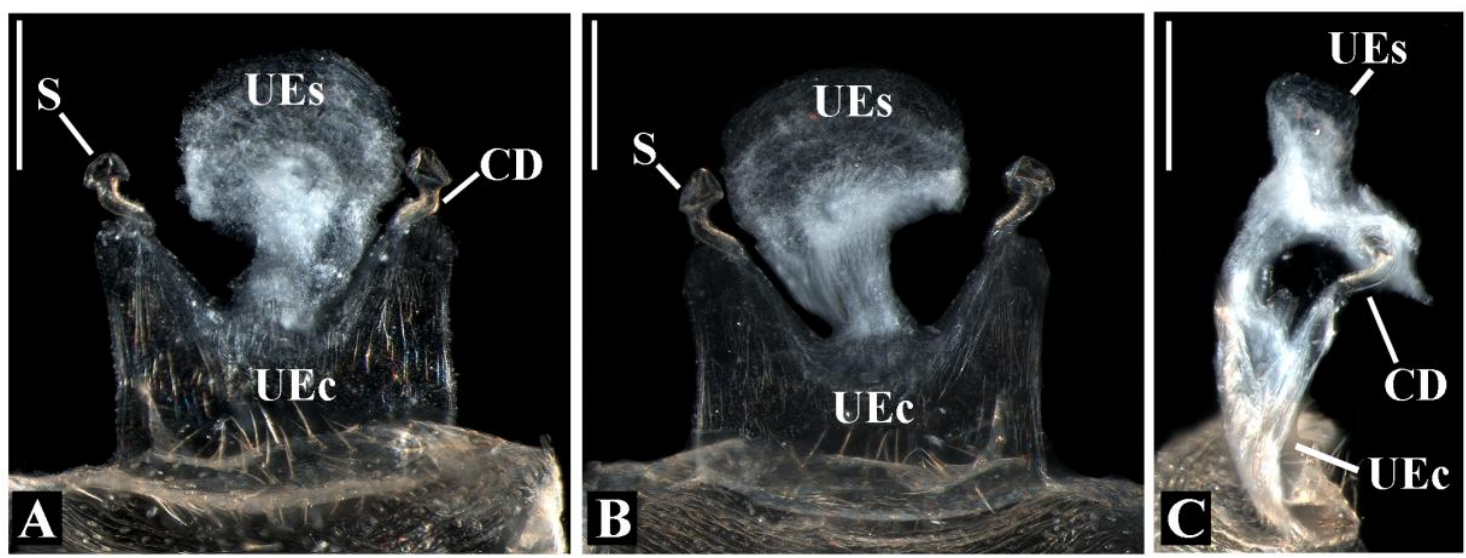

Figure 244. Glenognatha hirsutissima from Marquesas Islands (USNM). Female genitalia. A, dorsal. B, ventral. C, lateral. Scale bars, $200 \mu \mathrm{m}$. CD: copulatory duct. UEc: uterus externus chamber. UEs: uterus externus sac. S: spermathecae. 

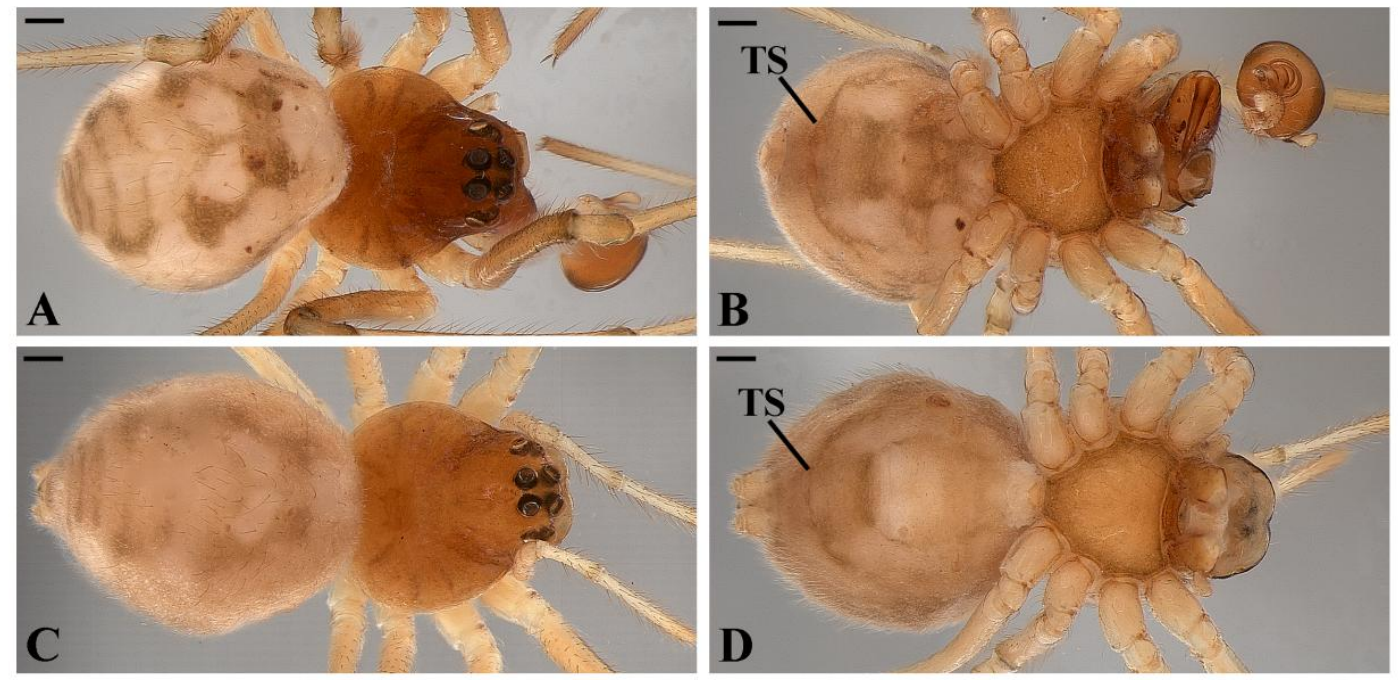

Figure 245. Glenognatha australis from Peru (CAS 9039650). A-B, Male habitus. A, dorsal. B, ventral. C-D, Female habitus. C, dorsal. D, ventral. Scale bars $200 \mu \mathrm{m}$. TS: tracheal spiracle. 

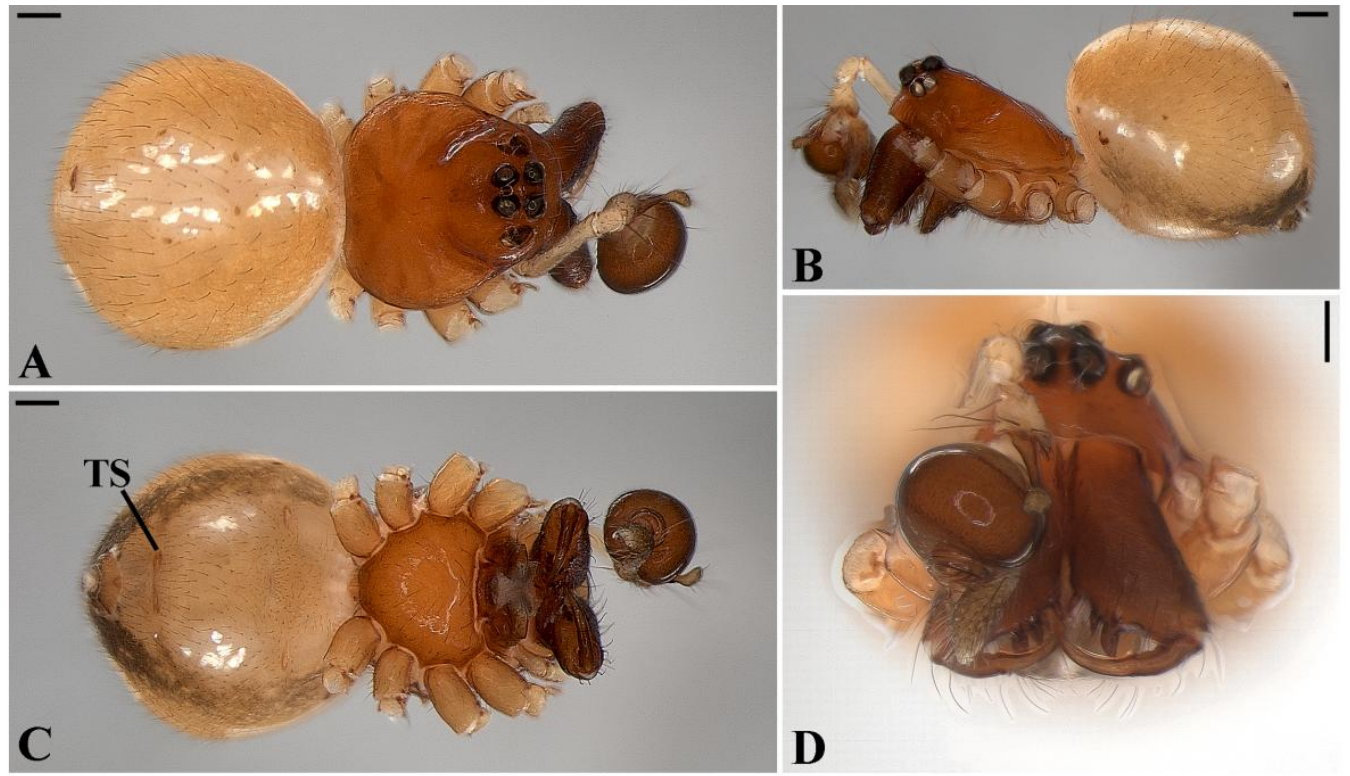

Figure 246. Glenognatha australis from Brazil (MCN 46671). Male habitus. A, dorsal. B, lateral. C, ventral. D, frontal. Scale bars, $200 \mu \mathrm{m}$. TS: tracheal spiracle.
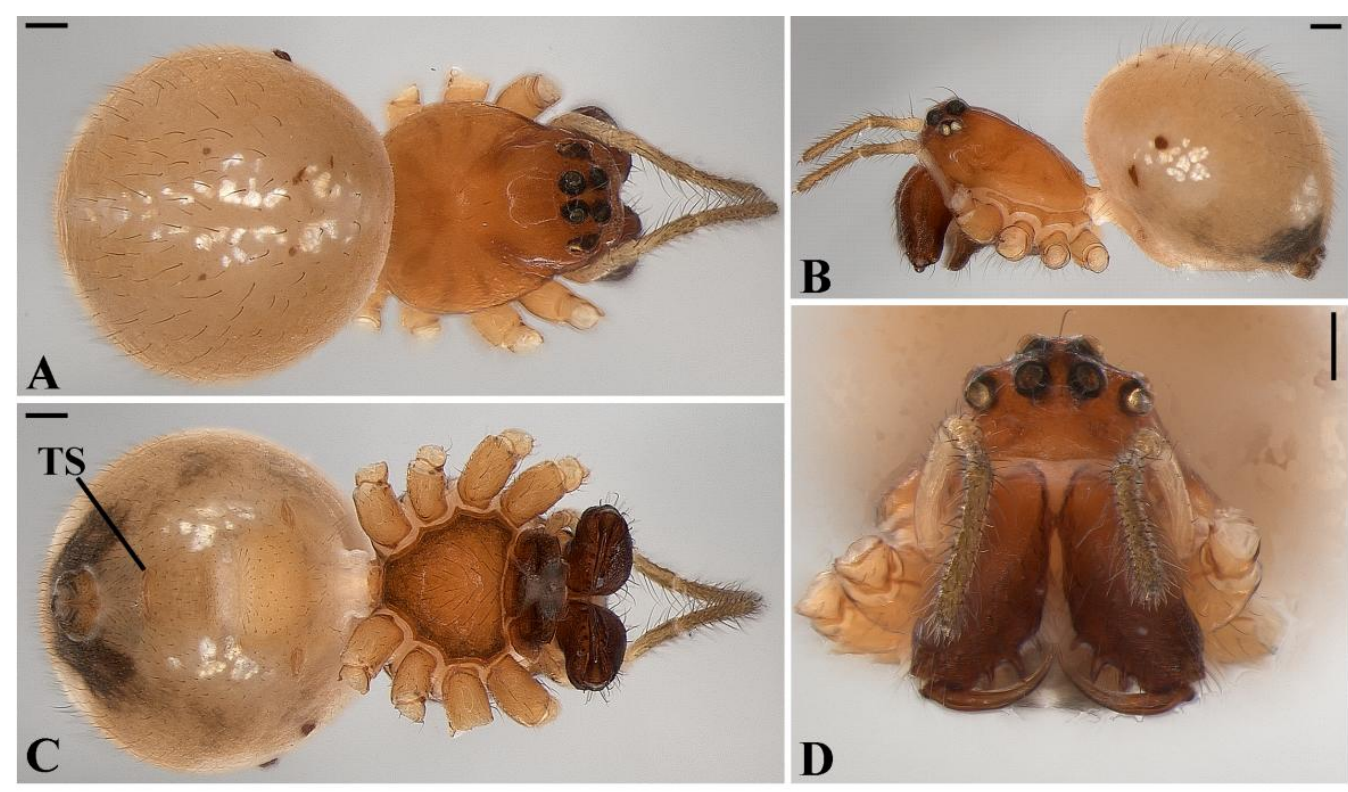

Figure 247. Glenognatha australis from Brazil (MCN 46671). Female habitus. A, dorsal. B, lateral. C, ventral. D, frontal. Scale bars, $200 \mu \mathrm{m}$. TS: tracheal spiracle. 


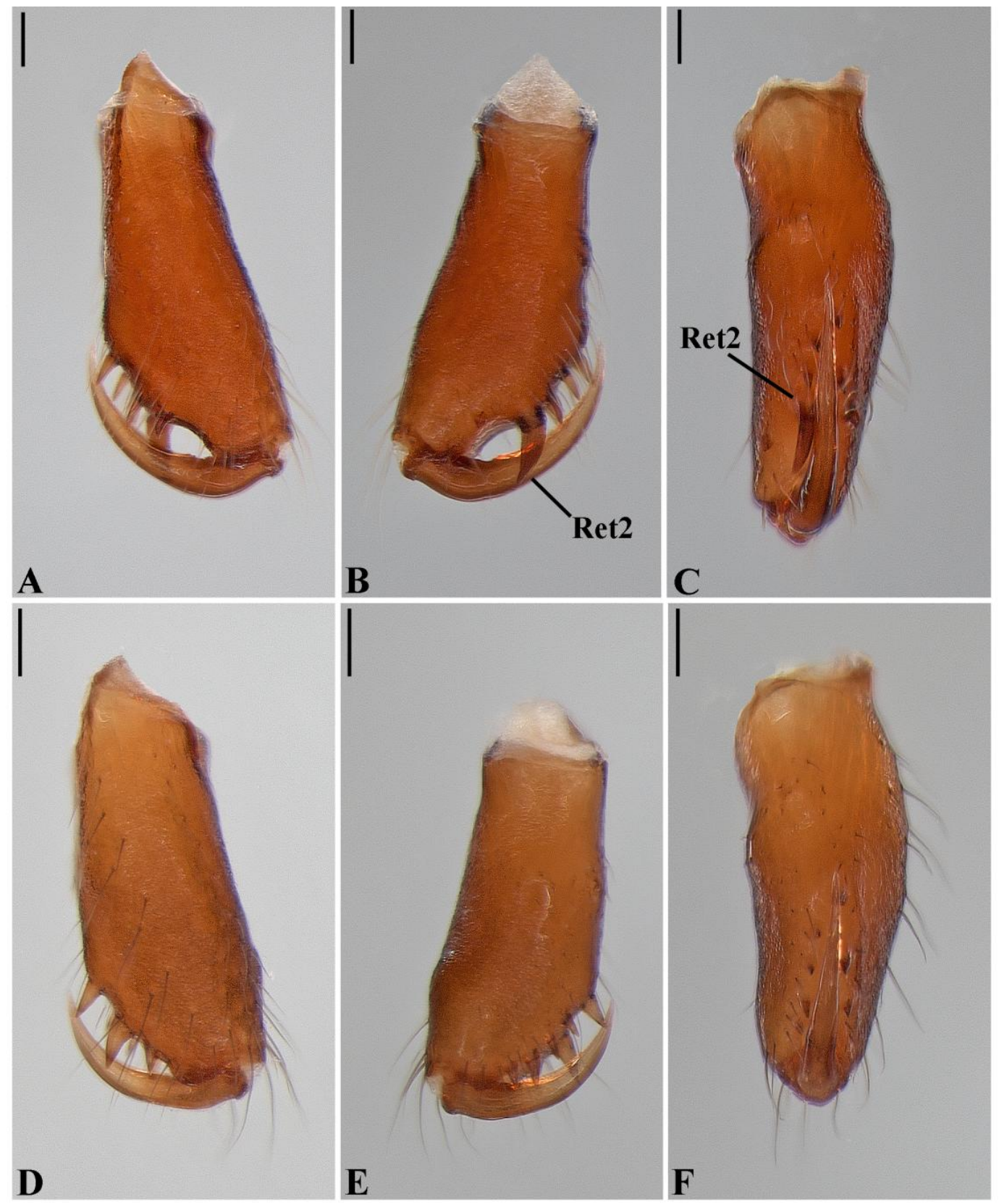

Figure 248. Glenognatha australis. A-C, Male left chelicerae from Argentina (MCT 838). A, anterior. B, posterior. C, mesal. D-F, Female left chelicerae from Brazil (MCN 42102). D, anterior. E, posterior. F, mesal. Scale bars, $100 \mu \mathrm{m}$. Ret: retromarginal tooth. 


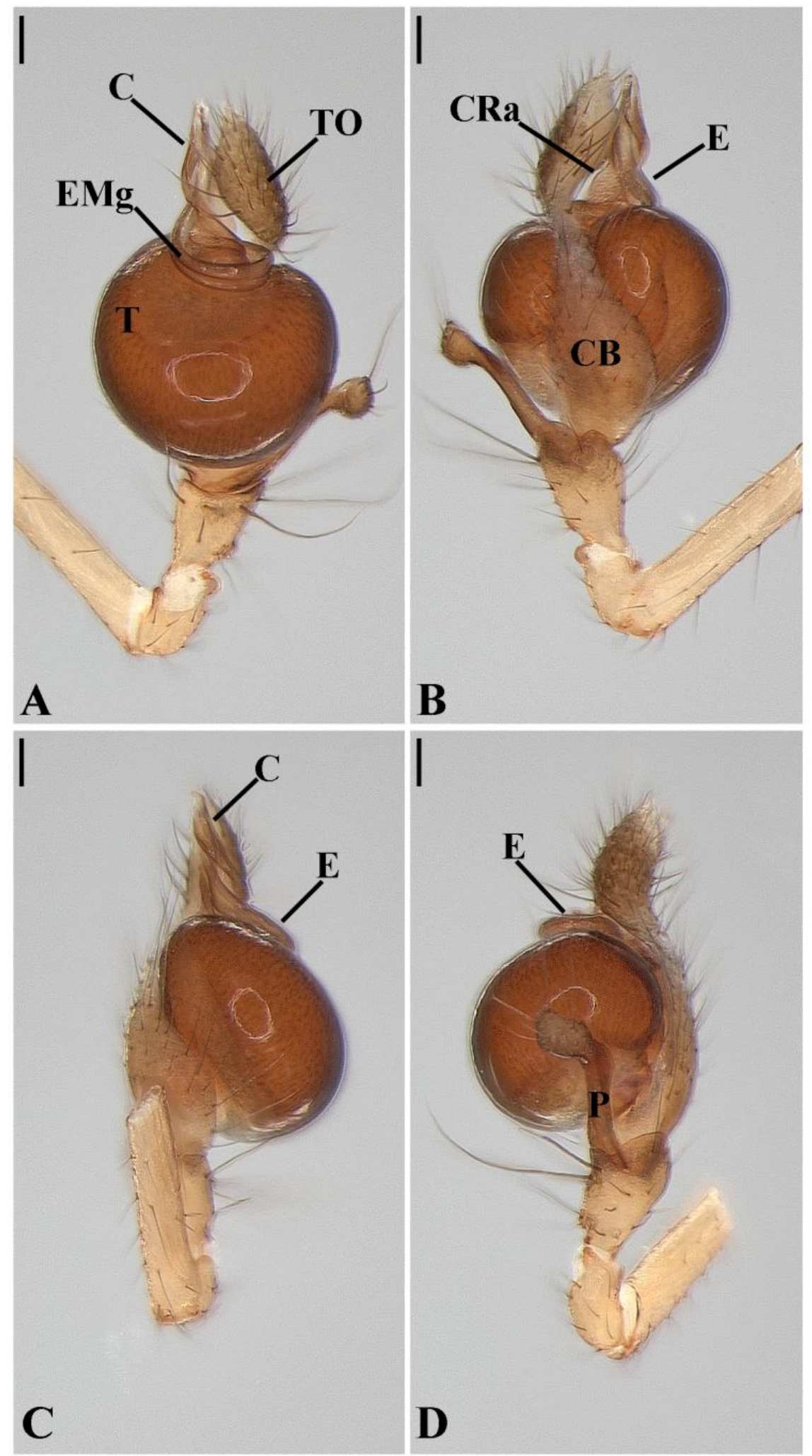

Figure 249. Glenognatha australis from Brazil (MCN 46671). Male left palp. A, ventral. B, dorsal. C, prolateral. D, retrolateral. Scale bars, $100 \mu \mathrm{m}$. C: conductor. CB: cymbium. CRa: conductor retrolateral apophysis. E: embolus. EMg: embolic medial groove. P: paracymbium. T: tegulum. TO: tarsal organ. 

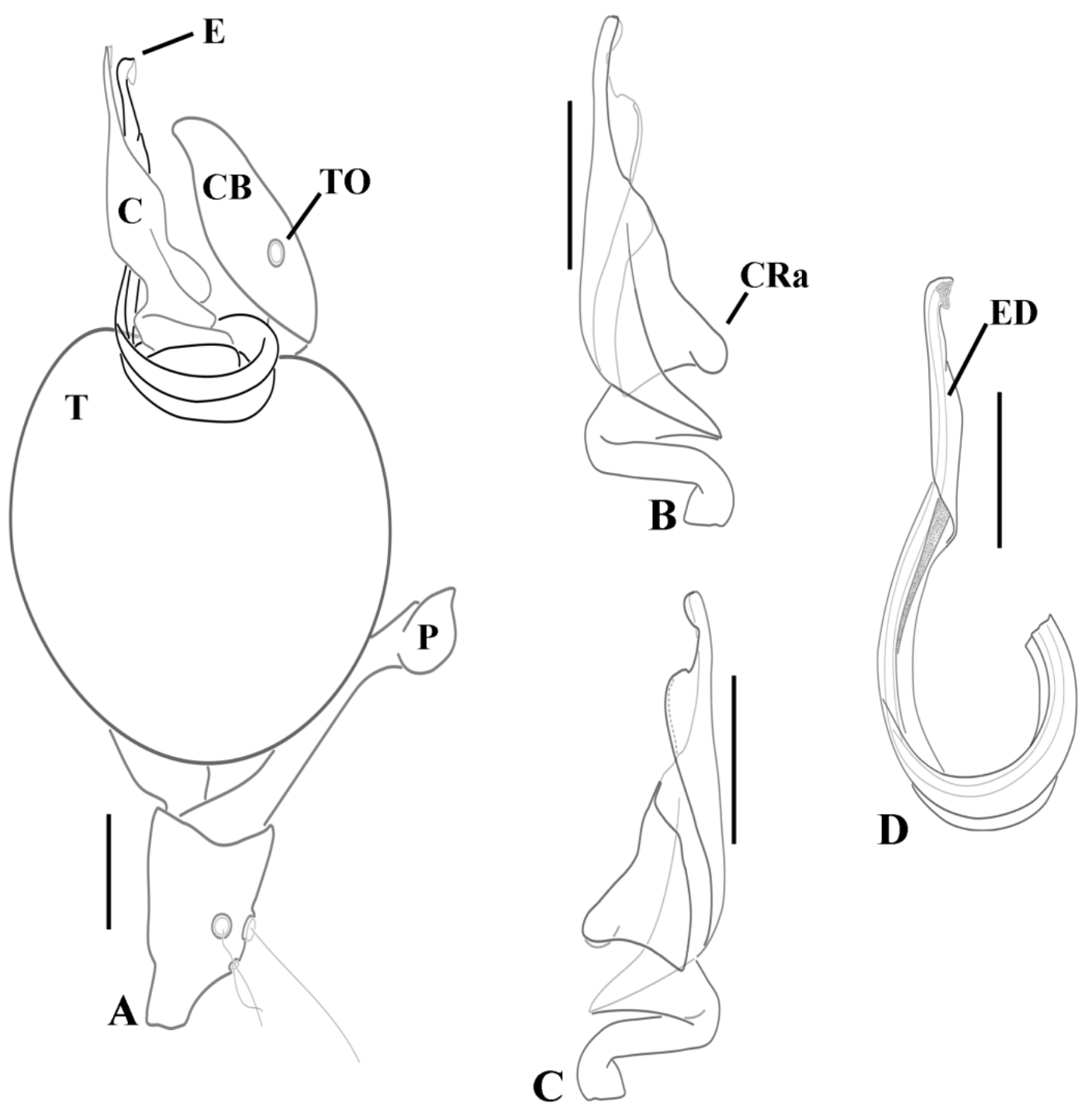

Figure 250. Glenognatha australis from Brazil (MCTP 841). Male left palp, schematic. A, ventral. B, conductor ventral. C, conductor dorsal. D, embolus ventral. Scale bars $100 \mu \mathrm{m}$. C: conductor. CB: cymbium. CRa: conductor retrolateral apophysis. E: embolus. ED: ejaculatory duct. P: paracymbium. T: tegulum. TO: tarsal organ. 


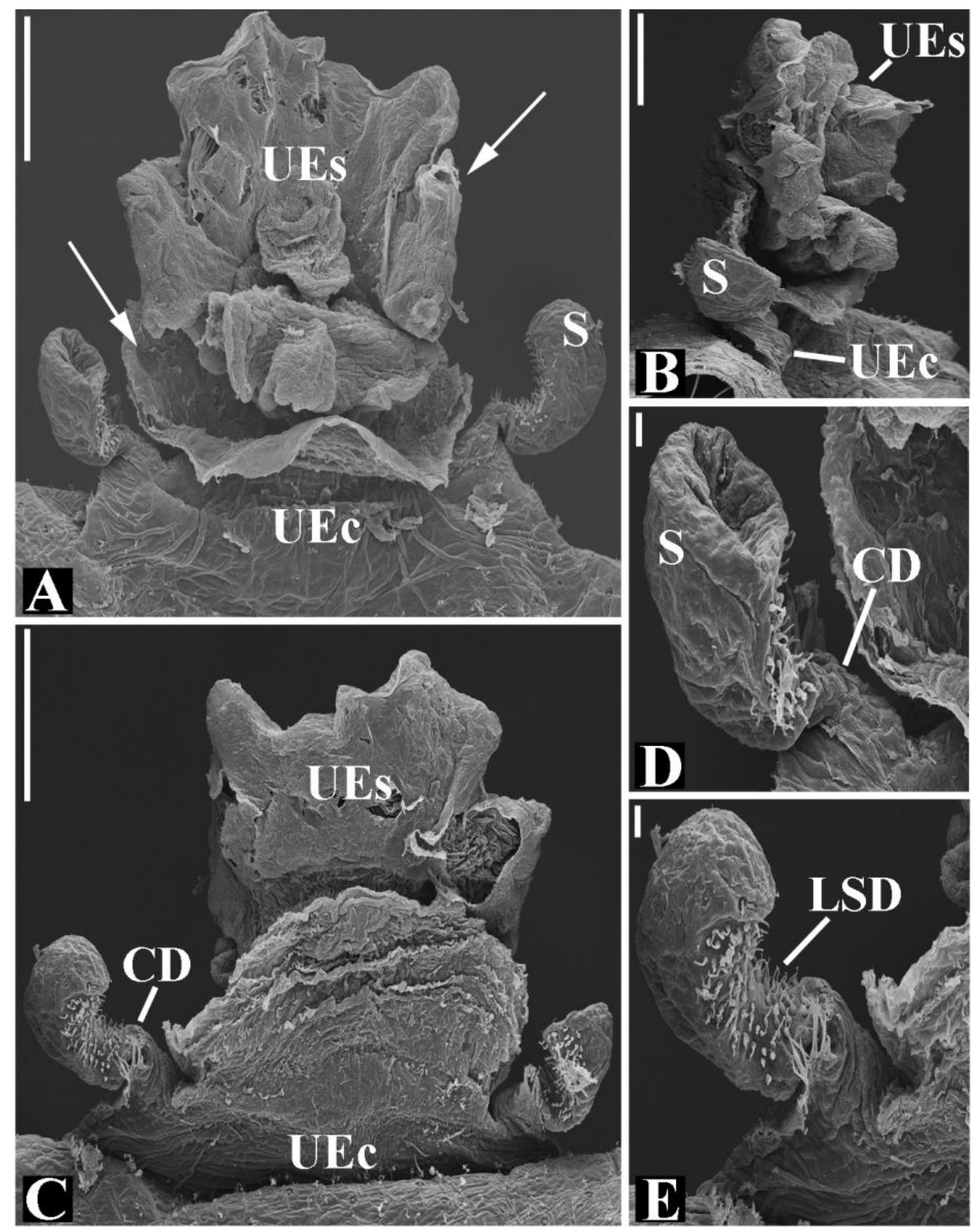

Figure 251. Glenognatha australis from Brazil. Female genitalia. A, dorsal (arrows, disc-shaped portions). B, lateral. C, ventral. D-E, spermathecae and copulatory duct. Scale bars, $100 \mu \mathrm{m}$ (A-C), $10 \mu \mathrm{m}$ (D, E). CD: copulatory duct. LSD: long-stem gland ducts. UEc: uterus externus chamber. UEs: uterus externus sac. S: spermathecae. 


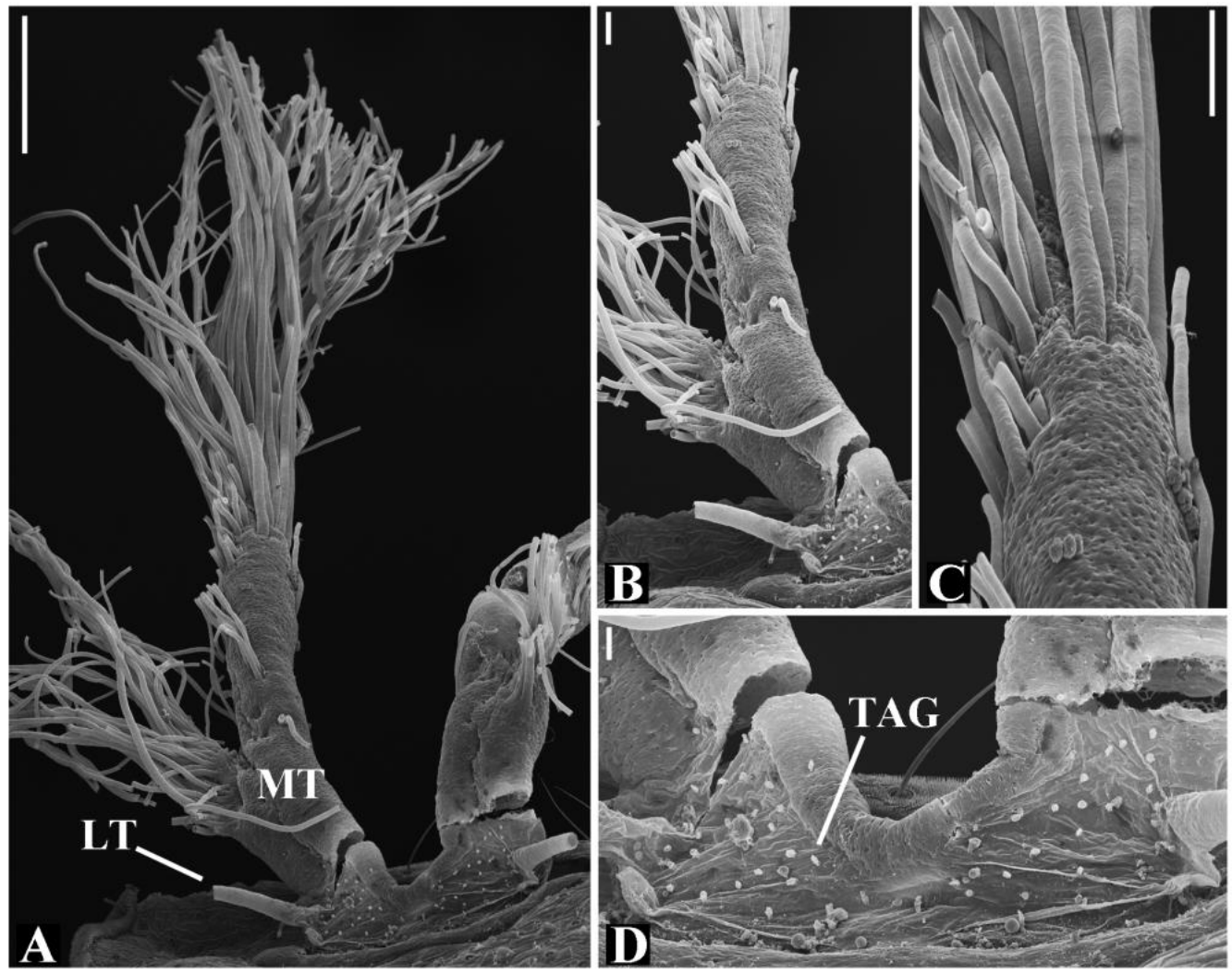

Figure 252. Glenognatha australis from Brazil. Female tracheal system. A, dorsal. B, median and lateral trunks. C, median trunk distal. D, tracheal spiracle posterior view. Scale bars, $100 \mu \mathrm{m}$ (A), $20 \mu \mathrm{m}$ (B-C), $10 \mu \mathrm{m}$ (D). LT: lateral tracheae. MT: median trunks. TAG: tracheal atrium gland. 


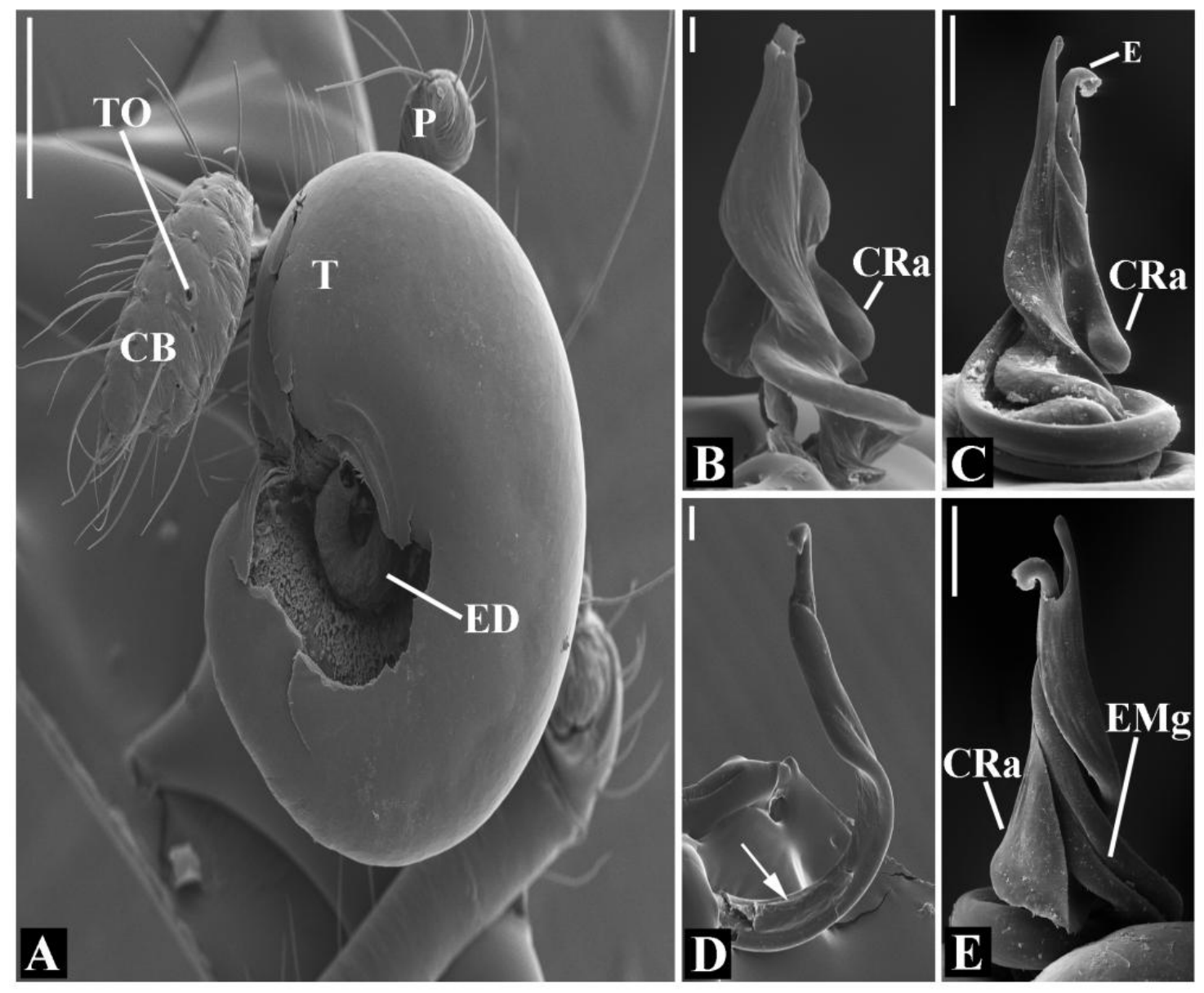

Figure 253. Glenognatha australis from Brazil. A, male left palp frontal. B, conductor ventral (tip broken). C, embolus and conductor ventral. D, embolus (arrow, inner membrane). E, embolus and conductor dorsal. Scale bars $100 \mu \mathrm{m}$ (A), $20 \mu \mathrm{m}$ (B,D), 50 $\mu \mathrm{m}(\mathrm{C}, \mathrm{E}) . \mathrm{CB}$ : cymbium. CRa: conductor retrolateral apophysis. E: embolus. EMg: embolic medial groove. P: paracymbium. T: tegulum. TO: tarsal organ. 

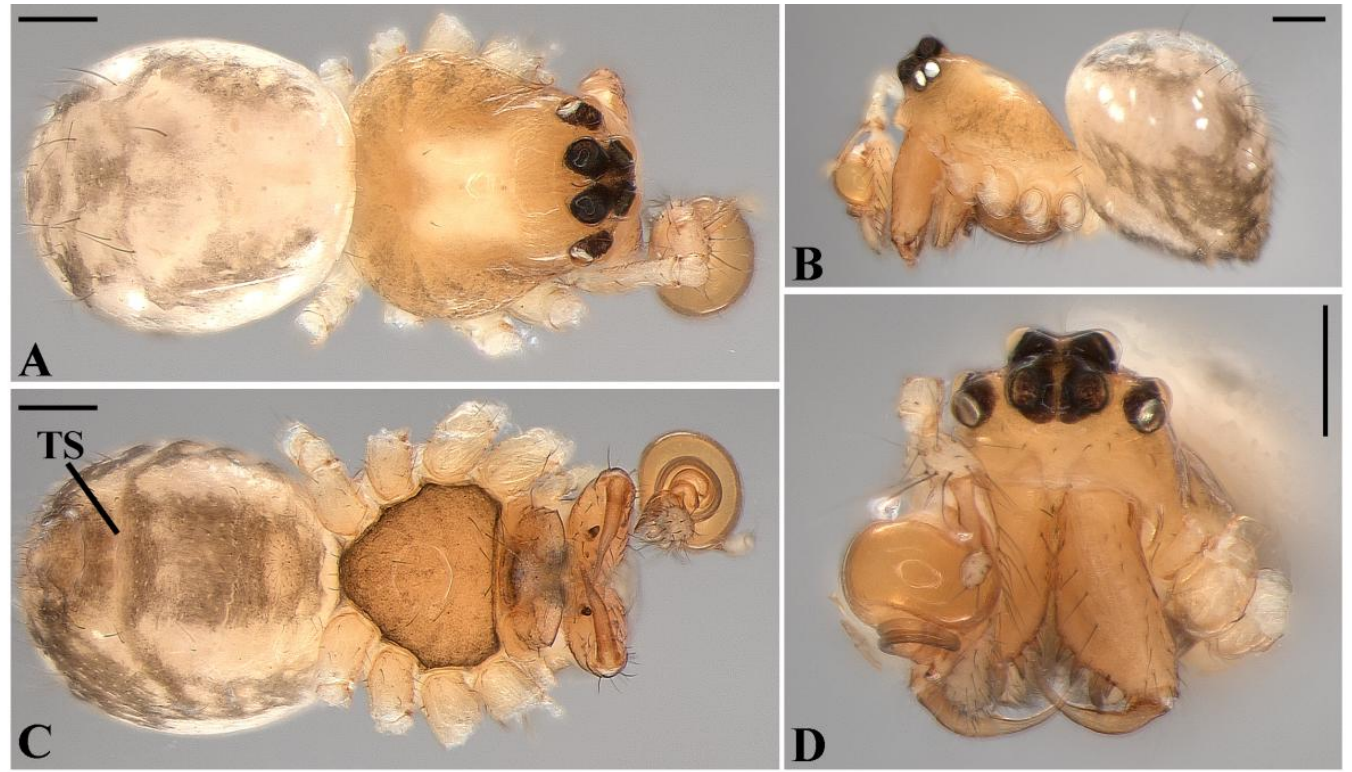

Figure 254. Glenognatha spherella from Mexico (CAS 9046858). Male habitus. A, dorsal. B, lateral. C, ventral. D, frontal. Scale bars, $200 \mu \mathrm{m}$. TS: tracheal spiracle.
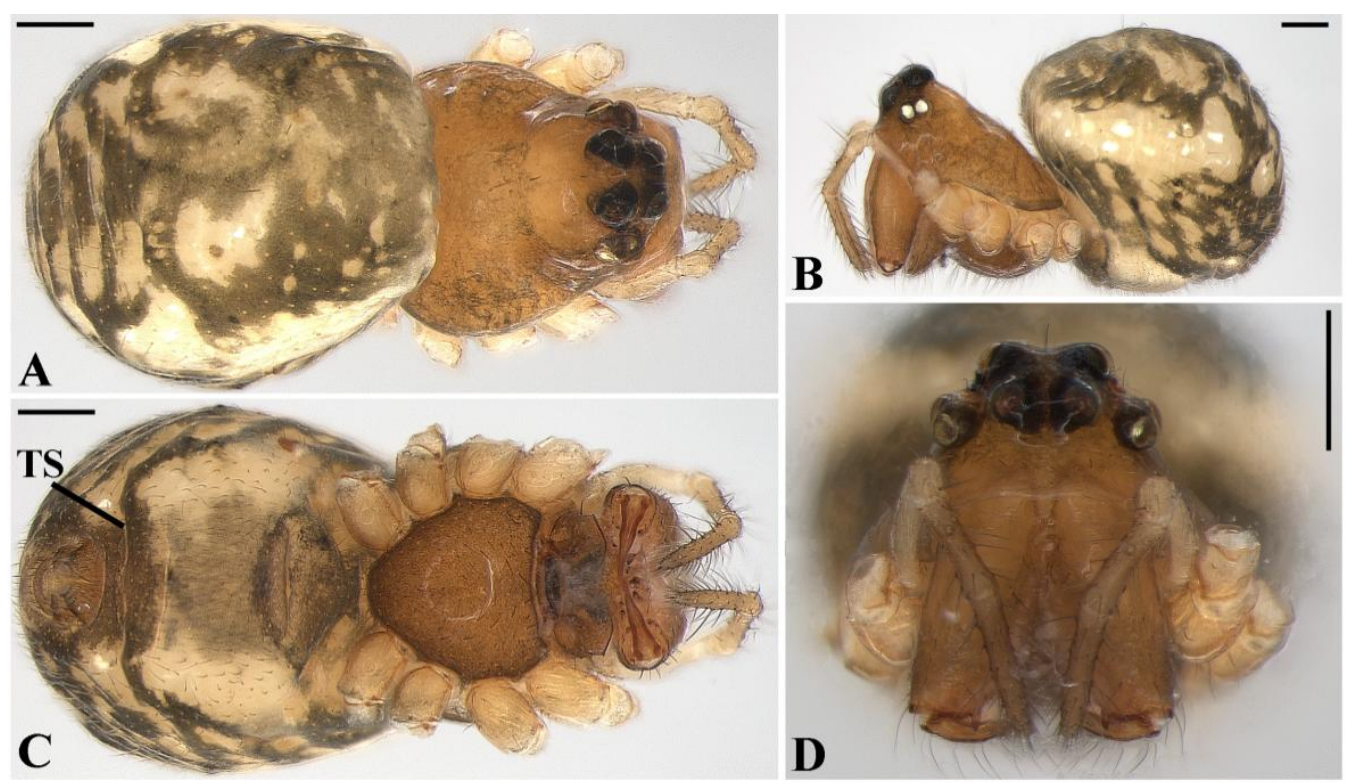

Figure 255. Glenognatha spherella from Mexico (CAS 9046857). Female habitus. A, dorsal. B, lateral. C, ventral. D, frontal. Scale bars, $200 \mu \mathrm{m}$. TS: tracheal spiracle. 

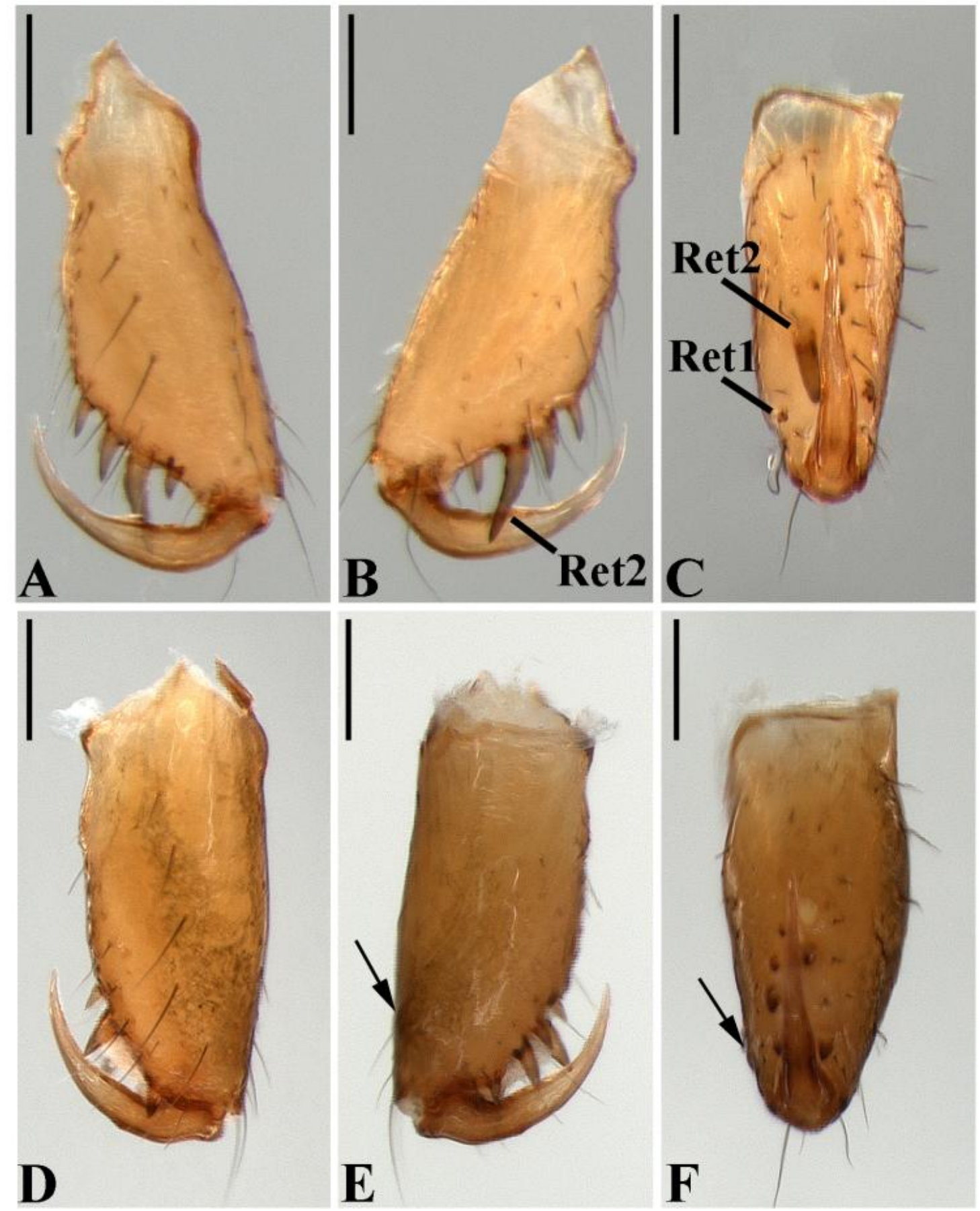

Figure 256. Glenognatha spherella from Mexico. A-C (CAS 9046858), Male left chelicerae. A, anterior. B, posterior. C, mesal. D-F (CAS 9046857), Female left chelicerae. D, anterior. E, posterior (arrow, postero-lateral bulge). F, mesal (arrow, postero-lateral bulge). Scale bars, $100 \mu \mathrm{m}$. Ret: retromarginal tooth. 


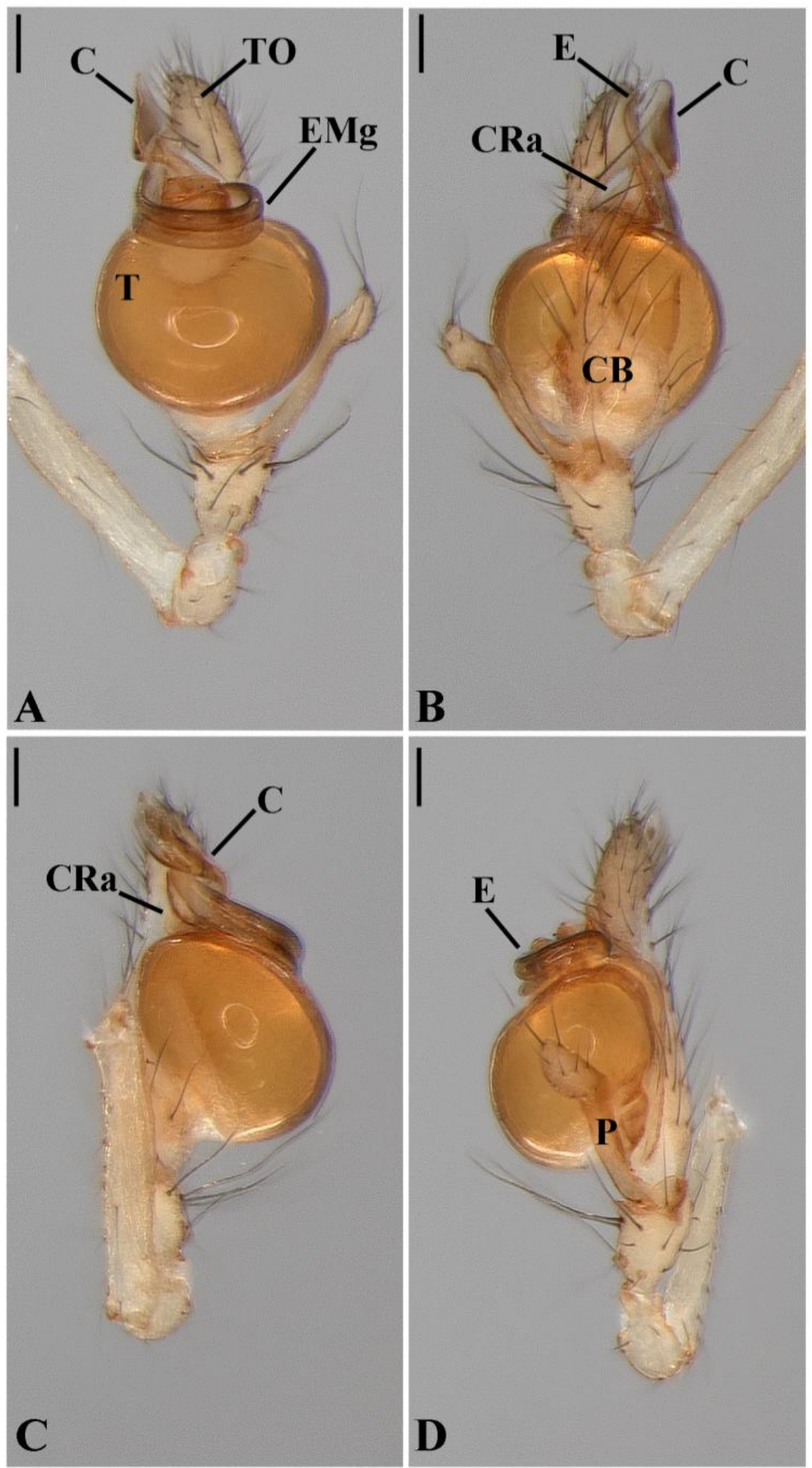

Figure 257. Glenognatha spherella from Mexico (CAS 9046858). Male left palp. A, ventral. B, dorsal. C, prolateral. D, retrolateral. Scale bars, $100 \mu \mathrm{m}$. C: conductor. CB: cymbium. CRa: conductor retrolateral apophysis. E: embolus. EMg: embolic medial groove. P: paracymbium. T: tegulum. TO: tarsal organ. 

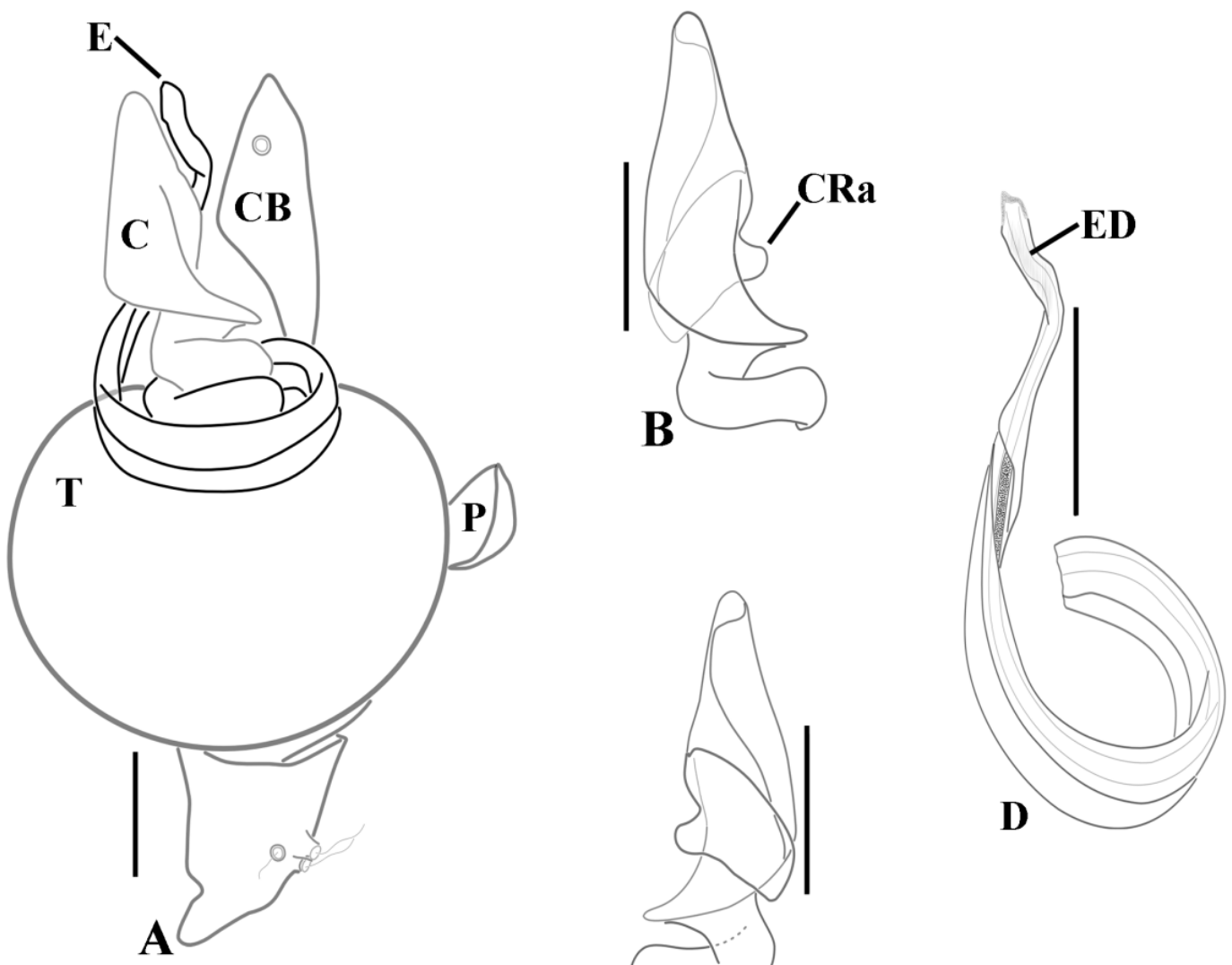

Figure 258. Glenognatha spherella from Mexico (AMNH). Male left palp, schematic. A, ventral. B, conductor ventral. C, conductor dorsal. D, embolus ventral. Scale bars $100 \mu \mathrm{m}$. C: conductor. CB: cymbium. CRa: conductor retrolateral apophysis. E: embolus. ED: ejaculatory duct. P: paracymbium. T: tegulum. 


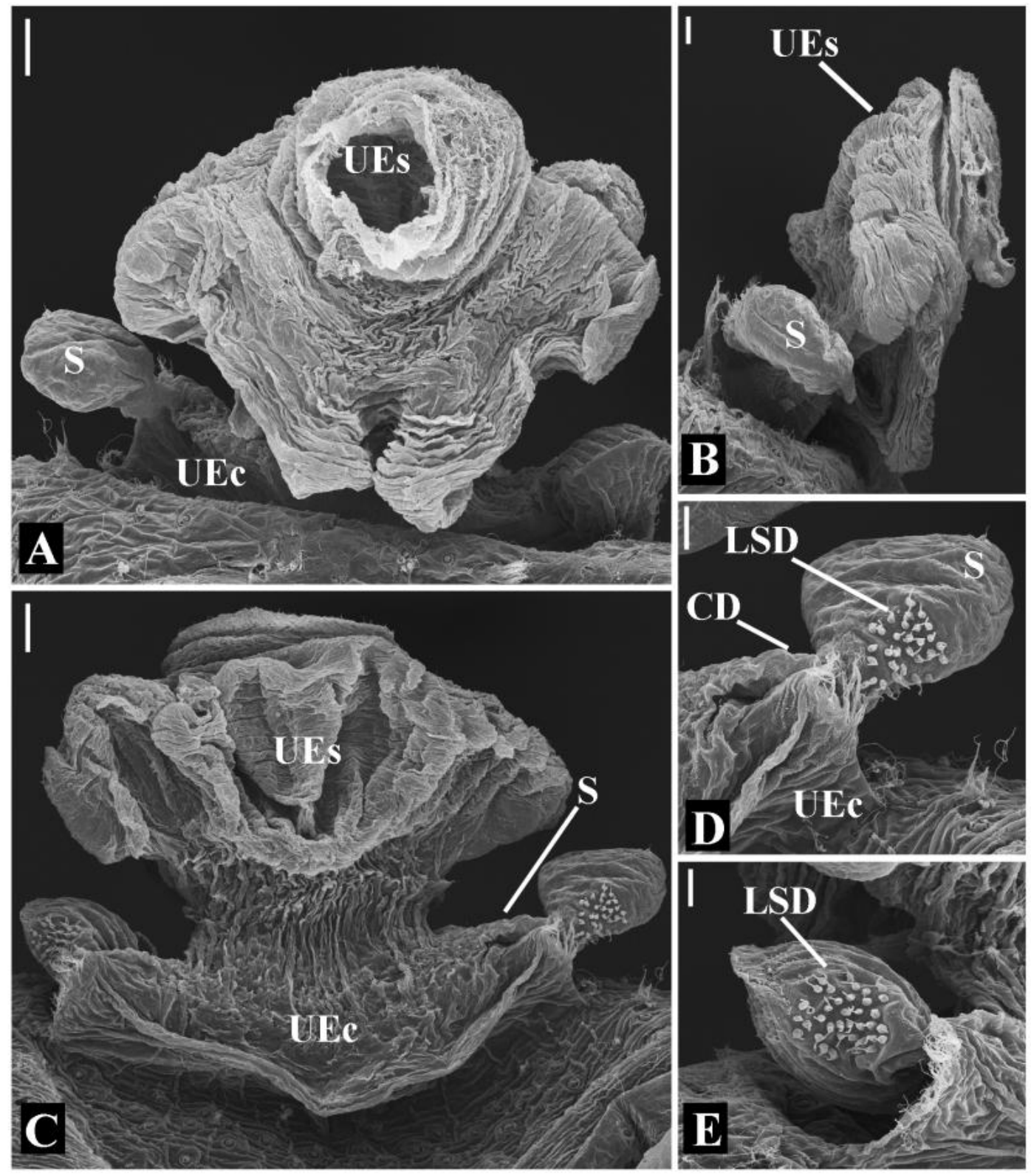

Figure 259. Glenognatha spherella from Costa Rica. Female genitalia. A, dorsal. B, lateral. C, ventral D-E, spermathecae and copulatory duct. Scale bars, $20 \mu \mathrm{m}$ (A, C), $10 \mu \mathrm{m}$ (B, D-E). CD: copulatory duct. LSD: long-stem gland ducts. UEc: uterus externus chamber. UEs: uterus externus sac. S: spermathecae. 


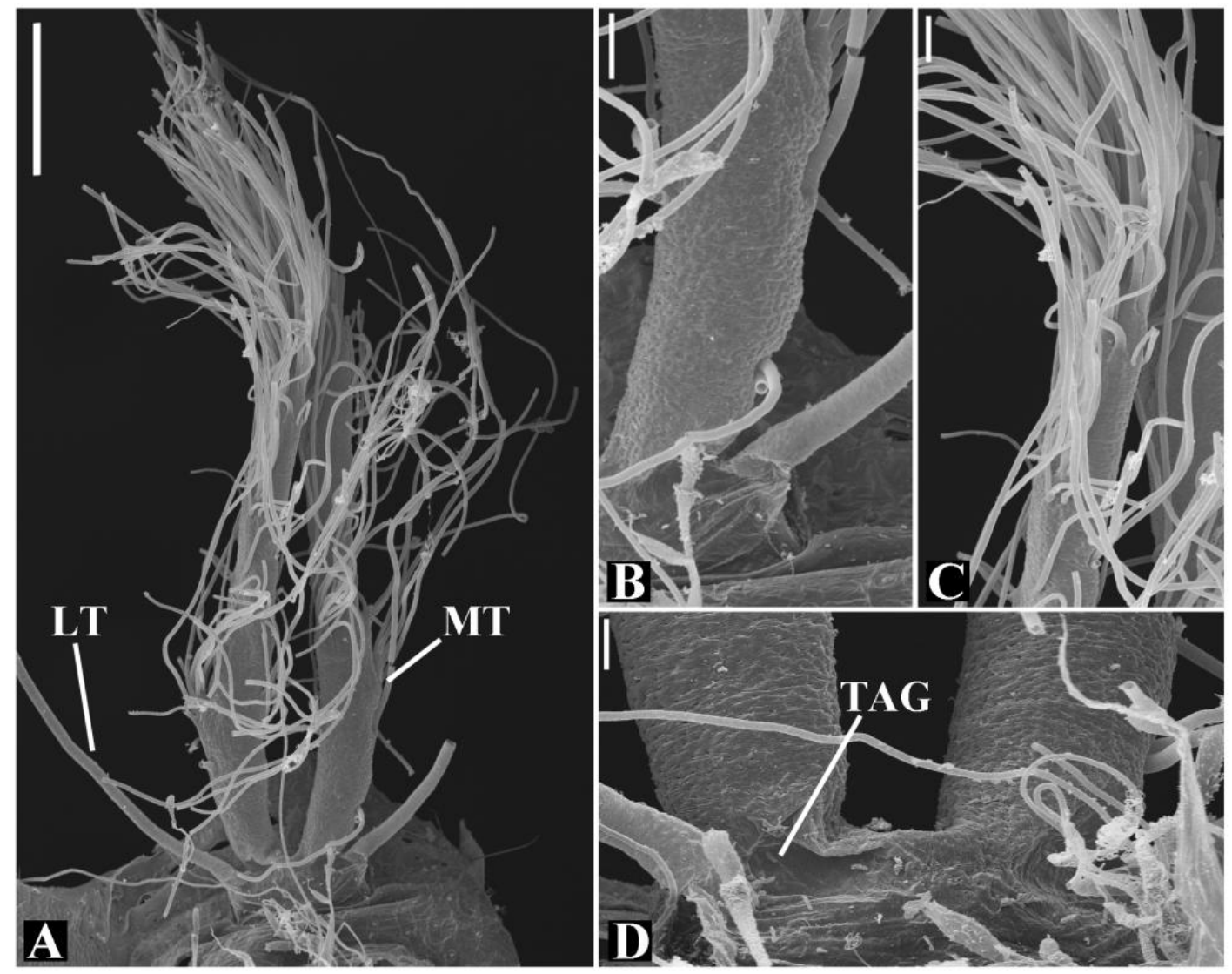

Figure 260. Glenognatha spherella from Mexico. Female tracheal system. A, dorsal. B, median and lateral trunks. C, median trunk distal. D, tracheal spiracle posterior view. Scale bars, $100 \mu \mathrm{m}$ (A), $20 \mu \mathrm{m}$ (B, C), $10 \mu \mathrm{m}$ (D). LT: lateral tracheae. MT: median trunks. TAG: tracheal atrium gland. 


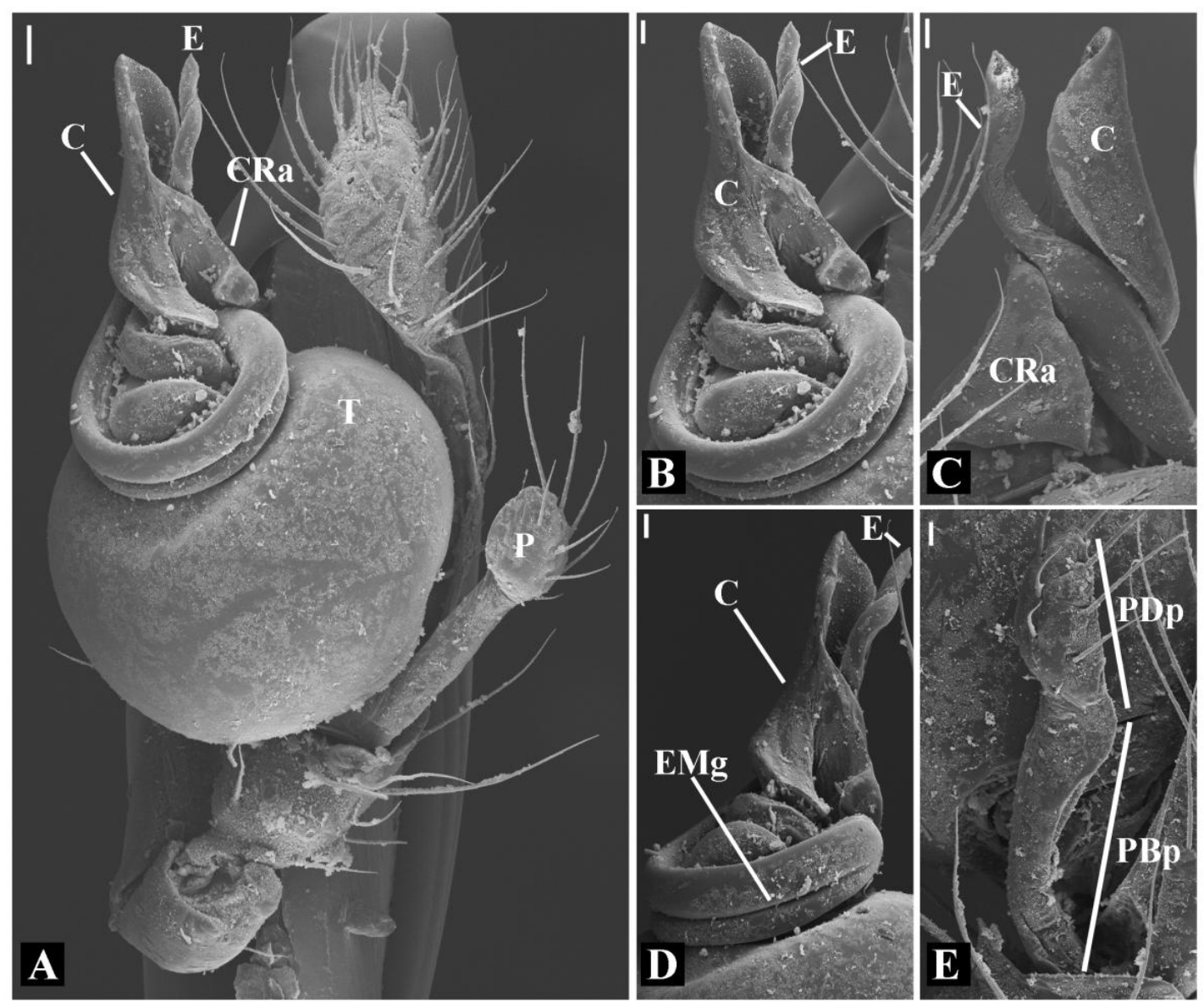

Figure 261. Glenognatha spherella from Costa Rica. A, male left palp ventral. B, embolus and conductor ventral. C, embolus and conductor dorsal. D, embolus and conductor retrolateral. E, paracymbium. Scale bars $20 \mu \mathrm{m}$ (A), $10 \mu \mathrm{m}$ (B-E). C: conductor. CRa: conductor retrolateral apophysis. E: embolus. EMg: embolic medial groove. P: paracymbium. PBp: paracymbium basal portion. PDp: paracymbium distal portion. T: tegulum. 

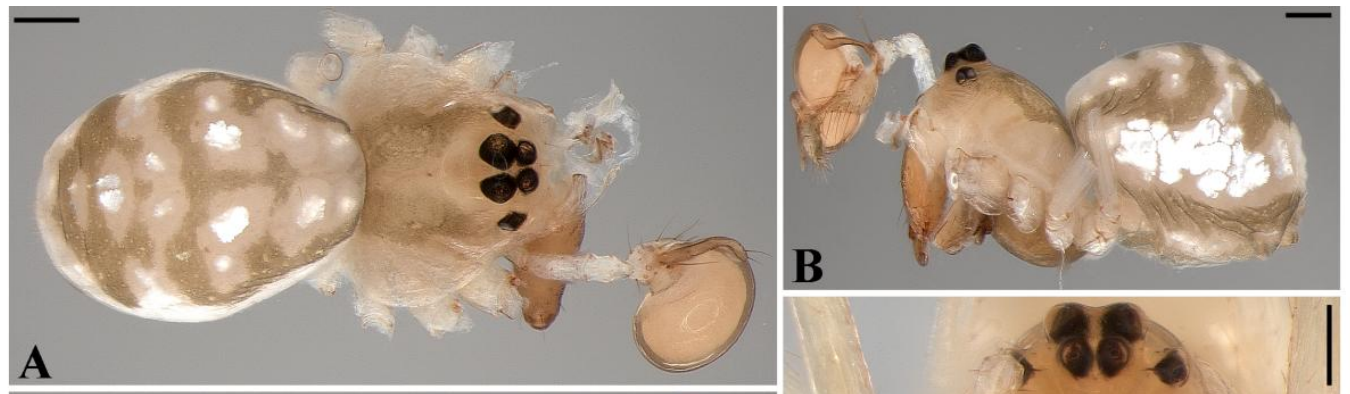

A

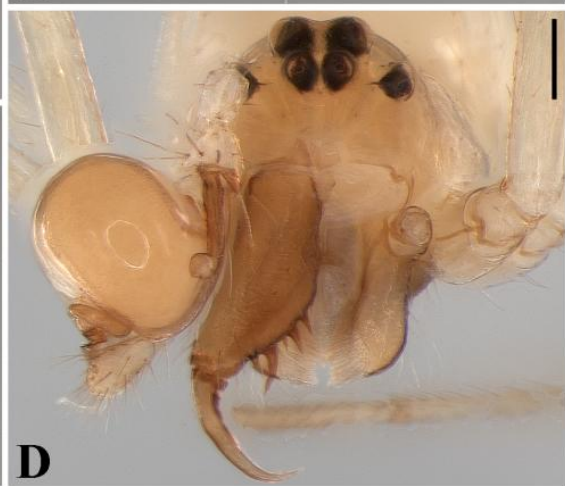

Figure 262. Glenognatha gloriae from Puerto Rico (MCZ 125485). Male habitus. A, dorsal. B, lateral. C, ventral. D, frontal (MCZ 125482). Scale bars, $200 \mu \mathrm{m}$ (A-C), 100 $\mu \mathrm{m}$ (D). TS: tracheal spiracle.

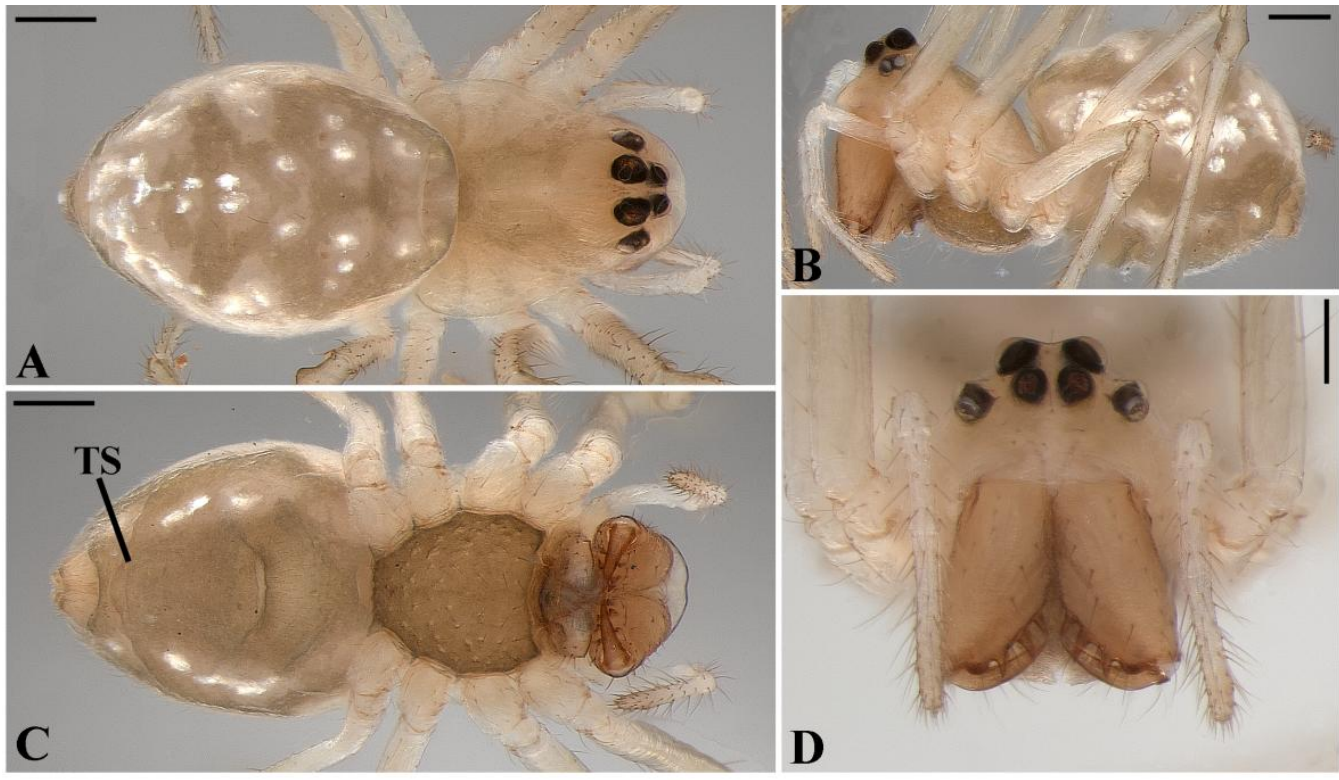

Figure 263. Glenognatha gloriae from Puerto Rico (MCZ 125485). Female habitus. A, dorsal. B, lateral. C, ventral. D, frontal. Scale bars, $200 \mu \mathrm{m}$ (A-C), $100 \mu \mathrm{m}$ (D). TS: tracheal spiracle. 


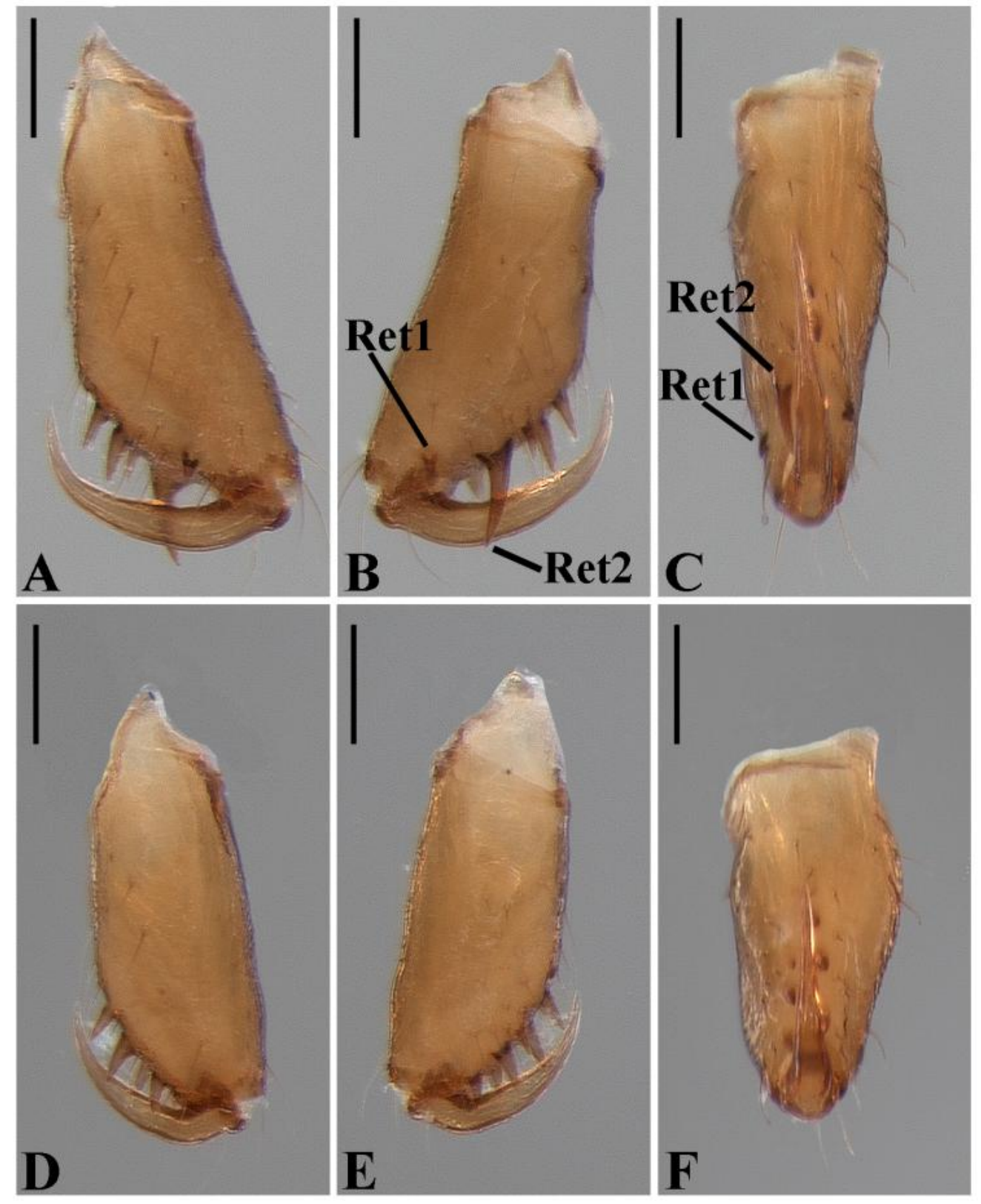

Figure 264. Glenognatha gloriae from Puerto Rico. A-C, Male left chelicerae (MCZ 125482). A, anterior. B, posterior. C, mesal. D-F, Female left chelicerae (MCZ 125482). D, anterior. E, posterior. F, mesal. Scale bars, $100 \mu \mathrm{m}$. Ret: retromarginal tooth. 


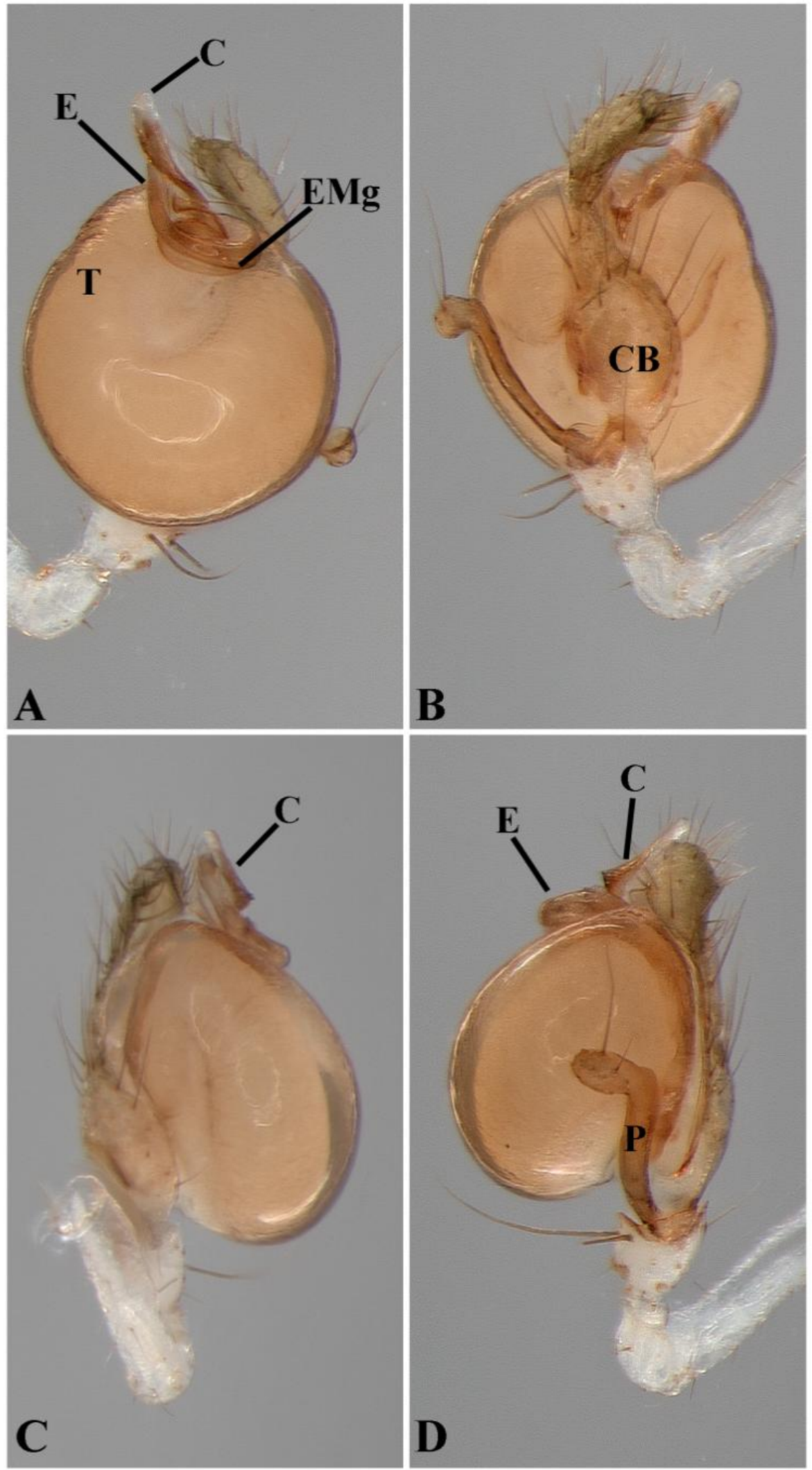

Figure 265. Glenognatha gloriae from Puerto Rico (MCZ 125485). Male left palp. A, ventral. B, dorsal. C, prolateral. D, retrolateral. Scale bars, $100 \mu \mathrm{m}$. C: conductor. CB: cymbium. E: embolus. EMg: embolic medial groove. P: paracymbium. T: tegulum. 

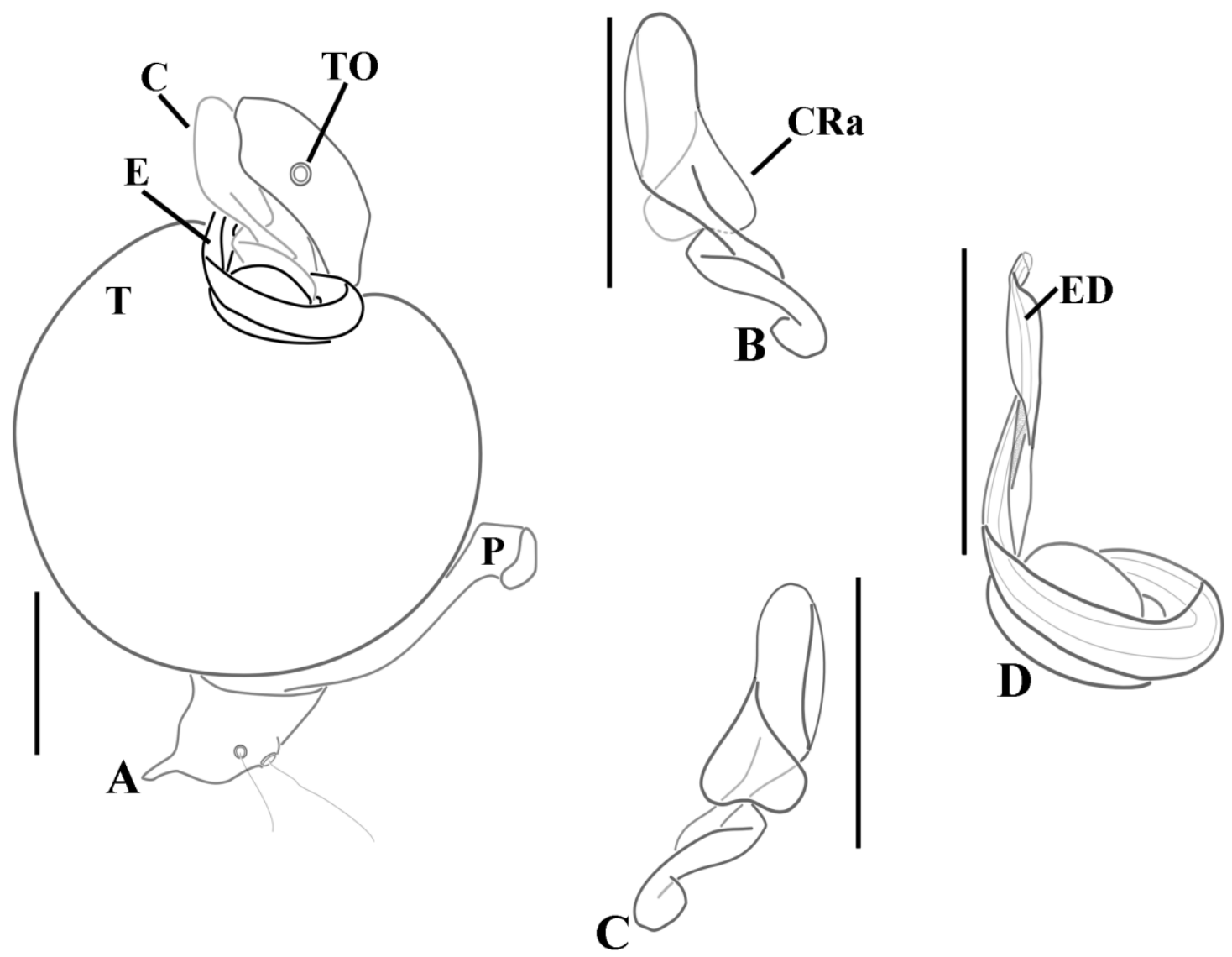

Figure 266. Glenognatha gloriae from Puerto Rico (MCZ 125485). Male left palp, schematic. A, ventral. B, conductor ventral. C, conductor dorsal. D, embolus ventral. Scale bars $100 \mu \mathrm{m}$. C: conductor. CRa: conductor retrolateral apophysis. E: embolus. ED: ejaculatory duct. P: paracymbium. T: tegulum. TO: tarsal organ. 

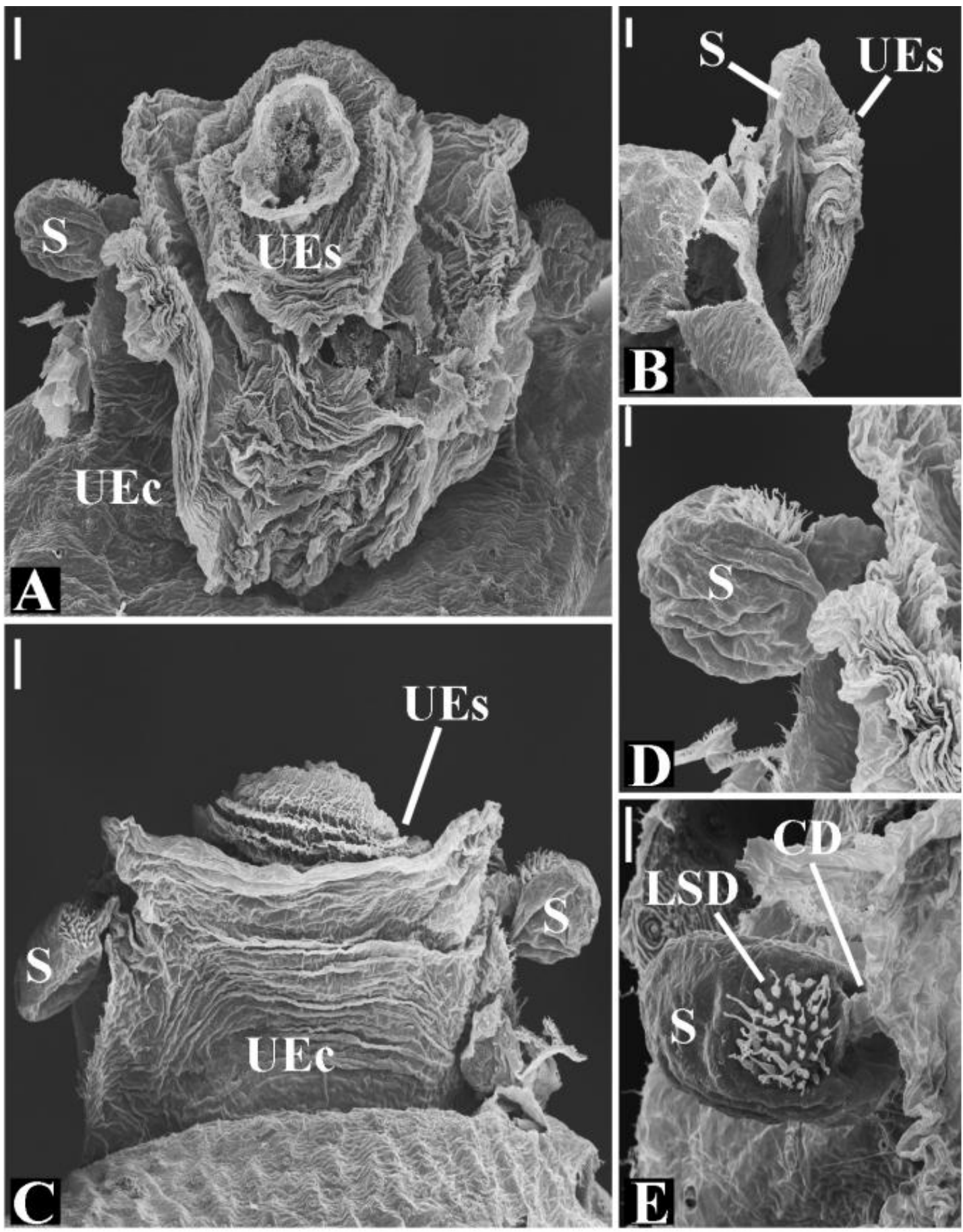

Figure 267. Glenognatha gloriae from Puerto Rico. Female genitalia. A, dorsal. B, lateral. C, ventral. D-E, spermathecae and copulatory duct. Scale bars, $20 \mu \mathrm{m}$ (A-C), $10 \mu \mathrm{m}$ (D, E). CD: copulatory duct. LSD: long-stem gland ducts. UEc: uterus externus chamber. UEs: uterus externus sac. S: spermathecae. 

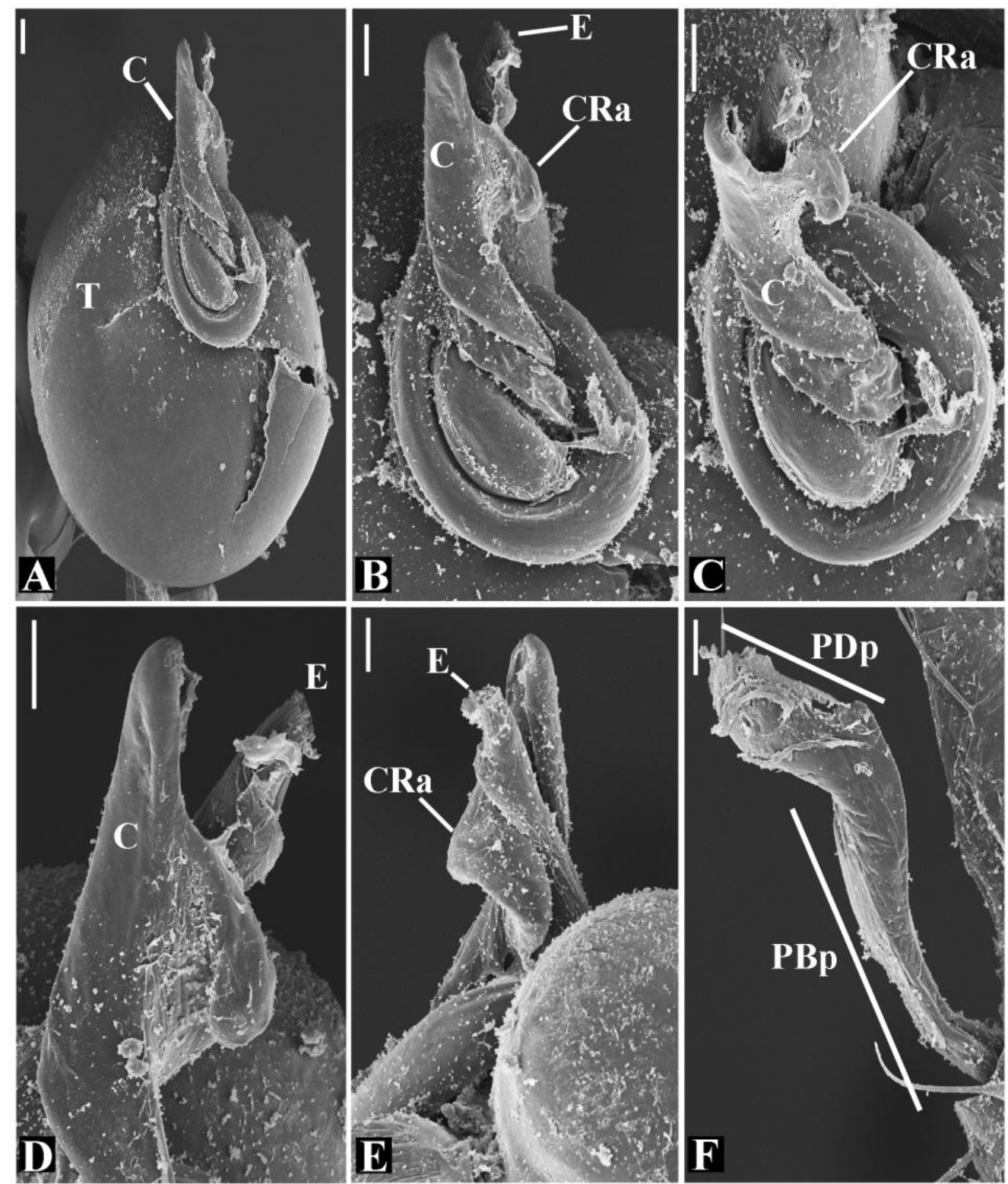

Figure 268. Glenognatha gloriae from Puerto Rico. A, Male left palp ventral. B-E, embolus and conductor. B, ventroretrolateral. C, ventroanterior. D, ventral. E, dorsal. F, paracymbium. Scale bars $20 \mu \mathrm{m}$. C: conductor. CRa: conductor retrolateral apophysis. E: embolus. PBp: paracymbium basal portion. PDp: Paracymbium distal portion. T: tegulum. 

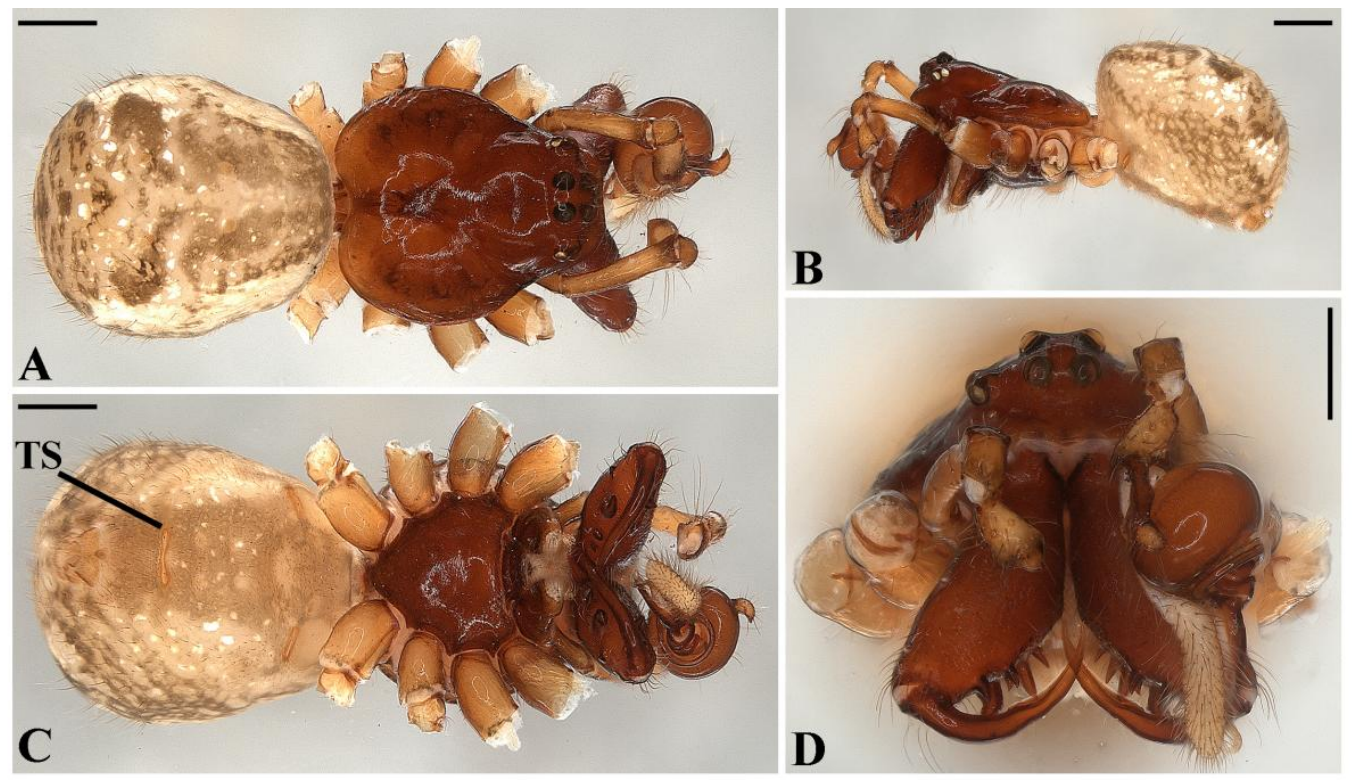

Figure 269. Glenognatha sp. nov. 11 from USA (Paratype, USNM). Male habitus. A, dorsal. B, lateral. C, ventral. D, frontal. Scale bars, $500 \mu \mathrm{m}$. TS: tracheal spiracle.
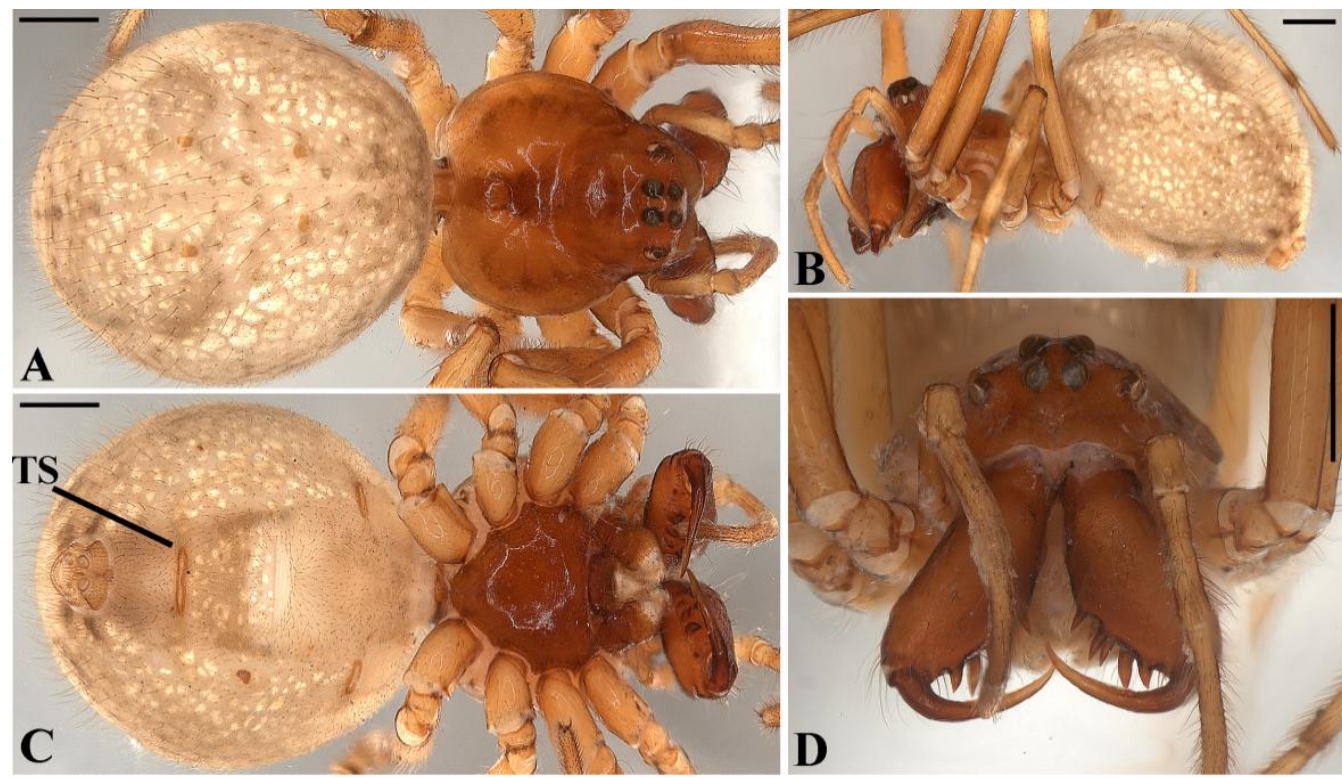

Figure 270. Glenognatha sp. nov. 11 from USA (Paratype, USNM). Female habitus. A, dorsal. B, lateral. C, ventral. D, frontal. Scale bars, $500 \mu \mathrm{m}$. TS: tracheal spiracle. 


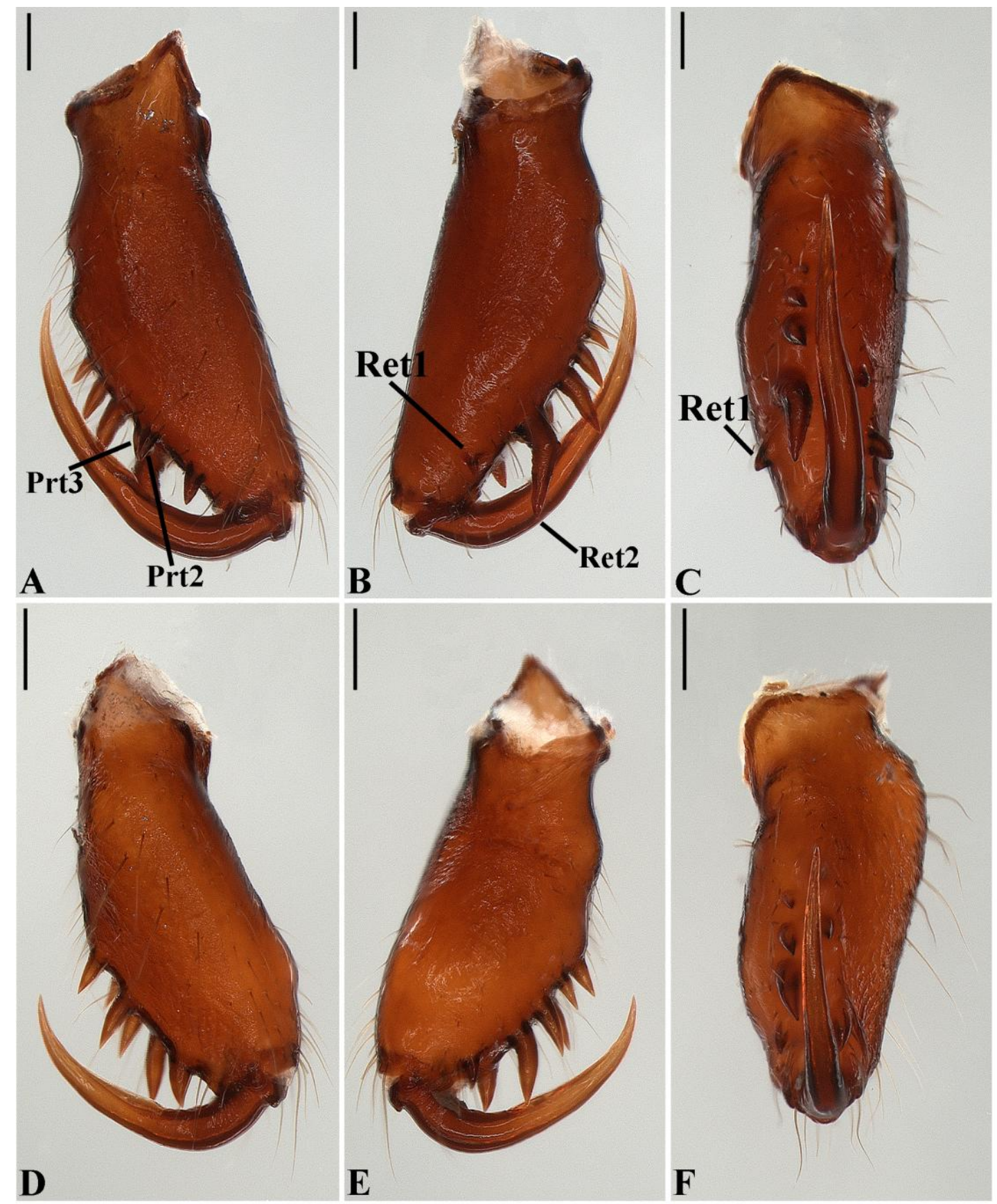

Figure 271. Glenognatha sp. nov. 11 from USA (Paratypes, USNM). A-C, Male left chelicerae. A, anterior. B, posterior. C, mesal. D-F, Female left chelicerae. D, anterior. E, posterior. F, mesal. Scale bars, $200 \mu \mathrm{m}$. Prt: promarginal tooth. Ret: retromarginal tooth. 


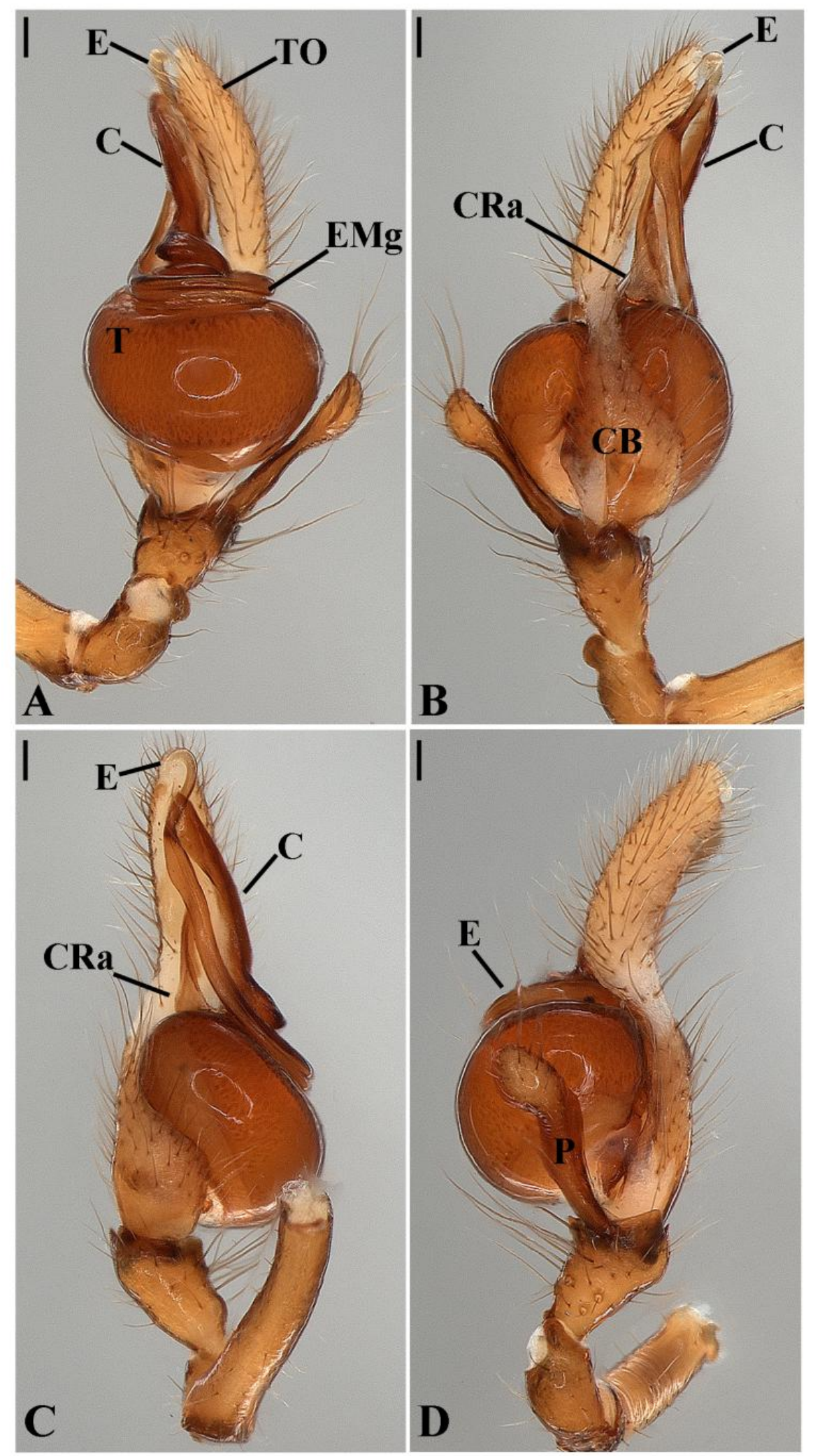

Figure 272. Glenognatha sp. nov. 11 from USA (Paratype, USNM). Male left palp. A, ventral. B, dorsal. C, prolateral. D, retrolateral. Scale bars, $100 \mu \mathrm{m}$. C: conductor. CB: cymbium. CRa: conductor retrolateral apophysis. E: embolus. EMg: embolic medial groove. P: paracymbium. T: tegulum. TO: tarsal organ. 

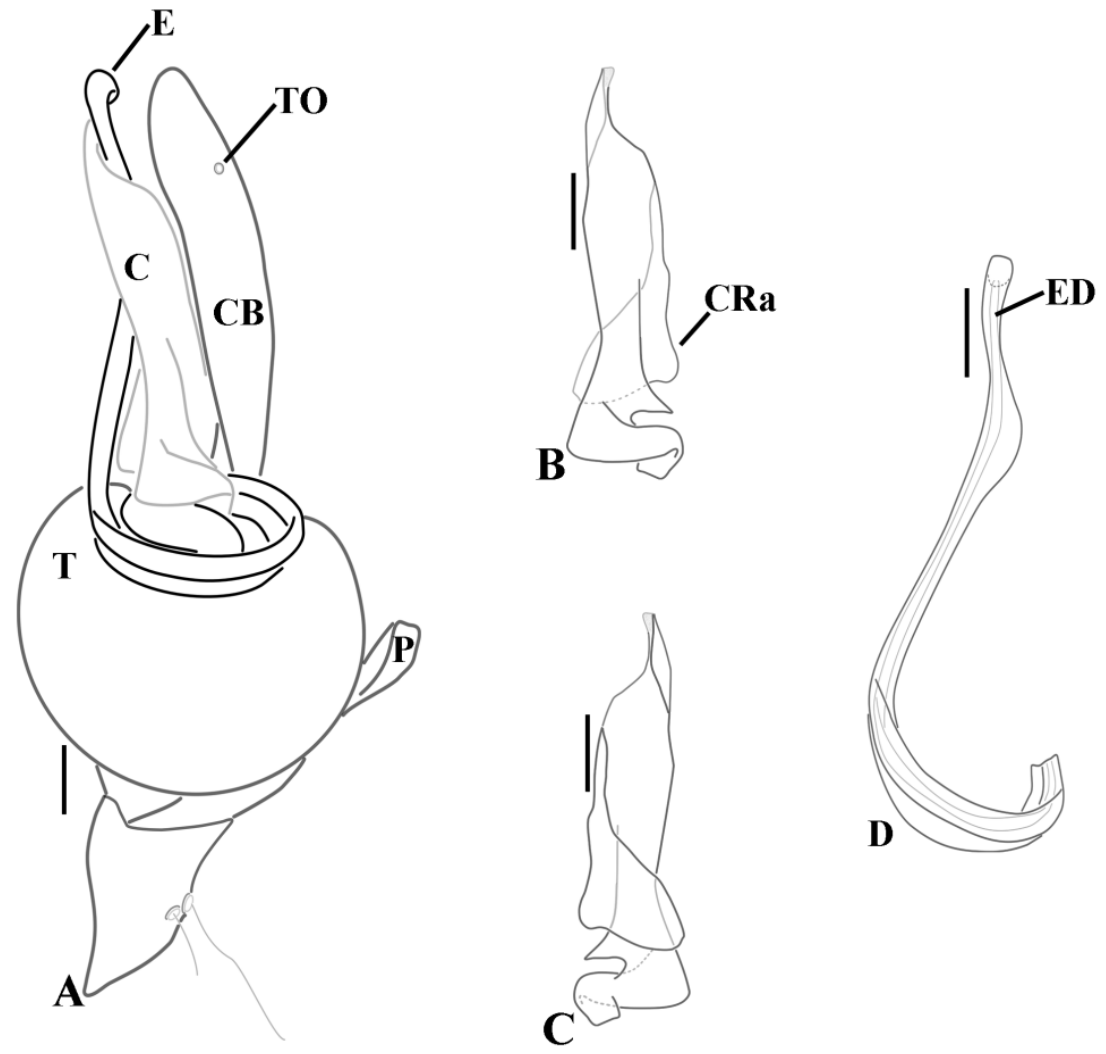

Figure 273. Glenognatha sp. nov. 11 from USA (USNM). Male left palp, schematic. A, ventral. B, conductor ventral. C, conductor dorsal. D, embolus ventral. Scale bars 100 $\mu \mathrm{m}$. C: conductor. CB: cymbium. CRa: conductor retrolateral apophysis. E: embolus. ED: ejaculatory duct. P: paracymbium. T: tegulum. TO: tarsal organ.

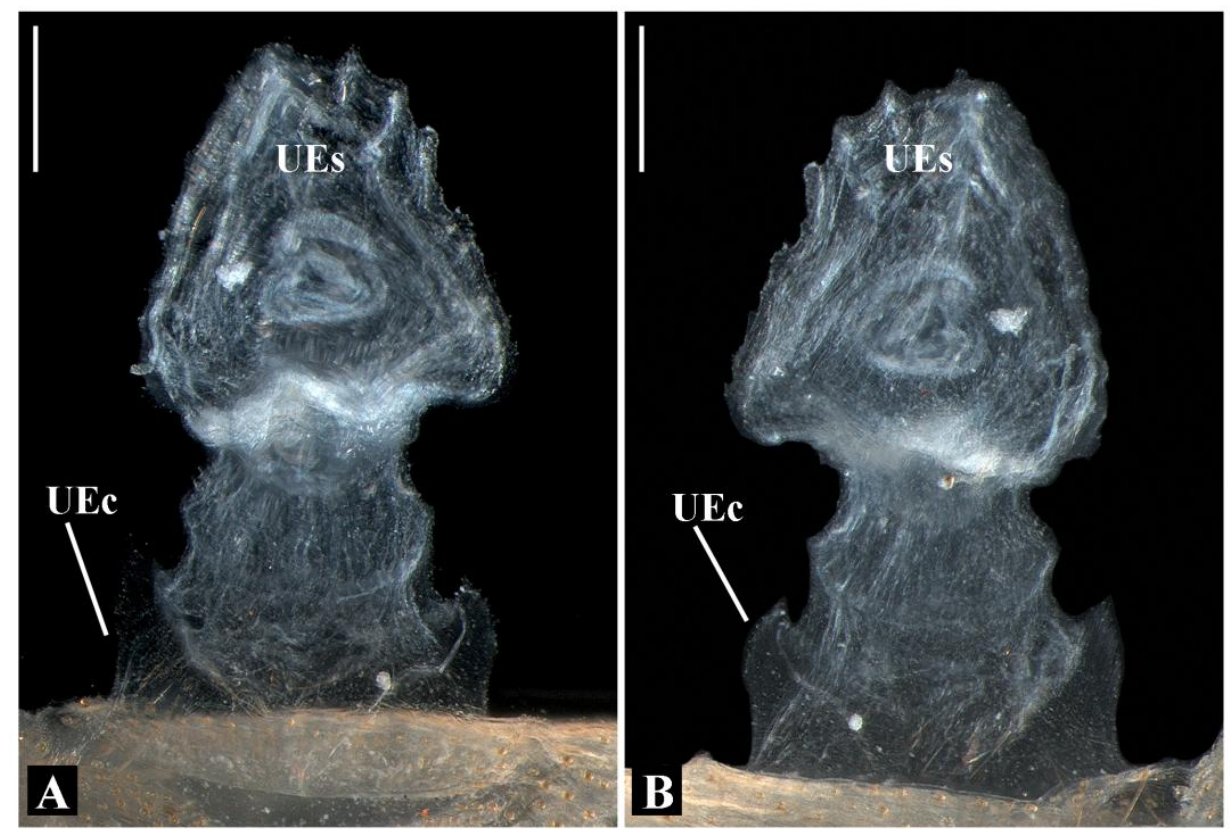

Figure 274. Glenognatha sp. nov. 11 from USA (Paratype, USNM). Female genitalia.

Female genitalia. A, dorsal. B, ventral. Scale bars, $100 \mu \mathrm{m}$. UEc: uterus externus chamber. UEs: uterus externus sac. 

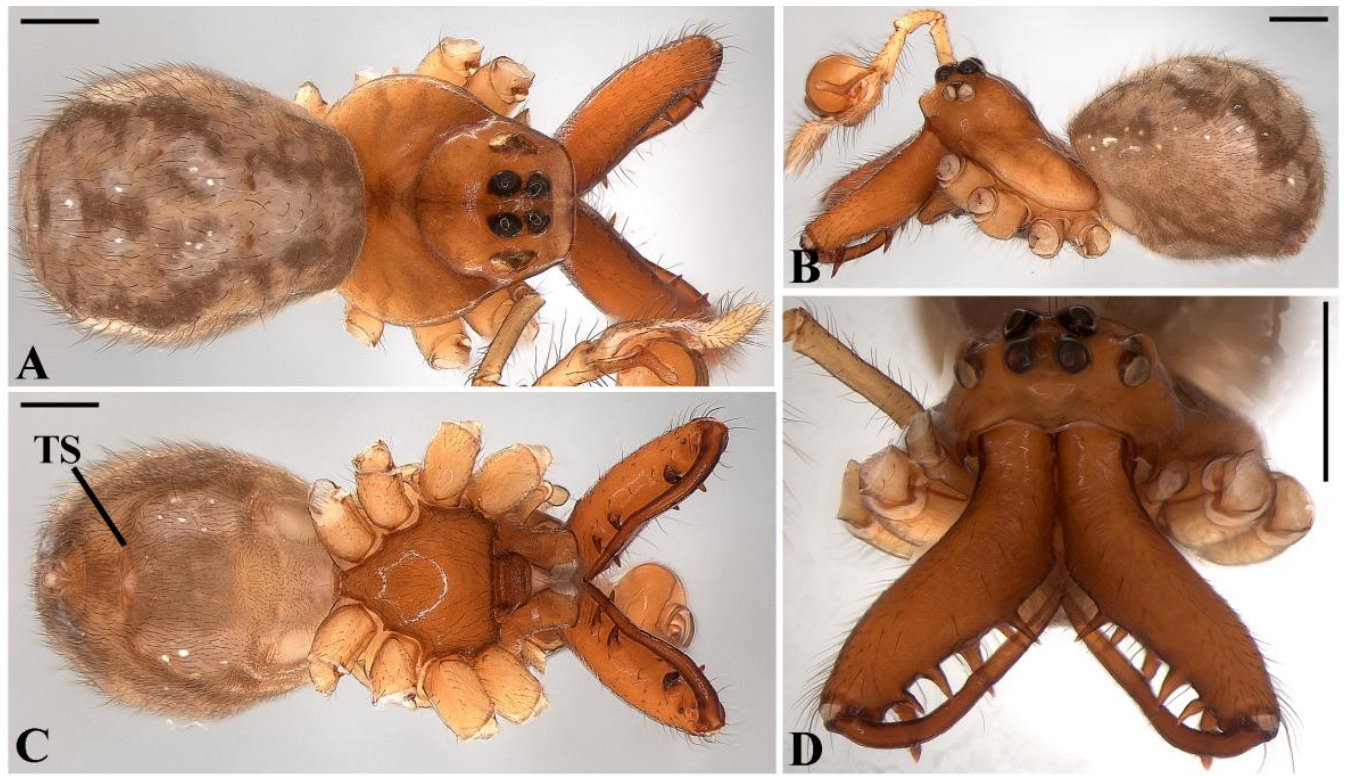

Figure 275. Glenognatha sp. nov. 7 from Brazil (MNRJ 6598). Male habitus. A, dorsal. B, lateral. C, ventral. D, frontal. Scale bars, $500 \mu \mathrm{m}$. TS: tracheal spiracle.

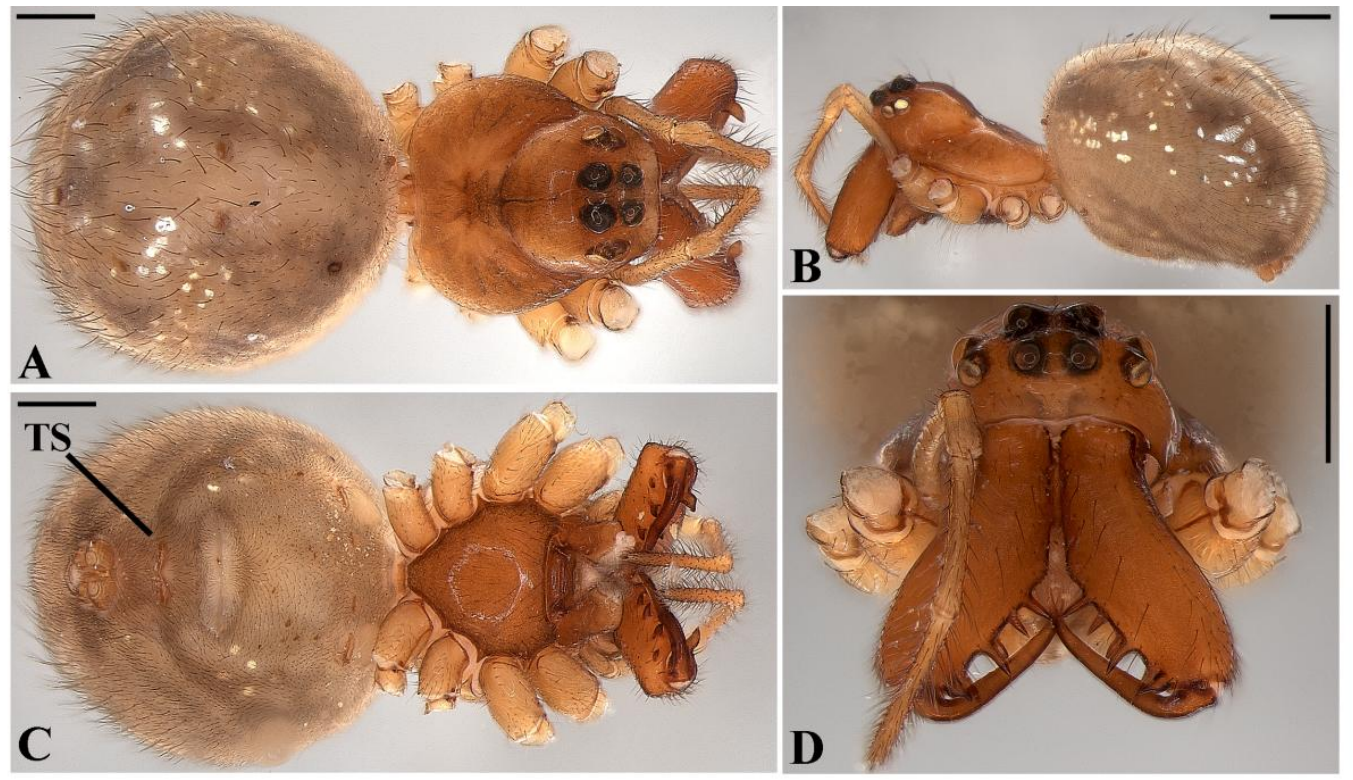

Figure 276. Glenognatha sp. nov. 7 from Brazil (MNRJ 6598). Female habitus. A, dorsal. B, lateral. C, ventral (arrow, pleural bar). D, frontal. Scale bars, $500 \mu \mathrm{m}$. TS: tracheal spiracle. 


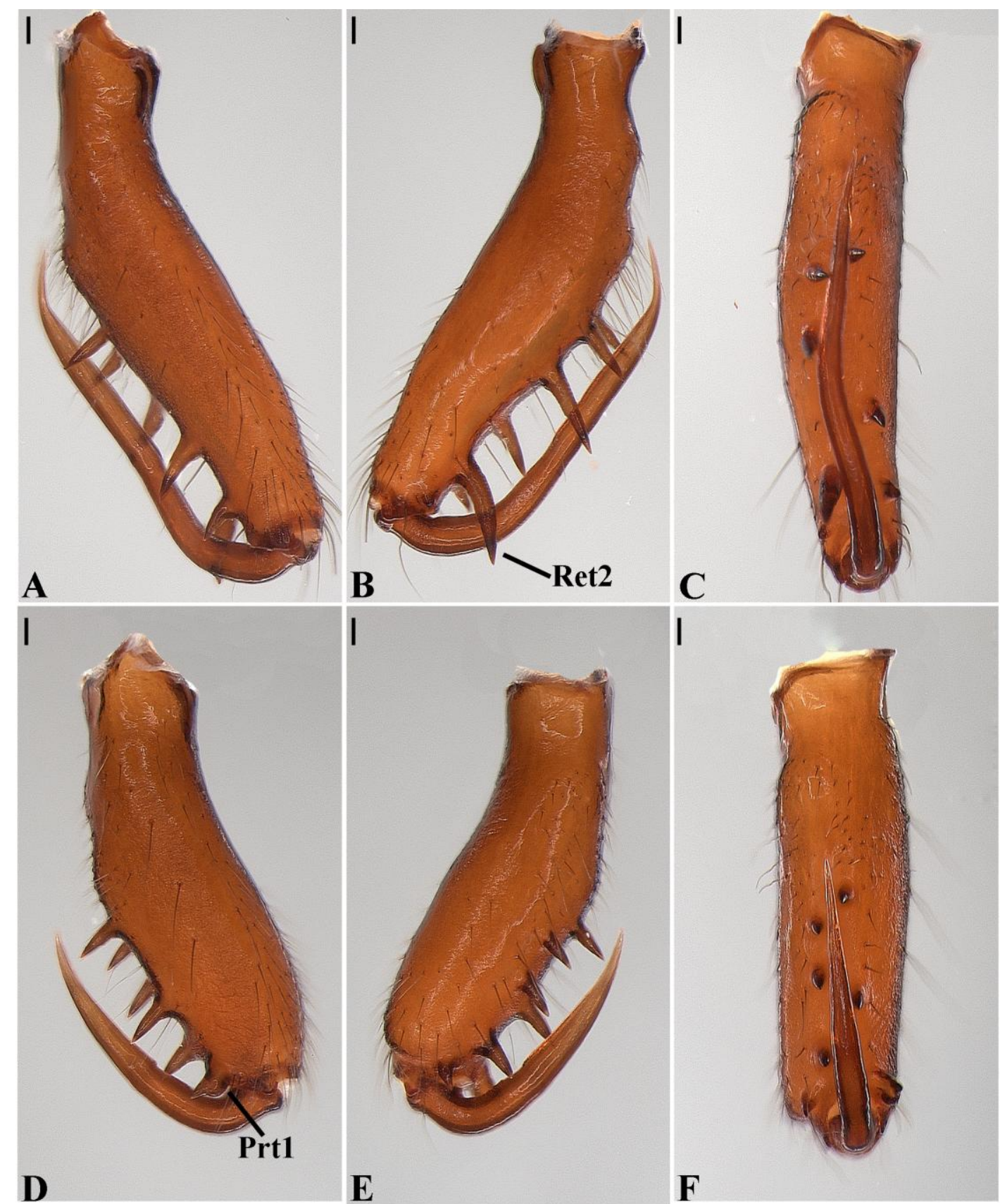

Figure 277. Glenognatha sp. nov. 7 from Brazil (MNRJ 6598). A-C, Male left chelicerae. A, anterior. B, posterior. C, mesal. D-F, female left chelicerae. D, anterior. E, posterior. F, mesal. Scale bars, $100 \mu \mathrm{m}$. Prt: promarginal tooth. Ret: retromarginal tooth. 


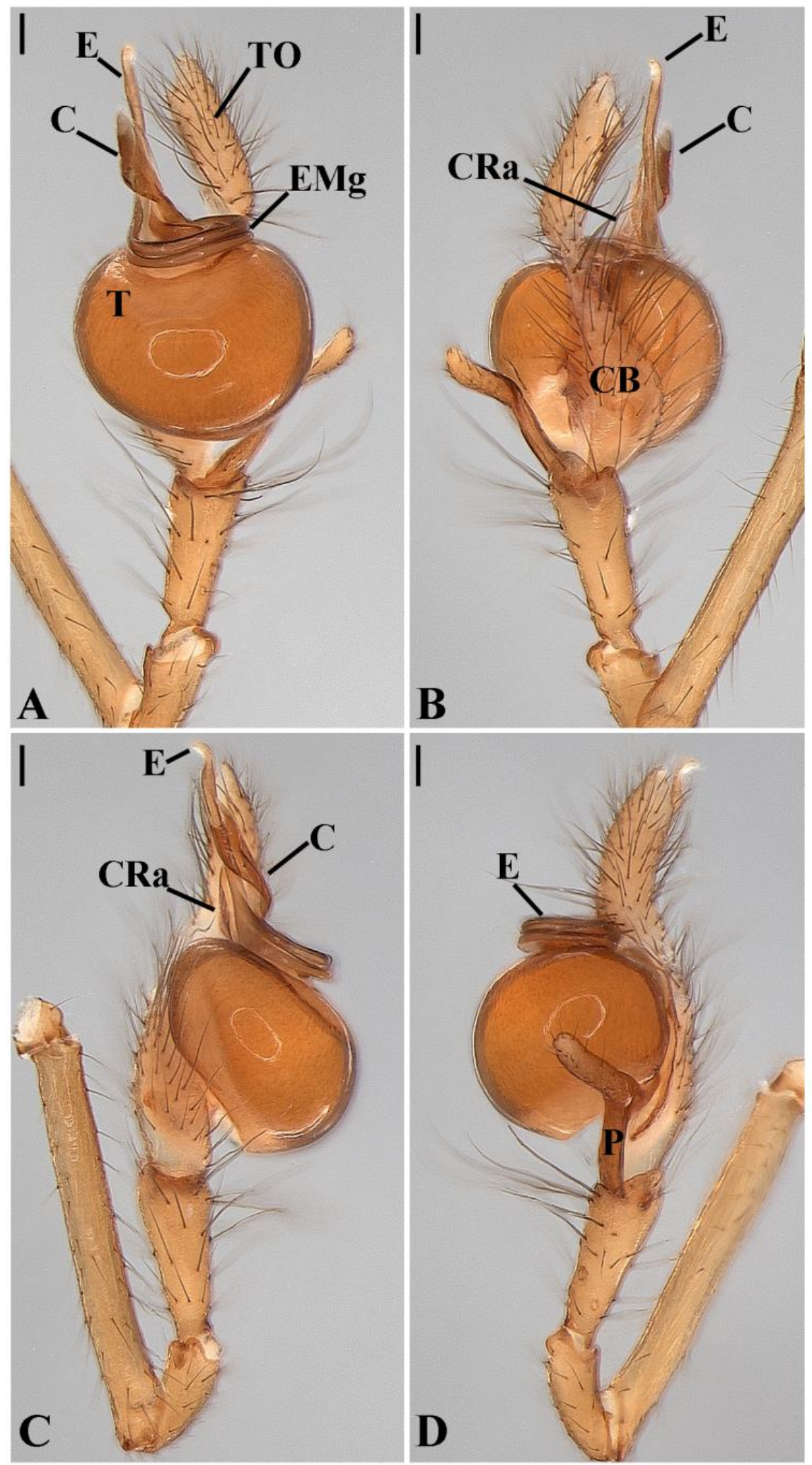

Figure 278. Glenognatha sp. nov. 7 from Brazil (MNRJ 6598). Male left palp. A, ventral. B, dorsal. C, prolateral. D, retrolateral. Scale bars, $100 \mu \mathrm{m}$. C: conductor. CB: cymbium. CRa: conductor retrolateral apophysis. E: embolus. EMg: embolic medial groove. P: paracymbium. T: tegulum. TO: tarsal organ. 

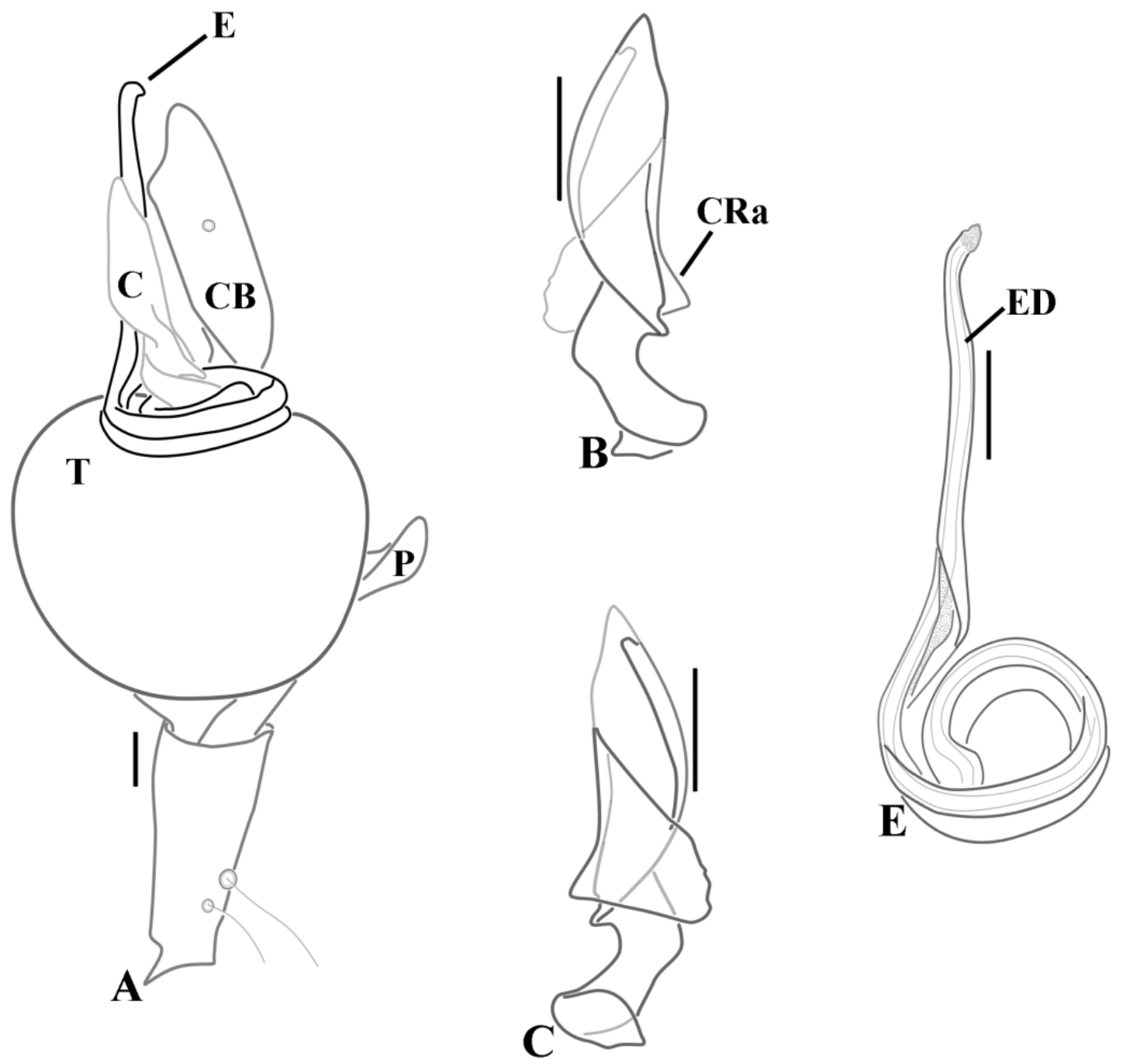

Figure 279. Glenognatha sp. nov. 7 from Brazil (MNRJ 6598). Male left palp, schematic. A, ventral. B, conductor ventral. C, conductor dorsal. D, embolus ventral. Scale bars $100 \mu \mathrm{m}$. C: conductor. CB: cymbium. CRa: conductor retrolateral apophysis. E: embolus. ED: ejaculatory duct. P: paracymbium. T: tegulum. 


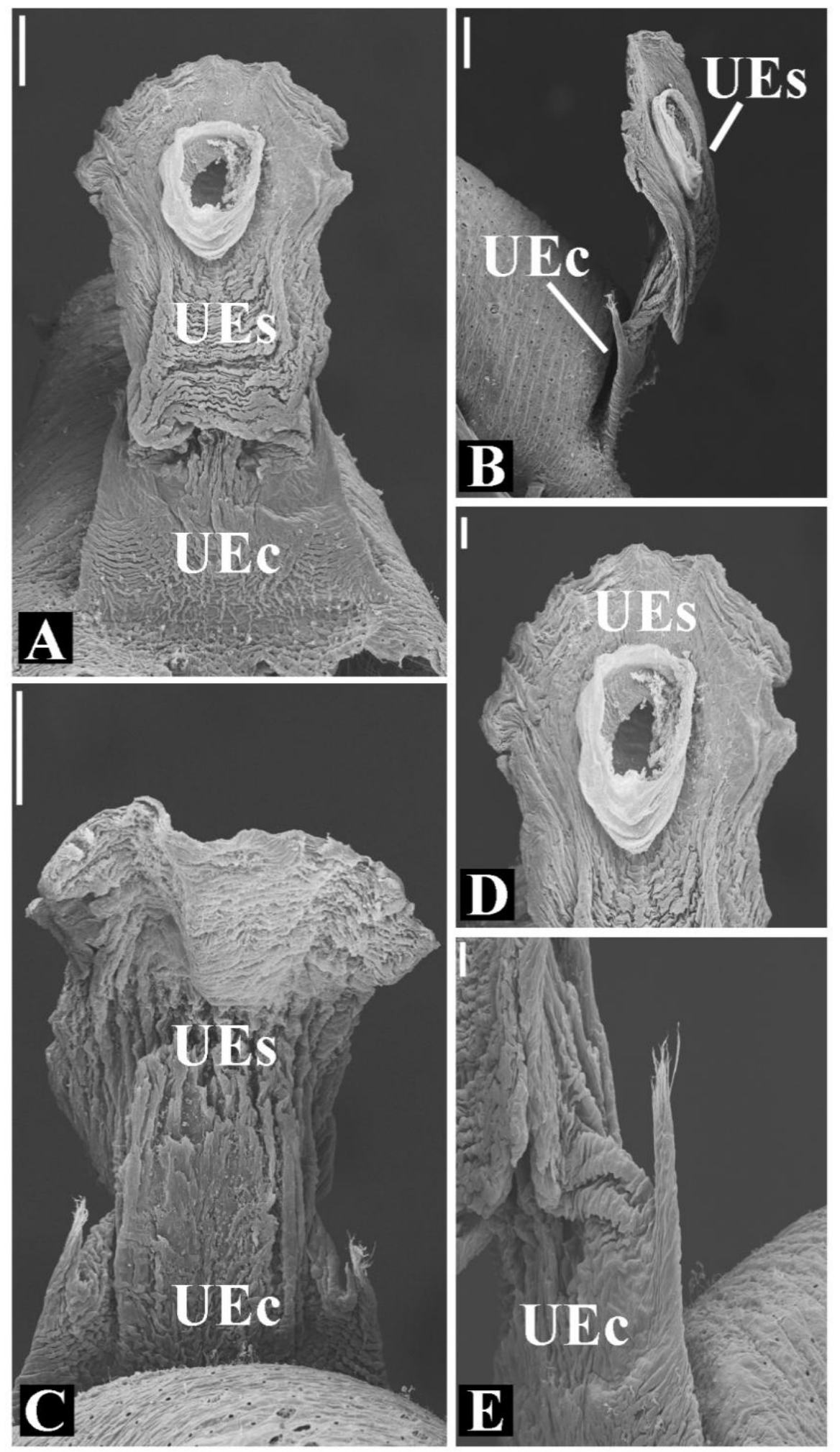

Figure 280. Glenognatha sp. nov. 7 from Brazil. Female genitalia. A, dorsal. B, lateral. C, ventral. D-E, uterus externus cuticle. Scale bars, $100 \mu \mathrm{m}$ (A-C), $30 \mu \mathrm{m}$ (D), $20 \mu \mathrm{m}$ (E). UEc: uterus externus chamber. UEs: uterus externus sac. 


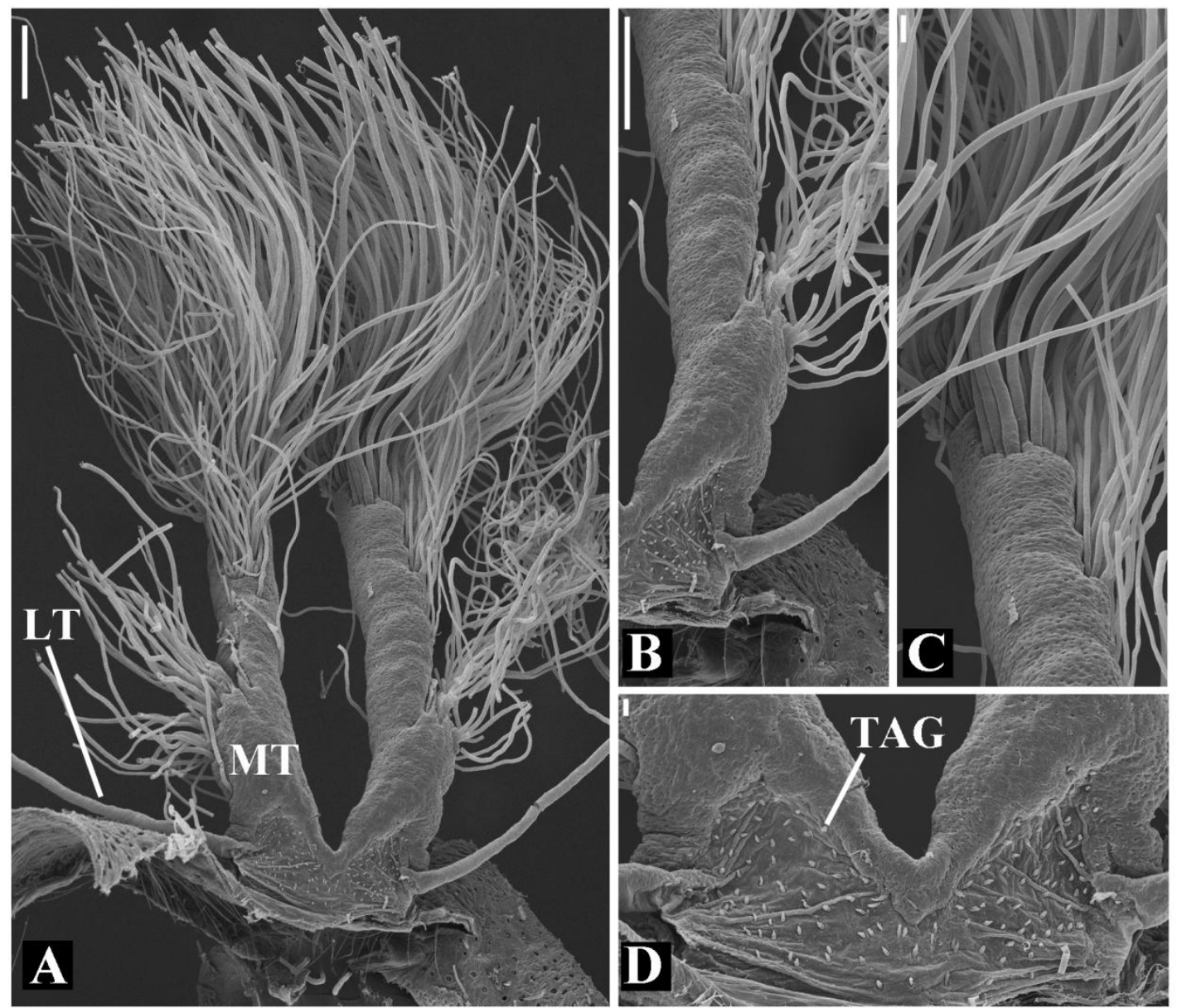

Figure 281. Glenognatha sp. nov. 7 from Brazil. Female tracheal system. A, dorsal. B, median and lateral trunks. C, median trunk distal. D, tracheal spiracle posterior view. Scale bars, $100 \mu \mathrm{m}$ (A, B), $20 \mu \mathrm{m}$ (C), $10 \mu \mathrm{m}$ (D). LT: lateral tracheae. MT: median trunks. TAG: tracheal atrium gland. 

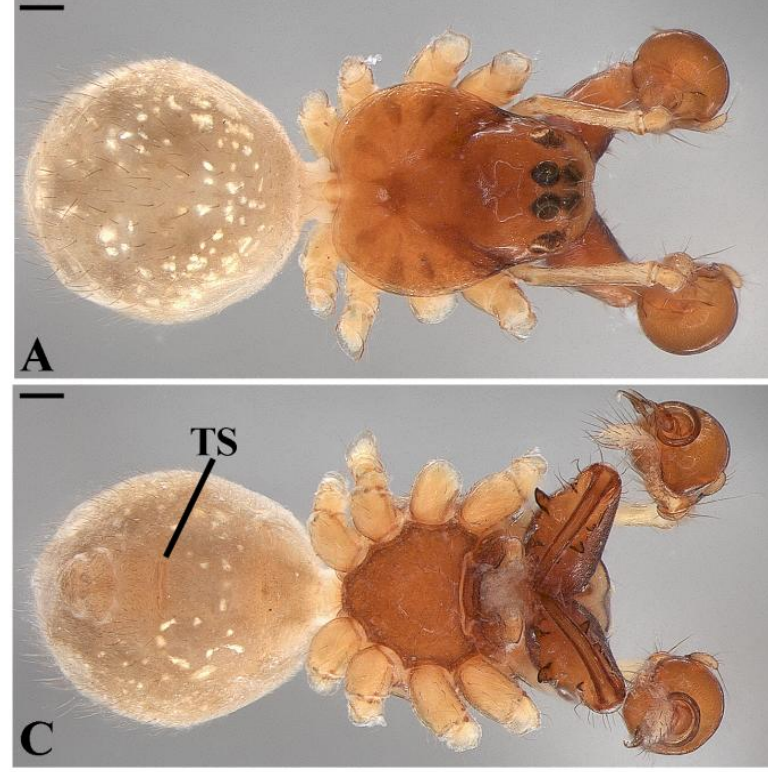

D
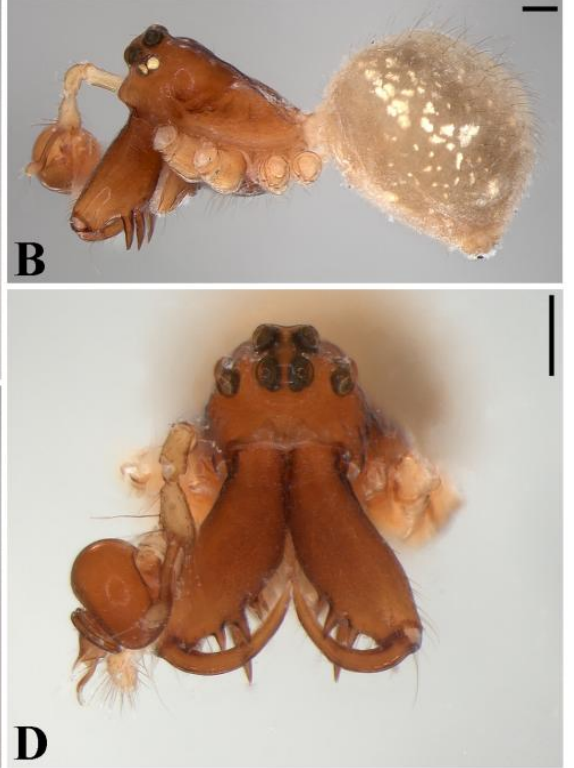

Figure 282. Glenognatha minuta from Mexico (AMNH). Male habitus. A, dorsal. B, lateral. C, ventral. D, frontal. Scale bars, $200 \mu \mathrm{m}$. TS: tracheal spiracle.

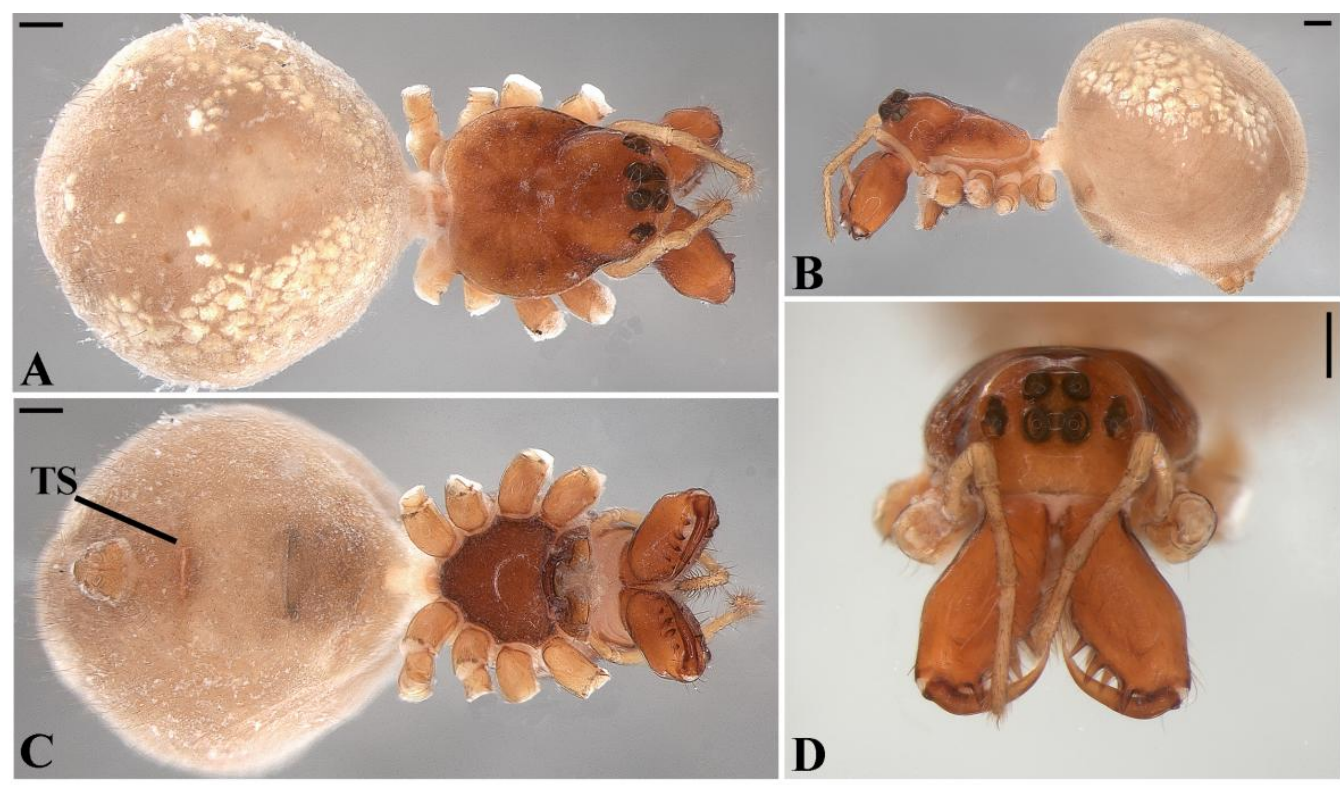

Figure 283. Glenognatha minuta from Mexico (AMNH). Female habitus. A, dorsal. B, lateral. C, ventral. D, frontal. Scale bars, $200 \mu \mathrm{m}$. TS: tracheal spiracle. 


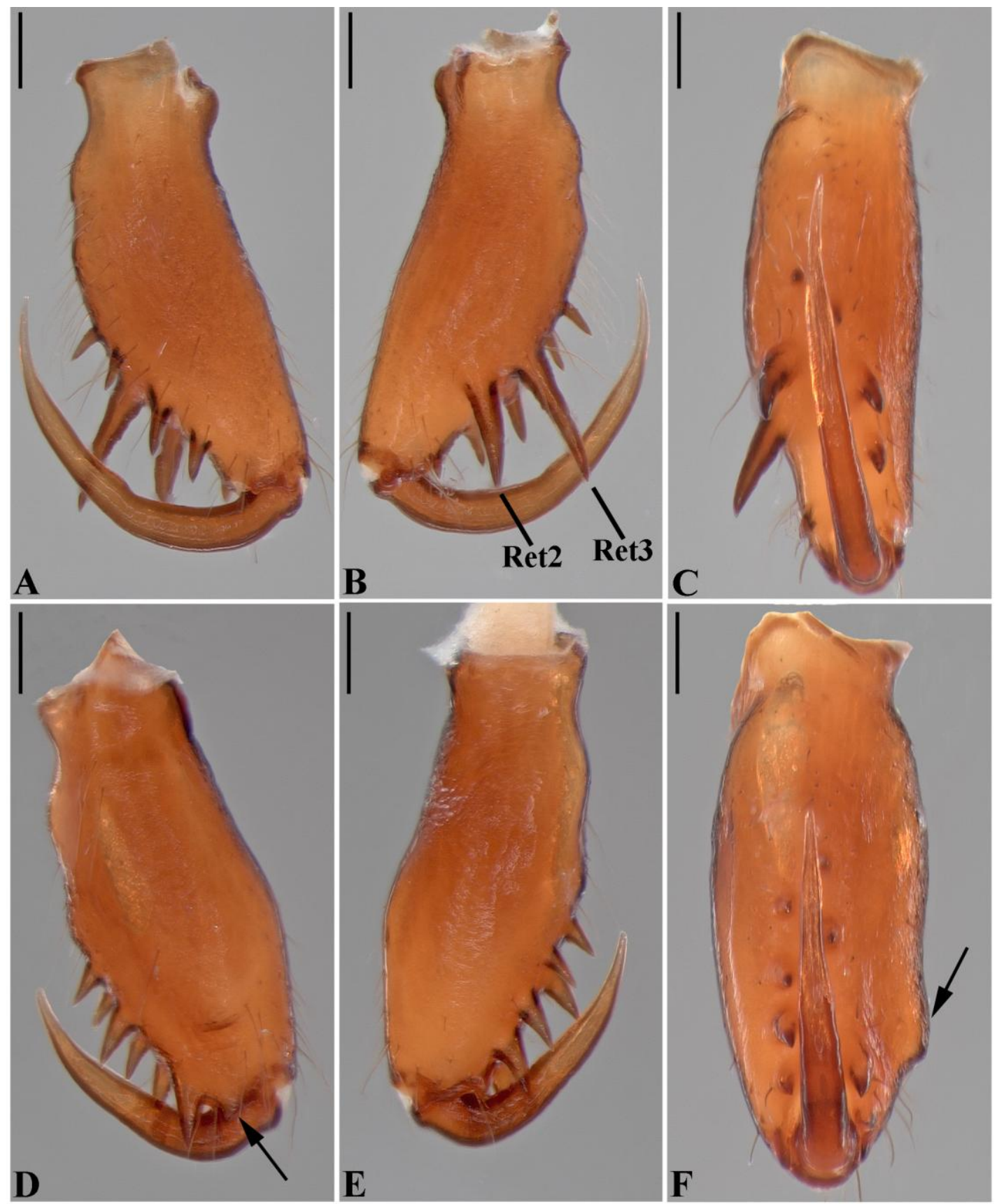

Figure 284. Glenognatha minuta. A-C, male left chelicerae from Panama (AMNH). A, anterior. B, posterior. C, mesal. D-F, female left chelicerae from Mexico (AMNH). D, anterior (arrow, cheliceral basal segment projection). E, posterior. F, mesal (arrow, cheliceral bulge). Scale bars, $100 \mu \mathrm{m}$. Ret: retromarginal tooth. 


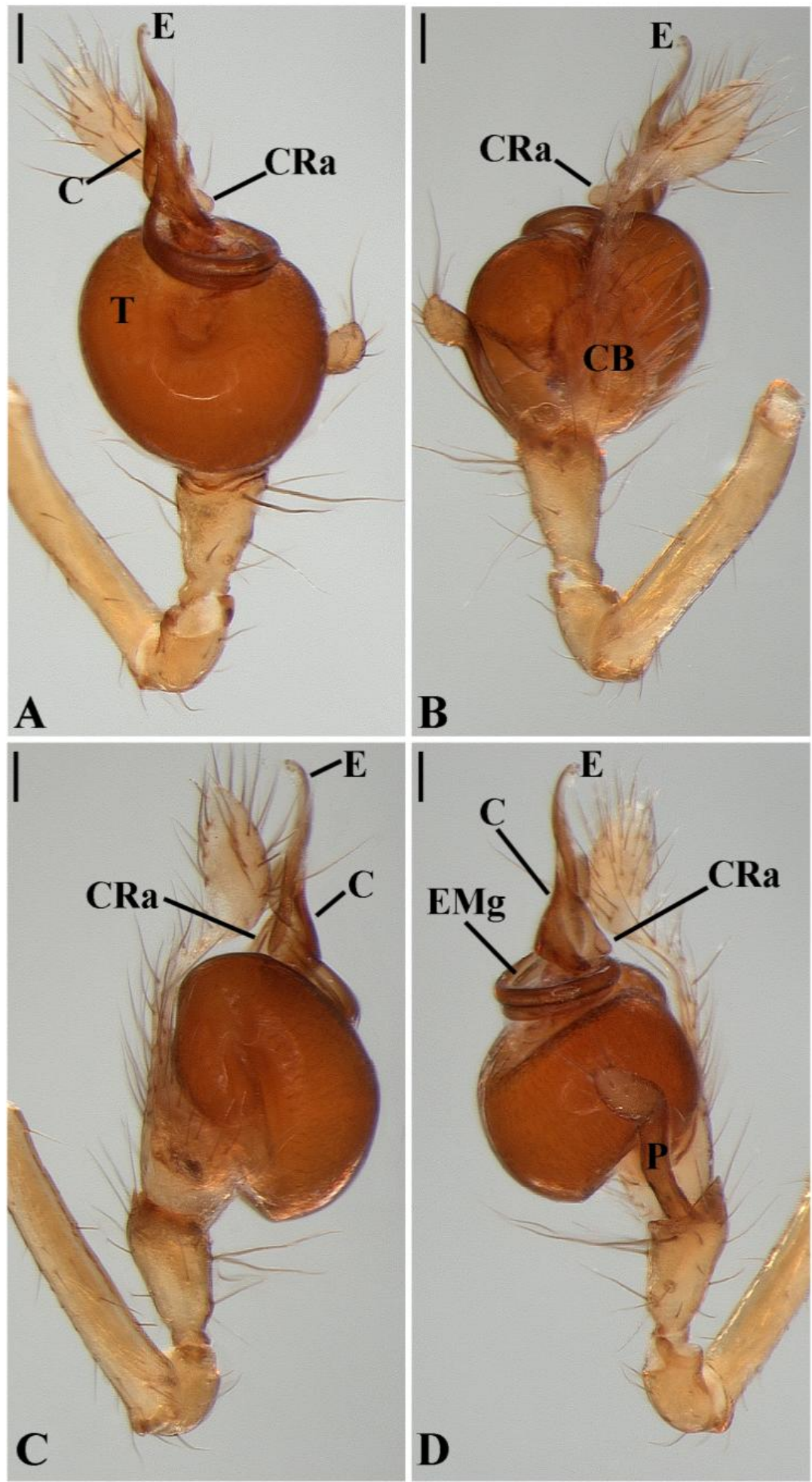

Figure 285. Glenognatha minuta from Mexico (AMNH). Male left palp. A, ventral. B, dorsal. C, prolateral. D, retrolateral. Scale bars, $100 \mu \mathrm{m}$. C: conductor. CB: cymbium. CRa: conductor retrolateral apophysis. E: embolus. EMg: embolic medial groove. P: paracymbium. T: tegulum. 

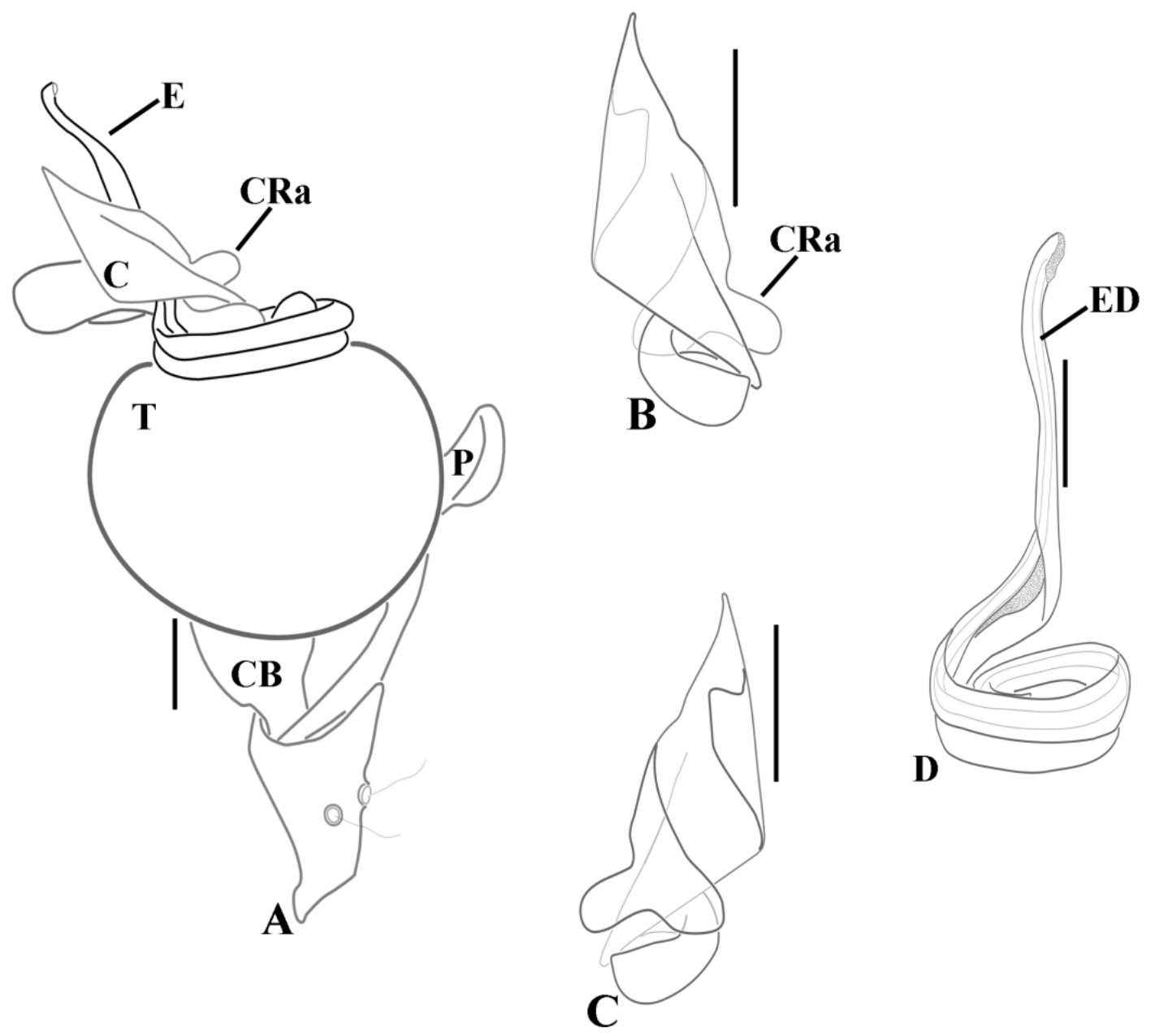

Figure 286. Glenognatha minuta from Mexico (AMNH). Male left palp, schematic. A, ventral. B, conductor ventral. C, conductor dorsal. D, embolus ventral. Scale bars 100 $\mu \mathrm{m}$. C: conductor. CB: cymbium. CRa: conductor retrolateral apophysis. E: embolus. ED: ejaculatory duct. P: paracymbium. T: tegulum. 

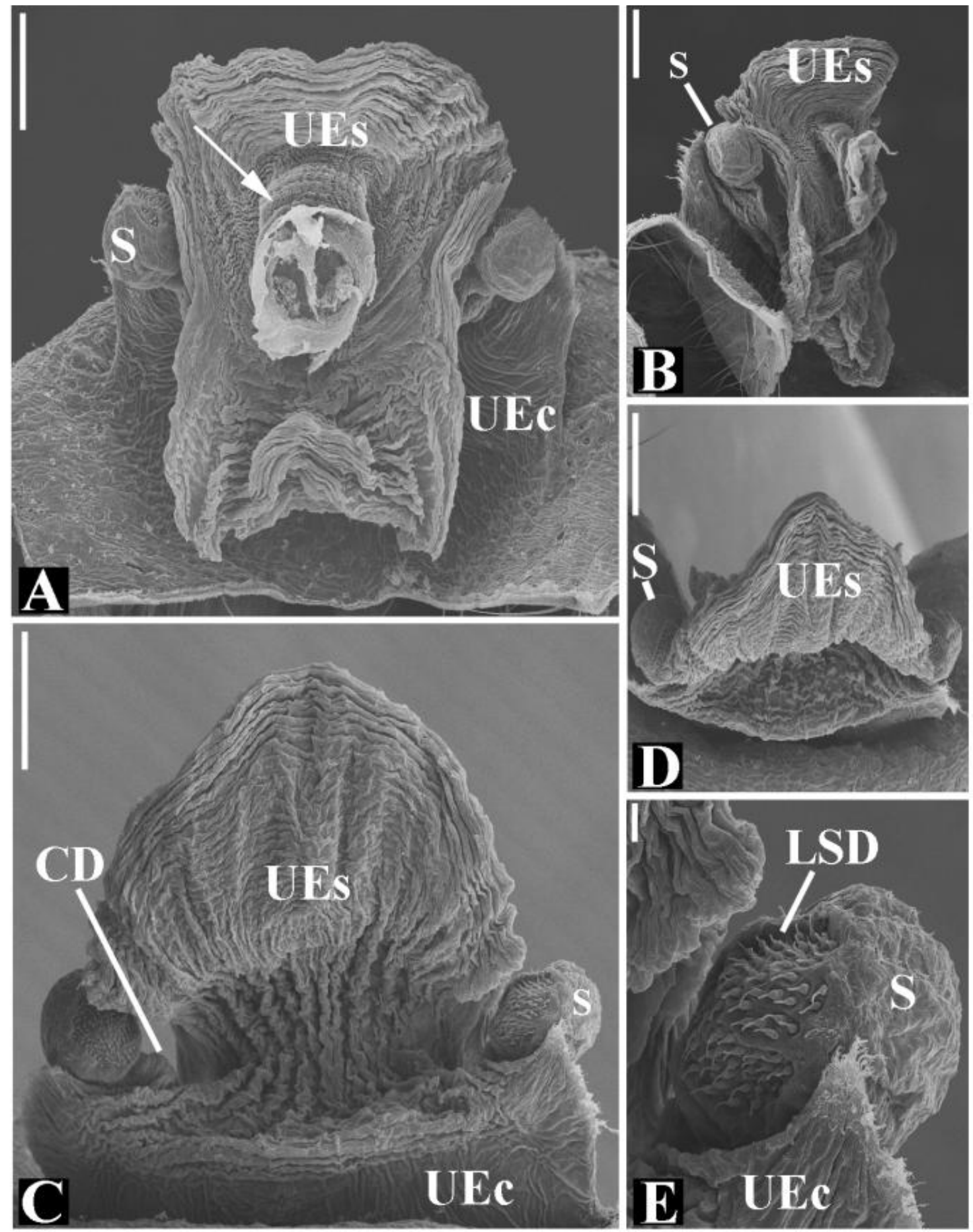

Figure 287. Glenognatha minuta from Mexico. Female genitalia. A, dorsal (arrow, tubiform projection). B, lateral. C, ventral D, anterior. E, spermathecae. Scale bars, $100 \mu \mathrm{m}$ (A-D), $10 \mu \mathrm{m}$ (E). CD: copulatory duct. LSD: long-stem gland ducts. UEc: uterus externus chamber. UEs: uterus externus sac. S: spermathecae. 


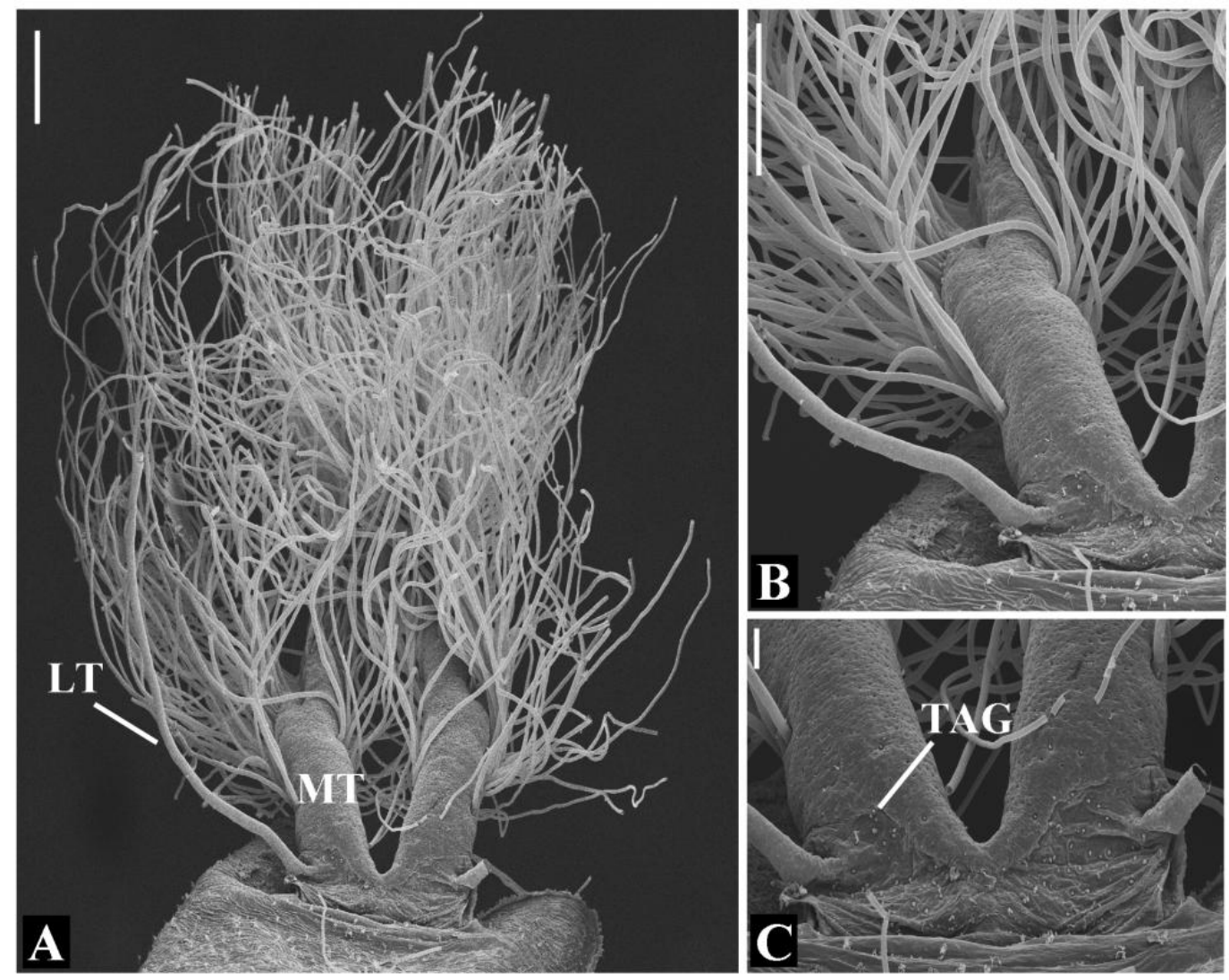

Figure 288. Glenognatha minuta from Mexico. Female tracheal system. A, dorsal. B, median and lateral trunks. C, tracheal spiracle posterior view. Scale bars, $100 \mu \mathrm{m}$ (AB), $20 \mu \mathrm{m}(\mathrm{C})$. LT: lateral tracheae. MT: median trunks. TAG: tracheal atrium gland.
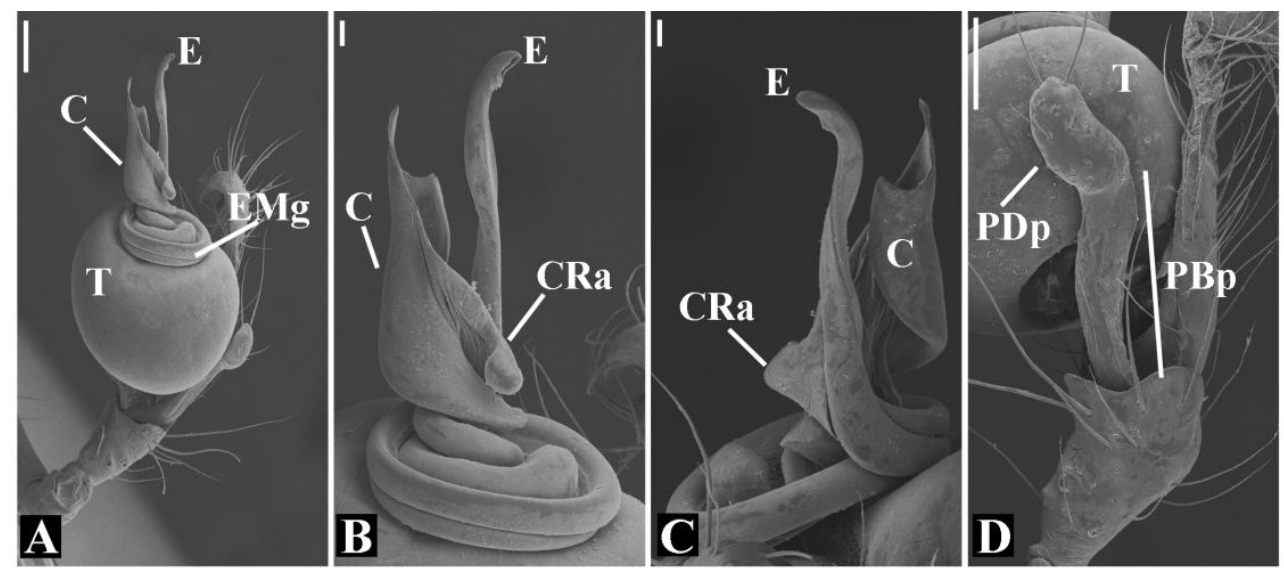

Figure 289. Glenognatha minuta from Panama. Male left palp. A, ventral. B, embolus and conductor ventral. C, embolus and conductor dorsal. D, paracymbium. Scale bars $100 \mu \mathrm{m}$ (A, D), $20 \mu \mathrm{m}$ (B-C). C: conductor. CRa: conductor retrolateral apophysis. E: embolus. EMg: embolic median groove. PBp: paracymbium basal portion. PDp: paracymbium distal portion. T: tegulum. 


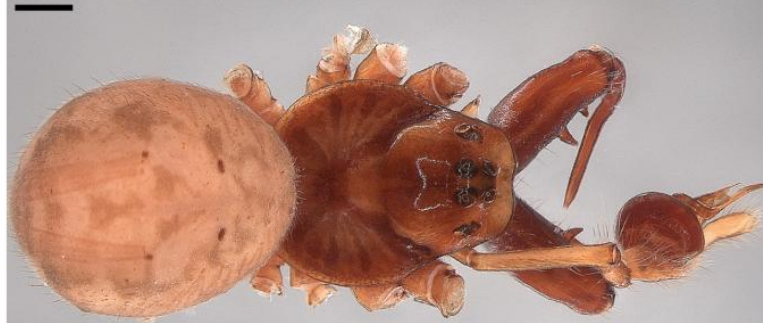

A

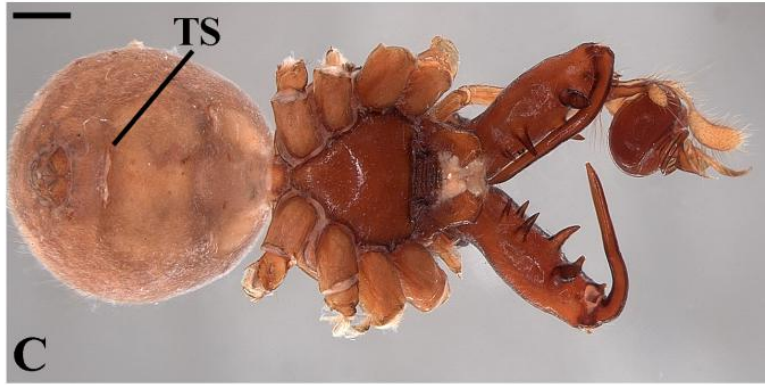

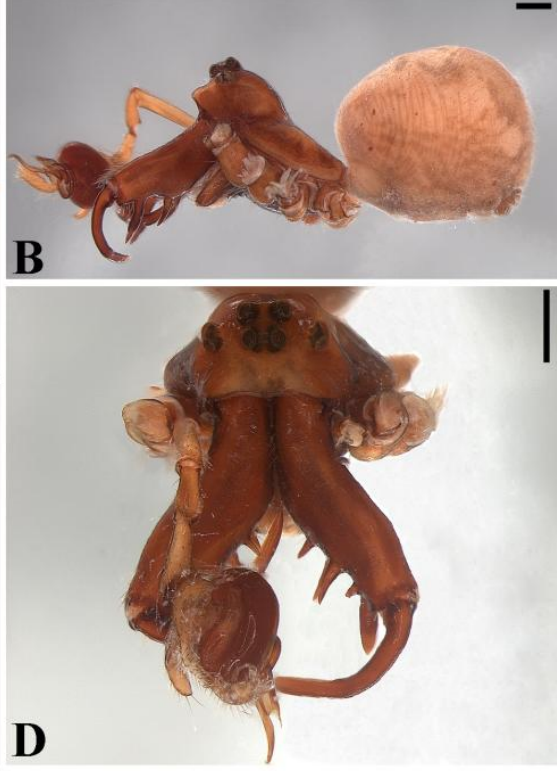

D

Figure 290. Glenognatha emertoni from USA (AMNH). Male habitus. A, dorsal. B, lateral. C, ventral. D, frontal. Scale bars, $500 \mu \mathrm{m}$. TS: tracheal spiracle.

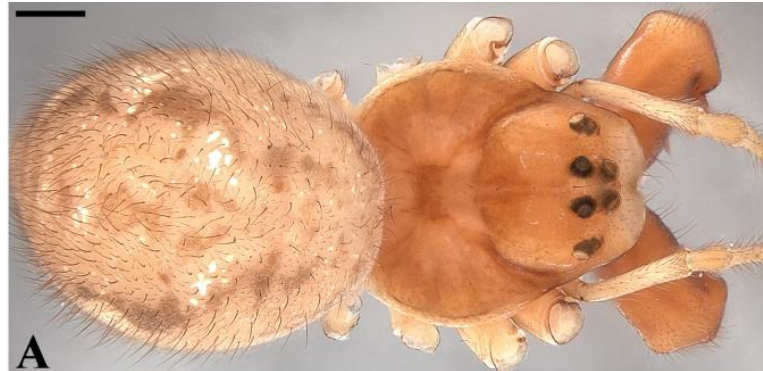

A

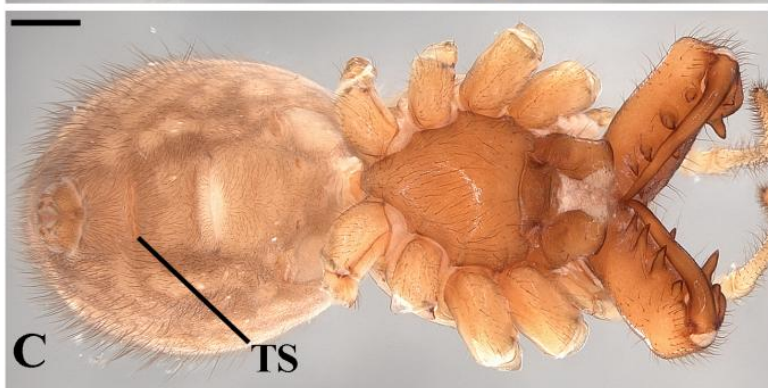

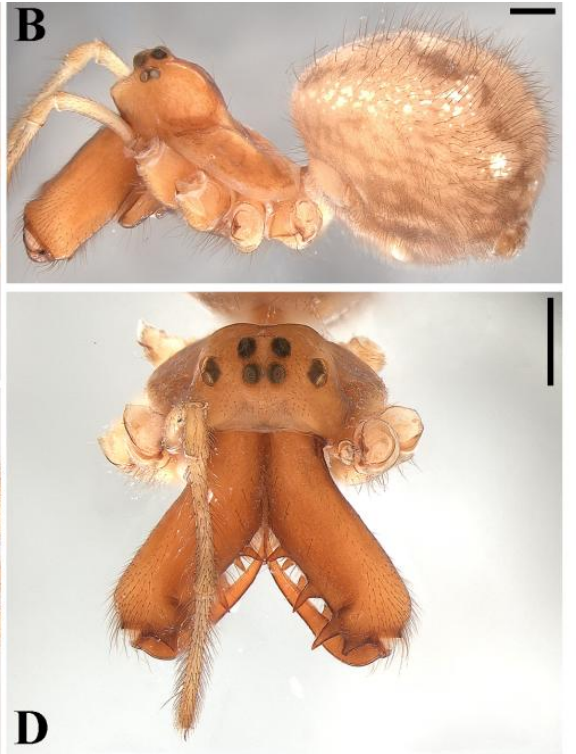

D

Figure 291. Glenognatha emertoni from USA (AMNH). Female habitus. A, dorsal. B, lateral. C, ventral. D, frontal. Scale bars, $500 \mu \mathrm{m}$. TS: tracheal spiracle. 


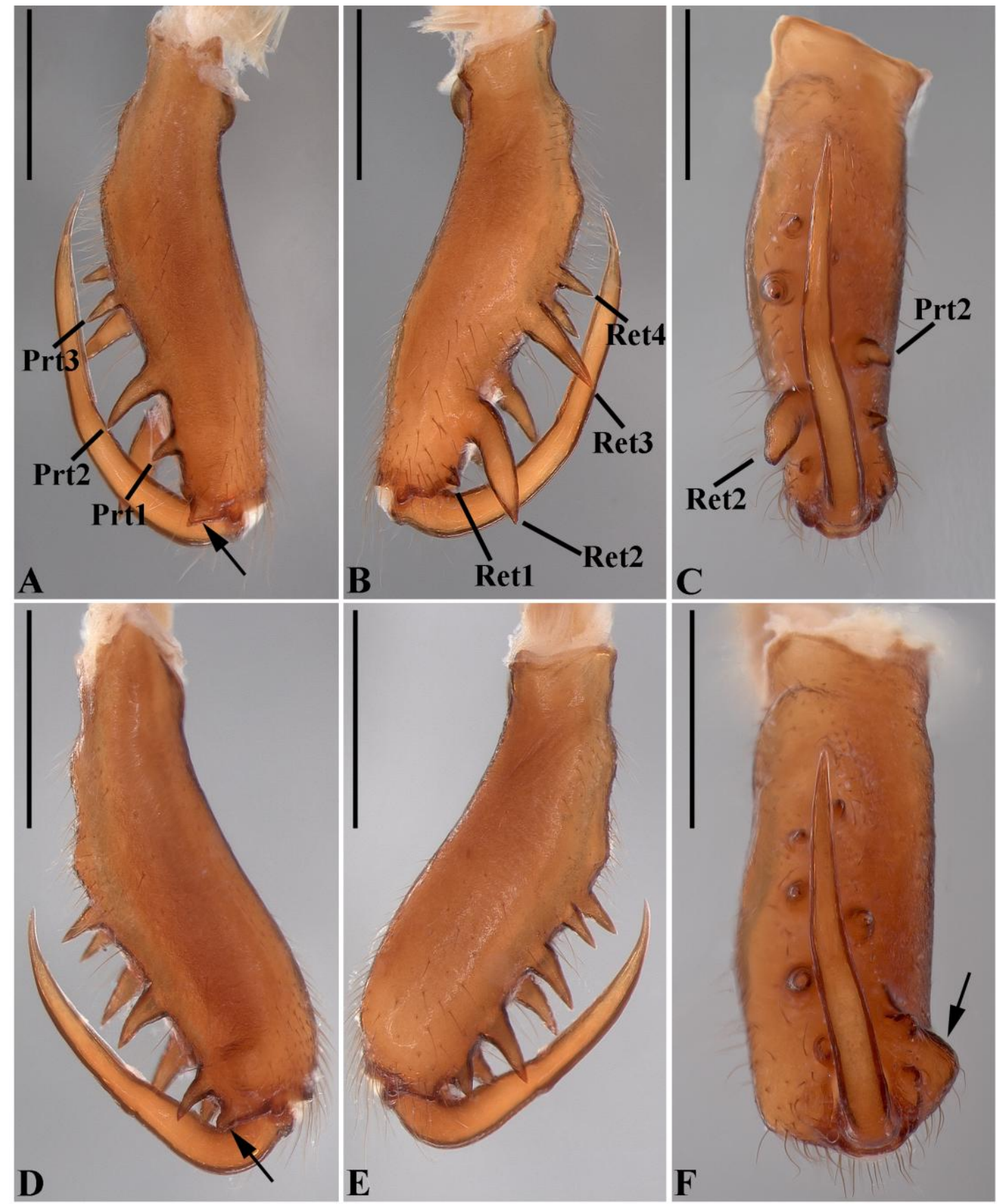

Figure 292. Glenognatha emertoni from USA (AMNH). A-C, Male left chelicerae. A, anterior (arrow, cheliceral basal segment projection). B, posterior. C, mesal. D-F, Female left chelicerae. D, anterior (arrow, cheliceral basal segment projection). E, posterior. F, mesal (arrow, cheliceral bulge). Scale bars, $500 \mu \mathrm{m}$. Prt: Promarginal tooth. Ret: retromarginal tooth. 


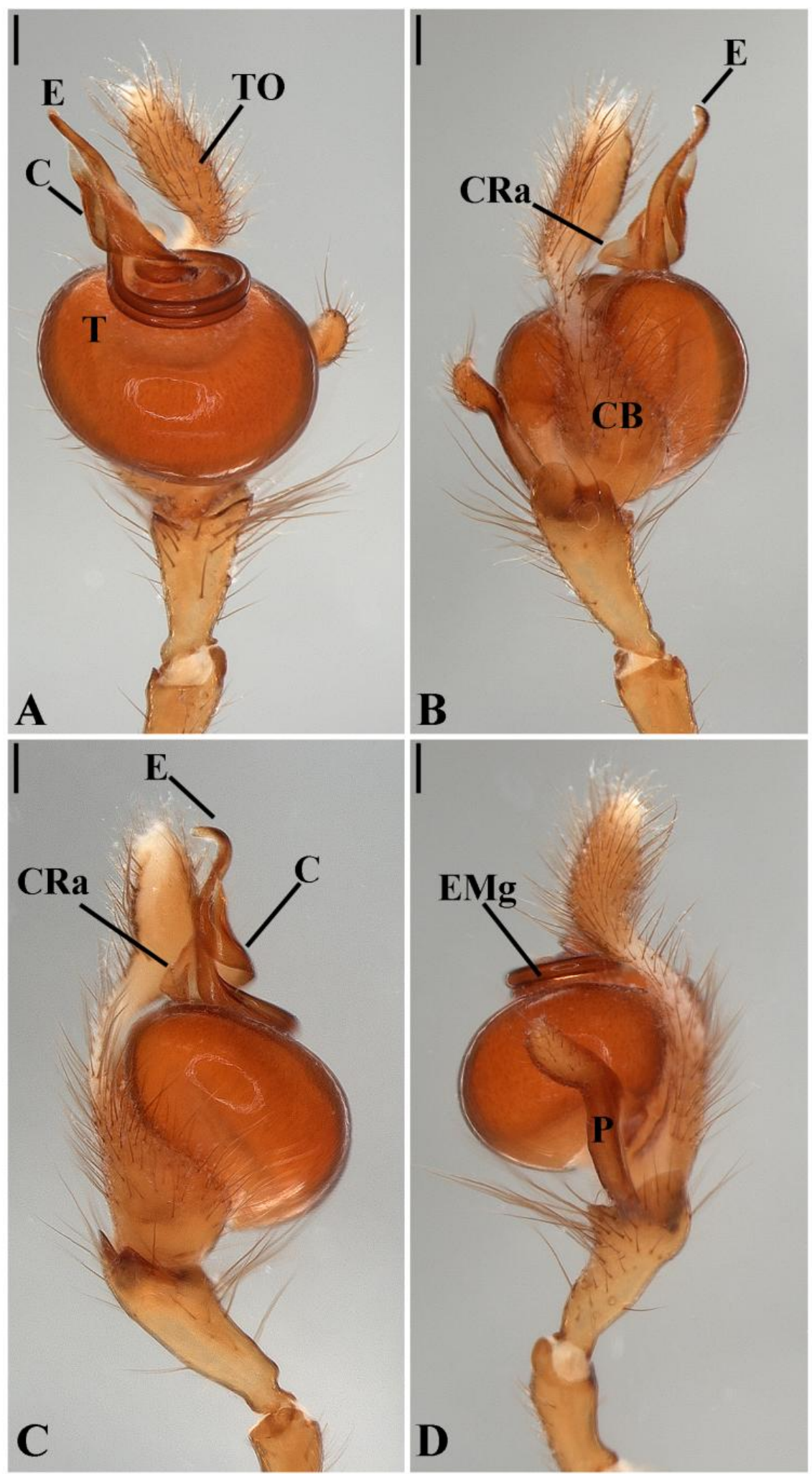

Figure 293. Glenognatha emertoni from USA (AMNH). Male left palp. A, ventral. B, dorsal. C, prolateral. D, retrolateral. Scale bars, $100 \mu \mathrm{m}$. C: conductor. CB: cymbium. CRa: conductor retrolateral apophysis. E: embolus. EMg: embolic medial groove. P: paracymbium. T: tegulum. TO: tarsal organ. 

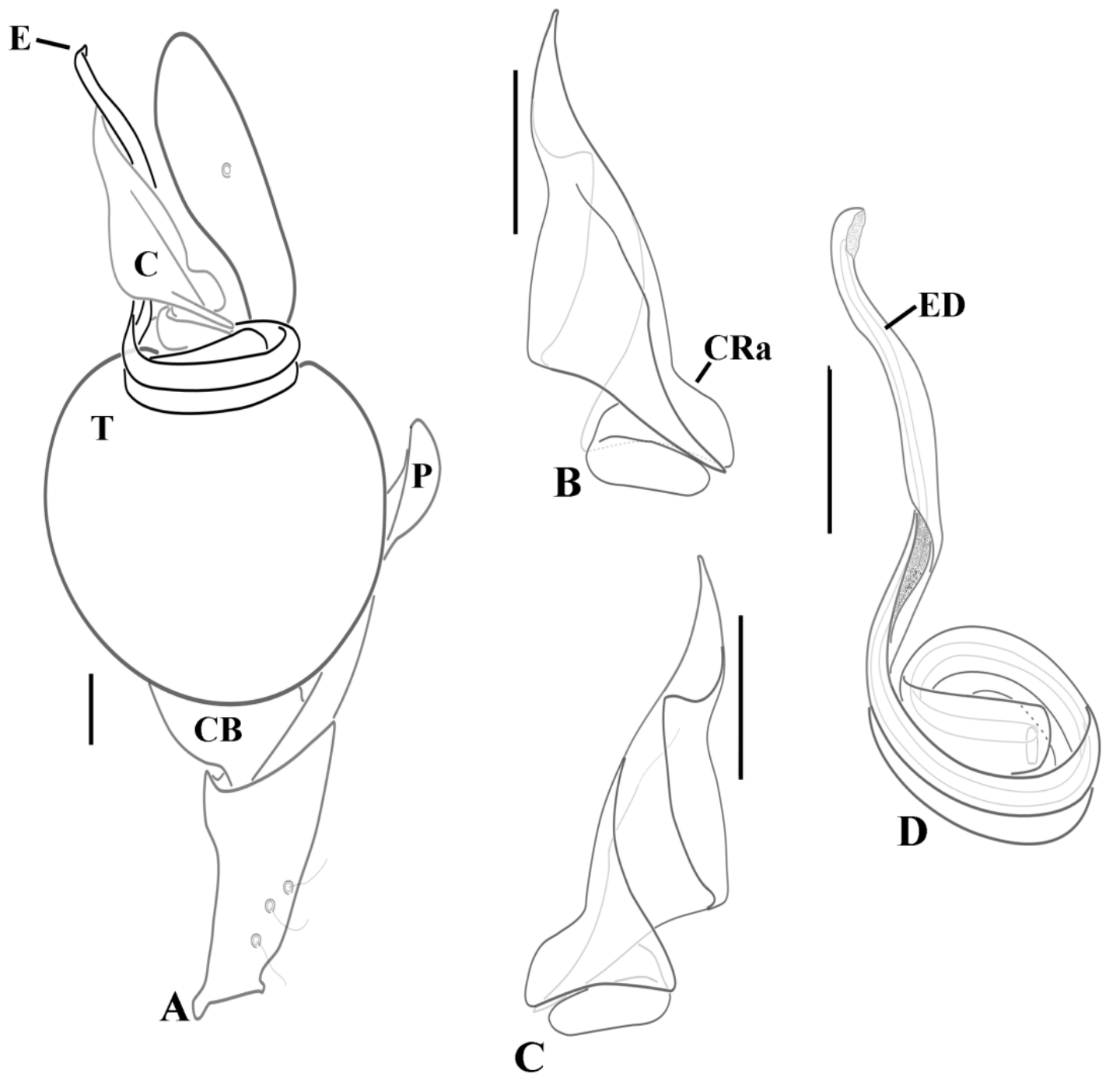

Figure 294. Glenognatha emertoni from USA (AMNH). Male left palp, schematic. A, ventral. B, conductor ventral. C, conductor dorsal. D, embolus ventral. Scale bars 100 $\mu \mathrm{m}$. C: conductor. CB: cymbium. CRa: conductor retrolateral apophysis. E: embolus. ED: ejaculatory duct. P: paracymbium. T: tegulum. 

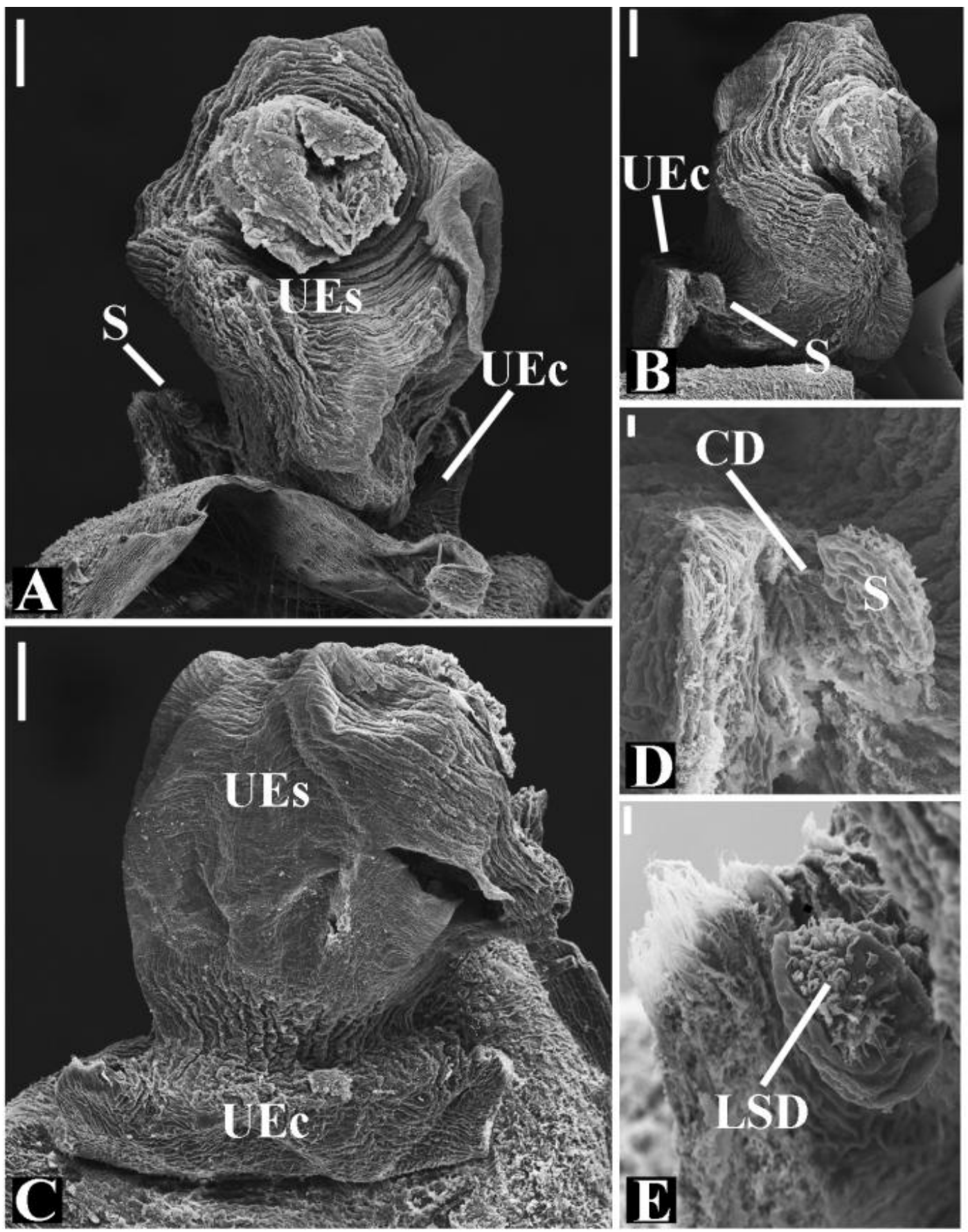

Figure 295. Glenognatha emertoni from USA. Female genitalia. A, dorsal. B, lateral. C, ventral. D-E, spermathecae and copulatory duct. Scale bars, $100 \mu \mathrm{m}$ (A-C), $10 \mu \mathrm{m}$ (D,E). CD: copulatory duct. LSD: long-stem gland ducts. UEc: uterus externus chamber. UEs: uterus externus sac. S: spermathecae. 


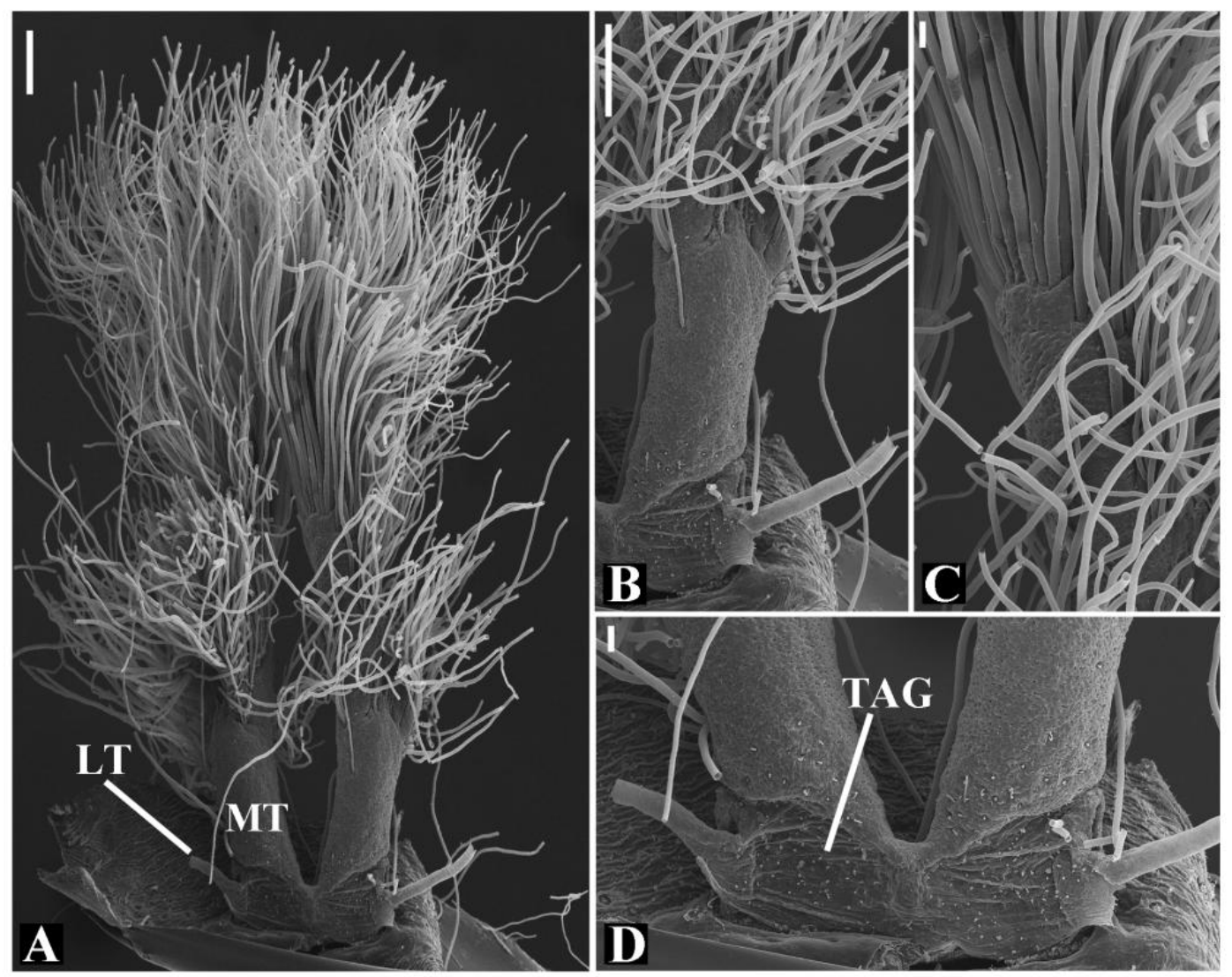

Figure 296. Glenognatha emertoni from USA. Female tracheal system. A, dorsal. B, median and lateral trunks. C, median trunk distal. D, tracheal spiracle posterior view. Scale bars, $100 \mu \mathrm{m}$ (A, B), $20 \mu \mathrm{m}$ (C, D). LT: lateral tracheae. MT: median trunks. TAG: tracheal atrium gland. 

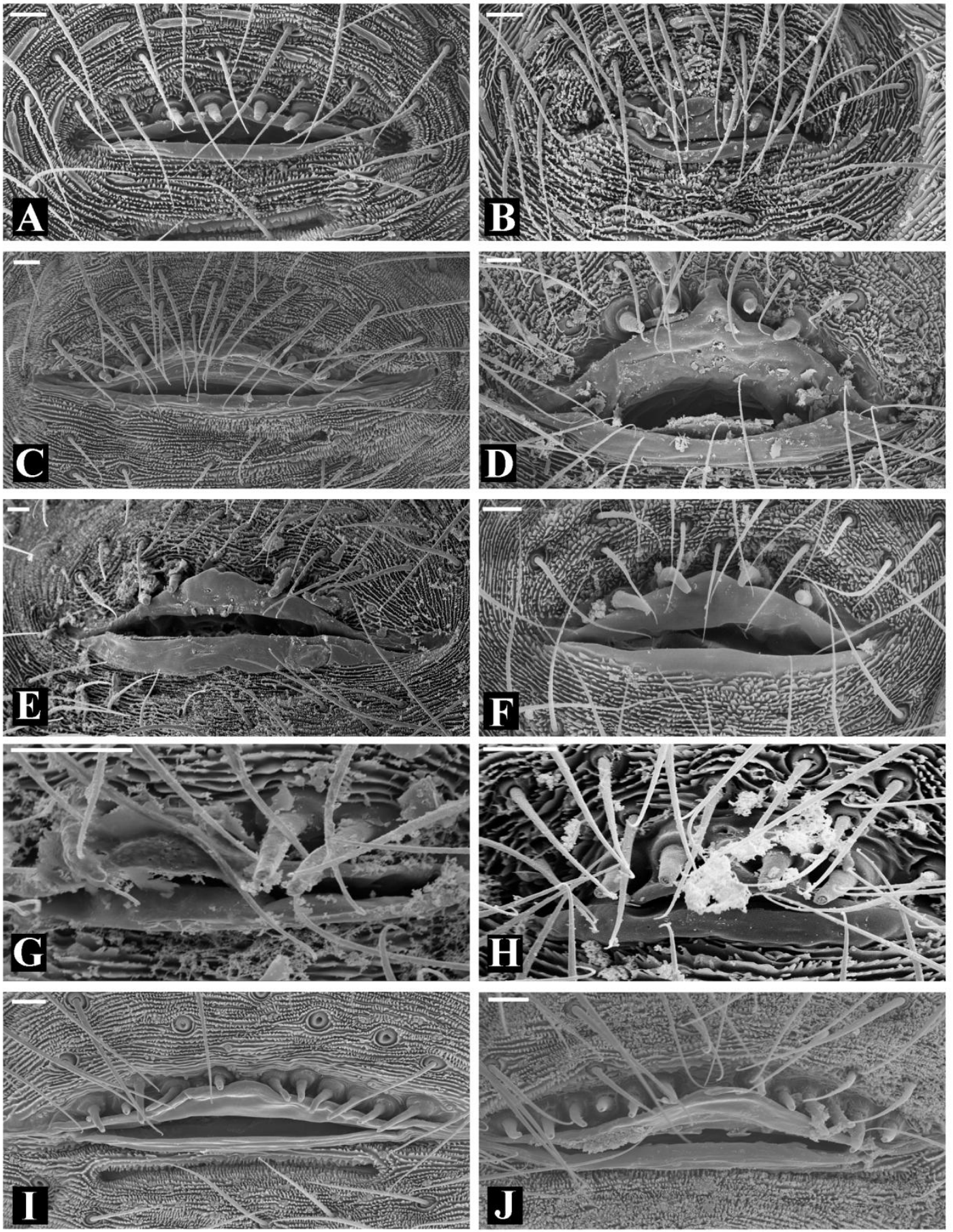

Figure 297. Epiandrous fusules. A, Glenognatha dentata. B, Glenognatha tangi. C, Glenognatha australis. D, Glenognatha gaujoni. E, Glenognatha globosa. F, Glenognatha sp. nov. 2. G, Glenognatha sp. nov. 3. H, Glenognatha sp. nov. 10. I, Glenognatha lacteovittata. J, Glenognatha sp. nov. 5. Scale bars $10 \mu \mathrm{m}$. 

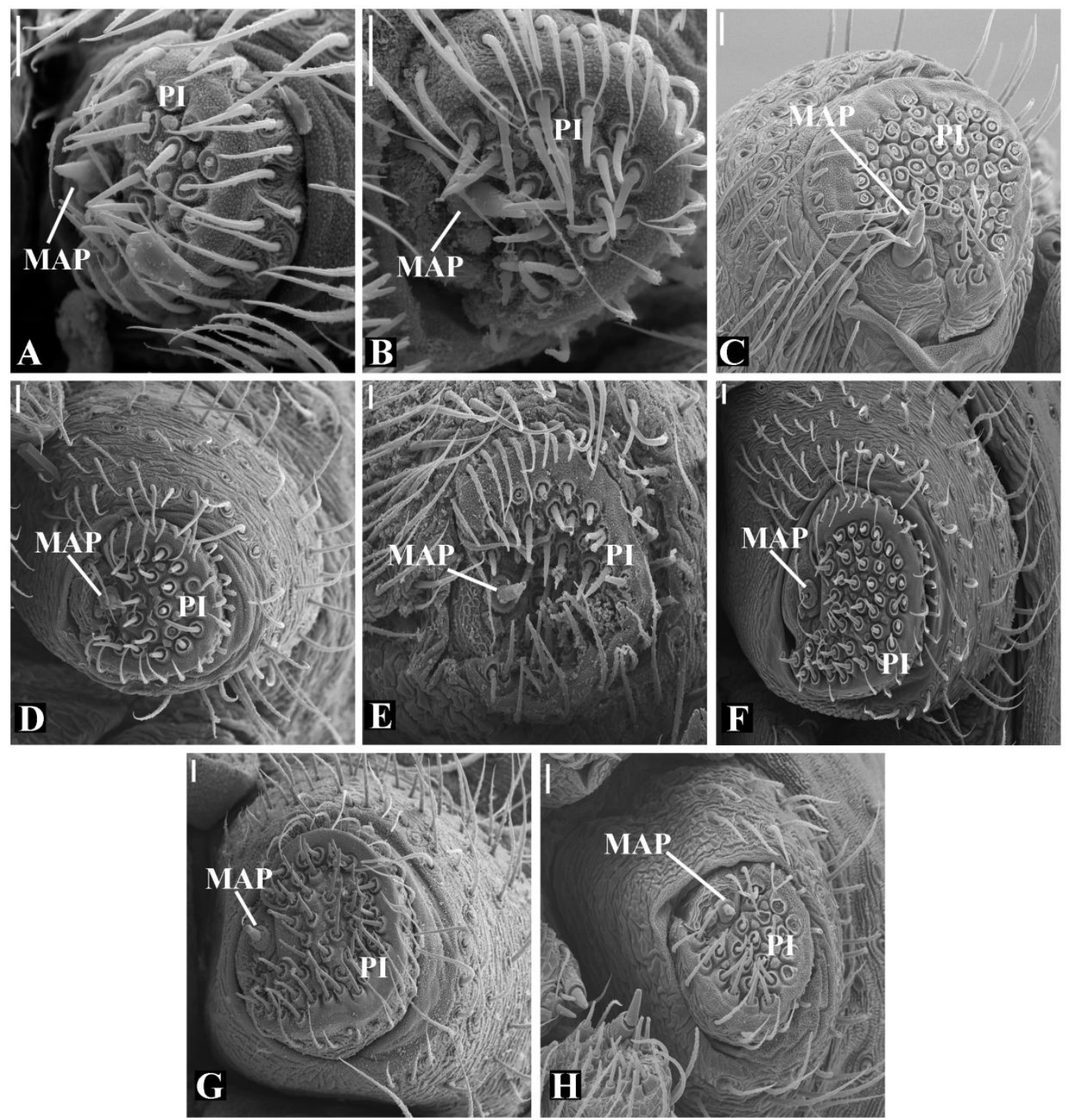

Figure 298. Female anterior lateral spinnerets. A, Glenognatha sp. nov. 1. B, Glenognatha sp. nov. 2. C, Glenognatha sp. nov. 7. D, Glenognatha australis. E, Glenognatha emertoni. F, Glenognatha gaujoni. G, Glenognatha globosa. H, Glenognatha lacteovittata. Scale bars $10 \mu \mathrm{m}$. 

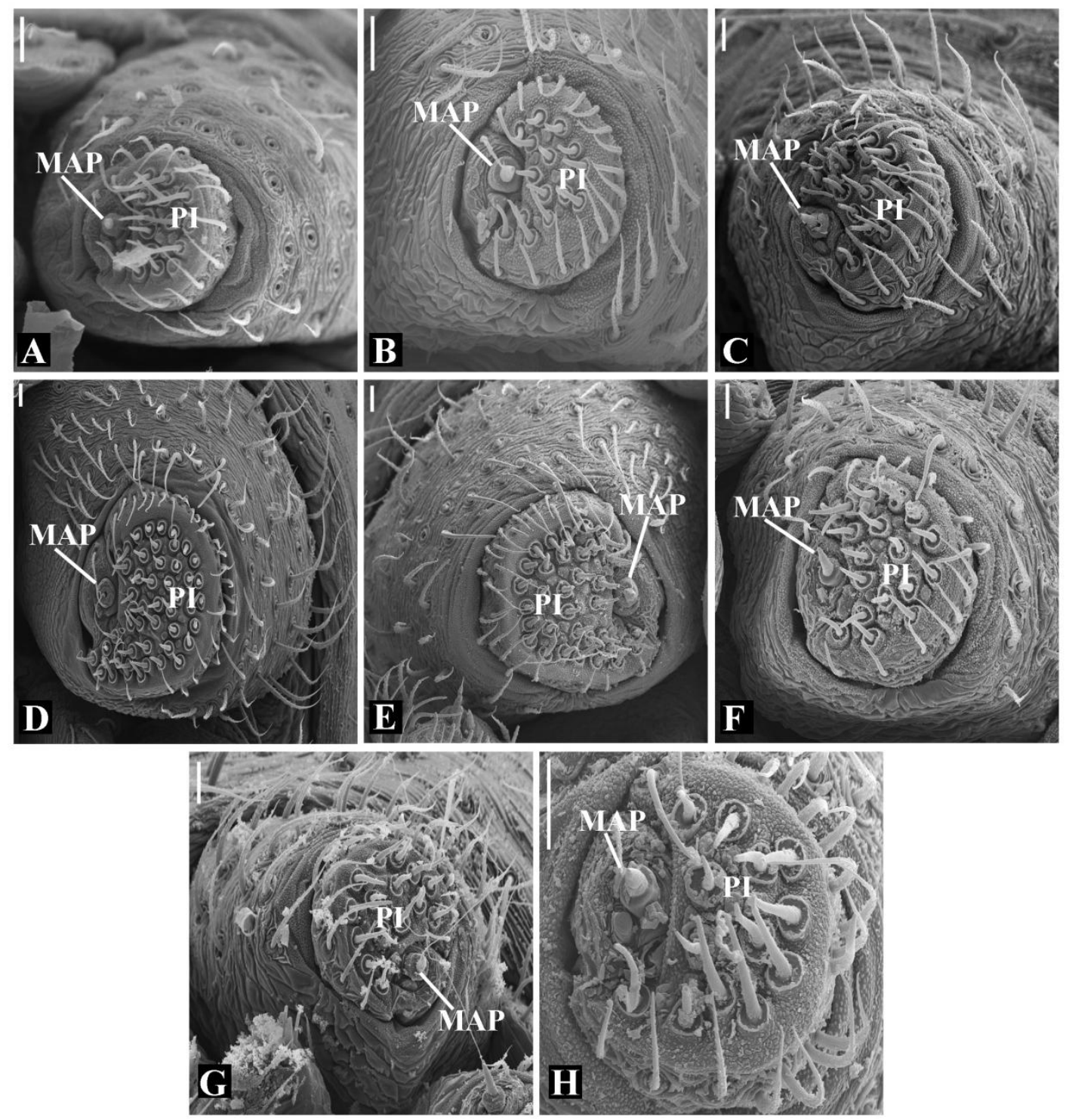

Figure 299. Male anterior lateral spinnerets. A, Glenognatha sp. nov. 1 B, Glenognatha sp. nov. 2. C, Glenognatha australis. D, Glenognatha gaujoni. E, Glenognatha globosa. F, Glenognatha lacteovittata. G, Glenognatha dentata. H, Glenognatha tangi. Scale bars $10 \mu \mathrm{m}$. 

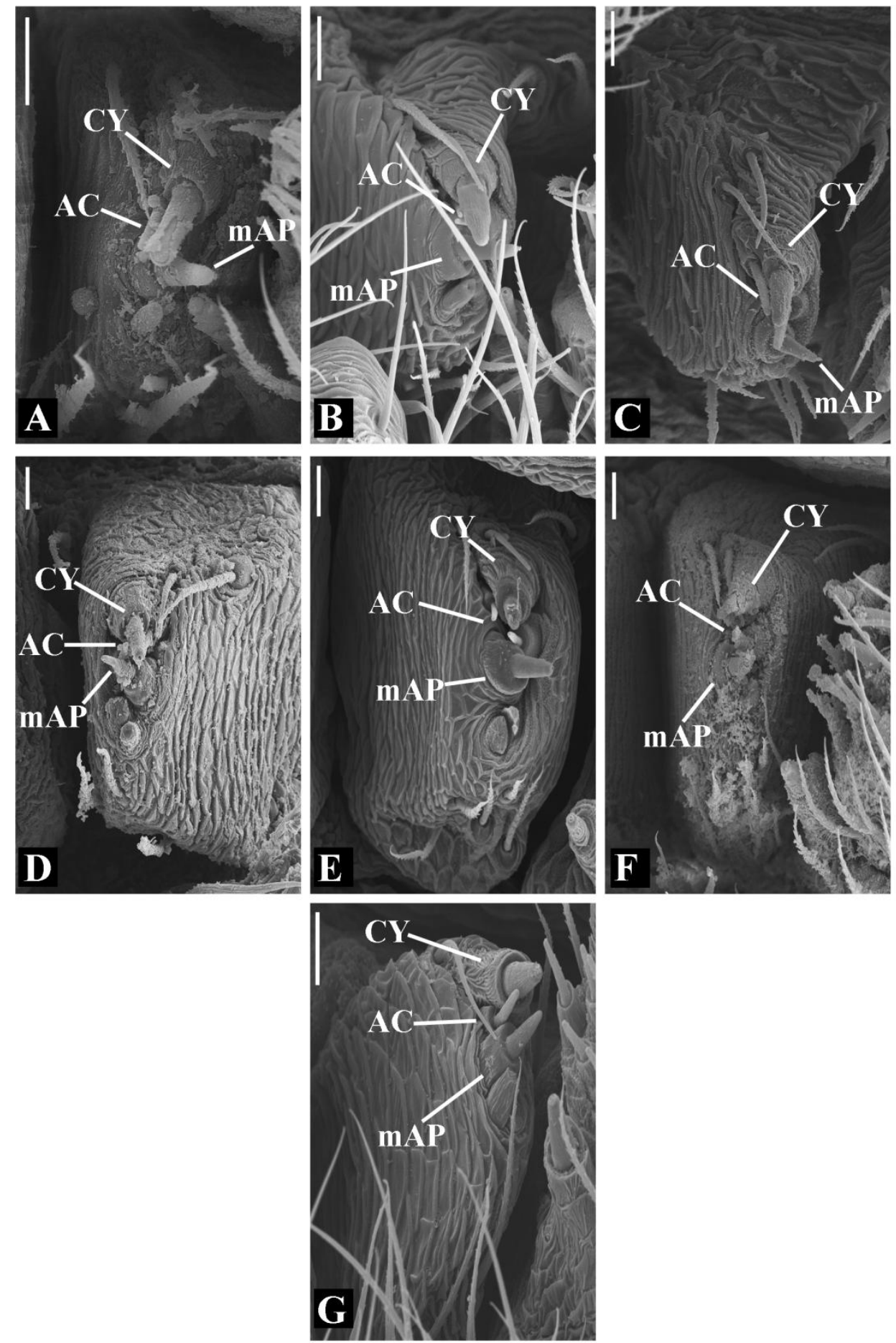

Figure 300. Female posterior median spinnerets. A, Glenognatha sp. nov. 2. B, Glenognatha sp. nov. 7. C, Glenognatha australis. D, Glenognatha emertoni. D, Glenognatha gaujoni. E, Glenognatha globosa. F, Glenognatha lacteovittata. Scale bars $10 \mu \mathrm{m}$. 

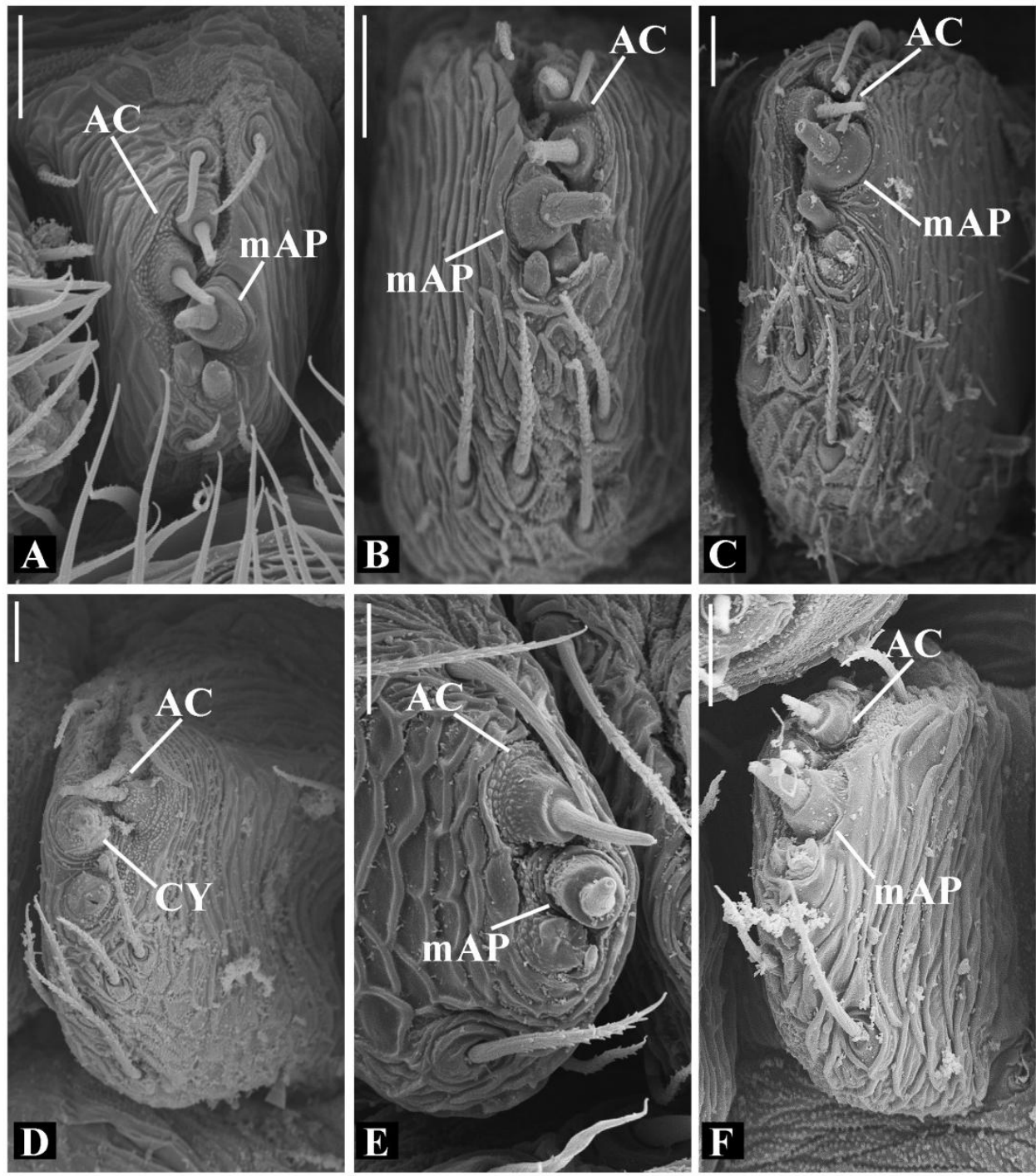

Figure 301. Male posterior median spinnerets. A, Glenognatha sp. nov. 2. B, Glenognatha australis. C, Glenognatha gaujoni. D, Glenognatha globosa. E, Glenognatha lacteovittata. F, Glenognatha tangi. Scale bars $10 \mu \mathrm{m}$. 

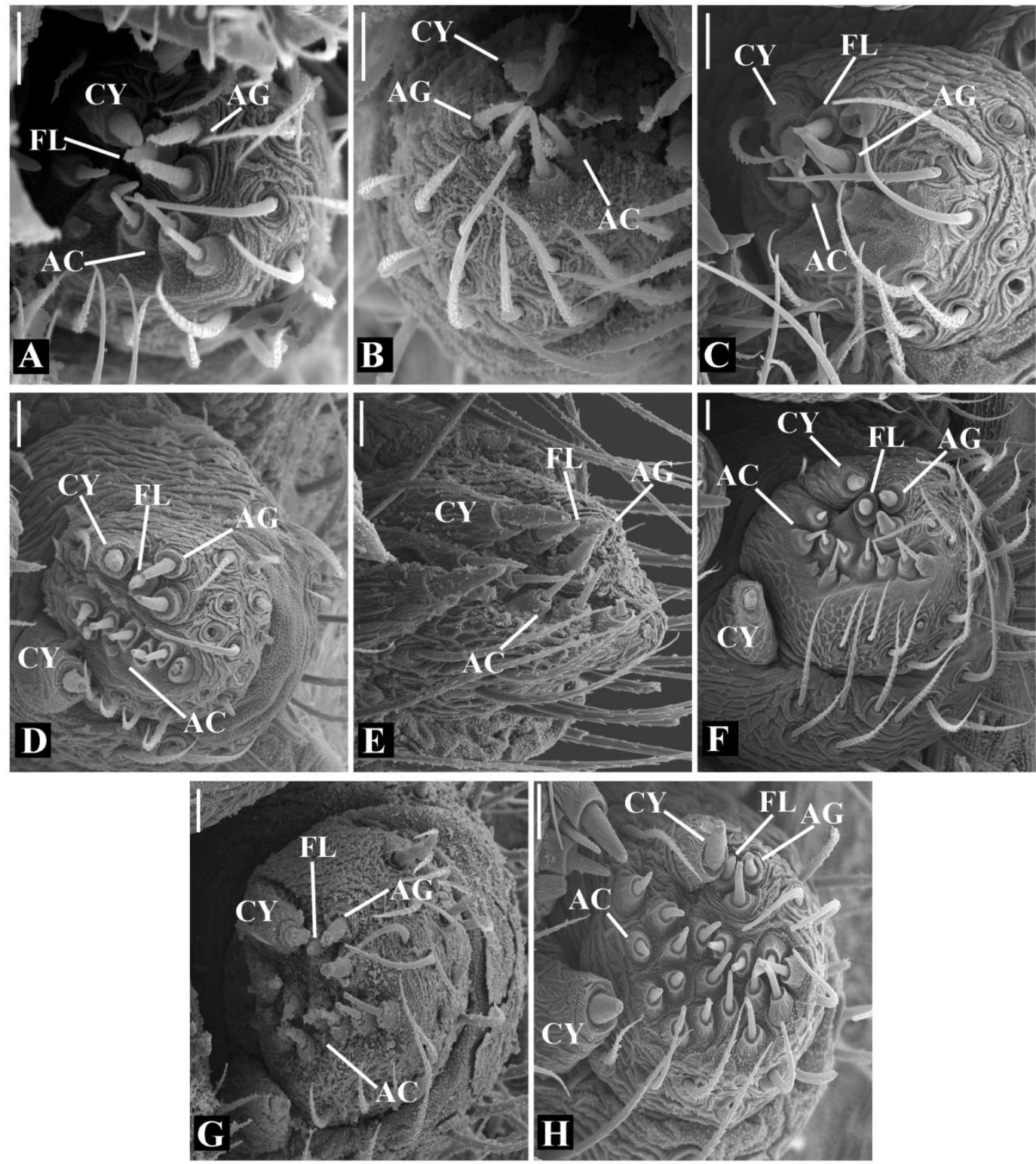

Figure 302. Female posterior lateral spinnerets. A, Glenognatha sp. nov. 1. B, Glenognatha sp. nov. 2. C, Glenognatha sp. nov. 7. D, Glenognatha australis. E, Glenognatha emertoni. F, Glenognatha gaujoni. G, Glenognatha globosa. H, Glenognatha lacteovittata. Scale bars $10 \mu \mathrm{m}$. 

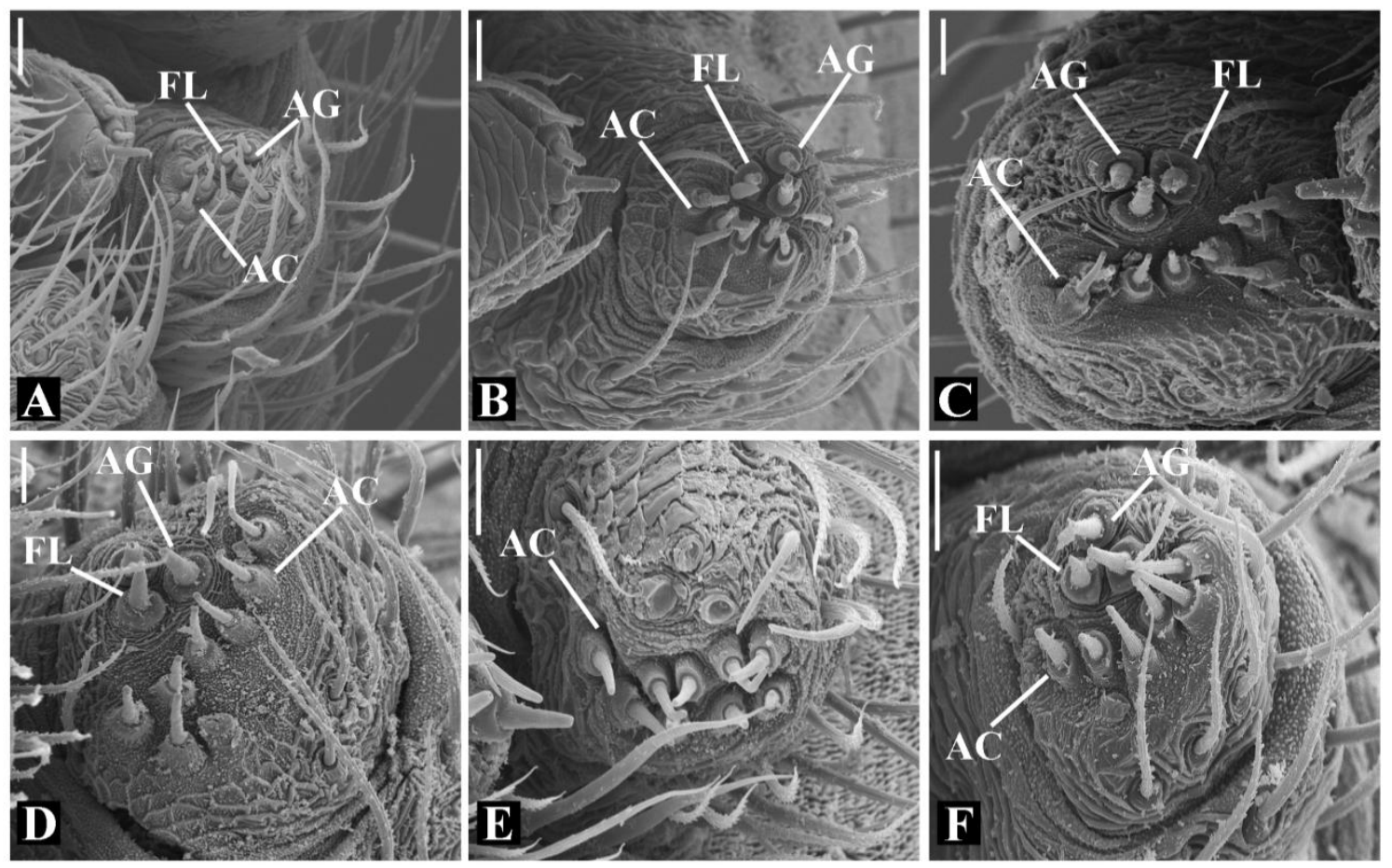

Figure 303. Male posterior lateral spinnerets. A, Glenognatha sp. nov. 2. B, Glenognatha australis. C, Glenognatha gaujoni. D, Glenognatha globosa. E, Glenognatha lacteovittata. F, Glenognatha tangi. Scale bars $10 \mu \mathrm{m}$. 


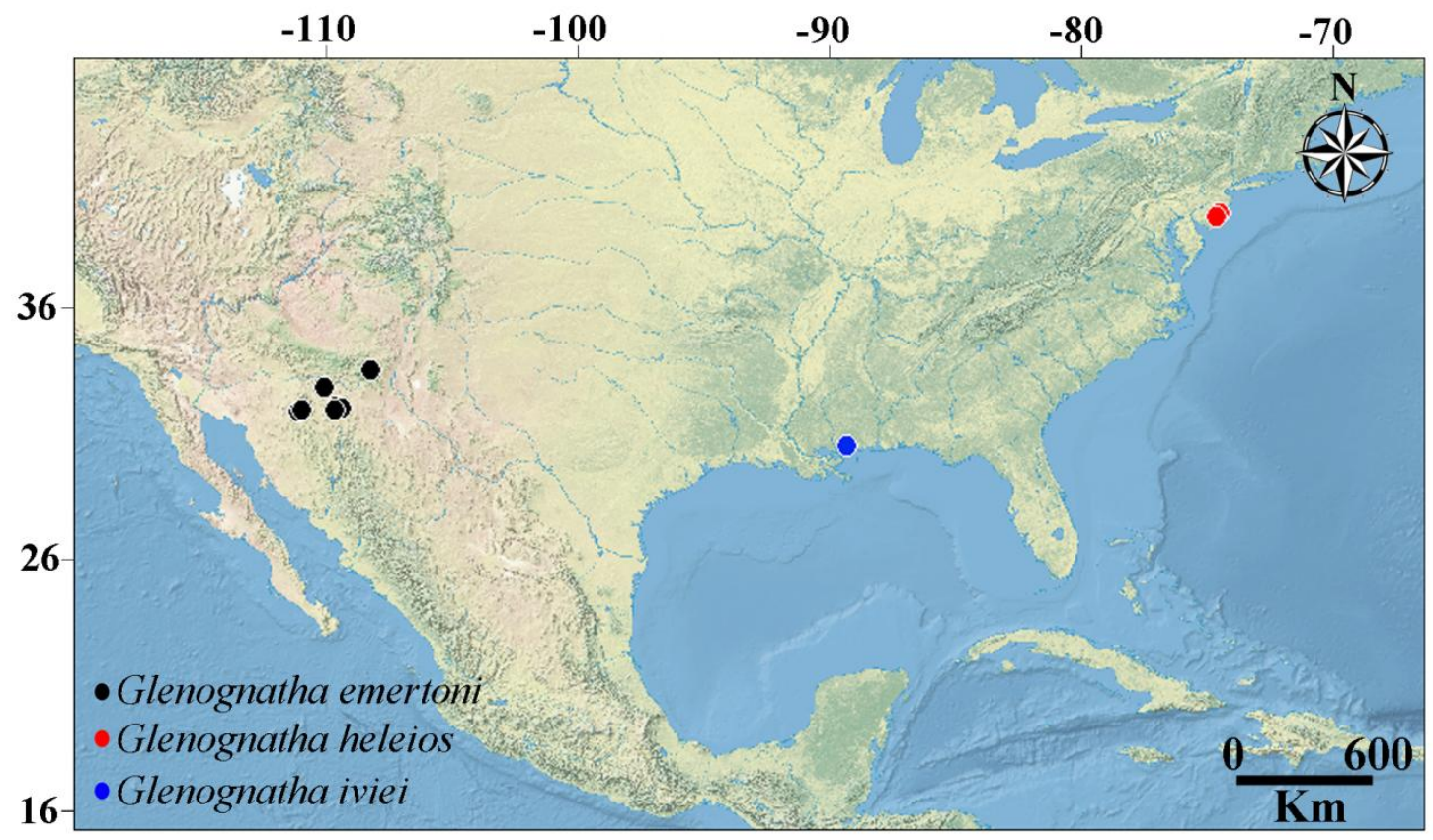

Figure 304. Distribution of G. emertoni, G. heleios and G. iviei.

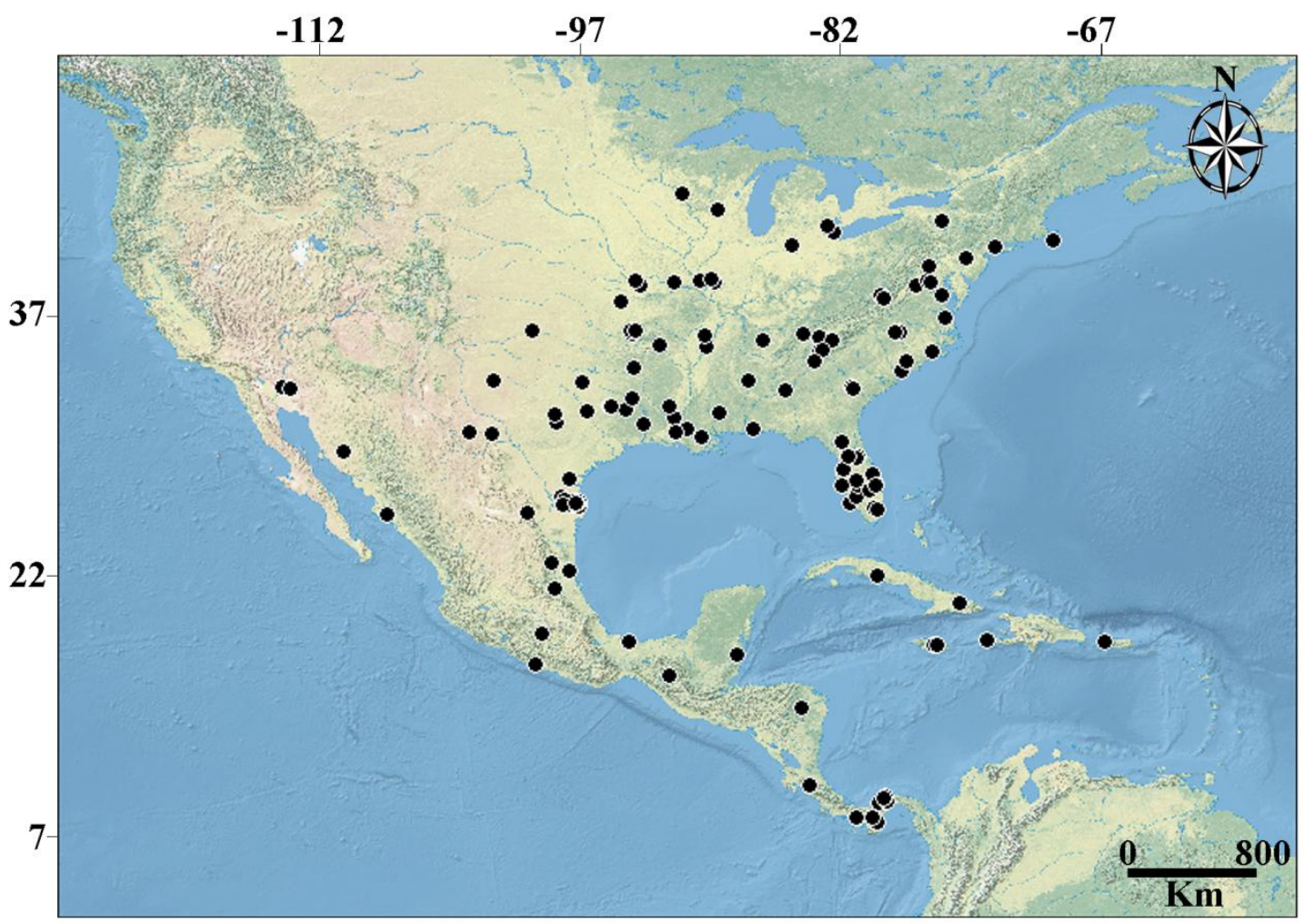

Figure 305. Distribution of G. foxi. 


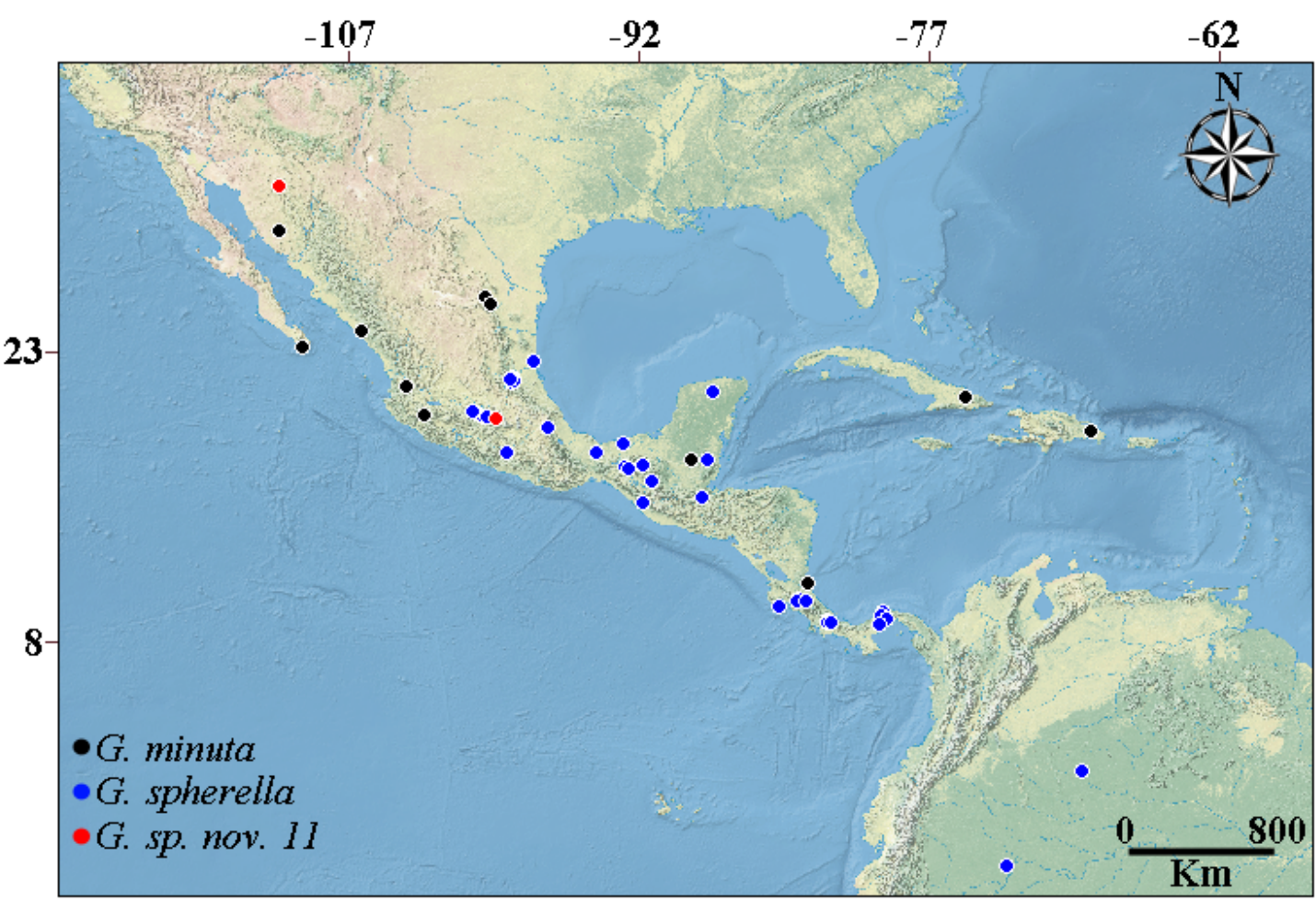

Figure 306. Distribution of G. minuta, G. spherella and G. sp. nov. 11.

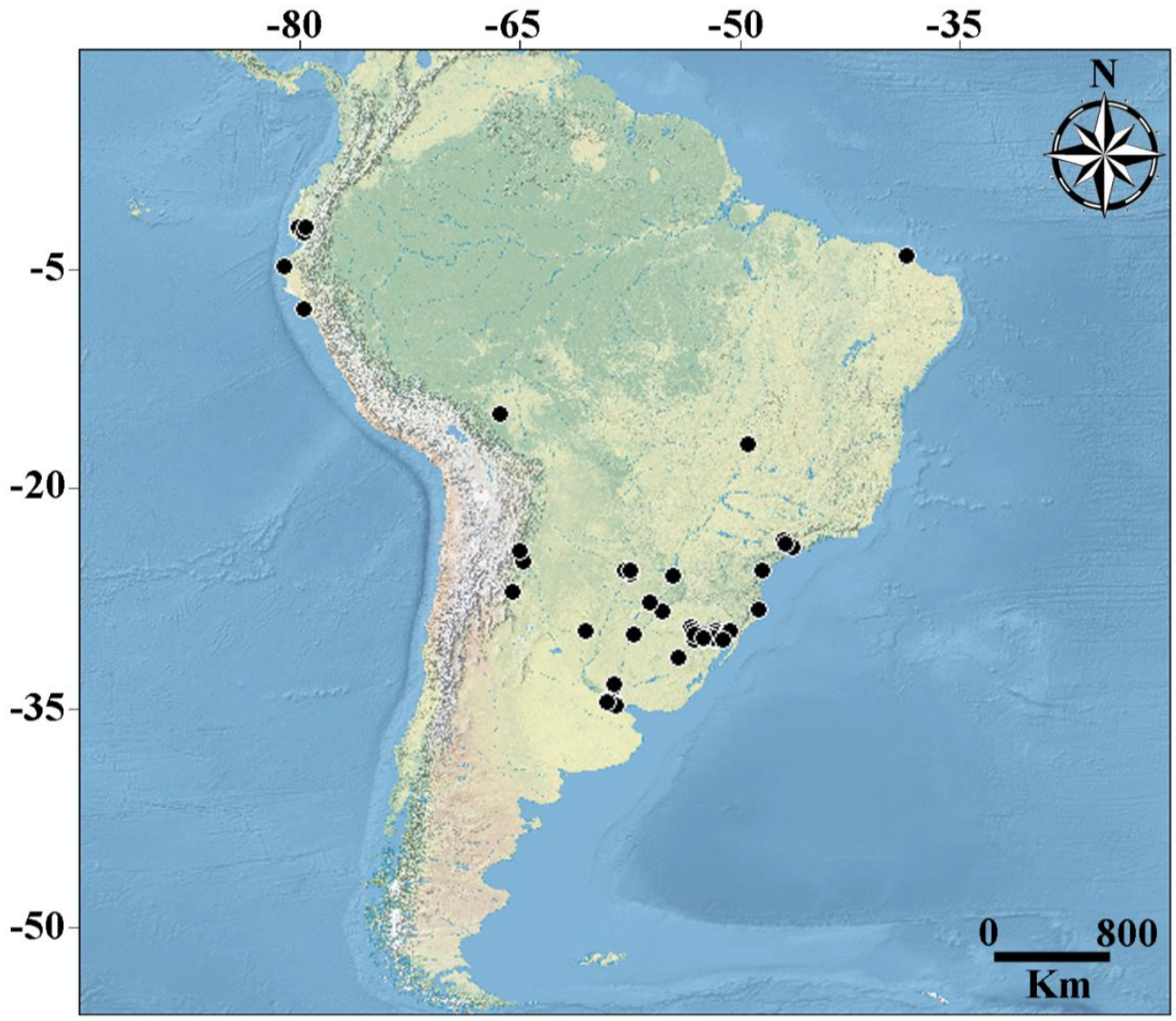

Figure 307. Distribution of G. australis. 


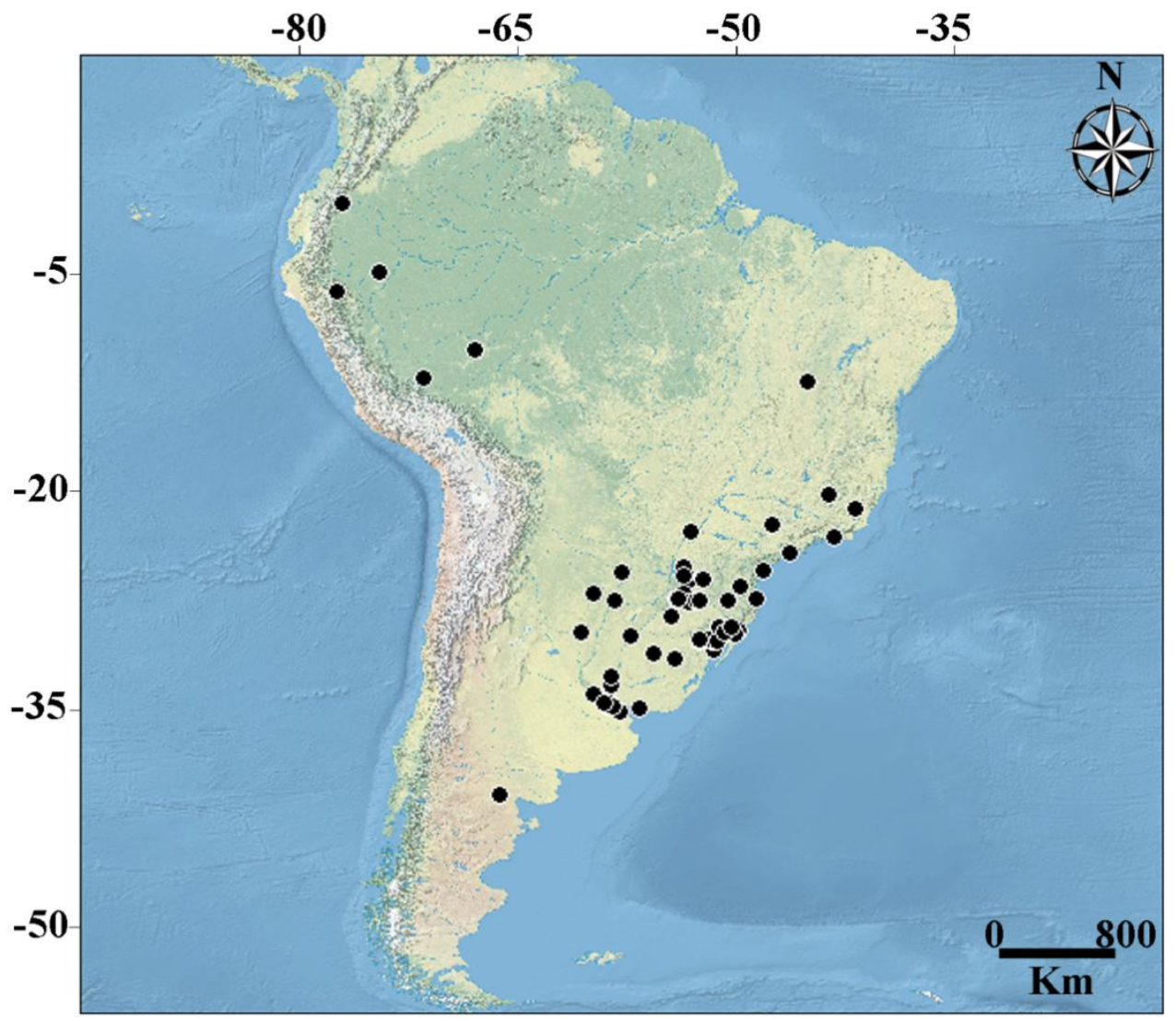

Figure 308. Distribution of G. lacteovittata.

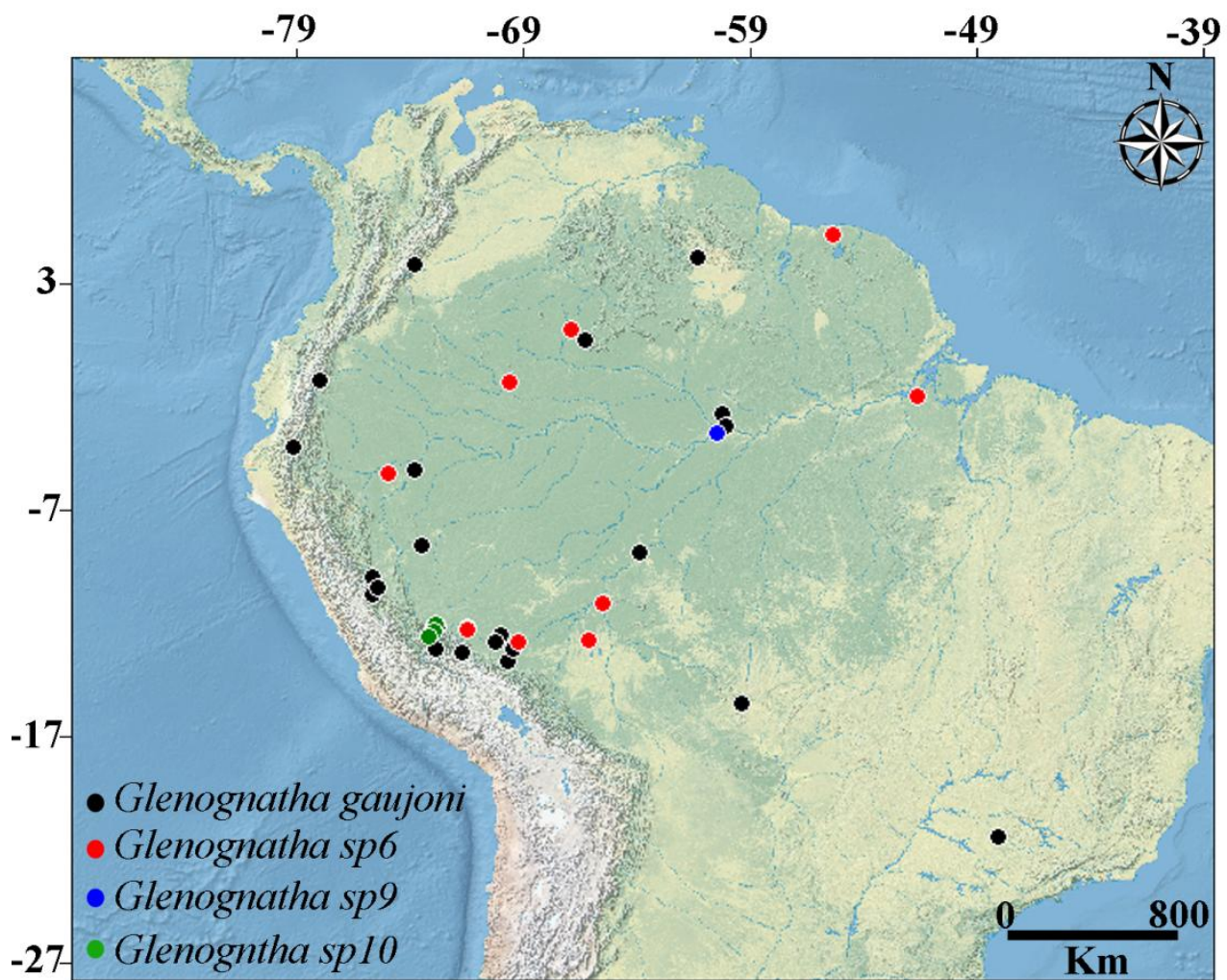

Figure 309. Distribution of G. gaujoni, G. sp. nov. 6, G. sp. nov. 9 and G. sp. nov. 10. 


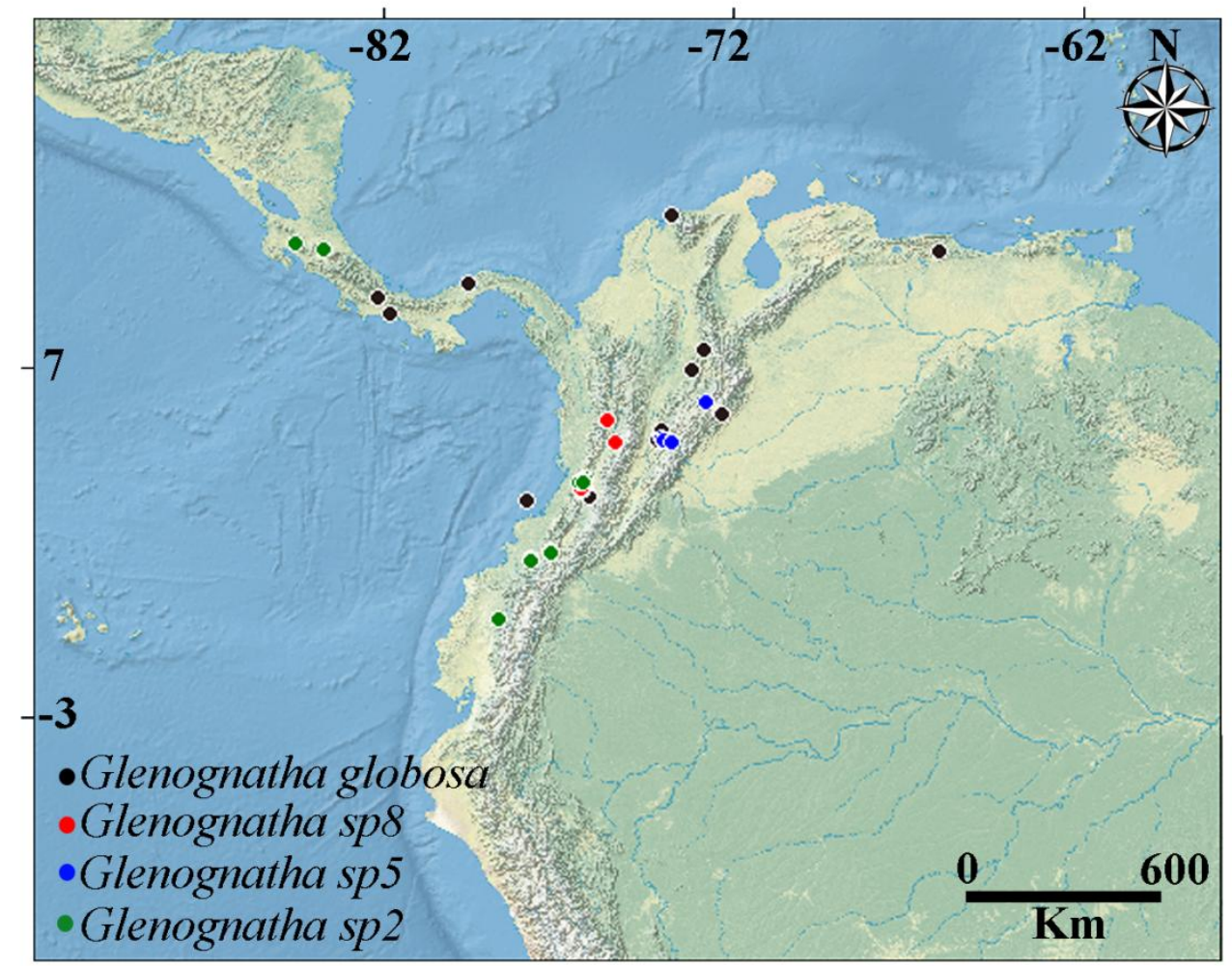

Figure 310. Distribution of G. globosa, G. sp. nov. 8, G. sp. nov. 5 and G. sp. nov. 2.

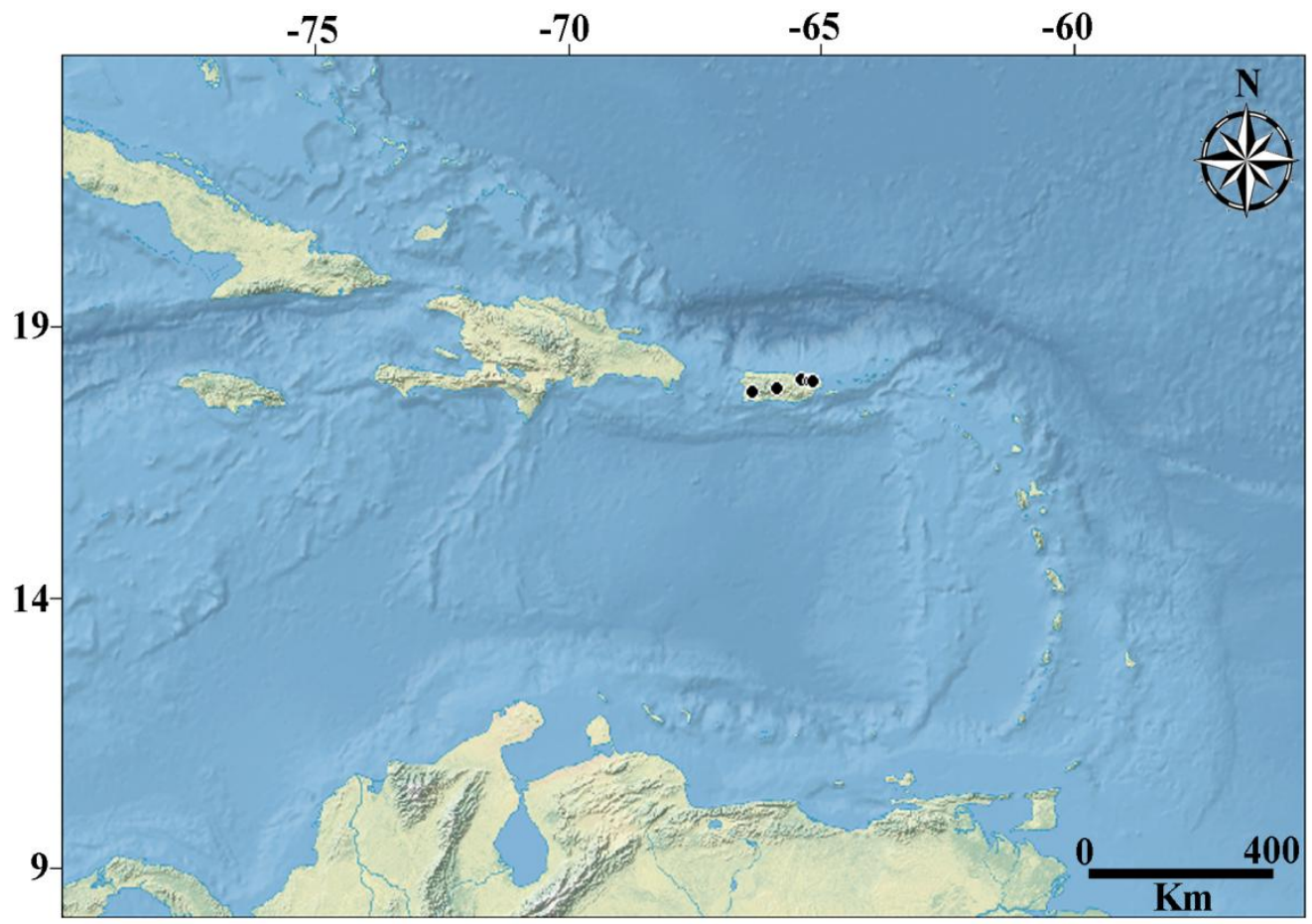

Figure 311. Distribution of G. gloriae. 


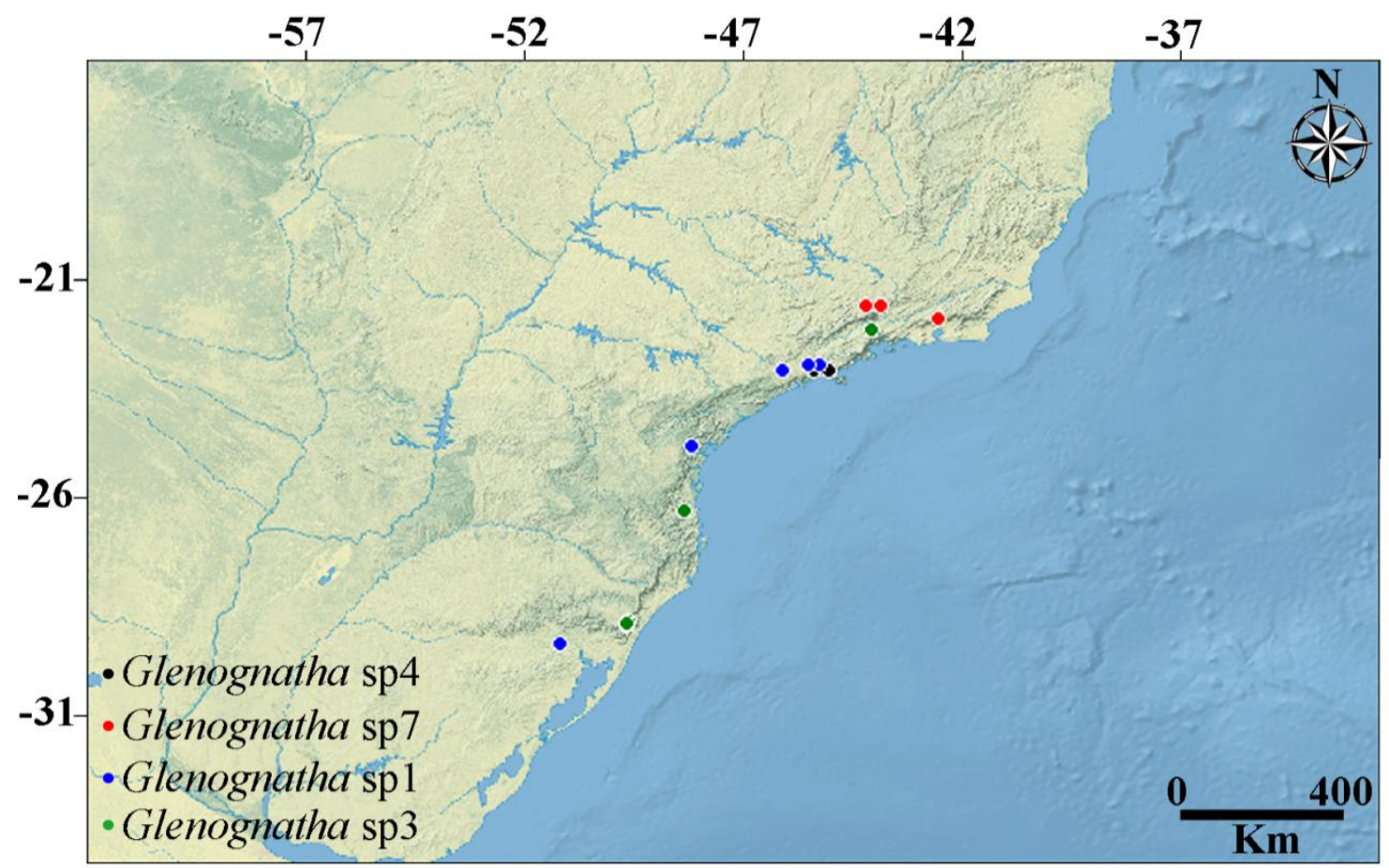

Figure 312. Distribution of G. sp. nov. 4, G. sp. nov. 7, G. sp. nov. 1 and G. sp. nov. 3.

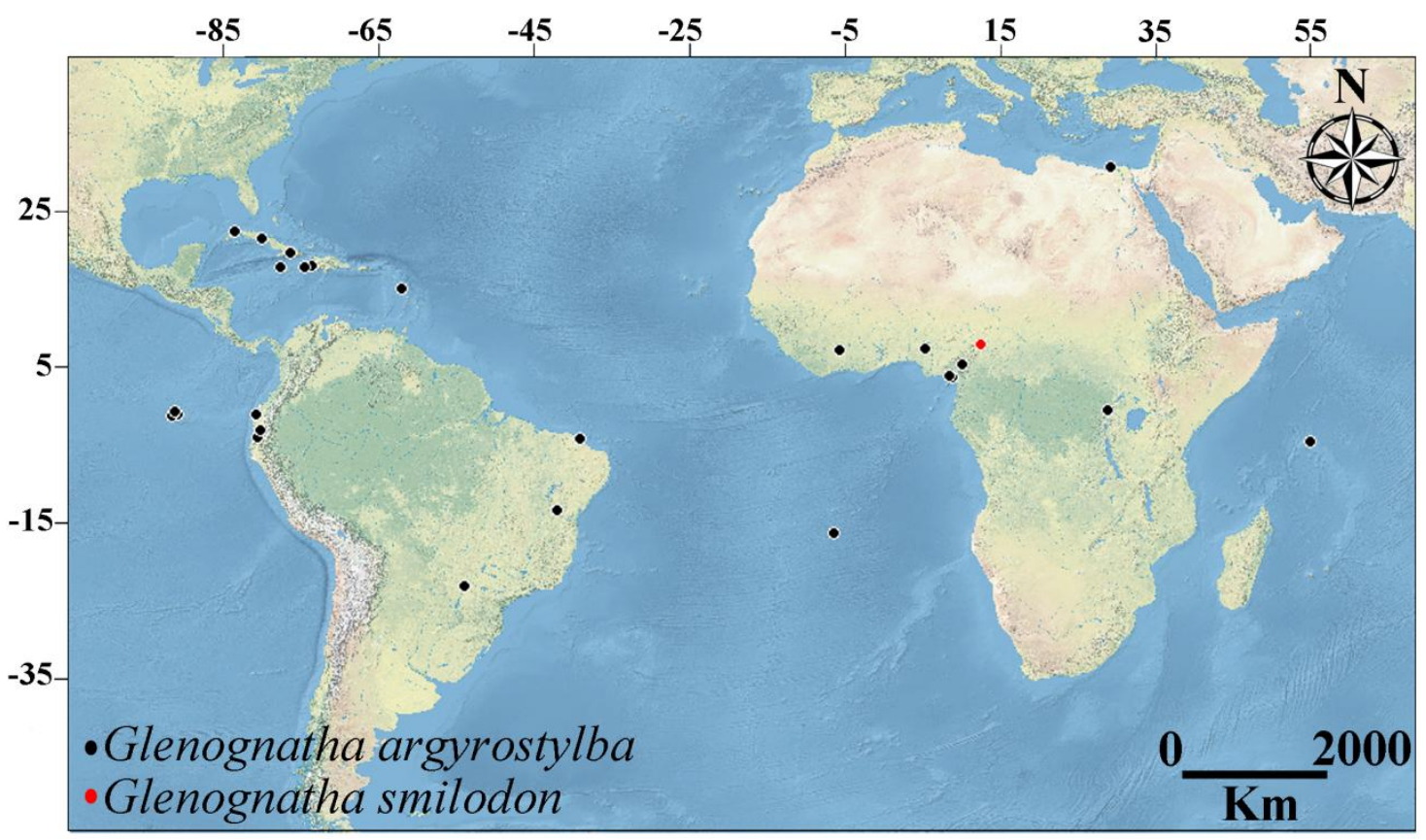

Figure 313. Distribution of G. argyrostilba and G. smilodon. 


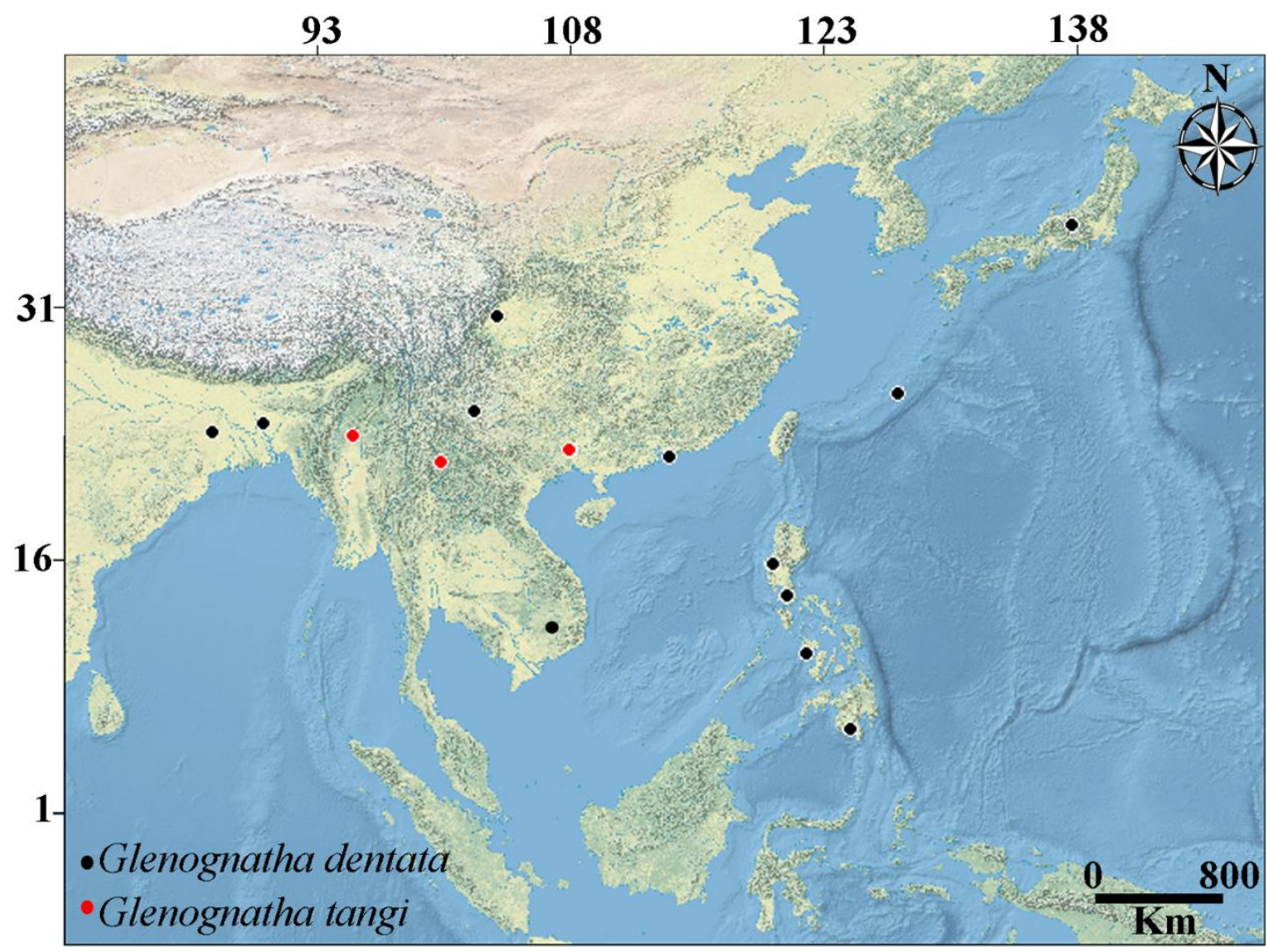

Figure 314. Distribution of $G$. dentata and G. tangi.

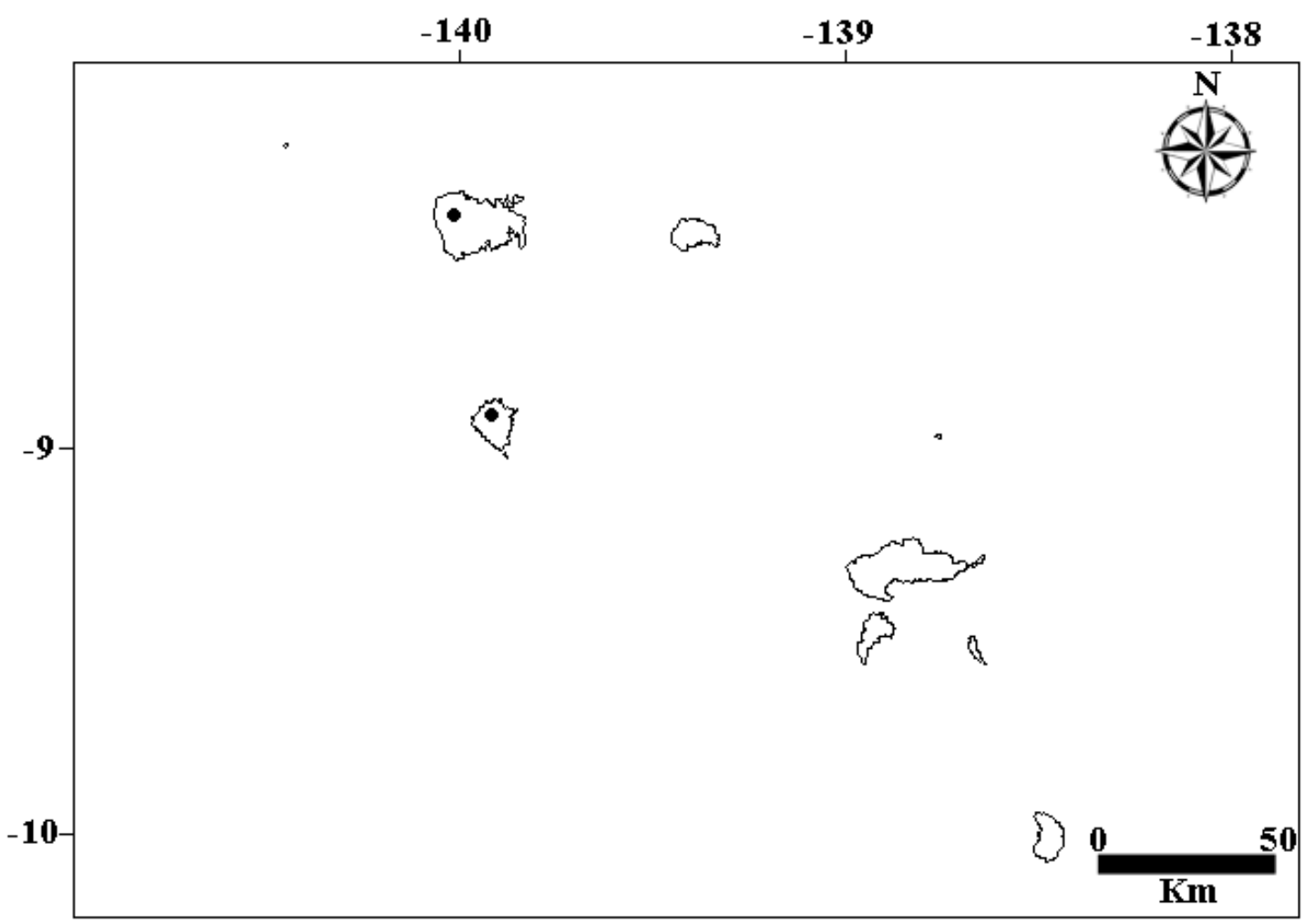

Figure 315. Distribution of G. hirsutissima. 